\title{
Rh-Based Mixed Alcohol Synthesis Catalysts: Characterization and Computational Report
}

$\begin{array}{ll}\text { KO Albrecht } & \text { V-A Glezakou } \\ \text { R Rousseau } & \text { MH Engelhard } \\ \text { T Varga } & \text { RJ Colby } \\ \text { JE Jaffe } & \text { X Li } \\ \text { D Mei } & \text { CF Windisch } \\ \text { SM Kathmann } & \text { T Lemmon } \\ \text { MJ Gray } & \text { TR Hart } \\ \text { BL Thompson } & \text { MA Gerber }\end{array}$

August 2013

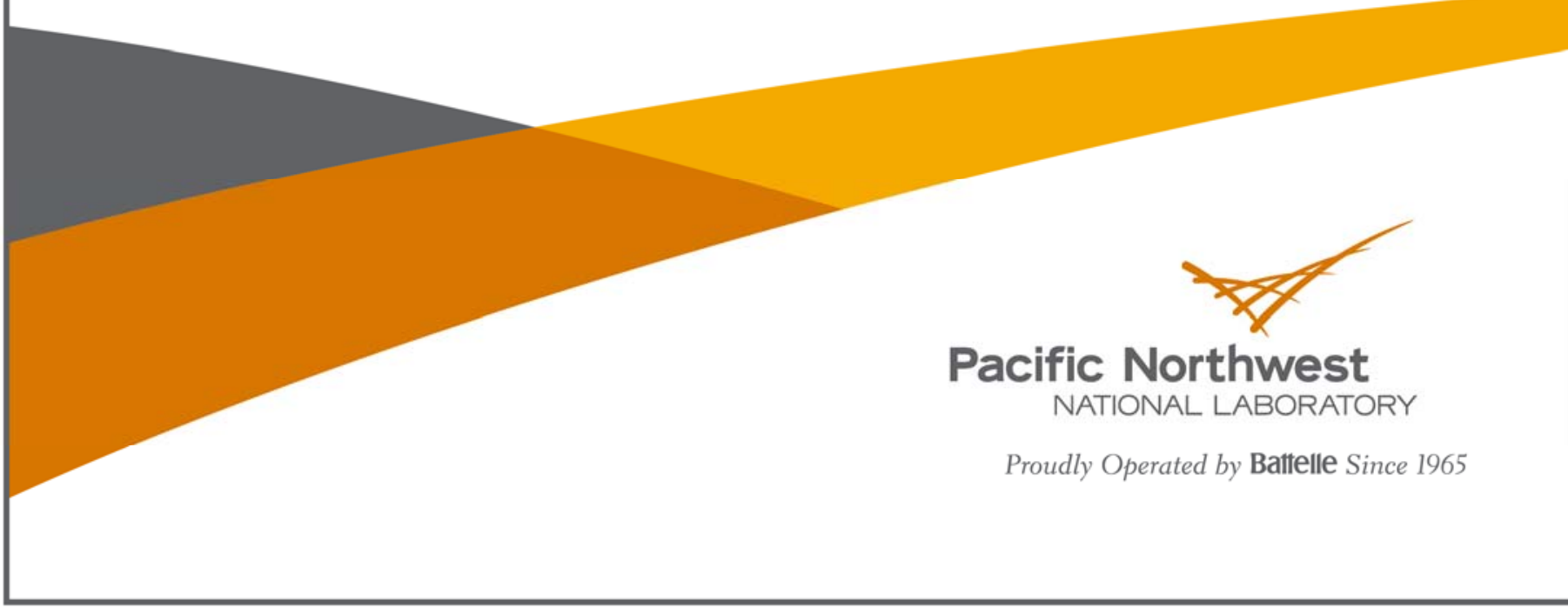




\title{
DISCLAIMER
}

This report was prepared as an account of work sponsored by an agency of the United States Government. Neither the United States Government nor any agency thereof, nor Battelle Memorial Institute, nor any of their employees, makes any warranty, express or implied, or assumes any legal liability or responsibility for the accuracy, completeness, or usefulness of any information, apparatus, product, or process disclosed, or represents that its use would not infringe privately owned rights. Reference herein to any specific commercial product, process, or service by trade name, trademark, manufacturer, or otherwise does not necessarily constitute or imply its endorsement, recommendation, or favoring by the United States Government or any agency thereof, or Battelle Memorial Institute. The views and opinions of authors expressed herein do not necessarily state or reflect those of the United States Government or any agency thereof.

\author{
PACIFIC NORTHWEST NATIONAL LABORATORY \\ operated by \\ BATTELLE \\ for the \\ UNITED STATES DEPARTMENT OF ENERGY \\ under Contract DE-AC05-76RL01830
}

Printed in the United States of America

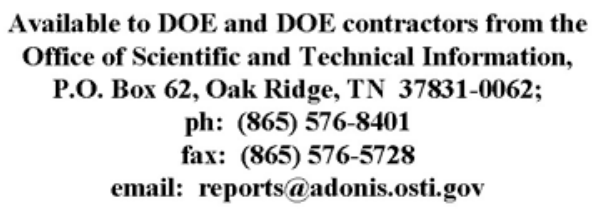

Available to the public from the National Technical Information Service 5301 Shawnee Rd., Alexandria, VA 22312 ph: (800) 553-NTIS (6847) email: orders@ntis.gov $<$ http://www.ntis.gov/about/form.aspx > Online ordering: http://www.ntis.gov 


\section{Rh-Based Mixed Alcohol Synthesis Catalysts: Characterization and Computational Report}

$\begin{array}{ll}\text { KO Albrecht } & \text { V-A Glezakou } \\ \text { R Rousseau } & \text { MH Engelhard } \\ \text { T Varga } & \text { RJ Colby } \\ \text { JE Jaffe } & \text { X Li } \\ \text { D Mei } & \text { CF Windisch } \\ \text { SM Kathmann } & \text { T Lemmon } \\ \text { MJ Gray } & \text { TR Hart } \\ \text { BL Thompson } & \text { MA Gerber }\end{array}$

August 2013

Prepared for

the U.S. Department of Energy

under Contract DE-AC05-76RL01830

Pacific Northwest National Laboratory

Richland, Washington 99352 



\section{Executive Summary}

The U.S. Department of Energy is conducting a program focused on developing a process for the conversion of biomass to bio-based fuels and co-products. The lignocellulosic biomass feedstock is first gasified to produce a product known as synthesis gas (syngas), which consists of $\mathrm{H}_{2}$ and CO. The syngas stream is subsequently converted thermochemically within a temperature range of 240 to $330^{\circ} \mathrm{C}$ and at elevated pressure (e.g., 1200 psig) over a catalyst. Ethanol is the desired reaction product, although other side compounds are produced, including $\mathrm{C}_{3}$ to $\mathrm{C}_{5}$ alcohols; higher (i.e., greater than $\mathrm{C}_{1}$ ) oxygenates such as methyl acetate, ethyl acetate, acetic acid and acetaldehyde; and higher hydrocarbon gases such as methane, ethane/ethene, propane/propene, etc. Saturated hydrocarbon gases (especially methane) are undesirable because they represent a diminished yield of carbon to the desired ethanol product and represent compounds that must be steam reformed at high energy cost to reproduce $\mathrm{CO}$ and $\mathrm{H}_{2}$. Ethanol produced by the thermochemical reaction of syngas could be separated and blended directly with gasoline to produce a liquid transportation fuel. Additionally, higher oxygenates and unsaturated hydrocarbon side products such as olefins also could be further processed to liquid fuels.

The goal of the current project is the development of an Rh-based catalyst with high activity and selectivity to $\mathrm{C}_{2}+$ oxygenates. Since the program began in 2005, significant improvements in activity and selectivity to the Rh-based catalyst have been achieved through empirical catalyst screening (e.g., support and promoter screening) and operational parametric screening. This report chronicles an effort to characterize numerous supports and catalysts to identify particular traits that could be correlated with the most active and/or selective catalysts. Characterization techniques were chosen to identify the properties of a variety of supports and catalysts. Carbon and silica were the general classes of supports analyzed. Analysis procedures used for several supports are identified below:

- Ash concentration of the carbon supports

- Elemental composition via inductively coupled plasma

- Surface area (Brunauer-Emmett-Teller) and pore size distribution (Barrett-Joyner-Halenda) via $\mathrm{N}_{2}$ adsorption and desorption

- Surface acidity measurements of the silica supports via $\mathrm{NH}_{3}$ desorption

- Carbon support surface functionality characterization via thermogravimetric analysis with mass spectrometer analysis of the effluent gas

- Diffuse reflectance infrared Fourier transform spectroscopy analysis of selected carbon supports to identify surface functionality.

Generally, analyses provided guidance in the selection of acceptable catalyst supports. For example, supports with high surface areas due to a high number of micropores were generally found to be poor at producing oxygenates, possibly because of mass transfer limitations of the products formed out of the micropores.

A number of prepared catalysts (i.e., with Rh and other metal promoters present) also were investigated with techniques such as X-ray photoelectron spectroscopy to identify the concentration of elements at the catalyst surface and the relative oxidation states; transmission electron microscopy to investigate metal particle size; $x$-ray diffraction to probe crystalline phases present in the catalysts; and 
$\mathrm{H}_{2}$ adsorption/desorption to measure the capacity of the catalysts to adsorb $\mathrm{H}_{2}$. Three select catalyst formulations were prepared and characterized at various points in the catalyst life cycle: as prepared, upon reduction, and after utilization in a reactor at low $\left(\sim 275^{\circ} \mathrm{C}\right)$ and high $\left(\sim 300^{\circ} \mathrm{C}\right)$ temperatures. General characteristics of catalysts presumably related to the activity of the catalysts were observed. For example, with Ir-promotion, the concentration of $\mathrm{Mn}$ at the surface of the Rh-Mn-Ir catalysts increased relative to the concentration of the Rh.

To probe fundamental aspects of the complicated reaction network of $\mathrm{CO}$ with $\mathrm{H}_{2}$, a computational/ theoretical investigation using quantum mechanical and $a b$ initio molecular dynamics calculations was initiated in 2009. Computational investigations were performed first to elucidate understanding of the nature of the catalytically active site. Thermodynamic calculations revealed that Mn likely exists as a metallic alloy with $\mathrm{Rh}$ in Rh-rich environments under reducing conditions at the temperatures of interest. After determining that reduced Rh-Mn alloy metal clusters were in a reduced state, the activation energy barriers of numerous transition state species on the catalytically active metal particles were calculated to compute the activation barriers of several reaction pathways that are possible on the catalyst surface. Comparison of calculations with an Rh nanoparticle versus an Rh-Mn nanoparticle revealed that the presence of $\mathrm{Mn}$ enabled the reaction pathway of $\mathrm{CH}$ with $\mathrm{CO}$ to form an adsorbed $\mathrm{CHCO}$ species, which was a precursor to $\mathrm{C}_{2}+$ oxygenates. The presence of Mn did not have a significant effect on the rate of $\mathrm{CH}_{4}$ production. Thus, a major early finding of the computational model was that the presence of $\mathrm{Mn}$ opened a reaction pathway to the formation of $\mathrm{C}_{2}+$ oxygenates that did not greatly affect the rate at which $\mathrm{CH}_{4}$ is formed, which is consistent with experimental observations. A Kinetic Monte Carlo model was developed to use the density functional theory-calculated activation energy barriers to examine kinetic rates for comparison with observed experimental selectivity. Results from the Kinetic Monte Carlo model were consistent with experimentally observed trends, such as an increase in methane selectivity with increasing temperature, further validating the density functional theory-based approach and developed reaction network.

Ir was observed during empirical catalyst screening experiments to improve the activity and selectivity of Rh-Mn catalysts. Thus, the addition of Ir to the Rh-Mn nanoparticles also was probed computationally. Simulations of Rh-Mn-Ir nanoparticles revealed that, with sufficient Ir concentrations, the Rh, Mn and Ir presumably would be well mixed within a nanoparticle. Activation barriers were calculated for Rh-Mn-Ir nanoparticles for several C-, H-, and O-containing transitional species on the nanoparticle surface. It was found that the presence of Ir opened yet another reactive pathway whereby $\mathrm{HCO}$ is formed and may undergo insertion with $\mathrm{CH}_{\mathrm{x}}$ surface moieties. The reaction pathway opened by the presence of Ir is in addition to the $\mathrm{CO}+\mathrm{CH}$ pathway opened by the presence of $\mathrm{Mn}$. Similar to $\mathrm{Mn}$, the presence of Ir was not found to not affect the rate of $\mathrm{CH}_{4}$ production.

This report aims to be a comprehensive account of the characterization and computational efforts performed in support of the development of the Rh-based mixed alcohol synthesis catalyst. A separate series of annual reports focuses specifically on the results of experimental laboratory testing with the Rh-

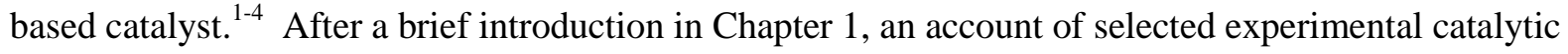
reactor tests is presented in Chapter 2 of this report in support of the prepared and tested catalysts. In addition to identifying relevant trends and characteristics of the investigated catalysts and supports, documentation of data collected is a goal of this report. Therefore, Chapter 2, Characterization, is extensive. The computational portion of the report is presented in Chapter 3 , and a brief summary of the work is provided in Chapter 4. References cited in the report are listed in Chapter 5, and Appendices A through $\mathrm{D}$ provide technical detail associated with our analytical efforts. 


\section{Acronyms and Abbreviations}

$\begin{array}{ll}\text { AIMD } & a b \text { initio molecular dynamics } \\ \text { BET } & \text { Brunauer-Emmett-Teller } \\ \text { DFT } & \text { density functional theory } \\ \text { DRIFTS } & \text { diffuse reflectance infrared Fourier transform spectroscopy } \\ \text { EDS } & \text { electronic dispersive spectroscopy } \\ \text { FCC } & \text { face-centered cubic } \\ \text { GHSV } & \text { gas hourly space velocity } \\ \text { HF } & \text { hydrofluoric acid } \\ \text { ICP } & \text { inductively coupled plasma } \\ \text { KMC } & \text { kinetic Monte Carlo } \\ \text { LRB } & \text { laboratory record book } \\ \text { MS } & \text { mass spectrometer } \\ \text { MWCNT } & \text { multi-walled carbon nanotube } \\ \text { PNNL } & \text { Pacific Northwest National Laboratory } \\ \text { SCCM } & \text { standard cubic centimeters per minute } \\ \text { STY } & \text { space-time yield } \\ \text { TCD } & \text { thermal conductivity detector } \\ \text { TEM } & \text { transmission electron microscopy } \\ \text { TG } & \text { thermogravimetric } \\ \text { TGA } & \text { thermogravimetric analyzer } \\ \text { TOF } & \text { turnover frequencies } \\ \text { TPD } & \text { temperature programmed desorption } \\ \text { XPS } & \text { X-ray photoelectron spectroscopy } \\ \text { XRD } & \\ & \end{array}$





\section{Contents}

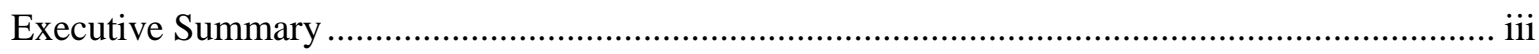

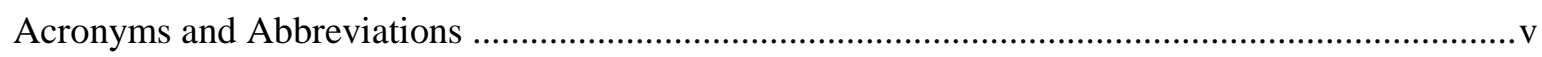

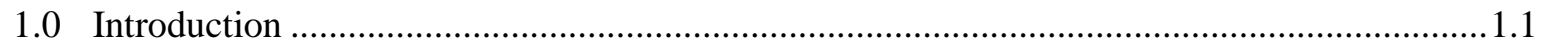

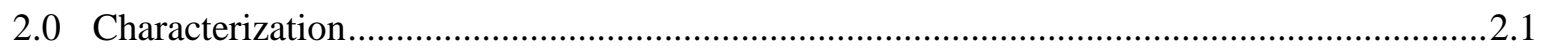

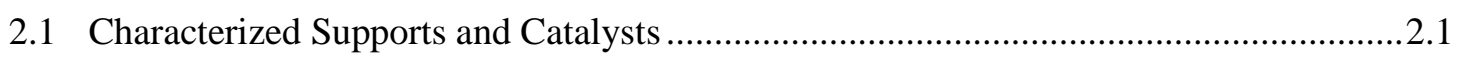

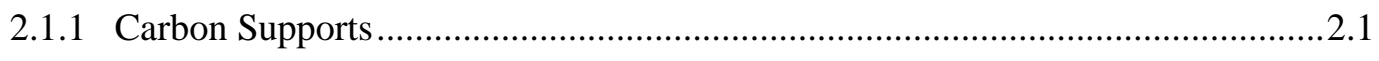

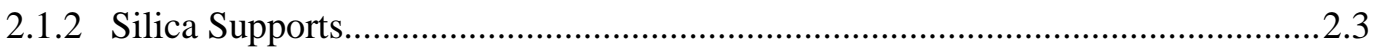

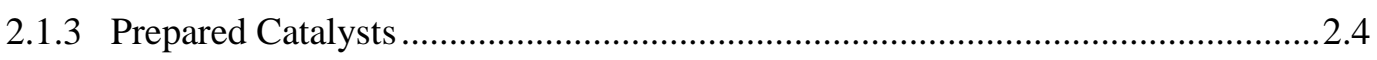

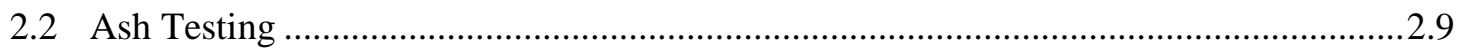

2.2.1 Carbon Supports KOA 1-15 ............................................................................ 2.9

2.2.2 HF-Washed and Graphitized Carbon Supports ................................................2.10

2.3 Elemental Analysis of Carbon and Silica Supports via Inductively Coupled Plasma... 2.12

2.3.1 Carbon Support Analysis ........................................................................... 2.12

2.3.2 ICP Analysis of the Low Pressure Methanol Synthesis Catalyst .......................2.23

2.3.3 ICP Analysis of Silica Mixed Alcohol Catalyst Supports...................................2.23

2.4 Surface and Pore Volume Measurements via Nitrogen Adsorption/Desorption............2.29

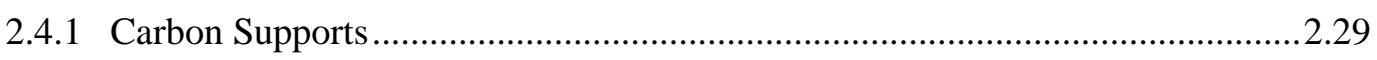

2.4.2 Demineralized and Graphitized Carbon Supports ............................................2.30

2.4 .3 Silica Supports.............................................................................................. 2.33

2.5 Surface Acidity Characterization of Selected Silica Mixed Alcohols Catalyst Supports via Ammonia Temperature Programmed Desorption ...................................................2.34

2.6 Carbon Support Surface Functionality Characterization through Temperature Programmed Desorption Utilizing a Coupled Thermogravimetric Analyzer and Mass

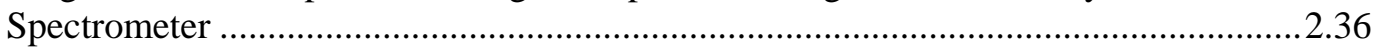

2.6.1 Unmodified Carbon Supports KOA 1-12 .......................................................2.36

2.6.2 HF-Washed, Graphitized, and $\mathrm{HNO}_{3}$ Functionalized Carbon Supports ............2.41

2.7 Diffuse Reflectance Infrared Fourier Transform Spectroscopy and Raman

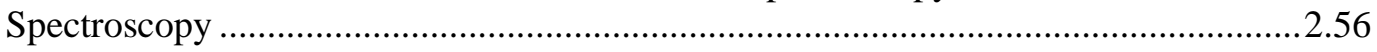

2.8 Hydrogen Chemisorption Testing on Select Mixed Alcohol Catalysts.........................2.58

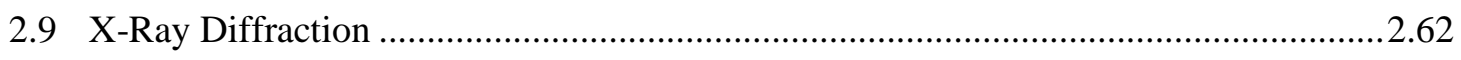

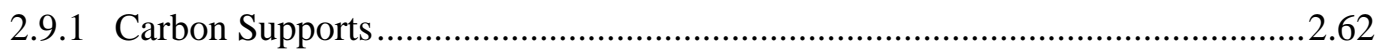

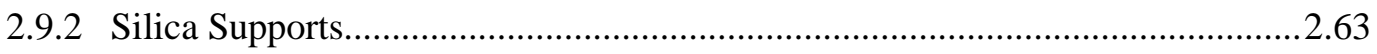

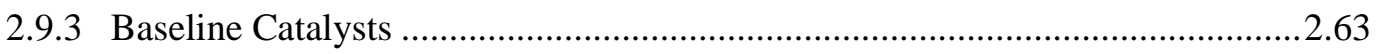

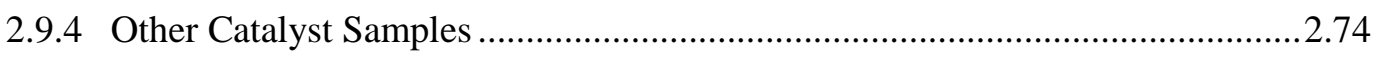

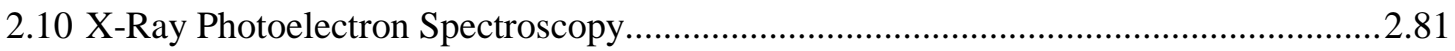

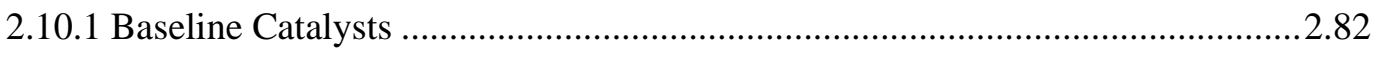

2.10.2 Other Fresh and Spent Catalysts .................................................................2.92 


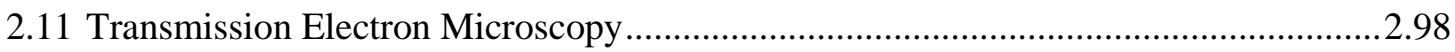

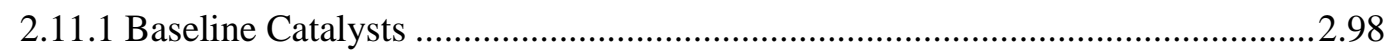

2.11.2 Other Fresh and Spent Catalysts …..................................................................2.118

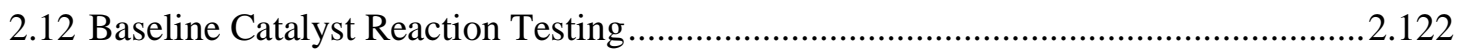

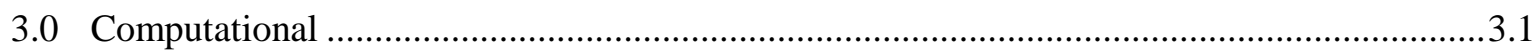

3.1 Catalytic Site Development.....................................................................................

3.1.1 Rh-Mn/ $\mathrm{SiO}_{2}$ Catalyst Characterization .......................................................... 3.1

3.1.2 Determination of Mn Oxidation State .................................................................3.3

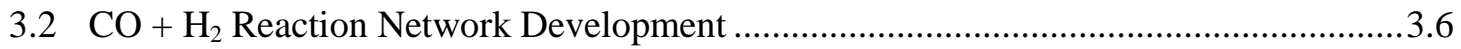

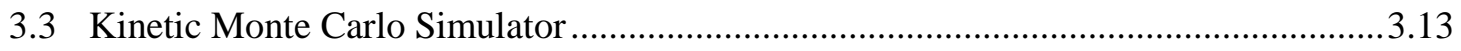

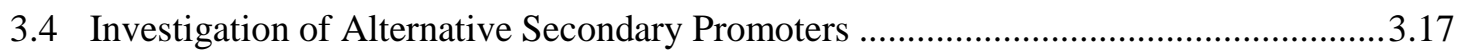

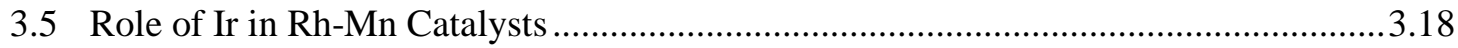

3.6 Effect of Catalyst Support on the Structure and Reactivity of Rh-Mn-Ir Catalyst

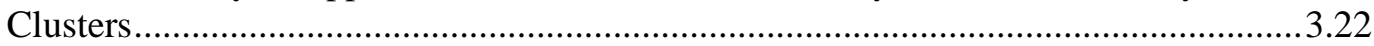

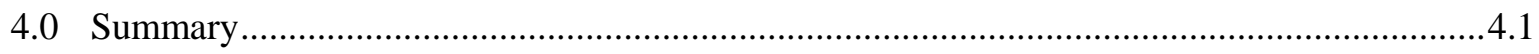

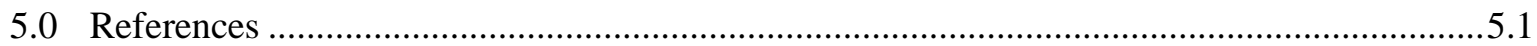

Appendix A - Pore Volume Distributions of Carbon Supports KOA1 through 15 Obtained from

$\mathrm{N}_{2}$ Adsorption/Desorption Testing ..................................................................................

Appendix $\mathrm{B}-\mathrm{NH}_{3}$ Temperature Programmed Desorption Traces of Silica Mixed Alcohol

Catalyst Supports ................................................................................................ B. 1

Appendix C - Thermogravimetric and Mass Spectrometer Data from Temperature Programmed

Desorption Testing on Samples KOA 1 through 12 ................................................................. C.1

Appendix D - X-Ray Photoelectron Spectroscopy (XPS) Spectra of the Metal Impregnated

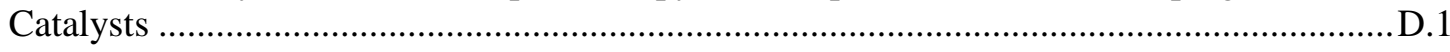




\section{Figures}

2.1 Simplified Diagram of the Reactor System used to Test Catalysts ......................................... 2.7

2.2 Ash Collected after Burning Various Treated Carbons ........................................................ 2.11

2.3 Pore size Distributions of Fresh, HF-Washed and Graphitized Samples of Norit ROX 0.8 ..... 2.31

2.4 Pore Size Distributions of Fresh, HF-Washed and Graphitized Samples of Norit Darco-LS ... 2.32

2.5 Pore Size Distributions of Fresh, HF-Washed and Graphitized Samples of the Pacco 90-100 CTC

2.6 Pore Size Distributions of Fresh, HF-Washed and Graphitized Samples of the Jacobi Ecosorb BX-Max

2.7 Overlay of the TG Curves Produced by TGA/MS Measurements of the Norit ROX 0.8 Carbon Support Prior to Modification and After Each Step of HF, Graphitization, and $\mathrm{HNO}_{3}$ Modification of the Support..

2.8 TG Curve (solid black line) and MS Traces of Relevant Compounds Observed in the Gas Phase Effluent during TGA/MS Testing of the Fresh (unmodified) Norit ROX 0.8 Carbon Support.

2.9 TG Curve (solid blue line) and MS Traces of Relevant Compounds Observed in the GasPhase Effluent during TGA/MS Testing of the HF-Treated (demineralized) Norit ROX 0.8 Carbon Support.

2.10 TG Curve (solid red line) and MS Traces of Relevant Compounds Observed in the Gas Phase Effluent during TGA/MS Testing of the HF-Treated (demineralized) and Graphitized Norit ROX 0.8 Carbon Support.

2.11 TG Curve (solid green line) and MS Traces of Relevant Compounds Observed in the GasPhase Effluent during TGA/MS Testing of the HF-Treated (demineralized), Graphitized, and Subsequently Re-Functionalized via $\mathrm{HNO}_{3}$ Treatment of the Norit ROX 0.8 Carbon Support .

2.12 Overlay of the TG Curves Produced by TGA/MS Measurements of the Norit Darco-LS Carbon Support Prior to Modification and After Each Step of HF Treatment, Graphitization, and $\mathrm{HNO}_{3}$ Modification of the Support.

2.13 TG Curve (solid black line) and MS Traces of Relevant Compounds Observed in the GasPhase Effluent during TGA/MS Testing of the Fresh (unmodified) Norit Darco-LS Carbon Support .

2.14 TG Curve (solid blue line) and MS Traces of Relevant Compounds Observed in the GasPhase Effluent during TGA/MS Testing of the HF-Treated (demineralized) Norit DarcoLS Carbon Support...

2.15 TG Curve (solid red line) and MS Traces of Relevant Compounds Observed in the GasPhase Effluent during TGA/MS Testing of the HF-Treated (demineralized), and Graphitized Darco-LS Carbon Support

2.16 TG Curve (solid green line) and MS Traces of Relevant Compounds Observed in the GasPhase Effluent during TGA/MS Testing of the HF-Treated (demineralized), Graphitized, and Subsequently Re-Functionalized via $\mathrm{HNO}_{3}$ Treatment of the Norit Darco-LS Carbon Support.

2.17 Overlay of TG Curves Produced by TGA/MS Measurements of the Pacco 90-100 CTC Carbon Support Prior to Modification and after Each Step of HF, Graphitization and $\mathrm{HNO}_{3}$ Modification of the Support. 
2.18 TG Curve (solid black line) and MS Traces of Relevant Compounds Observed in the GasPhase Effluent during TGA/MS Testing of the Fresh (unmodified) Pacco 90-100 CTC Carbon Support.

2.19 TG Curve (solid blue line) and MS Traces of Relevant Compounds Observed in the GasPhase Effluent during TGA/MS Testing of the HF-Treated (demineralized) Pacco 90-100 CTC Carbon Support.

2.20 TG Curve (solid red line) and MS Traces of Relevant Compounds Observed in the GasPhase Effluent during TGA/MS Testing of the HF-Treated (demineralized) and Graphitized Pacco 90-100 CTC Carbon Support.

2.21 TG Curve (solid green line) and MS Traces of Relevant Compounds Observed in the GasPhase Effluent during TGA/MS Testing of the HF-Treated (demineralized), Graphitized, and Subsequently Re-Functionalized via $\mathrm{HNO}_{3}$ Treatment of the Pacco 90-100 CTC Carbon Support.

2.22 DRIFTS Results on Carbon Supports KOA 6, 7, and 8

2.23 Raman Spectroscopy of the $\mathrm{C}=\mathrm{C}$ Stretch Bands in Samples KOA 6, 7, and 8.

2.24 Raman Investigation of C-H Stretching Mode of KOA 6, 7, and 8 ..... 2.57

$2.25 \mathrm{H}_{2}$ Chemisorption Results on Reduced Catalyst Samples. 2.58

2.26 Overlay of Diffractograms Collected through XRD Analysis of Samples KOA 1 through 12

2.27 Overlay of Diffractograms Collected through XRD Analysis of Various Silica MixedAlcohol Catalyst Supports. Davisil = Davisil 645; Merck = Merck Grade 60; Engelhard = Engelhard Mod D; Grace = Grace Grade 408; Norpro = Norpro 61138.

2.28 Diffractogram of Sample 60074-53-A, which was the Fresh Davisil 645 Silica-Supported Rh-Mn-Ir Catalyst in Table 2.4

2.29 Diffractogram Collected from Sample 60074-53-A-R, which was the Reduced Davisil 645 Silica-Supported Rh-Mn-Ir Baseline Catalyst in Table 2.4.

2.30 Diffractogram Collected from the Sample of Run 84, which was the Davisil 645 SilicaSupported Rh-Mn-Ir Baseline Catalyst Tested to $275^{\circ} \mathrm{C}$ in Table 2.4

2.31 Diffractogram Collected from the Sample of Run 86, which was the Davisil 645 SilicaSupported Rh-Mn-Ir Baseline Catalyst Tested to $300^{\circ} \mathrm{C}$ in Table 2.4

2.32 Diffractogram Collected from Sample 60074-70-C, which was the Fresh Hyperion Fibril MWCNT-Supported Rh-Mn Catalyst in Table 2.4

2.33 Diffractogram Collected from Sample 60074-70-C-R, which was the reduced Hyperion Fibril MWCNT-Supported Rh-Mn Catalyst in Table 2.4

2.34 Diffractogram Collected from the Sample from Run 80, which was the Hyperion Fibril MWCNT-Supported Rh-Mn Catalyst Tested to $276^{\circ} \mathrm{C}$ in Table 2.4

2.35 Diffractogram Collected from the Sample from Run 82, which was the Hyperion Fibril MWCNT-Supported Rh-Mn Catalyst Tested to $299^{\circ} \mathrm{C}$ in Table 2.4

2.36 Diffractogram Collected from Sample 60074-74-C, which was the fresh Hyperion Fibril MWCNT-Supported Rh-Mn-Ir Catalyst in Table 2.4

2.37 Diffractogram Collected from Sample 60074-74-C-R, which was the reduced Hyperion Fibril MWCNT-Supported Rh-Mn-Ir Catalyst in Table 2.4

2.38 Diffractogram Collected from the Sample from Run 89, which was the Hyperion Fibril MWCNT-Supported Rh-Mn-Ir Catalyst Tested to $275^{\circ} \mathrm{C}$ in Table 2.4 
2.39 Diffractogram Collected from the Sample from Run 88, which was the Hyperion Fibril MWCNT-Supported Rh-Mn-Ir Catalyst Tested to $314^{\circ} \mathrm{C}$ in Table 2.4 ................................. 2.74

2.40 XRD Analysis of Various Reduced Catalyst Samples Listed in Table 2.6............................ 2.75

2.41 Micro-XRD Results on Spent Catalyst Sample Run 31, which contained 11.78 wt\% Rh and $3.58 \mathrm{wt} \% \mathrm{Mn}$.

2.42 Micro-XRD Results on Spent Catalyst Sample Run 33, which contained 11.78 wt\% Rh, 3.58 wt\% Mn, and 2.19 wt\% Ir.

2.43 Micro-XRD Results on Spent Catalyst Sample Run 33, which contained 11.78 wt\% Rh, $3.58 \mathrm{wt} \% \mathrm{Mn}$, and $2.19 \mathrm{wt} \% \mathrm{Ir}$.

2.44 Micro-XRD Results on Spent Catalyst Sample Run 34, which contained 11.78 wt\% Rh, $3.58 \mathrm{wt} \% \mathrm{Mn}$, and $2.19 \mathrm{wt} \% \mathrm{Ir}$.

2.45 Micro-XRD Results on Spent Catalyst Sample Run 34, which contained 11.78 wt\% Rh, $3.58 \mathrm{wt} \% \mathrm{Mn}$, and $2.19 \mathrm{wt} \% \mathrm{Ir}$.

2.46 Micro-XRD Results on Spent Catalyst Sample Run 39, which contained 11.78 wt\% Rh, $3.58 \mathrm{wt} \% \mathrm{Mn}$, and $2.19 \mathrm{wt} \% \mathrm{Ir}$.

2.47 Micro-XRD Results on Spent Catalyst Sample Run 39, which contained 11.78 wt\% Rh, $3.58 \mathrm{wt} \% \mathrm{Mn}$, and $2.19 \mathrm{wt} \% \mathrm{Ir}$.

2.48 High-Energy-Resolution XPS Spectra of the Rh 3d Region of the 1x Rh-Mn-Ir on Davisil 645 Silica baseline catalyst.

2.49 High Energy-Resolution XPS from 0 to 510 eV Binding Energy of the Spent Davisil 645Supported Rh-Mn-Ir Catalysts (Run 84 and 86 in Table 2.4)

2.50 High-Energy-Resolution XPS spectra of the Mn 2p Region of the 1x Rh-Mn-Ir on Davisil 645 Silica Baseline Catalyst.

2.51 High-Energy-Resolution XPS Spectra of the Ir $4 \mathrm{f}$ region of the 1x Rh-Mn-Ir on the Davisil 645 Silica Baseline Catalyst.

2.52 High-Energy-Resolution XPS Spectra of the Rh 3d Region of the 2.11x Rh-Mn on Hyperion 384-82 MWCNT Baseline Catalyst.

2.53 High-Energy-Resolution XPS Spectra of the Mn 2p region of the 2.11x Rh-Mn on the Hyperion 384-82 MWCNT Baseline Catalyst.

2.54 High-Energy-Resolution XPS Spectra of the Rh 3d Region of the 2.11x Rh-Mn-Ir on the Hyperion 384-82 MWCNT Baseline Catalyst.

2.55 High-Energy-Resolution XPS Spectra of the Mn 2p Region of the 2.11x Rh-Mn-Ir on the Hyperion 384-82 MWCNT Baseline Catalyst...

2.56 High-Energy-Resolution XPS Spectra of the Ir 4f Region of the 2.11x Rh-Mn-Ir on the Hyperion 384-82 MWCNT Baseline Catalyst.

2.57 Rh 2p Electron Region of the Six Fresh (unreduced dashed lines) and Reduced (solid lines) Metal Impregnated Catalysts Listed in Table 2.6.....

2.58 High-Resolution XPS Spectra of the Rh 2p Electron Region of the Five Spent Catalysts Listed in Table 2.6.

2.59 High-Resolution XPS Spectra of the Mn 2p Electron Region of the Six Fresh (unreduced dashed) and reduced (solid) Metal Impregnated Catalysts in Table 2.6.

2.60 High-Resolution XPS Spectra of the Mn 2p Electron Region of the Five Spent Catalysts in Table 2.6. 
2.61 Ir 4f Electron Region of the Six Fresh (unreduced dashed lines) and Reduced (solid lines) Metal Impregnated Catalysts in Table 2.6.......................................................................... 2.97

2.62 Ir 4f Electron Region of the Five Spent Catalysts in Table 2.2 ............................................ 2.98

2.63 Comparison of the Same Micrograph Illustrating the Effect of Converting from Grayscale to Colorized Gradient Scale.....

2.64 TEM Micrograph of the Reduced Baseline Rh-Mn-Ir Sample 60074-53-A-R Supported on Davisil 645.

2.65 TEM Micrograph of the Reduced Baseline Rh-Mn-Ir Sample 60074-53-A-R Supported on Davisil 645.

2.66 TEM Micrograph of the Fresh (calcined but unreduced) Baseline Rh-Mn-Ir Catalyst Supported on Davisil 645 Silica.

2.67 TEM Micrograph of the Fresh (calcined but unreduced) Baseline Rh-Mn-Ir Catalyst Supported on Davisil 645 silica.

2.68 TEM Micrograph of the Baseline Rh-Mn-Ir Sample Tested to a Maximum Temperature of $275^{\circ} \mathrm{C}$ (Run 84 in Table 2.4) Supported on Davisil 645

2.69 TEM Micrograph of the Baseline Rh-Mn-Ir Sample Tested to a Maximum Temperature of $275^{\circ} \mathrm{C}$ (Run 84 in Table 2.4) supported on Davisil 645

2.70 TEM Micrograph of the Baseline Rh-Mn-Ir Sample Tested to a Maximum Temperature of $300^{\circ} \mathrm{C}$ (Run 86 in Table 2.4) Supported on Davisil 645.

2.71 TEM Micrograph of the Baseline Rh-Mn-Ir Sample Tested to a Maximum Temperature of $300^{\circ} \mathrm{C}$ (Run 86 in Table 2.4) Supported on Davisil 645

2.72 TEM Micrograph of the Freshly Prepared Baseline Rh-Mn Sample (sample 60074-70-C in Table 2.4) Supported on Hyperion Fibril MWCNT ....

2.73 Higher Magnification TEM Micrograph of the Freshly Prepared Baseline Rh-Mn Sample (sample 60074-70-C in Table 2.4) Supported on Hyperion Fibril MWCNT .

2.74 TEM Micrograph of the Reduced Baseline Rh-Mn Sample (sample 60074-70-C-R in Table 2.4) Supported on Hyperion Fibril MWCNT ...

2.75 Higher Magnification TEM Micrograph of the Reduced Baseline Rh-Mn Sample (sample 60074-70-C-R in Table 2.4) Supported on Hyperion Fibril MWCNT

2.76 TEM Micrograph of the Baseline Rh-Mn Sample Tested to $276^{\circ} \mathrm{C}$ (Run 80 in Table 2.4) Supported on Hyperion Fibril MWCNT

2.77 TEM Micrograph of the Baseline Rh-Mn Sample Tested to $276^{\circ} \mathrm{C}$ (Run 80 in Table 2.4) Supported on Hyperion Fibril MWCNT, Illustrating the Presence of Metallic Plates that were Observed throughout the Sample.

2.78 Higher Magnification TEM Micrograph of the Baseline Rh-Mn Sample Tested to $\sim 276^{\circ} \mathrm{C}$ (Run 80 in Table 2.4) Supported on Hyperion Fibril MWCNT with a Cross-Section View of a Metallic Plate Formed after Metallic Synthesis

2.79 Higher Magnification TEM Micrograph of the Baseline Rh-Mn Sample Tested to $\sim 276^{\circ} \mathrm{C}$ (Run 80 in Table 2.4) Supported on Hyperion Fibril MWCNT with a Top-View Picture of a Metallic Plate that Formed after Mixed Alcohol Synthesis.

2.80 TEM Micrograph of the Baseline Rh-Mn Sample Tested to $\sim 299^{\circ} \mathrm{C}$ (Run 82 in Table 2.4) Supported on Hyperion Fibril MWCNT. Note the presence of the plates similar to the micrographs from Run 80 (2.74 and Figure 2.75)..... 
2.81 Higher Magnification TEM Micrograph of the Baseline Rh-Mn Sample Tested to $299^{\circ} \mathrm{C}$ (Run 82 in Table 2.4) Supported on Hyperion Fibril MWCNT...

2.82 Higher Magnification TEM Micrograph of the Freshly Prepared Baseline Rh-Mn-Ir (sample 60074-74-C in Table 2.4) Supported on Hyperion Fibril MWCNT .

2.83 TEM Micrograph of the Reduced Baseline Rh-Mn-Ir (sample 60074-74-C-R in Table 2.4) Supported on Hyperion Fibril MWCNT

2.84 Higher Magnification TEM Micrograph of the Reduced Baseline Rh-Mn-Ir (sample 60074-74-C-R in Table 2.4) Supported on Hyperion Fibril MWCNT.

2.85 TEM Micrograph of the Baseline Rh-Mn-Ir that Experienced a Temperature of $275^{\circ} \mathrm{C}$ (Run 89 in Table 2.4) Supported on Hyperion Fibril MWCNT.

2.86 Higher Magnification TEM Micrograph of the Baseline Rh-Mn-Ir that Experienced a Temperature of $275^{\circ} \mathrm{C}$ (Run 89 in Table 2.4) Supported on Hyperion Fibril MWCNT

2.87 TEM Micrograph of the Baseline Rh-Mn-Ir that Experienced a Temperature of $314^{\circ} \mathrm{C}$ (Run 88 in Table 2.4) Supported on Hyperion Fibril MWCNT .

2.88 Higher Magnification TEM Micrograph of the Baseline Rh-Mn-Ir that Experienced a Temperature of $314^{\circ} \mathrm{C}$ (Run 88 in Table 2.4) Supported on Hyperion Fibril MWCNT.

2.89 TEM Micrograph of the Baseline Rh-Mn-Ir that Experienced a Temperature of $314^{\circ} \mathrm{C}$ (Run 88 in Table 2.4) Supported on Hyperion Fibril MWCNT.

2.90 TEM Micrograph of the Baseline Rh-Mn-Ir that Experienced a Temperature of $314^{\circ} \mathrm{C}$ (Run 88 in Table 2.4) Supported on Hyperion Fibril MWCNT, Showing Metal Particles (bright spots) that are Likely Inside the MWCNTs

2.91 TEM Micrograph of Catalyst 60074-56-A, which Consists of $8.34 \mathrm{wt} \% \mathrm{Rh}$ and $2.54 \mathrm{wt} \%$ Mn on Hyperion 395-08 .

2.92 TEM Micrograph of Catalyst 60074-56-A, which Consists of $8.34 \mathrm{wt} \%$ Rh and $2.54 \mathrm{wt} \%$ Mn on Hyperion 395-08

2.93 TEM Micrograph of Catalyst 60074-56-A, which Consists of 8.34 wt\% Rh and 2.54 wt\% Mn on Hyperion 395-08

2.94 TEM Micrograph of Catalyst 60074-59-C, which Consists of $11.778 \mathrm{wt} \% \mathrm{Rh}, 3.587 \mathrm{wt} \%$ Mn, and 2.189 wt\% Ir on Hyperion 384-82

2.95 TEM Micrograph of Catalyst 60074-59-C, which Consists of 11.778 wt\% Rh, 3.587 wt\% Mn, and 2.189 wt\% Ir on Hyperion 384-82

2.96 TEM Micrograph of Catalyst 60074-59-C, which Consists of 11.778 wt\% Rh, 3.587 wt\% Mn, and 2.189 wt\% Ir on Hyperion 384-82

2.97 STYs at $275^{\circ} \mathrm{C}$ and $7900 \mathrm{~L} / \mathrm{Lcat} / \mathrm{hr}$ GHSV for Low- and High-Temperature Test Runs (Runs 84 and 86, respectively) using the Davisil 645 Silica-Supported Rh-Mn-Ir Catalysts ... 2.122

2.98 Converted Carbon Selectivities at $275^{\circ} \mathrm{C}$ and $7900 \mathrm{~L} / \mathrm{Lcat} / \mathrm{hr}$ GHSV for Low- and HighTemperature Runs (Runs 84 and 86, respectively) using the Davisil 645 Silica-Supported Baseline Rh-Mn-Ir Catalyst.

2.99 STYs at $275^{\circ} \mathrm{C}$ and $7900 \mathrm{~L} / \mathrm{Lcat} / \mathrm{hr}$ GHSV for Hyperion Carbon Nanotube-Supported Rh-Mn (Run 80 for low temperature; Run 82 for high temperature) and Rh-Mn-Ir Catalysts (Run 89 for low temperature; Run 88 for high temperature).

2.100 Converted Carbon Selectivities at $275^{\circ} \mathrm{C}$ and $7900 \mathrm{~L} / \mathrm{Lcat} / \mathrm{hr}$ GHSV for Hyperion Carbon Nanotube-Supported Rh-Mn (Run 80 for low temperature; Run 82 for high temperature) and Rh-Mn-Ir Catalysts (Run 89 for low temperature; Run 88 for high temperature). 
3.1 TEM Images of Fresh and Used Rh/Mn/Silica Catalyst, Sample 3 (designated as S3)....

3.2 Calculated Equilibrium Constants for Elementary Reactions (2) through (7) as a Function of Temperature.

3.3 Structures of (MnO) $)_{50}$ (left) and $\left(\mathrm{Rh}_{2} \mathrm{O}_{3}\right)_{25}$ (right) Particles Extracted from HighTemperature AIMD Simulated Annealing Studies...

3.4 Elementary Reaction Diagram Showing Important Steps of Syngas Conversion as Complied from a Survey of the Literature and the Research Performed on this Project

3.5 Optimized Adsorption Configurations on the Rh47Mn3 Nanoparticle (partially shown for clarity): Rh (green), Mn (purple), C (gray), O (red), H (white).

3.6 Relative Energies and Reaction Barriers for the Methanation Reaction on the Three Different Clusters ....

3.7 Energetics for $\mathrm{CH}_{\mathrm{x}}+\mathrm{CO}$ Reactions in the Three Different Nanoparticles, $\mathrm{x}=1$ for (a); $\mathrm{x}=$ 2 for (b); and $\mathrm{x}=3$ for (c).

3.8 Snapshot of KMC Simulation of CO Hydrogenation to Ethanol: Si (yellow), O (red), Rh (cyan), Mn (grey), C (blue), and H (green).

3.9 Temperature Dependence on TOF of Major Products (a); CO Conversion (b); Methane and Acetaldehyde Selectivity (c); and Methanol, Ethanol, and Acetic Acid Selectivity (d).....

3.10 Pressure Dependence of Selectivity at $300^{\circ} \mathrm{C}$. Taken from Mei et al. ${ }^{7}$.

3.11 Arrhenius Plots (from the KMC model) Showing the T Dependence on the TOFs of (a) Methane and (b) Ethanol Production.

3.12 DFT Reaction Barriers as a Function of Electronegativity Differences between Rh and Metal Promoters.

3.13 Density Distributions from the Center of Mass of the Cluster.

3.14 Figure C 16. Coordination Numbers $(\mathrm{N})$ and Bond Fluctuations $(\delta)$ Plotted Versus Temperature for Two Cluster Compositions

3.15 Initial Reactive Steps Leading to $\mathrm{C}_{2}$ Oxygenates on a Thermalized and Annealed $\mathrm{Rh}_{40} \mathrm{Mn}_{6} \mathrm{Ir}_{4}$ Nanoparticle.

3.16 Elementary Reaction Diagram Showing Important Steps of Syngas Conversion.

3.17 Structure of $\mathrm{Rh}_{40} \mathrm{Ir}_{4} \mathrm{Mn}_{6}$ on Silica (left) and Carbon (right) Quenched from a 20 ps AIMD Simulation at T = $600 \mathrm{~K}$ : Rh (pink), Mn (white), Ir (blue), C (black), oxygen (red), Si (brown).

3.18 Structure of $\mathrm{Rh}_{40} \mathrm{Ir}_{4} \mathrm{Mn}_{6}$ on Silica (left) and Carbon (right) in the Presence of $\mathrm{H}_{2}$ and $\mathrm{CO}$ Adsorbates Quenched from a 20 ps AIMD Simulation at $\mathrm{T}=600 \mathrm{~K}$ 


\section{Tables}

2.1 Identification Numbers Assigned to Carbon Supports KOA 1 through16 with Descriptions, Lot Numbers, and PNNL Barcode or LRB Numbers.

2.2 Carbon Supports Subjected to Demineralization via Sequential HF Washing, HighTemperature Graphitization, and Surface Functionalization via Nitric Acid $\left(\mathrm{HNO}_{3}\right)$ Treatment.

2.3 Silica Samples Subjected to Various Analytical Measurements .

2.4 Baseline Catalysts Subjected to Various Characterization Tests.............................................. 2.5

2.5 Sequential Test Conditions used for Evaluating Baseline Catalysts ...................................... 2.8

2.6 Identification of Metal-Loaded Catalyst Samples with Descriptions and Metal Loadings and Support Identification

2.7 Gravimetrically Determined Concentrations of Ash after Firing the Carbon Supports at $700^{\circ} \mathrm{C}$ for $5 \mathrm{hr}$ in Air

2.8 Gravimetrically Determined Concentration of Ash after Firing the Carbon Supports in Air ...

2.9 Concentration of Inorganic Oxide Species Observed from ICP Analysis of the Ash Produced from High-Temperature Calcination of the Carbon Supports for Samples KOA 1 through 6.

2.10 Comparison of the $\mathrm{HNO}_{3}$ and Alternative $\mathrm{HNO}_{3} / \mathrm{HF}$ Methods of Acid Pretreatment on Sample KOA 2 for ICP Analysis.

2.11 Concentration in wt\% of Inorganic Oxide Species Observed from ICP Analysis on Selected Carbon Supports after Microwave Digestions using $\mathrm{HNO}_{3}$ and $\mathrm{HF}$.

2.12 ICP Analysis of the Inorganic Impurities Present in the Pacco 90-100 CT Lot 2007-2-22ACC, the Support after HF Washing, and after HF Washing and Graphitization in wt\%.......

2.13 ICP Analysis of Inorganic Impurities in the Norit ROX 0.8 Lot 520020, the Support after HF Washing, and after HF Washing and Graphitization in wt\%.

2.14 ICP Analysis of Inorganic Impurities in the Norit Darco-LS 12x20 Lot 854247, in the Support after HF Washing, and after HF Washing and Graphitization in wt\%.

2.15 ICP Analysis of Inorganic Impurities in the Jacobi Ecosorb BX-Max in Untreated Material, after HF Washing, and after HF Washing and Graphitization in wt\%.

2.16 ICP Analysis of Inorganic Impurities in the Untreated SGL Carbon Material.

2.17 Elemental Analysis Determined via ICP on the Engelhard Mod D, Davisil 645 and $\mathrm{HNO}_{3}$ Washed Davisil 645 Silica Mixed Alcohol Catalyst Supports .

2.18 Elemental Analysis Determined via ICP on the Merck Aldrich Grade 60, Sigma-Aldrich Grade 7734 and Sigma-Aldrich Grade 7754 Silica Mixed Alcohol Catalyst Supports.

2.19 Mixed Alcohol Synthesis Silica Catalyst Supports Digested in $\mathrm{HNO}_{3} / \mathrm{HF}$ and Analyzed with ICP.

2.20 Elemental Analysis Determined via ICP on the Merck Aldrich Grade 60, Sigma-Aldrich Grade 7734 and Davisil 645 silica Mixed Alcohol Catalyst Supports.

2.21 Surface Area and Porosity Data of Carbon Supports KOA 1 through 15 and the ICI Methanol Synthesis Catalyst

2.22 Surface-Area and Pore-Size Data for Various Fresh, HF-Washed, and Graphitized Carbon Supports..... 
2.23 Surface-Area and Porosity Data of Inorganic Mixed Alcohol Synthesis Supports

2.24 Total Amount of $\mathrm{NH}_{3}$ Desorbed during TPD Experiments With Silica Mixed Alcohol Synthesis Catalyst Supports

2.25 Oxygenated Surface Functionalities, Their Decomposition Species, and Temperature. Taken from Akosylu et al. ${ }^{6}$

2.26 Analysis of TPD Results on Carbon Supports KOA 1 through 6 ........................................... 2.39

2.27 Analysis of TPD Results on Carbon Supports KOA 7 through 12........................................ 2.39

2.28 Rh-Based Mixed Alcohol Catalysts Subjected to $\mathrm{H}_{2}$ Chemisorption Testing ......................... 2.59

2.29 Quantification (atomic percent) of Surface Elements on the Baseline Catalyst Samples as Determined by XPS Measurements.

2.30 Quantification (atomic percent) of Surface Elements on Various Samples Prior to Reduction and Reduced as Determined by XPS Measurements

2.31 Quantification (atomic percent) of Surface Elements on Various Spent Mixed Alcohol Catalysts.

3.1 Binding Energies of Reactive Intermediates on Three Different Nanoparticles. "Na" indicates non-adsorption site.

3.2 Activation Barriers For Reaction $\mathrm{CO}+\mathrm{H}_{2} \rightarrow$ Product. The "Theory" values are from "Progress in C1 Chemistry in Japan." ${ }^{\text {"18 }}$ Energies are reported in $\mathrm{kJ} / \mathrm{mol}$ 


\subsection{Introduction}

The U.S. Department of Energy is conducting a program focused on developing a process for converting biomass to bio-based fuels and co-products. In this process, a lignocellulosic biomass feedstock is first gasified to produce a product known as synthesis gas (syngas), which consists primarily of hydrogen $\left(\mathrm{H}_{2}\right)$ and carbon monoxide (CO). The syngas stream is subsequently thermochemically converted within a temperature range of 240 and $330^{\circ} \mathrm{C}$ and at an elevated pressure (e.g., 1200 psig) over a catalyst. The formation of ethanol via the following chemical reaction is the primary desired reaction pathway.

$$
2 \mathrm{CO}+4 \mathrm{H}_{2} \rightarrow \mathrm{C}_{2} \mathrm{H}_{5} \mathrm{OH}+\mathrm{H}_{2} \mathrm{O}
$$

However, several side reactions are also possible including the formation of $\mathrm{C}_{3}$ through $\mathrm{C}_{5}$ alcohols; higher (greater than $\mathrm{C}_{1}$ ) oxygenates such as methyl acetate, ethyl acetate, acetic acid and acetaldehyde; and higher hydrocarbon gases such as ethane/ethene, propane/propene, butane/butene and methane $\left(\mathrm{CH}_{4}\right)$. Methane and saturated higher hydrocarbons (i.e., alkanes) are the least desirable side products because, unlike the other products, the options for further processing such as deoxygenation, olefin oligomerization and/or alkylation are limited. From a process design standpoint, $\mathrm{CH}_{4}$ contributes significantly to the overall cost because it must be either burned to capture the heating cost (which is relatively low value and signifies lost carbon efficiency) or steam reformed back to a mixture of $\mathrm{CO}$ and $\mathrm{H}_{2}$ at a high energy cost.

Several different catalysts promote the formation of ethanol and higher alcohols. These catalysts include alkali-doped Co-Mo sulfide, modified Fischer-Tropsch catalysts, and modified methanol (Cu-Zn) catalysts. Several types of these catalysts were screened early in the program. ${ }^{4}$ Of the catalysts screened, Rh-based catalysts were selected from the standpoint that the production of $\mathrm{C}_{2}+$ alcohols and oxygenates were optimized in terms of selectivity and space-time yield (STY). In early investigations Mn, was determined to be a highly beneficial promoter to enhance the activity of the Rh catalyst for converting syngas to a mixed oxygenate stream. ${ }^{4}$ The addition of Ir as a promoter to the Rh-Mn catalyst was identified as a preferred secondary promoter in subsequent catalyst screening experiments. The STY of $\mathrm{C}_{2}+$ oxygenates increased from 110 to $150 \mathrm{~g} / \mathrm{L}_{\text {cat }} / \mathrm{hr}$ without Ir to 290 to $880 \mathrm{~g} / \mathrm{L}_{\text {cat }} / \mathrm{hr}$ with Ir. ${ }^{2}$ Initially, the Rh-based catalysts were supported almost exclusively on $\mathrm{SiO}_{2}$. Recent experiments revealed carbon supports may demonstrate greater activity and selectivity to mixed alcohols $\left(\mathrm{C}_{2}+\right)$. Thus, catalysts with Rh-Mn or Rh-Mn-Ir formulations supported on silica and carbon supports have been given most of attention in optimization and longevity studies throughout this catalyst development program.

In 2009, a Department of Energy Review Panel suggested a computational component be added to the experimental mixed alcohol catalyst development program. The computational aspect of the program was initiated to gain a fundamental understanding of the nature of the catalytic sites (i.e., the nature of the RhMn-Ir mixtures on the support surfaces) and the effect of promoters in the metal mixture. Using Kinetic Monte Carlo (KMC) and micro-kinetic models based on $a b$ initio results with computationally configured metal clusters, the development of a reaction pathway was initiated to understand the elementary reaction steps $\mathrm{CO}, \mathrm{H}_{2}$, and the numerous intermediates undergo when progressing toward ethanol, oxygenates, higher hydrocarbons, or methane. A characterization effort was initiated in parallel with the computational effort to validate the computationally generated results with laboratory observations. In addition to supporting the computational effort, the characterization effort also had the goal of identifying 
trends in physical and chemical data of supports and prepared catalysts that could be leveraged to make better catalysts.

The goal of this report is to provide a comprehensive account of the characterization and computational efforts in the development of the Rh-based mixed alcohol synthesis. A series of reports focusing on catalyst screening, development and reactor testing are presented separately. ${ }^{1-4}$ The results of the characterization and computational efforts are presented independently herein, but characterization results that support computational findings are referenced throughout the computational section and vice versa. The characterization section is especially detailed and lengthy because one goal of this report was to catalogue the data from the catalyst and support analyses. 


\subsection{Characterization}

\subsection{Characterized Supports and Catalysts}

Numerous silica and carbon supports and prepared catalysts containing Rh-Mn with and without Ir or other promoters were analyzed by various techniques. All of the samples characterized are presented in this section. While all samples characterized are listed in this section, not all samples were necessarily measured with a given analytical technique. Specifics on the methods for a given analytical technique as well as the samples measured are given under the separate heading of the technique later in the report.

\subsubsection{Carbon Supports}

Over the course of the program, several carbon catalyst supports were analyzed. To facilitate a common nomenclature among the several scientists performing various analytical techniques, the supports were assigned "trivial names" of KOA 1 through KOA16. Table 2.1 lists the 16 carbon supports analyzed along with manufacturer's names, product numbers and lot numbers, and Pacific Northwest National Laboratory (PNNL) chemical identification barcode numbers or laboratory record book (LRB) identification numbers.

Table 2.1. Identification Numbers Assigned to Carbon Supports KOA 1 through16 with Descriptions, Lot Numbers, and PNNL Barcode or LRB Numbers

\begin{tabular}{llll}
\hline $\begin{array}{c}\text { Sample } \\
\text { Name }\end{array}$ & \multicolumn{1}{c}{ Manufacturer's Name and Product Description } & Lot Number & \multicolumn{1}{c}{ LRB Code/ } \\
\hline KOA 1 & Norit ROX 0.8 & 520020 & 295055 \\
KOA 2 & Norit Darco-LS & 854247 & 288402 \\
KOA 3 & Sibunit & $2000-1$ & 295149 \\
KOA 4 & Englehard High Surface Area Graphite (HSAG-1) (Code 1642FHX4, & KA01004 & 247505 \\
& BM0501006), S/O 40022036 & & \\
KOA 5 & Englehard High Surface Area Graphite (HSAG-2) (Code 1642SX4), & 20000012 & 247506 \\
& S/O 40022029 & & \\
KOA 6 & Hyperion Fibril Catalyst Support CS-05C-063-XD 1/16 in. Extrudate & $395-08$ & N/A \\
KOA 7 & Hyperion Fibril Catalyst Support CS-07C-063-XD 1/16 in. Extrudate & $395-10$ & N/A \\
KOA 8 & Hyperion Fibril Catalyst Support CS-02C-063-XD 1/16 in. Extrudate & $384-82$ & 296477 \\
KOA 9 & Cummins-Moore 5559 S & N/A & 315886 \\
KOA 10 & Cummins-Moore 5586 AW & N/A & 315885 \\
KOA 11 & Norit Darco HF-Washed & N/A & $14388-88-2$ \\
KOA 12 & Norit ROX 0.8 HF-Washed & N/A & $14388-88-1$ \\
KOA 13 & Pacco Carbon 90-100 CTC 12 x 20 M & $2007-2-22 A C$ & 274766 \\
KOA 14 & Kureha Carbon - Bead Shaped A.C. & 04293 & N/A \\
& From Petroleum Pitch; Type: UBAC C-70 R; 0.5-1.0 mm & & \\
KOA 15 & TimCal Timrex HSAG 300 High S.A. Graphite Powder & AA-057 & 249338 \\
KOA 16 & Jacobi Ecosorb BX-Max & 6339 & 268728 \\
\hline
\end{tabular}




\subsubsection{Hydrofluoric Acid-Washed, Graphitized, and Nitric Acid Functionalized Carbon Supports}

KOA 1, 2, 13, and 16 were selected for modification through hydrofluoric acid (HF) washing to demineralize the carbons through dissolution of inorganic impurities in the HF. A portion of the HFwashed carbons also were subjected to high temperature treatment to convert a portion of the carbon present in the samples to graphite. Information for the various demineralized and graphitized samples is presented in Table 2.2.

Table 2.2. Carbon Supports Subjected to Demineralization via Sequential HF Washing, HighTemperature Graphitization, and Surface Functionalization via Nitric Acid $\left(\mathrm{HNO}_{3}\right)$ Treatment.

\begin{tabular}{lll}
\hline \multicolumn{1}{c}{ Carbon Support } & \multicolumn{1}{c}{ Treatment } & $\begin{array}{c}\text { PNNL Barcode Number/ } \\
\text { LRB Number }\end{array}$ \\
\hline Pacco 90-100 CTC & Fresh (KOA 13) & 274766 \\
Lot 2007-2-22-AC & HF-Washed & $60074-118-\mathrm{A}$ \\
& HF-Washed and Graphitized & $60074-133-2$ \\
& HF-Washed, Graphitized and $\mathrm{HNO}_{3}$ Treated & $60074-148-1$ \\
Norit Rox 0.8 & Fresh (KOA 1) & 295055 \\
Lot 520020 & HF-Washed & $60074-118-\mathrm{B}$ \\
& HF-Washed and Graphitized & $60074-133-3$ \\
Norit Darco-LS & HF-Washed, Graphitized and $\mathrm{HNO}_{3}$ Treated & $60074-148-2$ \\
12x20 Lot 854247 & Fresh (KOA 2) & 288402 \\
& HF-Washed & $60074-118-\mathrm{C}$ \\
& HF-Washed and Graphitized & $60074-133-1$ \\
Jacobi Ecosorb BX- & HF-Washed, Graphitized and $\mathrm{HNO}_{3}$ Treated & $60074-148-3$ \\
Max Lot 6339 & Fresh (KOA 16) & 268728 \\
& HF-Washed & $60074-159$ \\
\hline
\end{tabular}

The HF-washed (demineralized) samples were prepared by washing the carbon support in 25\% HF. The desired mass of carbon was measured out, and the bulk density of the carbon support was measured. The carbon was washed in the $25 \%$ HF solution through the addition of HF while stirring the mixture followed by a 1 hr soak at room temperature. The HF then was decanted off the carbon and sent to waste. The washing process was repeated two more times (three washes total). After the third HF wash, the carbon was rinsed with warm deionized water. For the first water wash, water at a volume twice that of the carbon was added and allowed to soak for $1 \mathrm{hr}$. For subsequent washes, a 1:1 volume ratio of water to carbon was added, stirred, and decanted immediately. The subsequent washes were performed until a constant $\mathrm{pH}$ was observed, which could take over 10 subsequent washes. Finally, the carbon was dried in a drying oven overnight.

The graphitization heating program consisted of the following procedure performed on samples that had been previously subjected to the demineralization procedure outlined above: 
1. $20^{\circ} \mathrm{C} / \mathrm{min}$ ramp from room temperature to $1500^{\circ} \mathrm{C}$

2. $5^{\circ} \mathrm{C} / \mathrm{min}$ ramp from $1500^{\circ} \mathrm{C}$ to $1800^{\circ} \mathrm{C}$

3. Soak for $8 \mathrm{hr}$ at $1800^{\circ} \mathrm{C}$

4. $5^{\circ} \mathrm{C} / \mathrm{min}$ ramp from $1800^{\circ} \mathrm{C}$ to $1500^{\circ} \mathrm{C}$

5. $10^{\circ} \mathrm{C} / \mathrm{min}$ ramp from $1500^{\circ} \mathrm{C}$ to $1000^{\circ} \mathrm{C}$

6. $1000^{\circ} \mathrm{C}$ to room temperature cooling period in the furnace cool.

Helium flowed into the chamber with the carbon in the furnace at 0.5 standard cubic feet per hour; the chamber volume was $0.085 \mathrm{ft}^{3}$. Hence, the chamber atmosphere turned over approximately 23 times per hour.

After demineralization and graphitization, the carbon supports were subjected to $\mathrm{HNO}_{3}$ washing in an attempt to re-functionalize the carbon surface with oxygen containing moieties in a controlled manner. About 8 to $9 \mathrm{~g}$ of carbon support was added to $100 \mathrm{~mL}$ of $70 \% \mathrm{HNO}_{3}$ in water in a $200 \mathrm{~mL}$ beaker. The solution was mixed at $500 \mathrm{rpm}$ and heated to $60^{\circ} \mathrm{C}$ and held for $1 \mathrm{hr}$. The support then was filter washed with deionized water and vacuum dried overnight at $120^{\circ} \mathrm{C}$.

\subsubsection{Silica Supports}

In addition to the carbon supports analyzed, several silica supports also were measured. In contrast to the carbon supports, the silica supports were not assigned "trivial names" and are simply referred to as their manufacturer given name throughout the report. Table 2.3 lists the silica supports subjected to analytical measurements with their lot numbers and PNNL chemical identification bar codes or LRB numbers for prepared samples as appropriate.

Table 2.3. Silica Samples Subjected to Various Analytical Measurements

\begin{tabular}{|c|c|c|}
\hline Manufacturers Name & Lot Number & $\begin{array}{l}\text { PNNL Barcode Number/ } \\
\text { LRB Sample Number }\end{array}$ \\
\hline Sigma-Aldrich Grade 7754 & S47688-428 & 326554 \\
\hline Perlkat 97-0 & N/A & 351151 \\
\hline Sigma-Aldrich Grade 7734 & STBB2202 & 326553 \\
\hline Merck Aldrich Grade 60 & 10922BE & 304178 \\
\hline Norpro SS 61137 & 2006120514 & N/A \\
\hline Grace Grade 408 & 5210080182 & 315840 \\
\hline Davisil 645 & 067189 & N/A \\
\hline Norpro SS 61138 & 200991005 & N/A \\
\hline Engelhard Mod D & 040800639AB & 247780 \\
\hline Perlkat 79-3 & 090602148-50 AnS & 304154 \\
\hline Perlkat 29-3 & 090602148-50 AnS & 304156 \\
\hline $\begin{array}{l}\text { CBV3014 CY1.6 } \\
\text { H-ZSM5 Zeolite }(\mathrm{Si} / \mathrm{Al}=30)^{(\mathrm{a})}\end{array}$ & N/A & N/A \\
\hline
\end{tabular}

(a) The H-ZSM5 zeolite sample is reported for a basis of comparison. H-ZSM5 is considered to be a strong solid acid. 


\subsubsection{Prepared Catalysts}

\subsubsection{Baseline Catalysts}

\section{Catalyst Preparation}

Catalyst samples containing $\mathrm{Rh}$ and $\mathrm{Mn}$ or $\mathrm{Rh}, \mathrm{Mn}$ and Ir supported on silica gel or carbon nanotubes were prepared for the purpose of characterizing fresh, reduced, and spent catalysts following testing under a nominal set of operating conditions.

The three selected baseline catalysts consisted of:

1. A Davisil 645 silica-supported Rh-Mn-Ir

2. A Hyperion Fibril Catalyst Support CS-02C-063-XD multi-walled carbon nanotube (MWCNT) supported Rh-Mn catalyst

3. A Hyperion Fibril Catalyst Support CS-02C-063-XD MWCNT supported Rh-Mn-Ir catalyst.

The names of the parent catalysts, LRB numbers, metal concentrations and short and long descriptions for each catalyst are listed in Table 2.4. Throughout this report, the catalysts listed in Table 2.4 are referred to as "baseline catalysts," as these catalysts served to provide a baseline for several analytical techniques that could be compared and contrasted with other catalysts deemed of interest. Each of the baseline catalysts were characterized after four distinct phases in the catalyst lifecycle. These phases are described below:

1. Fresh - The silica catalyst was calcined in air at $400^{\circ} \mathrm{C}$ for $2 \mathrm{hr}$. The carbon-supported catalysts were uncalcined, and the metals presumably remained in the nitrate phase.

2. Reduced - The catalysts were reduced in a $10 \% \mathrm{H}_{2} / \mathrm{N}_{2}$ gas mixture by heating the samples via a specific temperature ramp. Each reduced catalyst sample was recovered from the reactor under an $\mathrm{N}_{2}$ atmosphere (i.e., in a valve-isolated reactor loaded into a glove box) and stored in a sealed container for later characterization analyses.

3. Low Temperature Operation - The catalysts were used for the synthesis of mixed alcohols up to a nominal temperature of $275^{\circ} \mathrm{C}$. The sample was recovered from the reactor under an $\mathrm{N}_{2}$ atmosphere and stored in a sealed container under the $\mathrm{N}_{2}$ atmosphere.

4. High Temperature Run - The catalysts were used for the synthesis of mixed alcohols up to a nominal temperature of $300^{\circ} \mathrm{C}$. The sample was recovered from the reactor under an $\mathrm{N}_{2}$ atmosphere and stored in a sealed container under the $\mathrm{N}_{2}$ atmosphere. 
Table 2.4. Baseline Catalysts Subjected to Various Characterization Tests

\begin{tabular}{|c|c|c|c|c|c|}
\hline \multirow[t]{2}{*}{$\begin{array}{c}\text { Parent Catalyst } \\
\text { and Run Number }\end{array}$} & \multicolumn{3}{|c|}{ Metal Loading (wt\%) } & \multirow[t]{2}{*}{$\begin{array}{c}\text { Short } \\
\text { Description } \\
\end{array}$} & \multirow[t]{2}{*}{ Long Description $^{(\mathrm{a})}$} \\
\hline & $\mathrm{Rh}$ & Mn & Ir & & \\
\hline \multicolumn{6}{|c|}{ 1x Rh-Mn-Ir on Davisil 645 Silica } \\
\hline 60074-53-A & 5.56 & 1.69 & 1.04 & Fresh & Fresh catalyst calcined in air at $400^{\circ} \mathrm{C}$ for $2 \mathrm{hr}$. \\
\hline 60074-53-A-R & 5.56 & 1.69 & 1.04 & Reduced & Catalyst 60074-53-A after reduction. \\
\hline $\begin{array}{l}\text { 60074-53-A } \\
\text { Run } 84\end{array}$ & 5.56 & 1.69 & 1.04 & Spent_LT & Tested to a maximum temperature of $275^{\circ} \mathrm{C}$ \\
\hline $\begin{array}{l}\text { 60074-53-A } \\
\text { Run 86 }\end{array}$ & 5.56 & 1.69 & 1.04 & Spent_HT & Tested to a maximum temperature of $300^{\circ} \mathrm{C}$ \\
\hline \multicolumn{6}{|c|}{ 2.11x Rh-Mn on Hyperion Fibril Catalyst Support CS-02C-063-XD Lot 384-82 MWCNTs } \\
\hline $60074-70-\mathrm{C}$ & 11.78 & 3.58 & 0 & Fresh & Fresh, uncalcined sample. \\
\hline $60074-70-\mathrm{C}-\mathrm{R}$ & 11.78 & 3.58 & 0 & Reduced & Catalyst $60074-70-\mathrm{C}-\mathrm{R}$ after reduction. \\
\hline $\begin{array}{l}\text { 60074-70-C } \\
\text { Run } 80\end{array}$ & 11.78 & 3.58 & 0 & Spent_LT & Reaction tested up to $276^{\circ} \mathrm{C}$ \\
\hline $\begin{array}{l}60074-70-C \\
\text { Run } 82\end{array}$ & 11.78 & 3.58 & 0 & Spent_HT & Reaction tested up to $299^{\circ} \mathrm{C}$ \\
\hline \multicolumn{6}{|c|}{ 2.11x Rh-Mn-Ir on Hyperion Fibril Catalyst Support CS-02C-063-XD Lot 384-82 MWCNTs } \\
\hline 60074-74-C & 11.78 & 3.58 & 2.19 & Fresh & Fresh, uncalcined sample \\
\hline 60074-74-C-R & 11.78 & 3.58 & 2.19 & Reduced & Catalyst 60074-74-C after reduction. \\
\hline $\begin{array}{l}\text { 60074-74-C } \\
\text { Run } 89\end{array}$ & 11.78 & 3.58 & 2.19 & Spent_LT & Reaction tested up to $275^{\circ} \mathrm{C}$ \\
\hline $\begin{array}{l}\text { 60074-109-A } \\
\text { Run 88 } \\
\end{array}$ & 11.78 & 3.58 & 2.19 & Spent_HT & Reaction tested up to $314^{\circ} \mathrm{C} .^{(b)}$ \\
\hline \multicolumn{6}{|c|}{$\begin{array}{l}\text { (a) Greater specific description on testing conditions (e.g., temperature and gas hourly space velocity [GHSV]) } \\
\text { can be found in Table } 2.5 \text {. } \\
\text { (b) Average temperature over sampling period. The catalyst temperature was increased slowly from } 300 \text { to } \\
305^{\circ} \mathrm{C} \text { over } 8 \mathrm{hr} \text { and then increased to } 318^{\circ} \mathrm{C} \text { for the remaining } 16 \mathrm{hr} \text {. }\end{array}$} \\
\hline
\end{tabular}

All catalysts were prepared using the incipient wetness impregnation technique with all metals in the impregnation solution co-impregnated onto Hyperion CS-02C-63 multi-walled carbon nanotube extrudates that were crushed and screened to $-60 /+100$ mesh particles prior to impregnation. The appropriate quantities of rhodium nitrate solution (10 wt\% Rh concentration in solution), a 50\% manganese nitrate tetrahydrate solution, and, if used, dihydrogen hexachloroiridium (IV) hydrate solution (14\% Ir) were combined with enough deionized water to bring the total volume of the impregnation solution to $90 \%$ of the water adsorption pore volume of the support. The metal concentrations were selected to produce a reduced catalyst containing the specified concentrations in Table 2.4.

The metal solution was impregnated in drop-wise fashion onto nominally $3 \mathrm{~g}$ of support in a small vial placed upright on a vibrating table to keep the support solids in motion during impregnation. The impregnated catalysts were dried under an infrared lamp while being shaken until a steady weight was achieved, and then vacuum dried overnight at $110^{\circ} \mathrm{C}$ in a drying oven. 


\section{Baseline Catalyst Reaction Testing}

A subsample of each catalyst was placed in a reactor tube and reduced using a $10 \% \mathrm{H}_{2}$-in- $\mathrm{N}_{2}$ gas mixture. All catalysts were heated in the reducing atmosphere to $100^{\circ} \mathrm{C}$, then heated to $220^{\circ} \mathrm{C}$ at $2.5^{\circ} \mathrm{C} / \mathrm{min}$ ramp-up rate and held at that temperature for $1 \mathrm{hr}$. Next, the catalysts were heated from $220^{\circ} \mathrm{C}$ to $260^{\circ} \mathrm{C}$ at a rate of $2^{\circ} \mathrm{C} / \mathrm{min}$ and held at that temperature for $8 \mathrm{hr}$. Finally, the catalysts were heated to $\sim 350^{\circ} \mathrm{C}$ at a rate of $1.5^{\circ} \mathrm{C} / \mathrm{min}$ and held at that temperature for $2 \mathrm{hr}$ before cooling to ambient temperature under the reducing gas atmosphere. Each reduced catalyst sample was recovered from the reactor under an $\mathrm{N}_{2}$ atmosphere and stored in a sealed container for later analyses.

The bench-scale tubular reactor system used to test catalysts is designed to operate at pressures up to 1400 psig and temperatures up to $400^{\circ} \mathrm{C}$. The reaction system is shown in Figure 2.1. The catalyst chamber is $1.67 \mathrm{~cm}$ long and $0.635 \mathrm{~cm}$ in diameter. It is filled to a depth of $0.39 \mathrm{~cm}$ with porous metal frit holding the catalyst in place. A $0.159 \mathrm{~cm}$ outer diameter thermocouple sheath is extended through the center of the reactor, creating an annulus-shaped catalyst chamber. Two thermocouples are installed inside the sheath are spaced so one thermocouple is at the center of the catalyst bed and the other just upstream of the bed. The catalyst temperature monitored during a test is based on the thermocouple temperature at the center of the catalyst bed. The reactor is heated with hot oil to obtain better temperature control because this approach more efficiently removes the heat of reaction, thus minimizing a thermal excursion when the carbon conversion is increased.

The syngas fed to the reactor is metered through a mass flow controller. The system also meters reducing gas $\left(10 \% \mathrm{H}_{2}\right.$ in $\left.\mathrm{N}_{2}\right)$ to the reactor during catalyst reduction and $\mathrm{N}_{2}$ to purge the system of air before starting a test. The raw product gas leaving the reactor is passed through one of two cold traps to condense liquids at $0^{\circ} \mathrm{C}$ and through a back-pressure flow regulator that controls the system pressure. Gas flow is redirected from one trap to the other to isolate the former trap for liquid sample recovery. Once the isolated cold trap is emptied, it is backfilled with pressurized $\mathrm{N}_{2}$ (not shown in the figure) to the system operating pressure, to minimize pressure fluctuations when the cold trap is next used to collect a liquid sample.

The nominal feed rate to the reactor is determined by calibrating the mass flow controllers at system pressure before the tests. A Bios DryCal flow meter located downstream of the back-pressure flow regulator is used for this calibration. Flow-meter readings are corrected for standard pressure and temperature. The flow meter also is used to monitor the product gas flow rate downstream of the liquid sample cold traps during each test.

Dry product gas samples for analysis in a gas chromatograph are obtained downstream of the backpressure regulator in a line separate from that containing the DryCal flow meter, as shown in Figure 2.1. The reactor inlet, catalyst bed, cold sample trap, ambient temperature, and the upstream gas and ambient pressures are monitored during tests.

Gas cylinders containing a specified syngas mixture are used in the tests. The gas mixture consists of $\mathrm{H}_{2}, \mathrm{CO}, \mathrm{CO}_{2}$, and $\mathrm{N}_{2}$. Most of the tests conducted with the Rh-based catalysts use a gas that has a nominal $\mathrm{H}_{2}$ : $\mathrm{CO}$ ratio of 2:1. The nominal concentrations of $\mathrm{CO}_{2}$ and $\mathrm{N}_{2}$ are each $4 \%$ in the gas mixture. 


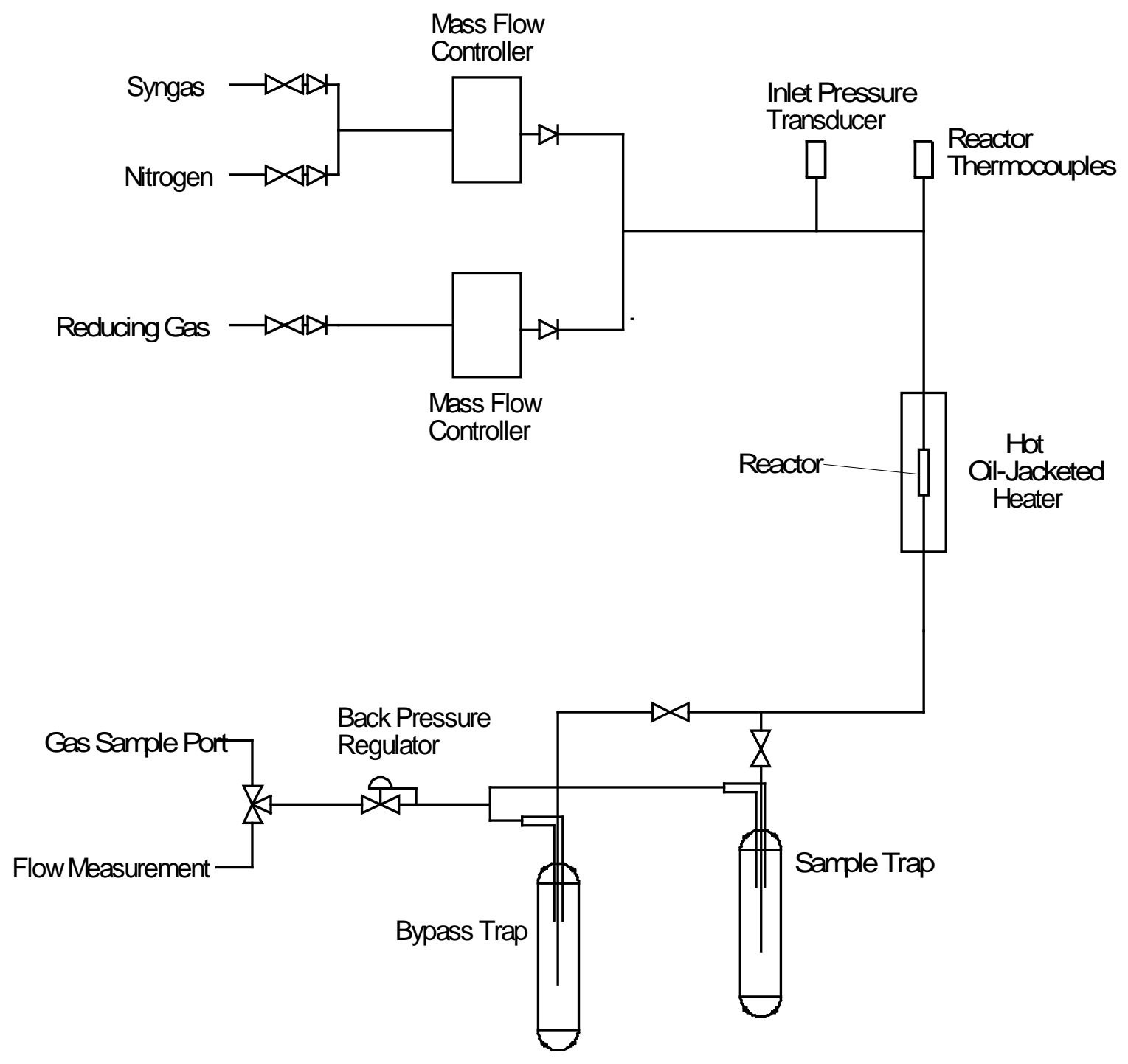

Figure 2.1. Simplified Diagram of the Reactor System used to Test Catalysts

A nominal $0.2 \mathrm{~g}$ subsample of a catalyst is loaded into the test reactor and reduced using a $10 \% \mathrm{H}_{2}$-in$\mathrm{N}_{2}$ gas mixture and using the same heating schedule previously described for preparing reduced catalysts. Once the reduced catalyst achieves ambient conditions, the reactor is flushed with the syngas at atmospheric pressure, pressurized to $1200 \mathrm{psig}$, and the desired flow rate is established. The reactor temperature is slowly raised to $240^{\circ} \mathrm{C}$ and maintained there for $\sim 30 \mathrm{hr}$ to allow the catalyst to age prior to conducting a test at those conditions. This temperature was selected for these tests because there is evidence that measurable reactions are taking place. The product stream is directed through one of the cold traps during this time. After aging the catalyst, the product stream is redirected through the other cold trap for a period sufficient for at least 10 bed volumes of gas feed (based on the operating pressure and gas feed rate) to pass through the cold trap. This period of time provides a representative gas sample and a sufficiently large liquid sample for subsequent analysis. The operating conditions are recorded before sampling with two or more grab samples of product gas obtained and analyzed in the gas chromatograph along with a feed gas sample and a calibration gas sample. The liquid recovered from the cold trap is weighed and, if two phases are present, separated into an aqueous phase and an organic phase. 
The weighed organic phase is not analyzed and is assumed to have a composition comparable to hexane for purposes of a carbon balance. The weighed aqueous phase is analyzed using a high-pressure liquid chromatograph to quantify the $\mathrm{C}_{1}$ to $\mathrm{C}_{5}$ oxygenates, which principally are alcohols, acids, aldehydes, esters, and any other products associated with significant peaks identified by the high-pressure liquid chromatograph. After sampling, a new set of test conditions (i.e., temperature and feed rate) are established through an empty cold trap and the sample collection period repeated to pass 10 bed volumes of gas through the trap. This procedure is repeated until a representative set of test conditions are obtained to evaluate catalyst performance in terms of $\mathrm{C}_{2}+$ oxygenate STY and carbon selectivity, and single-pass carbon conversion. At the completion of the test run the reactor is depressurized and cooled to room temperature in $\mathrm{N}_{2}$, isolated from the ambient air using valves upstream and downstream of the reactor, and then removed from the system. The catalyst is removed from the isolated reactor in an $\mathrm{N}_{2}$ environment and sealed in a container for later analysis. Table 2.5 lists the testing conditions for each test run.

Table 2.5. Sequential Test Conditions used for Evaluating Baseline Catalysts

\begin{tabular}{ccccccc}
\hline & Test Run & \multicolumn{5}{c}{ Test conditions - Temperature $\left({ }^{\circ} \mathrm{C}\right) ; \mathrm{GHSV}^{(\mathrm{a})},\left(\mathrm{L} / \mathrm{L}_{\text {cat }} / \mathrm{hr}\right)$} \\
\cline { 3 - 7 } Catalyst & No. & 1 & 2 & 3 & 4 & 5 \\
\hline $60074-53-A$ & 84 & $240 ; 7,900$ & $256 ; 7,900$ & $275 ; 7,900$ & $275 ; 7,900$ & $256 ; 7,900$ \\
$60074-53-A$ & 86 & $241 ; 7,900$ & $256 ; 7,900$ & $275 ; 7,900$ & $298 ; 12,000$ & $275 ; 7,900$ \\
$60074-70-C$ & 80 & $241 ; 7,900$ & $256 ; 7,900$ & $276 ; 7,900$ & $276 ; 7,900$ & $257 ; 7,900$ \\
$60074-70-C$ & 82 & $240 ; 7,900$ & $256 ; 7,900$ & $276 ; 7,900$ & $299 ; 12,000$ & $275 ; 7,900$ \\
$60074-74-C$ & 89 & $240 ; 7,900$ & $256 ; 7,900$ & $275 ; 7,900$ & $275 ; 7,900$ & $275 ; 7,900$ \\
$60074-109-A$ & 88 & $240 ; 7,900$ & $256 ; 7,900$ & $275 ; 7,900$ & $314^{(b)} ; 12,000$ & $275 ; 7,900$ \\
\hline
\end{tabular}

(a) GHSV $=$ feed flow rate at STP $\div$ volume of catalyst bed in the reactor

(b) Average temperature over sampling period. The catalyst temperature was increased slowly from 300 to $305^{\circ} \mathrm{C}$ for $8 \mathrm{hr}$, and then increased to $318^{\circ} \mathrm{C}$ for the remaining $16 \mathrm{hr}$.

\subsubsection{Prepared Catalysts of Interest}

Several other Rh-based catalysts deemed to be of interest due to high selectivity and/or STY to oxygenates were subjected to selected characterization techniques. Table 2.6 lists the identifying information for these catalysts. Of the catalysts characterized, six were fresh catalysts while another five were "spent" catalysts that had been used to produce mixed alcohols in a single tube reactor. The six fresh catalysts were tested as prepared (i.e., uncalcined) and in reduced form. The unreduced, fresh catalysts that were supported on carbon (e.g., MWCNTs) contained metals presumably still in nitrate form (for Rh and $\mathrm{Mn}$ ) or chloride form (for Ir). Reduction of the six fresh catalysts consisted of placing the catalysts in a $50 \mathrm{~mL} / \mathrm{min}$ stream of $10 \% \mathrm{H}_{2} / \mathrm{N}_{2}$ and heating to $100^{\circ} \mathrm{C}$; then in a $48 \mathrm{~min}$ ramp to $220^{\circ} \mathrm{C}$ with a $1 \mathrm{hr}$ soak, followed by ramping to $260^{\circ} \mathrm{C}$ for a $20 \mathrm{~min}$ period followed by an $8 \mathrm{hr}$ soak; and finally a ramp to $350^{\circ} \mathrm{C}$ for $1 \mathrm{hr}$ with a $2 \mathrm{hr}$ soak. This reduction procedure matches the reduction period for the activation of a mixed alcohol synthesis catalyst in a fixed-bed reactor. All of the reduced and spent catalysts were unloaded and transferred under inert conditions in a glove box and using Sample Saver ${ }^{\circledR}$ sealable sample containers to prevent contamination of the samples with air. 
Table 2.6. Identification of Metal-Loaded Catalyst Samples with Descriptions and Metal Loadings and Support Identification

\begin{tabular}{|c|c|c|c|c|c|c|}
\hline $\begin{array}{l}\text { LRB/Run } \\
\text { ID }\end{array}$ & Rh (wt\%) & $\begin{array}{c}\mathrm{Mn} \\
\text { (wt\%) }\end{array}$ & $\operatorname{Ir}(w t \%)$ & Os (wt $\%$ & Description & Support \\
\hline \multicolumn{7}{|c|}{ Fresh and Reduced Catalysts } \\
\hline 60074-13-A & 8.11 & 2.74 & 0 & 0 & 1.5x Rh-Mn on Hyperion 395-10 & KOA 7 \\
\hline 60074-52-B & 8.34 & 2.54 & 1.03 & 0 & 1.5x Rh-Mn, 1.0 x Ir on Hyperion 395-11 & $\mathrm{KOA} 8^{(\mathrm{a})}$ \\
\hline 60074-56-A & 8.34 & 2.54 & 0 & 0 & 1.5x Rh-Mn on Hyperion 395-08 & KOA 6 \\
\hline 60074-56-B & 8.34 & 2.54 & 0 & 0 & 1.5x Rh-Mn on Hyperion 395-10 & KOA 7 \\
\hline 60074-59-C & 11.778 & 3.587 & 2.189 & 0 & 2.11x Rh-Mn 2.11x Ir on Hyperion 384-82 & KOA 8 \\
\hline 60074-67-B & 8.34 & 2.54 & 1.56 & 0 & 1.5x Rh-Mn, 1.5x Ir on Hyperion 384-82 & KOA 8 \\
\hline \multicolumn{7}{|c|}{ Spent Catalysts } \\
\hline Run 31 & 11.78 & 3.58 & 0 & 0 & 2.11x Rh-Mn on Hyperion 384-82 & KOA 8 \\
\hline Run 32 & 8.34 & 2.54 & 0 & 1.53 & 1.5x Rh-Mn 1.0x Os on Hyperion 395-11 & KOA $8^{(\mathrm{a})}$ \\
\hline Run $33^{(\mathrm{b}, \mathrm{c})}$ & 11.778 & 3.587 & 2.189 & 0 & 2.11x Rh-Mn 2.11x Ir on Hyperion 384-82 & KOA 8 \\
\hline Run $34^{(\mathrm{d})}$ & 11.778 & 3.587 & 2.189 & 0 & 2.11x Rh-Mn 2.11x Ir on Hyperion 384-82 & KOA 8 \\
\hline Run $39^{c}$ & 11.778 & 3.587 & 2.189 & 0 & 2.11x Rh-Mn 2.11x Ir on Hyperion 384-82 & KOA 8 \\
\hline \multicolumn{7}{|c|}{$\begin{array}{l}\text { (a) Hyperion } 395-11 \text { is the same product as Hyperion } 384-82 \text {. } \\
\text { (b) Run } 33 \text { was subjected to a temperature excursion to } \sim 330^{\circ} \mathrm{C} \text {. } \\
\text { (c) Rh, Mn, and Ir were co-impregnated simultaneously. } \\
\text { (d) Rh and Mn were co-impregnated followed by Ir impregnation. }\end{array}$} \\
\hline
\end{tabular}

\subsection{Ash Testing}

\subsubsection{Carbon Supports KOA 1-15}

The concentrations of inorganic ash in carbon support samples KOA 1 through 15 (Table 2.1) were determined by heating the carbon supports in air. Porcelain crucibles with lids were used to ash the samples. The clean, empty crucibles were initially fired at $700^{\circ} \mathrm{C}$ for at least $1 \mathrm{hr}$ to remove any moisture and burn any other impurities present. Following the initial firing, a crucible was loaded with $\sim 5 \mathrm{~cm}^{3}(0.2-0.7 \mathrm{~g})$ of carbon support. The temperature was ramped from room temperature to $700^{\circ} \mathrm{C}$ at a rate of $1.5^{\circ} \mathrm{C} / \mathrm{min}$, soaked at $700^{\circ} \mathrm{C}$ for $5 \mathrm{hr}$, and returned to room temperature. Upon cooling, the mass was recorded. For samples KOA 1 through 12, the crucibles were filled and fired again. The wt\% ash reported is from the mass of the total ash after the second firing for samples KOA 1 through 12. Table 2.7 gives the results of the ash tests for samples KOA 1 through 15. 
Table 2.7. Gravimetrically Determined Concentrations of Ash after Firing the Carbon Supports at $700^{\circ} \mathrm{C}$ for $5 \mathrm{hr}$ in Air

\begin{tabular}{|c|c|c|}
\hline Trivial Name & Support Name & Ash, wt $\%$ \\
\hline KOA 1 & Norit ROX 0.8 & $4.5 \%$ \\
\hline KOA 2 & Norit Darco-LS & $13.8 \%$ \\
\hline KOA 3 & Sibunit & $0.2 \%$ \\
\hline KOA 4 & Engelhard High Surface Area Graphite (HSAG-1) & $0.1 \%$ \\
\hline KOA $5^{(a)}$ & Engelhard High Surface Area Graphite (HSAG-2) & $0.3 \%$ \\
\hline KOA 6 & Hyperion Fibril Catalyst Support CS-05C-063-XD; Lot 395-08 & $3.4 \%$ \\
\hline KOA 7 & Hyperion Fibril Catalyst Support CS-07C-063-XD;. Lot 395-10 & $3.7 \%$ \\
\hline KOA 8 & Hyperion Fibril Catalyst Support CS-02C-063-XD; Lot 384-82 & $1.0 \%$ \\
\hline KOA 9 & Cummins-Moore $5559 \mathrm{~S}$ & $2.3 \%$ \\
\hline KOA 10 & Cummins-Moore 5586 AW & $8.5 \%$ \\
\hline KOA 11 & Norit Darco HF-Washed & $4.7 \%$ \\
\hline KOA 12 & Norit ROX 0.8 HF-Washed & $0.3 \%$ \\
\hline $\mathrm{KOA} 13^{(\mathrm{b})}$ & Pacco Carbon 90-100 CTC 12 × $20 \mathrm{M}$ & $2.1 \%$ \\
\hline KOA $14^{(\mathrm{b})}$ & Kureha Carbon - Bead Shaped A.C. & $0.1 \%$ \\
\hline $\mathrm{KOA} 15^{(\mathrm{b})}$ & TimCal Timrex HSAG 300 High S.A. Graphite Powder & $0.5 \%$ \\
\hline
\end{tabular}

(a) KOA 5 only uses data from the second ashing because calculating the ash wt $\%$ from both the first and second ashing resulted in a negative value.

(b) Only one sample was ashed.

Ash was observed visually in all experiments even though the concentration of ash in some samples was very low. Ash in the carbon supports likely comes from the source of the carbon if it was a naturally occurring organic source (such as peat or pine wood) or from binder added by the manufacturer to aid formation of engineered shapes. Some samples, such as KOA 3, 4 and 5, had very low ash content. In the case of samples KOA 4 and 5, the manufacturing process potentially used a high-temperature graphitization furnace, which could have volatilized a large amount of inorganic material. Two samples that initially had high ash content-samples KOA 1 and 2-had substantially reduced amounts of ash present after washing with HF, which presumably dissolved much of the inorganic material. The carbons that are naturally occurring from sources such as peat, wood, and coconut shells such as samples KOA 1 , 2 and 13, respectively, exhibited greater concentrations of ash. Finally, samples KOA 6, 7, and 8, which are bound carbon nanotubes formed into engineered extrudates, showed relatively high ash concentrations when compared with other synthetic carbons. The high ash concentration is most likely due not only to the presence of a binder but also from a substrate used to grow the carbon nanotubes.

\subsubsection{HF-Washed and Graphitized Carbon Supports}

The demineralized and graphitized carbon supports were ashed in order to investigate the amount of ash present after the support modifications. About $5 \mathrm{~cm}^{3}$ of the samples were subjected to ash testing in covered alumina crucibles. All samples were heated to $700^{\circ} \mathrm{C}$ at a rate of $1.5^{\circ} \mathrm{C} / \mathrm{min}$ with a $5 \mathrm{hr}$ soak at $700^{\circ} \mathrm{C}$ in a muffle furnace in static air. Visual inspection of the graphitized samples revealed that the samples were not completely burned away after the $5 \mathrm{hr}$ soak. Therefore, the graphitized samples were heated again to $700^{\circ} \mathrm{C}$ at a rate of $3.0^{\circ} \mathrm{C} / \mathrm{min}$ with a $7 \mathrm{hr}$ soak. Visual inspection of the graphitized samples after the $12 \mathrm{hr}$ total soak revealed the samples were completely ashed. The results of the ashing of the fresh and modified carbon supports are presented in Table 2.8. 
Table 2.8. Gravimetrically Determined Concentration of Ash after Firing the Carbon Supports in Air

\begin{tabular}{llr}
\hline \multicolumn{1}{c}{ Carbon Support } & & $\begin{array}{r}\text { Ash, } \\
\text { wt\% }\end{array}$ \\
\hline Pacco 90-100 CTC & Fresh & 2.1 \\
Lot 2007-2-22-AC & HF-Washed & $<0.1$ \\
& HF-Washed and Graphitized & $<0.1$ \\
Norit Rox 0.8 & Fresh & 4.5 \\
Lot 520020 & HF-Washed & 0.1 \\
& HF-Washed and Graphitized & $<0.1$ \\
Norit Darco-LS 12x20 & Fresh & 13.8 \\
Lot 854247 & HF-Washed & 1.5 \\
& HF-Washed and Graphitized & 0.1 \\
Jacobi Ecosorb BX- & Fresh & 10.2 \\
Max Lot 6339 & HF-Washed & 1.4 \\
& HF-Washed (Duplicate) & 1.3 \\
& HF-Washed and Graphitized & 0.1 \\
& HF-Washed and Graphitized (Duplicate) & 0.3 \\
\hline
\end{tabular}

HF washing significantly reduced the amount of ash in each of the carbon supports. Graphitization further reduced the amount of ash present in the modified supports such that only $\sim 0.1 \mathrm{wt} \%$ of ash was typically left after ashing. While the concentration of ash in the modified samples was significantly diminished, ash was visually observed for all samples (see Figure 2.2).

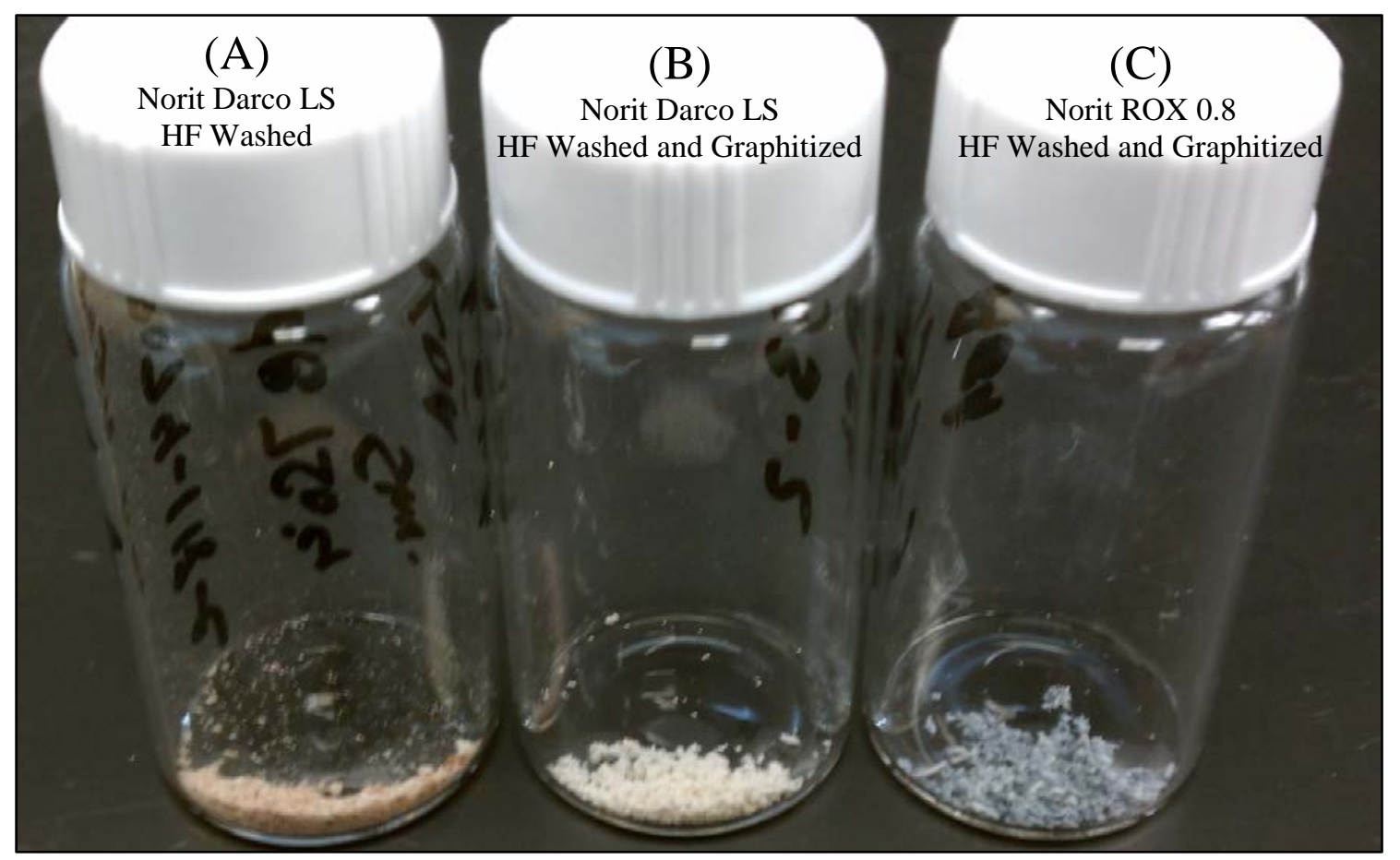

Figure 2.2. Ash Collected after Burning Various Treated Carbons. Sample A was produced by a burn at $700^{\circ} \mathrm{C}$ for $5 \mathrm{hr}$ while samples (B) and (C) were produced by cumulative burns totaling $12 \mathrm{hr}$ at $700^{\circ}$ C. (A): Sample 60074-118-C, which was Norit Darco-LS 12x20, Lot 854247 subjected to HF washing. (B): Sample 60074-133-3, which was Norit Darco-LS 12x20, Lot 854247 subjected to HF washing and subsequently graphitization. (C) Sample 60074-1332, which was Norit ROX 0.8, Lot 520020 subjected to HF washing and subsequent graphitization. 


\subsection{Elemental Analysis of Carbon and Silica Supports via Inductively Coupled Plasma}

\subsubsection{Carbon Support Analysis}

\subsubsection{1 $\mathrm{HNO}_{3}$ Digestion of Carbon Support Ash Residue}

Elemental analysis was conducted on the ash of samples KOA 1-6 using inductively coupled plasma (ICP). ICP analysis requires liquid solution; thus, the ash from KOA1-6 needed to be dissolved. First, concentrated $\mathrm{HNO}_{3}$ was added to the ash from a given sample and heated on a hot plate at $\sim 85^{\circ} \mathrm{C}$ for an extended period. However, even after extended heat treatment in $\mathrm{HNO}_{3}$, solids were still observed in all samples. This indicated that not all of the ash was dissolved, and, thus the quantitative nature of the ICP results from this digestion method should be questioned.

The ash tests and elemental analysis using ICP were conducted in two separate batches. Samples KOA 1 through 3 were analyzed in the first batch, and samples KOA 4 through 6 were analyzed in the second batch. In each batch, a blank ceramic crucible was subjected to heating using the ashing procedure and ICP analysis for quality assurance purposes. The blank from the KOA 1-3 set had low levels $\left(<50 \mathrm{ppm}\right.$ ) of $\mathrm{Ca}, \mathrm{Al}, \mathrm{Na}, \mathrm{K}$, and $\mathrm{Si}$ in the in the solution prepared from $\mathrm{HNO}_{3}$ treatment of the empty crucible. The blank from the KOA 4-6 set had very low ( $<5 \mathrm{ppm})$ levels of Ca and $\mathrm{Na}$ in the solution prepared from $\mathrm{HNO}_{3}$ treatment of the empty crucible. No other elements were detected in the analysis of the blank solutions.

The results of elemental analysis via ICP on samples KOA 1-6 are presented in Table 2.9. Table 2.9 is presented as the concentration of oxides that are present with the most stable oxidation state of the associated cation. The results are tabulated as oxides because this is the most likely form of the inorganic species after calcination at $700^{\circ} \mathrm{C}$ in air.

ICP analysis of the ash produced from high-temperature calcination of the carbon supports for samples KOA 1 through 6 revealed the presence of several inorganic species. Alkaline oxides, such as $\mathrm{Na}_{2} \mathrm{O}$ and $\mathrm{K}_{2} \mathrm{O}$ were observed in all samples. Previous testing in the mixed alcohols project has revealed $\mathrm{Na}$ and $\mathrm{K}$ are active catalyst components. Other inorganic species that could potentially affect catalytic performance such as Fe, Ca, Mg, Ba, P, and S were observed in several samples.

The results of Table 2.9 are qualitative in nature due to the fact that $\mathrm{HNO}_{3}$ was unable to sufficiently dissolve all of the ash present for analysis. Elemental analysis with ICP requires all of the solids present in a sample to be dissolved so the amount of ash present can be quantified. The lack of quantitative agreement between ash testing and ICP analysis is illustrated by the bottom row in Table 2.9, which shows very large differences between the oxides accounted for with ICP versus the concentration of ash for samples KOA 2, 3, 5, and 6. 
Table 2.9. Concentration of Inorganic Oxide Species Observed from ICP Analysis of the Ash Produced from High-Temperature Calcination of the Carbon Supports for Samples KOA 1 through 6

\begin{tabular}{|c|c|c|c|c|c|c|}
\hline & KOA 1, wt\% & KOA 2, wt\% & KOA 3, wt $\%$ & KOA 4, wt\% & KOA 5, wt $\%$ & KOA 6, wt $\%$ \\
\hline $\mathrm{Al}_{2} \mathrm{O}_{3}$ & 0.627 & 0.672 & 0.002 & 0.004 & 0.001 & 0.767 \\
\hline $\mathrm{As}_{2} \mathrm{O}_{5}$ & & 0.001 & & & & \\
\hline $\mathrm{B}_{2} \mathrm{O}_{3}$ & & & & & & 0.003 \\
\hline $\mathrm{BaO}$ & 0.001 & 0.020 & & 0.002 & & \\
\hline $\mathrm{CaO}$ & 0.593 & 0.242 & 0.040 & 0.040 & & 0.003 \\
\hline $\mathrm{Co}_{3} \mathrm{O}_{4}$ & & 0.001 & & & & \\
\hline $\mathrm{Cr}_{2} \mathrm{O}_{3}$ & 0.026 & & & & 0.007 & 0.001 \\
\hline $\mathrm{CuO}$ & 0.137 & 0.004 & & 0.001 & & 0.001 \\
\hline $\mathrm{Fe}_{2} \mathrm{O}_{3}$ & 0.325 & 0.258 & 0.010 & 0.010 & & 0.424 \\
\hline $\mathrm{K}_{2} \mathrm{O}$ & 0.116 & 0.033 & 0.041 & 0.005 & 0.002 & 0.001 \\
\hline $\mathrm{MgO}$ & 0.514 & 0.120 & 0.004 & 0.002 & & \\
\hline $\mathrm{MnO}$ & 0.011 & 0.003 & 0.001 & 0.002 & & 0.004 \\
\hline $\mathrm{MoO}_{3}$ & & & & & & 0.059 \\
\hline $\mathrm{Na}_{2} \mathrm{O}$ & 0.447 & 0.179 & 0.132 & 0.007 & 0.007 & 0.002 \\
\hline $\mathrm{NiO}$ & & 0.002 & & & & \\
\hline $\mathrm{P}_{2} \mathrm{O}_{5}$ & 0.310 & 0.003 & 0.003 & 0.006 & 0.001 & \\
\hline $\mathrm{SO}_{2}$ & 0.820 & 0.305 & 0.132 & 0.016 & 0.004 & \\
\hline $\mathrm{SiO}_{2}$ & 0.383 & 0.032 & 0.001 & 0.004 & 0.001 & 0.002 \\
\hline $\mathrm{SrO}$ & & 0.015 & & & & \\
\hline $\mathrm{TiO}_{2}$ & 0.033 & 0.038 & & & & \\
\hline $\mathrm{V}_{2} \mathrm{O}_{5}$ & & 0.004 & & & & \\
\hline $\mathrm{ZnO}$ & 0.082 & & & & & 0.001 \\
\hline $\mathrm{ZrO}_{2}$ & & 0.002 & & & & \\
\hline Total, Wt\% ${ }^{(\mathrm{a})}$ & 4.42 & 1.93 & 0.37 & 0.10 & 0.02 & 1.27 \\
\hline Ash Wt\% ${ }^{(\mathrm{b})}$ & 4.5 & 13.8 & 0.2 & 0.1 & 0.3 & 3.4 \\
\hline Difference, $\%^{(\mathrm{c})}$ & 7 & 86 & 85 & 0 & 93 & 62 \\
\hline \multicolumn{7}{|c|}{$\begin{array}{l}\text { (a) Sum of the oxides observed from ICP elemental analysis. } \\
\text { (b) Concentration of ash in the carbon samples determined via ash testing. } \\
\text { (c) Difference between the wt\% of oxides determined from ICP testing versus ash testing. }\end{array}$} \\
\hline
\end{tabular}

\subsubsection{2 $\mathrm{HNO}_{3} / \mathrm{HF}$ Digestion of Supports for ICP Analysis}

Initially, only samples KOA 1 through 6 were analyzed for elemental composition with ICP because of the inability of $\mathrm{HNO}_{3}$ and gentle heating (i.e., on a hot plate at $85^{\circ} \mathrm{C}$ ) to completely dissolve the ash from these samples. An alternative method to dissolve more of the inorganic material was initiated using sample KOA 2, which was chosen because of its high concentration of ash present after firing. The alternative method for preparing KOA 2 for elemental analysis began with the carbon support as opposed to the ash from the support after firing. We started to start with the carbon support instead of the ash to minimize the possibility of contamination by using a closed batch system during carbon support digestion in a sealed Teflon vessel that could be heated in a microwave oven. The closed vessel method facilitated a higher degree of confidence in the quantification of the impurities present in the carbon. 
For development of the method with sample KOA 2, the support was initially treated with hydrogen peroxide $\left(\mathrm{H}_{2} \mathrm{O}_{2}\right)$ to pre-oxidize the carbon support and make it more susceptible to oxidative attack from $\mathrm{HNO}_{3}$. Concentrated $\mathrm{HNO}_{3}$ then was added to the carbon support and heated to $200^{\circ} \mathrm{C}$ at a pressure of $200 \mathrm{psi}$ in the sealed Teflon container. The $\mathrm{HNO}_{3}$ application was performed to remove the carbon in the support via oxidation as well as to dissolve a portion of the inorganic material present. After $\mathrm{HNO}_{3}$ treatment, the sample was treated with concentrated $\mathrm{HF}$ at $200^{\circ} \mathrm{C}$ and $200 \mathrm{psi}$ in the same Teflon container in the microwave oven. After this treatment, some visible solids were still present, but we decided to conduct elemental analysis of the liquid with ICP using this sample and compare it with the previous application.

The next iteration in method development was to take the sample that was digested in $\mathrm{HNO}_{3}$ but still contained solids and add HF to the solution in order to digest the components not soluble in $\mathrm{HNO}_{3}$. About $1 \mathrm{mg}$ of $\mathrm{HF}$ was added to the mixture, weighed, and the solution was digested in the microwave again to $200^{\circ} \mathrm{C}$ at a pressure of $200 \mathrm{psi}$. Because HF is not compatible with the ICP apparatus, a saturated boric acid solution was added to the sample to complex the excess HF after the digestion.

The results of the elemental analysis from ICP on sample KOA 2 prepared via the ashing/ $\mathrm{HNO}_{3}$ method as well as the support/ $\mathrm{HNO}_{3} / \mathrm{HF}$ method are presented in Table 2.10. The results in Table 2.10 are again presented in terms of oxides.

The alternative method of $\mathrm{HNO}_{3}$ digestion with subsequent $\mathrm{HF}$ digestion on sample KOA 2 resulted in increased concentrations of refractory oxides such as alumina, silica, titania, and zirconia determined via ICP analysis. Thus, $11.1 \mathrm{wt} \%$ of sample KOA 2 was accounted for as inorganic oxides, which was in better agreement with the concentration of ash (13.8 wt\%) observed from calcining the sample at $700^{\circ} \mathrm{C}$ than from only the $\mathrm{HNO}_{3}$ treatment used previously.

After determining that a $\mathrm{HNO}_{3} / \mathrm{HF}$ digestion of the carbon supports was a superior method for quantifying the concentrations of impurities in the carbon supports, nine other carbon supports were analyzed using this method, albeit without the initial $\mathrm{H}_{2} \mathrm{O}_{2}$ pretreatment step, which should not be a compromising difference. Approximately $150 \mathrm{mg}$ of sample was digested in $10 \mathrm{~mL}$ concentrated $\mathrm{HNO}_{3}$ in the microwave oven. After this initial digestion, another digestion in the microwave oven was performed after the addition of $1 \mathrm{~mL}$ of concentrated HF. Samples then were transferred and diluted to $50 \mathrm{~mL}$ with deionized water and $1 \mathrm{~mL}$ of saturated boric acid solution was added. ICP analysis then was performed on the digestion solutions.

Table 2.11 lists the impurities observed in carbon supports KOA 1, 4 through 8, and 11 through 15 from ICP testing after using the $\mathrm{HNO}_{3} / \mathrm{HF}$ digestion method. The impurities are reported as the oxides most likely to be present in after ashing. In addition to the oxides reported in Table 2.11, scans were performed for $\mathrm{W}$ and $\mathrm{Mn}$ in the samples, but neither element was detected.

The results in Table 2.11 generally correlate well with the concentration of ash observed gravimetrically after firing at $700^{\circ} \mathrm{C}$ for $5 \mathrm{hr}$. While there is poor agreement with some supports such as KOA 4, 12, 14, and 15, this could be due to low ash concentrations observed after firing. In other words, the lack of agreement between ICP and gravimetrically determined ash numbers could be due to the small amount of ash left after firing at $700^{\circ} \mathrm{C}$, which makes accurate gravimetric determinations challenging. 
Table 2.10. Comparison of the $\mathrm{HNO}_{3}$ and Alternative $\mathrm{HNO}_{3} / \mathrm{HF}$ Methods of Acid Pretreatment on Sample KOA 2 for ICP Analysis

\begin{tabular}{|ccc|}
\hline & $\mathrm{HNO}_{3}$ & $\mathrm{HNO}_{3} / \mathrm{HF} \mathrm{Pretreatment}$ \\
\hline $\mathrm{Al}_{2} \mathrm{O}_{3}$ & $0.672 \%$ & $1.367 \%$ \\
$\mathrm{BaO}$ & $0.020 \%$ & $0.084 \%$ \\
$\mathrm{CaO}$ & $0.242 \%$ & $0.243 \%$ \\
$\mathrm{CuO}$ & $0.004 \%$ & $0.013 \%$ \\
$\mathrm{Cr}_{2} \mathrm{O}_{3}$ & $0.001 \%$ & $0.000 \%$ \\
$\mathrm{Fe}_{2} \mathrm{O}_{3}$ & $0.258 \%$ & $0.332 \%$ \\
$\mathrm{~K}_{2} \mathrm{O}$ & $0.033 \%$ & $0.031 \%$ \\
$\mathrm{MgO}$ & $0.121 \%$ & $0.057 \%$ \\
$\mathrm{MnO}$ & $0.003 \%$ & $0.004 \%$ \\
$\mathrm{Na} \mathrm{Na}_{2} \mathrm{O}$ & $0.179 \%$ & $0.243 \%$ \\
$\mathrm{NiO}$ & $0.002 \%$ & $0.000 \%$ \\
$\mathrm{P}_{2} \mathrm{O}_{5}$ & $0.003 \%$ & $0.000 \%$ \\
$\mathrm{SO}_{2}$ & $0.305 \%$ & $0.720 \%$ \\
$\mathrm{SiO}_{2}$ & $0.032 \%$ & $7.661 \%$ \\
$\mathrm{SrO}_{\mathrm{TiO}}$ & $0.015 \%$ & $0.020 \%$ \\
$\mathrm{~V}_{2} \mathrm{O}_{5}$ & $0.038 \%$ & $0.271 \%$ \\
$\mathrm{ZrO}_{2}$ & $0.004 \%$ & $0.005 \%$ \\
$\mathrm{Total}$ & $0.001 \%$ & $0.011 \%$ \\
\hline & $\mathbf{1 . 9 3 \%}$ & $\mathbf{1 1 . 1 \%}$ \\
\hline
\end{tabular}

It is interesting to note that only samples KOA 4, 5, and 15 were not completely digested by acid during microwave treatment. These samples are most likely highly graphitic in nature and, therefore, are most likely less susceptible to oxidative attack by $\mathrm{HNO}_{3}$ during the digestion process. The $\mathrm{H}_{2} \mathrm{O}_{2}$ pretreatment step may have helped with the digestion of the graphitic materials. However, it is likely that most of the inorganic material was digested by the multiple digestion method used in this method and only the refractory graphitic carbon was left after digestion.

KOA 6, 7, and 8 exhibited relatively high concentrations of Fe, which possibly remained after its use as a substrate for growing the carbon nanotubes. Alumina also was present at relatively high concentrations in KOA 6, 7, and 8, possibly from its use as a binder material in the carbon nanotubes. The relatively high concentration of Fe could be significant in that it could be catalytically active. Additionally, Fe could have been alloying with $\mathrm{Rh}, \mathrm{Mn}$, and/or $\mathrm{C}$ to form a mixed phase responsible for the unidentified HfIr analogue orthorhombic crystalline phase identified in Section 2.9.

HF washing reduced the amount of inorganic impurities present in the carbon supports. Comparison of samples KOA 1 and KOA 12, which were Norit ROX 0.8 before and after HF washing, respectively, showed that the concentration $\mathrm{Al}_{2} \mathrm{O}_{3}$ and $\mathrm{SiO}_{2}$ dropped significantly. This indicates that HF washing did remove a large amount of the inorganic impurities. Similarly, comparison of the inorganic concentrations in sample KOA 2 (Table 2.4) with sample KOA 11 showed that a large amount of $\mathrm{SiO}_{2}$ and $\mathrm{Al}_{2} \mathrm{O}_{3}$ was removed by washing the Norit Darco-LS with HF. 
Table 2.11. Concentration in wt\% of Inorganic Oxide Species Observed from ICP Analysis on Selected Carbon Supports after Microwave Digestions using $\mathrm{HNO}_{3}$ and $\mathrm{HF}$

\begin{tabular}{|c|c|c|c|c|c|c|c|c|c|c|c|}
\hline $\begin{array}{l}\text { Trivial } \\
\text { Name }\end{array}$ & KOA 1 & $\mathrm{KOA} 4^{(\mathrm{b})}$ & KOA $5^{(\mathrm{b})}$ & KOA 6 & KOA 7 & KOA 8 & KOA 11 & KOA 12 & KOA 13 & KOA 14 & $\mathrm{KOA} 15^{(\mathrm{b})}$ \\
\hline $\begin{array}{c}\text { Support } \\
\text { Description }\end{array}$ & $\begin{array}{c}\text { Norit } \\
\text { ROX } 0.8\end{array}$ & $\begin{array}{l}\text { Engelhard } \\
\text { HSAG-1 }\end{array}$ & $\begin{array}{l}\text { Engelhard } \\
\text { HSAG-2 }\end{array}$ & $\begin{array}{c}\text { Hyperion } \\
395-08\end{array}$ & $\begin{array}{c}\text { Hyperion } \\
395-10\end{array}$ & $\begin{array}{c}\text { Hyperion } \\
384-82\end{array}$ & $\begin{array}{c}\text { Norit Darco HF- } \\
\text { Washed }\end{array}$ & $\begin{array}{c}\text { Norit ROX } 0.8 \\
\text { HF-Washed }\end{array}$ & Рассо & Kureha & Timcal \\
\hline $\mathrm{Al}_{2} \mathrm{O}_{3}$ & 0.123 & 0.023 & 0.015 & 1.872 & 1.770 & 0.642 & 0.198 & 0.032 & 0.026 & 0.009 & 0.015 \\
\hline $\mathrm{BaO}$ & & 0.001 & & & & & 0.106 & & & & \\
\hline $\mathrm{CaO}$ & 0.038 & 0.056 & 0.017 & 0.000 & 0.000 & 0.000 & 0.185 & 0.020 & 0.062 & & 0.091 \\
\hline $\mathrm{Cr}_{2} \mathrm{O}_{3}$ & 0.001 & & & & & & & & & & \\
\hline $\mathrm{CuO}$ & & & & & 0.001 & & 0.004 & & & & \\
\hline $\mathrm{Fe}_{2} \mathrm{O}_{3}$ & 0.054 & 0.019 & & 1.185 & 1.101 & 0.154 & 0.010 & 0.020 & 0.014 & 0.001 & 0.036 \\
\hline $\mathrm{HgO}$ & 0.003 & 0.005 & 0.008 & & & 0.008 & & 0.008 & 0.012 & 0.065 & 0.005 \\
\hline $\mathrm{K}_{2} \mathrm{O}$ & 0.014 & 0.017 & 0.027 & & & & & & 0.891 & & \\
\hline $\mathrm{MgO}$ & 0.043 & 0.002 & & & & & 0.099 & 0.017 & 0.085 & & \\
\hline $\mathrm{MoO}_{3}$ & & & & 0.095 & 0.098 & 0.017 & & & & & \\
\hline $\mathrm{Na}_{2} \mathrm{O}$ & 0.080 & 0.030 & 0.028 & 0.019 & 0.016 & 0.015 & 0.050 & 0.022 & 0.113 & 0.018 & 0.022 \\
\hline $\mathrm{P}_{2} \mathrm{O}_{5}$ & 0.014 & & & & 0.025 & & & & 0.064 & & \\
\hline $\mathrm{SO}_{2}$ & 1.083 & 0.018 & 0.006 & 0.000 & 0.655 & 0.000 & 0.358 & 0.959 & 0.050 & 0.016 & 0.004 \\
\hline $\mathrm{SiO}_{2}$ & 3.6 & 1.3 & 0.9 & 0.9 & 0.9 & 0.6 & 1.3 & 1.1 & 1.1 & 1.1 & 1.7 \\
\hline $\mathrm{SrO}$ & & & & & & & 0.014 & & & & \\
\hline $\mathrm{TiO}_{2}$ & 0.018 & 0.002 & 0.002 & & & & 0.068 & 0.005 & & & 0.012 \\
\hline $\begin{array}{l}\text { Total, } \\
\mathrm{Wt}^{(\mathrm{c})}\end{array}$ & 5.1 & 1.5 & 1.0 & 4.1 & 4.6 & 1.4 & 2.4 & 2.2 & 2.4 & 1.2 & 1.9 \\
\hline Ash, Wt\% ${ }^{(\mathrm{d})}$ & 4.5 & 0.1 & 0.3 & 3.4 & 3.7 & 1 & 4.7 & 0.3 & 2.1 & 0.1 & 0.5 \\
\hline $\begin{array}{l}\text { (a) In additi } \\
\text { (b) Samples } \\
\text { (c) Sum of t } \\
\text { (d) Concentl }\end{array}$ & $\begin{array}{l}\text { to the oxid } \\
\text { ere not dige } \\
\text { oxides obs } \\
\text { ion of ash i }\end{array}$ & $\begin{array}{l}\text { reported, sca } \\
\text { ed completel } \\
\text { ed from ICF } \\
\text { he carbon sa }\end{array}$ & $\begin{array}{l}\text { for Mn and } \\
\text { and the solid } \\
\text { lemental ana } \\
\text { les determin }\end{array}$ & $\begin{array}{l}\text { also were p } \\
\text { quid mixtur } \\
\text { is } \\
\text { via ash tes }\end{array}$ & $\begin{array}{l}\text { ormed, but } \\
\text { as centrifu } \\
\text { This dat? }\end{array}$ & $\begin{array}{l}\text { her elemen } \\
\text { prior to ICP } \\
\text { o are report }\end{array}$ & $\begin{array}{l}\text { as observed. } \\
\text { alysis. } \\
\text { in Table 2.3. }\end{array}$ & & & & \\
\hline
\end{tabular}




\subsubsection{Demineralized and Graphitized Carbon Supports and SGL Carbon Material}

The demineralized (HF-washed) and graphitized supports listed in Table 2.2 were digested and analyzed via ICP. The $\mathrm{HNO}_{3}$ digestion method followed by $\mathrm{HF}$ treatment to digest the carbon and subsequently dissolve all inorganics, including $\mathrm{SiO}_{2}$, was utilized. The results for the four modified carbon supports are reported in Table 2.12 through Table 2.15. Included in the notes row of the tables are any notes that regarding samples that did not digest to completion (i.e had optically observable solids remaining after digestion). The graphitized samples were not digested to completion even after multiple (3-5) microwave cycles in concentrated $\mathrm{HNO}_{3}$.

The SGL carbon support was digested and analyzed in the same batch with the demineralized and graphitized material. Little was known initially of the SGL carbon support other than that it was relatively economical and abundant as a refinery co-product. The material was digested using the $\mathrm{HNO}_{3} / \mathrm{HF}$ method and subjected to ICP for elemental analysis. The results are given in Table 2.16. 
Table 2.12. ICP Analysis of the Inorganic Impurities Present in the Pacco 90-100 CT Lot 2007-2-22ACC, the Support after HF Washing, and after HF Washing and Graphitization in wt\%. ND indicates no data because the analytes were not specifically analyzed for. Blank cells indicate the analyte was not identified up to the specified detection limit.

\begin{tabular}{|c|c|c|c|c|c|c|}
\hline \multirow{2}{*}{$\frac{\text { Analyte }}{\mathrm{Wt} \%}$} & \multirow{2}{*}{$\begin{array}{c}\begin{array}{c}\text { Detection } \\
\text { Limit }\end{array} \\
\mathrm{Wt} \% \\
\end{array}$} & \multirow[t]{2}{*}{ Untreated } & \multicolumn{2}{|c|}{$\begin{array}{l}\text { HF-Washed } \\
\text { 60074-118-A }\end{array}$} & \multicolumn{2}{|c|}{$\begin{array}{l}\text { HF-Washed and Graphitized } \\
\text { 60074-133-1 }\end{array}$} \\
\hline & & & Primary & Duplicate & Primary & Duplicate \\
\hline $\mathrm{Al}_{2} \mathrm{O}_{3}$ & 0.004 & 0.026 & 0.007 & 0.008 & 0.010 & 0.011 \\
\hline $\mathrm{As}_{2} \mathrm{O}_{3}$ & 0.003 & ND & 0.003 & 0.003 & 0.002 & 0.002 \\
\hline $\mathrm{BaO}$ & 0.002 & & & & & \\
\hline $\mathrm{CaO}$ & 0.003 & 0.062 & 0.022 & 0.025 & 0.016 & 0.015 \\
\hline $\mathrm{CdO}$ & 0.002 & ND & & & & \\
\hline $\mathrm{CoO}$ & 0.003 & ND & & & & \\
\hline $\mathrm{Cr}_{2} \mathrm{O}_{3}$ & 0.003 & & & & & \\
\hline $\mathrm{CuO}$ & 0.003 & & 0.001 & 0.001 & 0.000 & 0.000 \\
\hline $\mathrm{Fe}_{2} \mathrm{O}_{3}$ & 0.003 & 0.014 & & & & \\
\hline $\mathrm{K}_{2} \mathrm{O}$ & 0.002 & 0.891 & 0.013 & 0.031 & 0.002 & 0.012 \\
\hline $\mathrm{MgO}$ & 0.003 & 0.085 & 0.009 & 0.011 & & \\
\hline $\mathrm{MnO}$ & 0.003 & & & & & \\
\hline $\mathrm{MoO}_{3}$ & 0.003 & & & & & \\
\hline $\mathrm{Na}_{2} \mathrm{O}$ & 0.003 & 0.113 & 0.011 & 0.039 & & 0.020 \\
\hline $\mathrm{NiO}$ & 0.003 & ND & & & & \\
\hline $\mathrm{P}_{2} \mathrm{O}_{5}$ & 0.005 & 0.064 & & & & \\
\hline $\mathrm{PbO}$ & 0.002 & ND & & & & \\
\hline PdO & 0.002 & ND & & & & \\
\hline $\mathrm{PtO}$ & 0.002 & ND & & & & \\
\hline $\mathrm{ReO}_{2}$ & 0.002 & ND & & & & \\
\hline $\mathrm{Rh}_{2} \mathrm{O}_{3}$ & 0.002 & ND & 0.010 & 0.011 & 0.010 & 0.012 \\
\hline $\mathrm{Ru}_{2} \mathrm{O}_{3}$ & 0.002 & ND & & & & \\
\hline $\mathrm{SO}_{2}$ & 0.004 & 0.050 & 0.036 & 0.037 & 0.010 & 0.010 \\
\hline $\mathrm{SiO}_{2}$ & 0.004 & 1.070 & 0.180 & 0.253 & 0.115 & 0.164 \\
\hline $\mathrm{SnO}$ & 0.002 & ND & 0.002 & 0.002 & 0.002 & 0.002 \\
\hline $\mathrm{SrO}$ & 0.002 & & & & & \\
\hline $\mathrm{TiO}_{2}$ & 0.003 & & & & & \\
\hline $\mathrm{V}_{2} \mathrm{O}_{5}$ & 0.004 & ND & & & & \\
\hline $\mathrm{WO}_{3}$ & 0.003 & & & & & \\
\hline $\mathrm{Y}_{2} \mathrm{O}_{3}$ & 0.003 & ND & & & & \\
\hline $\mathrm{ZnO}$ & 0.002 & ND & & & & \\
\hline $\mathrm{ZrO}_{2}$ & 0.003 & ND & & 0.004 & & \\
\hline Total $^{(a)}$ & & 2.4 & 0.3 & 0.4 & 0.2 & 0.3 \\
\hline $\mathrm{Ash}^{(\mathrm{b})}$ & & $2.1^{(\mathrm{b})}$ & & & & \\
\hline Notes & & & No $v$ & e solids & Small am & digested \\
\hline
\end{tabular}

(a) Total concentration of impurities reported as oxides as determined by ICP.

(b) Total concentration (wt\%) of ash as determined by firing at $700^{\circ} \mathrm{C}$ for $5 \mathrm{hr}$.

(c) Total concentration (wt\%) of ash as determined by firing at $700^{\circ} \mathrm{C}$ for $17 \mathrm{hr}$ total. 
Table 2.13. ICP Analysis of Inorganic Impurities in the Norit ROX 0.8 Lot 520020, the Support after HF Washing, and after HF Washing and Graphitization in wt\%. ND indicates no data because the analytes were not specifically analyzed for. Blank cells indicate the analyte was not identified to the specified detection limit.

\begin{tabular}{|c|c|c|c|c|c|c|}
\hline \multirow{2}{*}{$\frac{\text { Analyte }}{\mathrm{Wt} \%}$} & \multirow{2}{*}{$\begin{array}{c}\text { Detection } \\
\text { Limit } \\
\mathrm{Wt} \%\end{array}$} & \multirow[t]{2}{*}{ Untreated } & \multicolumn{2}{|c|}{$\begin{array}{c}\text { HF-Washed } \\
\text { 60074-118-B } \\
\end{array}$} & \multicolumn{2}{|c|}{$\begin{array}{l}\text { HF-Washed and Graphitized } \\
\text { 60074-133-3 } \\
\end{array}$} \\
\hline & & & Primary & Duplicate & Primary & Duplicate \\
\hline $\mathrm{Al}_{2} \mathrm{O}_{3}$ & 0.004 & 0.123 & 0.026 & 0.029 & 0.026 & 0.026 \\
\hline $\mathrm{As}_{2} \mathrm{O}_{3}$ & 0.003 & ND & 0.003 & 0.003 & & \\
\hline $\mathrm{BaO}$ & 0.002 & & & & & \\
\hline $\mathrm{CaO}$ & 0.003 & 0.0 .038 & 0.010 & 0.016 & 0.012 & 0.019 \\
\hline $\mathrm{CdO}$ & 0.002 & ND & & & & \\
\hline $\mathrm{CoO}$ & 0.003 & ND & & & & \\
\hline $\mathrm{Cr}_{2} \mathrm{O}_{3}$ & 0.003 & 0.001 & & & & \\
\hline $\mathrm{CuO}$ & 0.003 & & & & & \\
\hline $\mathrm{Fe}_{2} \mathrm{O}_{3}$ & 0.003 & 0.054 & 0.013 & 0.015 & 0.000 & 0.000 \\
\hline $\mathrm{K}_{2} \mathrm{O}$ & 0.002 & 0.014 & 0.044 & 0.044 & 0.014 & 0.011 \\
\hline $\mathrm{MgO}$ & 0.003 & 0.043 & 0.015 & 0.025 & & \\
\hline $\mathrm{MnO}$ & 0.003 & & & & & \\
\hline $\mathrm{MoO}_{3}$ & 0.003 & & & & & \\
\hline $\mathrm{Na}_{2} \mathrm{O}$ & 0.003 & 0.080 & 0.053 & 0.053 & 0.024 & 0.020 \\
\hline $\mathrm{NiO}$ & 0.003 & & & & & \\
\hline $\mathrm{P}_{2} \mathrm{O}_{5}$ & 0.005 & 0.014 & & & & \\
\hline $\mathrm{PbO}$ & 0.002 & ND & & & & \\
\hline PdO & 0.002 & ND & & & & \\
\hline $\mathrm{PtO}$ & 0.002 & ND & & & & \\
\hline $\mathrm{ReO}_{2}$ & 0.002 & ND & & & & \\
\hline $\mathrm{Rh}_{2} \mathrm{O}_{3}$ & 0.002 & ND & 0.011 & 0.011 & 0.012 & 0.010 \\
\hline $\mathrm{Ru}_{2} \mathrm{O}_{3}$ & 0.002 & ND & & & & \\
\hline $\mathrm{SO}_{2}$ & 0.004 & 1.083 & 0.771 & 0.760 & 0.045 & 0.055 \\
\hline $\mathrm{SiO}_{2}$ & 0.004 & 3.637 & 0.483 & 0.617 & 0.337 & 0.292 \\
\hline $\mathrm{SnO}$ & 0.002 & ND & 0.002 & 0.002 & 0.002 & 0.002 \\
\hline $\mathrm{SrO}$ & 0.002 & & & & & \\
\hline $\mathrm{TiO}_{2}$ & 0.003 & 0.018 & & & 0.004 & 0.006 \\
\hline $\mathrm{V}_{2} \mathrm{O}_{5}$ & 0.004 & ND & & & & \\
\hline $\mathrm{WO}_{3}$ & 0.003 & & & & & \\
\hline $\mathrm{Y}_{2} \mathrm{O}_{3}$ & 0.003 & ND & & & & \\
\hline $\mathrm{ZnO}$ & 0.002 & ND & & & & \\
\hline $\mathrm{ZrO}_{2}$ & 0.003 & ND & & & 0.003 & \\
\hline Total $^{(\mathrm{a})}$ & & 5.1 & 1.4 & 1.6 & 0.5 & 0.4 \\
\hline $\operatorname{Ash}^{(b)}$ & & $4.5^{(\mathrm{b})}$ & & & & \\
\hline Notes & & & No vis & e solids & Significant & it undigested \\
\hline
\end{tabular}

(a) Total concentration of impurities reported as oxides as determined by ICP.

(b) Total concentration ( $\mathrm{wt} \%$ ) of ash as determined by firing at $700^{\circ} \mathrm{C}$ for $5 \mathrm{hr}$.

(c) Total concentration (wt\%) of ash as determined by firing at $700^{\circ} \mathrm{C}$ for $17 \mathrm{hr}$ total. 
Table 2.14. ICP Analysis of Inorganic Impurities in the Norit Darco-LS 12x20 Lot 854247, in the Support after HF Washing, and after HF Washing and Graphitization in wt\%. ND indicates no data because the analytes were not specifically analyzed for. Blank cells indicate the analyte was not identified up to the specified detection limit.

\begin{tabular}{|c|c|c|c|c|c|c|}
\hline \multirow[t]{2}{*}{ Analyte } & \multirow{2}{*}{$\begin{array}{c}\begin{array}{c}\text { Detection } \\
\text { Limit }\end{array} \\
\mathrm{Wt} \%\end{array}$} & \multirow[t]{2}{*}{ Untreated } & \multicolumn{2}{|c|}{$\begin{array}{l}\text { HF-Washed } \\
\text { 60074-118-C }\end{array}$} & \multicolumn{2}{|c|}{$\begin{array}{l}\text { HF-Washed and Graphitized } \\
\text { 60074-133-2 }\end{array}$} \\
\hline & & & Primary & Duplicate & Primary & Duplicate \\
\hline $\mathrm{Al}_{2} \mathrm{O}_{3}$ & 0.004 & 1.367 & 0.026 & 0.029 & 0.014 & 0.015 \\
\hline $\mathrm{As}_{2} \mathrm{O}_{3}$ & 0.003 & ND & 0.003 & 0.003 & & \\
\hline $\mathrm{BaO}$ & 0.002 & 0.084 & & & 0.049 & 0.048 \\
\hline $\mathrm{CaO}$ & 0.003 & 0.243 & 0.010 & 0.016 & 0.038 & 0.039 \\
\hline $\mathrm{CdO}$ & 0.002 & ND & & & & \\
\hline $\mathrm{CoO}$ & 0.003 & ND & & & & \\
\hline $\mathrm{Cr}_{2} \mathrm{O}_{3}$ & 0.003 & & & & & \\
\hline $\mathrm{CuO}$ & 0.003 & 0.013 & & & & \\
\hline $\mathrm{Fe}_{2} \mathrm{O}_{3}$ & 0.003 & 0.332 & 0.013 & 0.015 & & \\
\hline $\mathrm{K}_{2} \mathrm{O}$ & 0.002 & 0.031 & 0.044 & 0.044 & 0.012 & 0.013 \\
\hline $\mathrm{MgO}$ & 0.003 & 0.057 & 0.015 & 0.025 & & \\
\hline $\mathrm{MnO}$ & 0.003 & 0.004 & & & & \\
\hline $\mathrm{MoO}_{3}$ & 0.003 & ND & & & & \\
\hline $\mathrm{Na}_{2} \mathrm{O}$ & 0.003 & 0.243 & 0.053 & 0.053 & 0.024 & 0.024 \\
\hline $\mathrm{NiO}$ & 0.003 & & & & & \\
\hline $\mathrm{P}_{2} \mathrm{O}_{5}$ & 0.005 & & & & & \\
\hline $\mathrm{PbO}$ & 0.002 & ND & & & & \\
\hline PdO & 0.002 & ND & & & & \\
\hline $\mathrm{PtO}$ & 0.002 & ND & & & & \\
\hline $\mathrm{ReO}_{2}$ & 0.002 & ND & & & & \\
\hline $\mathrm{Rh}_{2} \mathrm{O}_{3}$ & 0.002 & ND & 0.011 & 0.011 & 0.011 & 0.012 \\
\hline $\mathrm{Ru}_{2} \mathrm{O}_{3}$ & 0.002 & ND & & & & \\
\hline $\mathrm{SO}_{2}$ & 0.004 & 0.720 & 0.771 & 0.760 & 0.044 & 0.045 \\
\hline $\mathrm{SiO}_{2}$ & 0.004 & 7.661 & 0.483 & 0.617 & 0.201 & 0.275 \\
\hline $\mathrm{SnO}$ & 0.002 & ND & 0.002 & 0.002 & 0.002 & 0.002 \\
\hline $\mathrm{SrO}$ & 0.002 & 0.020 & & & 0.003 & 0.003 \\
\hline $\mathrm{TiO}_{2}$ & 0.003 & 0.271 & & & 0.069 & 0.090 \\
\hline $\mathrm{V}_{2} \mathrm{O}_{5}$ & 0.004 & 0.005 & & & & \\
\hline $\mathrm{WO}_{3}$ & 0.003 & $\mathrm{ND}$ & & & & \\
\hline $\mathrm{Y}_{2} \mathrm{O}_{3}$ & 0.003 & ND & & & & \\
\hline $\mathrm{ZnO}$ & 0.002 & ND & & & & \\
\hline $\mathrm{ZrO}_{2}$ & 0.003 & 0.011 & & & 0.004 & 0.004 \\
\hline Total $^{(\mathrm{a})}$ & & 11.1 & 1.4 & 1.6 & 0.5 & 0.6 \\
\hline Ash & & $13.8^{(\mathrm{b})}$ & & & & \\
\hline Notes & & & $\begin{array}{r}\text { Primar } \\
\text { particulate } \\
\text { vi }\end{array}$ & $\begin{array}{l}\text { ine black } \\
\text { licate had no } \\
\text { lids. }\end{array}$ & Significan & ndigested \\
\hline $\begin{array}{ll}\text { (a) Total } \\
\text { (b) Total } \\
\text { (c) Total }\end{array}$ & $\begin{array}{l}\text { centration } \\
\text { centration } \\
\text { centration }\end{array}$ & $\begin{array}{l}\text { urities rep } \\
\text { of ash as } \\
\text { of ash as }\end{array}$ & $\begin{array}{l}\text { s oxides as } \\
\text { ined by firi } \\
\text { ined by fir }\end{array}$ & $\begin{array}{l}\text { ined by ICP. } \\
00^{\circ} \mathrm{C} \text { for } 5 \mathrm{hr} \text {. } \\
00^{\circ} \mathrm{C} \text { for } 17 \mathrm{~h}\end{array}$ & & \\
\hline
\end{tabular}


Table 2.15. ICP Analysis of Inorganic Impurities in the Jacobi Ecosorb BX-Max in Untreated Material, after HF Washing, and after HF Washing and Graphitization in wt\%. Blank cells indicate the analyte was not identified up to the specified detection limit.

\begin{tabular}{|c|c|c|c|c|c|c|}
\hline \multirow[t]{2}{*}{ Analyte } & \multirow{2}{*}{$\begin{array}{c}\text { Detection } \\
\text { Limit }\end{array}$} & \multirow{2}{*}{$\frac{\text { Untreated }}{\text { Primary }}$} & \multicolumn{2}{|c|}{ HF-Washed } & \multicolumn{2}{|c|}{ HF-Washed and Graphitized } \\
\hline & & & Primary & Duplicate & Primary & Duplicate \\
\hline $\mathrm{AgO}$ & 0.002 & & & & & \\
\hline $\mathrm{Al}_{2} \mathrm{O}_{3}$ & 0.004 & 1.672 & 0.639 & 0.771 & 0.006 & 0.003 \\
\hline $\mathrm{As}_{2} \mathrm{O}_{3}$ & 0.003 & & & & & \\
\hline $\mathrm{Au}_{2} \mathrm{O}_{3}$ & 0.002 & & & & & \\
\hline $\mathrm{BaO}$ & 0.002 & 0.029 & 0.028 & 0.035 & 0.016 & 0.014 \\
\hline $\mathrm{CaO}$ & 0.003 & 0.323 & 0.266 & 0.319 & 0.050 & 0.039 \\
\hline $\mathrm{CdO}$ & 0.002 & & & & & \\
\hline $\mathrm{CoO}$ & 0.003 & & & & & \\
\hline $\mathrm{Cr}_{2} \mathrm{O}_{3}$ & 0.003 & & & & & \\
\hline $\mathrm{CuO}$ & 0.003 & & & & & \\
\hline $\mathrm{Fe}_{2} \mathrm{O}_{3}$ & 0.003 & 0.481 & 0.014 & 0.016 & & \\
\hline $\mathrm{K}_{2} \mathrm{O}$ & 0.002 & 0.056 & 0.007 & 0.008 & & \\
\hline $\mathrm{MgO}$ & 0.003 & 0.233 & 0.200 & 0.227 & & \\
\hline $\mathrm{MnO}$ & 0.003 & & & & & \\
\hline $\mathrm{MoO}_{3}$ & 0.003 & & & & & \\
\hline $\mathrm{Na}_{2} \mathrm{O}$ & 0.003 & 0.257 & 0.097 & 0.108 & & 0.009 \\
\hline $\mathrm{NiO}$ & 0.003 & & & & & \\
\hline $\mathrm{P}_{2} \mathrm{O}_{5}$ & 0.005 & & & & & \\
\hline $\mathrm{PbO}$ & 0.002 & & & & & \\
\hline $\mathrm{PdO}$ & 0.002 & & & & & \\
\hline $\mathrm{PtO}$ & 0.002 & & & & & \\
\hline $\mathrm{ReO}_{2}$ & 0.002 & & & & & \\
\hline $\mathrm{Rh}_{2} \mathrm{O}_{3}$ & 0.002 & & & & & \\
\hline $\mathrm{Ru}_{2} \mathrm{O}_{3}$ & 0.002 & & & & & \\
\hline $\mathrm{SO}_{2}$ & 0.004 & 0.355 & 0.445 & 0.440 & 0.047 & 0.041 \\
\hline $\mathrm{SiO}_{2}$ & 0.004 & 4.880 & 0.251 & 0.316 & 0.162 & 0.202 \\
\hline $\mathrm{SnO}$ & 0.002 & & & & & \\
\hline $\mathrm{SrO}$ & 0.002 & 0.003 & 0.002 & 0.003 & & \\
\hline $\mathrm{TiO}_{2}$ & 0.003 & 0.124 & 0.017 & 0.027 & 0.021 & 0.014 \\
\hline $\mathrm{V}_{2} \mathrm{O}_{5}$ & 0.004 & & & & & \\
\hline $\mathrm{WO}_{3}$ & 0.003 & & & & & \\
\hline $\mathrm{Y}_{2} \mathrm{O}_{3}$ & 0.003 & & & & & \\
\hline $\mathrm{ZnO}$ & 0.002 & & & & & \\
\hline $\mathrm{ZrO}_{2}$ & 0.003 & 0.005 & 0.003 & 0.003 & 0.003 & 0.003 \\
\hline \multicolumn{2}{|c|}{ Total $^{(a)}$} & 8.4 & 2.0 & 2.3 & 0.3 & 0.3 \\
\hline \multicolumn{2}{|c|}{ Ash } & $10.2^{(\mathrm{b})}$ & $1.4^{(\mathrm{b})}$ & $1.3^{(\mathrm{b})}$ & $0.1^{(\mathrm{c})}$ & $0.3^{(\mathrm{c})}$ \\
\hline \multicolumn{2}{|c|}{ Notes } & & & & \multicolumn{2}{|c|}{ Undigested black solids } \\
\hline
\end{tabular}

(a) Total concentration of impurities reported as oxides as determined by ICP.

(b) Total concentration (wt\%) of ash as determined by firing at $700^{\circ} \mathrm{C}$ for $5 \mathrm{hr}$.

(c) Total concentration (wt\%) of ash as determined by firing at $700^{\circ} \mathrm{C}$ for $17 \mathrm{hr}$ total. 
Table 2.16. ICP Analysis of Inorganic Impurities in the Untreated SGL Carbon Material. Blank cells indicate that the analyte was not identified up to the specified detection limit.

\begin{tabular}{|c|c|c|c|}
\hline Analyte & Detection Limit & & bon \\
\hline & $(\mathrm{wt} \%)$ & Primary & Duplicate \\
\hline $\mathrm{AgO}$ & 0.002 & & \\
\hline $\mathrm{Al}_{2} \mathrm{O}_{3}$ & 0.004 & 1.236 & 0.510 \\
\hline $\mathrm{As}_{2} \mathrm{O}_{3}$ & 0.003 & & \\
\hline $\mathrm{Au}_{2} \mathrm{O}_{3}$ & 0.002 & 0.003 & \\
\hline $\mathrm{BaO}$ & 0.002 & 0.010 & 0.011 \\
\hline $\mathrm{CaO}$ & 0.003 & 1.207 & 1.343 \\
\hline $\mathrm{CdO}$ & 0.002 & & \\
\hline $\mathrm{CoO}$ & 0.003 & & \\
\hline $\mathrm{Cr}_{2} \mathrm{O}_{3}$ & 0.003 & 0.012 & 0.029 \\
\hline $\mathrm{CuO}$ & 0.003 & & \\
\hline $\mathrm{Fe}_{2} \mathrm{O}_{3}$ & 0.003 & 0.097 & 0.215 \\
\hline $\mathrm{K}_{2} \mathrm{O}$ & 0.002 & 0.746 & 0.814 \\
\hline $\mathrm{MgO}$ & 0.003 & 0.194 & 0.231 \\
\hline $\mathrm{MnO}$ & 0.003 & 0.029 & 0.033 \\
\hline $\mathrm{MoO}_{3}$ & 0.003 & 0.007 & 0.007 \\
\hline $\mathrm{Na}_{2} \mathrm{O}$ & 0.003 & 0.229 & 0.214 \\
\hline $\mathrm{NiO}$ & 0.003 & 0.009 & 0.009 \\
\hline $\mathrm{P}_{2} \mathrm{O}_{5}$ & 0.005 & 0.102 & 0.131 \\
\hline $\mathrm{PbO}$ & 0.002 & & \\
\hline PdO & 0.002 & 0.002 & \\
\hline PtO & 0.002 & & \\
\hline $\mathrm{ReO}_{2}$ & 0.002 & & \\
\hline $\mathrm{Rh}_{2} \mathrm{O}_{3}$ & 0.002 & & \\
\hline $\mathrm{Ru}_{2} \mathrm{O}_{3}$ & 0.002 & & \\
\hline $\mathrm{SO}_{2}$ & 0.004 & 0.051 & 0.061 \\
\hline $\mathrm{SiO}_{2}$ & 0.004 & 0.078 & 0.085 \\
\hline $\mathrm{SnO}$ & 0.002 & & \\
\hline $\mathrm{SrO}$ & 0.002 & 0.006 & 0.007 \\
\hline $\mathrm{TiO}_{2}$ & 0.003 & 0.007 & 0.007 \\
\hline $\mathrm{V}_{2} \mathrm{O}_{5}$ & 0.004 & & \\
\hline $\mathrm{WO}_{3}$ & 0.003 & 0.003 & 0.003 \\
\hline $\mathrm{Y}_{2} \mathrm{O}_{3}$ & 0.003 & & \\
\hline $\mathrm{ZnO}$ & 0.002 & & \\
\hline $\mathrm{ZrO}_{2}$ & 0.003 & 0.006 & 0.005 \\
\hline \multicolumn{2}{|c|}{ Total $^{(a)}$} & 4.0 & 3.7 \\
\hline
\end{tabular}




\subsubsection{ICP Analysis of the Low Pressure Methanol Synthesis Catalyst}

ICP was also used to analyze the methanol synthesis catalyst developed by ICI (now Johnson Matthey). About $50 \mathrm{mg}$ of the catalyst was digested in a microwave oven in $10 \mathrm{~mL}$ of $\mathrm{HNO}_{3}$ at $200^{\circ} \mathrm{C}$. After digestion, the sample was diluted to $50 \mathrm{~mL}$ and analyzed for the $\mathrm{Zn}, \mathrm{Cu}, \mathrm{Mg}$, and $\mathrm{Al}$ concentrations.

ICP analysis determined the ICI catalyst to be $19.5 \mathrm{wt} \% \mathrm{ZnO}, 54.1 \mathrm{wt} \% \mathrm{CuO}, 1.0 \mathrm{wt} \% \mathrm{MgO}$, and $12.5 \mathrm{wt} \% \mathrm{Al}_{2} \mathrm{O}_{3}$. The sum of the identified oxides is $87.1 \mathrm{wt} \%$. It is possible that the concentration of $\mathrm{Al}_{2} \mathrm{O}_{3}$ is artificially low. It was previously observed that $\mathrm{HNO}_{3}$ is not the optimal acid for alumina digestion.

\subsubsection{ICP Analysis of Silica Mixed Alcohol Catalyst Supports}

Silica mixed alcohol catalyst supports were quantitatively analyzed for elemental analysis using ICP. For quantitative results to be obtained, samples were dissolved via acid digestion. The samples were digested in acid $\left(1 \mathrm{~mL} \mathrm{HF} / 9 \mathrm{~mL} \mathrm{HNO}_{3}\right.$ ) in polytetrafluoroethylene microwave vessels at $\sim 200^{\circ} \mathrm{C}$. After digestion in the $\mathrm{HF} / \mathrm{HNO}_{3}$ mixture, $0.5 \mathrm{~g}$ boric acid was added to each vessel to complex any remaining HF. The solutions were then transferred, diluted to $50 \mathrm{~mL}$, and subjected to ICP analysis. To determine Si amounts, the solutions were further diluted 100 times. Some supports were digested and analyzed in duplicate: Engelhard Mod D, Merck Aldrich Grade 60, Sigma-Aldrich Grade 7734, and Sigma-Aldrich Grade 7754. The Davisil 645 silica support also was analyzed in duplicate along with a sample of the Davisil 645 that had been washed in $10 \% \mathrm{HNO}_{3}$ at room temperature for $2 \mathrm{hr}$, washed, dried, and subsequently calcined at $500^{\circ} \mathrm{C}$ for $2 \mathrm{hr}$ in air.

The silica samples analyzed in duplicate are reported in Table 2.17 and Table 2.18. Other silica samples analyzed but not run in duplicate are reported in Table 2.19. The elements are reported as the wt\% oxides of the most stable oxidation state of the cation. In addition to the elements listed in the tables, Ba, Cd, Co, Cr, Mn, Mo, Ni, Pd, Pt, Re, Sn, V, W, Y, and Zn also were analyzed but were not observed down to the detection level of the instrument ( $\sim 25 \mathrm{ppm})$.

The silica catalyst supports had varying levels of water present. Thus, the silica samples were dried under vacuum for several hours, and the loss of water was determined gravimetrically. For the retest of selected samples (see Table 2.20), the samples were heated in a thermogravimetric analyzer (TGA) to determine the amount of water lost from a sample. These samples were heated from 30 to $300^{\circ} \mathrm{C}$ at $20^{\circ} \mathrm{C} / \mathrm{min}$ in flowing He. The samples then were soaked at $300^{\circ} \mathrm{C}$ for $20 \mathrm{~min}$ in flowing He. The percent mass lost from the initial loading through the end of the soak is reported in the non-shaded cells in Table 2.20.

All of the samples digested by the method outlined above were dissolved such that no solids were visible to the naked eye except for the Merck Aldrich Grade 60 samples and one of each of the duplicated samples of the Sigma-Aldrich Grade 7734 and $\mathrm{HNO}_{3}$-Washed Davisil 645. Regarding the Merck Aldrich Grade 60 samples, white, fluffy solids were still evident after the digestion. The reason that the Merck Aldrich Grade 60 failed to digest to completion is unknown. Regarding the Sigma-Aldrich Grade 7734 and $\mathrm{HNO}_{3}$-washed Davisil 645 silica supports, a greater concentration of silica was tested than could be digested by the HF added was believed to be the cause of the incomplete digestion. 
Data in Table 2.17 show that the Engelhard Mod D silica had a high concentration of $\mathrm{Al}_{2} \mathrm{O}_{3}$. Washing Davisil 645 with $10 \% \mathrm{HNO}_{3}$ did not eliminate the impurities present in the unwashed parent material, although $\mathrm{Ca}, \mathrm{Mg}$, and $\mathrm{Na}$ concentrations decreased. Some elements such as $\mathrm{Zr}$ and $\mathrm{Ti}$ appeared to increase in concentration, which may have resulted from contamination during the $\mathrm{HNO}_{3}$ washing process.

Table 2.18 illustrates the results of ICP testing on the Merck Aldrich Grade 60 and the Sigma-Aldrich Grade 7734 and Grade 7754 silica supports. Generally, the Merck Aldrich Grade 60 and Sigma-Aldrich Grade 7734 silica supports had similar impurities present at somewhat similar concentrations. The Sigma-Aldrich Grade 7754 silica support was found to contain impurities similar to the other supports shown in Table 18 but with decreased concentrations. Both of the Grade 7754 samples and one of the Grade 7734 samples were digested completely (the fact that the Grade 7734 sample did not digest completely was attributed to an inadvertently high amount of loaded silica). In contrast, neither of the Merck Aldrich Grade 60 samples digested completely.

Table 2.19 reports the data for the samples not analyzed in duplicate. The Perlkat 97-0, Grace Grade 408 and Norpro SS61138 silica supports had summed component concentrations at or above $93 \mathrm{wt} \%$. Thus, the analysis of these supports returned values that are most likely within $7 \%$ error, which, based on past experience with the ICP, is within the expected level of error. However, the Perlkat 97-3, Perlkat 293 and the Norpro 61137 returned summed oxide concentrations significantly lower than $100 \%$. The digestion and subsequent ICP analysis does not appear to be the source of the missing concentrations as the samples appeared visually to be completely digested. Furthermore, other samples run before and after analysis of these samples returned summed concentrations near $100 \%$. The three samples that returned low total oxide concentrations likely vented during microwave digestion, causing a loss of volatile Si-F complexes and subsequently returned an erroneously low Si concentration. Another contributing factor may be that these samples may have chemically-bound hydroxyl groups that were not dissociated by vacuum treatment at $200^{\circ} \mathrm{C}$, which would have resulted in an erroneously low concentration because water was present.

Table 2.20 shows the comparison of samples of the Davisil 645, Merck Aldrich Grade 60, and SigmaAldrich Grade 7734 tested in August 2011 (shaded cells) versus a retest of each of these silica supports in February-April 2012. In the retest performed in 2012, the silica samples were drawn directly from the parent bottles to ensure that the correct samples were analyzed. It is important to note that nearly all of the retested silica samples are within $5 \mathrm{wt} \%$ of $100 \mathrm{wt} \%$. This is likely due to improved methods of digestion and ICP such as properly sealing vessels to avoid inadvertent venting and better methods for adding boric acid to complex un-used HF. Generally, all of the non-silicon impurities reported during previous testing in 2011 were observed to be in the same range when retested in 2012. The two major differences are the water and $\mathrm{SiO}_{2}$ concentrations within the samples. The difference in the concentration of water on the supports is likely due to the more sensitive weight loss method employed in 2012 (TGA mass spectroscopy [TGA//MS]) versus a gravimetric method employing a vacuum oven in 2011. The higher concentration of Si observed in all samples is likely due to better sealing of the containers to avoid the escape of volatile Si-F complexes. 
Table 2.17. Elemental Analysis Determined via ICP on the Engelhard Mod D, Davisil 645 and $\mathrm{HNO}_{3^{-}}$ Washed Davisil 645 Silica Mixed Alcohol Catalyst Supports

\begin{tabular}{|c|c|c|c|c|c|c|}
\hline \multirow{2}{*}{$\frac{\mathrm{Wt} \%}{\text { Sample }}$} & \multicolumn{2}{|c|}{ Engelhard Mod D ${ }^{(a)}$} & \multicolumn{2}{|c|}{ Davisil $645^{(a)}$} & \multicolumn{2}{|c|}{$\begin{array}{c}61022-5-1 \\
\mathrm{HNO}_{3} \text { Washed Davisil 645 (a) }\end{array}$} \\
\hline & Primary $^{(a)}$ & Duplicate $^{(\mathrm{a})}$ & Primary $^{(a)}$ & Duplicate $^{(\mathrm{a})}$ & Primary $^{(a)}$ & Duplicate $^{(\mathrm{b})}$ \\
\hline $\mathrm{Al}_{2} \mathrm{O}_{3}$ & 1.903 & 1.616 & 0.011 & 0.009 & 0.019 & 0.017 \\
\hline \multicolumn{7}{|l|}{$\mathrm{As}_{2} \mathrm{O}_{3}$} \\
\hline $\mathrm{CaO}$ & 0.034 & 0.032 & 0.036 & 0.035 & 0.021 & 0.017 \\
\hline $\mathrm{CuO}$ & 0.001 & 0.001 & & & & \\
\hline $\mathrm{Fe}_{2} \mathrm{O}_{3}$ & 0.037 & 0.039 & 0.020 & 0.020 & & \\
\hline $\mathrm{K}_{2} \mathrm{O}$ & 0.007 & 0.006 & 0.007 & 0.006 & & \\
\hline $\mathrm{MgO}$ & 0.124 & 0.104 & 0.008 & 0.008 & 0.003 & 0.003 \\
\hline $\mathrm{Na}_{2} \mathrm{O}$ & 0.054 & 0.055 & 0.075 & 0.070 & 0.052 & 0.043 \\
\hline \multicolumn{7}{|l|}{$\mathrm{P}_{2} \mathrm{O}_{5}$} \\
\hline $\mathrm{PbO}_{2}$ & 0.001 & 0.001 & 0.002 & 0.002 & & \\
\hline $\mathrm{SrO}$ & 0.001 & 0.001 & 0.001 & 0.001 & & \\
\hline $\mathrm{TiO}_{2}$ & 0.015 & 0.013 & 0.002 & 0.002 & 0.021 & 0.018 \\
\hline \multicolumn{7}{|l|}{$\mathrm{Au}_{2} \mathrm{O}_{3}$} \\
\hline \multicolumn{7}{|l|}{$\mathrm{SO}_{2}$} \\
\hline $\mathrm{ZrO}_{2}$ & 0.005 & 0.005 & 0.008 & 0.008 & 0.014 & 0.011 \\
\hline $\mathrm{SiO}_{2}$ & 82.0 & 79.0 & 93.3 & 88.0 & 107.3 & 78.6 \\
\hline $\mathrm{H}_{2} \mathrm{O}$ & \multicolumn{2}{|c|}{$11.8^{(\mathrm{c})}$} & \multicolumn{2}{|c|}{$2.1^{(\mathrm{c})}$} & \multicolumn{2}{|c|}{$1.2^{(\mathrm{d})}$} \\
\hline Total & 96.0 & 92.7 & 95.6 & 90.3 & 108.6 & 79.9 \\
\hline
\end{tabular}

(a) Sample was completely digested such that no solids could be observed macroscopically.

(b) Silica sample size was greater than what could be dissolved in HF, and solids were observed.

(c) Determined by drying one sample of each silica material for 2 days under vacuum at $200^{\circ} \mathrm{C}$.

(d) Determined by drying a sample of silica over 3 days at $140^{\circ} \mathrm{C}$. 
Table 2.18. Elemental Analysis Determined via ICP on the Merck Aldrich Grade 60, Sigma-Aldrich Grade 7734 and Sigma-Aldrich Grade 7754 Silica Mixed Alcohol Catalyst Supports

\begin{tabular}{|c|c|c|c|c|c|c|}
\hline \multirow{2}{*}{$\begin{array}{l}\mathrm{Wt} \% \\
\text { Sample } \\
\end{array}$} & \multicolumn{2}{|c|}{ Merck Aldrich Grade 60} & \multicolumn{2}{|c|}{$\begin{array}{c}\text { Sigma-Aldrich Grade } 7734 \\
\text { Barcode \#326553 } \\
\end{array}$} & \multicolumn{2}{|c|}{$\begin{array}{c}\text { Sigma-Aldrich Grade } 7754 \\
\text { Barcode \#326554 }\end{array}$} \\
\hline & Primary $^{(\mathrm{a})}$ & Duplicate $^{(a)}$ & Primary $^{(b)}$ & Duplicate $^{(\mathrm{a})}$ & Primary $^{(b)}$ & Duplicate $^{(\mathrm{b})}$ \\
\hline $\mathrm{Al}_{2} \mathrm{O}_{3}$ & 0.038 & 0.036 & 0.030 & 0.045 & 0.009 & 0.007 \\
\hline \multicolumn{7}{|l|}{$\mathrm{As}_{2} \mathrm{O}_{3}$} \\
\hline $\mathrm{CaO}$ & 0.062 & 0.057 & 0.085 & 0.126 & 0.030 & 0.026 \\
\hline \multicolumn{7}{|l|}{$\mathrm{CuO}$} \\
\hline $\mathrm{Fe}_{2} \mathrm{O}_{3}$ & 0.024 & 0.021 & 0.005 & 0.008 & & \\
\hline $\mathrm{K}_{2} \mathrm{O}$ & 0.006 & 0.005 & & & & \\
\hline $\mathrm{MgO}$ & 0.010 & 0.010 & 0.014 & 0.022 & 0.008 & 0.006 \\
\hline $\mathrm{Na}_{2} \mathrm{O}$ & 0.158 & 0.162 & 0.158 & 0.218 & 0.033 & 0.032 \\
\hline \multicolumn{7}{|l|}{$\mathrm{P}_{2} \mathrm{O}_{5}$} \\
\hline $\mathrm{PbO}_{2}$ & 0.002 & 0.001 & & & & \\
\hline $\mathrm{SrO}$ & 0.001 & 0.001 & & & & \\
\hline $\mathrm{TiO}_{2}$ & 0.027 & 0.025 & 0.016 & 0.024 & 0.009 & 0.008 \\
\hline \multicolumn{7}{|l|}{$\mathrm{Au}_{2} \mathrm{O}_{3}$} \\
\hline $\mathrm{SO}_{2}$ & 0.034 & 0.042 & 0.059 & 0.088 & & \\
\hline $\mathrm{ZrO}_{2}$ & 0.007 & 0.007 & 0.012 & 0.015 & 0.026 & 0.018 \\
\hline $\mathrm{SiO}_{2}$ & 79.3 & 63.9 & 78.2 & 74.8 & 70.3 & 95.7 \\
\hline $\mathrm{H}_{2} \mathrm{O}$ & \multicolumn{2}{|c|}{$6.1^{(\mathrm{c})}$} & \multicolumn{2}{|c|}{$4.5^{(\mathrm{d})}$} & \multicolumn{2}{|c|}{$3.0^{(\mathrm{d})}$} \\
\hline Total & 85.8 & 70.3 & 83.1 & 79.8 & 73.4 & 98.8 \\
\hline
\end{tabular}

(a) Solids were observed after digestion.

(b) Sample was completely digested such that no solids could be observed macroscopically.

(c) Determined by drying one sample of each silica material for 2 days under vacuum at $200^{\circ} \mathrm{C}$.

(d) Determined by drying a sample of silica over 3 days at $140^{\circ} \mathrm{C}$. 
Table 2.19. Mixed Alcohol Synthesis Silica Catalyst Supports Digested in $\mathrm{HNO}_{3} / \mathrm{HF}$ and Analyzed with ICP

\begin{tabular}{ccccccc}
\hline $\mathrm{Wt} \%$ & $\begin{array}{c}\text { Perlkat } \\
97-0^{(\mathrm{a})}\end{array}$ & $\begin{array}{c}\text { Perlkat } \\
79-3^{(\mathrm{a})}\end{array}$ & $\begin{array}{c}\text { Grace } \\
\text { Grade } 408^{(\mathrm{a})}\end{array}$ & $\begin{array}{c}\text { Norpro } \\
\mathrm{SS61138}^{(\mathrm{a})}\end{array}$ & $\begin{array}{c}\text { Perlkat } \\
29-3^{(\mathrm{a})}\end{array}$ & $\begin{array}{c}\text { Norpro } \\
61137^{(\mathrm{a})}\end{array}$ \\
\hline $\mathrm{Al}_{2} \mathrm{O}_{3}$ & 0.057 & 1.941 & 0.004 & 0.268 & 1.285 & 0.125 \\
$\mathrm{As}_{2} \mathrm{O}_{3}$ & & 0.001 & & & & 0.001 \\
$\mathrm{CaO}$ & 0.007 & 0.036 & & 0.021 & 0.027 & 0.032 \\
$\mathrm{CuO}$ & 0.001 & 0.001 & 0.001 & 0.001 & 0.001 & 0.001 \\
$\mathrm{Fe}_{2} \mathrm{O}_{3}$ & 0.026 & 0.044 & 0.019 & 0.024 & 0.036 & 0.027 \\
$\mathrm{~K}_{2} \mathrm{O}$ & 0.010 & 0.034 & 0.014 & 0.012 & 0.011 & 0.011 \\
$\mathrm{MgO}$ & 0.705 & 0.027 & & 0.015 & 0.066 & 0.048 \\
$\mathrm{Na}_{2} \mathrm{O}$ & 0.057 & 0.299 & 0.100 & 0.102 & 0.058 & 0.101 \\
$\mathrm{P}_{2} \mathrm{O}_{5}$ & 0.005 & & & & \\
$\mathrm{PbO}_{2}$ & 0.002 & & 0.001 & 0.002 & & \\
$\mathrm{SrO}$ & 0.001 & 0.001 & 0.001 & 0.001 & 0.001 & 0.001 \\
$\mathrm{TiO}_{2}$ & 0.025 & 0.013 & 0.002 & 0.005 & 0.015 & 0.003 \\
$\mathrm{Au}_{2} \mathrm{O}_{3}$ & & 0.034 & & & & \\
$\mathrm{SO}_{2}$ & 0.066 & 0.028 & 0.044 & 0.028 & & \\
$\mathrm{ZrO}_{2}$ & 0.009 & 0.007 & 0.005 & 0.008 & 0.007 & 0.012 \\
$\mathrm{SiO}_{2}$ & 93.9 & 39.0 & 79.0 & 90.5 & 49.5 & 20.0 \\
$\mathrm{H}_{2} \mathrm{O}^{(\mathrm{b})}$ & 2.7 & 6.4 & 16.8 & 2.9 & 15.1 & 1.9 \\
$\mathrm{Total}$ & 97.6 & 47.9 & 96.0 & 93.9 & 66.1 & 22.2 \\
\hline
\end{tabular}

(a) Sample was completely digested such that no solids could be observed macroscopically.

(b) Determined by drying a sample for 2 days under vacuum at $200^{\circ} \mathrm{C}$. 
Table 2.20. Elemental Analysis Determined via ICP on the Merck Aldrich Grade 60, Sigma-Aldrich Grade 7734 and Davisil 645 silica Mixed Alcohol Catalyst Supports. Samples in un-shaded cells were tested in February-April 2012 while samples in shaded cells were tested in July-August 2011.

\begin{tabular}{|c|c|c|c|c|c|c|c|c|c|c|c|}
\hline \multirow{2}{*}{$\frac{\mathrm{Wt} \%}{\text { Sample }}$} & \multicolumn{3}{|c|}{ Davisil 645} & \multicolumn{4}{|c|}{ Sigma-Aldrich Grade 7734} & \multicolumn{4}{|c|}{ Merck Grade 60} \\
\hline & Primary $^{(\mathrm{a})}$ & Primary $^{(\mathrm{a})}$ & Duplicate $^{(\mathrm{a})}$ & Primary $^{(\mathrm{a})}$ & Duplicate $^{(\mathrm{a})}$ & Primary $^{(\mathrm{a})}$ & Duplicate $^{(\mathrm{b})}$ & Primary $^{(\mathrm{a})}$ & Duplicate $^{(\mathrm{a})}$ & Primary $^{(\mathrm{b})}$ & Duplicate $^{(\mathrm{b})}$ \\
\hline $\mathrm{Al}_{2} \mathrm{O}_{3}$ & 0.014 & 0.011 & 0.009 & 0.037 & 0.043 & 0.030 & 0.045 & 0.043 & 0.076 & 0.038 & 0.036 \\
\hline $\mathrm{CaO}$ & 0.054 & 0.036 & 0.035 & 0.103 & 0.084 & 0.085 & 0.126 & 0.084 & 0.086 & 0.062 & 0.057 \\
\hline $\mathrm{Fe}_{2} \mathrm{O}_{3}$ & $<0.005$ & 0.020 & 0.020 & 0.006 & 0.006 & 0.005 & 0.008 & 0.006 & 0.009 & 0.024 & 0.021 \\
\hline $\mathrm{K}_{2} \mathrm{O}$ & 0.013 & 0.007 & 0.006 & $<0.005$ & 0.016 & $<0.005$ & $<0.005$ & 0.016 & 0.013 & 0.006 & 0.005 \\
\hline $\mathrm{MgO}$ & 0.015 & 0.008 & 0.008 & 0.017 & 0.013 & 0.014 & 0.022 & 0.013 & 0.014 & 0.010 & 0.010 \\
\hline $\mathrm{Na}_{2} \mathrm{O}$ & 0.063 & 0.075 & 0.070 & 0.169 & 0.159 & 0.158 & 0.218 & 0.159 & 0.174 & 0.158 & 0.162 \\
\hline $\mathrm{TiO}_{2}$ & 0.009 & 0.002 & 0.002 & 0.020 & 0.033 & 0.016 & 0.024 & 0.033 & 0.034 & 0.027 & 0.025 \\
\hline $\mathrm{SO}_{2}$ & $<0.005$ & $<0.005$ & $<0.005$ & 0.064 & 0.082 & 0.059 & 0.088 & 0.082 & $<0.005$ & 0.034 & 0.042 \\
\hline $\mathrm{ZrO}_{2}$ & 0.008 & 0.008 & 0.008 & 0.005 & 0.007 & 0.012 & 0.015 & 0.007 & 0.007 & 0.007 & 0.007 \\
\hline $\mathrm{SiO}_{2}$ & 100.8 & 93.3 & 88.0 & 96.0 & 92.1 & 78.2 & 74.8 & 93.9 & 95.5 & 79.3 & 63.9 \\
\hline $\mathrm{H}_{2} \mathrm{O}$ & $0.5^{(\mathrm{c})}$ & \multicolumn{2}{|c|}{$2.1^{(\mathrm{d})}$} & \multicolumn{2}{|c|}{$1.3^{(\mathrm{c})}$} & \multicolumn{2}{|c|}{$4.5^{(\mathrm{e})}$} & \multicolumn{2}{|c|}{$2.0^{(\mathrm{c})}$} & \multicolumn{2}{|c|}{$6.1^{(\mathrm{d})}$} \\
\hline Total & 101.5 & 95.6 & 90.3 & 97.7 & 93.8 & 83.1 & 79.8 & 96.4 & 97.9 & 85.8 & 70.3 \\
\hline
\end{tabular}

(a) Sample was completely digested such that no solids could be observed macroscopically.

(b) Solids observed after digestion.

(c) Determined via thermogravimetric analysis mass spectrometry (TGA/MS) through ramping to $300^{\circ} \mathrm{C}$ at $20^{\circ} \mathrm{C} / \mathrm{min}$ in flowing $\mathrm{He}$ and soaking at $300^{\circ} \mathrm{C}$ for 20 min.

(d) Determined by drying one sample of each silica material for 2 days under vacuum at $200^{\circ} \mathrm{C}$.

(e) Determined by drying on sample of silica over 3 days at $140^{\circ} \mathrm{C}$. 


\subsection{Surface and Pore Volume Measurements via Nitrogen Adsorption/Desorption}

\subsubsection{Carbon Supports}

The surface area, pore size distribution, and pore volume of samples KOA 1 through 15 as well as the ICI low-pressure methanol synthesis catalyst were probed using $\mathrm{N}_{2}$ adsorption and desorption. Nitrogen adsorption/desorption tests were performed at $-196^{\circ} \mathrm{C}$. In a typical test, $\mathrm{N}_{2}$ first is adsorbed onto the catalyst starting from high vacuum conditions and progressing to atmospheric pressure, which fills the pores of the catalyst and creates subsequent monolayers of $\mathrm{N}_{2}$ on the catalyst surface. When the catalyst surface and pores become saturated with $\mathrm{N}_{2}$, a controlled vacuum is applied and desorption of the $\mathrm{N}_{2}$ from the surface and pores of the support is monitored. In this way, a hysteresis curve corresponding to the adsorption and desorption of $\mathrm{N}_{2}$ from the catalyst is created. The surface area reported below is the Brunauer-Emmett-Teller (BET) surface area. The pore size distribution was determined using the desorption isotherm of the adsorption/desorption hysteresis curve using the Barret-Joyner-Halenda method.

Table 2.21 lists data for supports KOA 1 through 15 and the ICI catalyst that includes the surface area, micropore surface area, and pore volume. Graphical representations of the pore size distributions for supports KOA 1 through 15 and the ICI catalyst are shown in Appendix A (Figures A.1 to A.16). It must be emphasized that, when analyzing the pore size distributions, the desorption peak at $38 \AA$ is not real; rather, it is an artifact of the $\mathrm{N}_{2}$ adsorption/desorption tests. ${ }^{5}$

For the purpose of definitions, the International Union of Pure and Applied Chemistry has defined micropores as pores with diameters less than $20 \mathrm{~A}$, mesopores as pores with diameters from 20 to $500 \mathrm{~A}$, and macropores as pores with diameters greater than $500 \mathrm{~A}$. Analysis of Table 2.21 and the pore size distribution plots provided in Appendix A reveals that samples KOA 1, 2, 4, 5, 9, 10, 11, 12, 13, and 14 all have pore volumes mostly in the micropore range, with a relatively small amount of mesopores. The pore volumes and diameters of samples KOA 6, 7, and 8 are significantly different than the other carbon supports. Samples KOA 6, 7, and 8 exhibit high pore volumes compared to the other supports, and the pore volumes are mainly from pores in the 100 to $1000 \AA$ region. Thus, samples KOA 6, 7, and 8 have larger pore volumes and pore diameters. Speculatively, this could allow for superior mass transport of reactants and products when using KOA 6, 7, and 8 as catalyst supports for the production of mixed alcohols from syngas. 
Table 2.21. Surface Area and Porosity Data of Carbon Supports KOA 1 through 15 and the ICI Methanol Synthesis Catalyst

\begin{tabular}{|c|c|c|c|c|}
\hline Trivial Name & Support Description & $\begin{array}{c}\text { Surface Area, } \\
\mathrm{m}^{2} / \mathrm{g}\end{array}$ & $\begin{array}{c}\text { Micropore } \\
\text { Surface Area, } \\
\mathrm{m}^{2} / \mathrm{g}\end{array}$ & $\begin{array}{c}\text { Pore } \\
\text { Volume, } \\
\mathrm{m}^{3} / \mathrm{g}\end{array}$ \\
\hline KOA 1 & Norit ROX 0.8 & 1071 & 787 & 0.42 \\
\hline KOA 2 & Norit Darco-LS & 729 & 348 & 0.67 \\
\hline KOA 3 & Sibunit & 547 & 22.5 & 0.93 \\
\hline KOA 4 & Englehard High Surface Area Graphite (HSAG-1) & 580 & 307 & 0.35 \\
\hline KOA 5 & Englehard High Surface Area Graphite (HSAG-2) & 640 & 67.6 & 0.5 \\
\hline KOA 6 & Hyperion 395-08 & 218 & 23.4 & 1.36 \\
\hline KOA 7 & Hyperion 395-10 & 335 & 0 & $2.47^{(\mathrm{b})}$ \\
\hline KOA 8 & Hyperion 384-82 & 466 & 0 & 1.24 \\
\hline KOA 9 & Cummins-Moore $5559 \mathrm{~S}$ & 1024 & 888 & 0.19 \\
\hline KOA 10 & Cummins-Moore 5586 AW & 1035 & 585 & 0.41 \\
\hline KOA 11 & Norit Darco HF-Washed & 776 & 380 & 0.68 \\
\hline KOA 12 & Norit ROX 0.8 HF-Washed & 1139 & 851 & 0.46 \\
\hline KOA 13 & Pacco Carbon & 1727 & 1074 & 0.41 \\
\hline KOA 14 & Kurhea Carbon & 1327 & 1109 & 0.23 \\
\hline KOA 15 & TimCal Timrex HSAG & 318 & 62.7 & 0.52 \\
\hline ICI Catalyst & ICI LP MeOH Synthesis Catalyst & 76.2 & 4.08 & 0.25 \\
\hline
\end{tabular}

\subsubsection{Demineralized and Graphitized Carbon Supports}

The demineralized (HF-washed) and graphitized carbon supports listed in Table 2.2 also were subjected to $\mathrm{N}_{2}$ adsorption/desorption testing. As shown in Table 2.22, HF washing of the carbon supports generally increased the BET surface area of the carbon supports. This was likely due to dissolving of minerals in the carbon, thus creating new pores. Interestingly, the micropore volume did not exhibit a similar general increase with HF washing and in two cases (Norit ROX 0.8 and Norit Darco-LS) actually decreased after HF washing. Thus, the domains of inorganic material removed by the HF may have had diameters larger than $20 \AA$ in the Norit carbon supports.

Graphitization of the HF-washed samples resulted in surface areas approximately 5 to 10 times less than the HF treated counterparts. As observed in the pore size distribution plots for the four samples (Figure 2.3 through Figure 2.6 below), nearly all of the loss of pore volume during graphitization was from the disappearance of pores less than $100 \AA$. Physically, it is likely that graphitization sealed the small pores, thus diminishing the pore volume and surface area of the supports. Alternatively, hightemperature treatment may have led to smaller pores coalescing into larger pores. However, none of the samples exhibited significant growth of pore volume related to pores between $100 \AA$ and $2000 \AA$ upon graphitization. Therefore, the pores formed by coalescence of smaller pores would have to be larger than $2000 \AA$. Mercury intrusion porosimetry could be employed to investigate the pore size distribution of pores greater than $2000 \AA$, which would determine if pore coalescence occurred during high-temperature treatment. Note that the spikes at $\sim 38 \AA$ are not real but an artifact of a tensile strength effect of $\mathrm{N}_{2}$ desorption on microporous materials as reported by Groen et al. ${ }^{5}$ 
Table 2.22. Surface-Area and Pore-Size Data for Various Fresh, HF-Washed, and Graphitized Carbon Supports

\begin{tabular}{|c|c|c|c|}
\hline Treatment Description & $\begin{array}{l}\text { BET Surface Area, } \\
\mathrm{m}^{2} / \mathrm{g}\end{array}$ & $\begin{array}{c}\text { Total Pore Volume, } \\
\mathrm{cm}^{3} / \mathrm{g}^{(\mathrm{a})}\end{array}$ & $\begin{array}{c}\text { Micropore Pore Volume, } \\
\mathrm{cm}^{3} / \mathrm{g}^{(\mathrm{b})}\end{array}$ \\
\hline \multicolumn{4}{|c|}{ Norit ROX 0.8} \\
\hline Fresh & 1071 & 0.395 & 0.074 \\
\hline HF-Washed & 1127 & 0.378 & 0.047 \\
\hline Graphitized & 138 & 0.240 & 0.013 \\
\hline \multicolumn{4}{|c|}{ Norit Darco-LS } \\
\hline Fresh & 729 & 0.678 & 0.060 \\
\hline HF-Washed & 1025 & 0.839 & 0.058 \\
\hline Graphitized & 206 & 0.465 & 0.032 \\
\hline \multicolumn{4}{|c|}{ Pacco 90-100 CTC } \\
\hline Fresh & 1727 & 0.263 & 0.140 \\
\hline HF-Washed & 1793 & 0.268 & 0.140 \\
\hline Graphitized & 218 & 0.104 & 0.047 \\
\hline \multicolumn{4}{|c|}{ Jacobi Ecosorb BX-Max } \\
\hline Fresh & 1511 & 0.922 & 0.317 \\
\hline HF-Washed & 1887 & 1.40 & 0.382 \\
\hline Graphitized & 301 & 0.284 & 0.033 \\
\hline
\end{tabular}

(a) Pore volume of pores from $\sim 0$ to $2000 \AA$.

(b) Contribution to the total pore volume from pores between 0 and $\sim 0 \AA$.

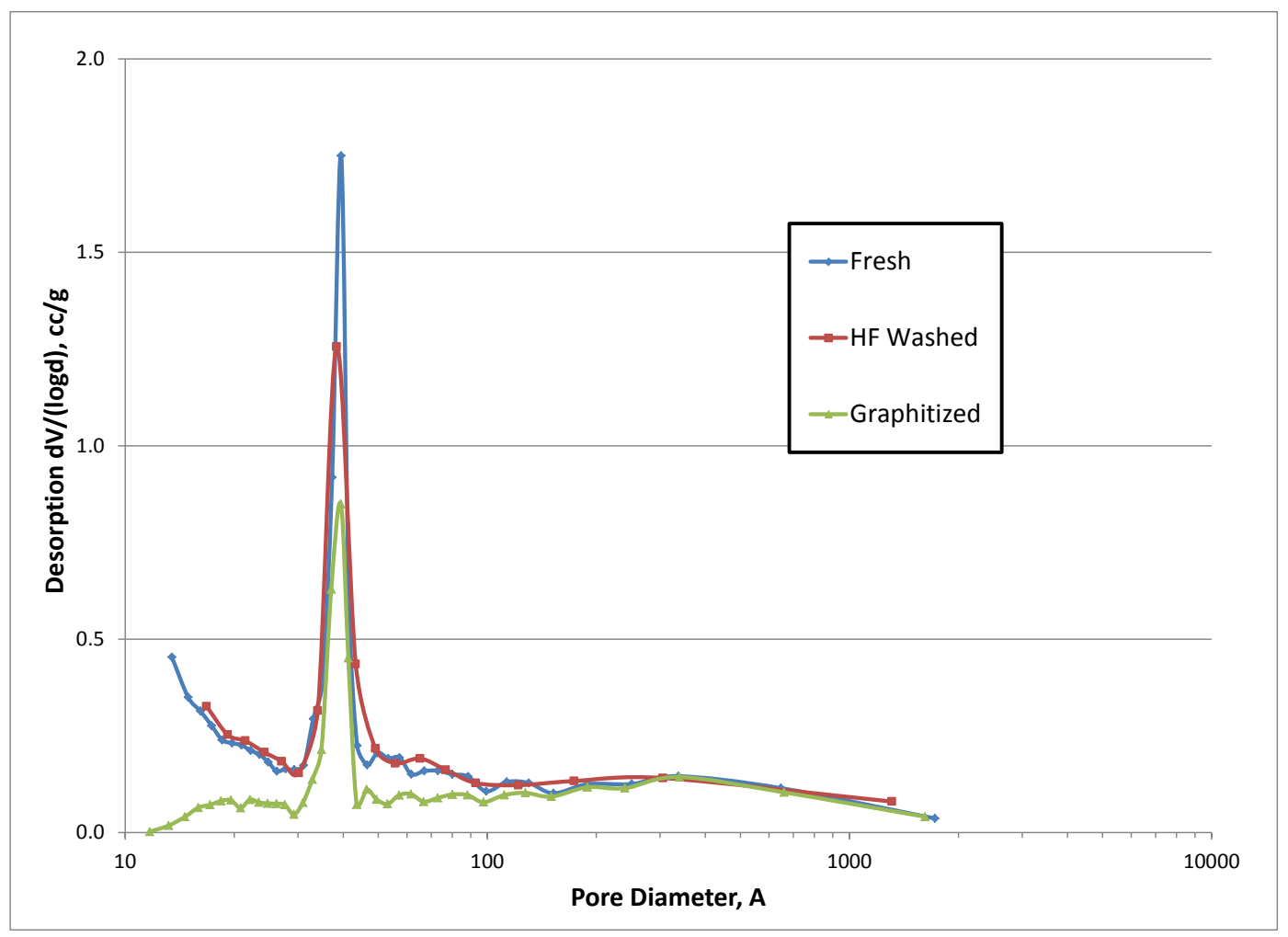

Figure 2.3. Pore size Distributions of Fresh, HF-Washed and Graphitized Samples of Norit ROX 0.8 


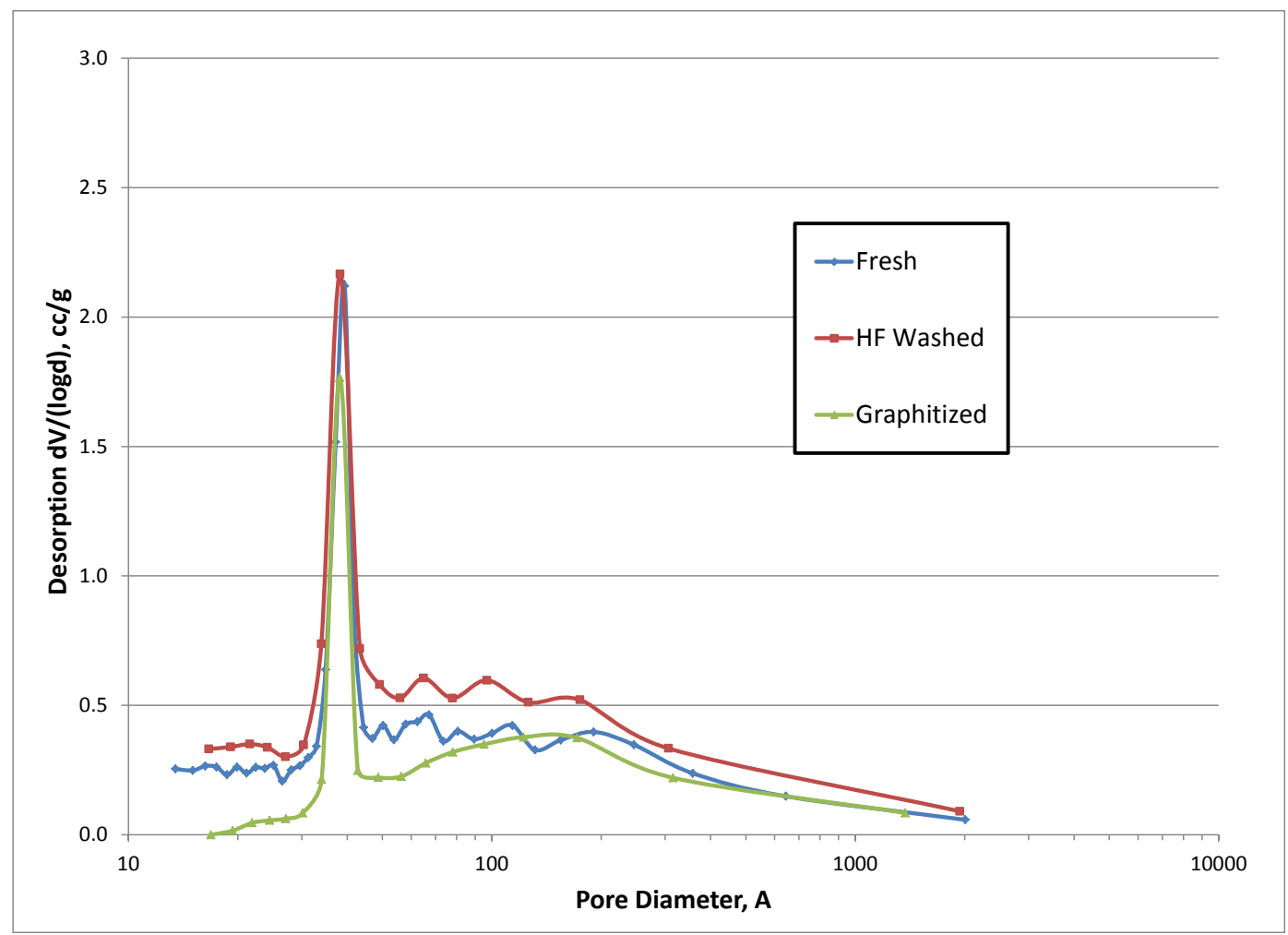

Figure 2.4. Pore Size Distributions of Fresh, HF-Washed and Graphitized Samples of Norit Darco-LS

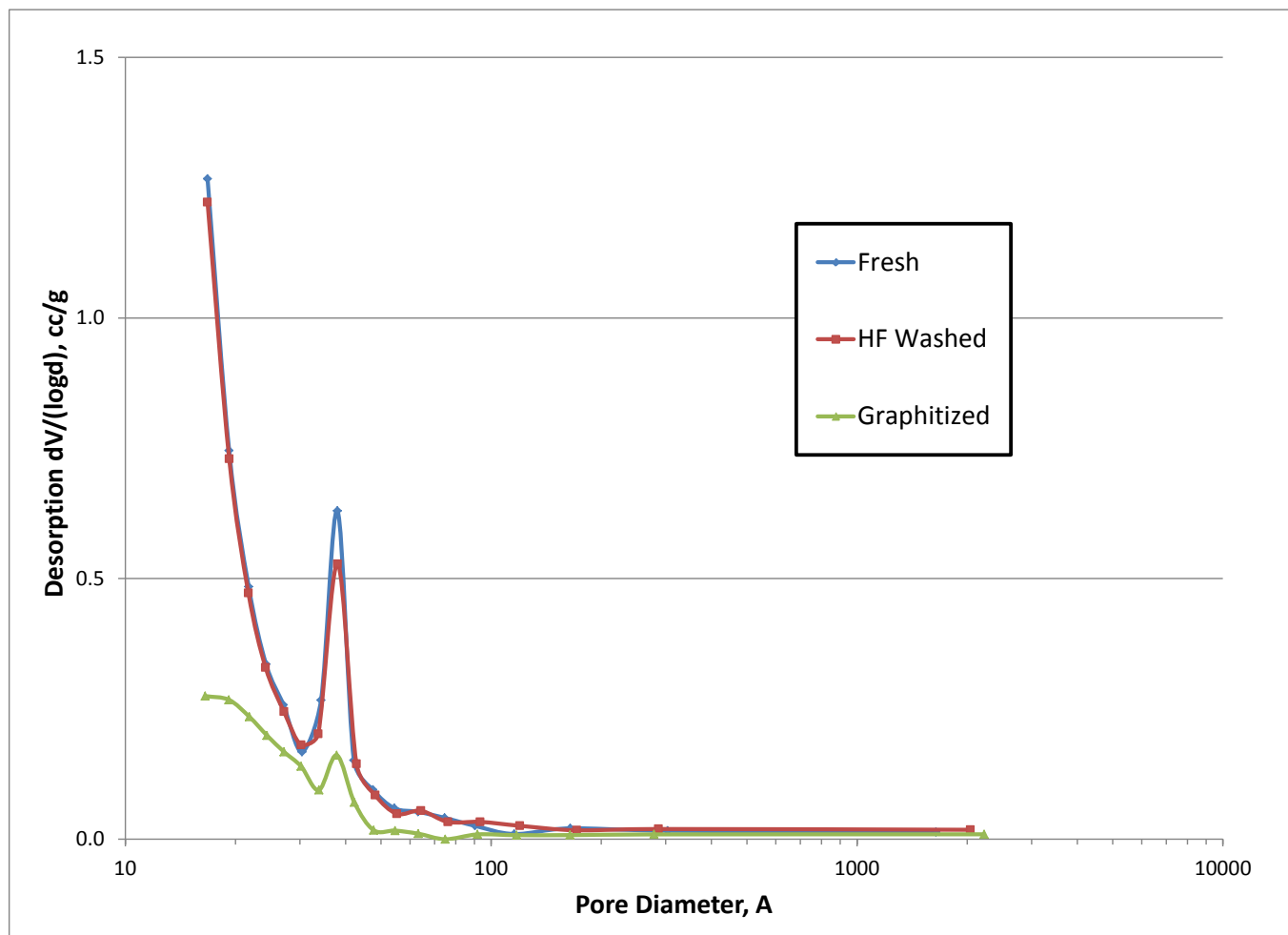

Figure 2.5. Pore Size Distributions of Fresh, HF-Washed and Graphitized Samples of the Pacco 90-100 CTC 


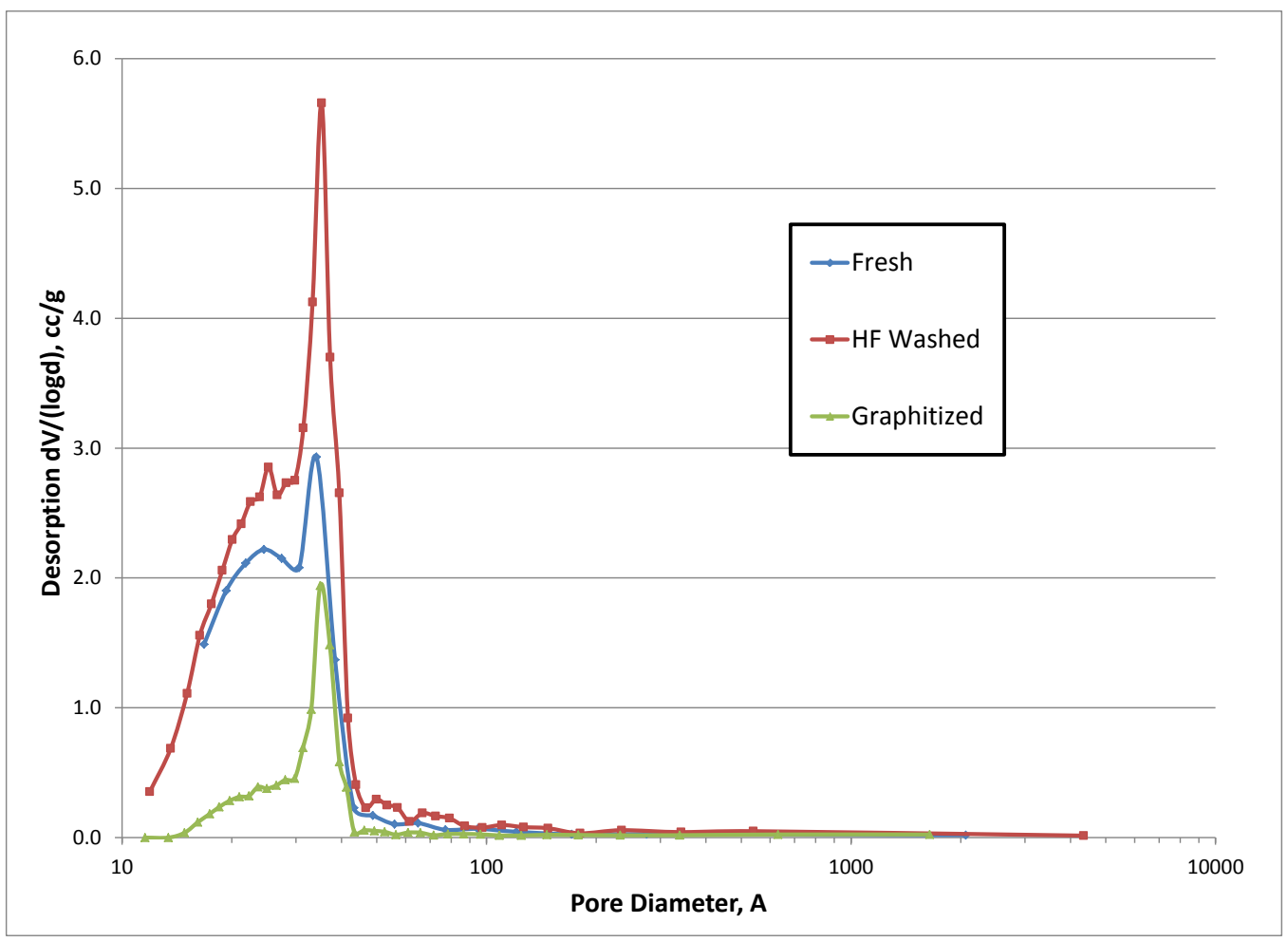

Figure 2.6. Pore Size Distributions of Fresh, HF-Washed and Graphitized Samples of the Jacobi Ecosorb BX-Max

\subsubsection{Silica Supports}

Table 2.23 lists $\mathrm{N}_{2}$ adsorption/desorption data for the inorganic mixed alcohol supports and includes the surface area, micropore surface area, and pore volume for each support investigated. Graphical representations of the pore size distributions are found in Appendix A in Figures A.17 through A.33. Again, it must be stressed that, when analyzing the pore size distributions, a sharp desorption peak at $38 \AA$ is not a real feature but is an artifact of pore expansion upon $\mathrm{N}_{2}$ desorption from micropores.

Two of the inorganic catalyst supports, the Norpro SA 5151 alumina and the ST 31119 titania did not have micro- or mesoporosity that could be probed by $\mathrm{N}_{2}$ adsorption/desorption. These materials could be macroporous, but to determine the pore size distribution of a macroporous material, other investigative techniques such as $\mathrm{Hg}$ porosimetry must be applied. Because the materials were essentially nonporous, no pore size distribution figures are associated with them.

Most of the supports do not have a large contribution to their total porosity from micropores, or from pores less than $20 \AA$. The supports that did have a significant volume of micropores were the Grace 408 silica and the Engelhard Mod D silica. The Perlkat 29-3 silica also had some micropores. Industrial catalytic supports typically minimize the amount of microporosity (unless shape selective chemistry is desired) to minimize internal mass transfer limitations. Overall, several of the supports appear to have pore size distributions centered near $\sim 100 \AA$. Pores of $100 \AA$ could be an optimal pore diameter for maximizing catalytic surface area while diminishing mass transfer limitations. 
Table 2.23. Surface-Area and Porosity Data of Inorganic Mixed Alcohol Synthesis Supports

\begin{tabular}{lccc}
\hline \multicolumn{1}{c}{ Support Description } & $\begin{array}{c}\text { Surface Area, } \\
\mathrm{m}^{2} / \mathrm{g}\end{array}$ & $\begin{array}{c}\text { Micropore Surface } \\
\text { Area, } \mathrm{m}^{2} / \mathrm{g}\end{array}$ & $\begin{array}{c}\text { Pore Volume, } \\
\mathrm{cm}^{3} / \mathrm{g}\end{array}$ \\
\hline Davisil 645 Silica & 284.8 & 22.1 & 1.2 \\
Engelhard Mod D Silica & 583.3 & 30.1 & 0.33 \\
PerlKat 97-0 Silica & 397.2 & 0 & 0.91 \\
PerlKat 79-3 Silica & 327.9 & 29.1 & 0.61 \\
Grace 408 Silica & 728 & 208.8 & 0.25 \\
Norpro SS61138 Silica & 276.4 & 14.2 & 1.04 \\
Perlkat 29-3 Silica & 618.6 & 79.5 & 0.42 \\
Norpro SS61137 Silica & 194.2 & 4 & 0.69 \\
Grace 980-25 Silica Alumina & 372 & 0 & 0.36 \\
BASF Al-5700 E 1/16 Ca on Alumina & 209 & 9.2 & 0.6 \\
Norpro ST 31119 Titania & 36.5 & 1.96 & 0.2 \\
SZ 31164 Zirconia & 97.1 & 1.29 & 0.34 \\
BASF 7264-109A MgO & 55.2 & 2.1 & 0.25 \\
Engelhard Al3945 E1/20 & 229.5 & 8.1 & 0.69 \\
Norpro SA5151 Alumina & 0.29 & 0.09 & nonporous \\
ST61165 Titania & 0.48 & 0 & nonporous \\
Merck Aldrich Grade 60 Silica & 535 & 0 & 0.73 \\
Sigma-Aldrich Silica Gel Grade 7734 & 530 & 0 & 0.79 \\
Sigma-Aldrich Silica Gel Grade 7754 & 449 & 0 & 0.79 \\
\hline
\end{tabular}

The three silica gels-Merck Aldrich Grade 60, Sigma-Aldrich Grade 7734. and Sigma-Aldrich Grade 7754-had similar pore size distributions as well as total pore volumes. While the Merck Aldrich Grade 60 and Sigma-Aldrich Grade 7734 had similar surface areas ( $\left.\sim 30 \mathrm{~m}^{2} / \mathrm{g}\right)$, the Sigma-Aldrich Grade 7754 surface area was $\sim 15 \%$ lower at $\sim 450 \mathrm{~m}^{2} / \mathrm{g}$.

\subsection{Surface Acidity Characterization of Selected Silica Mixed Alcohols Catalyst Supports via Ammonia Temperature Programmed Desorption}

The acidity of several silica mixed alcohol catalyst supports was probed with ammonia $\left(\mathrm{NH}_{3}\right)$ saturation with a subsequent temperature programmed desorption (TPD) routine. The Micromeritics AutoChem II 2920 was used for the $\mathrm{NH}_{3}$ saturation and subsequent TPD experiments. A Pfieffer Thermostar MS was used to quantify the amount of $\mathrm{NH}_{3}$ that was desorbed by each sample.

Approximately $100 \mathrm{mg}$ of catalyst was loaded into a sample quartz tube. Typically, the silica sample was pretreated prior to $\mathrm{NH}_{3}$ saturation in a stream of He flowing at 25 standard cubic centimeters per minute (SCCM) at $300^{\circ} \mathrm{C}$ for $2 \mathrm{hr}$. After pretreatment, the temperature was lowered to $120^{\circ} \mathrm{C}$ while $\mathrm{He}$ continued to flow. Once the sample had reached $120^{\circ} \mathrm{C}$, a stream of $15 \% \mathrm{NH}_{3} / \mathrm{He}$ was passed over the catalyst at 25 SCCM for $1 \mathrm{hr}$. Next, the gas stream was switched back to He flowing at 25 SCCM. Helium continued to pass over the sample for $2 \mathrm{hr}$ at $120^{\circ} \mathrm{C}$ to remove any physisorbed $\mathrm{NH}_{3}$. After passing He over the sample for $2 \mathrm{hr}$, the TPD experiment was initiated. The TPD experiment consisted of ramping the temperature of the sample from $120^{\circ} \mathrm{C}$ to $800^{\circ} \mathrm{C}$ at $10^{\circ} \mathrm{C} / \mathrm{min}$ intervals in He flowing at 25 SCCM. A 1 hr soak at $800^{\circ} \mathrm{C}$ also was employed as part of the TPD. The beginning of the $10^{\circ} \mathrm{C} / \mathrm{min}$ ramp is the beginning (i.e., $0 \mathrm{~min}$ ) of the TPD data presented below. 
A Thermostar MS was used to quantify the amount of $\mathrm{NH}_{3}$ desorbed from a silica sample. All of the samples desorbed water as well as $\mathrm{NH}_{3}$; therefore, it was not possible to use the thermal conductivity detector (TCD) that is typically used with the AutoChem II 2920 for quantification because it is not capable of distinguishing between different types of gases. Calibration of the MS was performed using the internal calibration feature of the AutoChem II 2920. The MS signal was correlated with the gas flow rate to produce a calibration curve of $\mathrm{NH}_{3}$ flow (SCCM) versus MS signal. The total amount of $\mathrm{NH}_{3}$ desorbed from a sample was determined by integrating the flow of $\mathrm{NH}_{3}$ with respect to time.

The Thermostar MS was calibrated several times over the period of time required to run the nine silica samples. During the course of approximately two weeks, we determined that the apparently random drift of the instrument was within $\pm 10 \%$ of the calibration used for quantification.

A mass of 16 was used for quantifying the MS data because the mass of 17, which is the parent ion of $\mathrm{NH}_{3}$, was quickly overwhelmed by the presence of water (17 is a major daughter ion of water). While the mass of 16 also is a daughter ion of water, its intensity relative to the 18 mass signal exhibited by water is only 3.5\% (compared with 17 at 30\%). However, in each sample, the presence of water being desorbed would eventually overwhelm the signal that could be attributed to $\mathrm{NH}_{3}$. A rigorous analysis of the signal at 16 was applied to determine if the signal was from $\mathrm{NH}_{3}$ or water. When the ratio of the signals of $16 / 18$ was greater than $5 \%$, the signal was attributed to $\mathrm{NH}_{3}$. When the ratio of the signals of 16/18 dipped below $5 \%$, the signal was attributed to water. When the signal was obscured because water was present, no $\mathrm{NH}_{3}$ flow is reported.

The silica catalyst supports can be grouped into two general categories:

1. Supports with a small number of relatively weak acidic sites

2. Supports with a greater number of acidic sites, including some stronger acid sites.

Table 2.24 reveals that that majority of the silica supports fell into the first category. The Davisil 645, $\mathrm{HNO}_{3}$ washed Davisil 645, Grace Grade 408, Merck Aldrich Grade 60, Norpro 61137 and 61138, Perlkat 97-0, and Sigma-Aldrich Grade 7734 and 7754 supports all desorbed less than $50 \mu \mathrm{mol}$ of $\mathrm{NH}_{3} / \mathrm{g}$ of support. Generally, $\mathrm{NH}_{3}$ desorption was complete below $250^{\circ} \mathrm{C}$, indicating that the few number of acid sites present are relatively weak (see the $\mathrm{NH}_{3}$ TPD profiles of the silica supports in Appendix B). Notable exceptions to this were the Grace Grade 408 (Figure B.8) and Norpro 61138 (Figure B.10) supports, which had a small number of stronger acid sites indicated by desorption of $\mathrm{NH}_{3}$ at temperatures greater than $\sim 300^{\circ} \mathrm{C}$.

Supports with more acid sites, including stronger acid sites, included the Engelhard Mod D silica gel as well as the Perlkat 79-3 and 29-3 supports. Many of the acid sites in the Engelhard Mod D were relatively weak and desorbed below 250 to $300^{\circ} \mathrm{C}$ (Figure B.11). However, the Perlkat 79-3 (Figure B.12) and Perlkat 29-3 (Figure B.13) had significant concentrations of strong acid sites as evidenced by the amount of $\mathrm{NH}_{3}$ desorbed above $\sim 300^{\circ} \mathrm{C}$. When compared with $\mathrm{H}-\mathrm{ZSM} 5$ zeolite strong acid catalyst (Figure B.14), the transition between weak and strong acid sites can be observed at $\sim 300^{\circ} \mathrm{C}$. However, the silica support with the greatest amount of $\mathrm{NH}_{3}$ desorbed (Perlkat 29-3) only had $~ 50 \%$ of the amount of $\mathrm{NH}_{3}$ desorbed compared with the H-ZSM5 zeolite, indicating that, in general, the silica supports are much less acidic than crystalline silica-aluminates as would be expected. 
Table 2.24. Total Amount of $\mathrm{NH}_{3}$ Desorbed during TPD Experiments With Silica Mixed Alcohol Synthesis Catalyst Supports

\begin{tabular}{cccc}
\hline & $\begin{array}{c}\text { Sample } \\
\text { Pretreatment } \\
\text { Temp, }{ }^{\circ} \mathrm{C}^{(\mathrm{a})}\end{array}$ & $\begin{array}{c}\text { Total NH } \text { Desorbed }_{3} \\
(\mu \mathrm{mol} / \mathrm{g})\end{array}$ \\
\hline Sample & 300 & 0.0759 & 12 \\
Sigma-Aldrich Grade 7754 & 300 & 0.1052 & 15 \\
Perlkat 97-0 & 300 & 0.0790 & 18 \\
Sigma-Aldrich Grade 7734 & 300 & 0.0835 & 21 \\
Merck Aldrich Grade 60 & 300 & 0.0907 & 21 \\
Davisil 645 (Washed in $\mathrm{HNO}_{3}$ ) & 300 & 0.0988 & 24 \\
Norpro 61137 & 120 & 0.0894 & 26 \\
Merck Aldrich Grade 60 & 300 & 0.0809 & 30 \\
Grace Grade 408 & 300 & 0.1090 & 50 \\
Davisil 645 & 300 & 0.0979 & 207 \\
Norpro 61138 & 300 & 0.0827 & 419 \\
Engelhard Mod D & 300 & 0.0916 & 514 \\
Perlkat 79-3 & 300 & 0.0839 & 987 \\
Perlkat 29-3 & 500 & 0.0995 & \\
\hline
\end{tabular}

(a) All pretreatments performed for $2 \mathrm{hr}$ in He flowing at $25 \mathrm{cc} / \mathrm{min}$.

(b) The H-ZSM5 zeolite sample is reported for a basis of comparison. H-ZSM5 is considered a strong solid acid.

Treatment of the Davisil 645 silica with $\mathrm{HNO}_{3}$ produced a silica support with slightly fewer acid sites than the parent silica. The Davisil 645 was stirred with $10 \% \mathrm{HNO}_{3}$, washed with deionized water, and subsequently calcined at $500^{\circ} \mathrm{C}$ for $2 \mathrm{hr}$. Because the amount of $\mathrm{NH}_{3}$ desorbed was low for both the $\mathrm{HNO}_{3}$-washed and unwashed samples, more testing would be required to ensure the differences are statistically significant. Qualitatively, the $\mathrm{HNO}_{3}$ washed sample may have fewer acid sites because the sample was calcined at $500^{\circ} \mathrm{C}$ in air for $2 \mathrm{hr}$ prior to $\mathrm{NH}_{3}$ TPD testing, which may have produced a more coordinated silica surface with fewer hydroxyl groups that exhibited acidity.

\subsection{Carbon Support Surface Functionality Characterization through Temperature Programmed Desorption Utilizing a Coupled Thermogravimetric Analyzer and Mass Spectrometer}

\subsubsection{Unmodified Carbon Supports KOA 1-12}

Oxygen functionality on the surface of samples KOA 1-12 was probed via TPD experiments using TGA/MS. Surface functionality, especially oxygenated surface functionality, on carbon surfaces can alter the catalytic properties by changing the hydrophobic nature of the support and by changing the mechanism of metal deposition when using wet impregnation techniques to prepare supported metal catalysts. ${ }^{6}$ 
The common oxygenated surface functionality on activated carbons listed in Table 2.6 decompose to form $\mathrm{CO}, \mathrm{CO}_{2}$, and/or $\mathrm{H}_{2} \mathrm{O}$. $^{6}$ Information about the decomposed species can be gained through the observed temperature and gaseous products of the decomposition. Aksoylu et al. ${ }^{6}$ reported that, in general, more acidic groups decompose as $\mathrm{CO}_{2}$ at lower temperatures. Additionally, these acidic groups decrease the hydrophobicity of a carbon support, making the surface more accessible to aqueous impregnation solutions.

For the TPD experiments, 100 to $300 \mathrm{mg}$ of the carbon support material was placed in a TGA. The TGA/MS is a Netzsch 409 STA C/CD. The temperature was ramped from 20 to $1300^{\circ} \mathrm{C}$ at $5^{\circ} \mathrm{C} / \mathrm{min}$, and the mass was recorded as a function of temperature. During the experiment, He flowing at $5 \mathrm{~mL} / \mathrm{min}$ was passed over the sample. An MS instrument sampled the gas stream the course of the test. Thus, the gas evolved during sample heating could be determined.

The thermogravimetric (TG) traces and MS responses for TPD experiments of samples KOA 1 through 12 are presented in Appendix C. The traces show the mass of the samples with respect to time and temperature. The mass spectrometer traces show 4 mass-to-charge ratios, or m/e, with respect to time and temperature. Water, with an atomic mass of 18 , is given by an $\mathrm{m} / \mathrm{e}$ of $18 . \mathrm{CO}_{2}$ has a $\mathrm{m} / \mathrm{e}$ response of 44 as the major peak but can be accompanied by a m/e response of 28 that is $10 \%$ the intensity of the $\mathrm{m} / \mathrm{e}$ response of 44 . CO has a $\mathrm{m} / \mathrm{e}$ response at 28 ; thus, $\mathrm{CO}_{2}$ is present when $\mathrm{m} / \mathrm{e}=44$ is present while $\mathrm{CO}$ is present when $\mathrm{m} / \mathrm{e}=28$ is greater than $10 \%$ of the $\mathrm{m} / \mathrm{e}=44$ response. The trace for $\mathrm{m} / \mathrm{e}=64$ most likely due to $\mathrm{SO}_{2}$. $\mathrm{SO}_{2}$ was observed in samples $\mathrm{KOA} 2,10,11$, and 12. Additionally, $\mathrm{SO}_{2}$ is very likely in sample KOA 1, but the mass spectrometer was not set to scan to a high enough value to observe an $\mathrm{m} / \mathrm{e}=64$. All of the MS traces are background subtracted based on the initial value for the given $\mathrm{m} / \mathrm{e}$ response at time zero (i.e., all responses are subtracted by the initial value).

Tables $7 \mathrm{a}$ and $7 \mathrm{~b}$ give qualitative observations based on the data presented in Appendix $\mathrm{C}$ and Table 2.6. Characterization of the oxygenated surface functionality on supports KOA 1 through 12 was performed by comparing the temperature and species desorbed during a period of weight loss on the carbon support. Samples KOA 1 and 2 exhibited physisorbed and chemisorbed water. Acidic functional groups, which desorbed as $\mathrm{CO}_{2}$ at relatively low temperature, also were observed. More stable, less hydrophilic oxygenated functional groups desorbed as $\mathrm{CO}$ at temperatures between 500 and $900^{\circ} \mathrm{C}$.

The high surface area graphites, samples KOA 4 and 5, exhibited no weight loss or gas desorbed during TPD, indicating very high carbon content with low oxygen surface functionality. Samples KOA 6 and 7 have very well defined oxygenated surface moieties, which were exhibited by a very rapid weight loss between 620 and $700^{\circ} \mathrm{C}$, which was most likely a phenol functionality. Sample KOA 8, which is more hydrophilic than sample $\mathrm{KOA} 6$, desorbed $\mathrm{CO}$ and $\mathrm{CO}_{2}$ between 580 and $680^{\circ} \mathrm{C}$, which is indicative of the possible presence of carboxylic anhydrides on the surface. Interestingly, samples KOA 7 and 8 should be hydrophilic, yet KOA 7 resembled hydrophobic KOA 6 much more closely than KOA 8. Samples KOA 9 and 10 have a high degree of physisorbed and chemisorbed water as evidenced by the large weight-loss peaks corresponding with the $\mathrm{m} / \mathrm{e}=18$ between 20 and $130^{\circ} \mathrm{C}$. Samples KOA 10 and 11 also exhibit $\mathrm{CO}_{2}$ and $\mathrm{CO}$ desorption at low temperature, indicating the presence of carboxylic acid or carboxylic anhydride functionality, which would increase the hydrophilicity of the support and conceptually agrees with the large amount of water desorbed. Samples KOA 9 and 10 exhibited hightemperature desorption patterns that are similar to samples KOA 1 and 2, indicating the likely presence of phenol, carbonyl, ether, and/or quinone oxygenated groups. Samples KOA 11 and 12, which are samples KOA 2 and 1 washed with HF, respectively, exhibited some different desorption characteristics after 
HF washing. Sample KOA 11, which is Norit Darco-LS, exhibited far less loss due to physisorbed and chemisorbed water at low temperatures after HF washing. Additionally, the amount of $\mathrm{SO}_{2}$ released was much less. Most significantly, the much smaller amount of $\mathrm{CO}_{2}$ released by sample KOA 11 leads to the possibility that HF destroyed a large amount of acidic, hydrophilic oxygen functionality on the surface of KOA 11. On the other hand, desorption of $\mathrm{CO}_{2}$ was similar (albeit at higher temperature) in sample KOA 12 versus KOA 1. Carbon monoxide desorption appeared to be similar in both cases, which could mean that HF washing did not affect the less acidic oxygenated surface functionality to as great an extent. HF washing did appear to diminish the amount of CO desorbed at high temperature between 1100 and $1300^{\circ} \mathrm{C}$. Thus, it is possible that the desorption experienced between 1100 and $1300^{\circ} \mathrm{C}$ in TPD measurements could have been from the dissociation inorganic species present (such as carbonates), which would have been largely dissolved and removed from the carbon supports during HF washing.

Table 2.25. Oxygenated Surface Functionalities, Their Decomposition Species, and Temperature. Taken from Akosylu et al. ${ }^{6}$

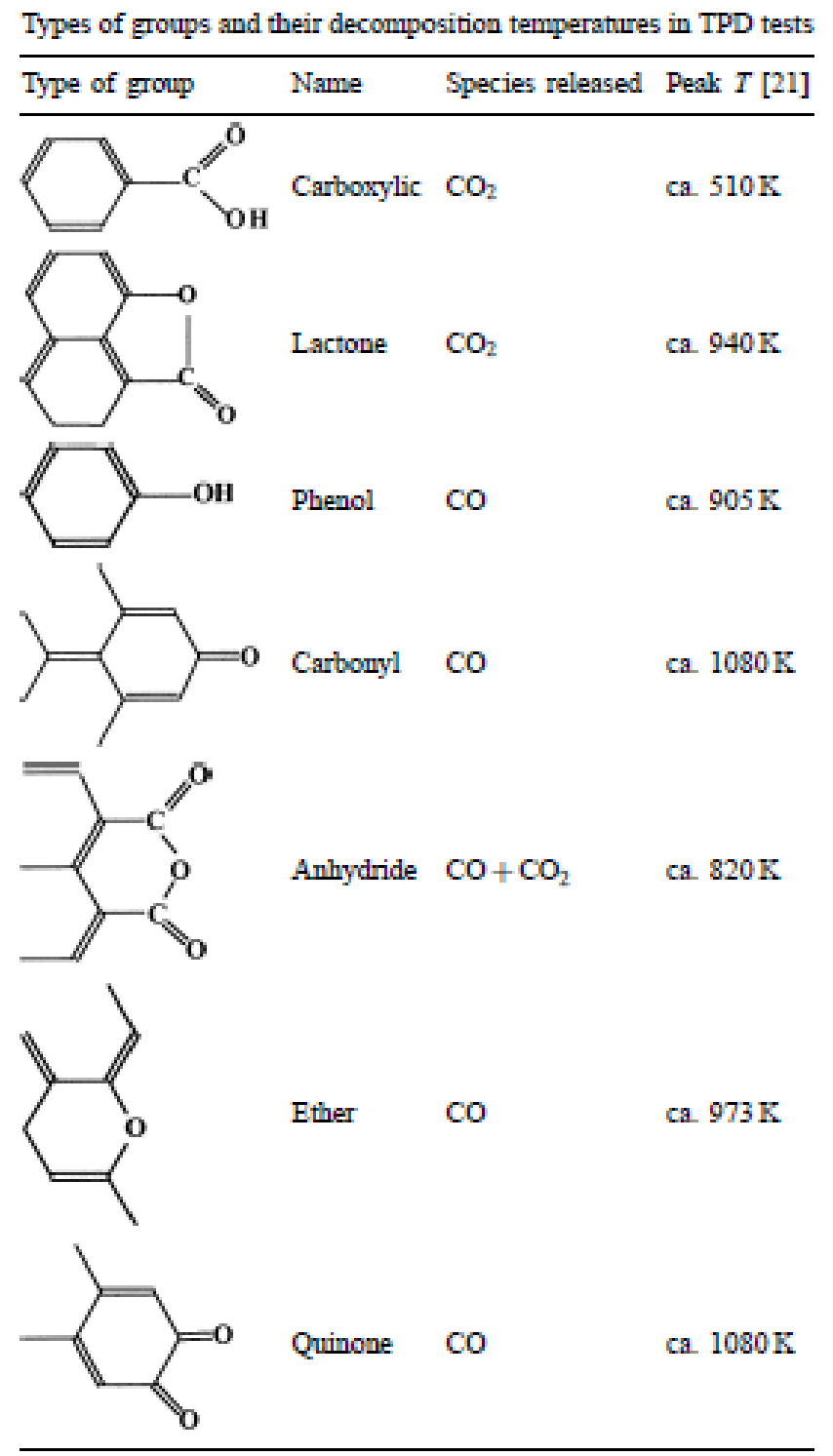


Table 2.26. Analysis of TPD Results on Carbon Supports KOA 1 through 6

\begin{tabular}{|c|c|c|c|c|c|c|c|c|c|c|c|c|c|}
\hline \multirow[b]{2}{*}{ Sample } & \multirow[b]{2}{*}{$\begin{array}{c}\text { Support } \\
\text { Description }\end{array}$} & \multicolumn{3}{|c|}{ Weight Loss Period 1} & \multicolumn{3}{|c|}{ Weight Loss Period 2} & \multicolumn{3}{|c|}{ Weight Loss Period 3} & \multicolumn{3}{|c|}{ Weight Loss Period 4} \\
\hline & & Range & Species & $\begin{array}{l}\text { Possible } \\
\text { Surface } \\
\text { Species }^{\mathrm{a}}\end{array}$ & Range & Species & $\begin{array}{c}\text { Possible } \\
\text { Surface } \\
\text { Species }\end{array}$ & Range & Species & $\begin{array}{c}\text { Possible } \\
\text { Surface } \\
\text { Species }\end{array}$ & Range & Species & $\begin{array}{c}\text { Possible } \\
\text { Surface } \\
\text { Species }\end{array}$ \\
\hline KOA 1 & Norit ROX 0.8 & $65-115$ & $\mathrm{H}_{2} \mathrm{O}$ & $\mathrm{A}$ & $160-335$ & $\mathrm{CO}_{2}$ & $\mathrm{~B}$ & $575-950$ & $\mathrm{CO}$ & E, H & $1150-1300$ & $\mathrm{CO}, \mathrm{H}_{2} \mathrm{O}$ & Unknown \\
\hline KOA 2 & Norit Darco-LS & $35-95$ & $\mathrm{H}_{2} \mathrm{O}$ & A & $450-700$ & $\mathrm{CO}, \mathrm{CO}_{2}$ & $\mathrm{~F}$ & $700-915$ & $\mathrm{CO}$ & $\mathrm{E}, \mathrm{H}$ & $1050-1300$ & $\mathrm{CO}, \mathrm{H}_{2} \mathrm{O}$ & Unknown \\
\hline KOA 3 & Sibunit & $60-100$ & $\mathrm{H}_{2} \mathrm{O}$ & A & $800-1050$ & $\mathrm{CO}$ & E, $\mathrm{H}$ & & & & & & \\
\hline KOA 4 & HSAG-1 & & & & & & & & & & & & \\
\hline KOA 5 & HSAG-2 & & & & & & & & & & & & \\
\hline KOA 6 & $\begin{array}{c}\text { Hyperion } \\
395-08\end{array}$ & $620-700$ & $\mathrm{CO}$ & $\mathrm{D}$ & $700-1300$ & $\mathrm{CO}$ & E, G, H & & & & & & \\
\hline
\end{tabular}

Table 2.27. Analysis of TPD Results on Carbon Supports KOA 7 through 12

\begin{tabular}{|c|c|c|c|c|c|c|c|c|c|c|c|c|c|}
\hline \multirow[b]{2}{*}{ Sample } & \multirow[b]{2}{*}{$\begin{array}{c}\text { Support } \\
\text { Description }\end{array}$} & \multicolumn{3}{|c|}{ Weight Loss Period 1} & \multicolumn{3}{|c|}{ Weight Loss Period 2} & \multicolumn{3}{|c|}{ Weight Loss Period 3} & \multicolumn{3}{|c|}{ Weight Loss Period 4} \\
\hline & & Range & Species & $\begin{array}{l}\text { Possible } \\
\text { Surface } \\
\text { Species }^{(\mathrm{a})}\end{array}$ & Range & Species & $\begin{array}{l}\text { Possible } \\
\text { Surface } \\
\text { Species }\end{array}$ & Range & Species & $\begin{array}{c}\text { Possible } \\
\text { Surface } \\
\text { Species }\end{array}$ & Range & Species & $\begin{array}{l}\text { Possible } \\
\text { Surface } \\
\text { Species }\end{array}$ \\
\hline KOA 7 & $\begin{array}{c}\text { Hyperion } \\
395-10\end{array}$ & $620-700$ & $\mathrm{CO}$ & $\mathrm{D}$ & $\begin{array}{l}700- \\
1300\end{array}$ & $\mathrm{CO}$ & E, $\mathrm{H}$ & & & & & & \\
\hline KOA 8 & $\begin{array}{c}\text { Hyperion } \\
384-82\end{array}$ & $30-120$ & $\mathrm{H}_{2} \mathrm{O}$ & A & $580-680$ & $\mathrm{CO}, \mathrm{CO}_{2}$ & F & $680-980$ & $\mathrm{CO}$ & E, $\mathrm{H}$ & & & \\
\hline KOA 9 & $\begin{array}{c}\text { Cummins-Moore } \\
5559 \mathrm{~S}\end{array}$ & $40-130$ & $\mathrm{H}_{2} \mathrm{O}$ & A & $130-600$ & $\mathrm{CO}, \mathrm{CO}_{2}$ & F & $600-750$ & $\mathrm{CO}$ & D, G & 780-980 & $\mathrm{CO}$ & E, H \\
\hline KOA 10 & $\begin{array}{l}\text { Cummins-Moore } \\
5586 \text { AW }\end{array}$ & $20-120$ & $\mathrm{H}_{2} \mathrm{O}$ & A & $120-400$ & $\mathrm{CO}_{2}$ & B & $500-980$ & $\mathrm{CO}$ & $\mathrm{D}, \mathrm{E}, \mathrm{G}, \mathrm{H}$ & $\begin{array}{l}1100- \\
1300\end{array}$ & $\mathrm{CO}$ & Unknown \\
\hline KOA 11 & $\begin{array}{l}\text { Norit Darco HF- } \\
\text { Washed }\end{array}$ & $60-120$ & $\mathrm{H}_{2} \mathrm{O}$ & A & $180-260$ & $\mathrm{CO}_{2}$ & B & $500-880$ & $\mathrm{CO}$ & $\mathrm{D}, \mathrm{E}, \mathrm{G}, \mathrm{H}$ & $\begin{array}{l}1150- \\
1300\end{array}$ & $\begin{array}{l}\mathrm{CO} \\
\mathrm{H}_{2} \mathrm{O}\end{array}$ & Unknown \\
\hline KOA 12 & $\begin{array}{c}\text { Norit ROX } 0.8 \\
\text { HF-Washed }\end{array}$ & $140-500$ & $\begin{array}{c}\mathrm{H}_{2} \mathrm{O}, \mathrm{CO}, \\
\mathrm{CO}_{2}\end{array}$ & $\mathrm{~A}, \mathrm{~B}, \mathrm{~F}$ & $500-980$ & $\mathrm{CO}$ & $\mathrm{D}, \mathrm{E}, \mathrm{G}, \mathrm{H}$ & $\begin{array}{l}1150- \\
1300\end{array}$ & $\mathrm{CO}$ & Unknown & & & \\
\hline
\end{tabular}

(a) Possible surface species desorbed: $\mathrm{A}=\mathrm{H}_{2} \mathrm{O}$; $\mathrm{B}=$ Carboxylic Acid; $\mathrm{C}=$ Lactone; $\mathrm{D}=$ Phenol; $\mathrm{E}=$ Carbonyl; $\mathrm{F}=\mathrm{Anydride}$; $\mathrm{G}=$ Ether; $\mathrm{H}=\mathrm{Quinone}$ 



\subsubsection{HF-Washed, Graphitized, and $\mathrm{HNO}_{3}$ Functionalized Carbon Supports}

Selected carbon supports listed in Table 2.2 also were subjected to TGA/MS analysis to investigate surface functionality. Specifically, the Pacco, Norit ROX 0.8, and Norit Darco-LS carbon supports were modified by consecutively subjecting the carbon supports to demineralization (HF washing), hightemperature graphitization, and finally $\mathrm{HNO}_{3}$ washing to re-functionalize the surface. The supports were characterized with TGA/MS after each step in the modification procedure.

A Netzsch STA 409 C/CD instrument was used to collect the TGA and MS spectra. About $150 \mathrm{mg}$ of sample was tested in a ceramic crucible inside the heated zone of the TGA/MS. The temperature program employed ramped from 30 to $1300^{\circ} \mathrm{C}$ at $5^{\circ} \mathrm{C} / \mathrm{min}$. The atmosphere was purged continuously with $100 \mathrm{~mL}$ of ultra-high pressure He. The MS sampled the gas just above the crucible to provide real-time data on the constituents of the gases evolved during the thermal ramp.

\subsubsection{Norit ROX 0.8}

An overlay of the TG traces of the Norit ROX 0.8 carbon support after each modification step is shown in Figure 2.7. Generally, HF treatment diminished the weight loss associated with decomposition of the carbon support above $\sim 1000^{\circ} \mathrm{C}$ (black versus blue curves). Prior to $1000^{\circ} \mathrm{C}$, the general shapes of the curves are similar. Graphitization of the support (red trace) results in a sample that loses very little mass during the TGA/MS experiment. Finally, $\mathrm{HNO}_{3}$ treatment of the graphitized support does reinstitute some surface functionality that is effectively decomposed by $200^{\circ} \mathrm{C}$. Above $200^{\circ} \mathrm{C}$, the trace of the $\mathrm{HNO}_{3}$-treated sample exhibits a slight downward curve similar to the graphitized sample. The slight downward trend is likely an effect of increasing buoyancy of the gas moving through the reactor as the furnace is heated.

An overlay of the TG curve overlaid with the curves of relevant gas phase compounds as identified by MS (water [m = 18], $\mathrm{CO}[\mathrm{m}=28]$, and $\mathrm{CO}_{2}[\mathrm{~m}=44]$ ) of the unmodified Norit ROX 0.8 are shown in Figure 2.8. The data in Figure 2.8 are similar to the data for sample KOA 1 reported above in Table 26, and the results are similar. A period of water loss up to $\sim 150^{\circ} \mathrm{C}$ is initially observed. Next, a period of weight loss accompanied by the observation of $\mathrm{CO}_{2}$ in the effluent occurred from $\sim 160$ through $350^{\circ} \mathrm{C}$. This period of weight loss likely results from decomposition of carboxylic acid-like moieties on the carbon surface. Water accompanies the initial portion of the decomposition indicating the moieties that decompose may have bound water or decompose up to $\sim 240^{\circ} \mathrm{C}$ may have bound water or dehydrate/ decompose to form water. Next, periods of weight loss occurring from $\sim 550$ to $1000^{\circ} \mathrm{C}$ and 1100 to $1300^{\circ} \mathrm{C}$ are observed with only $\mathrm{CO}$ in the gas phase. Note that the baseline for mass $=28$ drifts upward throughout the temperature program. A probable baseline for the MS trace with a mass $=28$ is shown in Figure 2.8. The mass loss from 550 to $1000^{\circ} \mathrm{C}$ accompanied by the observation of CO may result from decomposition of carbonyl or quinone type surface species. The mass loss between 1100 and $1300^{\circ} \mathrm{C}$ is likely due to a mineral (e.g., a carbonate) within the Norit ROX 0.8 support. The mineral may decompose directly to a metal oxide, although in the case of a carbonate, $\mathrm{CO}_{2}$ and not $\mathrm{CO}$ would be expected unless the mineral and the carbon support were interacting to produce $\mathrm{CO}$ upon decomposition instead of $\mathrm{CO}_{2}$. 


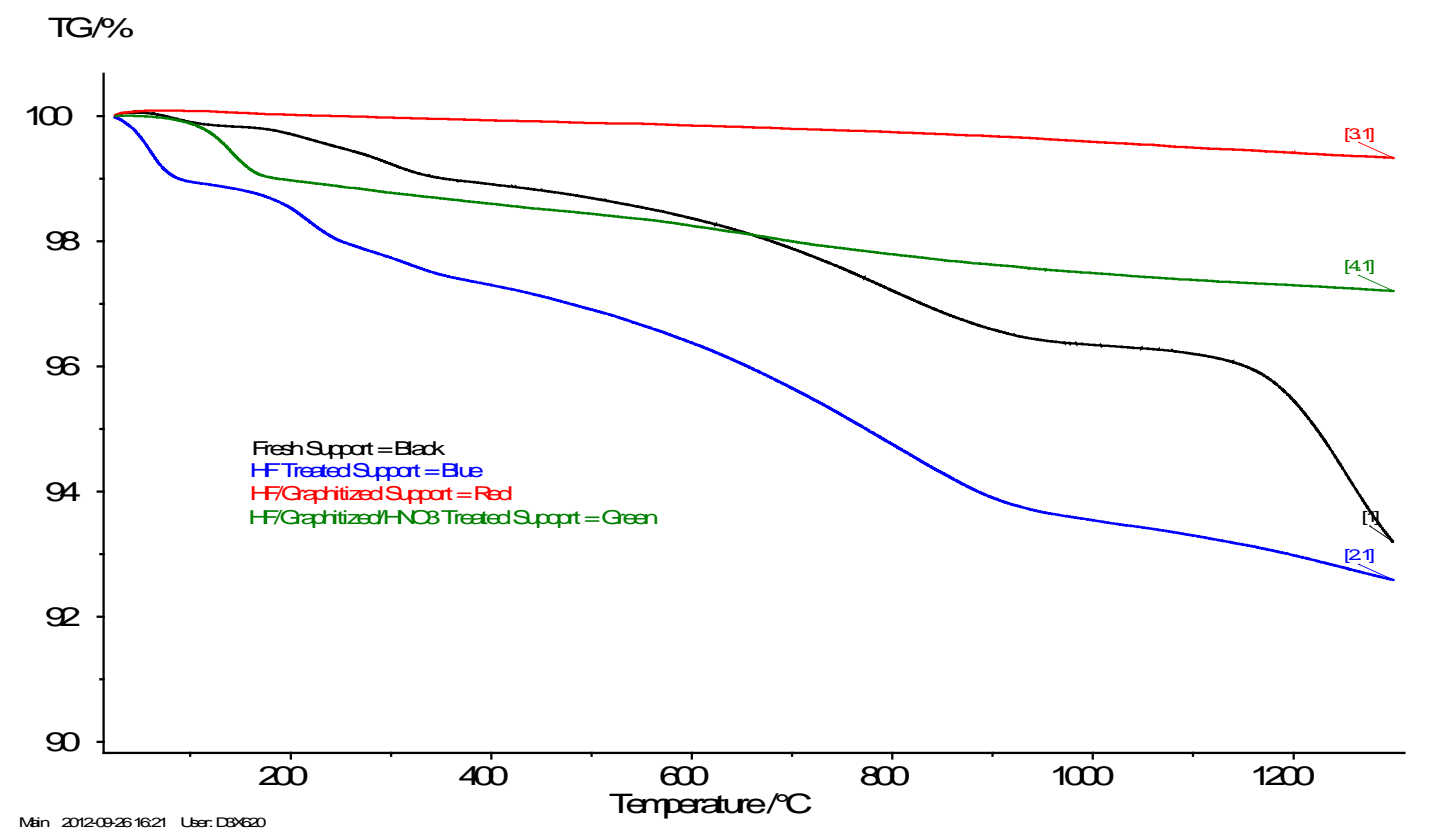

Figure 2.7. Overlay of the TG Curves Produced by TGA/MS Measurements of the Norit ROX 0.8 Carbon Support Prior to Modification and After Each Step of HF, Graphitization and $\mathrm{HNO}_{3}$ Modification of the Support

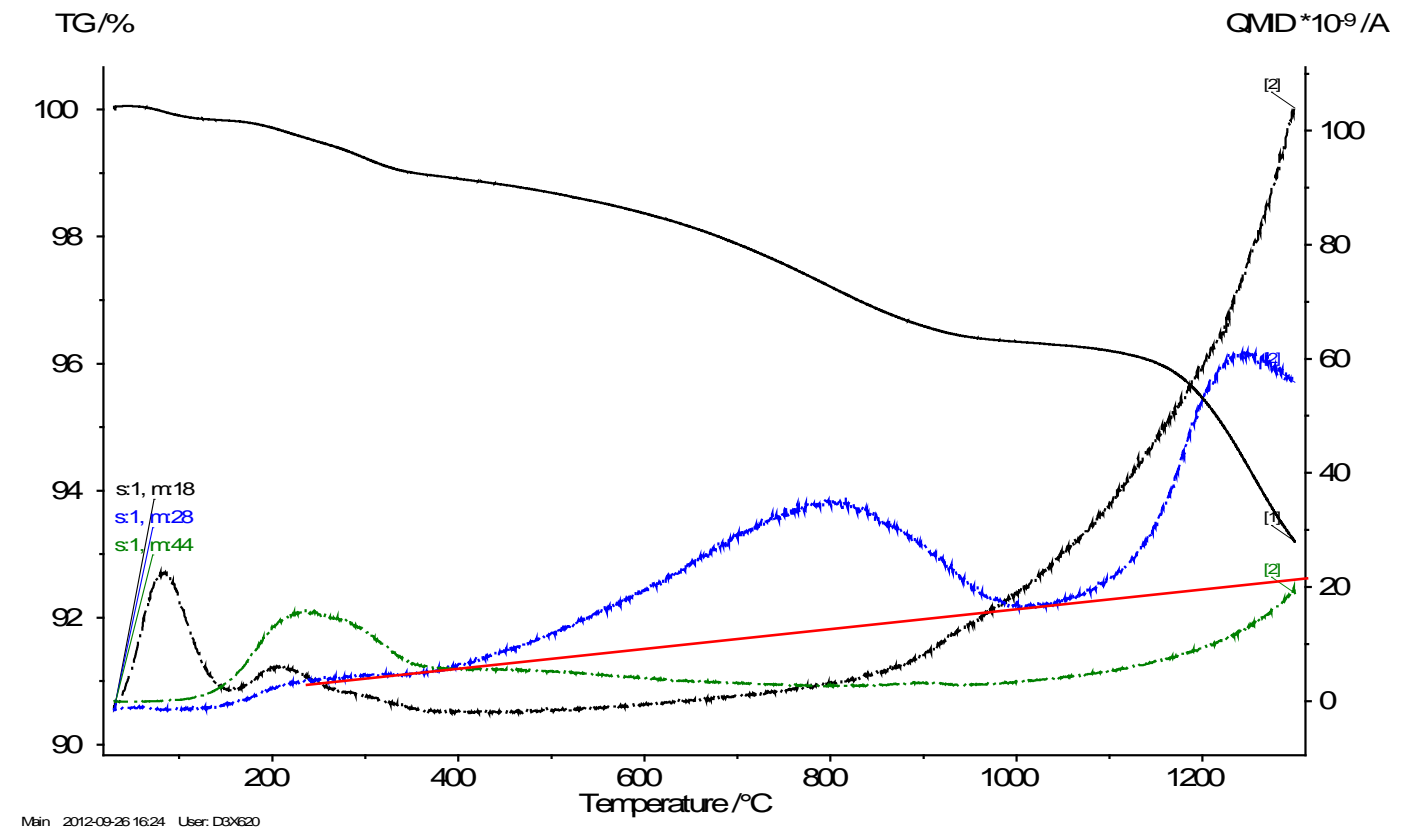

Figure 2.8. TG Curve (solid black line) and MS Traces of Relevant Compounds Observed in the Gas Phase Effluent during TGA/MS Testing of the Fresh (unmodified) Norit ROX 0.8 Carbon Support. The black dashed line (s1, m:18) corresponds to water, the blue dashed line (s1, $\mathrm{m}: 28)$ corresponds to $\mathrm{CO}$ and the green dashed line (s1, m:44) corresponds to $\mathrm{CO}_{2}$. The solid red line represents the likely baseline of the s1, m:28 response, which appeared to drift upward during the experiment. 
Generally, the TGA/MS traces of the HF-washed (demineralized) Norit ROX 0.8 carbon support were similar to those of the unmodified support. As shown in Figure 2.9, the HF-washed support did have a greater concentration of physisorbed and chemisorbed water compared with the fresh support as evidenced by the greater mass loss from $30^{\circ} \mathrm{C}$ to $\sim 150^{\circ} \mathrm{C}$. The amount of water present was high initially. Thus, the MS trace of the water, which was background subtracted to the first reading, dips below the starting point once all of the water has been removed from the support. Periods of mass loss with $\mathrm{CO}_{2}$ and water are again observed from $\sim 160^{\circ} \mathrm{C}$ to $\sim 350^{\circ} \mathrm{C}$, although the MS response for the $\mathrm{CO}_{2}$ is smaller than in the fresh sample. A similar period of weight loss accompanied by CO conversion is observed from $\sim 550$ to $950^{\circ} \mathrm{C}$. Note that the $\mathrm{CO}(\mathrm{m}=28)$ baseline again drifts upward similar to in Figure 2.8 , but the likely baseline is not drawn in Figure 2.9. Finally, the period of mass loss simultaneously observed with CO from $\sim 1100$ to $1300^{\circ} \mathrm{C}$ is either severely diminished or lacking completely in the HF-treated sample shown in Figure 2.9. Although strong conclusions are difficult to draw because of drift in the m/e = $28 \mathrm{MS}$ response and drift in the TG trace possibly due to gas buoyancy of the cup, it is possible to state that the sharp loss of mass in the fresh sample is not observed in the HF-treated sample. Therefore, it is likely that the $\mathrm{HF}$ treatment did remove the compound as the mass loss above $1100^{\circ} \mathrm{C}$ was not observed as shown in Figure 2.8.

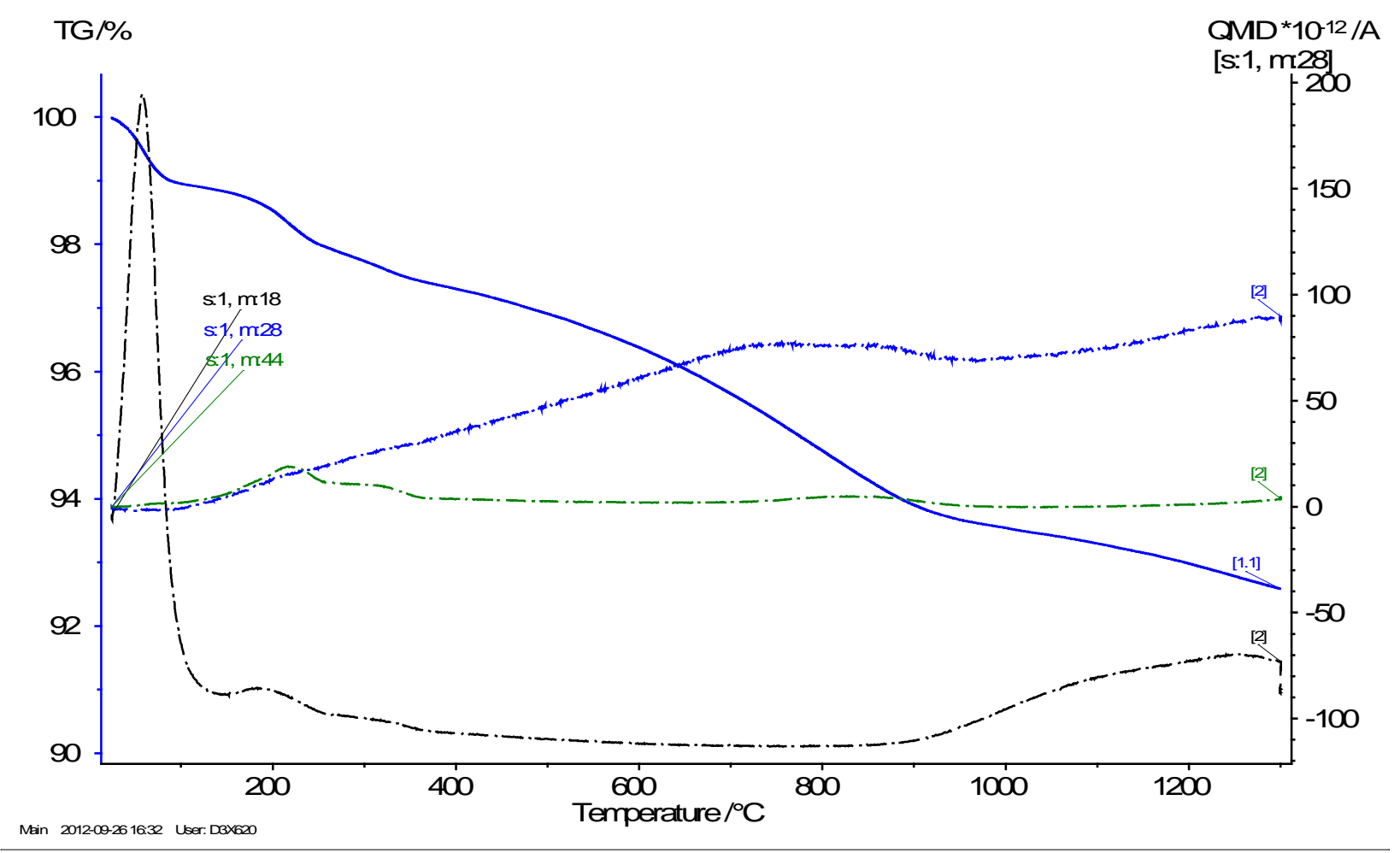

Figure 2.9. TG Curve (solid blue line) and MS Traces of Relevant Compounds Observed in the GasPhase Effluent during TGA/MS Testing of the HF-Treated (demineralized) Norit ROX 0.8 Carbon Support. The black dashed line (s1, m:18) corresponds to water, the blue dashed line $(\mathrm{s} 1, \mathrm{~m}: 28)$ corresponds to $\mathrm{CO}$, and the green dashed line (s1, m:44) corresponds to $\mathrm{CO}_{2}$. 
A portion of the Norit ROX 0.8 sample subjected to demineralization through HF washing was next subjected to graphitization via high-temperature treatment at $1800^{\circ} \mathrm{C}$ for $8 \mathrm{hr}$. Results of TGA/MS measurements on the HF-washed and graphitized Norit ROX 0.8 support is shown in Figure 2.10. Neither mass loss nor the presence of $\mathrm{H}_{2} \mathrm{O}$, $\mathrm{CO}$, or $\mathrm{CO}_{2}$ was observed in the gas phase during the temperature program up to $1300^{\circ} \mathrm{C}$. The lack of mass loss and gases corresponding with decomposed moieties is expected because the sample had been heated to such high temperature previously. The slight decrease of the TG trace due to buoyance and drifting CO baseline throughout the course of the temperature program can be readily seen in in Figure 2.10.

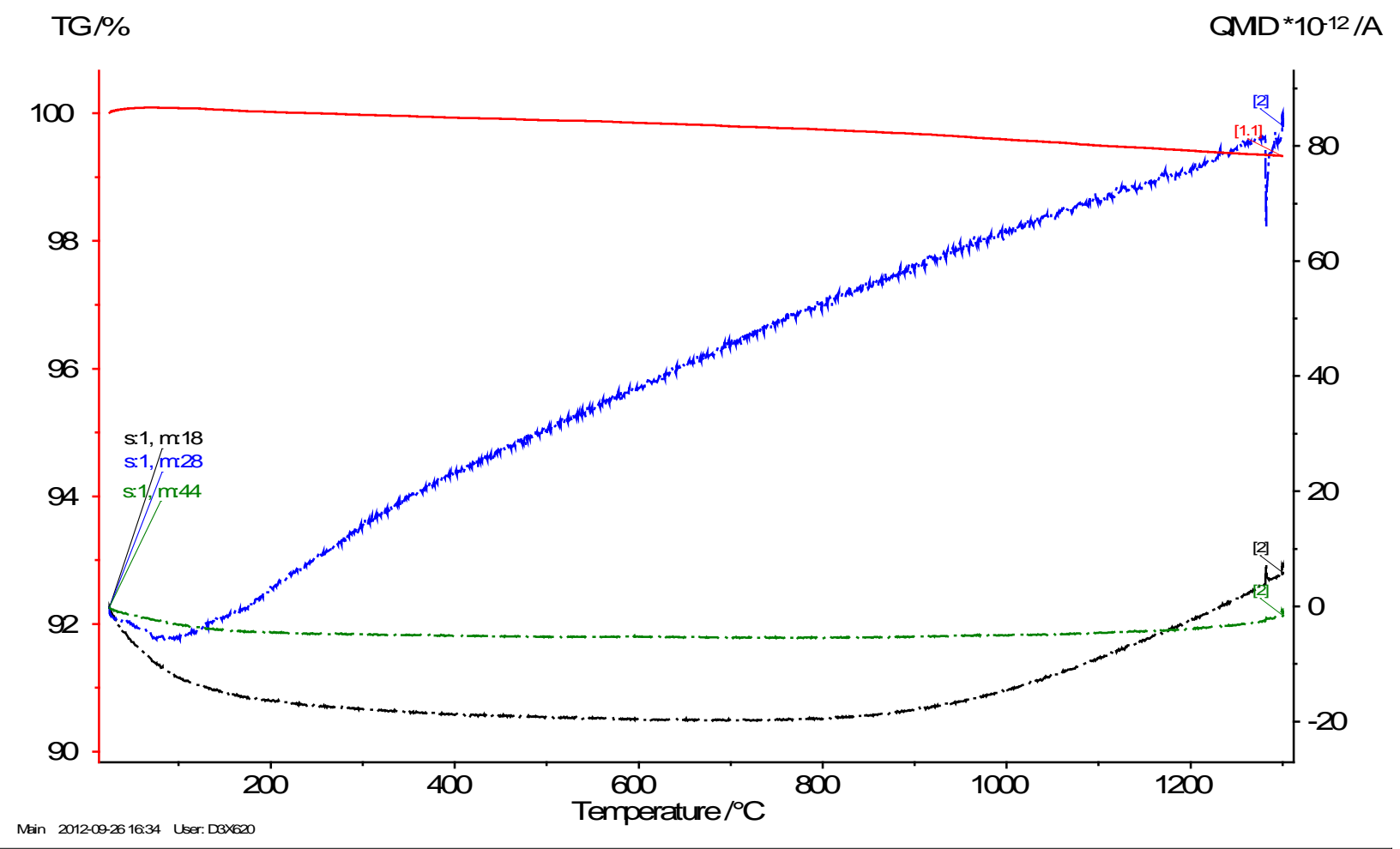

Figure 2.10. TG Curve (solid red line) and MS Traces of Relevant Compounds Observed in the Gas Phase Effluent during TGA/MS Testing of the HF-Treated (demineralized) and Graphitized Norit ROX 0.8 Carbon Support. The black dashed line (s1, m:18) corresponds to water, the blue dashed line (s1, m:28) corresponds to CO, and the green dashed line (s1, m:44) corresponds to $\mathrm{CO}_{2}$.

A portion of the Norit ROX 0.8 carbon support subjected to demineralization and graphitization was further modified through exposure to $\mathrm{HNO}_{3}$ to re-functionalize the carbon surface in a controlled manner. The TGA and MS traces of the $\mathrm{HNO}_{3}$-treated carbon support are shown in Figure 2.11; the TG trace is the solid green line. In addition to water, $\mathrm{CO}$ and $\mathrm{CO}_{2}$, the red dashed line corresponding to $\mathrm{NO}_{\mathrm{x}}$ species $(\mathrm{m}=30)$ is plotted. The MS trace illustrates that water (likely physisorbed water) is observed to desorb from the support below $\sim 100^{\circ} \mathrm{C}$. From a period of $\sim 100$ to $200^{\circ} \mathrm{C}$ about $1 \mathrm{wt} \%$ of the mass of the support is lost. This period of mass loss corresponds with the presence of water, $\mathrm{CO}_{2}$, and $\mathrm{NO}_{\mathrm{x}}$. Thus, the $\mathrm{HNO}_{3}$ treatment did appear to oxidize the surface of the carbon support as indicated by the evolution of $\mathrm{CO}_{2}$. However, some $\mathrm{N}_{2}$ containing groups also may have been incorporated into the carbon/oxygen moieties 
formed by the $\mathrm{HNO}_{3}$. Alternatively, the presence of $\mathrm{NO}_{x}$ and water observed simultaneously may be from $\mathrm{HNO}_{3}$ that was not completely rinsed out of the pores.

The oxidized species formed via $\mathrm{HNO}_{3}$ treatment of the HF-washed and graphitized Norit ROX 0.8 were relatively unstable and had decomposed before a temperature of $200^{\circ} \mathrm{C}$ had been reached.

Production of more stable (albeit less well defined) oxygenated species could likely be produced through $\mathrm{HNO}_{3}$ treatment at higher temperature or through application of a stronger oxidizer such as $\mathrm{H}_{2} \mathrm{O}_{2}$.

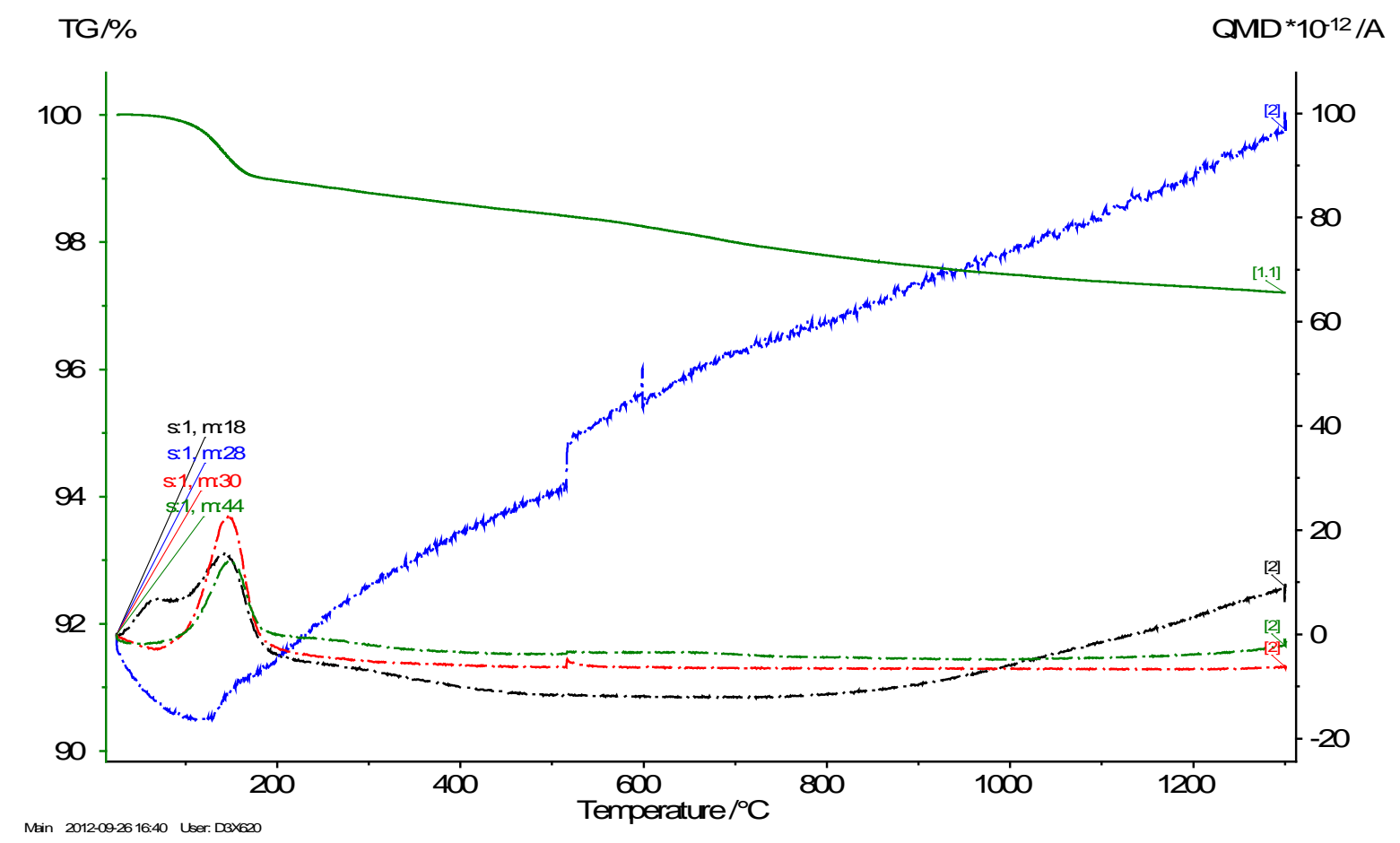

Figure 2.11. TG Curve (solid green line) and MS Traces of Relevant Compounds Observed in the GasPhase Effluent during TGA/MS Testing of the HF-Treated (demineralized), Graphitized, and Subsequently Re-Functionalized via $\mathrm{HNO}_{3}$ Treatment of the Norit ROX 0.8 Carbon Support. The black dashed line (s1, m:18) corresponds to water, the blue dashed line (s1, m:28) corresponds to CO, the red dashed line (s1, m:30) corresponds to $\mathrm{NO}_{\mathrm{x}}$ species, and the green dashed line (s1, m:44) corresponds to $\mathrm{CO}_{2}$. Note that the discontinuity in the $\mathrm{CO}$ trace at $\sim 575^{\circ} \mathrm{C}$ is likely an instrument artifact and, therefore, is not real.

\subsubsection{Darco-LS}

Similar to the Norit ROX 0.8, the Darco-LS carbon catalyst support was also subjected to demineralization through HF washing, graphitization, and surface re-functionalization via $\mathrm{HNO}_{3}$ washing. The carbon support was measured with TGA/MS after each modification step. The TG traces illustrating the weight losses of the fresh support and after each modification step are overlaid in Figure 2.12. The results of TGA/MS testing of the modified Darco-LS samples are similar to the same observations with the Norit ROX 0.8. The fresh and HF-treated (black versus blue traces) samples are similar except for the lack of weight loss above $\sim 1100^{\circ} \mathrm{C}$. The graphitized sample (red trace) shows only a very slow decline over the course of the temperature program, which likely results from gas buoyancy in the furnace and not actual sample weight loss. Finally, the $\mathrm{HNO}_{3}$ sample (green trace) shows an initial rapid weight loss that 
is completed by $\sim 200^{\circ} \mathrm{C}$. In contrast to the $\mathrm{HNO}_{3}$ treated support, there also appears to be a period of weight loss at $\sim 600$ and $800^{\circ} \mathrm{C}$. Each of the TG traces is analyzed in greater detail with the MS traces of relevant compounds in the gas phase below.

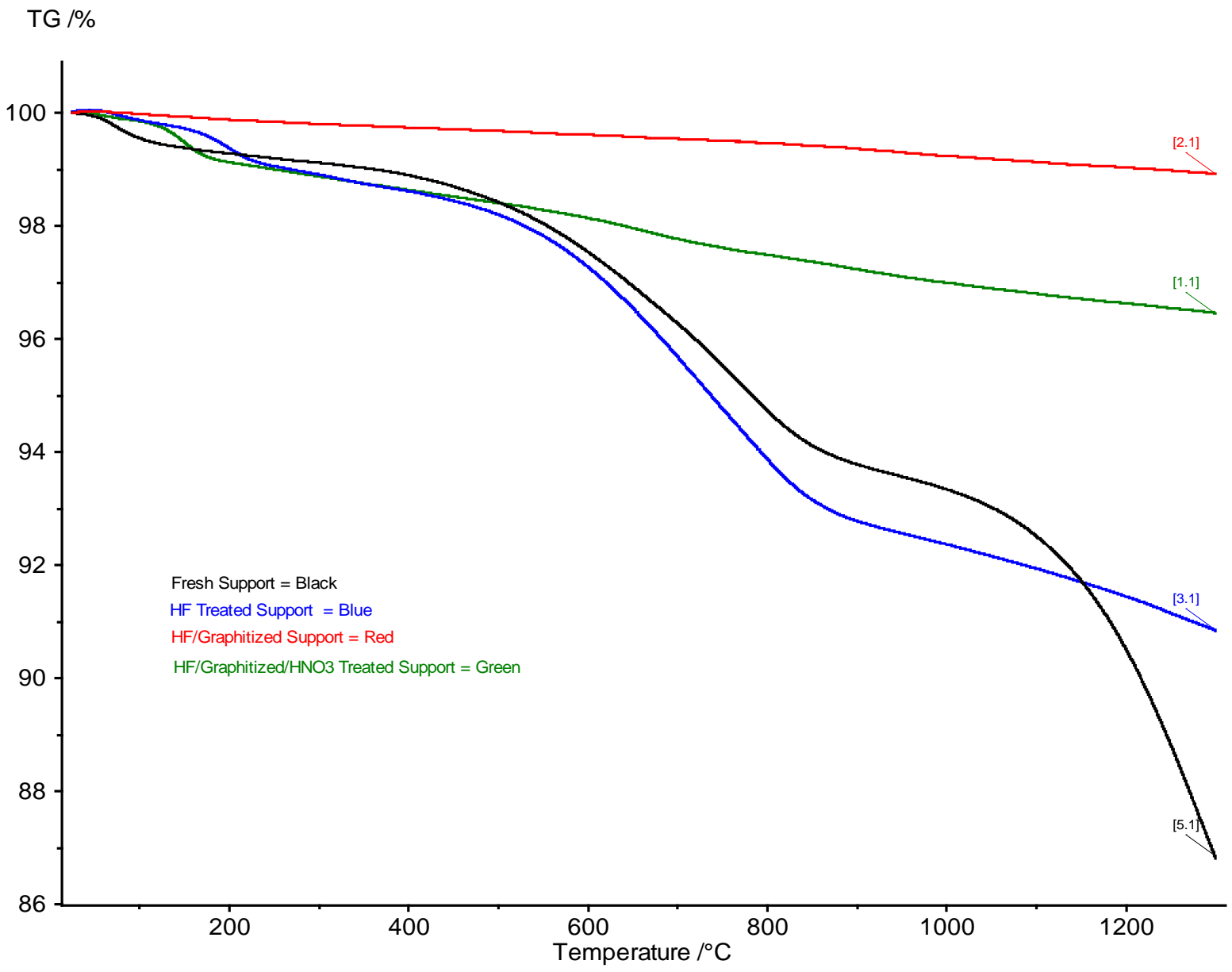

Figure 2.12. Overlay of the TG Curves Produced by TGA/MS Measurements of the Norit Darco-LS Carbon Support Prior to Modification and After Each Step of HF Treatment, Graphitization, and $\mathrm{HNO}_{3}$ Modification of the Support

The results of the TGA/MS measurements on the fresh (unmodified) Norit Darco-LS support are shown in Figure 2.13. An initial period of weight loss due to physisorbed and chemisorbed water is observed up to $\sim 150^{\circ} \mathrm{C}$. The TG trace remains relatively unchanged up to $\sim 400^{\circ} \mathrm{C}$ when a period of weight loss accompanied by $\mathrm{CO}$ and $\mathrm{CO}_{2}$ present in the gas-phase effluent is observed. Note that the $\mathrm{CO}$ baseline drifts up during the course of the baseline. The solid red line in Figure 2.13 is a likely baseline for the $\mathrm{CO}\left(\mathrm{m}=28\right.$ trace). The period of weight loss between 400 and $800^{\circ} \mathrm{C}$ coupled with $\mathrm{CO}$ and $\mathrm{CO}_{2}$ in the gas phase may be due to the decomposition of oxygenated surface species such as the anhydride species suggested in Table 2.25. Interestingly, water appears above $800^{\circ} \mathrm{C}$, which may result from dehydration of a mineral impurity in the Darco-LS. Finally, similar to the fresh Norit ROX 0.8 sample, a period of weight loss with CO observed in the gas phase occurs from $\sim 1100$ to $1300^{\circ} \mathrm{C}$. This period of weight loss may result from decomposition of a mineral in the carbon support. 


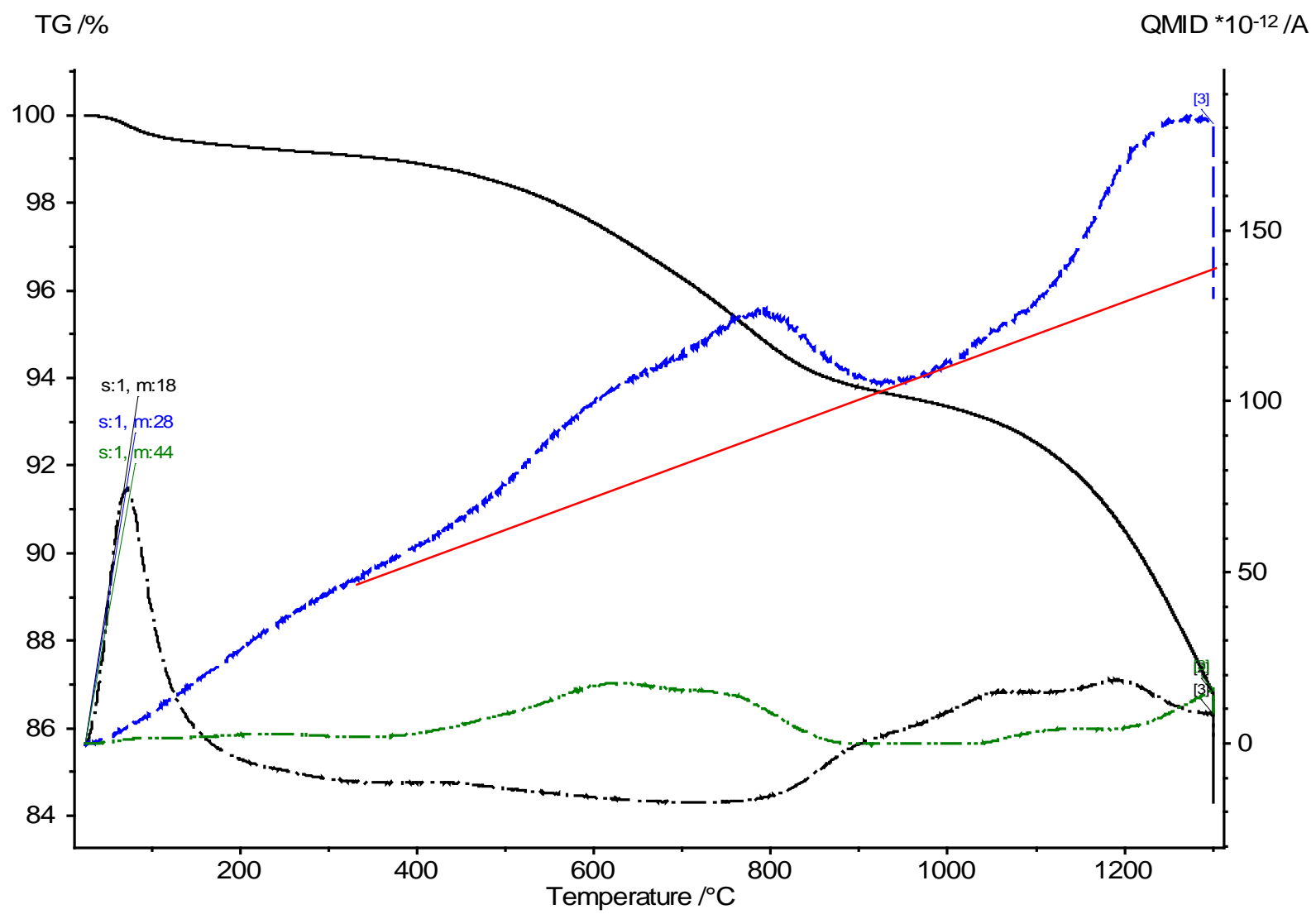

Figure 2.13. TG Curve (solid black line) and MS Traces of Relevant Compounds Observed in the GasPhase Effluent during TGA/MS Testing of the Fresh (unmodified) Norit Darco-LS Carbon Support. The black dashed line (s1, m:18) corresponds to water, the blue dashed line (s1, m:28) corresponds to $\mathrm{CO}$, and the green dashed line (s1, m:44) corresponds to $\mathrm{CO}_{2}$. The solid red line represents the likely baseline of the s1, m:28 response, which appeared to drift upward through the experiment.

Washing of the Darco-LS carbon support with HF to dissolve the minerals present in the support did not have a significant effect on the periods of mass loss in the support up to $\sim 1100^{\circ} \mathrm{C}$. As seen in Figure 2.14, the most significant difference compared to the fresh support was the lack of mass loss from 1100 to $1300^{\circ} \mathrm{C}$ associated with $\mathrm{CO}$ in the gas-phase effluent. The lack of this mass loss period lends support to the hypothesis that the mass lost during this phase is from a decomposing mineral in the fresh support that is removed by the demineralization treatment. 


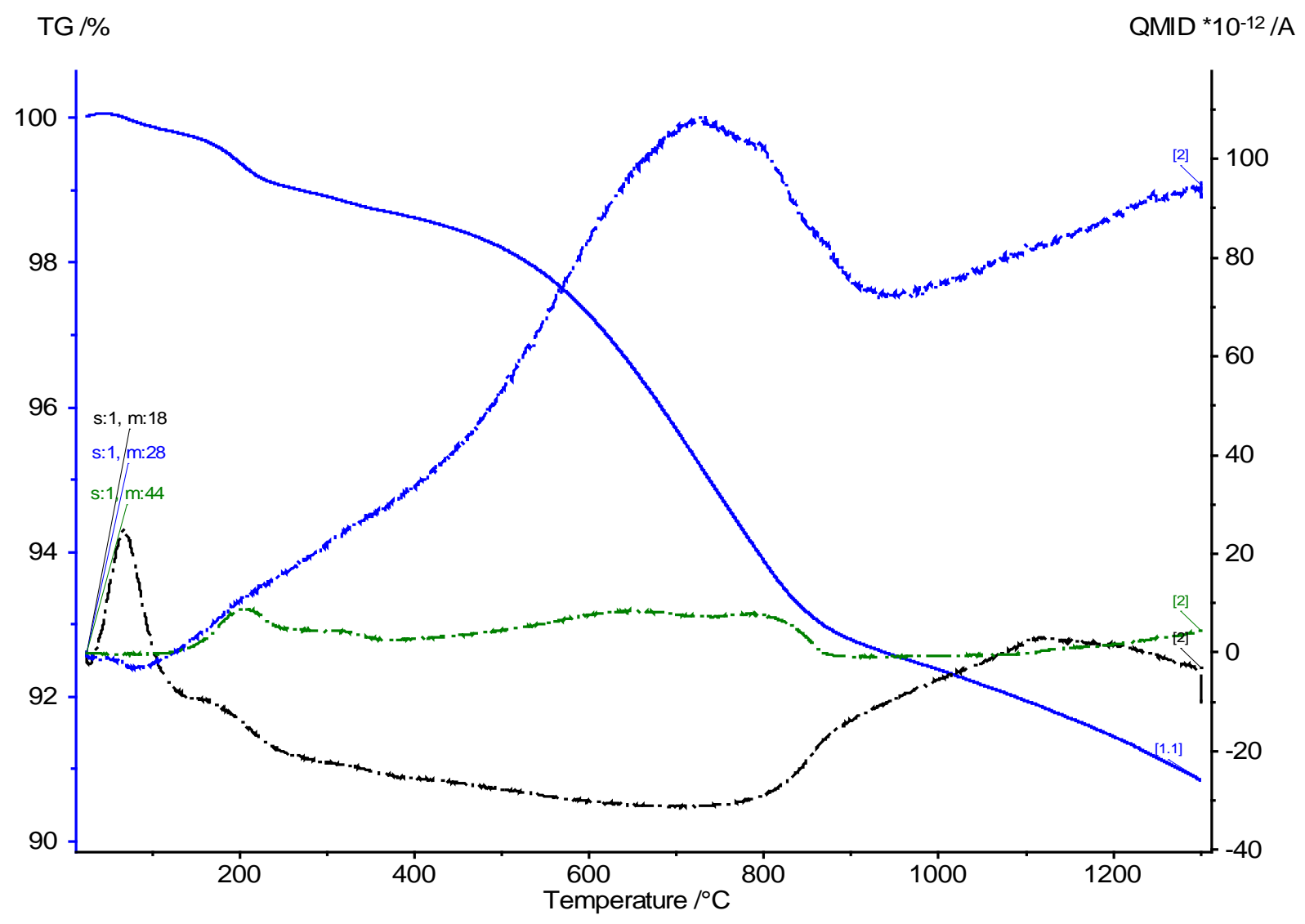

Figure 2.14. TG Curve (solid blue line) and MS Traces of Relevant Compounds Observed in the GasPhase Effluent during TGA/MS Testing of the HF-Treated (demineralized) Norit Darco-LS Carbon Support. The black dashed line (s1, m:18) corresponds to water, the blue dashed line (s1, m:28) corresponds to CO, and the green dashed line (s1, m:44) corresponds to $\mathrm{CO}_{2}$.

The TGA/MS traces for the HF-washed and graphitized Norit Darco-LS sample are shown in Figure 2.15. The TG trace is relatively flat with only a slight loss throughout the course of the temperature program most likely resulting from the buoyancy of the sample carrier in the heating furnace. The traces for $\mathrm{CO}$ and $\mathrm{H}_{2} \mathrm{O}$ are relatively flat, while the increasing baseline of the $\mathrm{CO}$ can again be observed. The data in Figure 2.15 illustrate that graphitization has removed any oxygenated functionality from the surface of the Darco-LS support. 


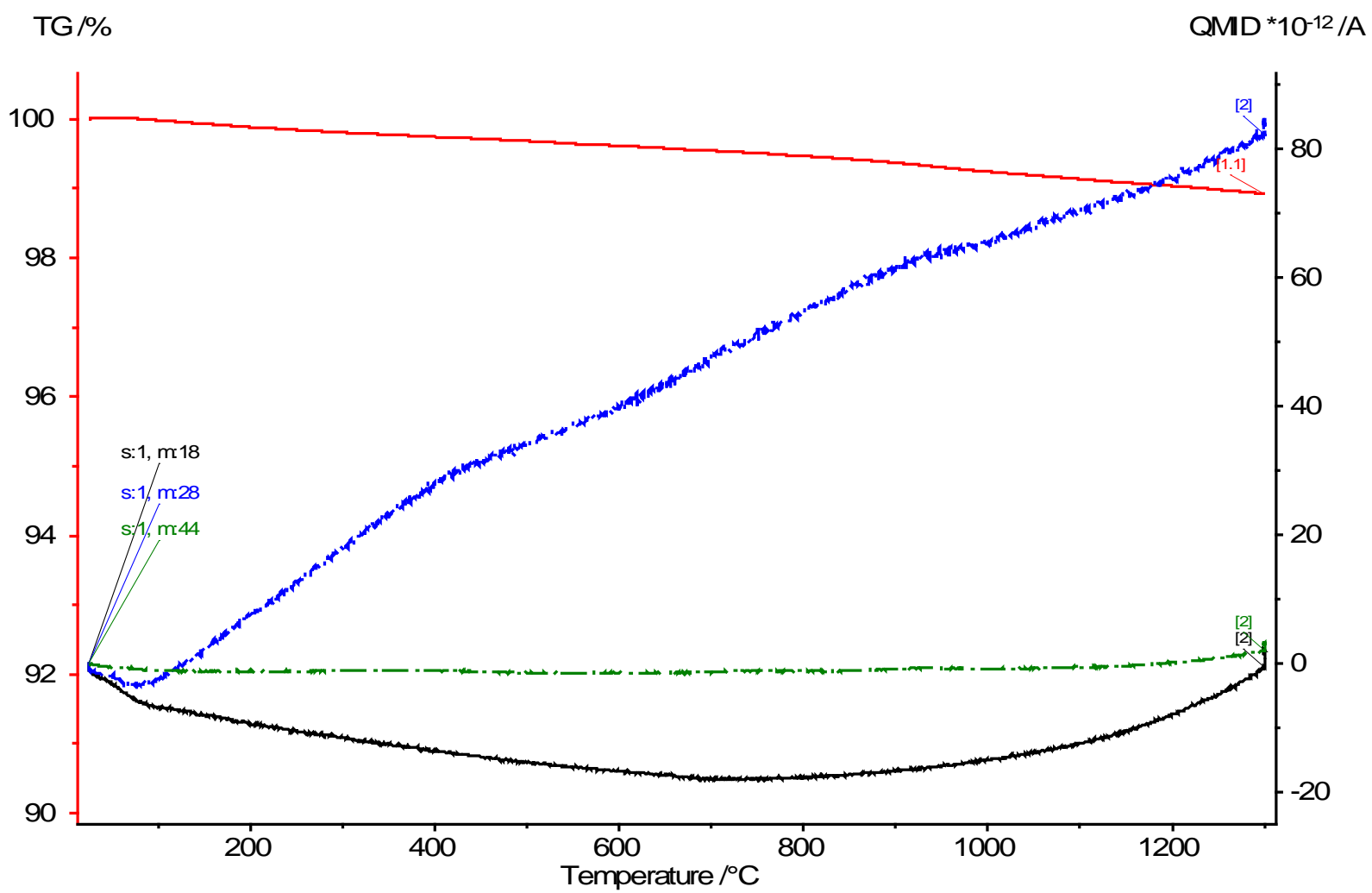

Figure 2.15. TG Curve (solid red line) and MS Traces of Relevant Compounds Observed in the Gas-Phase Effluent during TGA/MS Testing of the HF-Treated (demineralized), and Graphitized Darco-LS Carbon Support. The black dashed line (s1, m:18) corresponds to water, the blue dashed line (s1, m:28) corresponds to $\mathrm{CO}$, and the green dashed line (s1, m:44) corresponds to $\mathrm{CO}_{2}$.

Figure 2.16 shows the TGA/MS results of the Darco-LS carbon support subjected to $\mathrm{HNO}_{3}$ treatment after HF washing and graphitization. The TG trace decreases in mass throughout the temperature program as a result of buoyancy in the flowing gas. The CO background also increases throughout the temperature program. A period of small weight loss due to adsorbed water is observed up to $\sim 120^{\circ} \mathrm{C}$. Next, a period of weight loss from $\sim 100$ to $200^{\circ} \mathrm{C}$ is observed with $\mathrm{H}_{2} \mathrm{O}, \mathrm{CO}_{2}$, and $\mathrm{NO}_{\mathrm{x}}(\mathrm{m}=30)$ present. The weight loss may indicate oxidized surface species with $\mathrm{N}_{2}$ incorporated into the moieties that decompose in this temperature range. Alternatively, $\mathrm{HNO}_{3}$ may still be present in the pores from incomplete washing of the support after treatment.

In contrast to the $\mathrm{HNO}_{3}$-washed Norit ROX 0.8, Figure 2.16 illustrates that the Darco-LS carbon had a period of very small weight loss accompanied with $\mathrm{CO}$ and $\mathrm{CO}_{2}$. This result suggests that the $\mathrm{HNO}_{3}$ treatment of the Darco-LS carbon resulted in the formation of a small amount of oxygenated surface moieties such as the anhydride species suggested in Table 2.25 . 


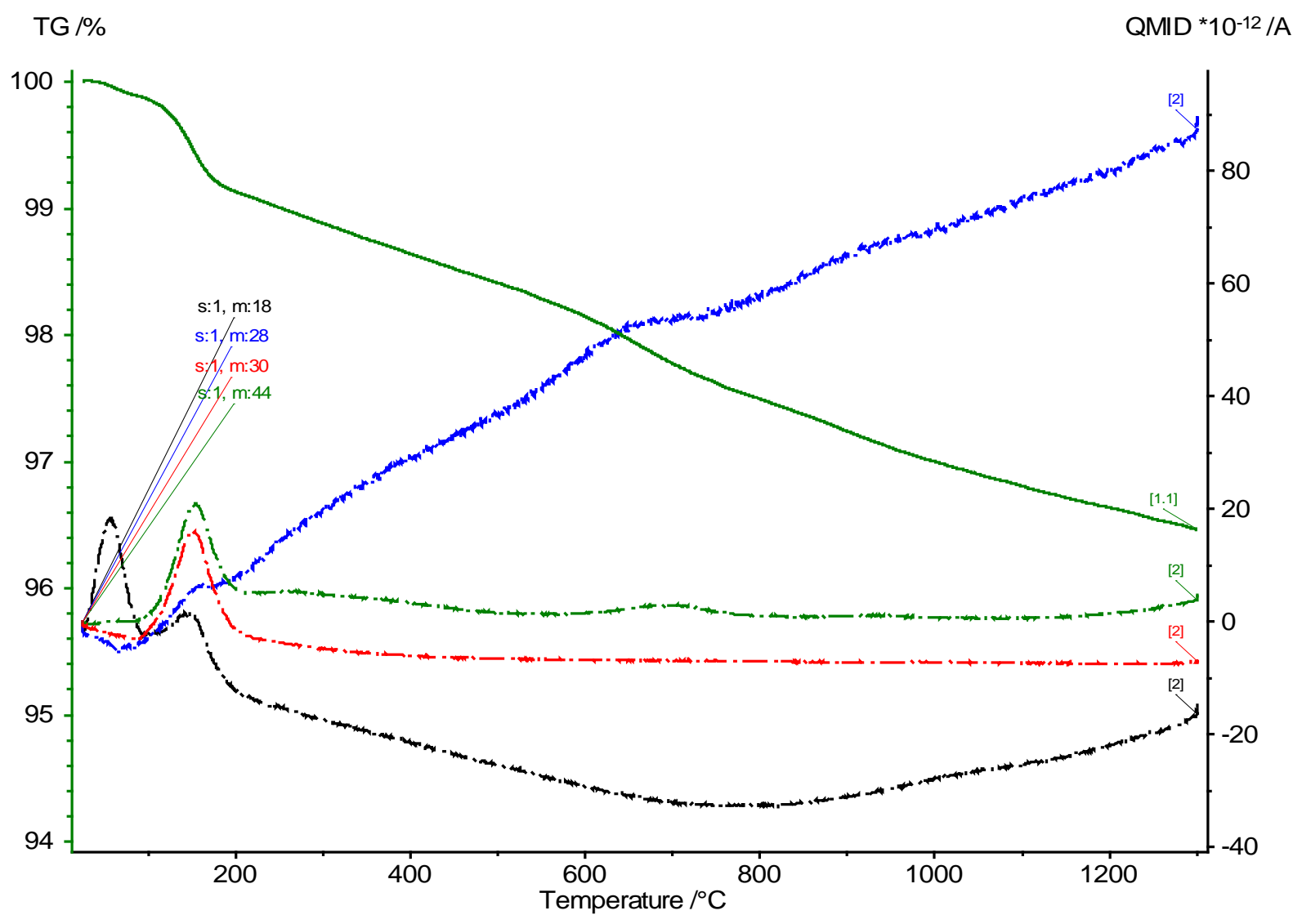

Figure 2.16. TG Curve (solid green line) and MS Traces of Relevant Compounds Observed in the GasPhase Effluent during TGA/MS Testing of the HF-Treated (demineralized), Graphitized, and Subsequently Re-Functionalized via $\mathrm{HNO}_{3}$ Treatment of the Norit Darco-LS Carbon Support. The black dashed line (s1, m:18) corresponds to water, the blue dashed line (s1, m:28) corresponds to CO, the red dashed line (s1, m:30) corresponds to $\mathrm{NO}_{\mathrm{x}}$ species, and the green dashed line (s1, m:44) corresponds to $\mathrm{CO}_{2}$.

\subsubsection{Pacco 90-100 CTC Carbon Support}

The Pacco 90-100 CTC carbon support was the third and final support subjected to TGA/MS measurements after demineralization (HF washing), graphitization, and $\mathrm{HNO}_{3}$ washing. Figure 2.17 illustrates the overlay of the TG traces collected after each modification procedure. The fresh (unmodified) and HF-washed samples are qualitatively similar except for a period of weight loss beginning at $1100^{\circ} \mathrm{C}$ observed in the fresh (black trace) sample. The HF-washed and graphitized sample (red trace) exhibits a significant period of weight loss at 600 to $850^{\circ} \mathrm{C}$. The graphitized Pacco CTC 90100 carbon support sample is significantly different than the analogous Norit ROX 0.8 and Norit DarcoLS samples. Interestingly, the $\mathrm{HNO}_{3}$-treated sample (that had undergone graphitization prior to $\mathrm{HNO}_{3}$ treatment) does not show a similar decrease in the 600 to $850^{\circ} \mathrm{C}$ temperature range. It is highly likely that the sample labeled Pacco CTC 90-100 HF-Treated/Graphitized was labeled incorrectly and was not the sample analyzed. 


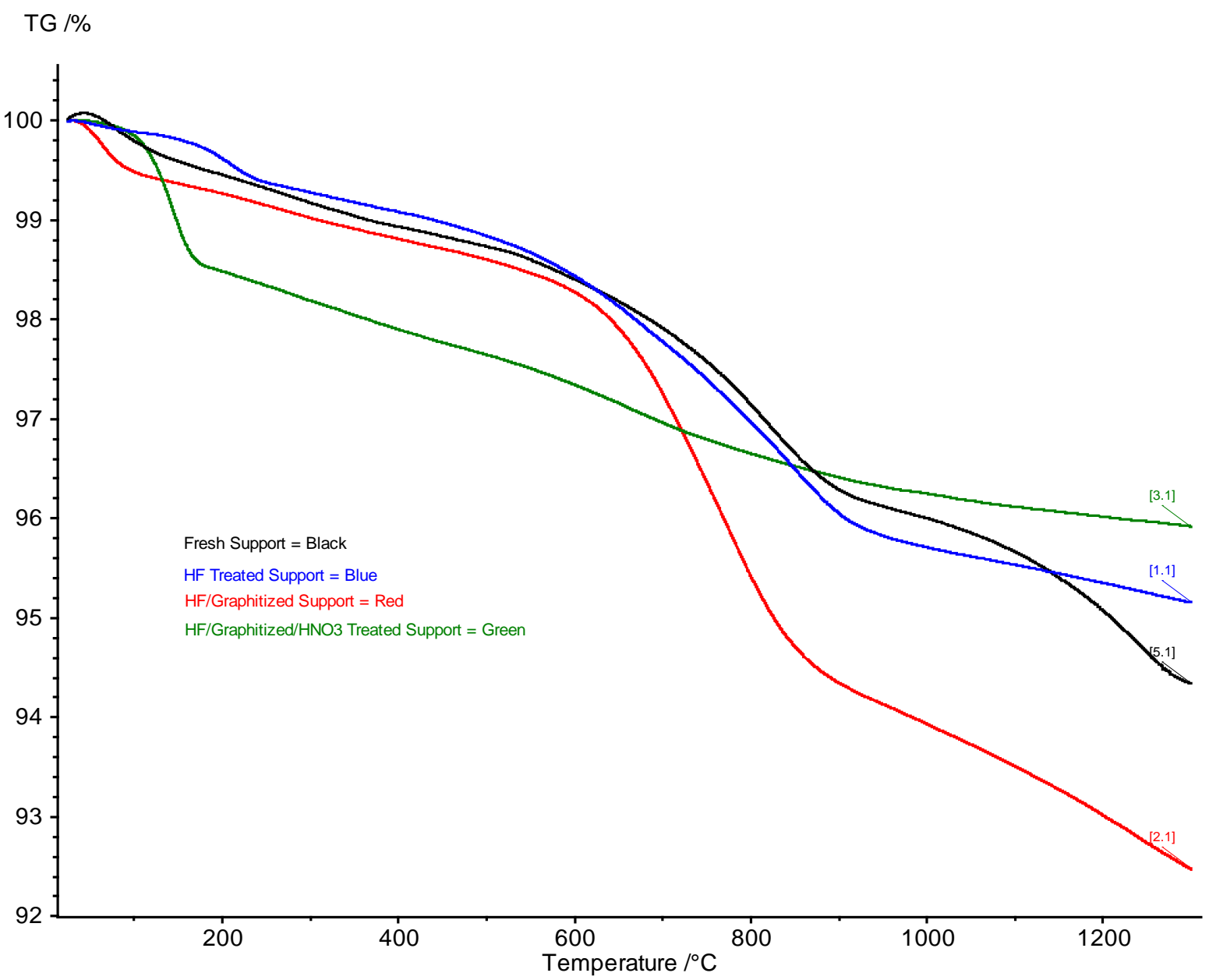

Figure 2.17. Overlay of TG Curves Produced by TGA/MS Measurements of the Pacco 90-100 CTC Carbon Support Prior to Modification and after Each Step of HF, Graphitization and $\mathrm{HNO}_{3}$ Modification of the Support.

The results of the TGA/MS measurements on the fresh (unmodified) Pacco CTC 90-100 carbon support are shown in Figure 2.18. An initial period of weight loss due to physisorbed and chemisorbed water is observed up to $\sim 150^{\circ} \mathrm{C}$. The TG trace remains relatively unchanged up to $\sim 500^{\circ} \mathrm{C}$ when a period of weight loss accompanied by observation of $\mathrm{CO}$ and initially a small amount of $\mathrm{CO}_{2}$. Note that the $\mathrm{CO}$ baseline drifts up during the course of the baseline. The solid red line in Figure 2.18A is a likely baseline for the $\mathrm{CO}\left(\mathrm{m}=28\right.$ trace). The beginning of the period of weight loss between $\sim 500$ to $625^{\circ} \mathrm{C}$ coupled with $\mathrm{CO}$ and $\mathrm{CO}_{2}$ in the gas phase may result from decomposition of oxygenated surface species such as the anhydride species suggested in Table 2.25. From 625 to $900^{\circ} \mathrm{C}, \mathrm{CO}$ primarily is observed in the gas phase and may be due to the decomposition of phenol, carbonyl, ether, or quinone type species as suggested in Table 2.25. Interestingly, water appears above $1000^{\circ} \mathrm{C}$, which may result from dehydration of a mineral impurity. Finally, similar to the fresh Norit ROX 0.8 and Norit Darco-LS samples, a period of weight loss with CO observed in the gas phase occurs from $\sim 1100$ and $1300^{\circ} \mathrm{C}$. This period of weight loss may be due to the decomposition of a mineral in carbon support. 


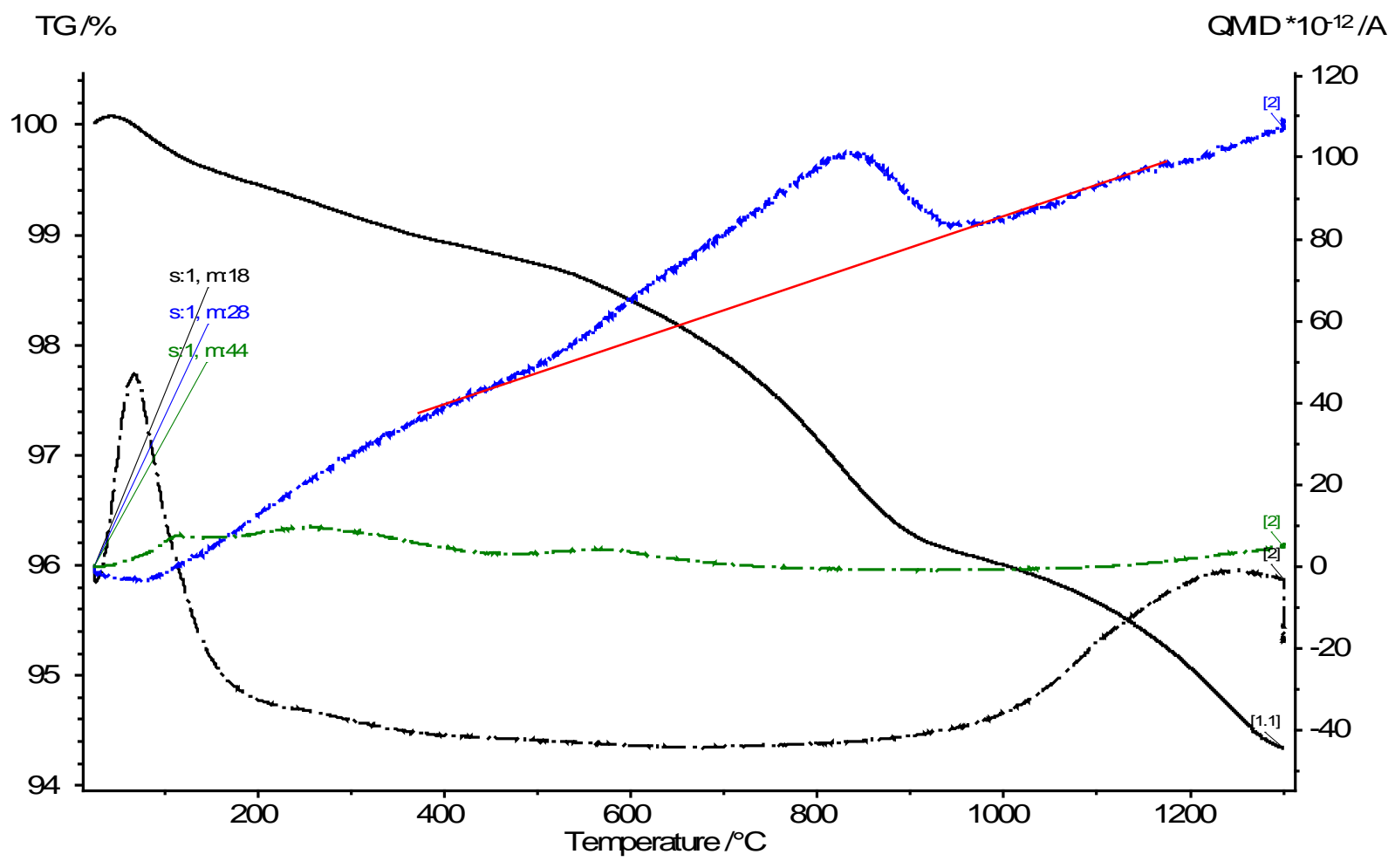

Figure 2.18. TG Curve (solid black line) and MS Traces of Relevant Compounds Observed in the GasPhase Effluent during TGA/MS Testing of the Fresh (unmodified) Pacco 90-100 CTC Carbon Support. The black dashed line (s1, m:18) corresponds to water, the blue dashed line (s1, m:28) corresponds to CO, and the green dashed line (s1, m:44) corresponds to $\mathrm{CO}_{2}$. The solid red line represents the likely baseline of the s1, m:28 response, which appeared to drift upward through the experiment.

Results of TGA/MS measurement of the HF-treated Pacco CTC 90-100 carbon sample is shown in Figure 2.19. An initial period of small weight loss attributed to adsorbed water being released is observed up to $\sim 100^{\circ} \mathrm{C}$. A period of weight loss from 160 to $225^{\circ} \mathrm{C}$ accompanied by $\mathrm{CO}_{2}$ and water is observed in the HF-treated sample. A similar period was not observed in the fresh sample. In this temperature range, Table 2.25 suggests that a carboxylic acid group on the surface may be decomposing. Another possibility is that a mineral could be decomposing in this temperature range. The mineral may have been incompletely dissolved by HF treatment. The portion of the mineral that was not dissolved in HF may have been more accessible to the open atmosphere through the partial HF digestion. The greater accessibility of the mineral may account for the sharper period of mass loss with a more definitive observation of water and $\mathrm{CO}_{2}$ in the gas phase.

The period of mass loss from $\sim 500$ to $900^{\circ} \mathrm{C}$ is again observed in the HF-treated Pacco 90-100 CTC sample in Figure 2.19. In contrast to the unmodified Pacco sample, $\mathrm{CO}_{2}$ can be observed in the gas phase for the duration of the weight loss period, which may result from decomposition of an incompletely dissolved carbonate mineral. The shape of the $\mathrm{CO}$ trace remains qualitatively similar to the unmodified sample. Hence, the same surface functional groups (anhydride, phenol, anhydride, and quinone shown in Table 2.25) are proposed for the HF-treated sample. The period of mass loss above $1100^{\circ} \mathrm{C}$ is absent from the HF-washed sample, indicating that the mass loss in this temperature range is likely due to a mineral that is susceptible to dissolution in HF and is completely removed. 


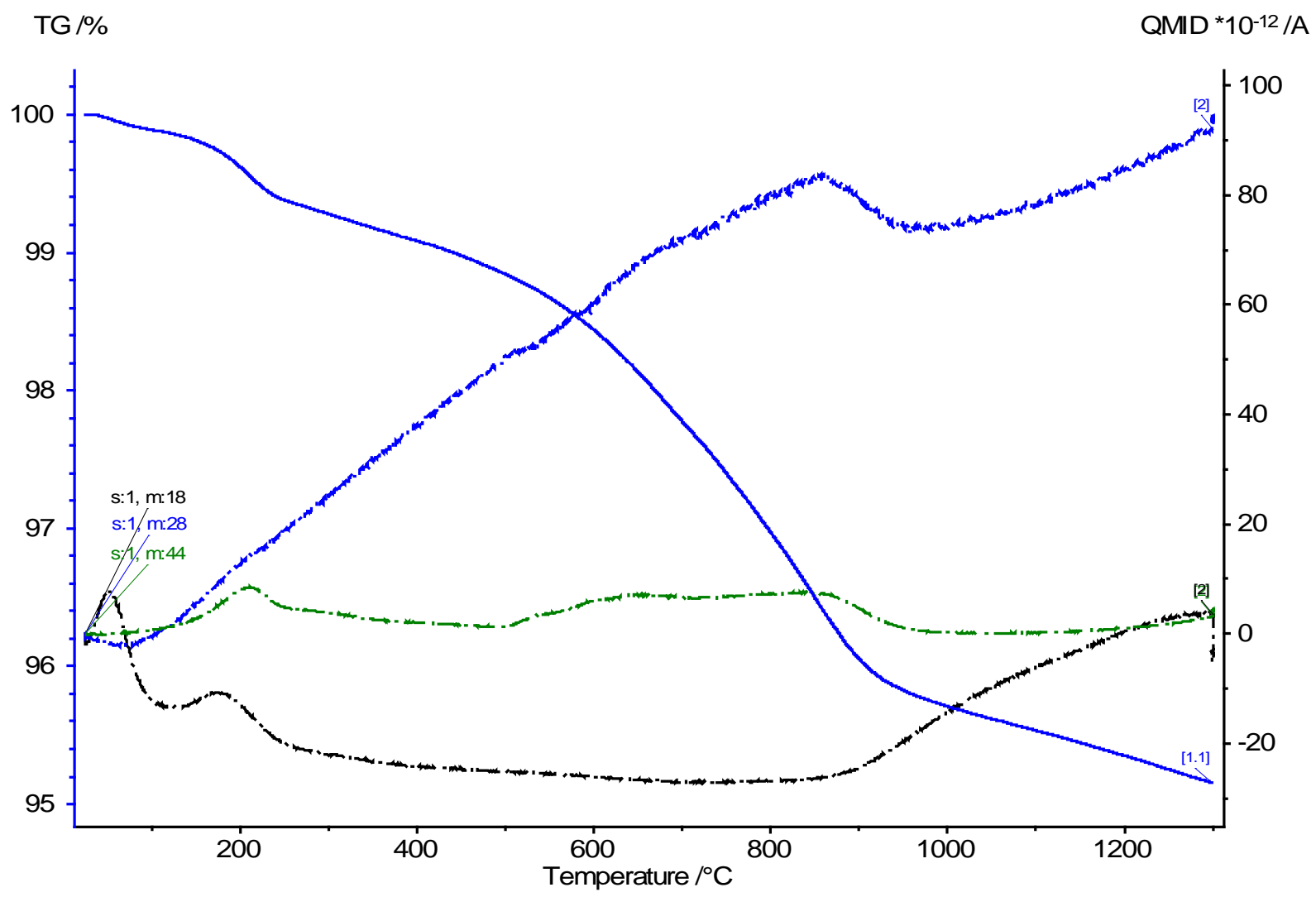

Figure 2.19. TG Curve (solid blue line) and MS Traces of Relevant Compounds Observed in the GasPhase Effluent during TGA/MS Testing of the HF-Treated (demineralized) Pacco 90-100 CTC Carbon Support. The black dashed line (s1, m:18) corresponds to water, the blue dashed line (s1, m:28) corresponds to CO, and the green dashed line (s1, m:44) corresponds to $\mathrm{CO}_{2}$.

The HF-washed and graphitized Pacco 90-100 CTC carbon support sample TGA/MS traces are shown in Figure 2.20. In contrast to the graphitized Norit ROX 0.8 and Norit Darco-LS samples, the graphitized Pacco sample exhibited significant mass losses throughout the temperature program. Indeed, the TG and MS traces appear to be qualitatively similar to the HF-washed sample shown in Figure 2.19. Therefore, it is highly likely that the graphitized Pacco 90-100 CTC sample was accidentally gathered from a sample that was marked for graphitization but that never actually was graphitized. Interestingly, TGA/MS measurements on the $\mathrm{HNO}_{3}$ treated sample illustrated in Figure 2.21 illustrate a sample in line with what would be expected for a sample that was graphitized. Therefore, it is likely that the Pacco 90-100 CTC sample was graphitized and treated with $\mathrm{HNO}_{3}$ and only the sample submitted for TGA/MS analysis was erroneous. 


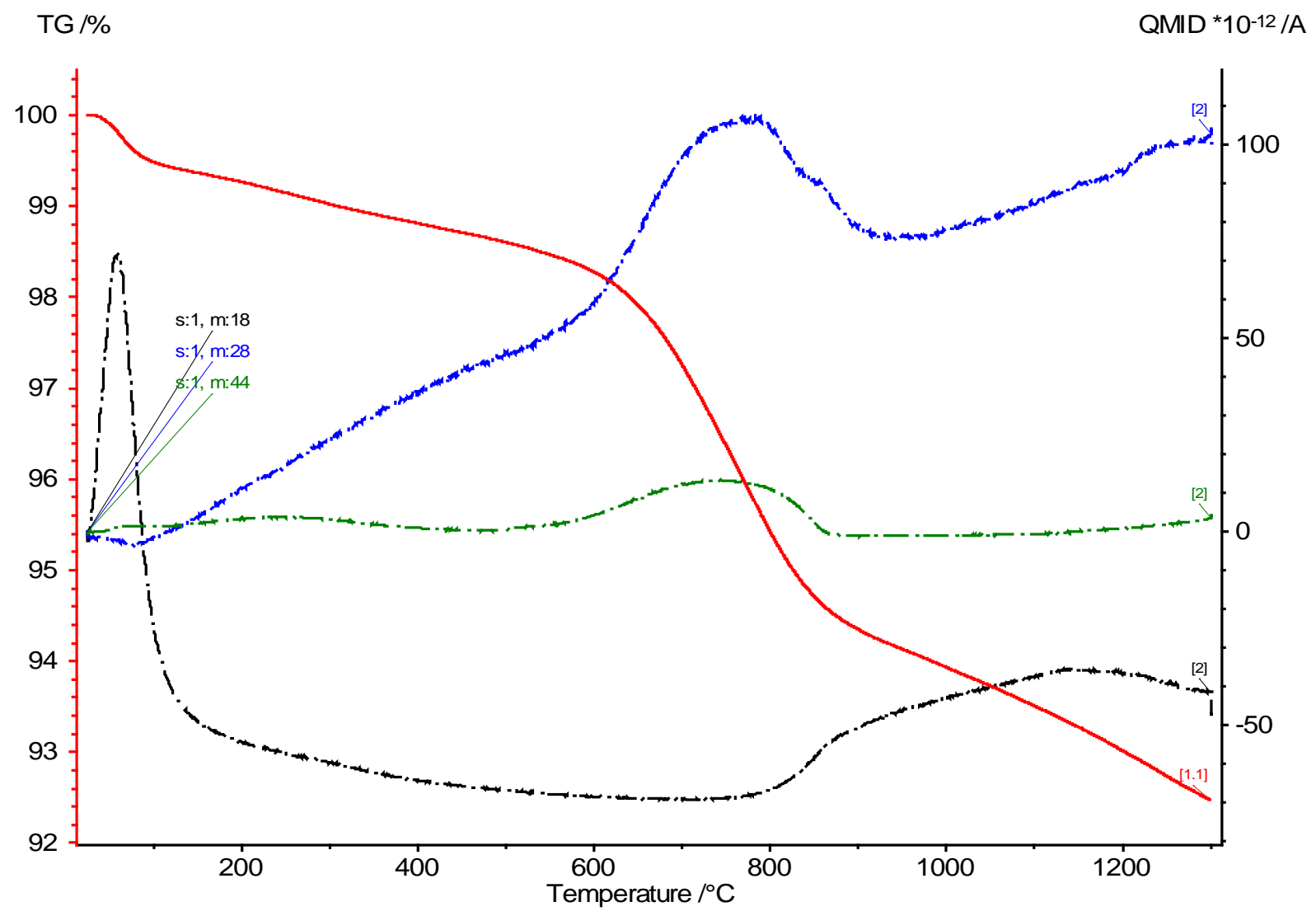

Figure 2.20. TG Curve (solid red line) and MS Traces of Relevant Compounds Observed in the GasPhase Effluent during TGA/MS Testing of the HF-Treated (demineralized) and Graphitized Pacco 90-100 CTC Carbon Support. The black dashed line (s1, m:18) corresponds to water, the blue dashed line (s1, m:28) corresponds to CO, and the green dashed line (s1, m:44) corresponds to $\mathrm{CO}_{2}$.

The $\mathrm{HNO}_{3}$-treated Pacco 90-100 CTC sample illustrated in Figure 2.21 is similar to the Norit DarcoLS. The TG trace is the solid green line in Figure 2.21. In addition to water, $\mathrm{CO}$, and $\mathrm{CO}_{2}$, the red dashed line corresponding to $\mathrm{NO}_{\mathrm{x}}$ species $(\mathrm{m}=30)$ is plotted. The MS trace illustrates that water (likely physisorbed water) is observed to desorb from the support below $\sim 100^{\circ} \mathrm{C}$. Over a temperature range of $\sim 100$ to $200^{\circ} \mathrm{C}$, about $1.5 \mathrm{wt} \%$ of the mass of the support is lost. This period of mass loss corresponds with the presence of water, $\mathrm{CO}, \mathrm{CO}_{2}$, and $\mathrm{NO}_{\mathrm{x}}$. Thus, the $\mathrm{HNO}_{3}$ treatment did appear to oxidize the surface of the carbon support as evidenced by the evolution of $\mathrm{CO}_{2}$. However, some $\mathrm{N}_{2}$ containing groups also may have been incorporated into the carbon/oxygen moieties formed by the $\mathrm{HNO}_{3}$. Alternatively, the presence of $\mathrm{NO}_{\mathrm{x}}$ and water observed simultaneously may be from $\mathrm{HNO}_{3}$ that was not completely rinsed out of the pores. A period of small mass loss accompanied by a period during which $\mathrm{CO}$ and a small amount of $\mathrm{CO}_{2}$ were present occurred between 600 and $700^{\circ} \mathrm{C}$. This period of mass loss may have been due to oxygenated surface species such as a lactone, phenol, or ether moieties as suggested by Table 2.25. However, the concentration of these moieties is very small and a stronger oxidant (e.g., $\mathrm{H}_{2} \mathrm{O}_{2}$ ) or greater $\mathrm{HNO}_{3}$ treatment temperatures would likely be required to create a greater degree of surface functionality. 


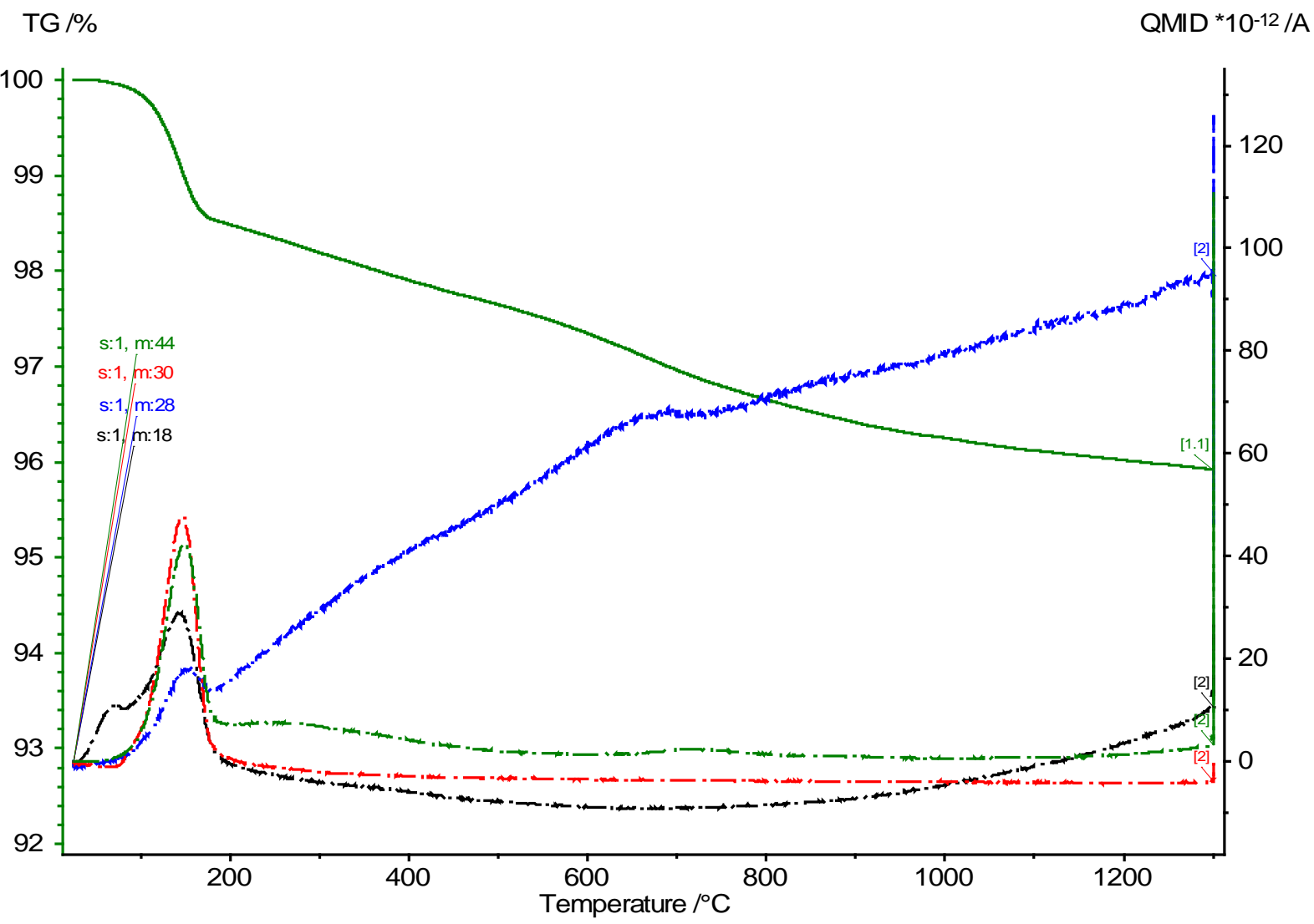

Figure 2.21. TG Curve (solid green line) and MS Traces of Relevant Compounds Observed in the GasPhase Effluent during TGA/MS Testing of the HF-Treated (demineralized), Graphitized, and Subsequently Re-Functionalized via $\mathrm{HNO}_{3}$ Treatment of the Pacco 90-100 CTC Carbon Support. The black dashed line (s1, m:18) corresponds to water, the blue dashed line (s1, m:28) corresponds to CO, the red dashed line (s1, m:30) corresponds to $\mathrm{NO}_{\mathrm{x}}$ species, and the green dashed line (s1, m:44) corresponds to $\mathrm{CO}_{2}$.

The following general observations can be made from the modification of the Norit ROX 0.8, DarcoLS and Pacco 90-100 CTC carbon supports. HF washing does not significantly affect the oxygenated surface functionality but is reasonably efficient at removing a large portion of mineral impurities present. Graphitization is efficient at removing all oxygenated surface functionality. The graphitized carbon also appears to be hydrophobic based on the lack of water observed in the temperature program up to $\sim 150^{\circ} \mathrm{C}$ in samples that were certainly subjected to graphitization. Finally, the $\mathrm{HNO}_{3}$ treatment produced relatively unstable oxygenated surface species that likely resembled carboxylic acid moieties and decomposed generally by $\sim 200^{\circ} \mathrm{C}$. More investigation is warranted to determine if the presence of $\mathrm{NO}_{\mathrm{x}}$ species observed in re-functionalized samples is due to $\mathrm{N}_{2}$ incorporated into surface moieties or from $\mathrm{HNO}_{3}$ left in pores from incomplete washing of the carbon supports. 


\subsection{Diffuse Reflectance Infrared Fourier Transform Spectroscopy and Raman Spectroscopy}

Diffuse reflectance infrared Fourier transform spectroscopy (DRIFTS) and Raman spectroscopy were performed on samples KOA 6, 7, and 8, which are Hyperion carbon supports 395-08, 395-10, and 384-82, respectively. These experiments were performed using spectroscopy techniques to determine surface functionality, specifically oxygenated surface functionality, on samples KOA 6, 7, and 8. At a basic level, the techniques are performed by radiation that interacts in a characteristic manner through absorption or scattering with the different functional groups on the carbon supports. Stretching and bending modes of $\mathrm{C}=\mathrm{C}, \mathrm{C}-\mathrm{H}$, and $\mathrm{C}-\mathrm{O}$ are commonly observed with DRIFTS and Raman spectroscopy. Thus, DRIFTS and Raman spectroscopy were meant to complement earlier TPD work with the TGA/MS to characterize surface functionality on samples KOA 6, 7, and 8.

DRIFTS data proved to be inconclusive, as can be seen in Figure 2.22. All spectra are dominated by increasing intensity background toward lower frequencies. Irregularities at ca. 3500, 3000, and $1500 \mathrm{~cm}^{-1}$ are likely due to an over-correction of the background. Thus, DRIFTS did not yield information about any functional groups present on carbon supports KOA 6, 7, and 8.

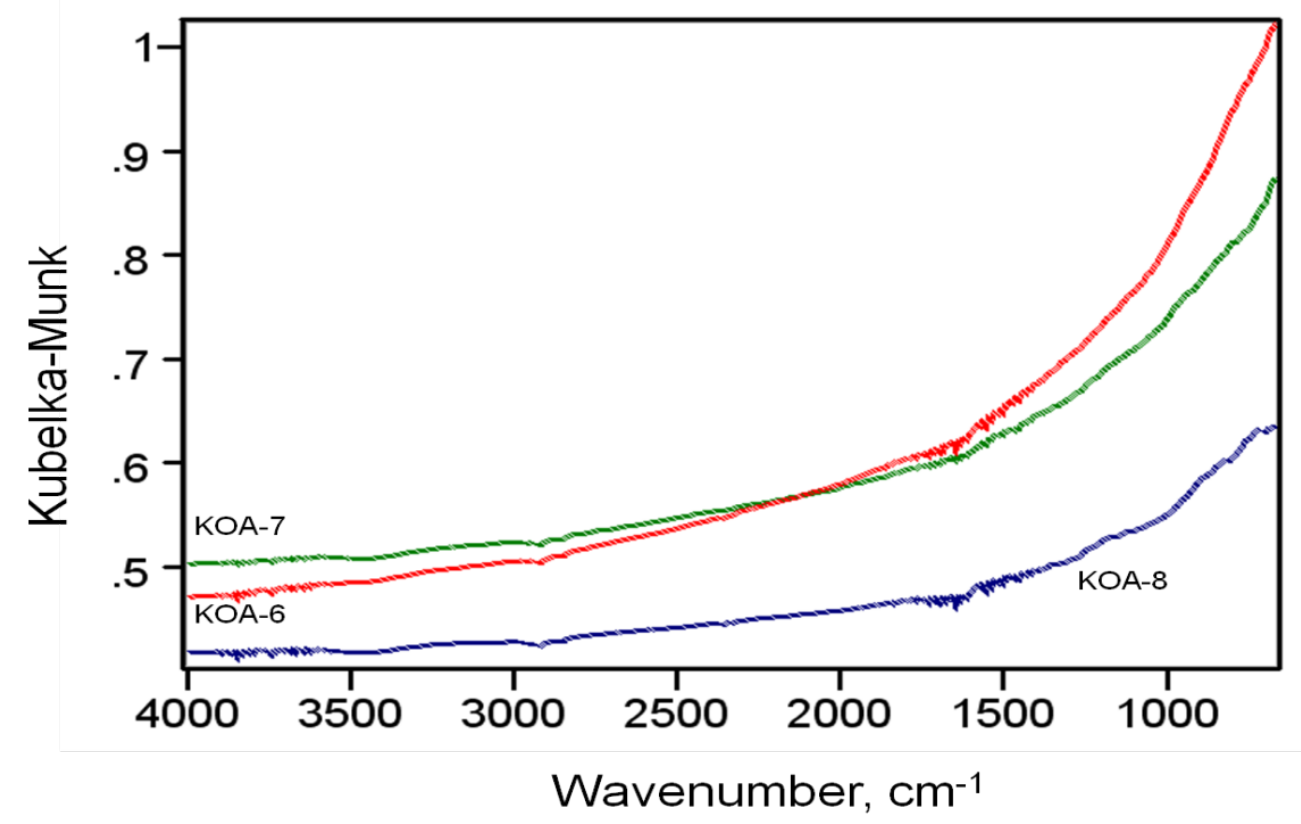

Figure 2.22. DRIFTS Results on Carbon Supports KOA 6, 7, and 8

Raman spectra on KOA 6, 7, and 8 were obtained using ultraviolet (244-nm) excitation radiation. Figure 2.23 shows the $\mathrm{C}=\mathrm{C}$ bands of the three carbon support samples. The $\mathrm{G}$ band shifts in frequency from low to high as follows: KOA-7 < KOA-6 < KOA-8. This shift appears to be real, not an artifact of the support or technique. The insert in Figure 2.23 shows two small bands in all spectra at 947 and $1090 \mathrm{~cm}-1$. Assignments for these bands cannot be confirmed at this time. The latter may arise from a small amount of C-O, although isotopic shift (carbon-13) may be possible. There is a slight broadening of the D band to lower frequency that might be explained by a small amount of D band (ca., $1360 \mathrm{~cm}-1$ ) that appears slightly greater for sample KOA 6 than for samples KOA 7 and 8. 


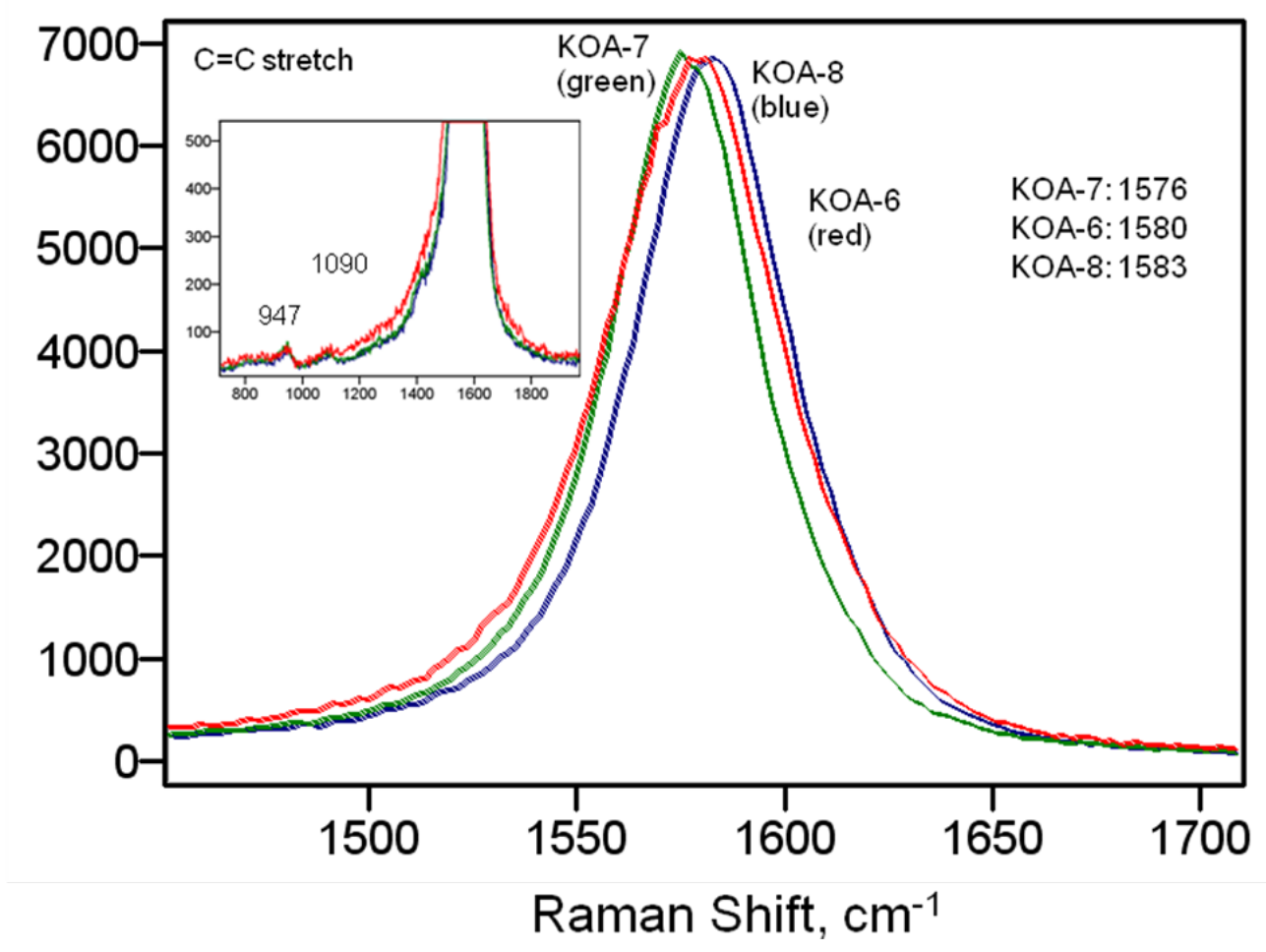

Figure 2.23. Raman Spectroscopy of the $\mathrm{C}=\mathrm{C}$ Stretch Bands in Samples KOA 6, 7, and 8.

Figure 2.24 shows smaller but significant bands between 2800 and $3300 \mathrm{~cm}^{-1}$ in the spectra for samples KOA 6, 7, and 8. Graphite does not have known overtones or combination bands in this region; thus, these features must come from a C-H stretch. Because the spectra are normalized with respect to the $1580 \mathrm{~cm}^{-1}$ band, by comparing the intensities, a case can be made that there is more $\mathrm{C}-\mathrm{H}$ stretch for the samples in the following order: KOA- $8<$ KAO- $6<$ KOA-7. This trend should be interpreted with caution, however, because multiple measurements on KOA-8 gave different results, although both KOA-8 spectra had C-H peaks that were weaker than the other two. There also appears to be a more distinct high-frequency feature at ca. $3225 \mathrm{~cm}-1$ for KOA-8. Although there is wide variation between hydrocarbons, aromatic C-H is usually at higher frequency, ca. $3000-3200 \mathrm{~cm}-1$, while aliphatic lower is at 2800 to $3000 \mathrm{~cm}-1$. This may indicate that KOA-8 has a higher proportion of aromatic C-H structures than the other samples. Finally, the peak of the $\mathrm{C}-\mathrm{H}$ stretch envelope shifts to higher frequency with the sample, in the same order as the G band: KOA-7 < KOA-6 < KOA-8. 


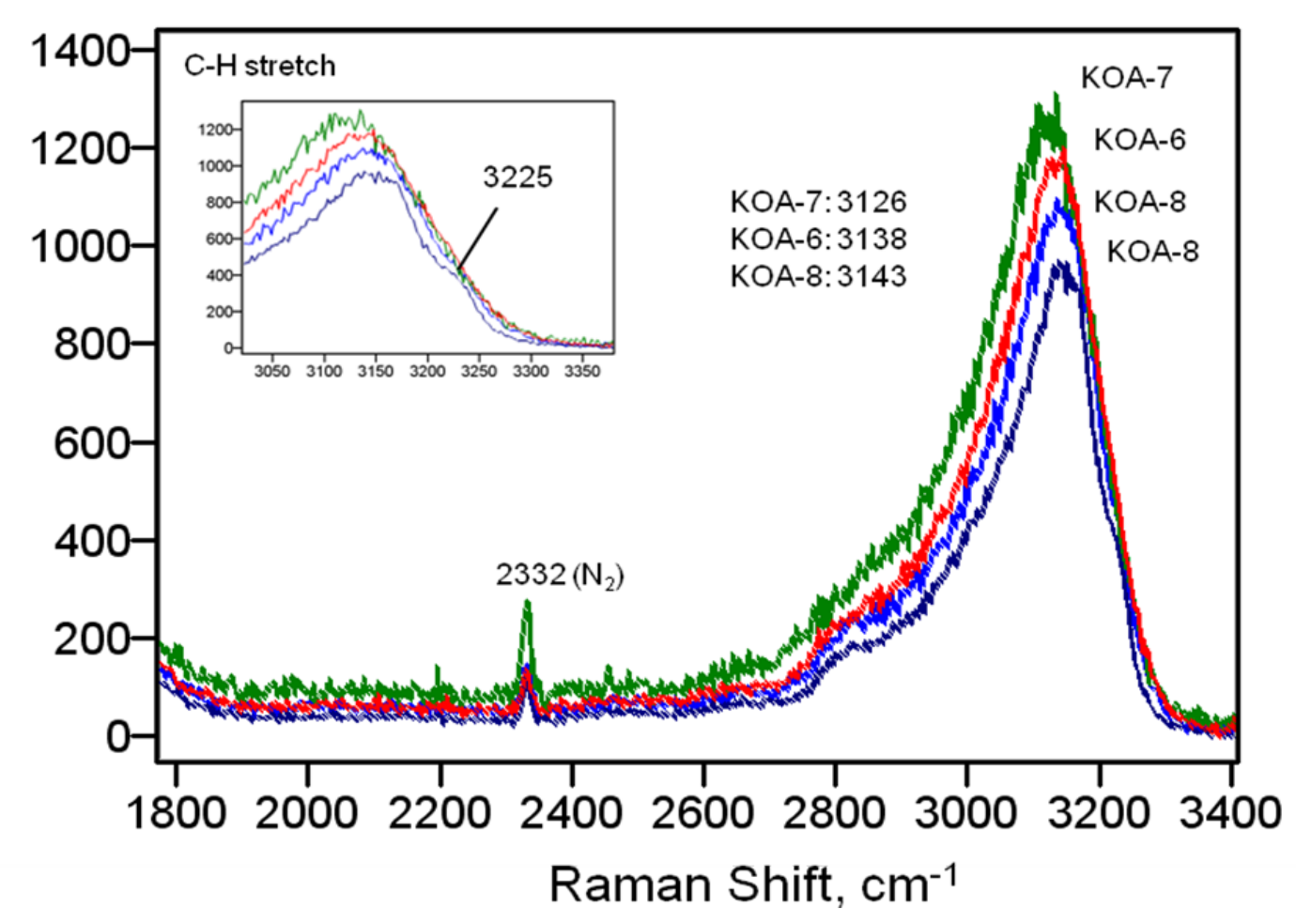

Figure 2.24. Raman Investigation of C-H Stretching Mode of KOA 6, 7, and 8

Overall, the results of DRIFTS and Raman spectroscopy on KOA 6, 7 and 8 were inconclusive. TPD results demonstrated that the oxygen functionality of KOA 6-8 was in the range of 2-5 wt\%, which could be below the detection limit of DRIFTS. Experiments involving Raman spectroscopy showed shifts in the stretch $\mathrm{C}=\mathrm{C}$ mode of the carbon supports. Additionally, some C-O stretching modes could be present in all three samples, although isotopic effects could also be present.

\subsection{Hydrogen Chemisorption Testing on Select Mixed Alcohol Catalysts}

Several Rh-based mixed alcohols catalysts were subjected to $\mathrm{H}_{2}$ chemisorption testing. Hydrogen chemisorption testing is performed by applying in pulses a known concentration of $\mathrm{H}_{2}$ over a reduced catalyst sample. The sample adsorbs the $\mathrm{H}_{2}$ onto the surface of the catalyst until the surface is saturated. The amount of $\mathrm{H}_{2}$ adsorbed is measured via a calibrated response using a TCD.

The samples subjected to $\mathrm{H}_{2}$ chemisorption testing are listed in Table 2.28. Catalysts supported on Davisil 645 and Sigma-Aldrich Grade 7734 silica as well as Hyperion CS-02C-063-XD bound carbon nanotube catalysts were tested. Various Rh, Mn, and Ir concentrations on each support were investigated. In Table 2.28, the "Impregnation" column notes whether Rh, Mn, and Ir were added to the catalyst in a single impregnation step (Step 1) or if Rh and Mn were added and then Ir impregnated onto the catalyst in a subsequent step (Step 2). Three of the catalysts (60074-53-A, 60074-73-A, and 60074-109-A) were each tested twice to test the reproducibility of the catalysts to adsorb $\mathrm{H}_{2}$. 
Table 2.28. Rh-Based Mixed Alcohol Catalysts Subjected to $\mathrm{H}_{2}$ Chemisorption Testing

\begin{tabular}{|c|c|c|c|c|c|}
\hline Test Number & Sample & $\mathrm{Rh}$ & Mn & Ir & Impregnation \\
\hline \multicolumn{6}{|c|}{ Davisil 645 Silica-Supported Catalysts } \\
\hline 1 & 60074-10-A & 5.35 & 1.63 & 0.00 & 1 \\
\hline 2 & 60074-53-A & 5.56 & 1.69 & 1.03 & 1 \\
\hline 3 & 60074-53-A & 5.56 & 1.69 & 1.03 & 1 \\
\hline 4 & 60074-73-A & 5.56 & 1.69 & 1.03 & 2 \\
\hline 5 & 60074-73-A & 5.56 & 1.69 & 1.03 & 2 \\
\hline 6 & $61022-10-6$ & 8.30 & 2.50 & 1.50 & 1 \\
\hline 7 & 60074-74-B & 8.34 & 2.54 & 1.55 & 2 \\
\hline \multicolumn{6}{|c|}{ Sigma-Aldrich Grade 7734 Silica-Supported Catalysts } \\
\hline 8 & 60074-121-E & 5.56 & 1.69 & 0.00 & 1 \\
\hline 9 & 60074-121-A & 5.56 & 1.69 & 1.03 & 1 \\
\hline 10 & $61022-10-6$ & 8.30 & 2.50 & 1.50 & 1 \\
\hline 11 & 60074-119-D & 8.34 & 2.54 & 1.55 & 1 \\
\hline 12 & $61022-10-5$ & 11.10 & 3.40 & 2.10 & 2 \\
\hline \multicolumn{6}{|c|}{ Hyperion CS-02C-063-XD (Lot 384-82) Carbon-Supported Catalysts } \\
\hline 13 & 61022-4-5 & 11.70 & 3.60 & 2.20 & 1 \\
\hline 14 & $60074-122-4$ & 11.70 & 3.60 & 3.80 & 1 \\
\hline 15 & 60074-109-A & 11.78 & 3.58 & 2.19 & 1 \\
\hline 16 & 60074-109-A & 11.78 & 3.58 & 2.19 & 1 \\
\hline
\end{tabular}

Approximately 0.05 to $0.10 \mathrm{~g}$ of each catalyst was loaded into a quartz tube and mounted on the Micromeritics AutoChem II 2920. Each sample was reduced prior to the $\mathrm{H}_{2}$ chemisorption testing in 9.8\% $\mathrm{H}_{2} /$ Ar flowing at $25 \mathrm{SCCM}$. The following temperature programed reduction profile was employed for each sample:

1. Baseline temperature $\left(\sim 35^{\circ} \mathrm{C}\right)$ to $100^{\circ} \mathrm{C}$ at $10^{\circ} \mathrm{C} / \mathrm{min}$

2. Immediately proceed from 100 to $220^{\circ} \mathrm{C}$ at $2.5^{\circ} \mathrm{C} / \mathrm{min}$

3. Hold at $220^{\circ} \mathrm{C}$ for $60 \mathrm{~min}$

4. Heat from 220 to $260^{\circ} \mathrm{C}$ at $2.0^{\circ} \mathrm{C} / \mathrm{min}$

5. Hold at $260^{\circ}$ for $480 \mathrm{~min}$

6. Heat from 260 to $350^{\circ} \mathrm{C}$ at $1.5^{\circ} \mathrm{C} / \mathrm{min}$

7. Hold at $350^{\circ} \mathrm{C}$ for $120 \mathrm{~min}$.

After the last reduction step, each sample was held at $350^{\circ} \mathrm{C}$ for $60 \mathrm{~min}$ in flowing Ar to remove any $\mathrm{H}_{2}$ from the surface.

The $\mathrm{H}_{2}$ chemisorption portion of the test was initiated by cooling the sample from $350^{\circ} \mathrm{C}$ to $45^{\circ} \mathrm{C}$ in flowing Ar. When the temperature was stabilized in the stream of flowing $\mathrm{Ar}$, a stream of $9.8 \% \mathrm{H}_{2} / \mathrm{Ar}$ was pulsed over the sample every 5 min. Initially, the catalysts took up almost all of the $\mathrm{H}_{2}$ in the stream. As the surface of the sample because saturated with $\mathrm{H}_{2}$ after several pulses, a response in the TCD curve was observed, indicating that $\mathrm{H}_{2}$ was present in the Ar stream passing over the detector. When the area of two of the pulsed peaks were similar (only 3\% different), the sample was declared to have a saturated surface because no more $\mathrm{H}_{2}$ was being adsorbed and the experiment was ended. 
The concentration of $\mathrm{H}_{2}$ taken up by the catalyst was determined via a calibration method conducted automatically by the Micromeritics instrument whereby the $9.8 \mathrm{H}_{2} / \mathrm{Ar}$ stream is internally mixed to different concentrations and the TCD response recorded. The automatic calibration was performed several times over the duration of dates used to collect the reported data and was found to vary $~ 6 \%$ over the course of the experiments.

The results of $\mathrm{H}_{2}$ chemisorption testing on the samples outlined in Table 2.28 are presented in Figure 2.25. Note that the "Test Number" column in Table 2.28 references the "Test Number" row in Figure 2.25. First, it should be noted that there was a relatively large spread in the results from tests performed to check the reproducibility of the method. Each of the catalyst samples tested in duplicate on the Davisil 645 silica returned results that differed by $\sim 15 \%$. The duplicate tests on the catalyst sample supported on the Hyperion-02C carbon exhibited a greater degree of difference with the two tests resulting in values differing by $\sim 40 \%$. As mentioned previously, the scatter observed during the calibration of the TCD resulted in scatter of $\sim 6 \%$. Thus, the scatter observed during sample testing could be the result of heterogeneity within the samples themselves. Several more tests on a single sample would need to be run to calculate the statistical confidence interval that could be applied to the samples as well as the method, but the 15 to $40 \%$ differences shown in the duplicates should inspire caution when making strong conclusions based on the data in Figure 2.25.

The $\mathrm{H}_{2}$ chemisorption tests on silica-supported samples in Figure 2.25 exhibited similar trends. First, the results suggest that catalysts containing $\mathrm{Rh}$ and $\mathrm{Mn}$ but no Ir have the highest amount of $\mathrm{H}_{2}$ adsorbed. The addition of Ir diminishes the amount of $\mathrm{H}_{2}$ that is adsorbed for catalyst prepared on both Davisil 645 and Sigma-Aldrich 7734 silica supports. Originally it appeared that adding the Ir in a separate step resulted in less $\mathrm{H}_{2}$ adsorbed than when the $\mathrm{Rh}, \mathrm{Mn}$, and Ir were added simultaneously (Test Numbers 2 and 3 versus 4 and 5 as well as Test Number 11 versus 12). However, the higher concentration of Rh, Mn, and Ir on the Davisil 645 appears to disagree with this trend (Test Number 6 versus 7). Overarching general trends are difficult to identify based on the concentration of Ir added to the Rh and Mn, but it does appear that the amount of $\mathrm{H}_{2}$ adsorbed is similar for similar concentrations of the various metals on each of the silica supports (e.g., Test Number 1 versus 8, 2 and 3 versus 9, as well as 6 versus 10 and 11).

The results of $\mathrm{H}_{2}$ chemisorption testing on the Hyperion CS-02C-063-XD (Lot 384-82) exhibited no visible trends with respect to metal loadings or impregnation order. This may be due to the small number of samples run or the possibility of large variation in the returned results suggested by the duplicate test. Generally, even though there is more than two times (by weight) the amount of Rh, Mn, and Ir on the carbon-supported samples, the $\mathrm{H}_{2}$ uptake was lower compared to the silica-supported samples with less metal. A silica sample with similar metal concentrations (Test Number 12) did exhibit similar $\mathrm{H}_{2}$ uptake when compared with some of the carbon samples (Test Numbers 13 and 16). 


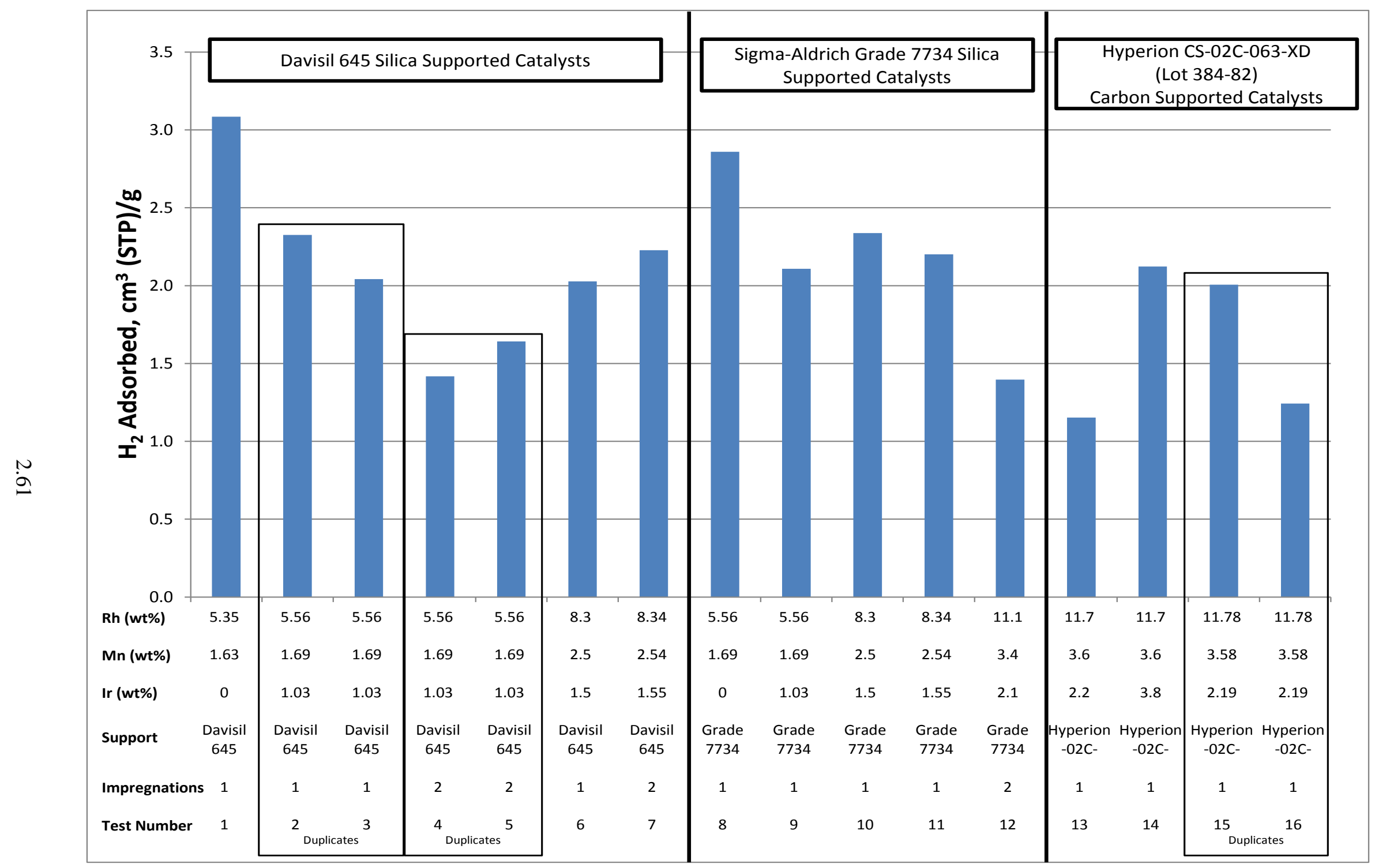

Figure 2.25. $\mathrm{H}_{2}$ Chemisorption Results on Reduced Catalyst Samples. Note that the test numbers in Figure 2.25 reference the test numbers in Table 2.28. 


\subsection{X-Ray Diffraction}

\subsubsection{Carbon Supports}

X-ray diffraction (XRD) was performed on carbon supports KOA 1 through 12 (Table 1) to determine the crystalline nature of the carbon in the supports. Specifically, XRD was performed to determine the relative amount of graphite present in the carbon supports. XRD was performed using $\mathrm{Cr}$ radiation. A scan range from 20 to $150^{\circ} \mathrm{C} 2$-Theta was used.

Results of XRD testing on carbon supports KOA 1 through 12 are shown in Figure 2.26. Unfortunately, quartz and graphite have nearly overlapping strong reflections at $\sim 40^{\circ} 2$-Theta. Because quartz (likely as an analytical artifact) was observed in all samples, it is difficult to draw meaningful conclusions on the graphitic nature of the samples from Micro-XRD data. It is possible that the nature of the carbon is more graphitic in samples KOA 3 and 6 through 8, which can be observed from taller, more narrow peaks around $40^{\circ}$ 2-Theta in these samples. However, because of the presence of quartz, strong conclusions cannot be made.

XRD did produce some notable features. First, support KOA 8 exhibited a reflection at $\sim 79^{\circ} 2$-Theta that was not observed in sample KOA 6 or 7, even though all three supports are carbon nanotubes. However, this reflection could potentially be from a binder or carbon nanotube substrate (such as Ni or $\mathrm{Fe}$ ) not found in samples KOA 6 and 7. HF washing of samples KOA 1 and 2 to produce samples KOA 12 and 11, respectively, did not completely remove the reflection attributed to quartz, although several other reflections present in KOA 1 and 2 did not appear in KOA 11 and 12. It is likely that HF washing of samples KOA 1 and 2 dissolved crystalline inorganic material, which resulted in XRD spectra of samples KOA 11 and 12 with fewer reflections due to removed inorganic impurities.

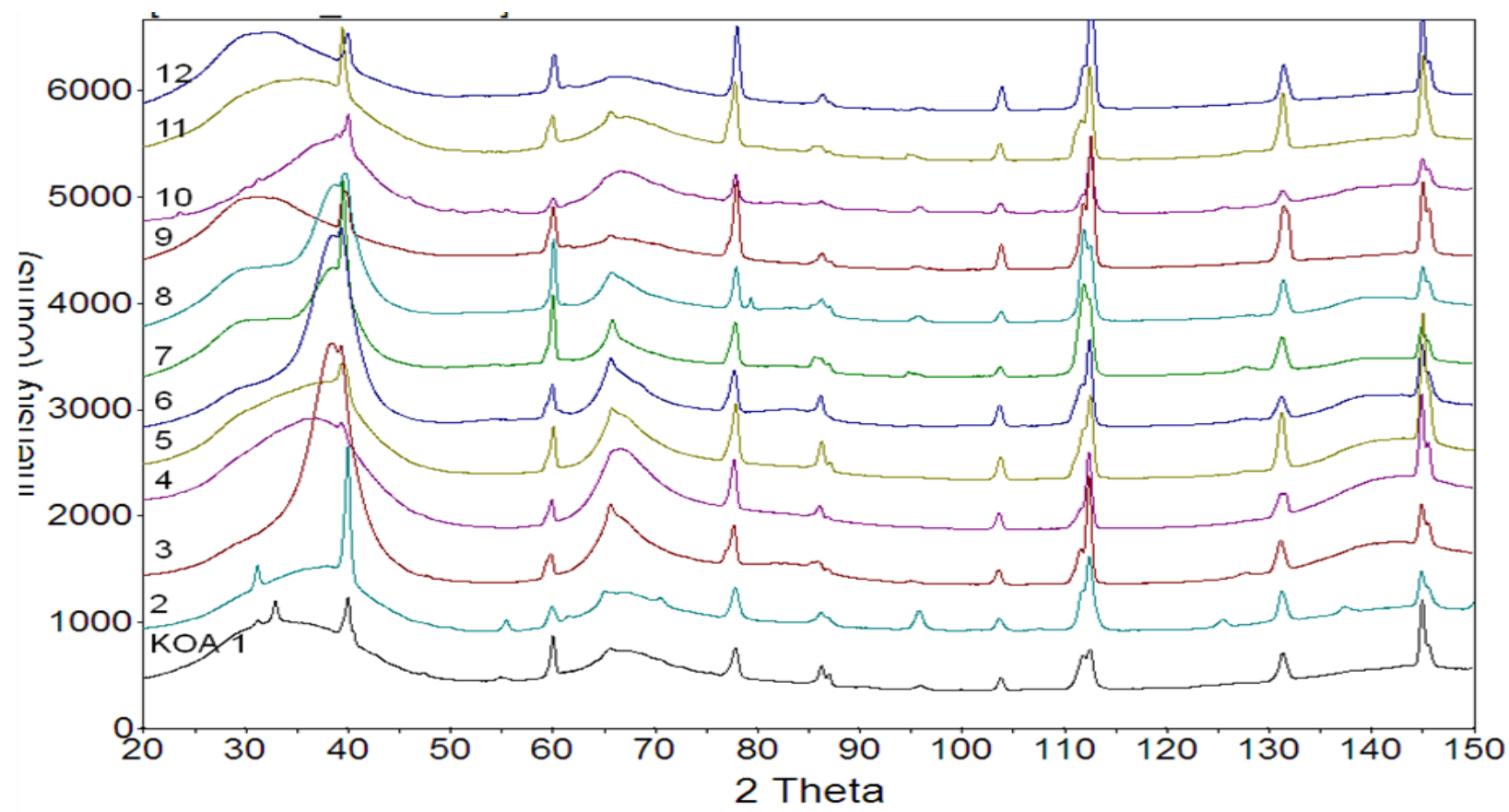

Figure 2.26. Overlay of Diffractograms Collected through XRD Analysis of Samples KOA 1 through 12 


\subsubsection{Silica Supports}

Several silica samples listed in Table 2.3 also were subjected to XRD measurements using $\mathrm{Cr}$ radiation. An overlay of the results for all of the supports is presented in Figure 2.27. The red lines indicating the reflection for quartz also are present in the overlay. The Davisil (645), Merck (Grade 60), and Engelhard (Mod D) had the least amount of quartz present with non-existent or very small reflections of the main quartz peak observed at $\sim 40^{\circ}$ 2-Theta. The Perlkat 29-3 exhibited the largest reflection of the main quartz peak, and appeared to be the most crystalline support in general. The top four reflections in Figure 2.27 (Norpro 61137, Perlkat 29-3, Norpro (61138), and Grace (Grade 408)) exhibited an unidentified phase at $\sim 67^{\circ} \mathrm{C}$ 2-Theta, which was not observed in the other silica supports. The reflection may be due to another crystalline $\mathrm{SiO}_{2}$ phase, which may suggest that these supports are polycrystalline.

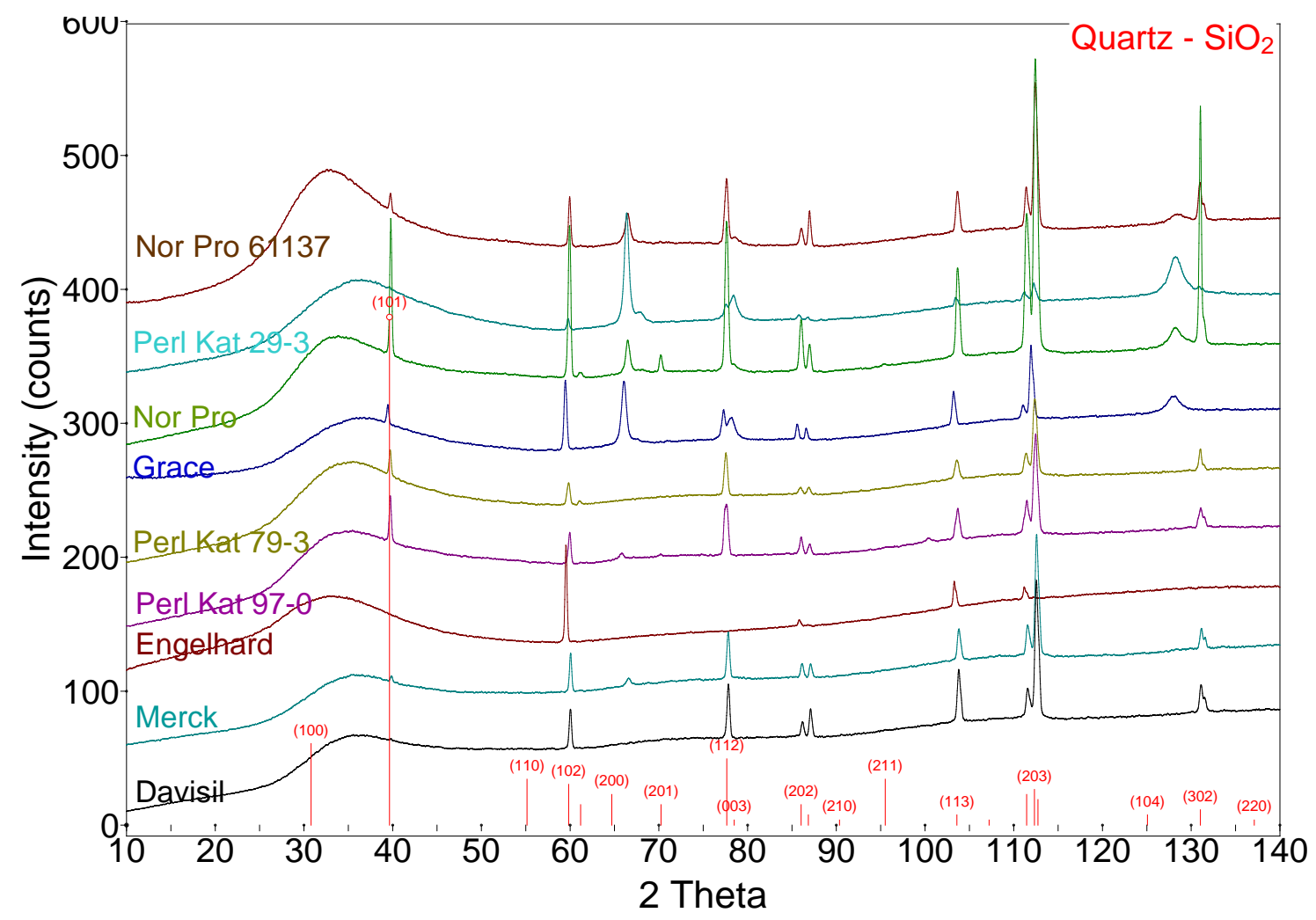

Figure 2.27. Overlay of Diffractograms Collected through XRD Analysis of Various Silica MixedAlcohol Catalyst Supports. Davisil = Davisil 645; Merck = Merck Grade 60; Engelhard = Engelhard Mod D; Grace = Grace Grade 408; Norpro = Norpro 61138.

\subsubsection{Baseline Catalysts}

Each of the baseline catalysts in Table 2.4 were measured with XRD to probe the crystalline phases present at various stages in the catalyst lifecycle. As with the $\mathrm{C}$ and $\mathrm{Si}$ samples discussed above, $\mathrm{Cr}$ was used as the radiation source in these measurements. The measurements ranged from 10 to $160^{\circ} 2$-Theta using a Micro XRD. Micro XRD was preferred because samples could be sealed within the glove box in a capillary with wax on either end prior to measurements in the open atmosphere. Thus, the atmospheric integrity of the samples was preserved during measurements. 
Several phases are overlaid in the following diffractograms. However, because of the complexity of the diffractograms, assertive conclusions on the phases present were difficult in nearly every case. In several instances, some of the major reflections of a possible crystalline match are present while others are clearly lacking. Therefore, it is especially informative to compare the relative differences in the diffractograms with similar samples at other points in the catalyst life cycle while regarding the overlaid phases in the diffractograms as qualitative suggestions on phases that may be present.

\subsubsection{Davisil 645 Silica-Supported Catalysts}

The diffractogram collected on the fresh Rh-Mn-Ir catalyst supported on Davisil 645 silica is illustrated in Figure 2.28. The sample was calcined at $400^{\circ} \mathrm{C}$ for $2 \mathrm{hr}$ in air as part of the material preparation. Thus, the $\mathrm{Rh}$ and $\mathrm{Mn}$ would be expected to be in an oxide phase. Indeed, the reflection at $\sim 53^{\circ}$ 2-Theta does match reasonably well with $\mathrm{Rh}_{2} \mathrm{O}_{3}$ (blue lines), although other reflections at $\sim 75$ and $100^{\circ}$ 2-Theta are not present. The reflection reported in the literature for the $\mathrm{Mn}_{2} \mathrm{O}_{3}$ mineral phase named Bixbyite also is a reasonable match under the broad hump shared with the literature reference for $\mathrm{Rh}_{2} \mathrm{O}_{3}$.

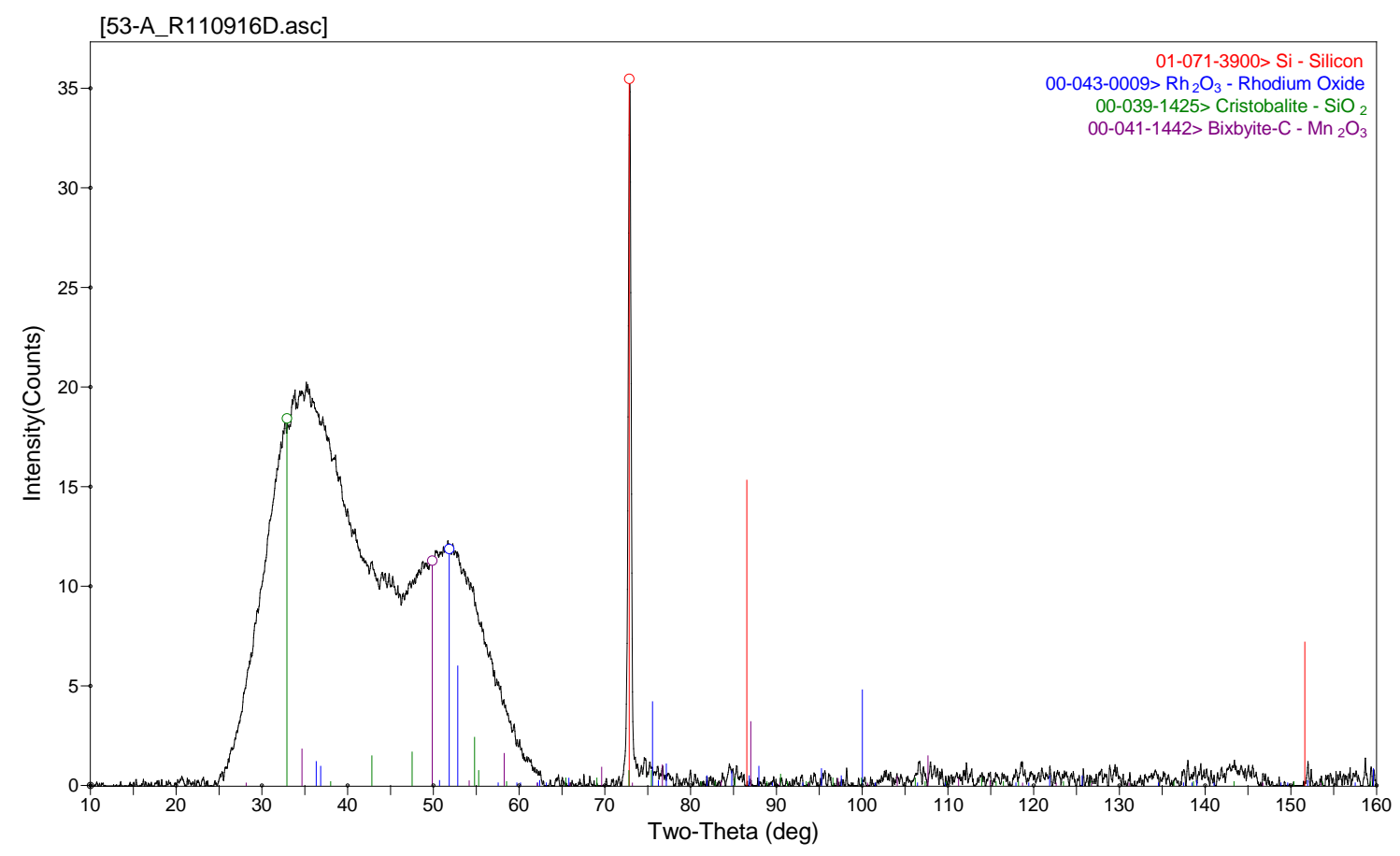

Figure 2.28. Diffractogram of Sample 60074-53-A, which was the Fresh Davisil 645 Silica-Supported Rh-Mn-Ir Catalyst in Table 2.4

The reflection at $\sim 72^{\circ}$ 2-Theta aligns reasonably well with silicon (i.e., not silica). Interestingly, this reflection was observed in each of the fresh baseline catalyst samples (Figure 2.28, Figure 2.32, and Figure 2.36) regardless of whether the catalyst was supported on silica or carbon nanotubes. The reflection also disappears in each of the baseline catalysts upon reduction, and is not observed in the reduced or spent catalysts. The presence of the phase remains a mystery, and impurities or instrument artifacts cannot be ruled out.

The reflection reported in the literature for the $\mathrm{SiO}_{2}$ phase Cristobalite in Figure 2.28 is present under the broad reflection centered at $\sim 35^{\circ} 2$-Theta. However, it is likely that the broad reflection in Figure 
2.28 is indeed $\mathrm{SiO}_{2}$ in a transition between the gel progressing toward Cristabolite. In Figure 2.29, the Cristobalite peak lines up more satisfactorily with a more narrow reflection centered at $33^{\circ} 2$-Theta.

Analysis of the fresh/uncalcined Davisil 645 silica in Figure 2.27 (Davisil) through heat treatment at $400^{\circ} \mathrm{C}$ in air in Figure 2.28 (60074-53-A) and finally through a sample that has undergone the reduction treatment in Figure 2.29 (60074-53-A-R) suggests that the Davisil 645 starts relatively amorphous as a silica gel and increases in crystallinity through the calcination and reduction phases, ending with a $\mathrm{SiO}_{2}$ phase closer to Cristobalite than quartz. Further analysis of the silica phase in Run 84 (Figure 2.20) and Run 86 (Figure 2.31) spent catalyst suggests that, from a crystalline perspective, the Cristobalite phase is stable after reduction as the peak does not shift or narrow significantly.

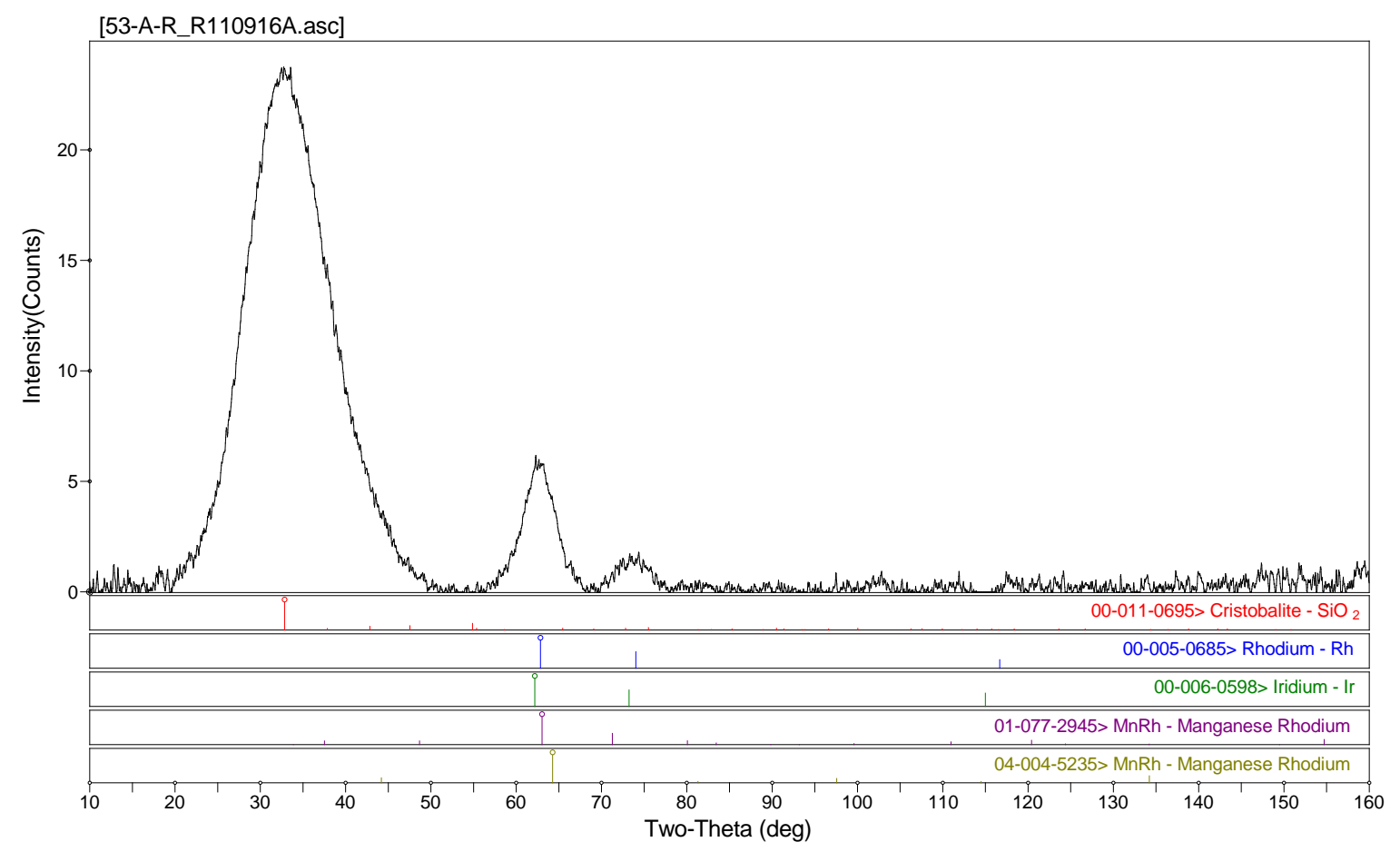

Figure 2.29. Diffractogram Collected from Sample 60074-53-A-R, which was the Reduced Davisil 645 Silica-Supported Rh-Mn-Ir Baseline Catalyst in Table 2.4

The metallic phases in the reduced (Figure 2.29) and spent (Figure 2.30 and Figure 2.31) silicasupported baseline catalysts do appear to be fully reduced. In Figure 2.29, the reflection centered at $\sim 63^{\circ}$ 2-Theta is the major reflection for a face-centered cubic (FCC) crystal. Many metals, including Rh and Ir, have FCC crystals in the zero-valence state. Interestingly, analysis of the XRD data revealed some reported Rh-Mn alloy phases also have reflections in the same vicinity of the diffractorgam. Therefore, it cannot be confirmed or ruled out that the Rh-Mn alloy exists after reduction. The Rh-Mn alloy has been proposed by computational investigations carried out in the mixed alcohol catalyst development program. $^{7}$

The reflections of the metals in the spent catalysts do appear to grow slightly more crystalline during reaction testing. For example, in Figure 2.30, higher order reflections at $120^{\circ} 2$-Theta are apparent that also are likely due to the presence of FCC metals such as Rh, Ir, or the Rh-Mn alloy. The lack of the high-angle reflections in the reduced sample (Figure 2.29) and their appearance in the low-temperature spent sample may suggest that the metal crystals exhibit crystallinity on a longer length scales. 
Furthermore, the peaks associated with the FCC reflections do appear to narrow slightly when the lowtemperature and high-temperature (Figure 2.31) spent catalysts are compared, which may further suggest a small amount of crystalline size growth in the higher temperature-tested catalyst. Overall, the differences in the low-temperature diffractogram versus the high temperature diffractogram are subtle, suggesting only small differences in the overall crystallinity of the two samples.

Finally, an HfIr reflection is included in Figure 2.30 and Figure 2.31. The inclusion of this reflection is to help in comparison with the reflections on the MWCNT-supported baseline catalysts discussed later. The HrIr phase was observed in the MWCNT-supported baseline catalysts. However, the phase is not actually expected to be present in the catalyst; it is coincidentally isomorphic with an unidentifiable phase and only coincidentally includes Ir. The HfIr reflection is a poor match in the silica-supported samples and, thus, the phase responsible for the reflection in the MWCNT-supported catalysts does not appear to be present in the silica-supported catalysts.

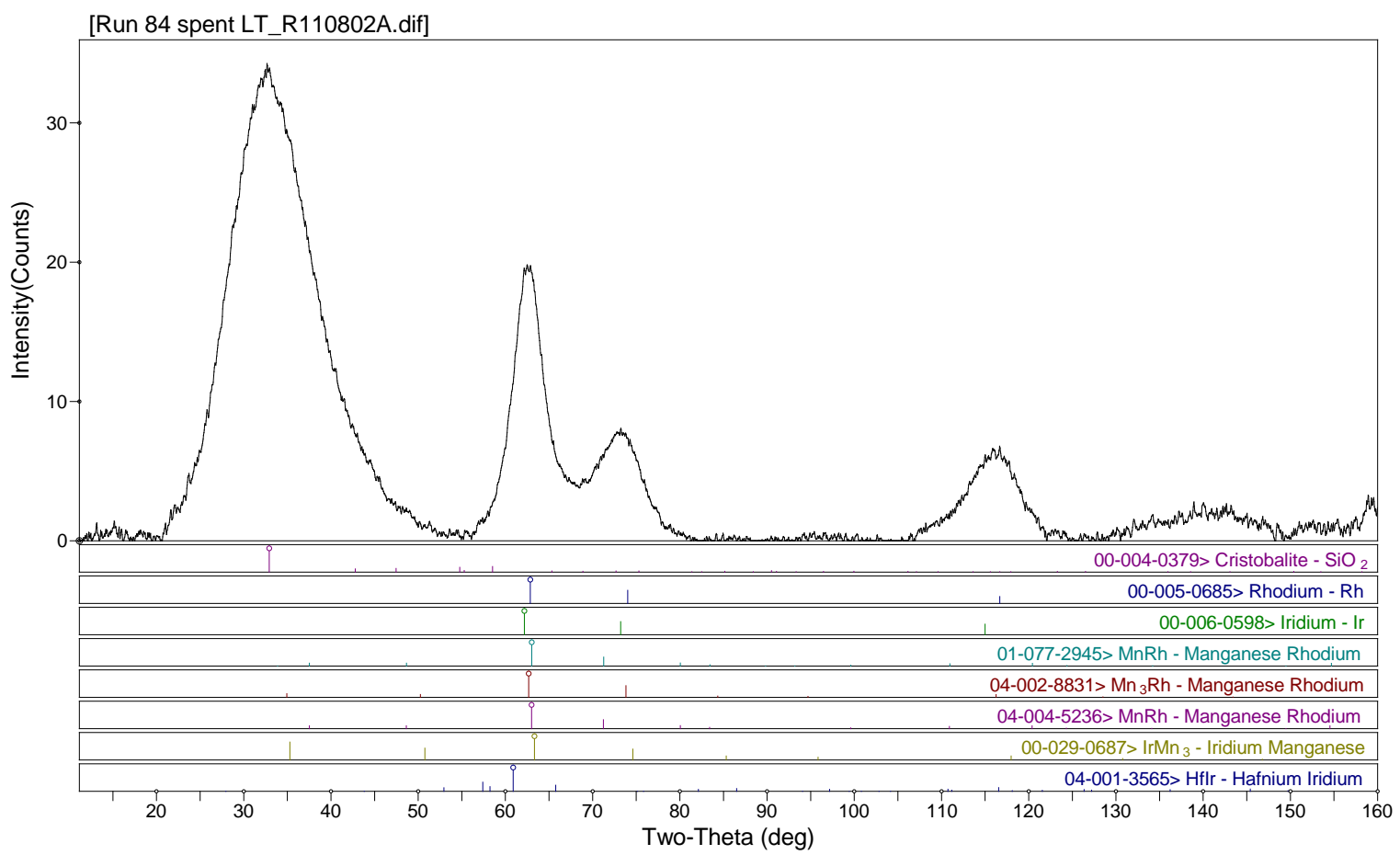

Figure 2.30. Diffractogram Collected from the Sample of Run 84, which was the Davisil 645 SilicaSupported Rh-Mn-Ir Baseline Catalyst Tested to $275^{\circ} \mathrm{C}$ in Table 2.4 


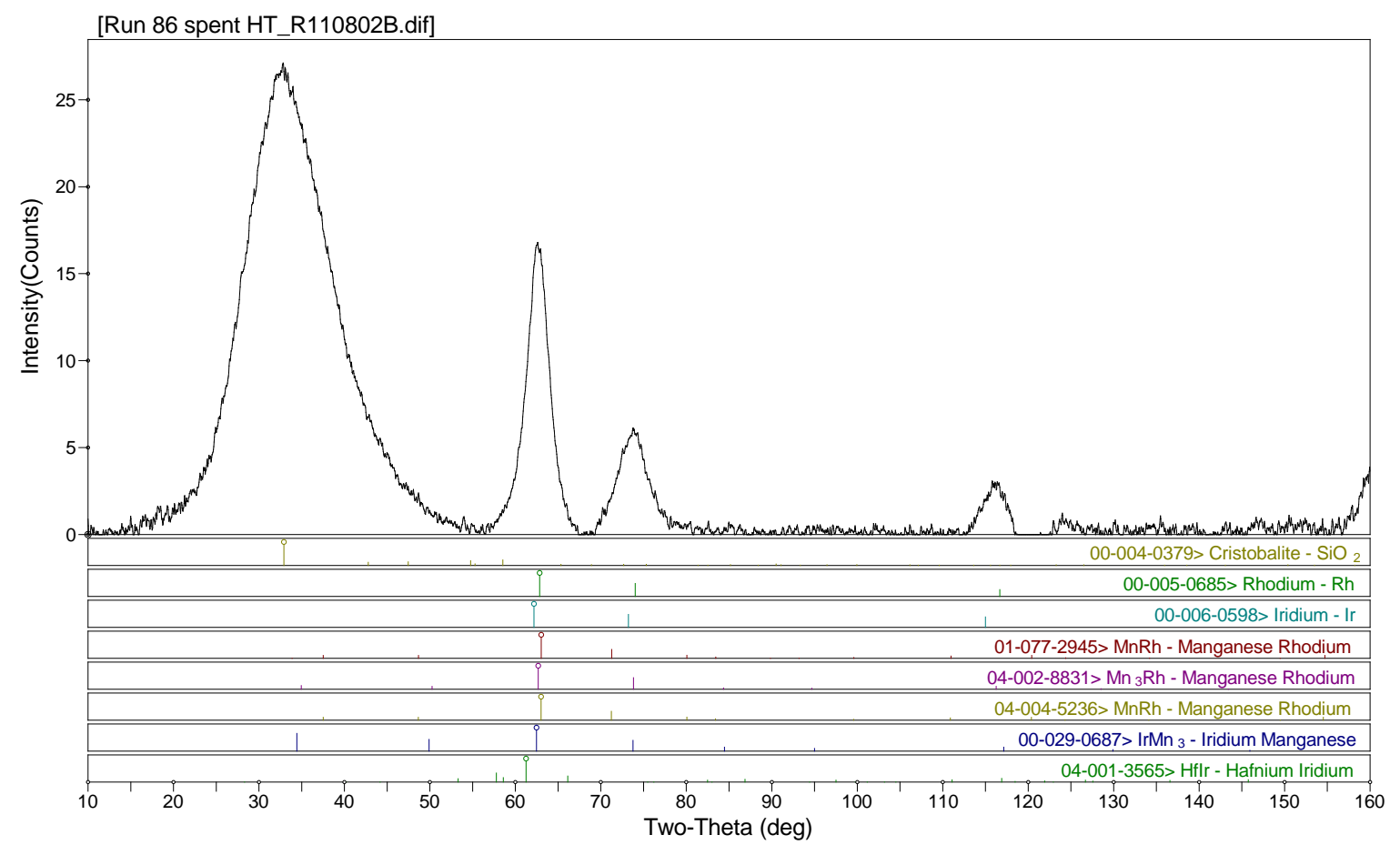

Figure 2.31. Diffractogram Collected from the Sample of Run 86, which was the Davisil 645 SilicaSupported Rh-Mn-Ir Baseline Catalyst Tested to $300^{\circ} \mathrm{C}$ in Table 2.4

\subsubsection{Hyperion Fibril MWCNT-Supported Rh-Mn Baseline Catalyst}

The fresh Hyperion Fibril MWCNT-supported Rh-Mn catalyst was measured with XRD, and the results are presented in Figure 2.32. The unidentified phase at $72^{\circ} \mathrm{C}$ that matches the major reflection of silicon is again observed similar to the other fresh baseline catalysts. Other reflections align satisfactorily with a series of reflections reported for carbon nanotubes.

In contrast to the silica-supported catalysts discussed above, the MWCNT-supported samples are not calcined as part of catalyst preparation. Therefore, the Rh and Mn present on the support likely are present as nitrates. There are other minor reflections unaccounted for in the fresh catalyst diffractogram (e.g., between 30 to $35^{\circ}$ 2-Theta), but no reference reported in the literature for rhodium nitrate or manganese nitrate could be found in the XRD analysis software. In general, it would appear that the metals are amorphous on the MWCNT support prior to reduction. Transmission electron microscope (TEM) measurements on a similar sample (Figure 2.73) suggest that, if metal clusters are present in the sample, they are very small (likely $<1 \mathrm{~nm}$ ). 


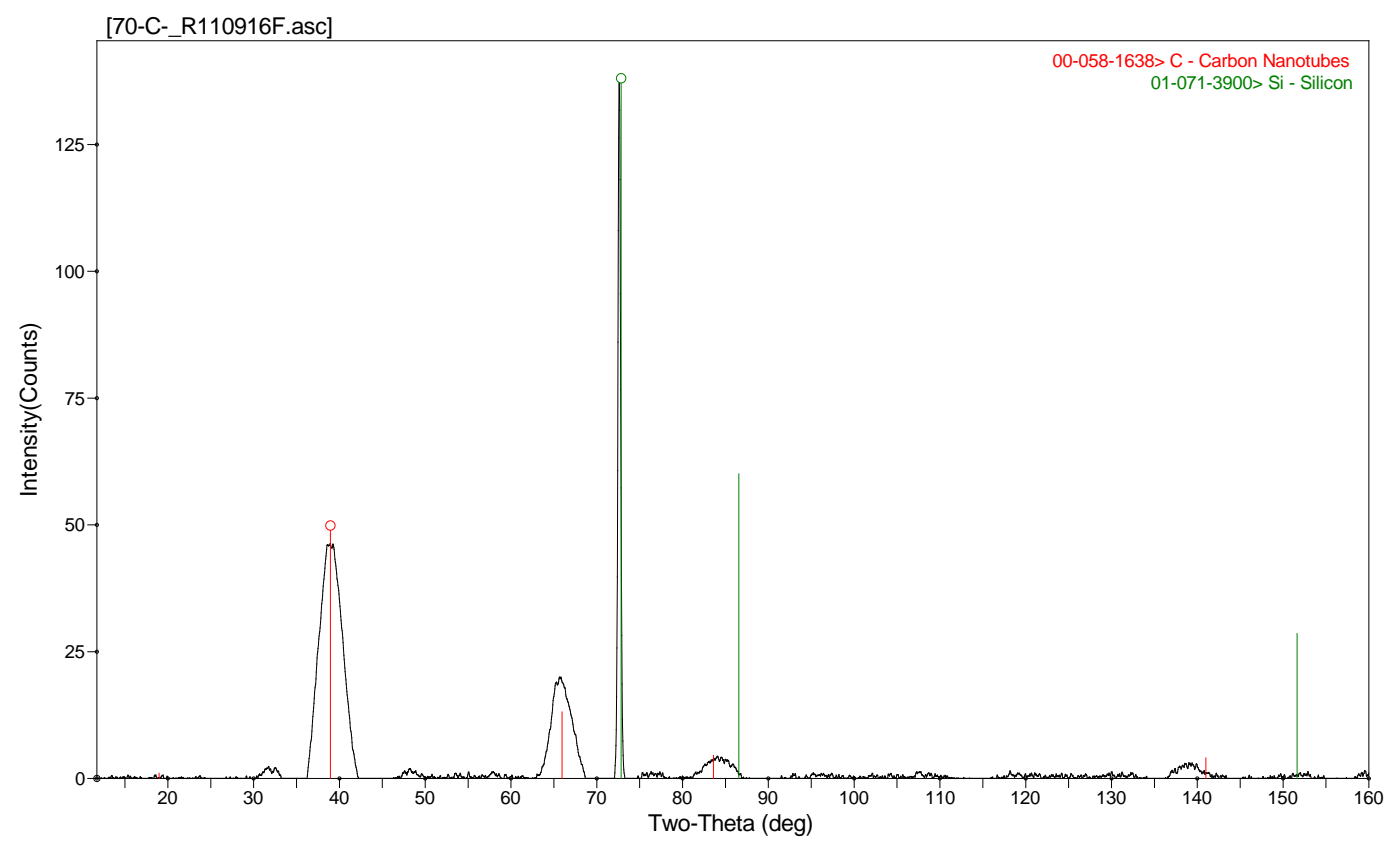

Figure 2.32. Diffractogram Collected from Sample 60074-70-C, which was the Fresh Hyperion Fibril MWCNT-Supported Rh-Mn Catalyst in Table 2.4

The diffractogram of the reduced Rh-Mn supported MWCNT-supported catalyst is significantly different than the fresh sample. Similar to the fresh catalyst, the peaks associated with carbon nanotubes are again observed in Figure 2.33. However, a broad hump is now present on the left side of the major nanotube reflection. This broad hump may be due to a lesser reflection of quartz (as shown by the blue line in Figure 2.34 and Figure 2.35). The quartz may be present in the sample as a minor impurity due to its presence as a catalyst support in the mixed alcohols reactor. On the other hand, the reflection is observed in all diffractograms of reduced and spent MWCNT-supported samples and may be a weakly crystalline metallic phase.

Figure 2.33 also illustrates a broad series of reflections that extend from 50 to $90^{\circ} \mathrm{C} 2$-Theta. This broad hump likely contains reflections from several weakly crystalline phases. Rh, the Rh-Mn alloy, and the unidentified phase isomorphic with HfIr are all likely candidates. An extensive literature search for a phase to explain the presence of the HfIr phase that would correlate with any of the elements present in the MWCNT-supported catalysts proved unfruitful. However, because the phase is present or could be suggested in nearly any of the MWCNT-supported catalysts, the isomorphic analogue of the HfIr phase is plotted in these diffractograms for the sake of comparison. Note that the phase almost certainly does not actually contain Ir as it is present in catalysts without Ir.

Interestingly, one of the phases that could be suggested for a match in Figure 2.33 is metallic Mn (green reflection lines). Crystalline metallic Mn within the catalyst seems unlikely. Metallic Mn has been suggested by computational work within Rh-Mn particles to be active for the production of mixed alcohols, but the zero-valence state Mn exists stably as an alloy with $\mathrm{Rh}^{7}{ }^{\mathrm{X}} \mathrm{X}$-ray photoelectron spectroscopy (XPS) results on sample 60074-70-C-R were inconclusive as to the oxidation state of Mn due to the wide and overlapping range of Mn oxidation states (Figure 2.53). However, the XPS results suggest that the $\mathrm{Mn}$ at the surface is nearer $\mathrm{MnO}$ than metallic Mn. Thus, more work is warranted to 
determine if the reflection of Mn in the MWCNT-supported samples is truly due to metallic Mn or is coincidentally isomorphic with another phase.

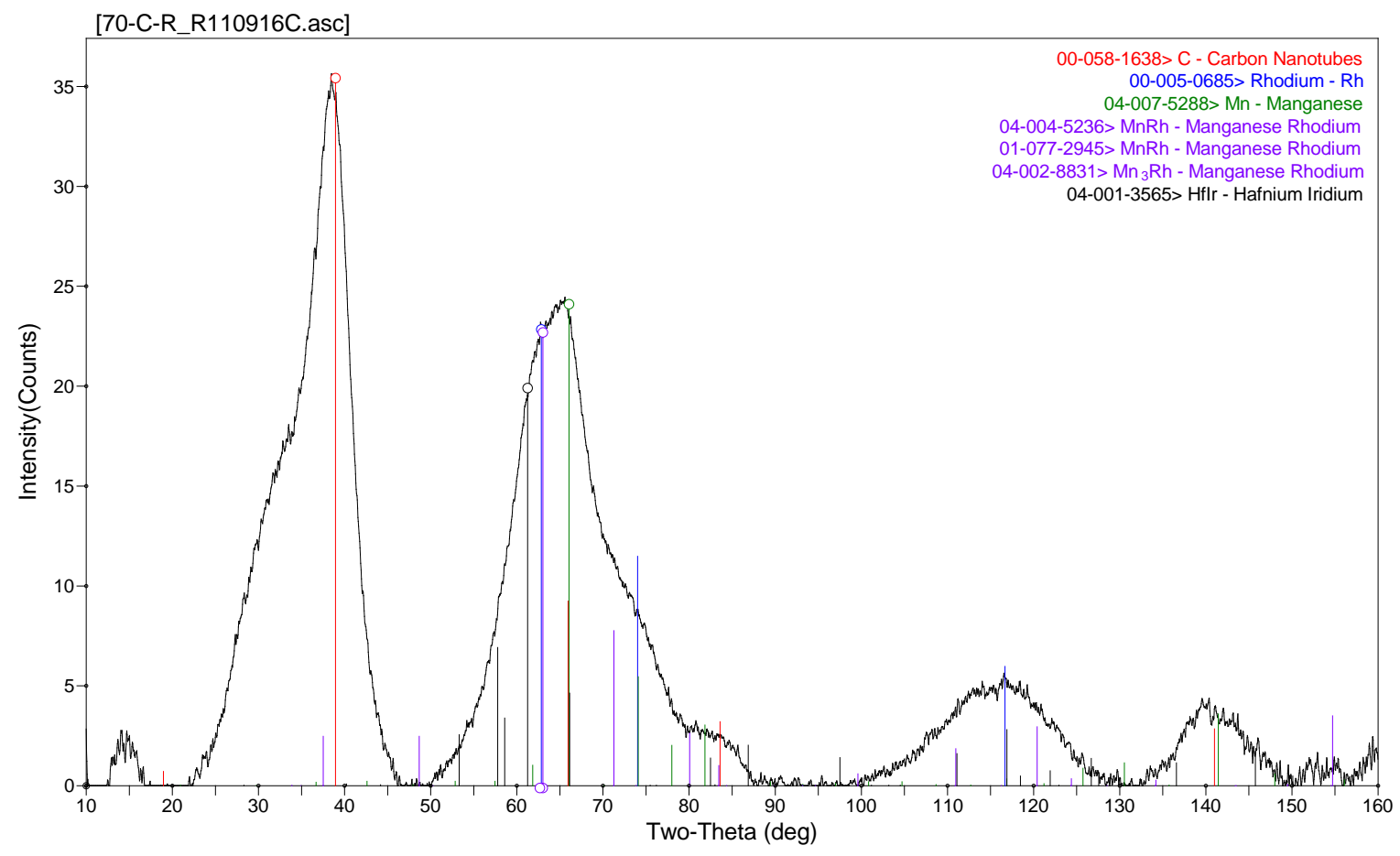

Figure 2.33. Diffractogram Collected from Sample 60074-70-C-R, which was the reduced Hyperion Fibril MWCNT-Supported Rh-Mn Catalyst in Table 2.4

Use of the MWCNT-supported Rh-Mn catalyst for the synthesis of mixed alcohols further increases the crystallinity of the phases likely present after Figure 2.33 to reflections with sharper features in Figure 2.34 and Figure 2.35. Most of the reflections suggested in Figure 2.34 and Figure 2.35 are the same reflections suggested after reduction in Figure 2.33. One notable difference is the appearance of $\mathrm{MnCO}_{3}$ in the spent samples. The presence of $\mathrm{MnCO}_{3}$ could be significant as it may act as a "sink" for the $\mathrm{Mn}$, resulting in the removal of a necessary catalytic component from the active phase. It is unclear if the formation of $\mathrm{MnCO}_{3}$ is from an interaction with the carbon support or through a carbonation reaction with the syngas, which contains $4 \% \mathrm{CO}_{2}$. Note that $4 \% \mathrm{CO}_{2}$ at 1200 psig is $\sim 3.3 \mathrm{~atm}$ partial pressure of $\mathrm{CO}_{2}$, making a carbonation reaction a distinct possibility.

Generally, the sample subjected to mixed alcohols synthesis at higher temperature (Figure 2.35) is more crystalline than its lower temperature analogue (Figure 2.34). For instance, the HrIr phase analogue becomes increasingly crystalline when the catalyst is subjected to mixed alcohol synthesis conditions as evidenced by the sharper, narrower peaks. The imperfect match of the HfIr phase to the several reflections observed in Figure 2.35 is illustrated as bright green lines that do not align exactly with the observed reflections. 


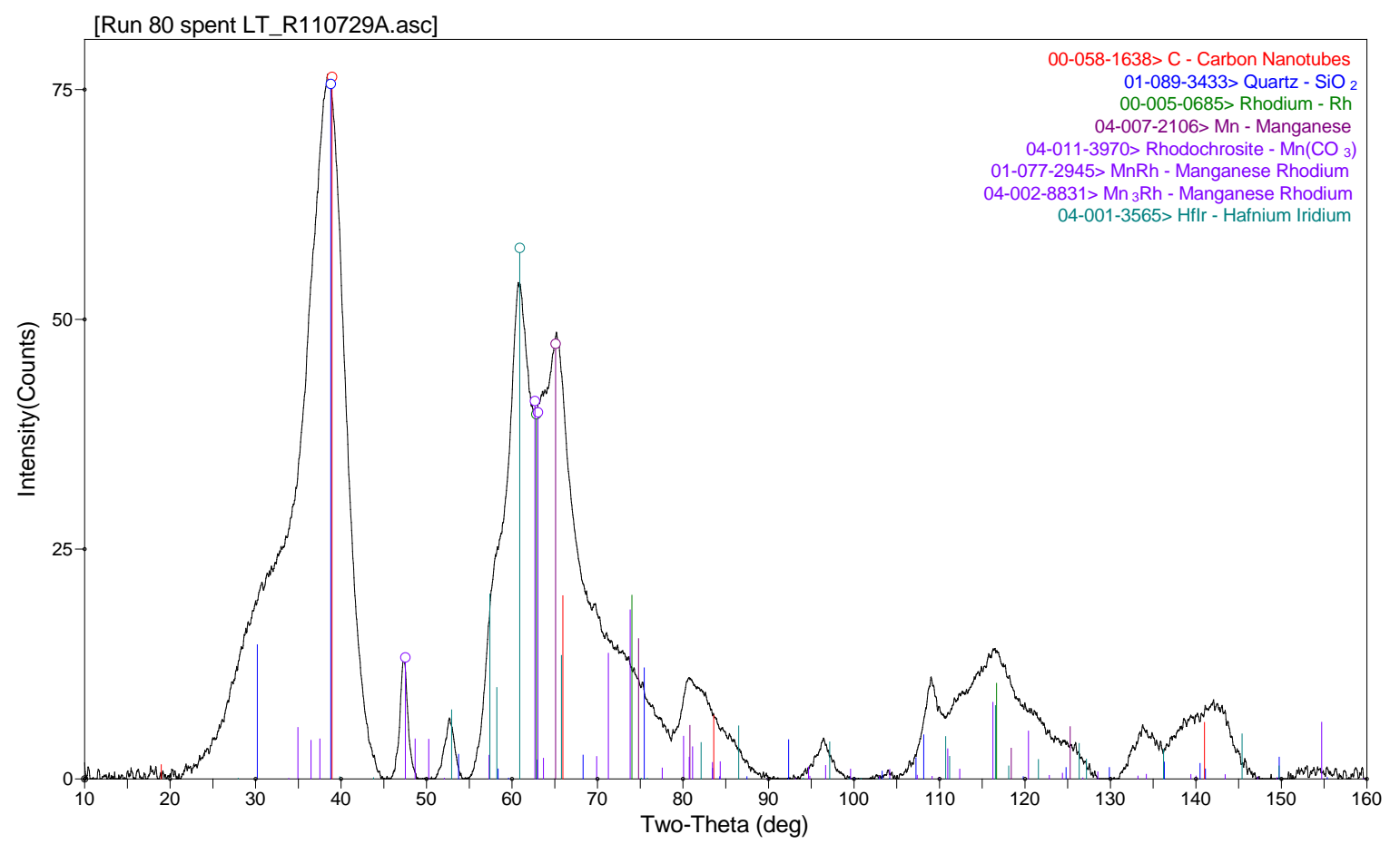

Figure 2.34. Diffractogram Collected from the Sample from Run 80, which was the Hyperion Fibril MWCNT-Supported Rh-Mn Catalyst Tested to $276^{\circ} \mathrm{C}$ in Table 2.4

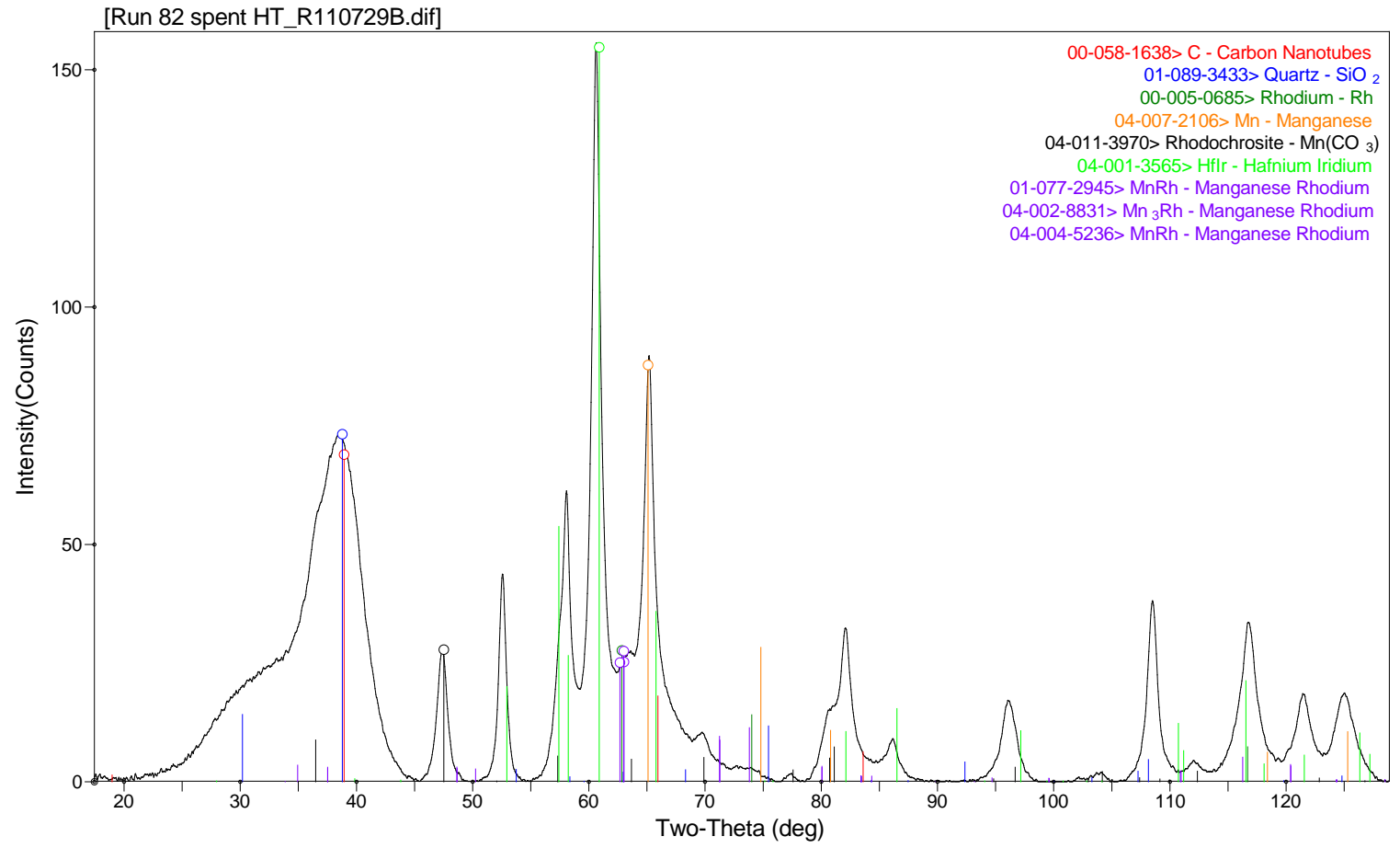

Figure 2.35. Diffractogram Collected from the Sample from Run 82, which was the Hyperion Fibril MWCNT-Supported Rh-Mn Catalyst Tested to $299^{\circ} \mathrm{C}$ in Table 2.4 


\subsubsection{Hyperion Fibril MWCNT-Supported Rh-Mn-Ir Baseline Catalyst}

The fresh Hyperion Fibril MWCNT support Rh-Mn-Ir catalyst was measured with XRD and the results are presented in Figure 2.36. The unidentified phase at $72^{\circ} 2$-Theta matching the major reflection of Si again is observed to be similar to the other fresh baseline catalysts. Similar to the MWCNTsupported Rh-Mn catalyst, other reflections line up satisfactorily with a series of reflections reported for carbon nanotubes. Indeed, the diffractograms of both MWCNT-supported catalysts are similar.

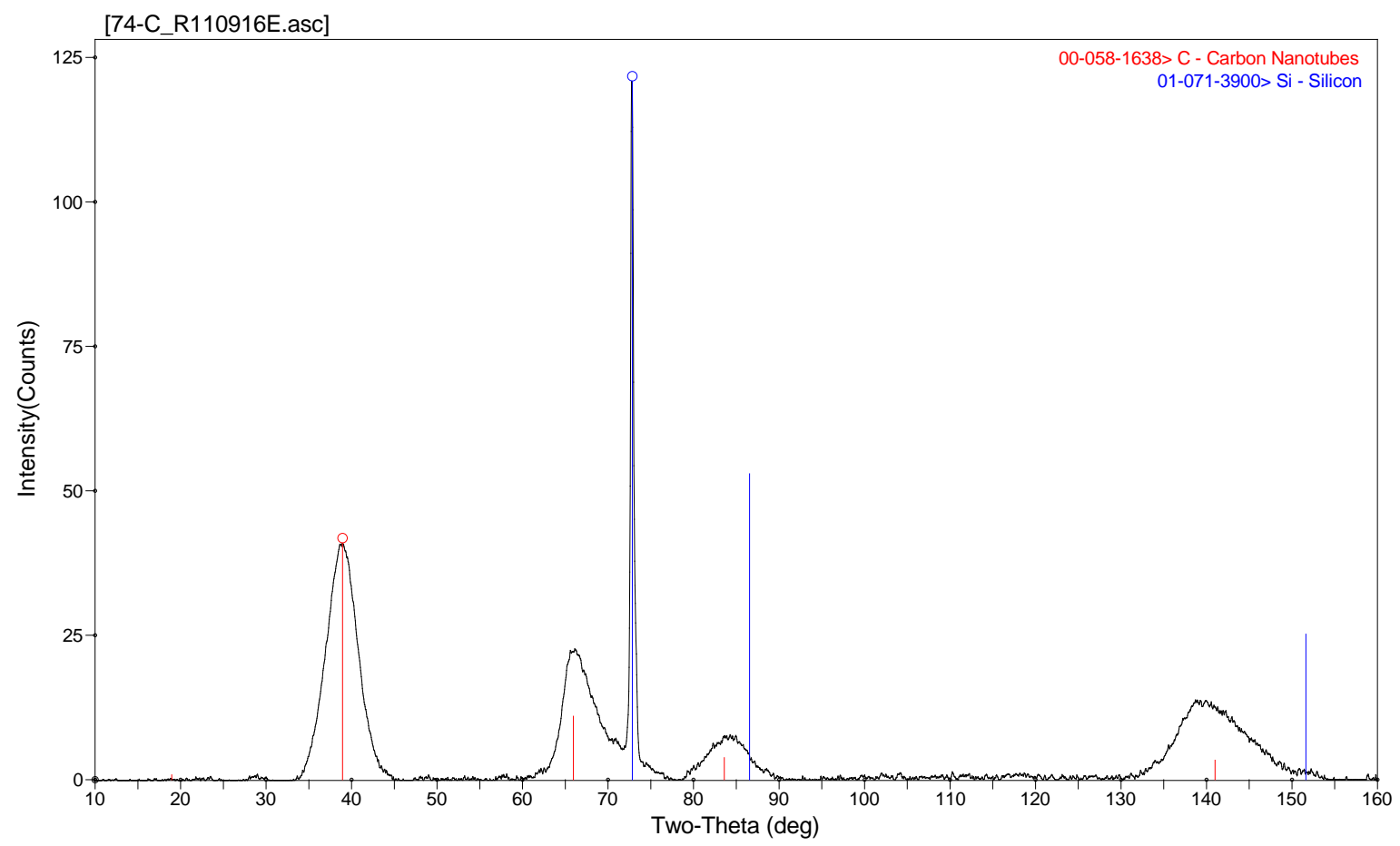

Figure 2.36. Diffractogram Collected from Sample 60074-74-C, which was the fresh Hyperion Fibril MWCNT-Supported Rh-Mn-Ir Catalyst in Table 2.4

The diffractogram of the reduced MWCNT-supported Rh-Mn-Ir catalyst is shown in Figure 2.37. Similar to the fresh catalyst, the reduced Rh-Mn-Ir catalyst is strikingly similar to the Rh-Mn catalyst. Similar broad reflections that may indicate the presence of metallic Rh, Rh-Mn, and even metallic Mn are again suggested in Figure 2.37. $\mathrm{MnCO}_{3}$ is not yet present in the diffractogram, again suggesting that the formation of the carbonate may be from a carbonation reaction with $\mathrm{CO}_{2}$ from the syngas feed than through interaction with the carbon support. 


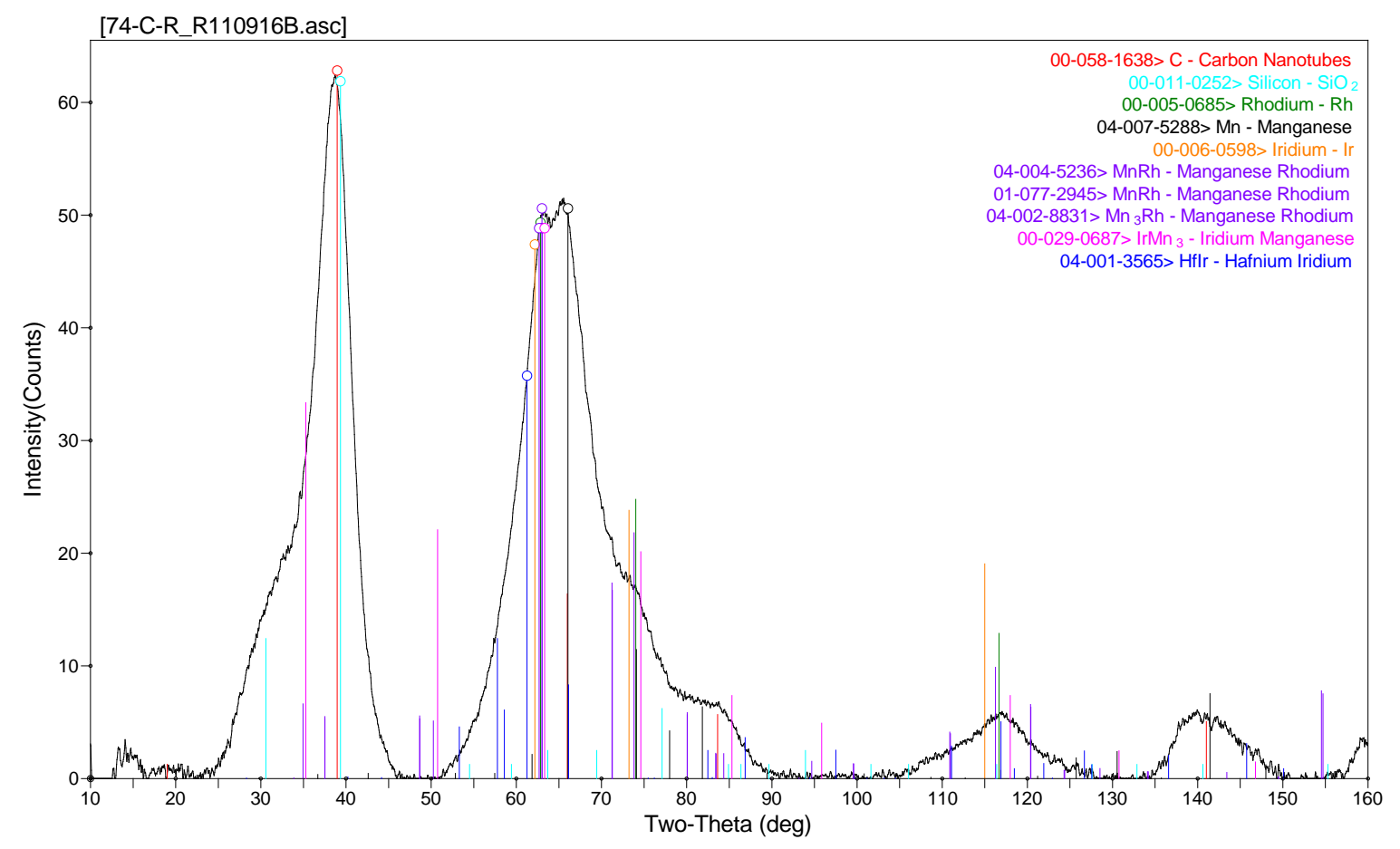

Figure 2.37. Diffractogram Collected from Sample 60074-74-C-R, which was the reduced Hyperion Fibril MWCNT-Supported Rh-Mn-Ir Catalyst in Table 2.4

The diffractogram of the spent Rh-Mn-Ir catalyst supported on MWCNTs tested up $275^{\circ} \mathrm{C}$ (Run 89) shown in Figure 2.38 is similar to its Rh-Mn analogue in Figure 2.34. Some peaks do develop at the top of the broad reflection between 60 and $70^{\circ}$ 2-Theta. One notable difference is the development of a sharp peak on the right side of the broad reflection at $70^{\circ}$ 2-Theta. This peak may be due to the $\mathrm{MnCO}_{3}$ reflection (bright green line under the peak), although the reflection seems to be more intense than information from the literature reference would suggest.

Another interesting observation in the low temperature (Run 89; Figure 2.38) and high temperature (Run 88; Figure 2.39) spent Rh-Mn-Ir catalysts is that that the Mn peak, which aligned well in the spent Rh-Mn catalysts, now appears to be shifted to the right of the reflection. The black reference lines at $\sim 66^{\circ}$ 2-Theta is not as good a match with the peak now located at $\sim 65^{\circ} 2$-Theta. A similar crystal may be responsible for the reflection in both the Rh-Mn and Rh-Mn-Ir catalysts, but may be shifted in the RhMn-Ir catalyst due to the inclusion of another atom in the crystal (e.g., Ir). In any case, the presence of metallic Mn was unlikely in either instance, but the value in tracking a peak with an isomorphic analogue to detect a shift in the various diffractograms can be observed. 


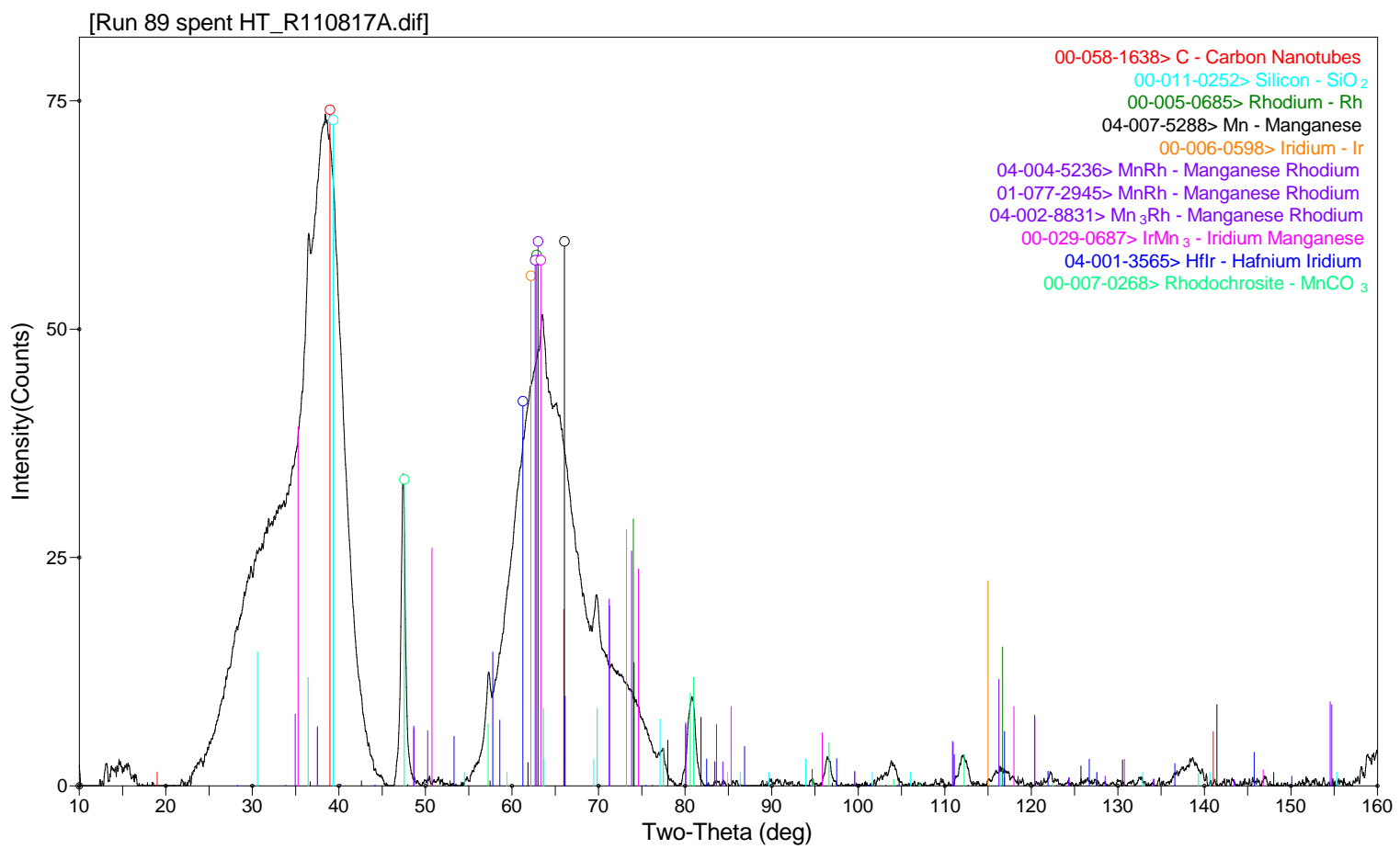

Figure 2.38. Diffractogram Collected from the Sample from Run 89, which was the Hyperion Fibril MWCNT-Supported Rh-Mn-Ir Catalyst Tested to $275^{\circ} \mathrm{C}$ in Table 2.4

The HfIr phase analogue in the high temperature spent Rh-Mn-Ir catalyst illustrated in Figure 2.39 is not as well defined in the similar Rh-Mn catalyst (Figure 2.35). Without Ir present, the phase appears to grow in crystallinity and become well defined at higher temperature. The growth and definition of the HfIr phase may indeed be a clue as to the deactivation mechanism of the catalyst. In other words, Ir may not only open new reaction pathways to oxygenates as suggested by the computational investigations, ${ }^{8}$ but may also inhibit the crystallization of phase that is detrimental to the activity of the catalyst. 


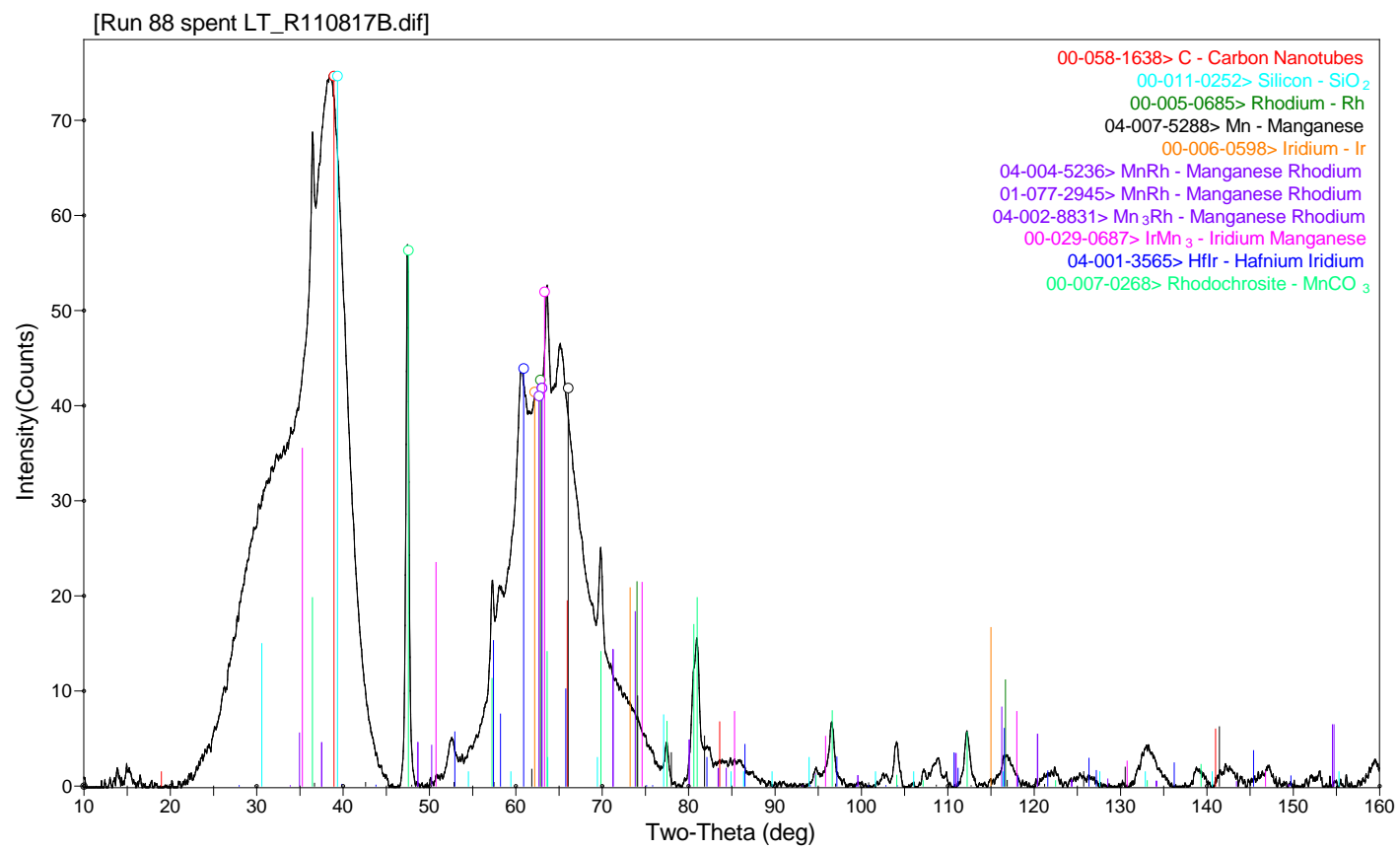

Figure 2.39. Diffractogram Collected from the Sample from Run 88, which was the Hyperion Fibril MWCNT-Supported Rh-Mn-Ir Catalyst Tested to $314^{\circ} \mathrm{C}$ in Table 2.4

\subsubsection{Other Catalyst Samples}

XRD measurements also were performed on the fresh metal impregnated catalyst samples listed in Table 2.6. The fresh carbon-supported samples shown in Figure 2.40 characterized with XRD were reduced through a procedure that was identical in the production of the baseline reduced catalysts. As shown in Figure 2.40, the diffractograms of theses samples were similar to the diffractograms produced during analysis of the carbon supports.

The most significant observation in Figure 2.40 is that the FCC structure expected for metallic Rh may be present as a relatively broad reflection centered at $\sim 63^{\circ} 2$-Theta. However, the sample with the highest concentration of Rh and Ir, sample 60074-59-C, exhibits a more pronounced FCC reflection at $\sim 63^{\circ}$ 2-Theta, which indicates larger and/or more ordered crystalline Rh and/or Ir. These tests suggest that the reduction of the metal precursors (i.e., nitrates or chloride) without prior calcination leads to welldispersed metals on the catalyst surface, which was confirmed through TEM images on the similar baseline catalysts. 


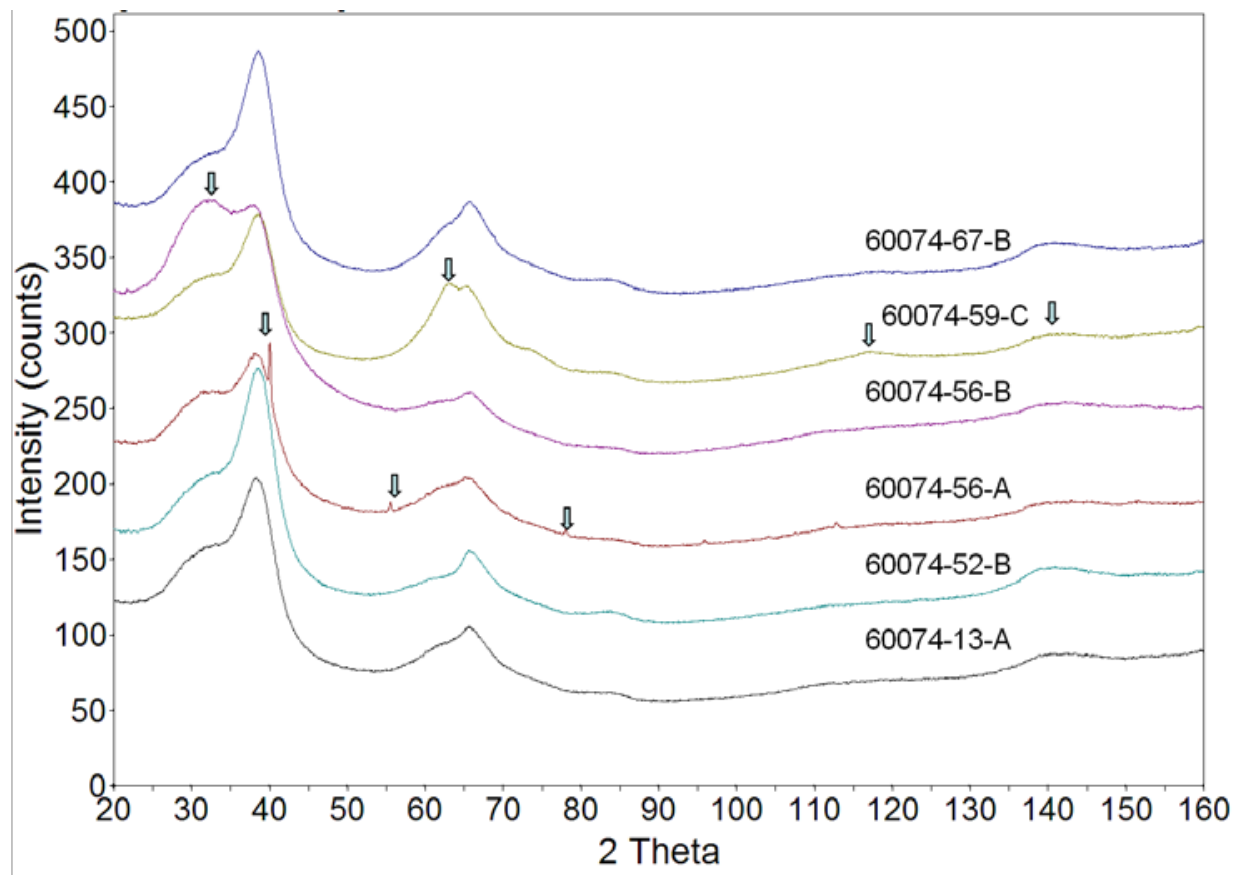

Figure 2.40. XRD Analysis of Various Reduced Catalyst Samples Listed in Table 2.6. The arrows point out possible differences in these diffractograms from the XRD analysis of the supports (samples KOA 6, 7 or 8) in Figure 2.26.

XRD analysis of the spent catalysts listed in Table 2.6 is given in Figure 2.41 through Figure 2.47. Figure 2.41 shows results of XRD testing on the catalyst from Run 31, which contained $11.78 \mathrm{wt} \% \mathrm{Rh}$ and $3.58 \mathrm{wt} \% \mathrm{Mn}$. Another sample, labeled Run 32, contained $8.34 \mathrm{wt} \% \mathrm{Rh}, 2.54 \mathrm{wt} \% \mathrm{Mn}$, and $1.53 \mathrm{wt} \%$ Os. Both catalysts were supported on KOA 8 (Hyperion Fibril Catalyst Support CS-02C-063XD Lot 384-82 MWCNTs). The result of XRD testing on Run 32 was virtually identical to the result from Run 31; hence, Micro-XRD results from Run 32 are not shown in Figure 2.41.

The diffractogram in Figure 2.41 is very similar to the high temperature baseline catalyst (Run 82) Rh-Mn MWCNT-supported catalyst shown in Figure 2.35, which is consistent as these catalysts are the same composition on the same support. Thus, the discussion above and phases matched in Figure 2.35 are appropriate also for the diffractogram in Figure 2.41. The fact that the diffractogram from Run 32 (not shown) was nearly identical to the diffractogram in Figure 2.41 indicates that Os did not have a significant effect on the crystal structure of the catalyst. $\mathrm{MnCO}_{3}$ was identified as a crystalline phase similar to the spent Rh-Mn baseline catalyst. An elementally unconstrained search of the Powder Diffraction Files Database returned a crystalline phase with a structure analogous to an orthorhombic HfIr phrase. While Hf and Ir are not present in this sample, it could give a hint as to the structure of the crystal structure of the unidentified phase. The crystal structure observed that was unable to be matched did not correlate with metallic Rh, Mn or any known mixed phase of Rh and Mn. 


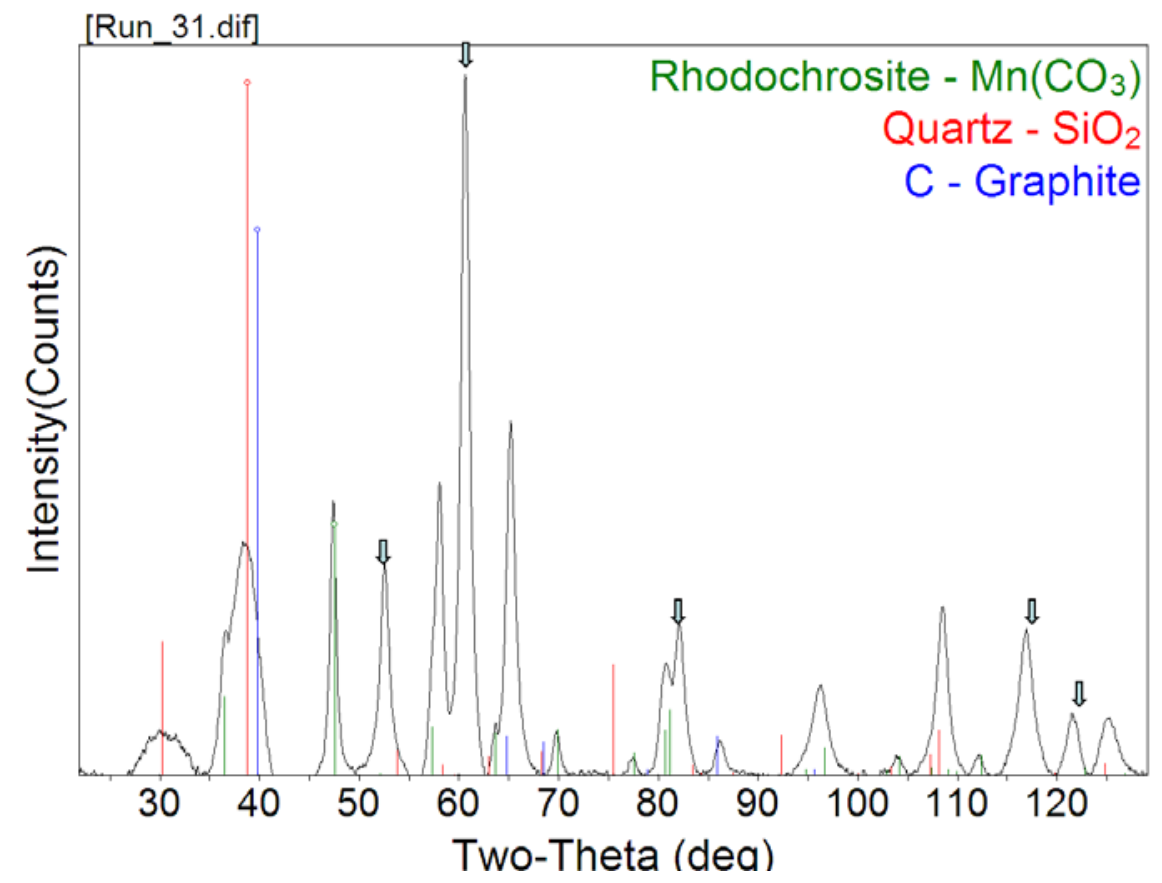

Figure 2.41. Micro-XRD Results on Spent Catalyst Sample Run 31, which contained 11.78 wt\% Rh and $3.58 \mathrm{wt} \% \mathrm{Mn}$. The arrows indicate a crystalline phase that was unsuccessfully phase matched with Rh and Mn but correlates to an orthorhombic HfIr phase.

Samples from Runs 33, 34 and 39 (see Table 2.6) were also analyzed via Micro-XRD. These samples had Ir present as a promoter. The catalysts from Runs 33 and 39 were prepared by simultaneously impregnating Rh, Mn, and Ir. In contrast, the catalyst used in Run 34 was prepared by impregnating Rh and Mn simultaneously onto the catalyst first and then followed by Ir impregnation. Most notably, the catalyst used in Run 33 exhibited a brief temperature excursion to $330^{\circ} \mathrm{C}$ at one point during testing. The catalysts in both Run 33 and 34 were considered to have been run at relatively high temperature (312 to $315^{\circ} \mathrm{C}$ ) during one condition of testing. In contrast, Run 39 was run for a longer period of time but at relatively cooler temperatures with $297^{\circ} \mathrm{C}$ having been the maximum operating temperature. Thus, major points for comparison of the catalysts can be summed up by stating Run 33 experienced a brief high temperature excursion, Run 34 was prepared via sequential metal impregnations of Rh and Mn followed by Ir while Run 39 experienced extended albeit cooler operating conditions.

The results of Micro-XRD testing on Runs 33, 34 and 39 are presented in Figure 2.42 through Figure 47.

Figure 2.42 and Figure 2.43 are identical diffractograms with alternate potential phases (colored lines) overlaid. Similarly, Figure 2.44 and Figure 2.45 are identical results as are Figure 2.46 and Figure 2.47.

Similar to the baseline catalysts and the catalysts from Runs 31 and 32, the catalysts from Runs 33, 34, and 39 all contained $\mathrm{MnCO}_{3}$ as a major crystalline phase as evidenced by the results in Figure 2.42 through Figure 2.47. The presence of crystalline $\mathrm{MnCO}_{3}$ could be significant, because if the $\mathrm{Mn}$ is present as a carbonate in crystalline form, it may not be associated with Rh in a form active for the synthesis of ethanol. 
The HfIr orthorhombic analogue was again present in the catalysts from Runs 33, 34, and 39. While Ir was present in the catalysts from Runs 33, 34, and 39, the presence of the HfIr analogue phase in the catalysts from Runs 31 and 32, neither of which contained Ir, suggests that the HfIr analogue crystalline phase does not contain Ir. Investigation of other potential phases including $\mathrm{Mn}$ or Fe carbides (Fe is present in the MWCNT supports per ICP results) did not reveal the true identity of the HfIr phase.

In Figure 2.42, the red arrow illustrates the location of a reflection that could correlate with the $\mathrm{MnRh}$ or $\mathrm{Mn}_{3} \mathrm{Rh}$ crystalline phase. The reflection, which correlates with the shoulder on the peak at $\sim 63^{\circ}$ 2-Theta, can be observed to match with the $\mathrm{MnRh}$ and $\mathrm{Mn}_{3} \mathrm{Rh}$ peaks (green and blue lines) in Figure 2.42. Per similar phase matching in the baseline catalysts (e.g., Figure 2.39), it also is possible that the reflection is due to metallic $\mathrm{Rh}$ and/or Ir. It is significant to note that the strong $\mathrm{MnRh}$ (or $\mathrm{Mn}_{3} \mathrm{Rh}$ ) reflection present in Figure 2.43 does not appear in diffractograms taken of samples from Runs 34 and 39 (see Figure 2.45 and Figure 2.47). Additionally, the peak at $73^{\circ}$ 2-Theta is not present in the diffractogram from Runs 34 and 39. The peak at $117^{\circ}$ 2-Theta is present in Figure 2.45 and Figure 2.47, but the presence of this peak also can be attributed to the HfIr analogue phase, as can be observed in Figure 2.44 and Figure 2.46 .

The possibility of Rh, Ir, MnRh or $\mathrm{Mn}_{3} \mathrm{Rh}$ peak from Run 33 could correlate with the fact that the catalyst in Run 33 exhibited a temperature excursion to $330^{\circ} \mathrm{C}$. Qualitatively, the temperature excursion in Run 33 may have resulted in diminished activity. Because the reflection is not exhibited by the catalysts in Runs 34 and 39, it is not likely that the crystalline phase did not develop as extensively during operation at 312 to $315^{\circ} \mathrm{C}$ because Run 34 also was run at these temperatures. Additionally, Run 39 was run for a longer time on stream (albeit at cooler temperatures than Run 33) and does not exhibit the phase. Thus, a high-temperature excursion, even though the excursion only lasted for a few moments, could induce the formation of a crystalline $\mathrm{Rh}$ or $\mathrm{Rh}-\mathrm{Mn}$ phase that is not conducive to mixed alcohol synthesis. This may be consistent with the baseline catalyst tests as the Rh-Mn and Rh-Mn-Ir catalysts tested to higher temperature (Run 82 in Figure 2.35 and Run 88 in Figure 2.39) appear to have more crystalline phases attributed to MnRh. 


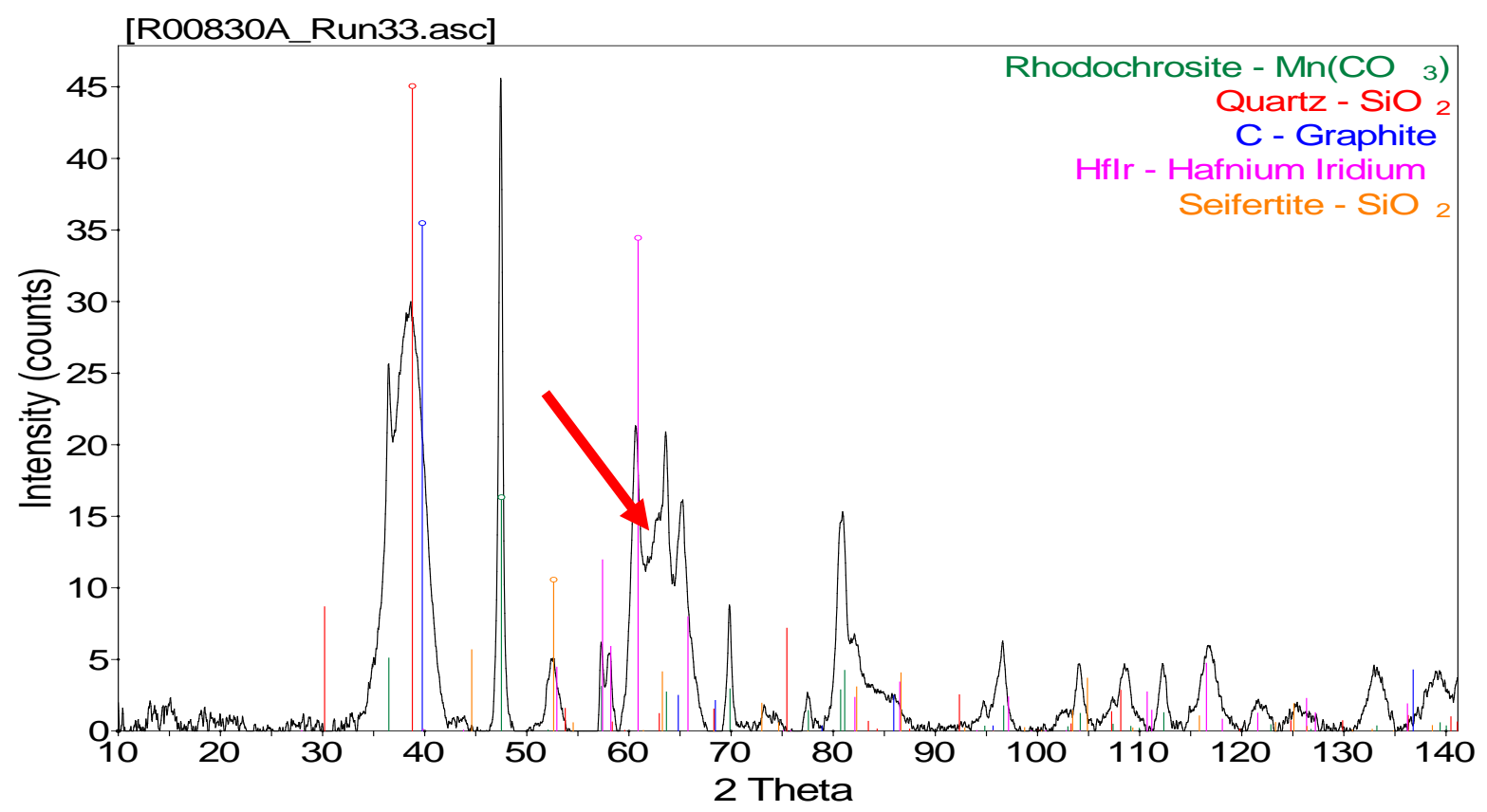

Figure 2.42. Micro-XRD Results on Spent Catalyst Sample Run 33, which contained 11.78 wt\% Rh, $3.58 \mathrm{wt} \% \mathrm{Mn}$, and $2.19 \mathrm{wt} \%$ Ir. The metals were simultaneously co-impregnated. This catalyst sample was subjected to a temperature excursion to $\sim 330^{\circ} \mathrm{C}$. Potential crystalline phases are shown by the color-coded lines. The red arrow denotes the presence of the reflection indicating the possible presence of a MnRh crystalline phase.

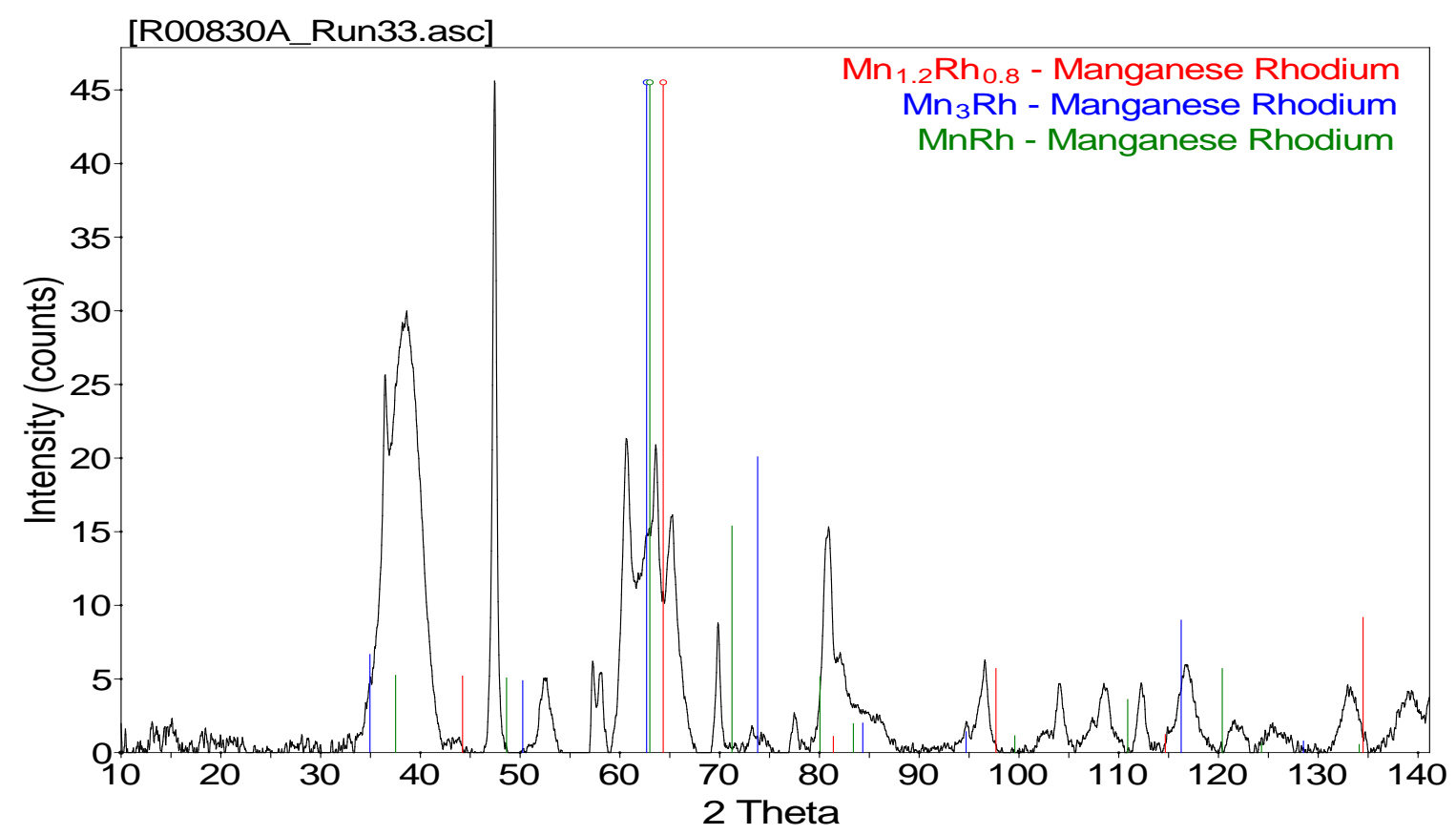

Figure 2.43. Micro-XRD Results on Spent Catalyst Sample Run 33, which contained 11.78 wt\% Rh, $3.58 \mathrm{wt} \% \mathrm{Mn}$, and $2.19 \mathrm{wt} \%$ Ir. The metals were simultaneously co-impregnated. Potential crystalline phases are shown by the color-coded lines. The XRD trace is identical to the trace in Figure 2.42. 


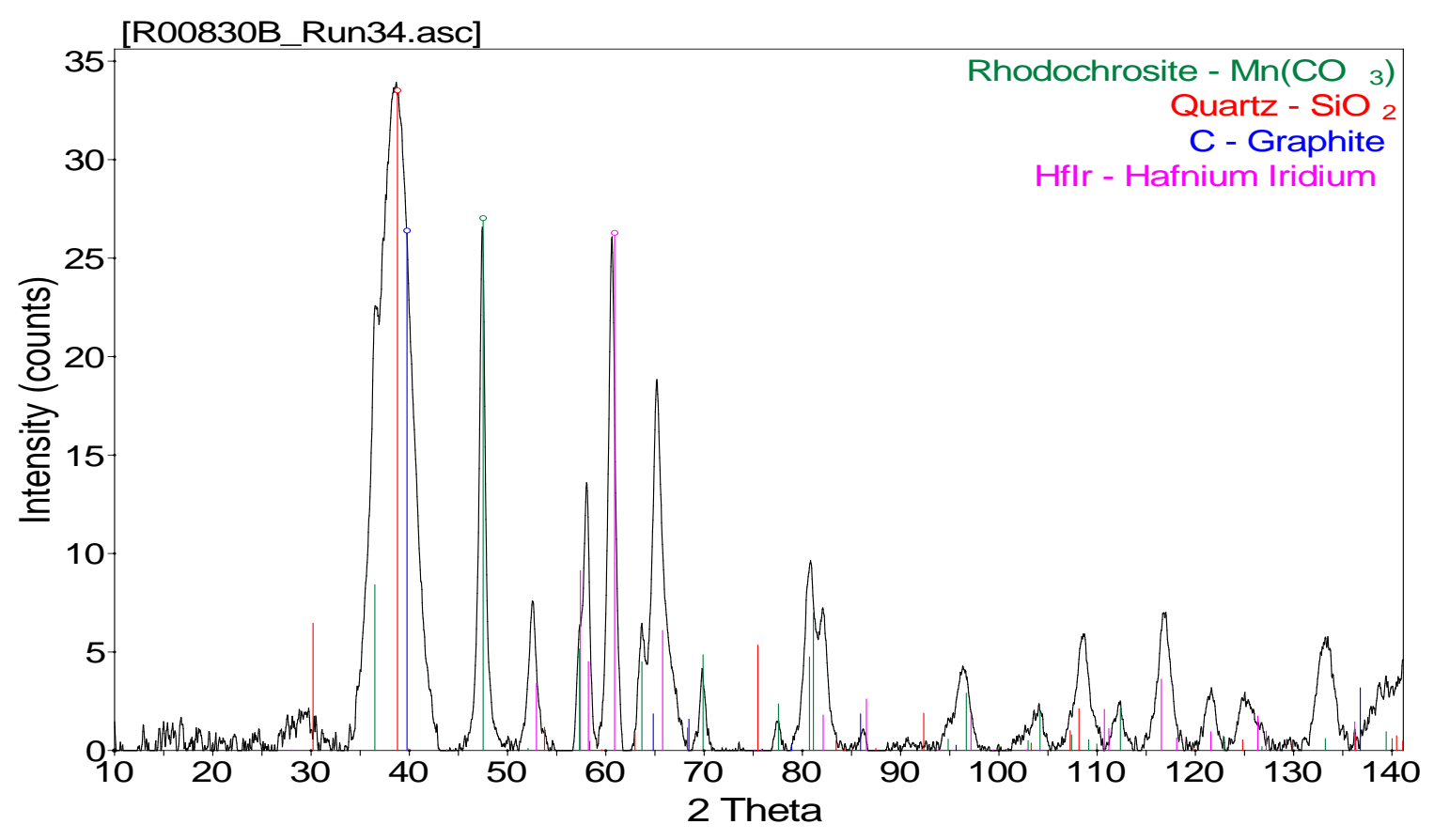

Figure 2.44. Micro-XRD Results on Spent Catalyst Sample Run 34, which contained 11.78 wt\% Rh, $3.58 \mathrm{wt} \% \mathrm{Mn}$, and $2.19 \mathrm{wt} \%$ Ir. The Rh and Mn were co-impregnated, followed by Ir impregnation. Potential crystalline phases are shown by the color-coded lines.

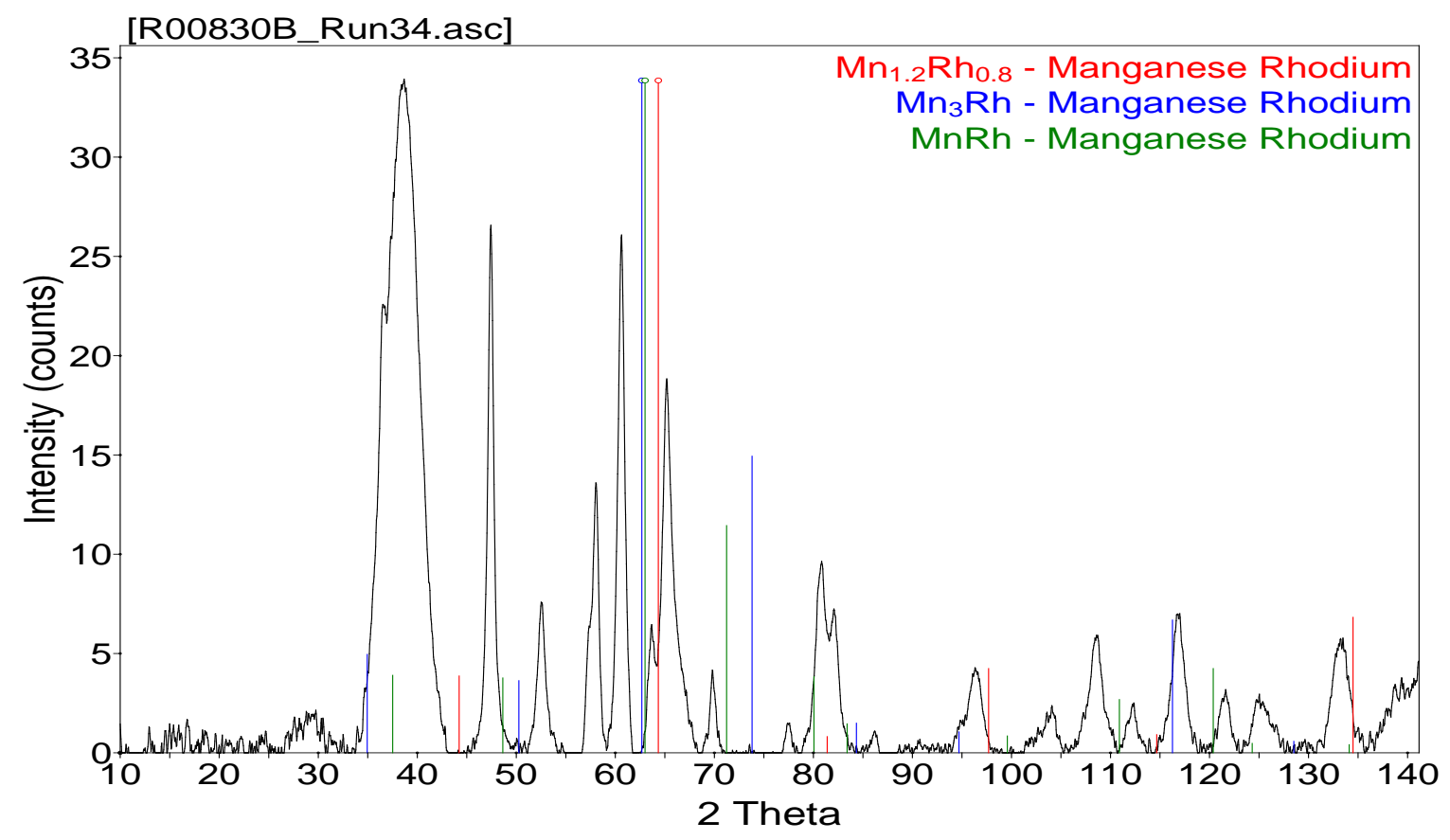

Figure 2.45. Micro-XRD Results on Spent Catalyst Sample Run 34, which contained 11.78 wt\% Rh, $3.58 \mathrm{wt} \% \mathrm{Mn}$, and $2.19 \mathrm{wt} \%$ Ir. The Rh and Mn were co-impregnated, followed by Ir impregnation. Potential crystalline phases are shown by the color-coded lines. The XRD trace is identical to the trace in Figure 2.44. 


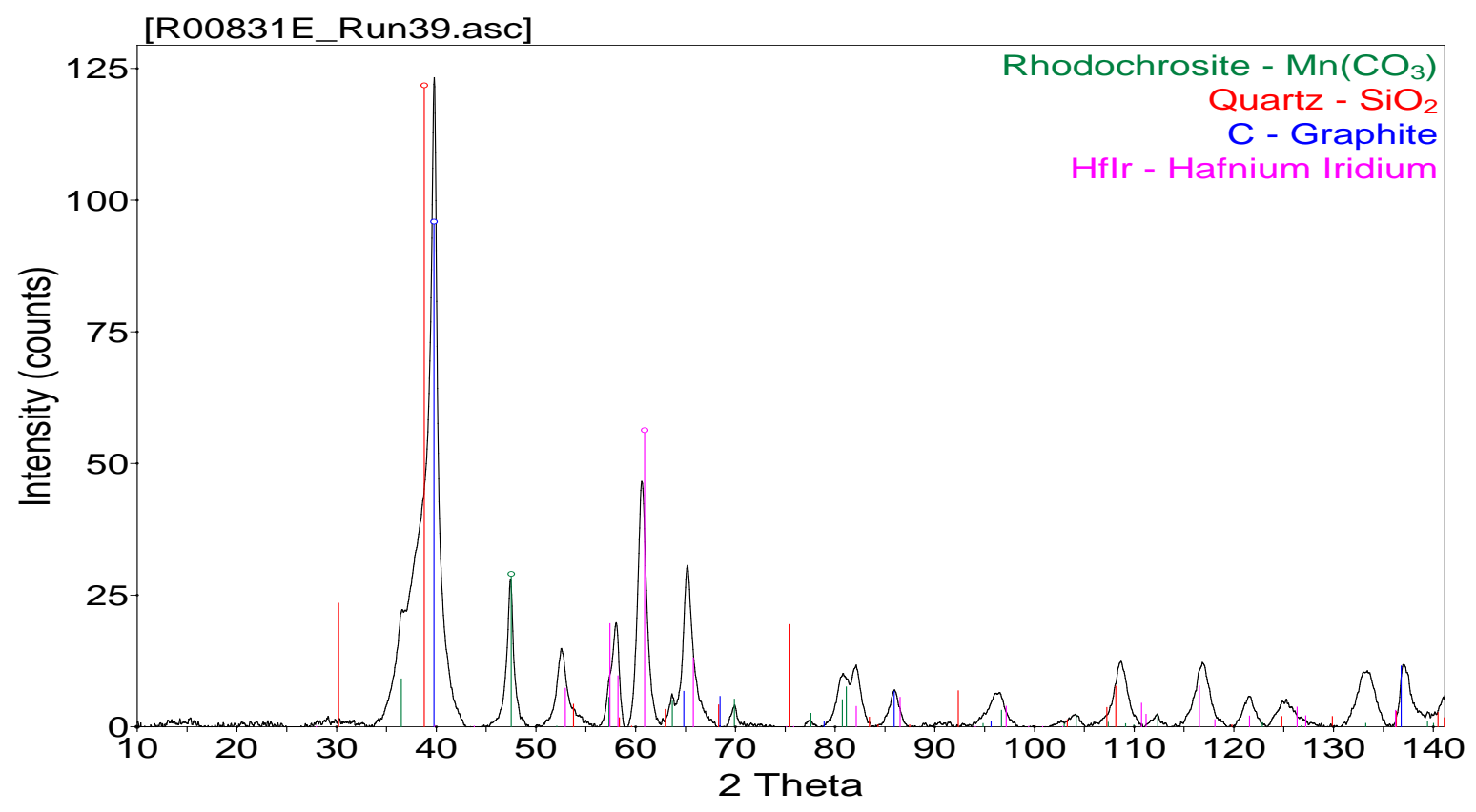

Figure 2.46. Micro-XRD Results on Spent Catalyst Sample Run 39, which contained 11.78 wt\% Rh, $3.58 \mathrm{wt} \% \mathrm{Mn}$, and $2.19 \mathrm{wt} \%$ Ir. The metals were simultaneously co-impregnated. Potential crystalline phases are shown by the color-coded lines.

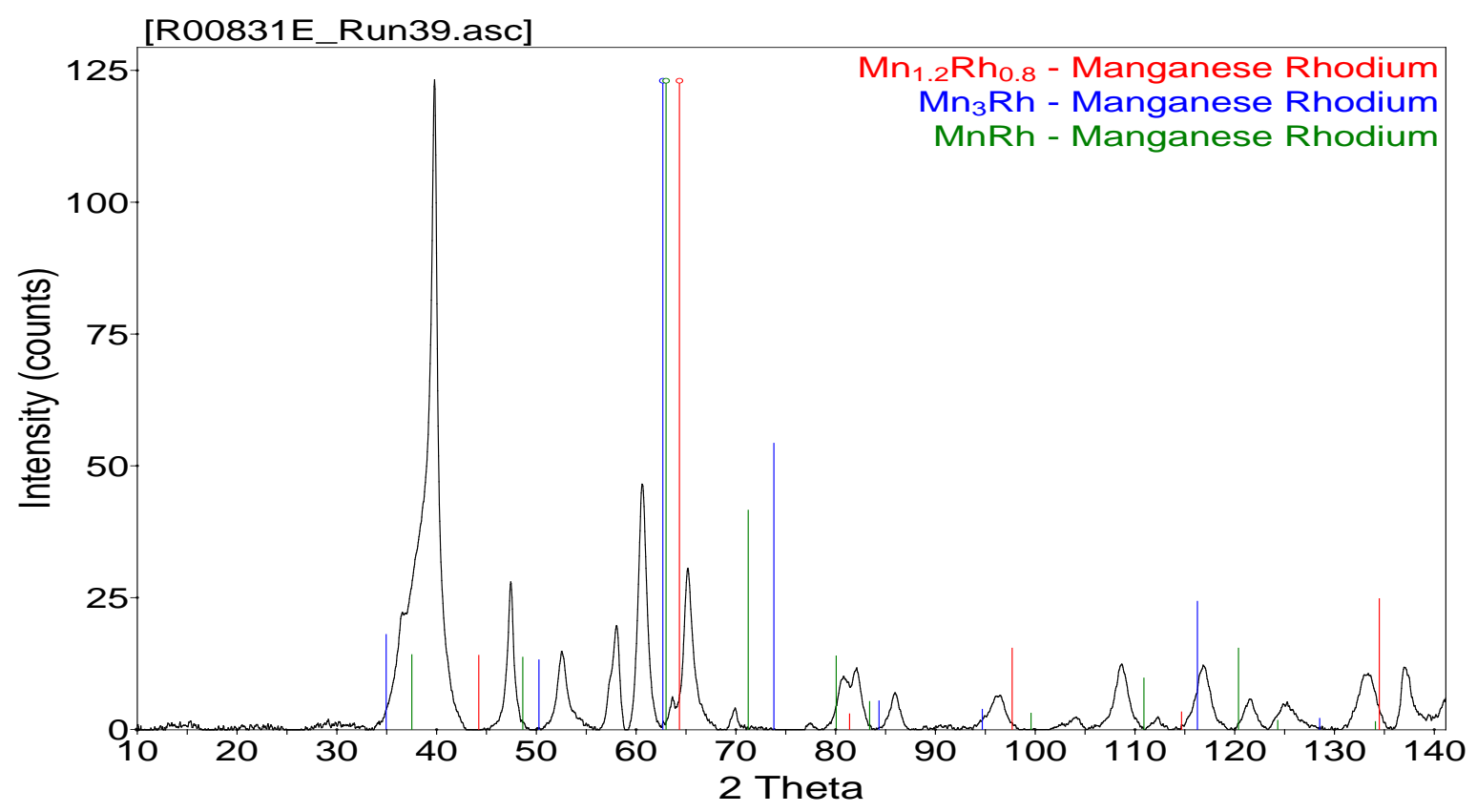

Figure 2.47. Micro-XRD Results on Spent Catalyst Sample Run 39, which contained 11.78 wt\% Rh, $3.58 \mathrm{wt} \% \mathrm{Mn}$, and $2.19 \mathrm{wt} \%$ Ir. The metals were simultaneously co-impregnated. Potential crystalline phases are shown by the color-coded lines. The XRD trace is identical to the trace in Figure 2.46. 


\subsection{X-Ray Photoelectron Spectroscopy}

XPS is a characterization technique that probes the energy state of electrons released by irradiation with an X-ray beam. The energy of the released electrons is characteristic of the atom from which they were released. Thus, qualitative information on the atoms present and the oxidation state of the atoms as well as quantitative elemental concentration information can be obtained from XPS measurements. Additionally, because the electrons released from the sample are relatively low energy, only electrons released at or very near the surface of the sample can escape the sample and be detected; electrons released deeper in the sample are deflected because of their low energy. Thus, XPS is a surface-sensitive technique that gives quantitative information on the atomic population of a sample at the surface. XPS also can yield information on the oxidation state of atomic species at the surface of the sample through shifts in the characteristic energy of the electrons released.

Because XPS is a surface characterization technique, care was taken to prevent exposure of the reduced and spent samples to air in order to minimize contamination of the catalyst surfaces. Reduced and spent samples were unloaded and transported in an inert atmosphere. The catalysts were received and stored inside a $\mathrm{N}_{2}$ purged recirculated glove box. Powdered catalyst samples were mounted for analysis inside a $\mathrm{N}_{2}$-recirculated glove box operated at $<1 \mathrm{ppm} \mathrm{O}_{2}$. The catalyst powder was pressed onto clean double-sided Nichiban tape supported by $1 \mathrm{~cm}$ x $3 \mathrm{~cm}$ flat Si wafers. The Si wafer pieces containing the catalysts were sealed into clean trace clean sample vials from VWR International for the short 1-min transfer into the glove bag attached to the XPS system. The Si wafers containing the catalysts were mounted for XPS inside a $\mathrm{N}_{2}$ purged glove bag from $\mathrm{I}^{2} \mathrm{R}$ Inc. attached to the XPS sample introduction chamber. The samples were exposed to the glove bag environment for only $\sim 1$ to 2 min during the transfer into the spectrometer. The glove bag was continually purged with $\mathrm{N}_{2}\left(<1 \mathrm{ppm}\right.$ oxygen) from $\mathrm{LN}_{2}$ evaporation. The oxygen concentration in the glove bag during the XPS sample mounting was measured at $<30 \mathrm{ppm}$ oxygen. The sample holder was then placed into the XPS vacuum introduction system and pumped to $<1 \times 10^{-6}$ Torr using a turbomolecular pumping system prior to introduction into the main ultrahigh vacuum system. The main vacuum system pressure is maintained at $<5 \times 10^{-9}$ Torr during analysis and pumped using a series of sputter ion pumps.

XPS measurements were performed using a Physical Electronics Quantum 2000 scanning electron spectroscopy for chemical analysis microprobe. This system uses a focused monochromatic Al Ka X-rays $(1486.7 \mathrm{eV})$ source and a spherical section analyzer. The instrument has a 16-element multichannel detector (32-element multichannel detector for the baseline catalysts). The x-ray beam used was a $100 \mathrm{~W}$, $100 \mu \mathrm{m}$ diameter beam that was rastered over a $1.3 \mathrm{~mm}$ by $0.1 \mathrm{~mm}$ rectangle on the sample for the baseline catalysts and $1.3 \mathrm{~mm}$ by $0.2 \mathrm{~mm}$ for the other samples analyzed. The x-ray beam is incident normal to the sample and the photoelectron detector was at $45^{\circ}$ off-normal. Wide scan data were collected using a pass energy of $117.4 \mathrm{eV}$. For the $\mathrm{Ag} \mathrm{d}_{5 / 2}$ line, these conditions produce a full width at half maximum of better than $1.6 \mathrm{eV}(0.91 \mathrm{eV}$ for the baseline analyses). The high energy resolution photoemission spectra were collected using a pass energy of $46.95 \mathrm{eV}$ (69.0 eV for the baseline catalysts). For the $\mathrm{Ag} \mathrm{d}_{5 / 2}$ line, these conditions produced a full width at half maximum of better than $0.98 \mathrm{eV}$. The binding energy scale is calibrated using the $\mathrm{Cu} 2 \mathrm{p}_{3 / 2}$ feature at $932.62 \pm 0.05 \mathrm{eV}$ and $\mathrm{Au} 4 \mathrm{f}$ at $83.96 \pm 0.05$ $\mathrm{eV}$ for known standards. The samples experienced variable degrees of charging. Low energy electrons at $\sim 1 \mathrm{eV}, 20 \mu \mathrm{A}$ and low energy $\mathrm{Ar}^{+}$ions were used to minimize this charging. 
The most significant source of possible error in XPS measurements is in the charge reference chosen for a given spectrum. The electron beam applied to the sample can cause the sample to charge through a build-up of electrons, thus changing the energy of the ejected electrons. All of the electrons within a spectrum will be shifted equally, but choosing the best reference is crucial to accurately compare spectra consisting of different materials (e.g., Rh on silica versus Rh on MWCNTs). For the MWCNTs, the samples were charge referenced to the $\mathrm{C} 1 \mathrm{~s}$ electron line at $284.4 \mathrm{eV}$ based on XPS measurements of the MWCNT supports without metal loading. The charge reference of $284.4 \mathrm{eV}$ is consistent within the effective $\pm 0.1 \mathrm{eV}$ resolution of the instrument with the $284.5 \mathrm{eV}$ reported for MWCNTs by Okpalugo et al. ${ }^{9}$

Selection of an accurate charge reference for the silica-supported samples was more challenging because of the insulating nature of the support in addition to a wide variety of binding energies reported for different polymorphs of silica. Davisil 645 is a silica gel. The Handbook of X-Ray Photoelectron Spectroscopy reports that the binding energy of the $\mathrm{Si} 3 / 2 \mathrm{p}$ line of quartz is $103.7 \mathrm{eV}$ and silica gel is $103.4 \mathrm{eV} .{ }^{10}$ The National Institute of Standards and Technology database also was reviewed, and the numerous references given for silica gel had an average of $103.6 \mathrm{eV} .^{11}$ Because of the wide range in values given for even silica gel, we decided that the spectra should be charge referenced at $103.5 \mathrm{eV}$, which is the mean of the references for silica gel from the Handbook of X-Ray Photoelectron Spectroscopy and values from the National Institute of Standards and Technology database. While every effort was taken to appropriately charge reference the silica-supported samples, the relative uncertainty in the selection causes the effective resolution of the spectra be considered to be $\pm 0.2 \mathrm{eV}$ (which covers the low references of silica gel at $103.3 \mathrm{eV}$ up to quartz at $103.7 \mathrm{eV}$ ) when comparing narrow scan spectra of silica and MWCNT samples instead of the typical $\pm 0.1 \mathrm{eV}$ effective resolution.

\subsubsection{Baseline Catalysts}

The concentrations of elements at the surface of the three baseline catalysts after each of the four distinct catalyst lifecycle points are listed in Table 2.29. The ratio of Mn:Rh at the surface of the catalysts also was tracked to determine enrichment of metals at the surface. The table contains all of the quantification data for each of the samples for ease of comparison. The individual high-energy resolution spectra for each metal will be discussed individually below to elucidate the binding energy and the implications on the oxidation state and environment of the metals. 
Table 2.29. Quantification (atomic percent) of Surface Elements on the Baseline Catalyst Samples as Determined by XPS Measurements

\begin{tabular}{|cccccccc|}
\hline Sample & Rh & Mn & Ir & C & Si & O & $\begin{array}{c}\text { Mn:Rh } \\
\text { Ratio }\end{array}$ \\
\hline \multicolumn{7}{c}{ 1x Rh-Mn-Ir on Davisil 645 Silica } \\
\hline Fresh & 0.82 & 0.06 & 0.02 & 2.6 & 25.2 & 71.3 & 0.1 \\
60074-53-A & 0.87 & 0.08 & 0.02 & 1.4 & 26.1 & 71.5 & 0.1 \\
Reduced & 0.93 & 0.16 & 0.03 & 6.8 & 24.7 & 67.4 & 0.2 \\
60074-53-A-R & 0.76 & 0.20 & 0.03 & 6.5 & 26.0 & 66.6 & 0.3 \\
Spent_LT & 0.33 & 0.09 & 0.02 & 7.1 & 25.3 & 67.2 & 0.3 \\
Run 84 & 0.30 & 0.09 & 0.02 & 11.5 & 24.1 & 64.0 & 0.3 \\
Spent_HT & 1.53 & 0.12 & 0.06 & 10.1 & 24.9 & 63.3 & 0.1 \\
Run 86 & 1.32 & 0.07 & 0.05 & 15.7 & 23.1 & 59.8 & 0.1 \\
\hline 2.11x Rh-Mn on Hyperion Fibril Catalyst Support CS-02C-063-XD Lot 384-82 MWCNTs & \\
\hline Fresh & 2.26 & 0.88 & & 79.7 & 0.0 & 17.1 & 0.4 \\
60074-70-C & 2.12 & 0.85 & & 80.7 & 0.0 & 16.3 & 0.4 \\
Reduced & 2.39 & 1.70 & & 90.4 & 0.1 & 5.5 & 0.7 \\
60074-70-C-R & 2.23 & 1.38 & & 91.1 & 0.1 & 5.2 & 0.6 \\
Spent_LT & 1.54 & 1.20 & & 90.3 & 0.4 & 6.6 & 0.8 \\
Run 80 & 1.30 & 0.94 & & 91.6 & 0.3 & 5.9 & 0.7 \\
Spent_HT & 2.07 & 0.98 & & 87.9 & 1.3 & 7.8 & 0.5 \\
Run 82 & 1.69 & 0.96 & & 89.6 & 0.9 & 6.9 & 0.6 \\
\hline 2.11x Rh-Mn-Ir on Hyperion Fibril Catalyst Support CS-02C-063-XD Lot 384-82 MWCNTs & \\
\hline Fresh & 2.41 & 1.13 & 0.10 & 82.2 & 0.1 & 14.1 & 0.5 \\
60074-74-C & 2.35 & 0.90 & 0.11 & 82.4 & 0.1 & 14.1 & 0.4 \\
Reduced & 1.90 & 1.20 & 0.08 & 93.1 & 0.2 & 3.5 & 0.6 \\
60074-74-C-R & 1.80 & 1.10 & 0.08 & 94.0 & 0.0 & 3.0 & 0.6 \\
Spent_LT & 1.26 & 1.65 & 0.04 & 86.1 & 1.8 & 9.2 & 1.3 \\
Run 89 & 1.43 & 1.39 & 0.05 & 90.4 & 0.6 & 6.1 & 1.0 \\
Spent_HT & 1.44 & 1.15 & 0.07 & 91.1 & 0.5 & 5.7 & 0.8 \\
Run 88 & 1.46 & 1.71 & 0.08 & 90.3 & 0.3 & 6.2 & 1.2 \\
\hline
\end{tabular}

\subsubsection{Davisil 645 Silica-Supported Rh-Mn-Ir Baseline Catalyst}

Figure 2.48 illustrates the high energy resolution of the $\mathrm{Rh} 3 \mathrm{~d}$ region of the silica-supported catalyst in Table 2.4. The spectra for two areas on each sample are presented. $\mathrm{Rh}_{2} \mathrm{O}_{3}$ has a reported binding energy of $309.2 \mathrm{eV}$, which agrees reasonably well with the observed $309.5 \mathrm{eV}$ peak of the fresh sample in Figure 2.48 given the $\pm 0.2 \mathrm{eV}$ effective resolution of the silica-supported samples. ${ }^{7}$ The $\mathrm{Rh}_{5 / 2}$ peak shifted to the right to a value of $306.8 \mathrm{eV}$ indicating that the $\mathrm{Rh}$ in the reduced and spent samples was reduced. However, the reported value for reduced $\mathrm{Rh}$ is $307.4 \mathrm{eV}{ }^{7}$ Furthermore, testing of a piece of sputtered $\mathrm{Rh}$ foil in the XPS collected under similar conditions as the data collected in Figure 2.48 gave a binding energy of $307.2 \mathrm{eV}$. Therefore, while the Rh was reduced, it had a lower binding energy than what would be expected for metallic Rh. One possible reason may be differential charging. When large 
conductive crystals become electronically insulated due to the insulating nature of the catalyst support, a negative shift in the binding energy below the expected binding energy expected for a metal can be observed. ${ }^{12}$ Indeed, Rh on alumina has been reported to exhibit a binding energy of $306.8 \mathrm{eV} .^{13}$ However, it should be mentioned that the Rh in that study was on the order of $130 \mathrm{~nm}$, which is much larger than the maximum metal crystal sizes of $\sim 20 \mathrm{~nm}$ observed in the TEM analysis of the catalysts used in Runs 84 and 86 (see, for example, Figure 2.70. If differential charging is indeed the cause for the unexpectedly low binding energy of Rh it would suggest the active metals and the support are electronically decoupled.

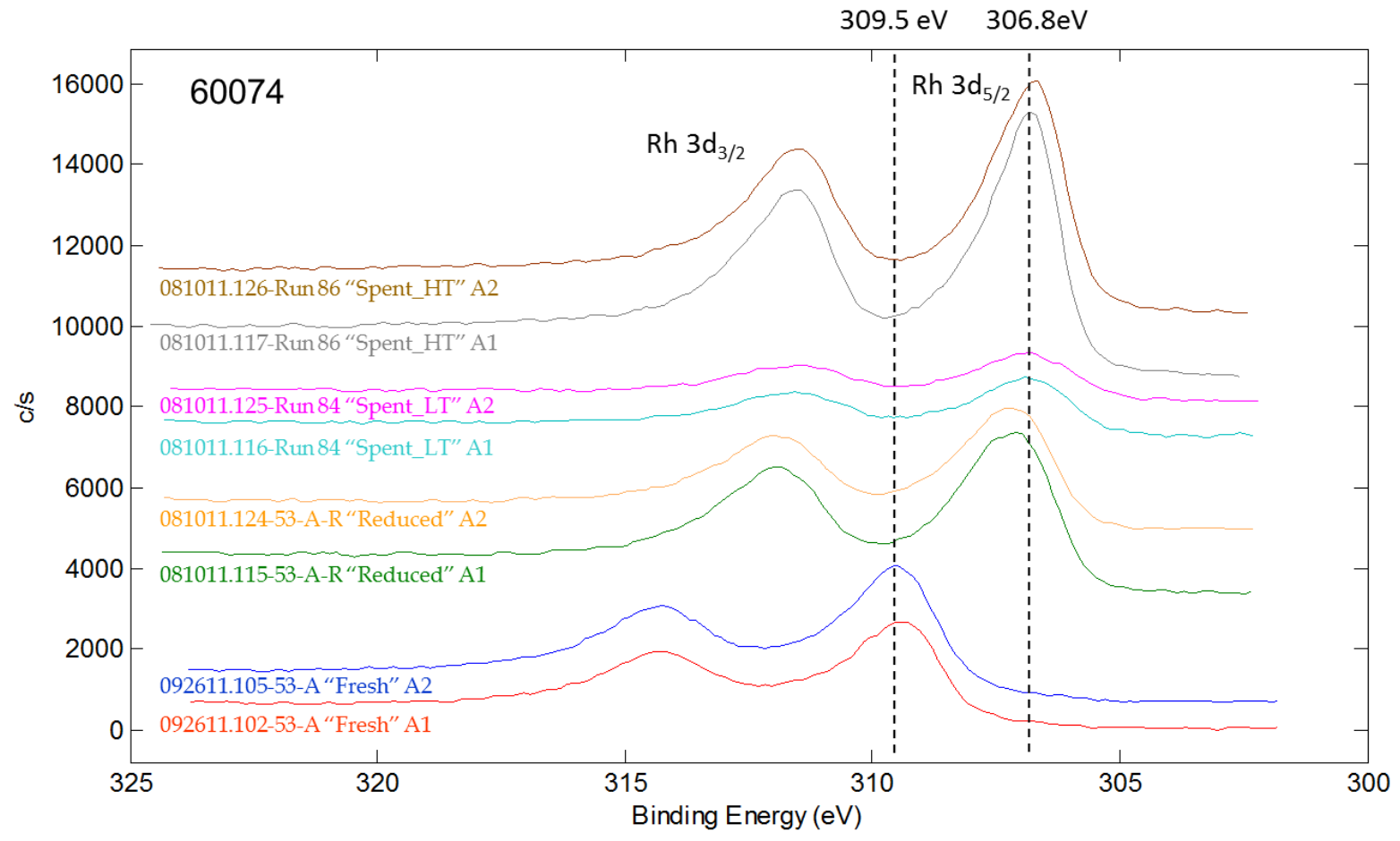

Figure 2.48. High Energy Resolution XPS Spectra of the Rh 3d Region of the 1x Rh-Mn-Ir on Davisil 645 Silica baseline catalyst. The spectra are charge referenced using the Si $2 p$ line at $103.5 \mathrm{eV}$.

The observance of Rh with an unexpectedly low binding energy at $306.8 \mathrm{eV}$ appears to be unique to the spent catalysts (Run 84 and Run 86) and not the freshly reduced catalyst. The freshly reduced catalyst exhibits a peak with higher binding energy nearer the expected value for $\mathrm{Rh}^{(0)}$ at $307.4 \mathrm{eV}$. The shift in binding energy from freshly reduced to spent catalyst may be an indication of sintering of the Rh from small but electronically coupled particles to larger Rh particles that are electronically isolated from the silica support.

An alternative explanation for the unexpectedly low binding energy of Rh may be that the Ir is altering the state of the Rh after use as a mixed alcohols synthesis catalyst. Mei et al. ${ }^{7}$ observed the expected value for $\mathrm{Rh}^{(0)}(307.4 \mathrm{eV})$ on a catalyst similar to 60074-53-A, except the catalyst analyzed did not contain Ir. 


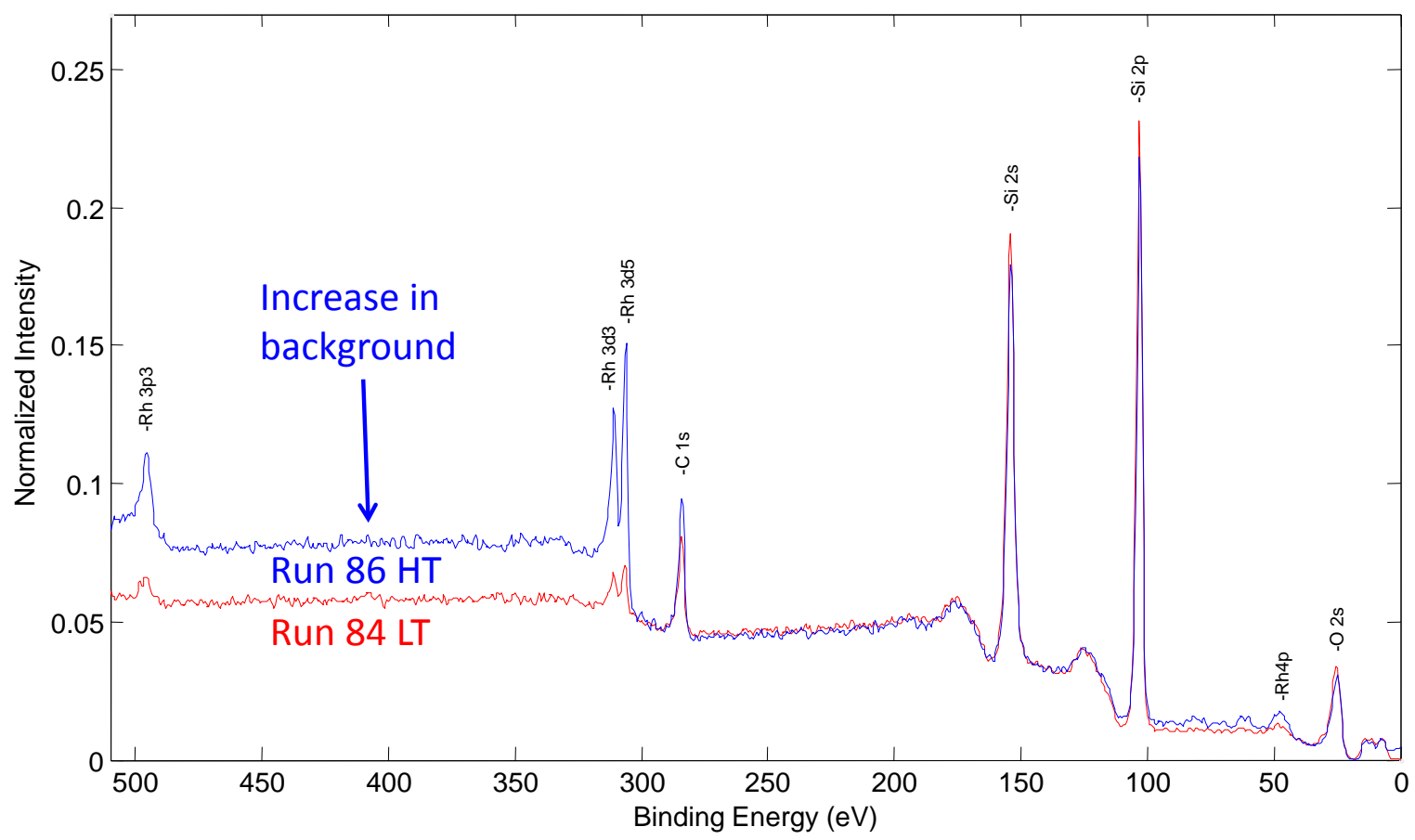

Figure 2.49. High Energy-Resolution XPS from 0 to 510 eV Binding Energy of the Spent Davisil 645Supported Rh-Mn-Ir Catalysts (Run 84 and 86 in Table 2.4)

The difference in operating temperature between Run $84\left(275^{\circ} \mathrm{C}\right)$ and Run $86\left(300^{\circ} \mathrm{C}\right)$ resulted in an apparent increase in the concentration of $\mathrm{Rh}$ at the surface of the silica-supported baseline catalyst. The concentration of Rh jumped from $\sim 0.3$ at\% to 1.3 to 1.5 at\% at the higher temperature (Table 2.29).

Further confirmation of the higher Rh concentration at the surface in the Run 86 sample is apparent in the shifted background above $\sim 320 \mathrm{eV}$ in Figure 2.50 .

The Mn concentration at the surface of the silica-supported baseline samples was low as illustrated in Table 2.29 and Figure 2.50. Data in Table 2.29 may suggest there is less $\mathrm{Mn}$ at the surface after use for mixed alcohol synthesis compared with the reduced but unused catalyst (60074-53-A-R versus Run 84 and/or Run 86). However, with the low Mn concentration values, strong conclusions are difficult to draw. The lack of Mn at the surface may allude to the possibility that Mn is drawn into the silica support under reaction conditions. Indeed, manganese silicates do exist as stable phases, ${ }^{14}$ although it is unclear if the reaction temperature range of 275 to $300^{\circ} \mathrm{C}$ is sufficient to produce such a phase from these XPS results. 


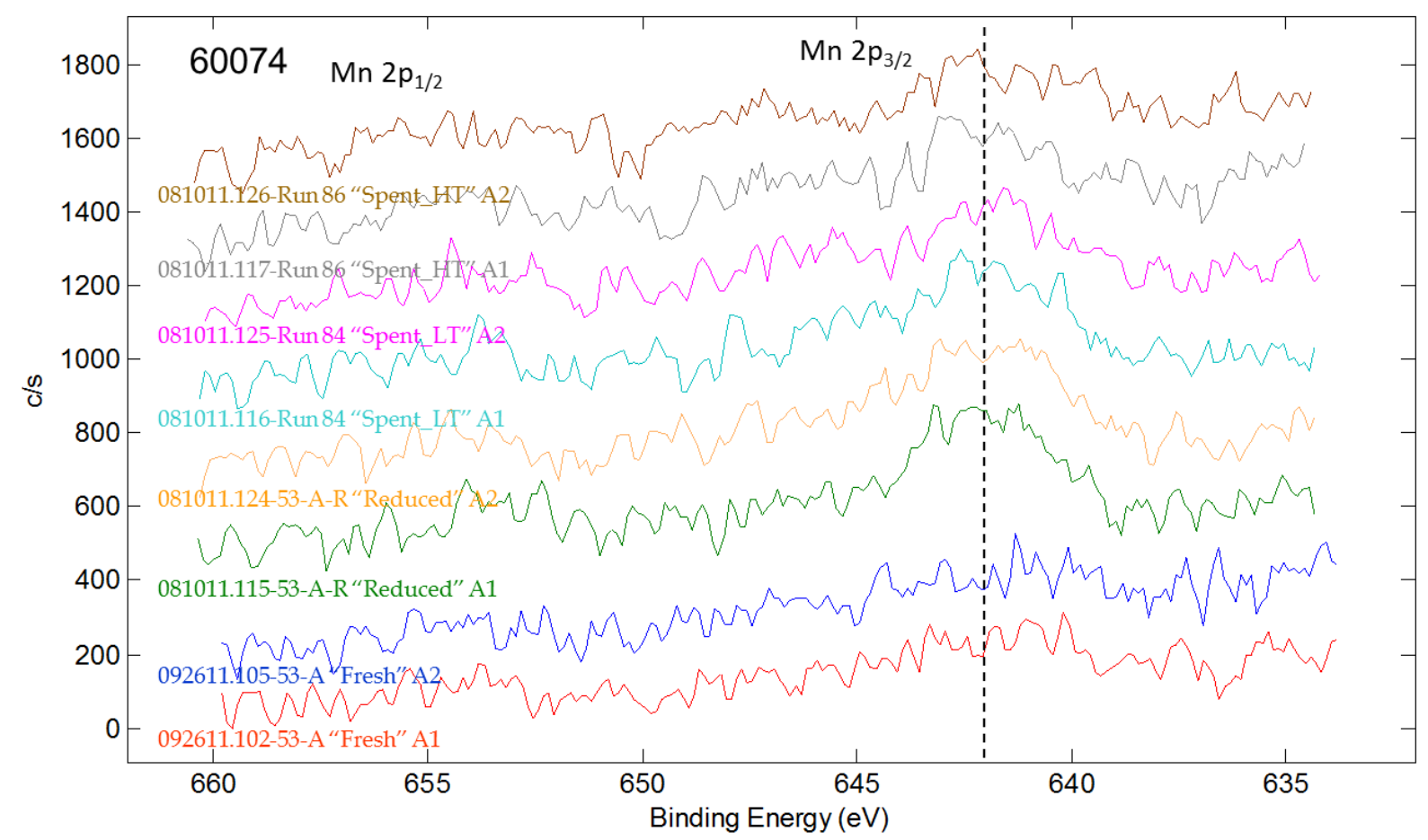

Figure 2.50. High Energy Resolution XPS spectra of the Mn 2p Region of the 1x Rh-Mn-Ir on Davisil 645 Silica Baseline Catalyst. The spectra are charge referenced using the Si 2p line at $103.5 \mathrm{eV}$.

The Ir concentration at the surface of the silica-supported baseline catalysts increased upon reduction of the fresh catalyst (60074-53-A versus 60074-53-A-R). The Ir concentration is lower at the surface with only 0.02 at\% detected in the low temperature sample (Run 84). However, similar to Rh, there is a significant increase in the concentration of Ir at the surface when the Run 84 and Run 86 (tested to $300^{\circ} \mathrm{C}$ ) samples are compared with samples tested at lower temperatures.

The Ir $4 f_{7 / 2}$ electron peak in the high-resolution energy scan (Figure 2.51) clearly shifted to a lower binding energy upon reduction, indicating a reduced state as compared with the fresh catalyst. The peaks of the binding energy measurements for the $\mathrm{Ir} 4 \mathrm{f}_{7 / 2}$ electron in the reduced and spent (Run 84 and Run 86) catalysts illustrate a binding energy at $60.3 \mathrm{eV}$. Similar to the Rh binding energy mentioned above, the $60.3 \mathrm{eV}$ for the Ir electron is lower than what would be expected for metallic Ir. A piece of metallic Ir foil measured with the XPS instrument under identical parameters as the sample collected revealed metallic Ir had a measured binding energy of $60.9 \mathrm{eV}$. The lower-than-expected value of the Ir binding energy may again be due to differential charging of large metallic particles insulated from the grounded XPS sample stage by the silica support. If in fact the Rh and Ir are differentially charging, large metallic clusters of $\mathrm{Rh}$ and Ir may be present on the silica support. 


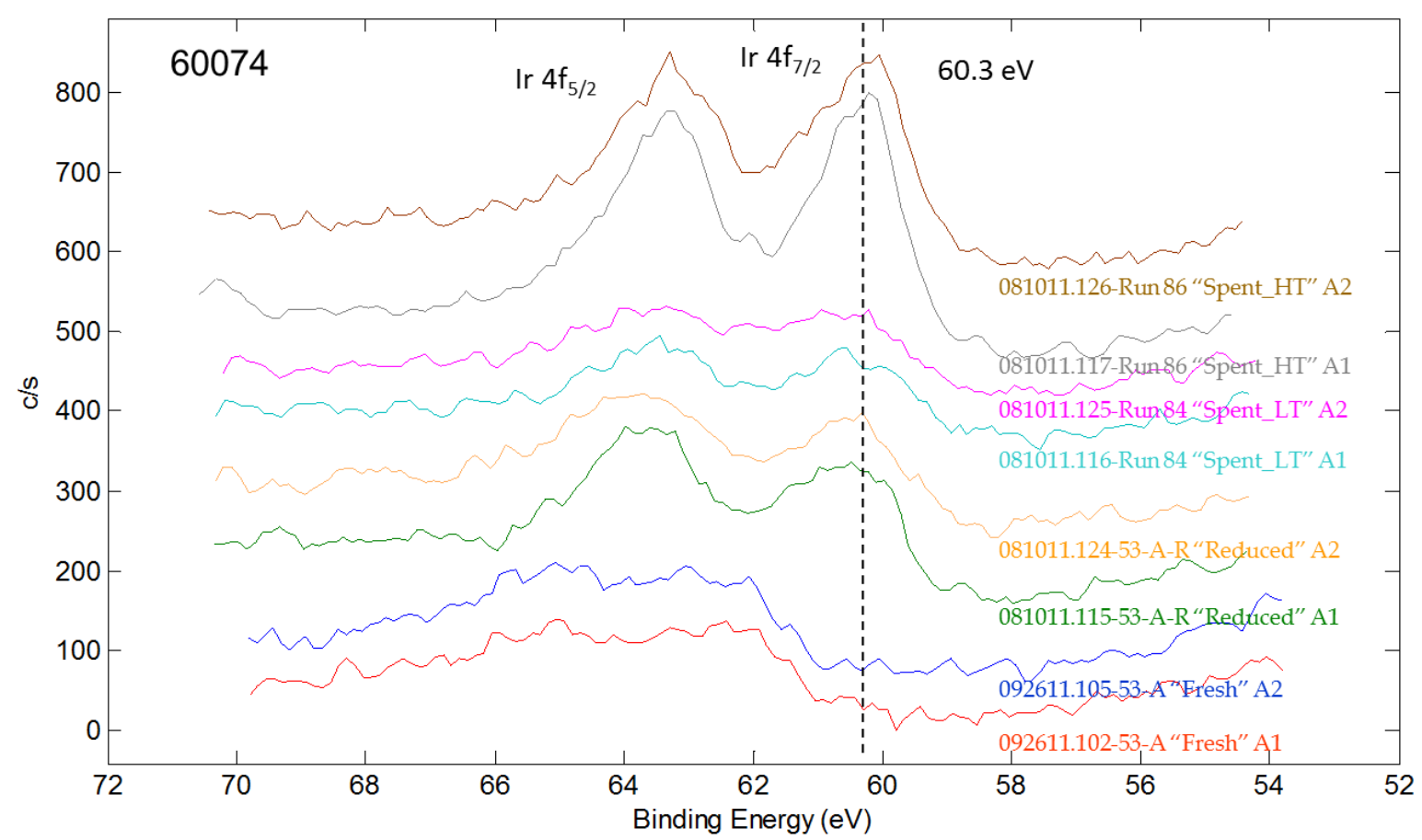

Figure 2.51. High-Energy Resolution XPS Spectra of the Ir 4f region of the $1 x$ Rh-Mn-Ir on the Davisil 645 Silica Baseline Catalyst. The spectra are charge referenced using the Si $2 p$ line at $103.5 \mathrm{eV}$.

The oxygen (Figure D.1), silicon (Figure D.2), and carbon (Figure D.3) high-energy resolution scans of the silica-supported baseline catalysts are shown in Appendix D. There is not a large variation in the type of oxygen present in the catalyst as illustrated by the relative similarity and peak location of the $\mathrm{O} 1 \mathrm{~s}$ electron measurements. The same statement can be generally made about the type of carbon on the catalyst except that the fresh (unreduced catalyst) does not contain much carbon. The Si high-energy resolution scans are aligned at $103.5 \mathrm{eV}$ as expected because each of the spectra are shifted (i.e., charge referenced) to $103.5 \mathrm{eV}$ as discussed above.

\subsubsection{Hyperion Fibril MWCNT-Supported Rh-Mn Baseline Catalyst}

The concentration of $\mathrm{Rh}$ and $\mathrm{Mn}$ (as well as $\mathrm{C}, \mathrm{O}$, and $\mathrm{Si}$ ) on the MWCNT support Rh-Mn baseline catalyst (60074-70-C, 60074-70-C-R, Run 80 and Run 82) also are presented in Table 2.29. The Rh present at the surface does not appear to change significantly between the fresh and reduced catalyst (60074-70-C versus 60074-70-C-R). The Rh concentration at the surface after being used for mixed alcohol synthesis does appear to decrease to 1.3 to 1.5 at\%, although the concentration increases again after use at the higher temperature. Figure 2.52 shows that the Rh present on the catalyst after reduction as well as on the spent catalysts is in a reduced state. The binding energy of $307.3 \mathrm{eV}$ is within the effective instrument resolution $( \pm 0.1 \mathrm{eV})$ of both the value reported in the literature $(307.4 \mathrm{eV})^{7}$ and the reference measurement performed on a sample of $\mathrm{Rh}$ foil under identical measurement parameters (307.2 eV). Furthermore, the binding energy is the expected value for metallic Rh on the MWCNTsupported sample, indicating that, not only is Rh in its metallic state, it is also in good electrical contact with the grounded XPS sample stage. Therefore, the MWCNT support is not acting as an insulator as was hypothesized with the silica support. 


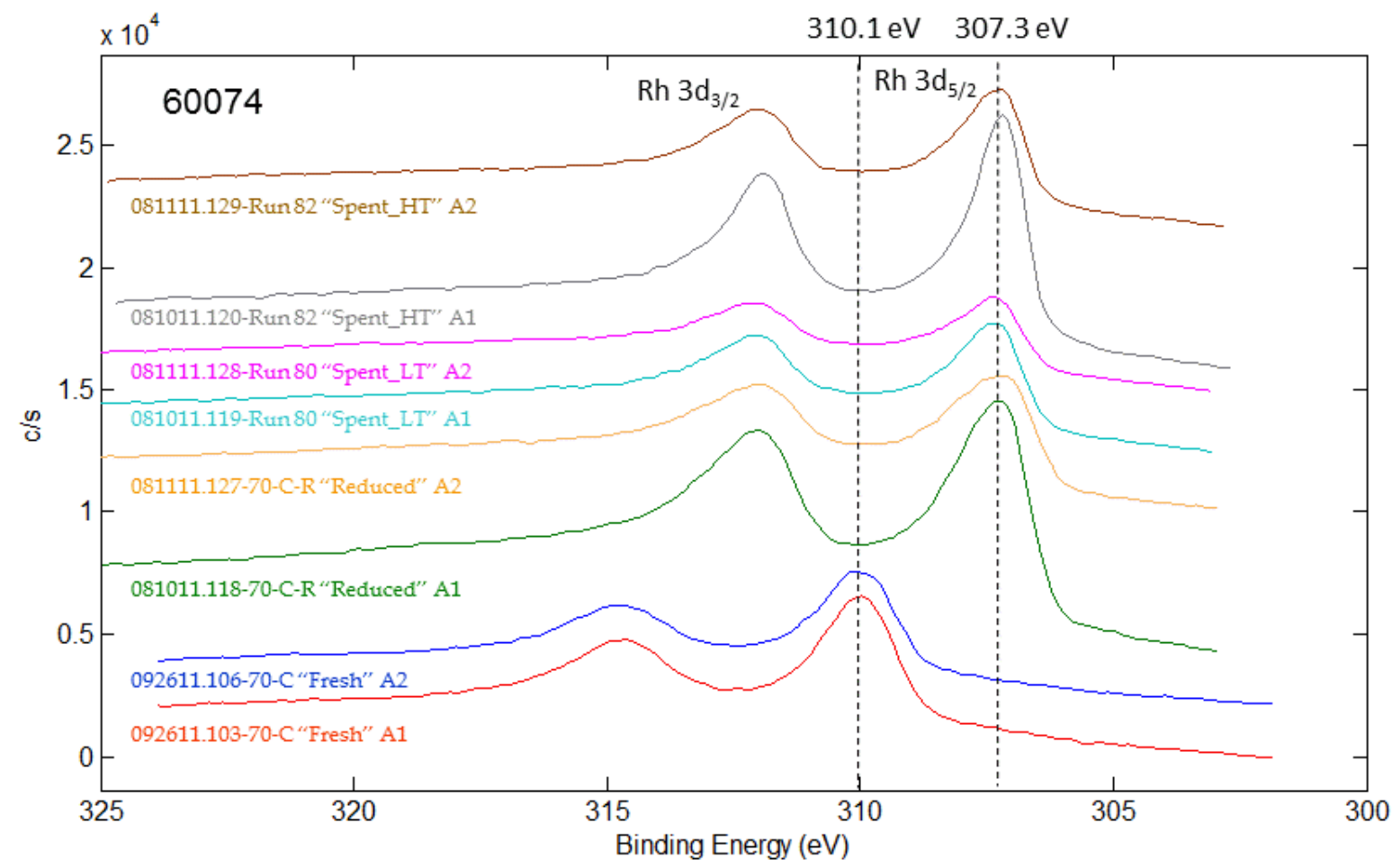

Figure 2.52. High-Energy Resolution XPS Spectra of the Rh 3d Region of the 2.11x Rh-Mn on Hyperion 384-82 MWCNT Baseline Catalyst. The spectra are charge referenced using the $\mathrm{C} 1 \mathrm{~s}$ line at $284.4 \mathrm{eV}$.

In contrast to Rh, the Mn at the surface does appear to increase by a factor of approximately two when the fresh catalyst is reduced. The increase in Mn concentration at the surface post-reduction may be explained by the removal of a large amount of oxygen (from 16.3-17.1 at\% to 5.2-5.5 at\% after reduction). The removal of oxygen may be consistent with reduction of the Mn. However, the highenergy resolution results in the Mn region are ambiguous as to the oxidation state of the Mn (Figure 2.53). The ambiguity of the Mn oxidation state is due to the similarity in binding energies and wide range of binding energy of $\mathrm{Mn}^{(0)}$ and $\mathrm{MnO}$. Some of the Mn may be reduced but the binding energy of the electrons liberated from $\mathrm{Mn}$ are very similar for $\mathrm{Mn}$ and $\mathrm{MnO}(\mathrm{Mn}=638.8$ to $641.0 \mathrm{eV}$ and $\mathrm{MnO} 640.3$ to $642.5 \mathrm{eV}$ ). The broad Mn high energy resolution peak is reasonably well aligned at $641.7 \mathrm{eV}$, although the spent catalysts (especially the Run 80 LT spent catalyst) appear to be shifted to slightly higher binding energy, indicating a slightly higher oxidation state.

$\mathrm{MnCO}_{3}$ was also observed during XRD analysis of the spent (Run 80 and Run 82) MWCNTsupported Rh-Mh samples (Figure 2.34 and Figure 2.35). To investigate if the Mn at the surface on the spent catalysts was present as $\mathrm{MnCO}$, a reference of $\mathrm{MnCO}_{3}$ analyzed with the XPS instrument using parameters identical to those used in the measurements of the spent Rh-Mn catalyst samples revealed that the $\mathrm{Mn}$ binding energy $\mathrm{MnCO}_{3}$ is $641.4 \mathrm{eV}$. Because the main contribution of $\mathrm{Mn}$ at the surface in the spent catalyst samples is close to (or slightly greater than) $641.7 \mathrm{eV}$, it appears that the majority of the Mn at the surface is not present as $\mathrm{MnCO}_{3}$. However, XPS is a surface technique while XRD is a bulk technique. Thus, a large fraction of the Mn present may indeed be $\mathrm{MnCO}_{3}$ while only the $\mathrm{Mn}$ at the surface may be a different more highly oxidized species of Mn due to the slight shift in binding energy to higher values. 


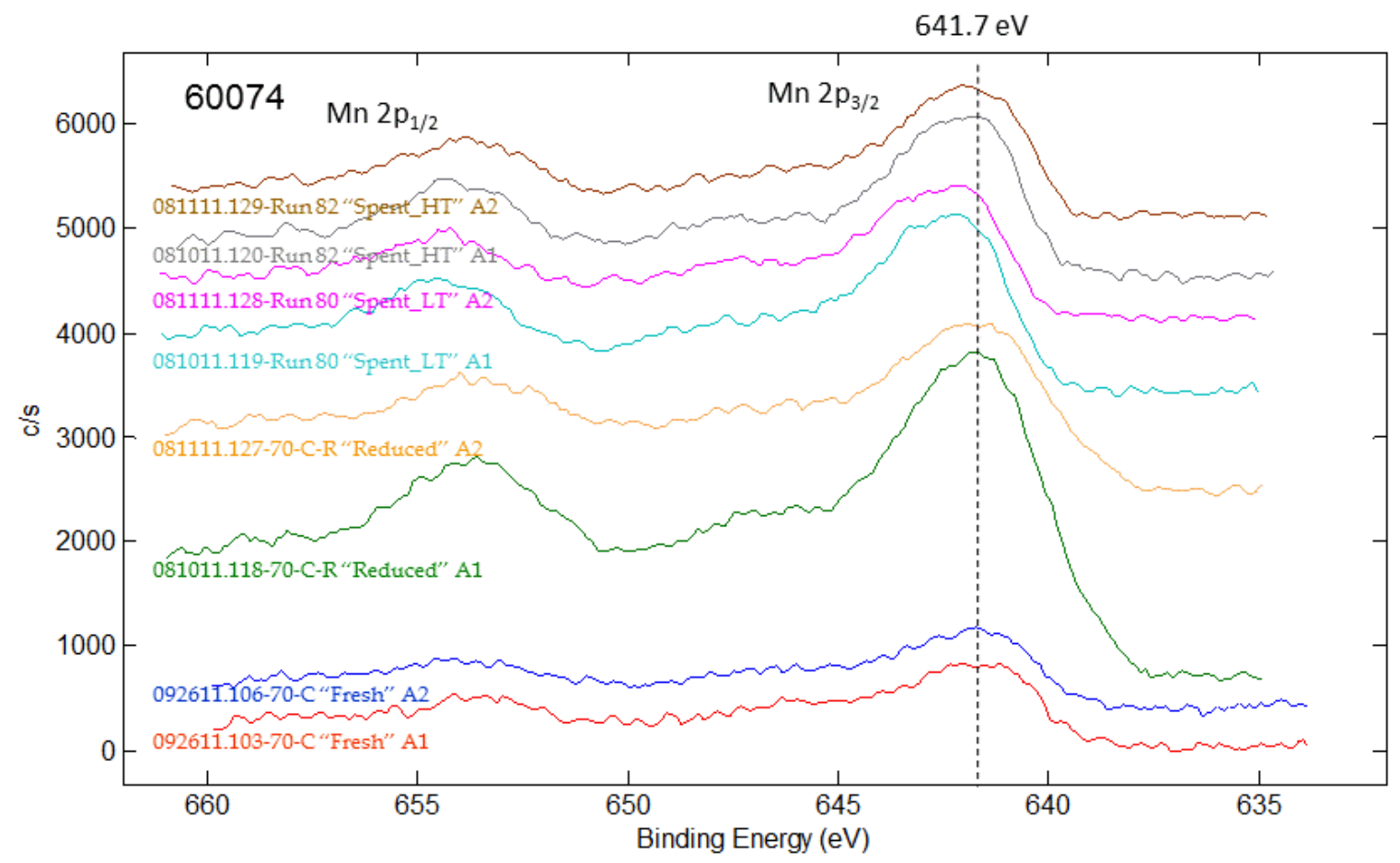

Figure 2.53. High Energy-Resolution XPS Spectra of the Mn 2p region of the 2.11x Rh-Mn on the Hyperion 384-82 MWCNT Baseline Catalyst. The spectra are charge referenced using the $\mathrm{C} 1 \mathrm{~s}$ line at $284.4 \mathrm{eV}$.

The oxygen (Figure D.4), carbon (Figure D.5), and silicon (Figure D.6) high-energy resolution spectra for the MWCNT-supported Rh-Mn catalysts are presented in Appendix D. The oxygen in the fresh 60074-74-C sample is likely present to a large extent as nitrates associated with the uncalcined metals (which are loaded on the catalyst as nitrates). Upon reduction, much of the oxygen is removed. The samples from Runs 80 and 82 exhibit a greater concentration of oxygen, which may be due to the presence of oxygenated-metallic intermediates on the surface that are necessary for the formation of mixed oxygenates. The oxygen in the spent samples is shifted slightly to the left (higher binding energy) when compared with the oxygen present in the fresh catalyst.

The C in Figure D.5 illustrates the major peaks aligned at $284.4 \mathrm{eV}$ because of the charge referencing of the sample to this value. Investigation of the $\mathrm{MnCO}_{3}$ reference sample indicated that if carbonate were present, it would exhibit a C 1s peak at $289.3 \mathrm{eV}$. Figure D.5 illustrates that while there is no sharply defined peak in this region, there may be a broad peak centered at $\sim 291 \mathrm{eV}$. Thus, no well-defined carbonate is observed at the surface of the catalyst. $\mathrm{MnCO}_{3}$ could be present (based on XRD analysis) but may be located under other species (e.g., $\mathrm{C}$ or Rh) making it invisible to XPS.

Si should only be a contaminant in the MWCNT-supported catalysts. It is likely the Si in observed in Run 82 in Figure D.6 is contamination from the quartz wool used to support the catalyst bed in the mixed alcohol synthesis fixed-bed reactor. The other samples did not exhibit Si as would be expected. 


\subsubsection{Hyperion Fibril MWCNT-Supported Rh-Mn-Ir Baseline Catalyst}

The high-energy resolution region of Rh on the MWCNT-supported Rh-Mn-Ir baseline catalyst is illustrated in Figure 2.54. Similar to the MWCNT-supported Rh-Mn catalyst, the Rh in the reduced (60074-74-C-R) and spent catalysts (Run 89 and Run 88) has the binding energy of metallic Rh (307.4 eV, which is the expected value found in the literature). ${ }^{7}$ Generally, the concentration of Rh at the surface as illustrated in Table 2.29 also follows similar trends with the Rh concentration at the surface, decreasing from the time the catalyst is reduced to after use for mixed alcohol synthesis.

In contrast to the Rh-Mn catalyst, the concentration of the Mn at the surface on the Rh-Mn-Ir catalyst is slightly greater. The biggest difference in the concentration of metals at the surface can be observed in the $\mathrm{Mh}: \mathrm{Rh}$ ratio of the two types of catalyst (Rh-Mn versus Rh-Mn-Ir). While the $\mathrm{Mn}: \mathrm{Rh}$ ratio is consistently less than 1.0, which indicates a Rh-rich surface in the Rh-Mn system, the ratio is 1.0 to 1.3 for the lower temperature-tested Rh-Mn-Ir catalyst and 0.8 to 1.2 for the Rh-Mn-Ir catalyst tested at higher temperature. The diminished $\mathrm{Mn}$ :Rh ratio may be a hint of a deactivation mode. If $\mathrm{Mn}$ is indeed needed for carbon coupling, less $\mathrm{Mn}$ at the surface may be detrimental to activity. In the same vein, the presence of Ir appears to cause higher concentrations of Mn at the surface, which may be a reason for an increase in the STY of $\mathrm{C}_{2}+$ oxygenates when Ir is present. This observation fits well with recent computational results that suggested the presence of Ir in Rh-Mn-Ir clusters forces Mn to the outside of a metallic cluster. ${ }^{8}$

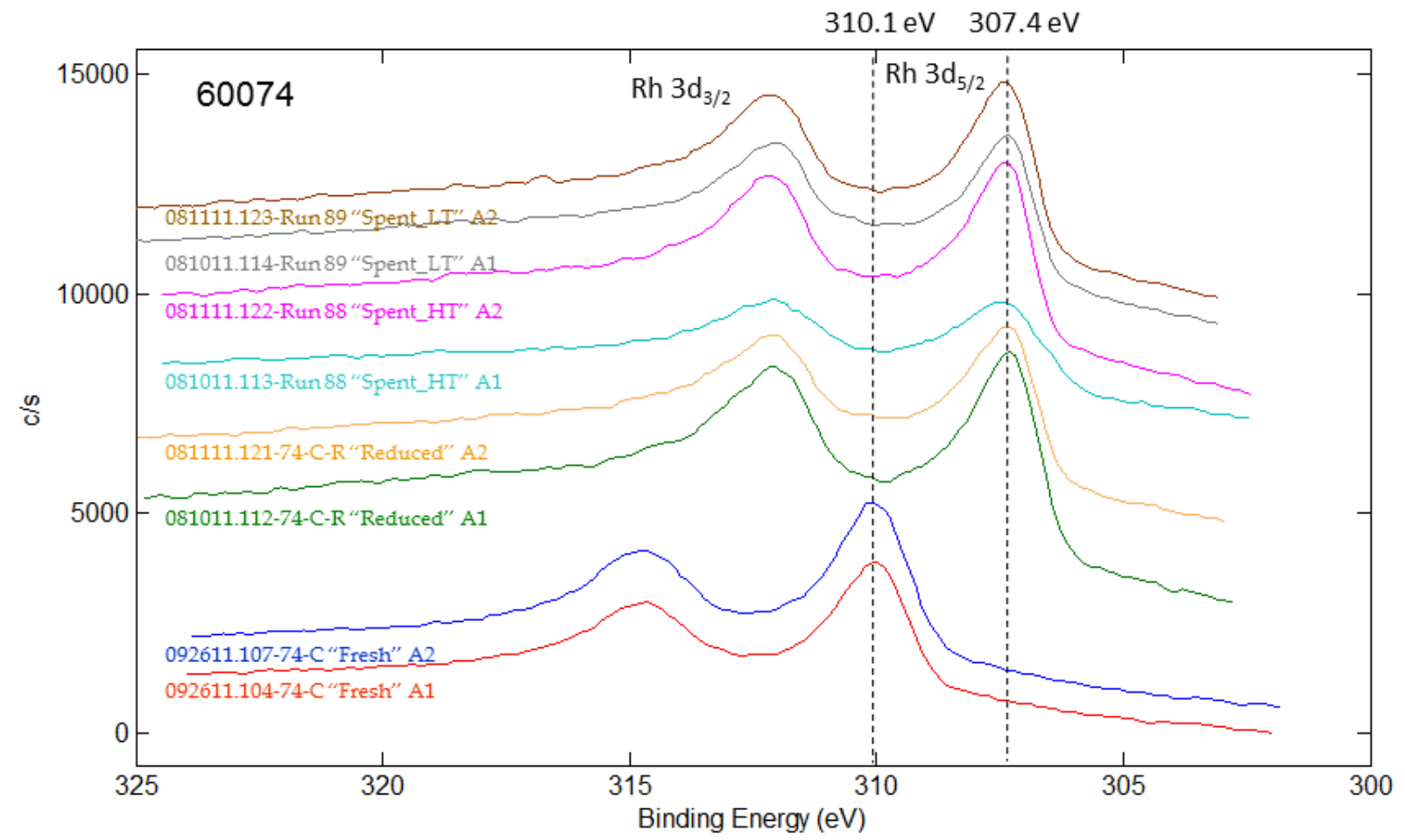

Figure 2.54. High-Energy Resolution XPS Spectra of the Rh 3d Region of the 2.11x Rh-Mn-Ir on the Hyperion 384-82 MWCNT Baseline Catalyst. The spectra are charge referenced using the $\mathrm{C} 1 \mathrm{~s}$ line at $284.4 \mathrm{eV}$. 
In addition to the higher ratio of Mn:Rh at the surface of the Rh-Mn-Ir catalyst supported on MWCNTs, the Mn also appears to be in a more oxidized state compared to its Rh-Mn counterpart. For example, in

Figure 2.55, the Run 89 low-temperature spent catalyst peaks at $642.3 \mathrm{eV}$, which is a higher binding energy (more oxidized) when compared with its counterpart (Run 80) in Figure 2.53 (641.4 eV). These values are similar for the reduced catalysts in each case. The samples subjected to higher temperature in the fixed-bed reactor appear to be nearer each other at around $641.7 \mathrm{eV}$ each. The implications on the differences in the oxidation state on the activity of the catalysts are currently unclear but such a significant difference $(\sim 1.0 \mathrm{eV})$ between the low temperature samples when Ir is present may lead to greater understanding of the mechanism in future experimental and computational work.

The high-energy resolution spectra in the range of Ir for the MWCNT-supported Rh-Mn-Ir catalyst are shown in Figure 2.56. As with Rh on the MWCNT samples, the binding energy observed for Ir is that expected for metallic Ir $(60.9 \mathrm{eV})$ based on experiments on a piece of Ir foil in the XPS instrument under identical sample collection parameters. The fact that the Rh and Ir both exhibit binding energies for the expected metals indicates that the majority of the metals at the surface of the catalyst are reduced to the 0 valence state. In contrast with the silica-supported catalyst supports, the fact that Rh and Ir do exhibit the values expected for the metallic state indicates that the metals are in good electrical contact with the ground XPS stage and that the MWCNT support is not acting as an insulator as was hypothesized with the silica support.

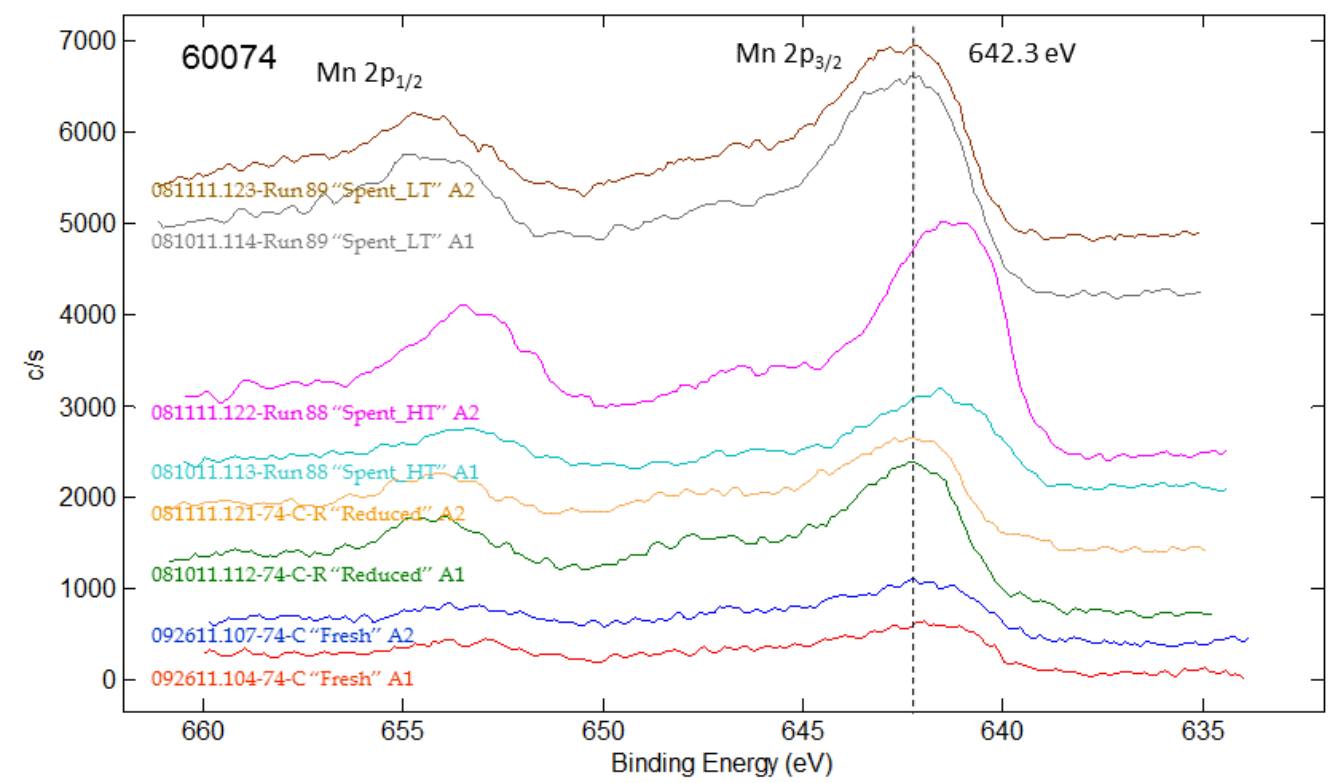

Figure 2.55. High Energy-Resolution XPS Spectra of the Mn 2p Region of the 2.11x Rh-Mn-Ir on the Hyperion 384-82 MWCNT Baseline Catalyst. The spectra are charge referenced using the $\mathrm{C} 1 \mathrm{~s}$ line at $284.4 \mathrm{eV}$. 


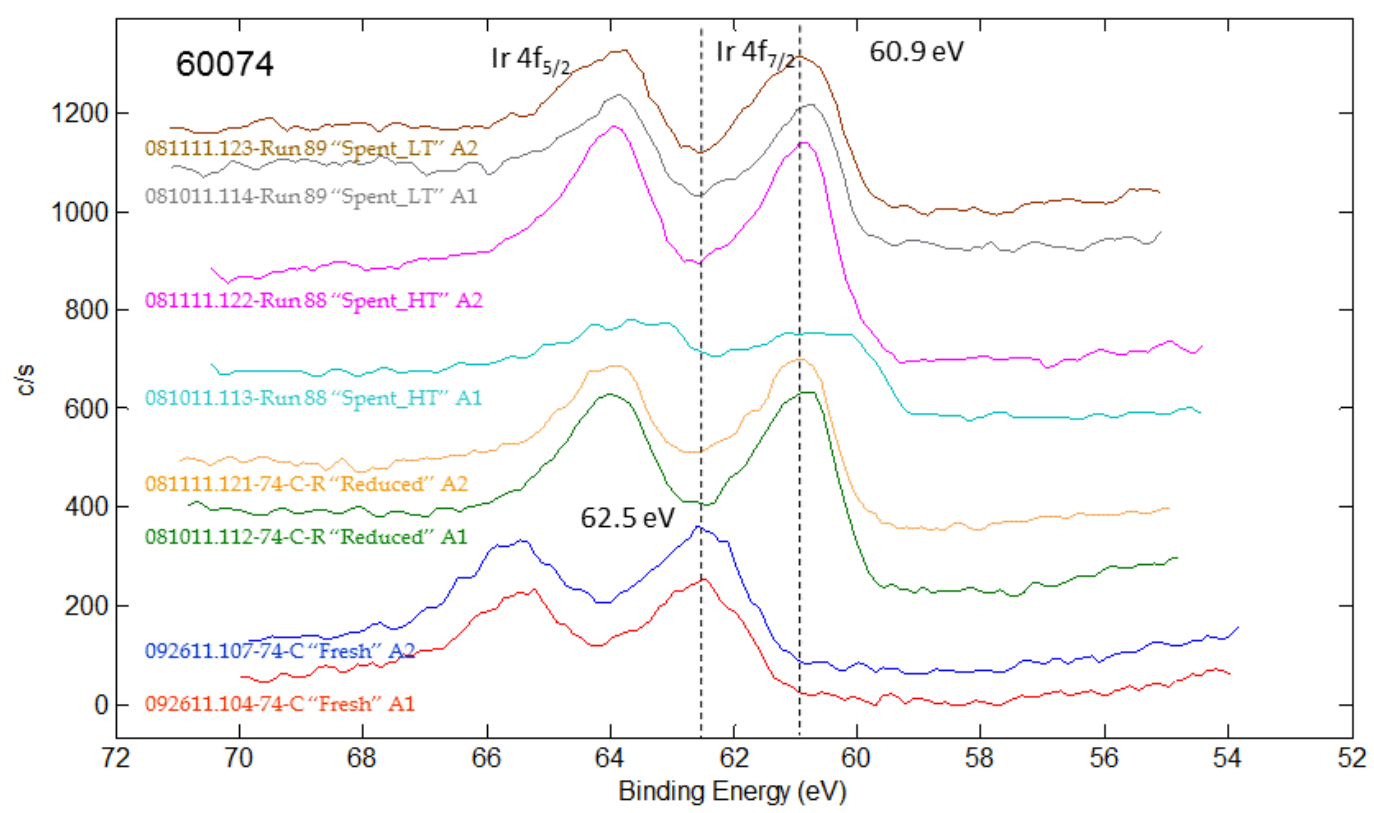

Figure 2.56. High-Energy Resolution XPS Spectra of the Ir 4f Region of the 2.11x Rh-Mn-Ir on the Hyperion 384-82 MWCNT Baseline Catalyst. The spectra are charge referenced using the $\mathrm{C} 1 \mathrm{~s}$ line at $284.4 \mathrm{eV}$.

The oxygen (Figure D.7), silicon (Figure D.8), and carbon (Figure D.9) high-resolution energy scans are provided in Appendix D. Similar to the Rh-Mn catalyst, the oxygen concentration diminishes substantially upon reduction of the fresh catalyst, probably because of the removal of oxygen via the decomposition of nitrate metal salt precursors. The oxygen does reappear on the catalyst surface after utilization for mixed alcohols synthesis but in a form with higher binding energy compared with the fresh catalyst.

The Si signal illustrated in Figure D.8 is again likely due to contamination of the sample with quartz wool either from the reduction furnace or the fixed-bed reactors used for mixed alcohol synthesis.

Finally, the $\mathrm{C} 1 \mathrm{~s}$ region again shows the peaks lined up at $384.4 \mathrm{eV}$ due to the charge referencing applied during data analysis. The Spent HT sample does show an interesting peak slightly to the right (lower binding energy) of the CNT C 1s line, but the scan of a different area on the same line (Spent_HT A2) indicates it may have been an anomaly or, more likely, a pocket of adventitious carbon (C-C or C-H) on the catalyst, possibly from coking or some other phenomenon. Similar to the Rh-Mn catalyst, a broad hump at $291 \mathrm{eV}$ may again be observed, but no strong indication of $\mathrm{MnCO}_{3}$ (expected in the $\mathrm{C} 1 \mathrm{~S}$ region at $289.3 \mathrm{eV}$ ) can be observed. While $\mathrm{MnCO}_{3}$ was observed in the XRD results (Figure 2.39), it does not appear to be a major constituent of the carbon at the surface.

\subsubsection{Other Fresh and Spent Catalysts}

XPS measurements also were performed on the six fresh catalysts and the five spent catalysts listed in Table 2.6. The catalysts were analyzed with XPS before and after reduction. The amounts of elements present at the surface of the samples are reported in Table 2.30 and Table 2.31. A notable outlier in the XPS data was 60074-52-B (not shown in Table 2.30), which did not exhibit a signal for Rh or Mn. It is 
highly probable that a carbon support sample with no metals loaded was inadvertently analyzed instead of sample 60074-52-B.

The catalyst samples were prepared using rhodium nitrate and manganese nitrate. Iridium chloride also was used on two samples. XPS measurements revealed that the $\mathrm{N}_{2}$ was removed from the samples after reduction, indicating that the nitrates were decomposed during reduction. However, $\mathrm{Cl}$ was detected in samples before and after reduction, indicating that reduction did not remove all of the $\mathrm{Cl}$ from the catalyst with Ir present. However, XPS analysis of the spent catalysts with Ir present revealed no residual $\mathrm{Cl}$ present on the surface of the catalyst. Thus, the $\mathrm{Cl}$ was removed from the surface of the catalysts during alcohol synthesis.

The most surprising observation is the Mn:Rh ratio on the surface of the catalyst for the reduced samples in Table 2.30 versus the spent samples in Table 2.31. The Mn:Rh ratios in all of the reduced samples in Table 2.30 are between 0.4 and 0.7 , indicating that the surfaces of the reduced catalysts are initially $\mathrm{Rh}$ rich, as would be expected because the atomic concentration of $\mathrm{Rh}$ is $\sim 1.7$ times greater than the concentration of the $\mathrm{Mn}$ on a given catalyst. Thus, the $\mathrm{Mn}: \mathrm{Rh}$ ratio should be $\sim 0.59$. When $\mathrm{Ir}$ is not present in a catalyst sample as in Run 31 above, the ratio of $\mathrm{Mn}: \mathrm{Rh}$ remains relatively similar on the catalysts after reduction and after mixed alcohol synthesis at 0.7 to 0.8 , indicating a Rh-rich surface. However, when Ir is present in the catalysts from Runs 33, 34, and 39, the ratio of Mn:Rh increases dramatically to 1.5 to 3.7. The effect of Os also is similar as shown in the catalyst from Run 32, in which the Mn:Rh ratio increases to 1.2 to 1.5. Thus, it appears that Ir and Os may have a similar effect whereby a Mn-rich surface is developed during mixed alcohol synthesis conditions.

Table 2.30. Quantification (atomic percent) of Surface Elements on Various Samples Prior to Reduction and Reduced as Determined by XPS Measurements

\begin{tabular}{ccccccccc}
\hline Sample & Description & Sample Treatment & C & O & Mn & Rh & Ir $\begin{array}{c}\text { Mn:Rh } \\
\text { Atomic } \\
\text { Ratio }\end{array}$ \\
\hline 60074-13A & 1.5x Rh-Mn on & Prior to reduction & 80.5 & 16.9 & 0.54 & 1.99 & 0.00 & 0.3 \\
& Hyperion 395-10 & Reduced & 94.6 & 3.1 & 0.71 & 1.69 & 0.00 & 0.4 \\
& & Reduced & 94.5 & 3.0 & 0.77 & 1.70 & 0.00 & 0.5 \\
60074-56A & 1.5x Rh-Mn on & Prior to reduction & 71.9 & 24.2 & 1.21 & 2.67 & 0.01 & 0.5 \\
& Hyperion 395-08 & Reduced & 90.9 & 4.9 & 1.74 & 2.50 & 0.00 & 0.7 \\
& & Reduced & 90.9 & 4.9 & 1.79 & 2.41 & 0.00 & 0.7 \\
60074-56B & 1.5x Rh-Mn on & Prior to reduction & 76.7 & 20.1 & 0.98 & 2.20 & 0.00 & 0.4 \\
& Hyperion 395-10 & Reduced & 92.9 & 4.2 & 1.10 & 1.83 & 0.00 & 0.6 \\
& & Reduced & 93.8 & 3.5 & 0.93 & 1.72 & 0.00 & 0.5 \\
60074-59C & 2.11x Rh-Mn 2.11x & Prior to reduction & 81.8 & 15.4 & 0.73 & 2.01 & 0.09 & 0.4 \\
& Ir on Hyperion 384- & Reduced & 93.3 & 3.7 & 1.22 & 1.76 & 0.06 & 0.7 \\
& 82 & Reduced & 93.6 & 3.8 & 0.98 & 1.67 & 0.05 & 0.6 \\
60074-67B & 1.5x Rh-Mn, 1.5x Ir & Prior to reduction & 90.4 & 8.1 & 0.34 & 1.07 & 0.04 & 0.3 \\
& on Hyperion 384-82 & Reduced & 95.6 & 3.1 & 0.40 & 0.94 & 0.03 & 0.4 \\
& & Reduced & 93.1 & 5.3 & 0.60 & 0.94 & 0.02 & 0.6 \\
\hline
\end{tabular}


Table 2.31. Quantification (atomic percent) of Surface Elements on Various Spent Mixed Alcohol Catalysts. Two separate analysis areas are presented for each sample.

\begin{tabular}{|c|c|c|c|c|c|c|c|}
\hline Sample & Sample Description & $\mathrm{C}$ & $\mathrm{O}$ & Mn & $\mathrm{Rh}$ & Ir & $\begin{array}{c}\text { Mn:Rh } \\
\text { Atomic } \\
\text { Ratio }\end{array}$ \\
\hline \multirow[t]{2}{*}{ Run 31} & 2.11x Rh-Mn on Hyp. 384-82 & 90.7 & 5.8 & 1.36 & 1.75 & 0.00 & 0.8 \\
\hline & & 87.1 & 8.3 & 1.18 & 1.64 & 0.00 & 0.7 \\
\hline \multirow[t]{2}{*}{ Run 32} & 1.5x Rh-Mn 1.0x Os on Hyp.395-11 & 85.1 & 9.9 & 2.12 & 1.75 & $0.01^{(a)}$ & 1.2 \\
\hline & & 79.1 & 14.0 & 3.38 & 2.26 & $0.02^{(a)}$ & 1.5 \\
\hline \multirow[t]{2}{*}{ Run 33} & 2.11x Rh Mn 2.11x Ir on Hyp. 384-82 & 81.5 & 11.7 & 3.32 & 2.20 & 0.09 & 1.5 \\
\hline & & 85.7 & 9.5 & 2.53 & 1.80 & 0.08 & 1.4 \\
\hline \multirow[t]{2}{*}{ Run 34} & 2.11x Rh Mn 2.11x Ir on Hyp. 384-82 & 81.0 & 12.5 & 2.96 & 1.51 & 0.09 & 2.0 \\
\hline & & 82.2 & 11.9 & 3.65 & 1.71 & 0.10 & 2.1 \\
\hline \multirow[t]{2}{*}{ Run 39} & 2.11x Rh Mn 2.11x Ir on Hyp. 384-82 & 73.4 & 18.7 & 4.29 & 1.17 & 0.06 & 3.7 \\
\hline & & 71.6 & 19.7 & 3.82 & 1.39 & 0.05 & 2.7 \\
\hline
\end{tabular}

(a) These values should be zero as Ir was not present on the spent catalyst from Run 32.

Figure 2.57 illustrates that the binding energy of $\mathrm{Rh}$ in the fresh, unreduced samples in Table 2.30 did not match the expected binding energy for $\mathrm{Rh}_{2} \mathrm{O}_{3}$. $\mathrm{Rh}_{2} \mathrm{O}_{3}$ has a reported binding energy of $309.2 \mathrm{eV}{ }^{7}$ XPS measurements observed a binding energy of $310.3 \mathrm{eV}$ on the dried only catalyst. This is most likely because the $\mathrm{Rh}$ in the fresh, unreduced samples is present as $\mathrm{Rh}\left(\mathrm{NO}_{3}\right)_{2}$. XPS also revealed that, after the reduction process, all of the $\mathrm{Rh}$ at the surface was reduced. The value reported in the literature for reduced $\mathrm{Rh}$ is $307.4 \mathrm{eV} .^{7}$ Thus, all reduced and spent catalysts (Figure 2.58) have Rh present in the reduced state.

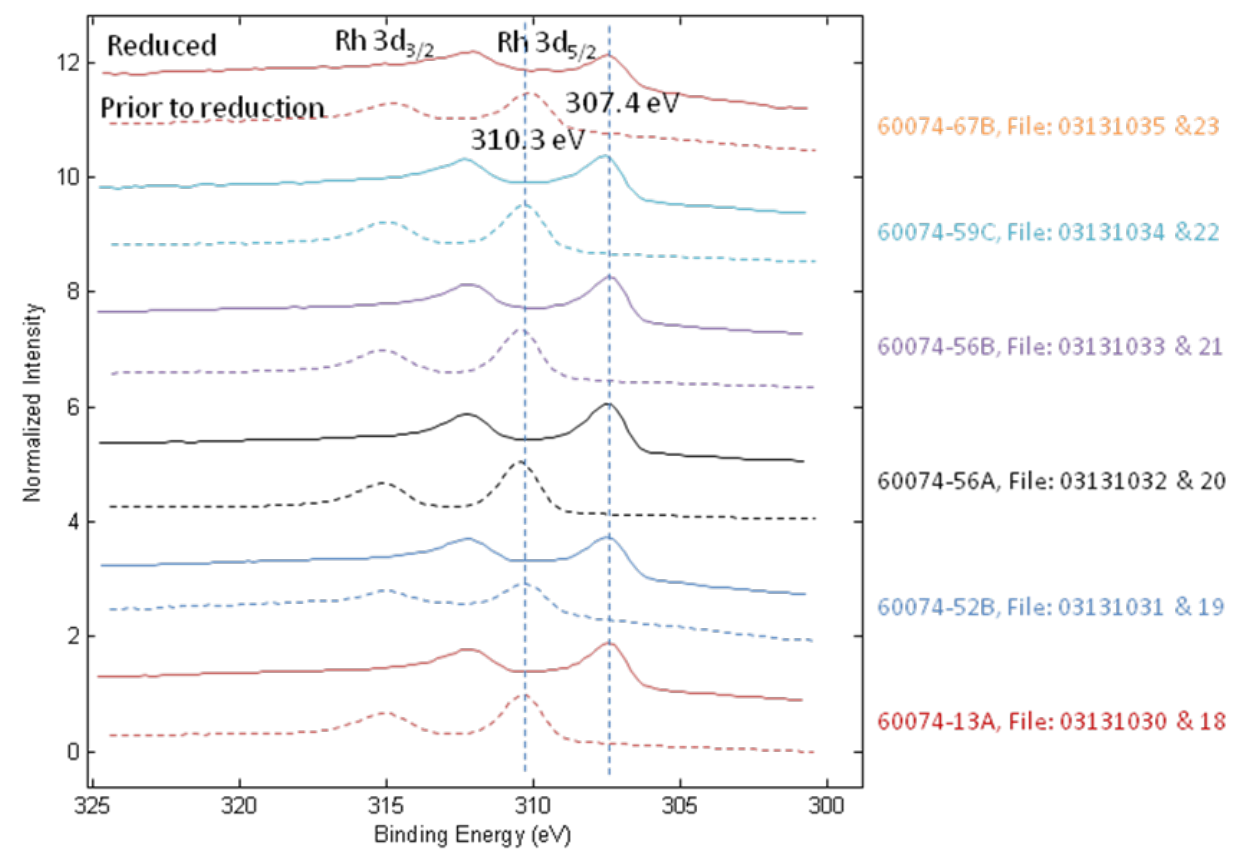

Charge referenced using the $\mathrm{C} 1 \mathrm{~s}$ line at $284.5 \mathrm{eV}$ Reference: D. Kazachkin, et. al., "High resolution XPS characterization of chemical functionalized MWCNTs and SWCNTs “ Carbon, V43, 2005 P 153.

Figure 2.57. Rh 2p Electron Region of the Six Fresh (unreduced dashed lines) and Reduced (solid lines) Metal Impregnated Catalysts Listed in Table 2.6 


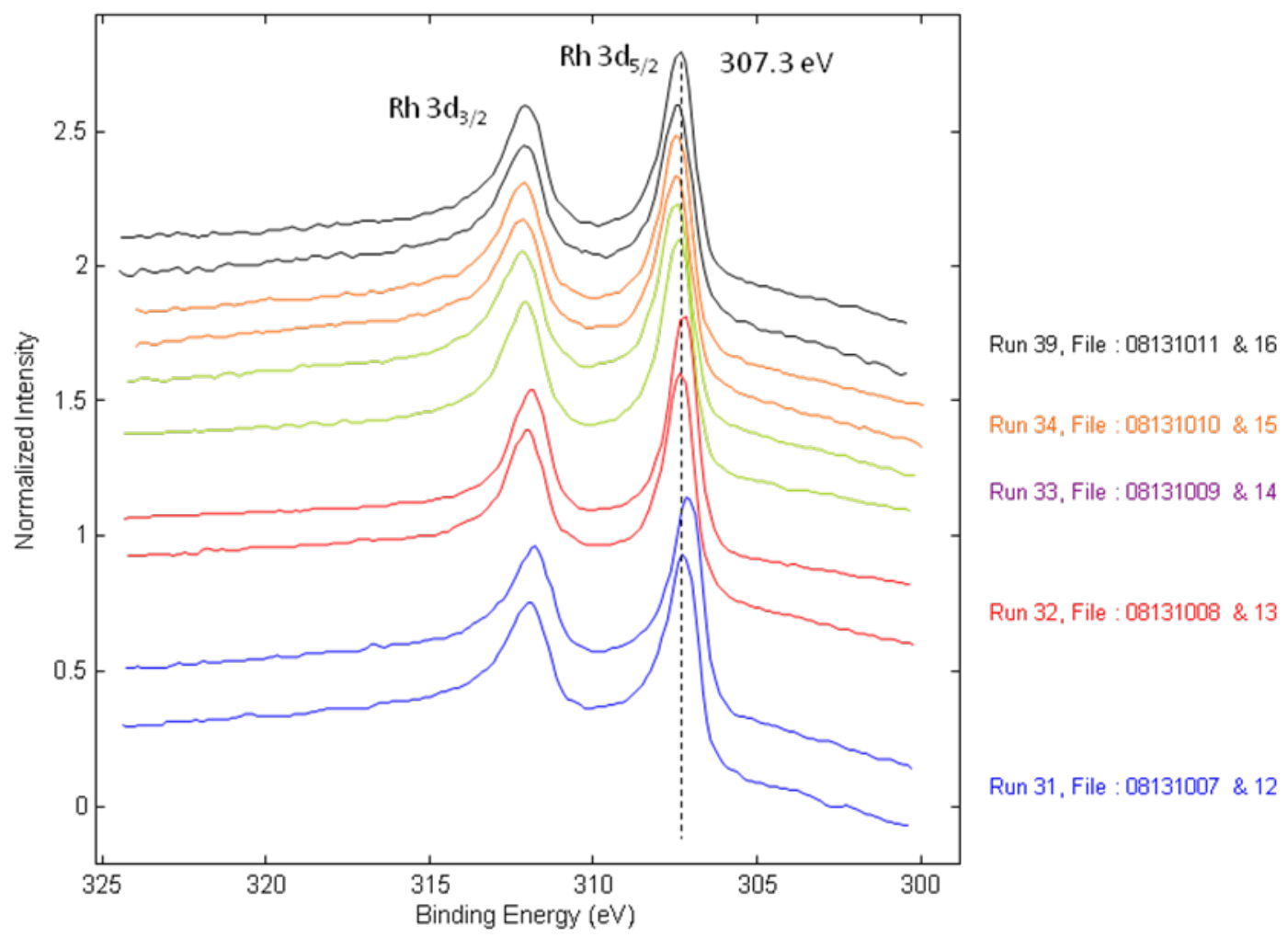

Charge referenced using the $C 1$ s line at $284.5 \mathrm{eV}$ Reference: D. Kazachkin , et. Al., "High resolution XPS characterization of chemical functionalised MWCNTs and SWCNTs " Carbon. V43. 2005 P 153

Figure 2.58. High-Resolution XPS Spectra of the Rh 2p Electron Region of the Five Spent Catalysts Listed in Table 2.6

Comparison of Figure 2.59 and Figure 2.60 revealed that the Mn 2p region was similar in the unreduced, reduced, and spent catalysts listed in Table 2.6. The acme of the Mn $2 \mathrm{p}_{3 / 2}$ electron peak in reduced and spent catalysts remained at $641.8-642.0 \mathrm{eV}$, which is within the functional resolution of the XPS instrument. The ambiguity of the Mn oxidation state most likely results from the similarity in binding energies of $\mathrm{Mn}^{(0)}$ and $\mathrm{MnO}$. The broad nature of the peaks in the Mn spectra may suggest that some of the $\mathrm{Mn}$ is reduced when used in the mixed alcohols reactor, but the binding energy of the electrons liberated from $\mathrm{Mn}$ are very similar for $\mathrm{Mn}$ and $\mathrm{MnO}(\mathrm{Mn}=638.8$ to $641.0 \mathrm{eV}$ and $\mathrm{MnO}=640.3$ to $642.5 \mathrm{eV}$ ). However, the fact that the peak in the spent catalyst is centered at $641.8 \mathrm{eV}$ suggests that the majority of the Mn at the surface is oxidized. More detailed analysis through the use of Mn references in various known oxidation states would be required to better understand the state of $\mathrm{Mn}$ on the surface of the reduced and spent samples. 


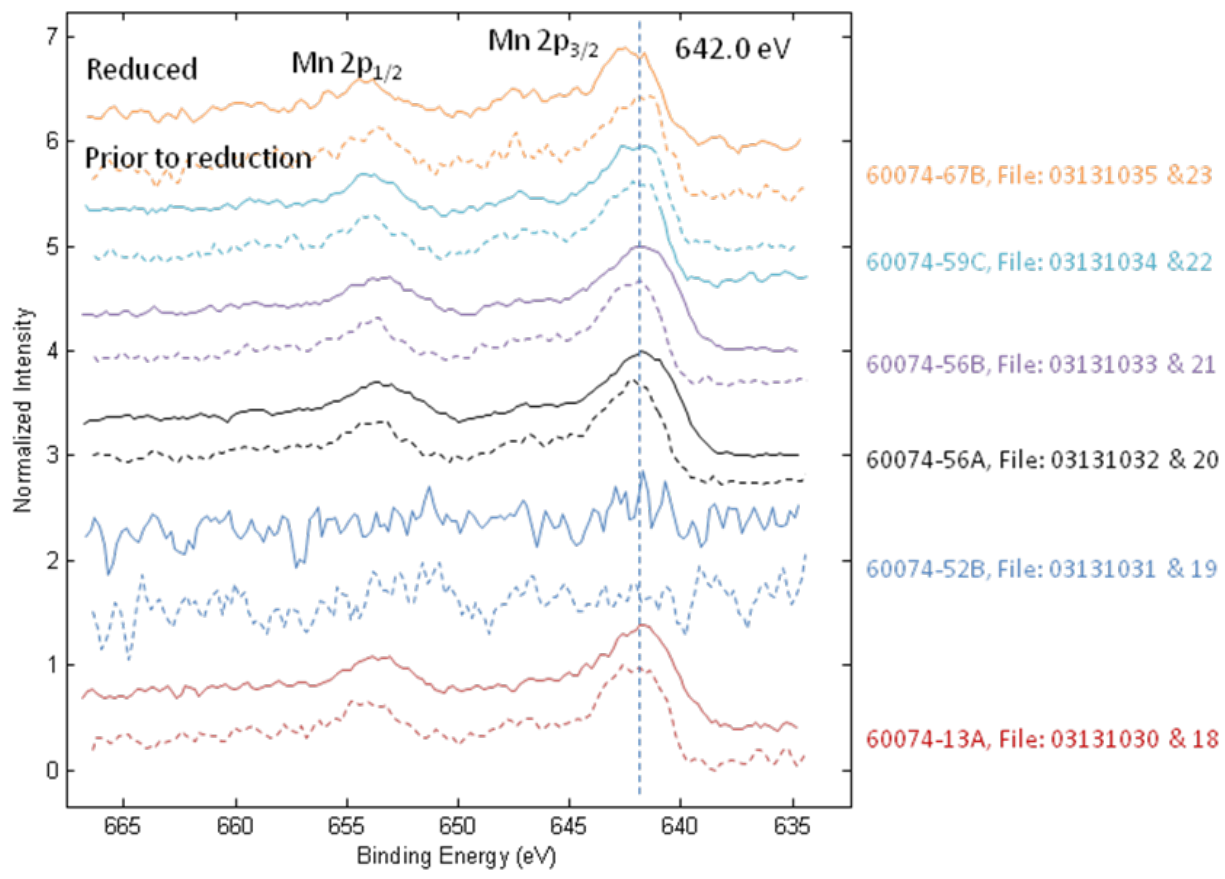

Charge referenced using the $\mathrm{C} 1 \mathrm{~s}$ line at $284.5 \mathrm{eV}$ Reference: D. Kazachkin, et. al., "High resolution XPS characterization of chemical functionalized MWCNTs and SWCNTs "Carbon, V43, 2005 P 153.

Figure 2.59. High-Resolution XPS Spectra of the Mn 2p Electron Region of the Six Fresh (unreduced dashed) and reduced (solid) Metal Impregnated Catalysts in Table 2.6

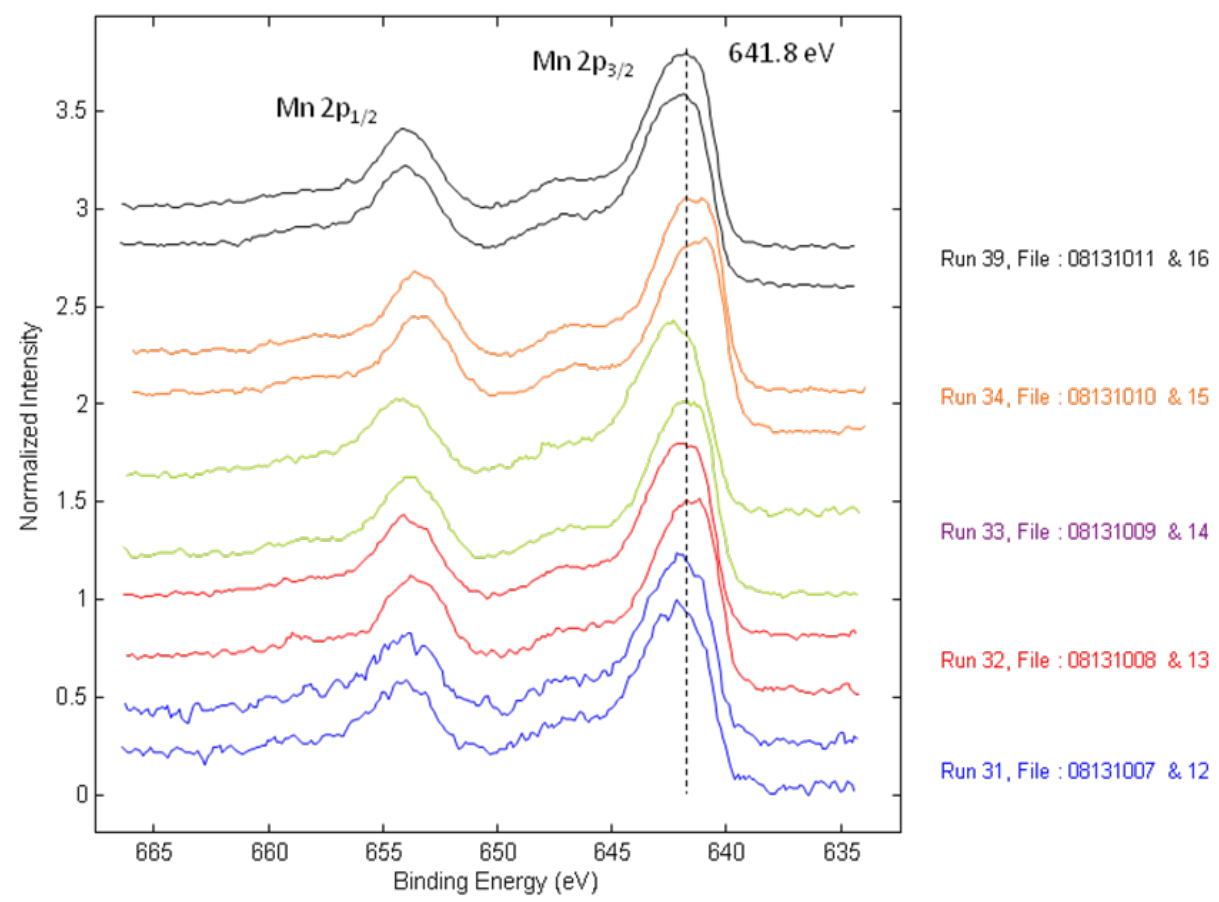

Charge referenced using the $\mathrm{C} 1 \mathrm{~s}$ line at $284.5 \mathrm{eV}$ Reference: D. Kazachkin ,et. Al., "High resolution XPS characterization of chemical functionalised MWCNTs and SWCNTs" Carbon. V43. 2005 P 153

Figure 2.60. High-Resolution XPS Spectra of the Mn 2p Electron Region of the Five Spent Catalysts in Table 2.6 
X-ray diffraction results on the spent samples in Table 2.6 revealed that $\mathrm{MnCO}_{3}$ is likely present in the spent catalysts (see Figure 2.41 to Figure 2.47). Analysis of a $\mathrm{MnCO}_{3}$ reference sample with XPS indicated that $\mathrm{Mn}$ has a binding energy of $641.4 \mathrm{eV}$ when present as carbonate. Because of the broad nature of the XPS spectra in Figure 2.60 it is possible that some Mn exists as $\mathrm{MnCO}_{3}$ at the surface, but the majority of the Mn appears to have a higher binding energy. The relative narrow nature of the peaks in XRD would suggest that the $\mathrm{MnCO}_{3}$ in the spent catalyst samples is composed of large and/or welldefined crystals.

Analysis of Figure 2.61 shows that Ir clearly shifts to lower binding energies after reduction, indicating a more reduced state. In contrast to the freshly reduced catalysts, the state of the Ir on the spent catalysts in Figure 2.62 may be more slightly reduced. Because both the reduced and spent samples are charge referenced to the same $\mathrm{C} 1 \mathrm{~s}$ binding energy, the binding energy of the $\mathrm{Ir} 4 \mathrm{f}_{7 / 2}$ electron at $61.2 \mathrm{eV}$ versus the spent catalysts at $60.9 \mathrm{eV}$ is significant and outside the typical functional resolution limit of the XPS ( 0.1 to $0.2 \mathrm{eV})$. One possible explanation for slightly more reduced Ir on the spent catalysts is that $\mathrm{Cl}$ remaining from the $\mathrm{IrCl}_{3}$ precursor was removed in the mixed alcohol synthesis reactor. Chloride, which was present even after initial reduction according to XPS experiments on freshly reduced samples, was likely removed by the harsh reducing conditions present in the mixed alcohol synthesis reactor, resulting in a more reduced state of Ir.

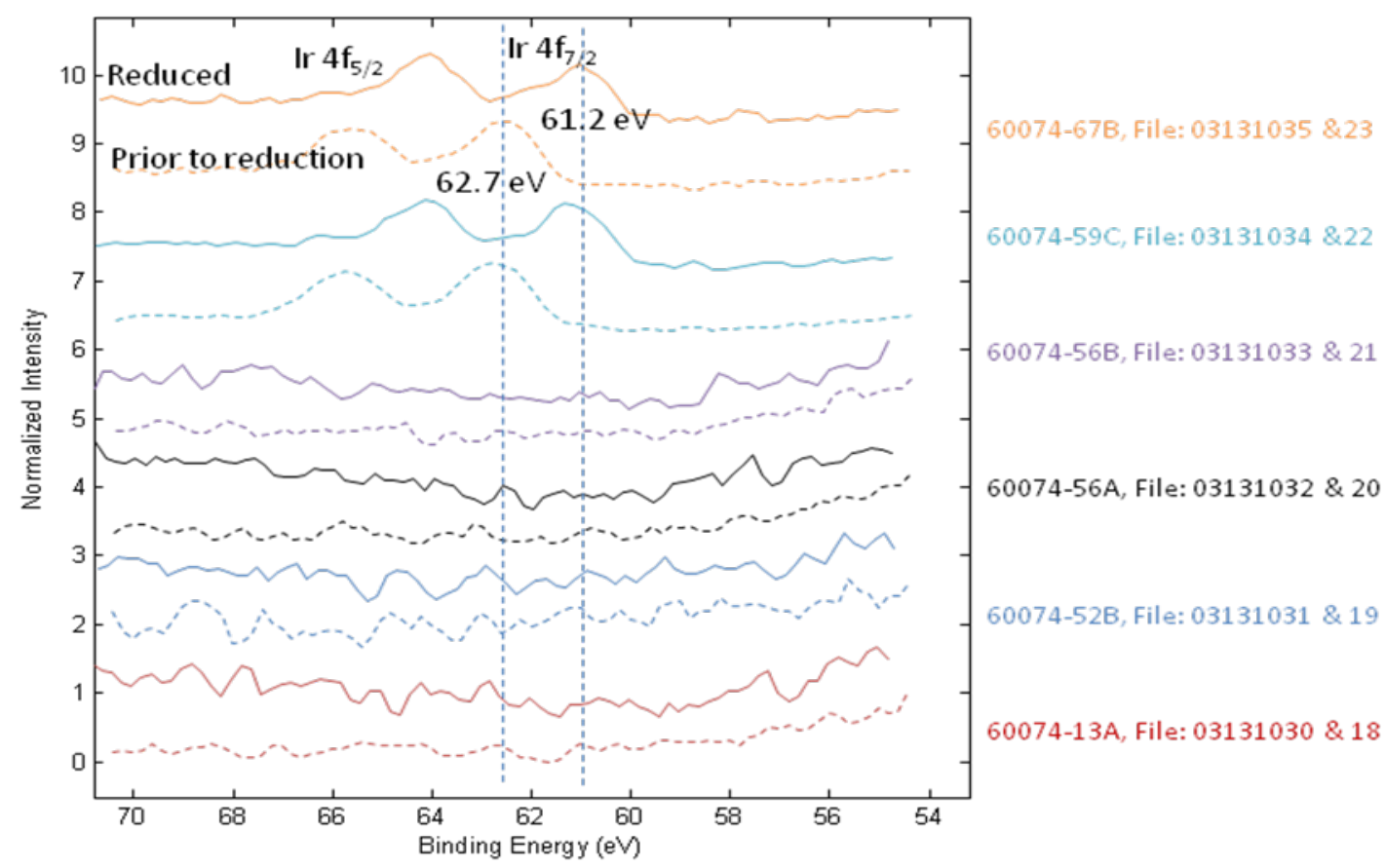

Charge referenced using the $\mathrm{C} 1$ s line at $284.5 \mathrm{eV}$ Reference: D. Kazachkin, et. al., "High resolution XPS characterization of chemical functionalized MWCNTs and SWCNTs " Carbon, V43, 2005 P 153.

Figure 2.61. Ir 4f Electron Region of the Six Fresh (unreduced dashed lines) and Reduced (solid lines) Metal Impregnated Catalysts in Table 2.6 


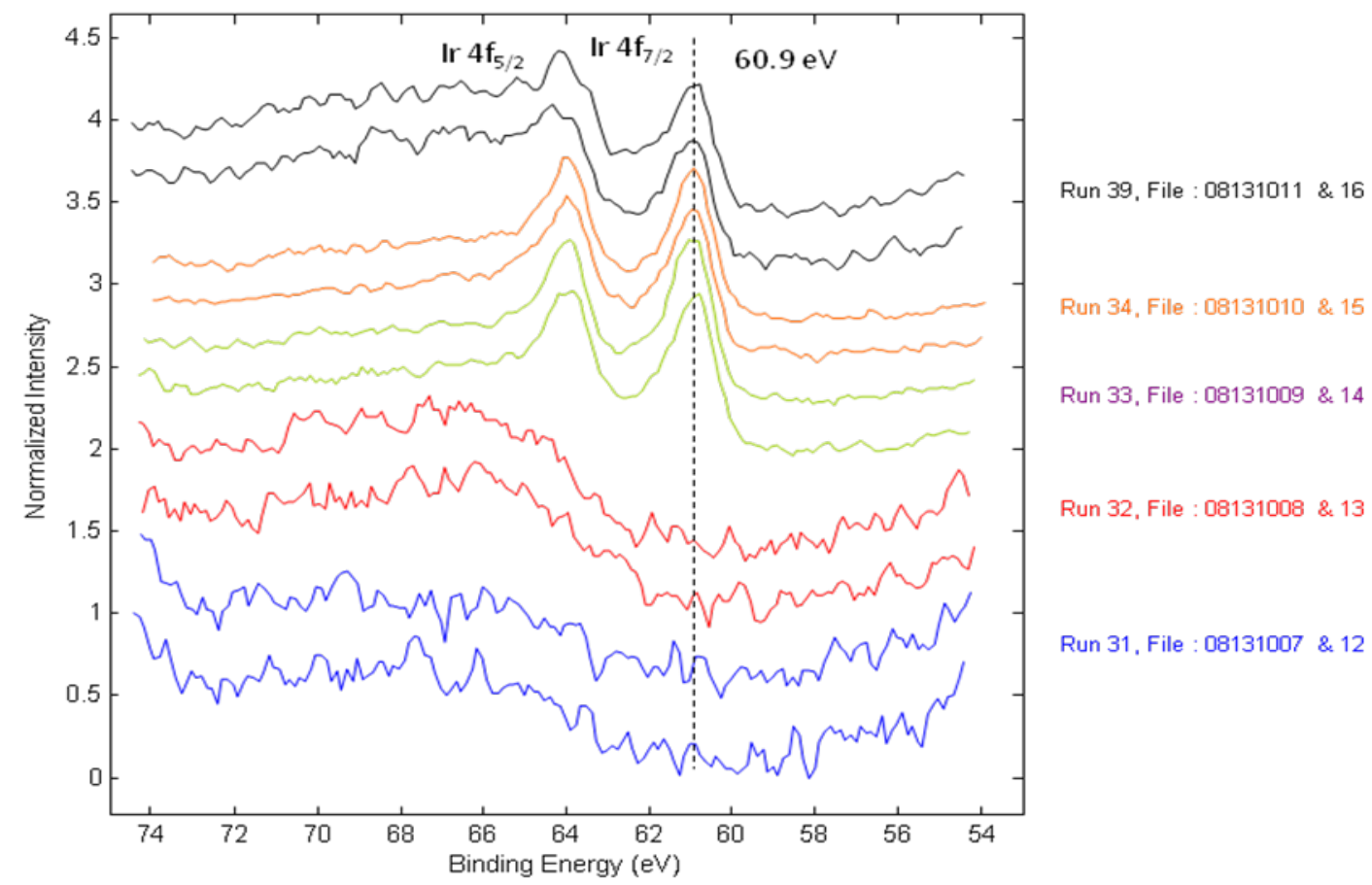

Charge referenced using the C $1 \mathrm{~s}$ line at $284.5 \mathrm{eV}$ Reference: D. Kazachkin , et. Al., "High resolution XPS characterization of chemical functionalised MWCNTs and SWCNTs" Carbon. V43. 2005 P 153

Figure 2.62. Ir 4f Electron Region of the Five Spent Catalysts in Table 2.2

Included in Appendix D are stack-style plots for the fresh, reduced and spent samples in Table 2.6 for the elements of carbon and oxygen. The $\mathrm{C} 1$ s binding energies are shown in Figures D.10 and D.11 while the $\mathrm{O}$ 1s binding energies are shown in Figures D.12 and D.13. The XPS data for the catalysts are charge referenced to $284.5 \mathrm{eV}$ in a similar manner for both the fresh and spent catalysts. Thus, the $\mathrm{C} 1 \mathrm{~s}$ lines stack up at exactly $284.5 \mathrm{eV}$. The O 1s region in Figures D.12 and D.13 show slight differences in the binding energies of the oxygen present on the surface of the catalyst but no correlations with catalyst performance are apparent.

\subsection{Transmission Electron Microscopy}

\subsubsection{Baseline Catalysts}

The baseline catalysts listed in Table 2.4 were subjected to TEM. TEM was performed to visually observe the morphology and metal particle sizes of the catalysts on the 1 to $100 \mathrm{~nm}$ length scale.

To better illustrate some of the effects present in the TEM micrographs, selected images have been converted from grayscale to colorized gradient scale. Figure 2.63 illustrates the enhancement in converting a micrograph from grayscale to colorized gradient scale so that features are sharper and are more easily distinguished by the human eye. In Figure 2.63, the metal clusters appear as brighter dots and the boundaries of the carbon nanotube support are more readily distinguished as the orange color from the black background. 


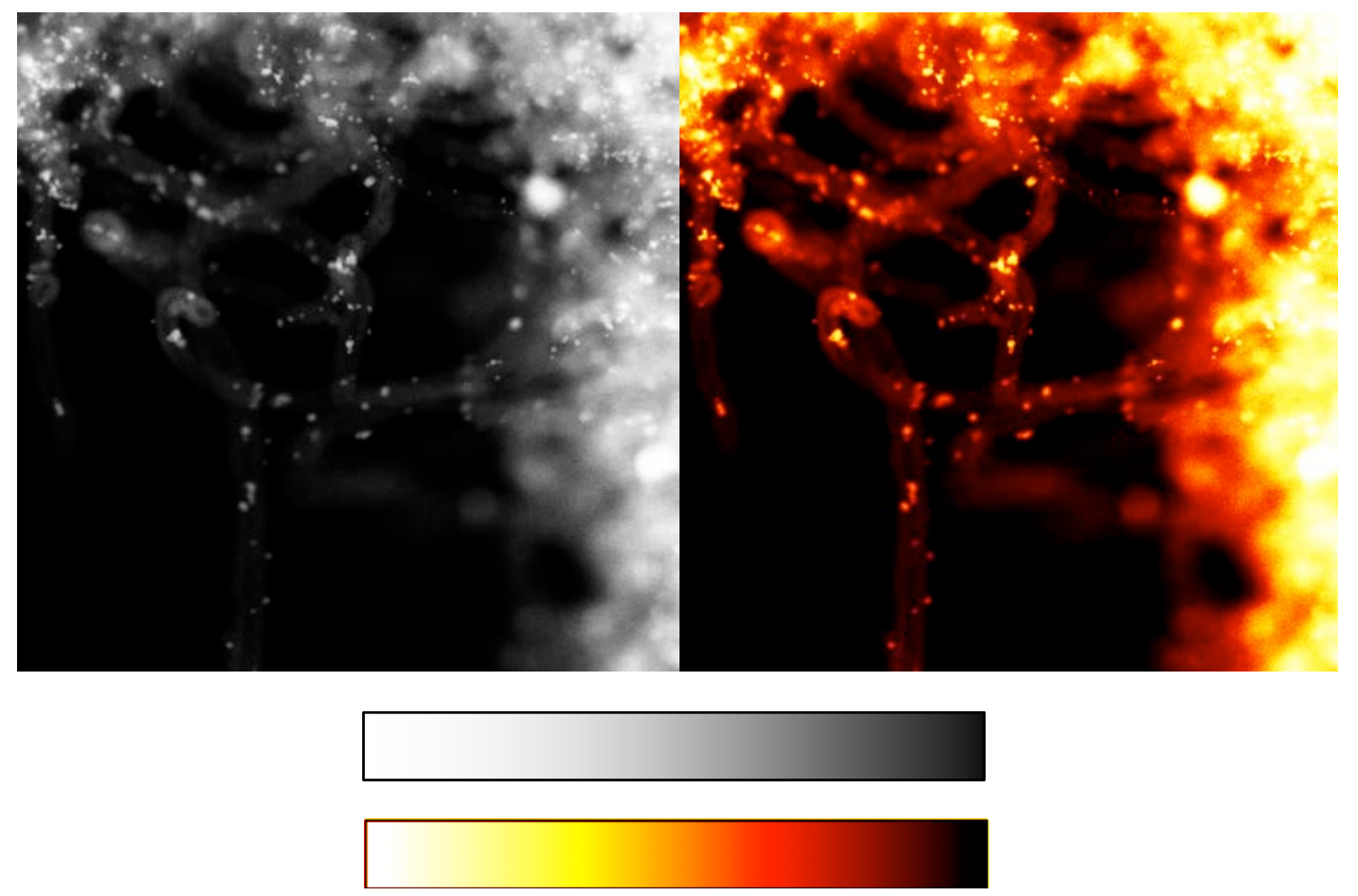

Figure 2.63. Comparison of the Same Micrograph Illustrating the Effect of Converting from Grayscale to Colorized Gradient Scale

\subsubsection{Davisil 645 Silica-Supported Rh-Mn-Ir Baseline Catalysts}

The Davisil 645 silica-supported catalysts listed in Table 2.4 were first analyzed with TEM. The silica-supported baseline catalysts consisted of $5.56 \mathrm{wt} \% \mathrm{Rh}, 1.69 \mathrm{wt} \% \mathrm{Mn}$, and $1.04 \mathrm{wt} \% \mathrm{Ir}$. The micrograph of the fresh silica-supported catalysts illustrates small dark clusters of metal on the gray silica support. The loading of Mn and Ir on the catalyst was below the detection limits of electronic dispersive spectroscopy (EDS). Thus, Mn and Ir could not be detected anywhere on the samples and attempts to identify the elemental concentrations of individual metal particles were unsuccessful. Thus, the metal particles are probably rich in Rh but even the Rh loading level was such that the EDS technique had difficulty identifying $\mathrm{Rh}$ because of the great relative concentration of silica.

TEM micrographs of the reduced baseline catalyst on Davisil 645 (60074-56-A-R in Table 2.4) are shown in Figure 2.65 and Figure 2.65. The micrographs are slightly out of focus because of instrumentation challenges when the samples were collected. However, comparison with the fresh (calcined) samples in Figure 2.66 and Figure 2.67 does reveal coarsening of some metal particles during reduction. For example, only 3 to $5 \mathrm{~nm}$ to clusters were observed in the fresh samples while clusters greater $5 \mathrm{~nm}$ and some up $\sim 7 \mathrm{~nm}$ may be observed in the reduced samples. While this is certainly a degree of coarsening, there is still a large distribution of metal clusters that are in the 3 to $5 \mathrm{~nm}$ range in the reduced sample. Furthermore, some of the larger metal clusters in the reduced samples may have started exhibiting facets that would be indicative of metallic crystallinity. 


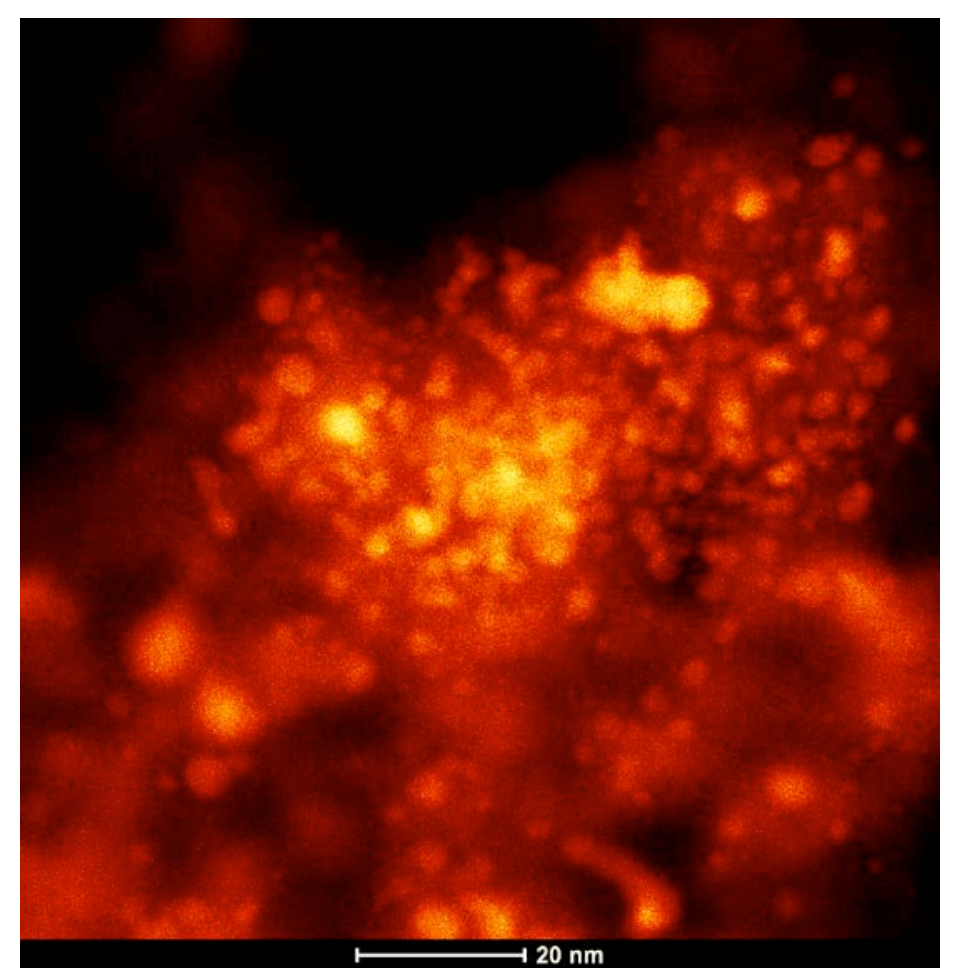

Figure 2.64. TEM Micrograph of the Reduced Baseline Rh-Mn-Ir Sample 60074-53-A-R Supported on Davisil 645

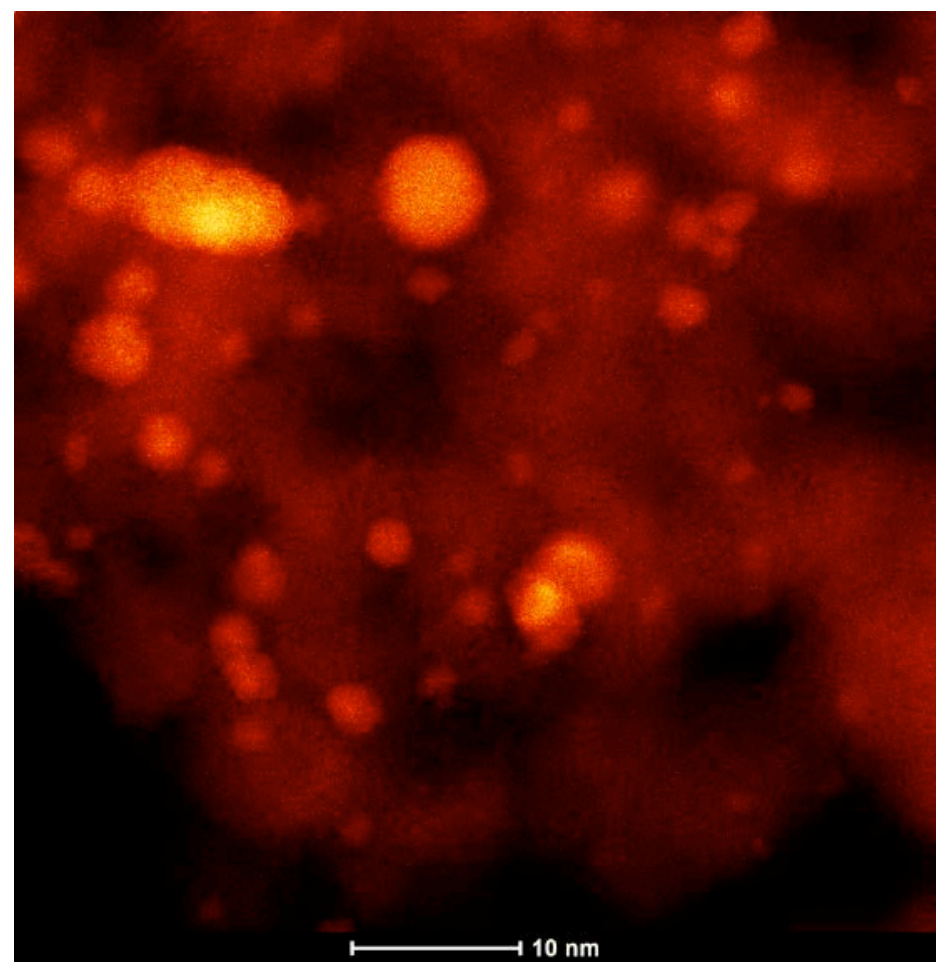

Figure 2.65. TEM Micrograph of the Reduced Baseline Rh-Mn-Ir Sample 60074-53-A-R Supported on Davisil 645 


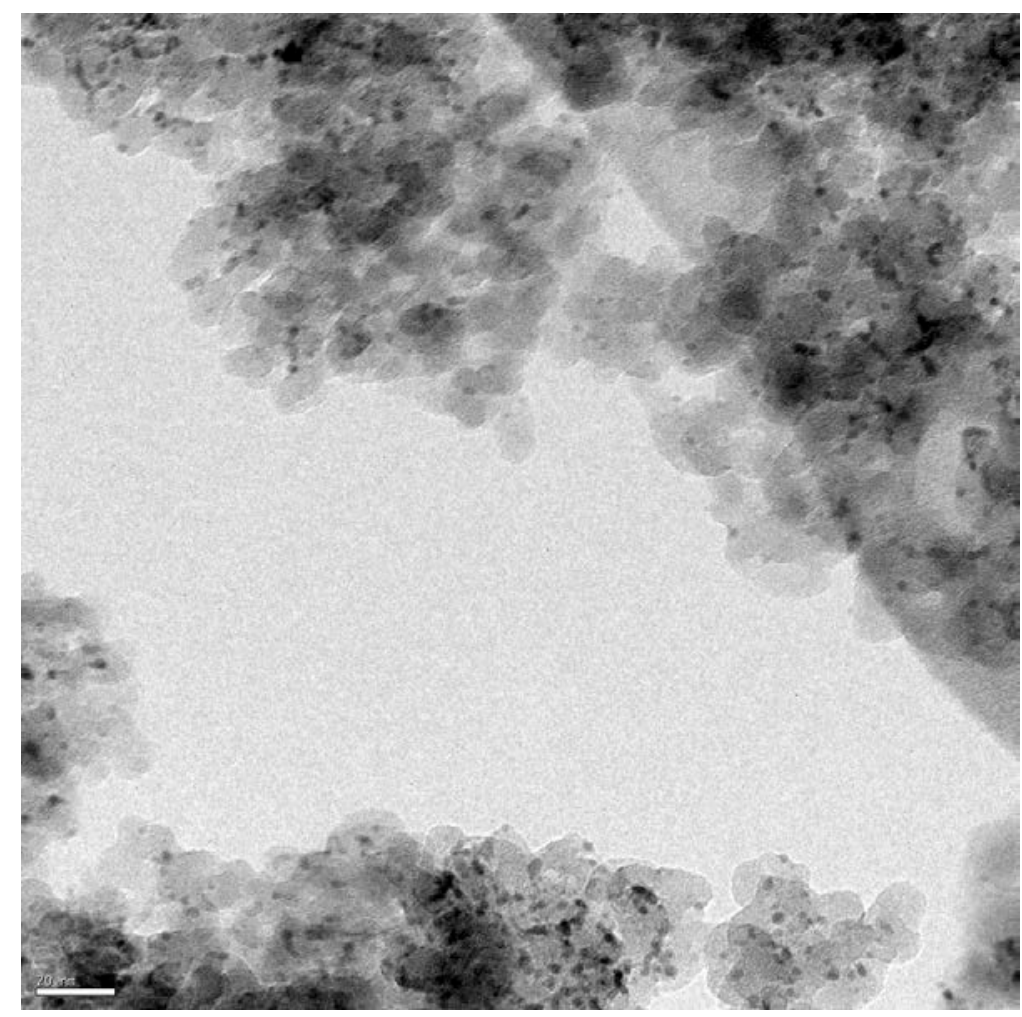

Figure 2.66. TEM Micrograph of the Fresh (calcined but unreduced) Baseline Rh-Mn-Ir Catalyst Supported on Davisil 645 Silica. The scale bar in the micrograph is $20 \mathrm{~nm}$.

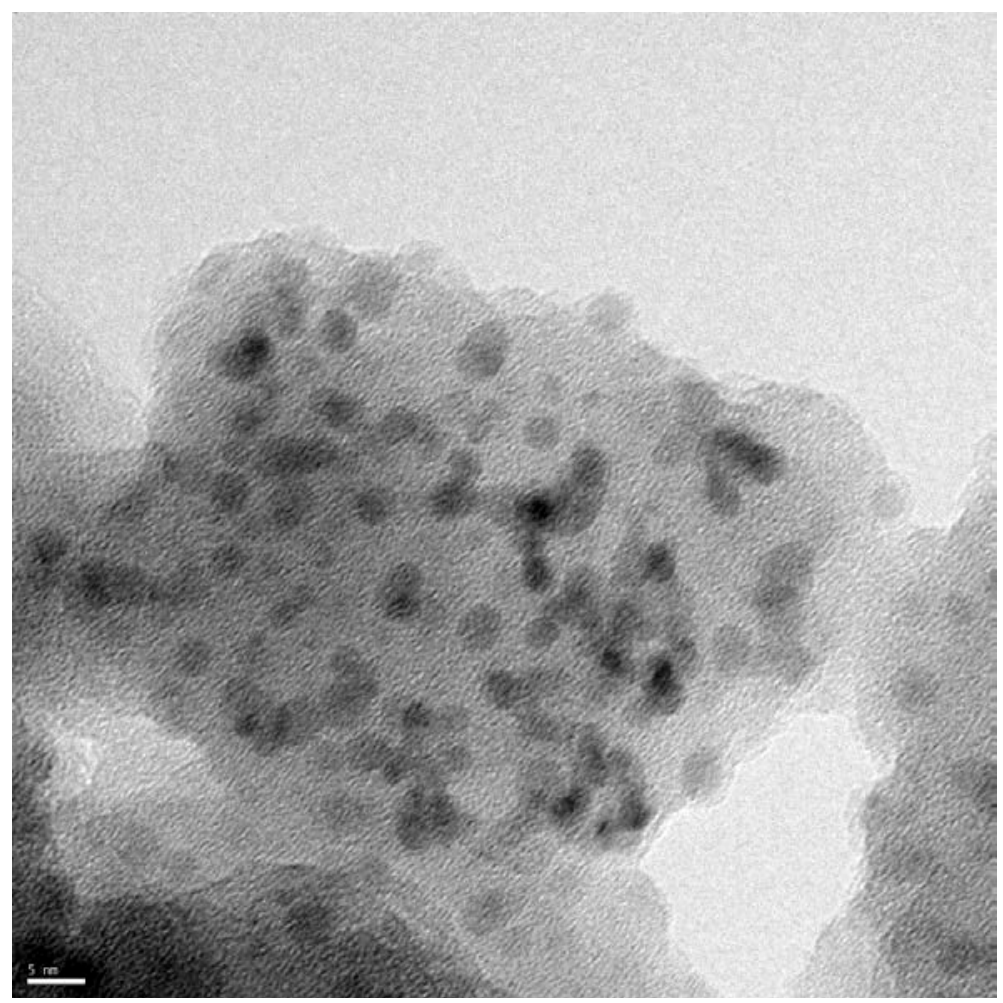

Figure 2.67. TEM Micrograph of the Fresh (calcined but unreduced) Baseline Rh-Mn-Ir Catalyst Supported on Davisil 645 silica. The scale bar in the micrograph is $5 \mathrm{~nm}$. 
Figure 2.68 and Figure 2.69 illustrate samples of the Davisil 645 silica-supported baseline catalysts after mixed alcohols synthesis testing to a maximum temperature of $275^{\circ} \mathrm{C}$. Coarsening of some of the metal particles as compared to the reduced samples can clearly be observed in Figure 2.68. Furthermore, many particles that are 3 to $5 \mathrm{~nm}$ in size have crystalized as indicated by the straight edges (facets) on the outside of the particles (see Figure 2.69). In many of the larger metal particles, Rh was readily observed by EDS but the lack of observed Mn and Ir may be because they are truly not present or simply because the concentration of these metals is below the detection limit of the EDS instrument.

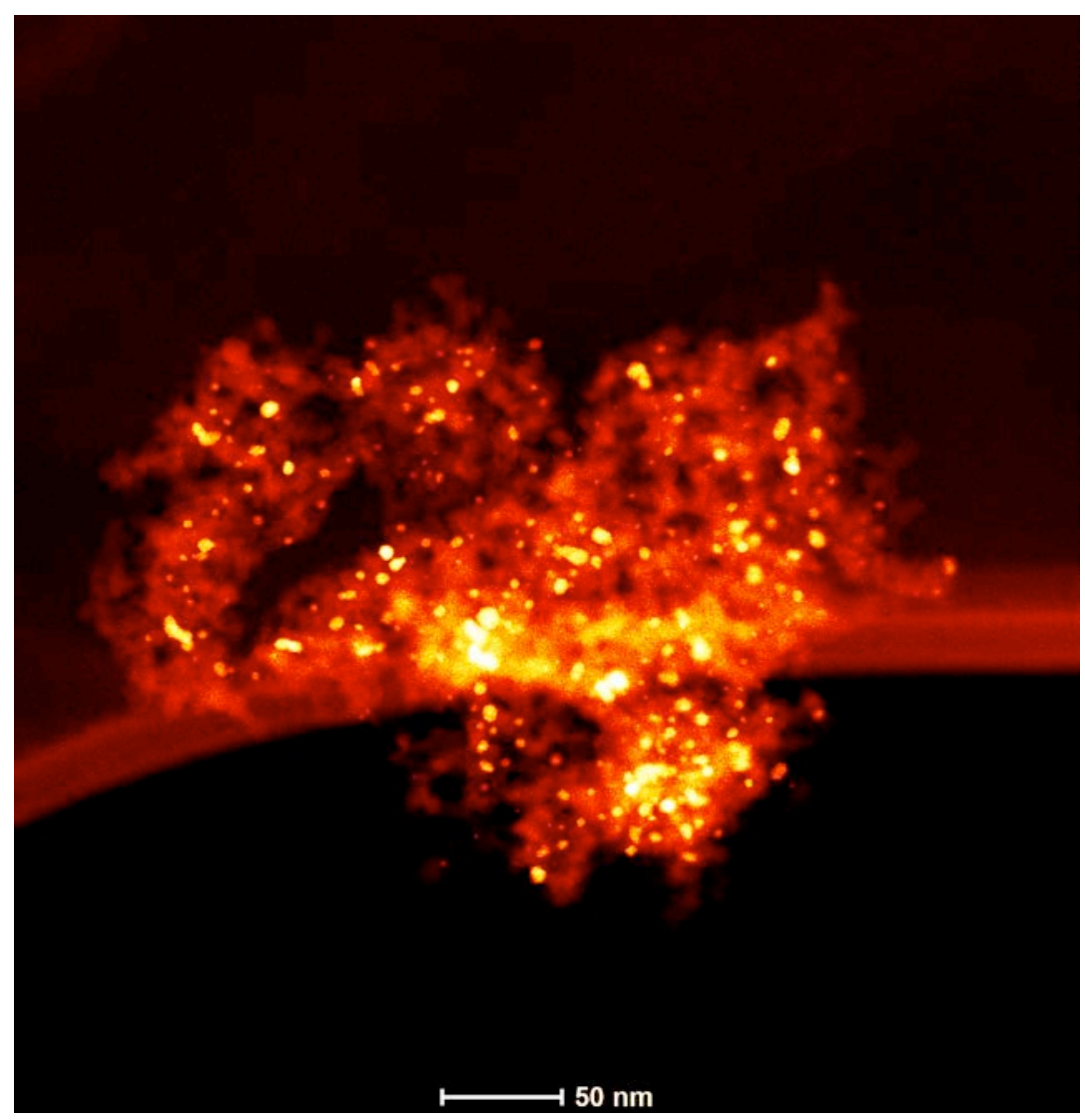

Figure 2.68. TEM Micrograph of the Baseline Rh-Mn-Ir Sample Tested to a Maximum Temperature of $275^{\circ} \mathrm{C}$ (Run 84 in Table 2.4) Supported on Davisil 645 


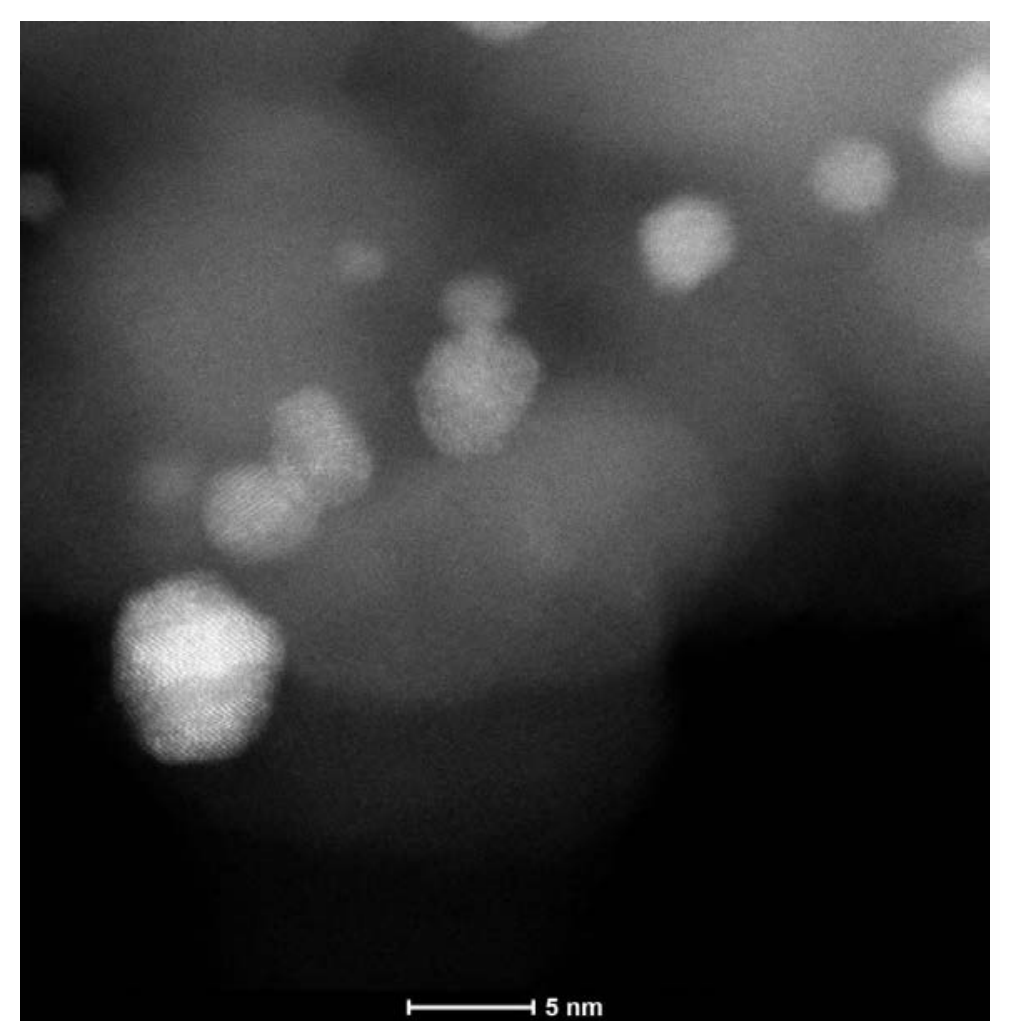

Figure 2.69. TEM Micrograph of the Baseline Rh-Mn-Ir Sample Tested to a Maximum Temperature of $275^{\circ} \mathrm{C}$ (Run 84 in Table 2.4) supported on Davisil 645

TEM micrographs of the silica-supported baseline catalysts tested as mixed alcohol synthesis catalysts to $300^{\circ} \mathrm{C}$ are shown in Figure 2.70 and Figure 2.71. Analysis of micrographs of samples similar to that shown in Figure 2.70 revealed that, in general, the size of the clusters were similar between the Run 84 and Run 86 samples in Table 2.4 with the exception of the growth of some very large clusters in the Run 86 samples. One such cluster is seen in Figure 2.70 as pointed out by the blue arrow. Figure 2.71 illustrates again the formation of straight facets on a metal cluster, indicating that the metal particles were becoming crystalline after reduction and sometime during reaction testing. EDS measurements on the metal particle in Figure 2.71 indicated the particle was mostly Rh with a trace amount of Ir possibly present. 


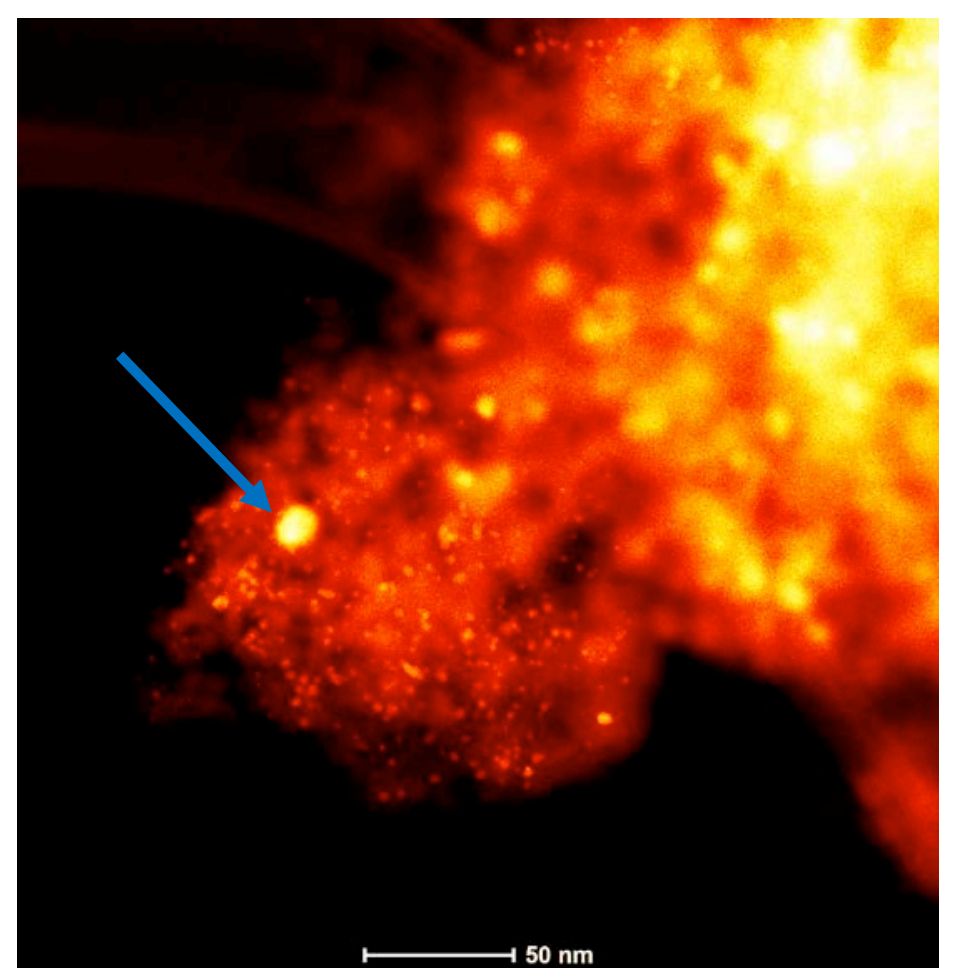

Figure 2.70. TEM Micrograph of the Baseline Rh-Mn-Ir Sample Tested to a Maximum Temperature of $300^{\circ} \mathrm{C}$ (Run 86 in Table 2.4) Supported on Davisil 645. The blue arrow points to a representative large cluster that was observed in Run 86 samples but not in Run 84 samples.

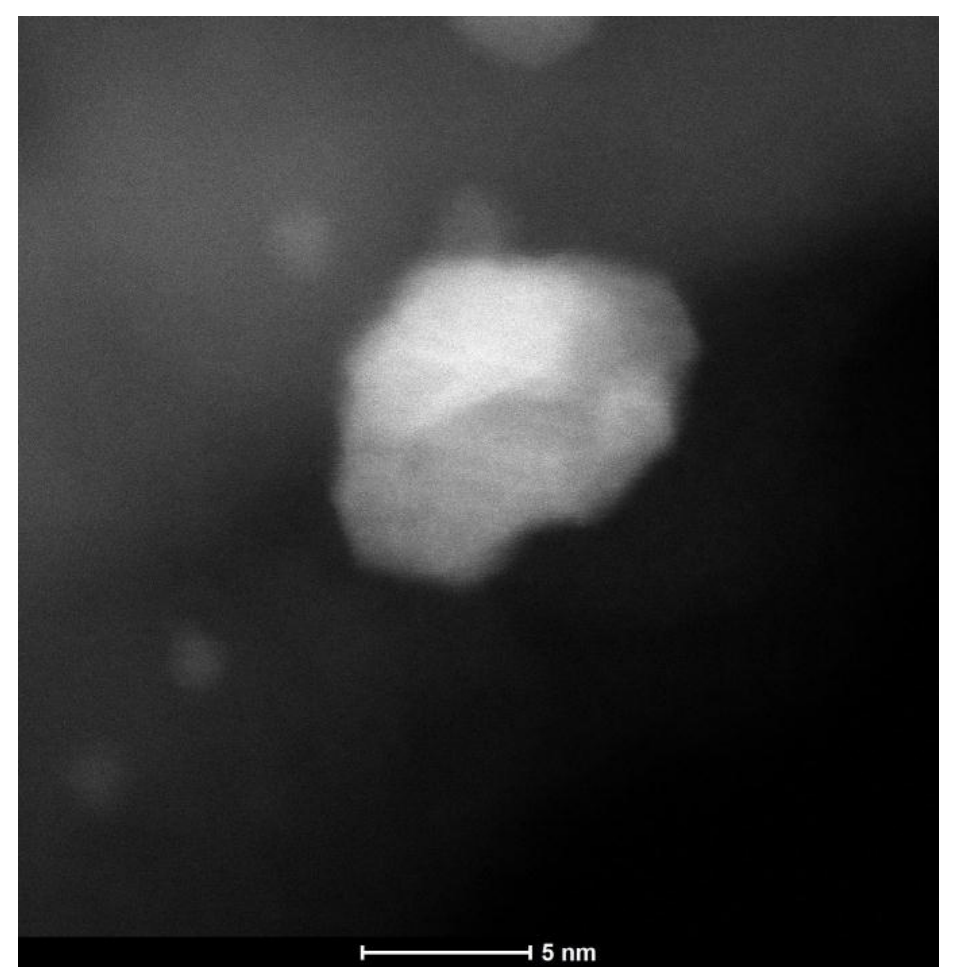

Figure 2.71. TEM Micrograph of the Baseline Rh-Mn-Ir Sample Tested to a Maximum Temperature of $300^{\circ} \mathrm{C}$ (Run 86 in Table 2.4) Supported on Davisil 645 


\subsubsection{Hyperion Fibril MWCNT-Supported Rh-Mn Baseline Catalysts}

The set of Rh-Mn baseline catalysts supported on Hyperion Fibril CS-02C-063-XD MWCNT in Table 2.4 were next analyzed with TEM. The MWCNT-supported catalyst consisted of $11.78 \mathrm{wt} \% \mathrm{Rh}$ and $3.58 \mathrm{wt} \% \mathrm{Mn}$. No Ir was present. Micrographs of the catalysts (Figure 2.72 and Figure 2.73) show the tube-like nature of the carbon nanotubes with small black metallic dots. Generally, the particles appear to be well dispersed and generally $\sim 2 \mathrm{~nm}$ in size. EDS analysis was attempted on some metal clusters but it quickly became apparent that the MWCNTs are readily damaged by the electron beam. Furthermore, the EDS spectra would change with time while the electron beam was held over a certain spot. The changing spectra may have been from beam-induced reduction or even from vaporization/ deposition of metals near the beam. Therefore, detailed EDS analysis was mostly infeasible for the MWCNT samples.

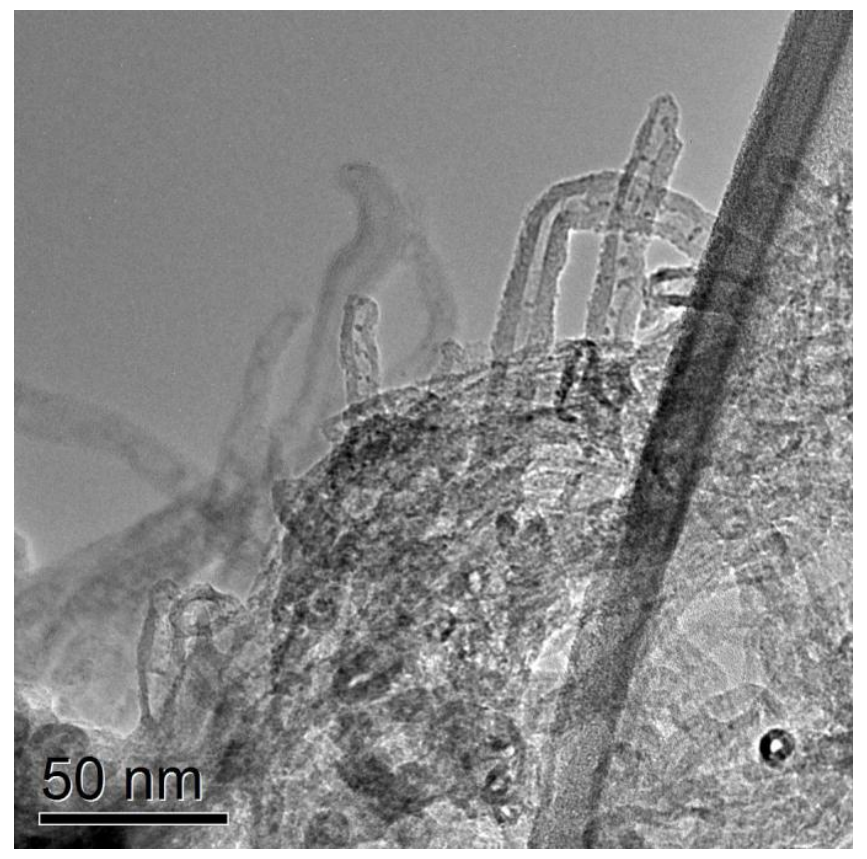

Figure 2.72. TEM Micrograph of the Freshly Prepared Baseline Rh-Mn Sample (sample 60074-70-C in Table 2.4) Supported on Hyperion Fibril MWCNT

Figure 2.74 and Figure 2.75 are TEM micrographs of the reduced Hyperion Fibril-supported Rh-Mn catalyst corresponding with sample 60074-70-C-R in Table 2.4. Similar to the freshly prepared catalyst, the metal particles appear to be well distributed on the carbon nanotubes with metal clusters generally $\sim 2 \mathrm{~nm}$ in size. The metal particles did not appear to sinter during the reduction process. 


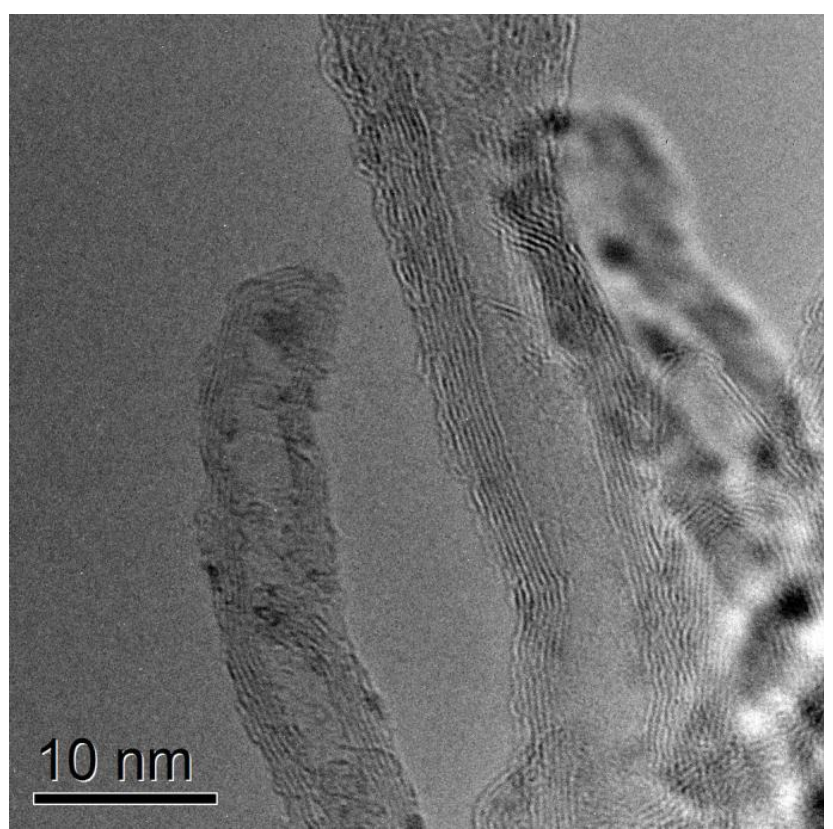

Figure 2.73. Higher Magnification TEM Micrograph of the Freshly Prepared Baseline Rh-Mn Sample (sample 60074-70-C in Table 2.4) Supported on Hyperion Fibril MWCNT

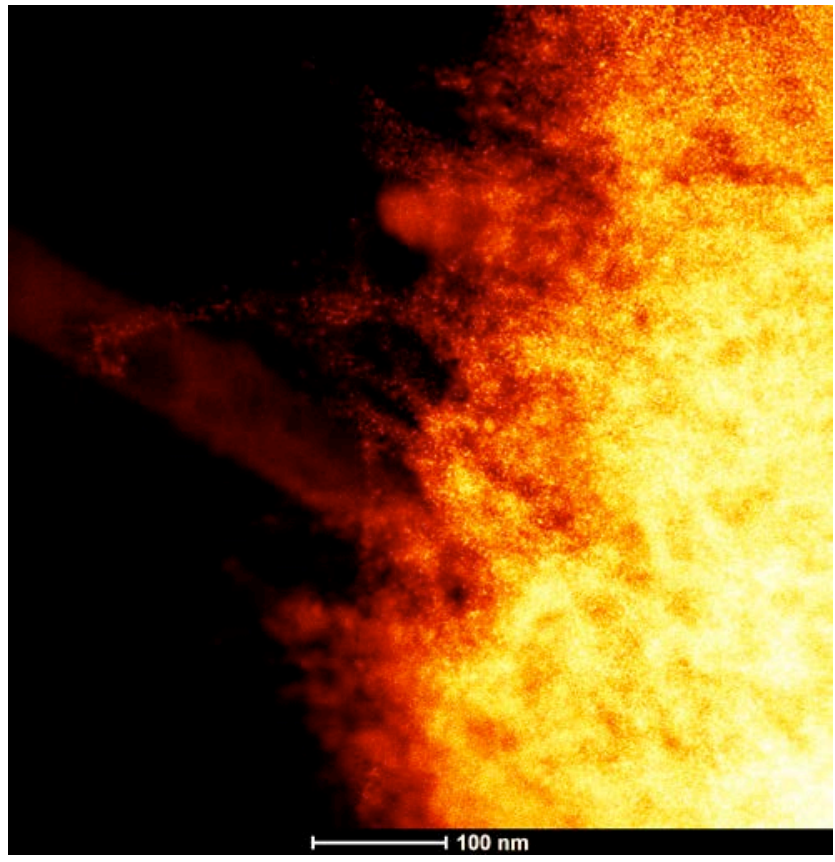

Figure 2.74. TEM Micrograph of the Reduced Baseline Rh-Mn Sample (sample 60074-70-C-R in Table 2.4) Supported on Hyperion Fibril MWCNT 


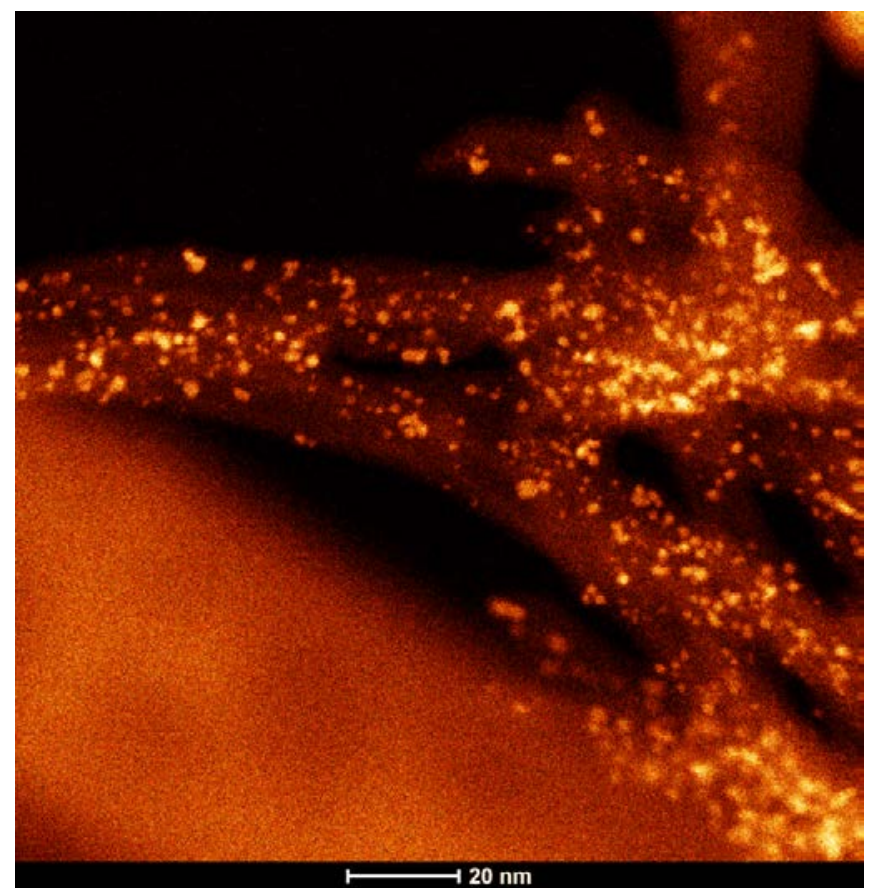

Figure 2.75. Higher Magnification TEM Micrograph of the Reduced Baseline Rh-Mn Sample (sample 60074-70-C-R in Table 2.4) Supported on Hyperion Fibril MWCNT

TEM micrographs of the MWCNT-supported Rh-Mn catalyst used to synthesize mixed alcohols up to a temperature of $276^{\circ} \mathrm{C}$ (Run 80 in Table 2.4) are illustrated in Figure 2.76 through Figure 2.80.

Figure 2.76 illustrates that the metal did start to sinter after using the catalysts for mixed alcohols synthesis. While there are clearly some metal particles in the 2 to $5 \mathrm{~nm}$ range present as light dots in Figure 2.76, there also are metal clusters that are significantly larger. Thus, the metals have sintered.

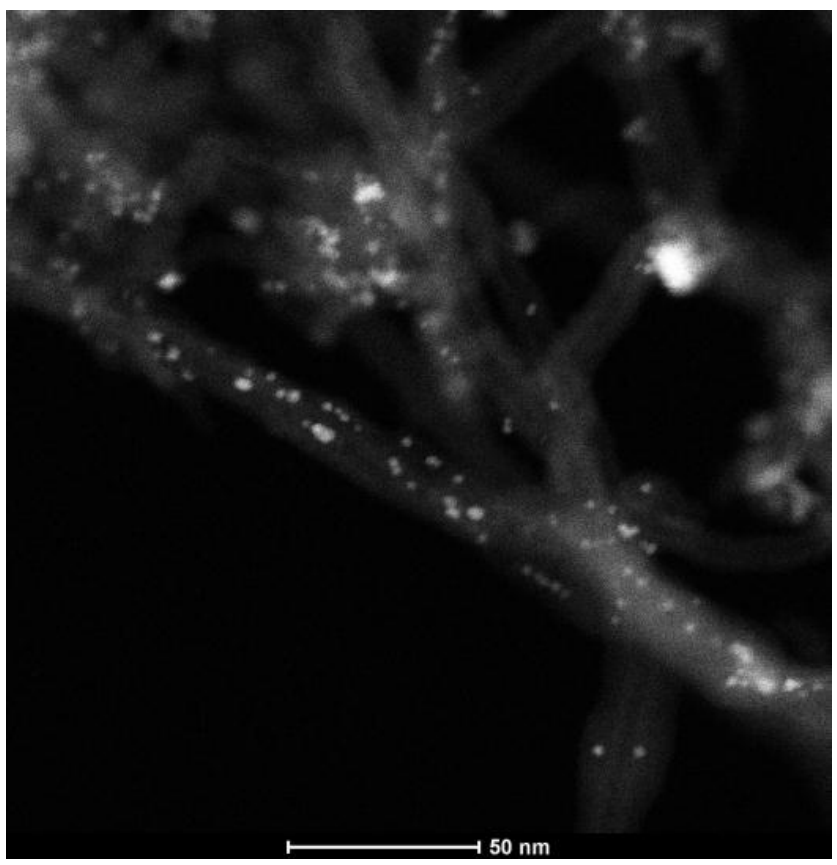

Figure 2.76. TEM Micrograph of the Baseline Rh-Mn Sample Tested to $\sim 276^{\circ} \mathrm{C}$ (Run 80 in Table 2.4) Supported on Hyperion Fibril MWCNT 
Perhaps most interesting in the spent MWCNT-supported Rh-Mn catalysts was the appearance of plate-like species of considerable size (note the relatively large-scale bar of $200 \mathrm{~nm}$ in Figure 2.77). The plates appear to be interwoven into the MWCNT nests, making contamination unlikely (see Figure 2.77). The plates appear to have a highly defined layered orientation as illustrated in Figure 2.78. The plates also appear to have a highly ordered rectangular shape (Figure 2.79).

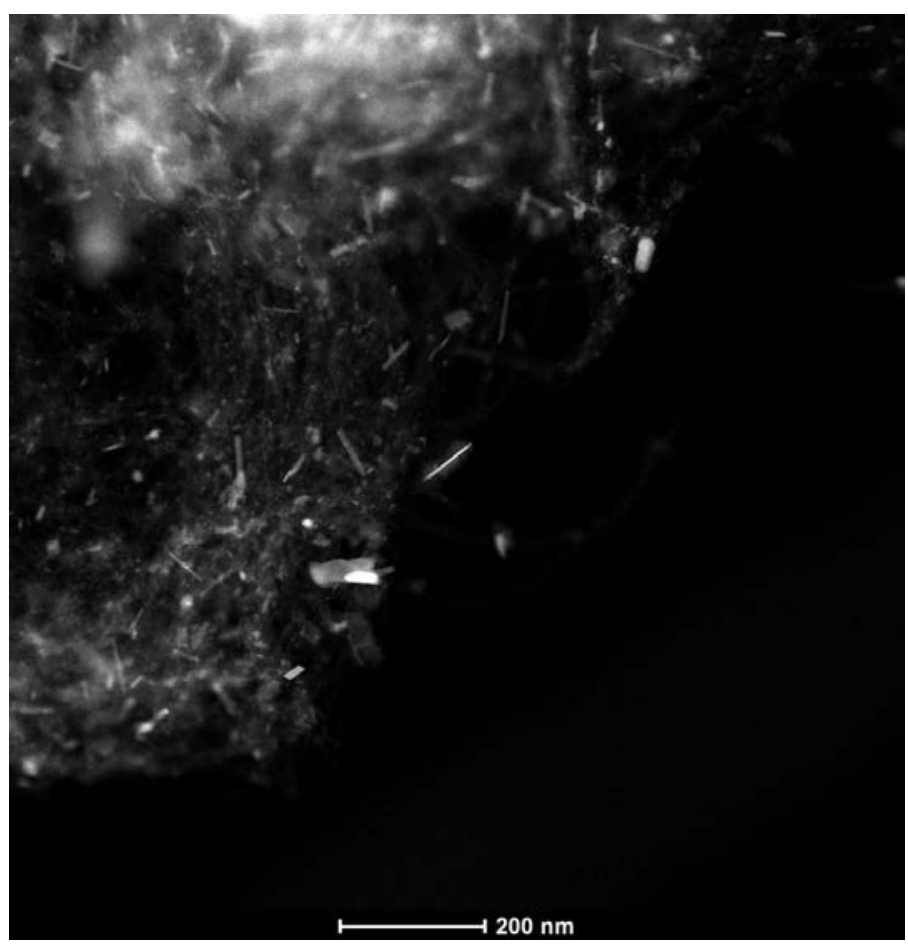

Figure 2.77. TEM Micrograph of the Baseline Rh-Mn Sample Tested to $\sim 276^{\circ} \mathrm{C}$ (Run 80 in Table 2.4) Supported on Hyperion Fibril MWCNT, Illustrating the Presence of Metallic Plates that were Observed throughout the Sample 


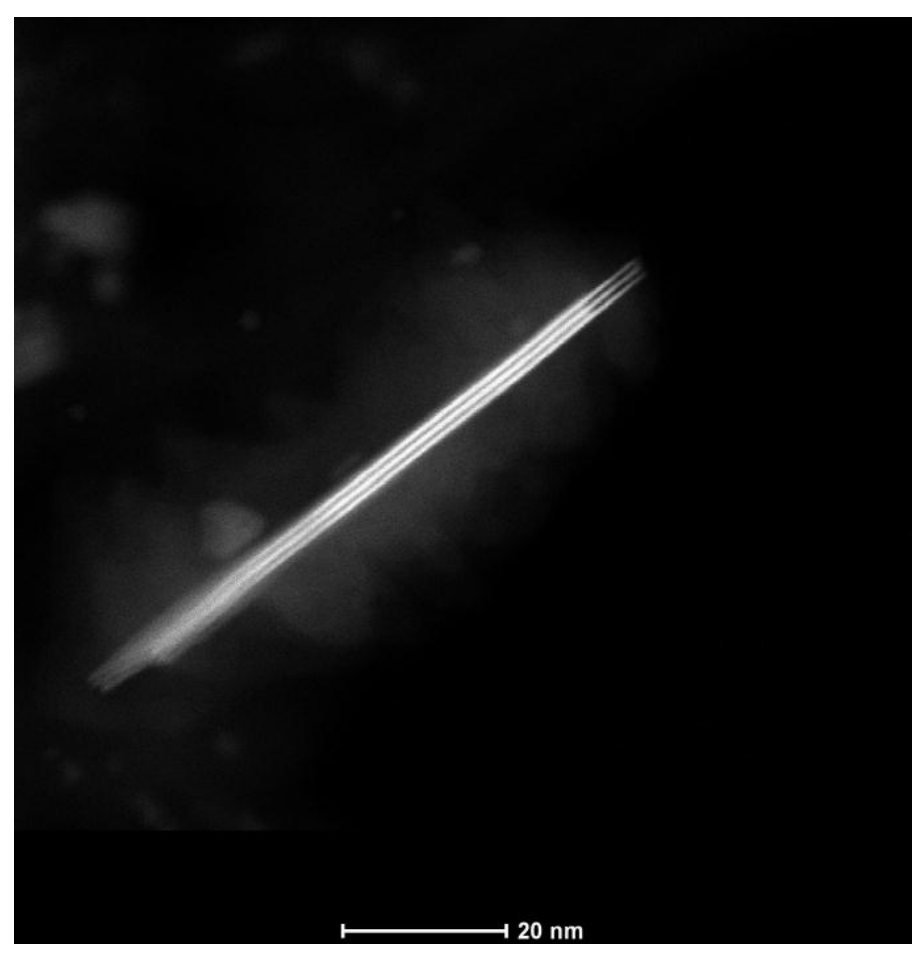

Figure 2.78. Higher Magnification TEM Micrograph of the Baseline Rh-Mn Sample Tested to $\sim 276^{\circ} \mathrm{C}$ (Run 80 in Table 2.4) Supported on Hyperion Fibril MWCNT with a Cross-Section View of a Metallic Plate Formed after Metallic Synthesis

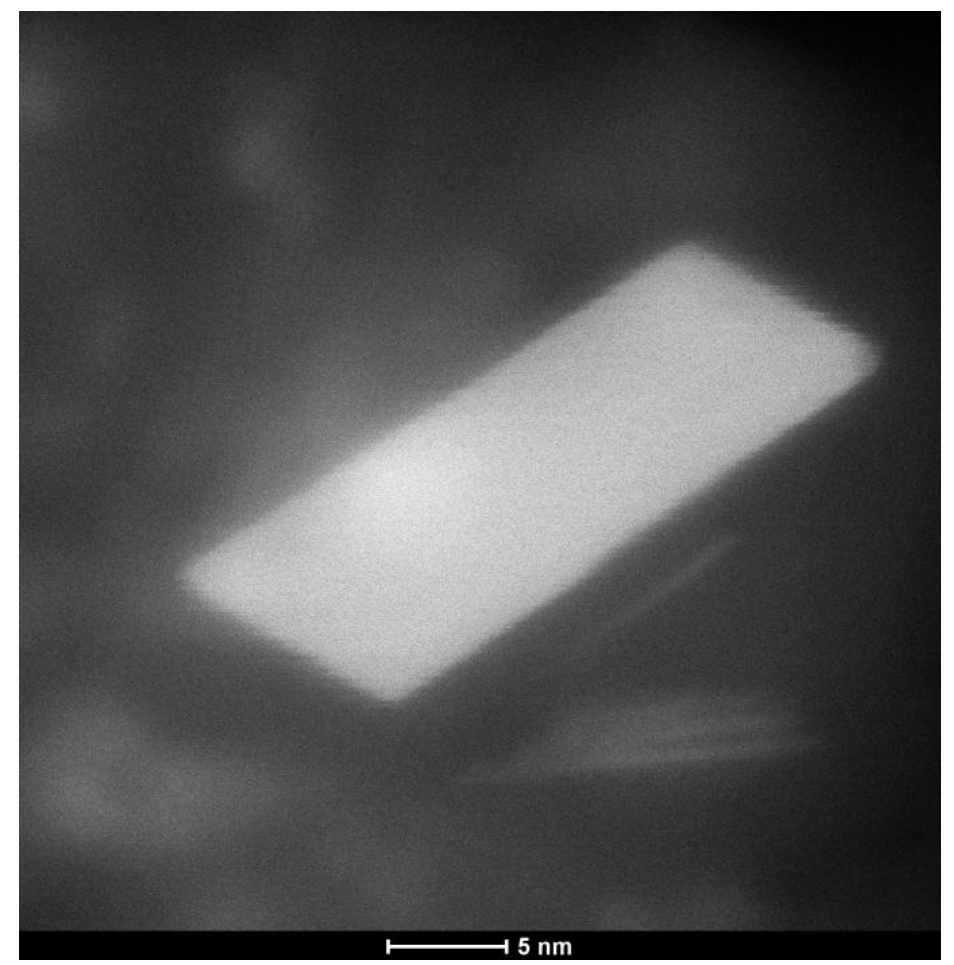

Figure 2.79. Higher Magnification TEM Micrograph of the Baseline Rh-Mn Sample Tested to $\sim 276^{\circ} \mathrm{C}$ (Run 80 in Table 2.4) Supported on Hyperion Fibril MWCNT with a Top-View Picture of a Metallic Plate that Formed after Mixed Alcohol Synthesis 
The plates also appear in the MWNCT-supported Rh-Mn catalysts tested in a single-tube reactor to a temperature of $299^{\circ} \mathrm{C}$ (Run 82 in Table 2.4), further reducing the likelihood that the plates are a symptom of contamination (Figure 2.80). The higher magnification micrograph in Figure 2.81 shows that there are still a number of small metal particles attached to the CNTs even after testing at $299^{\circ} \mathrm{C}$. EDS analysis of the plates was again challenging for reasons discussed previously. However, it can be generally stated that while significant coarsening and growth of the metallic plates was observed after reaction testing compared with the reduced catalyst 60074-70-C-R, there was not a significant difference in coarsening between the Run $80\left(276^{\circ} \mathrm{C}\right)$ and Run $82\left(299^{\circ} \mathrm{C}\right)$ samples.

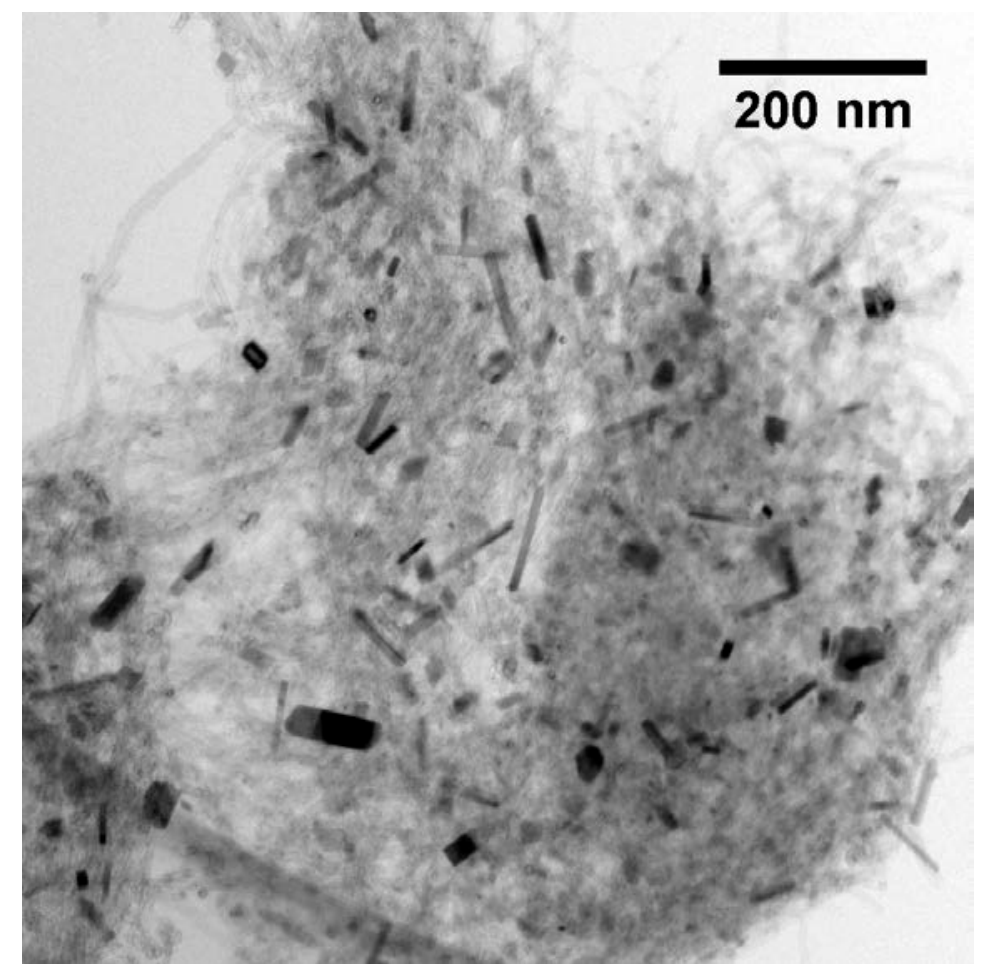

Figure 2.80. TEM Micrograph of the Baseline Rh-Mn Sample Tested to $\sim 299^{\circ} \mathrm{C}$ (Run 82 in Table 2.4) Supported on Hyperion Fibril MWCNT. Note the presence of the plates similar to the micrographs from Run 80 (Figure 2.74 and Figure 2.75). 


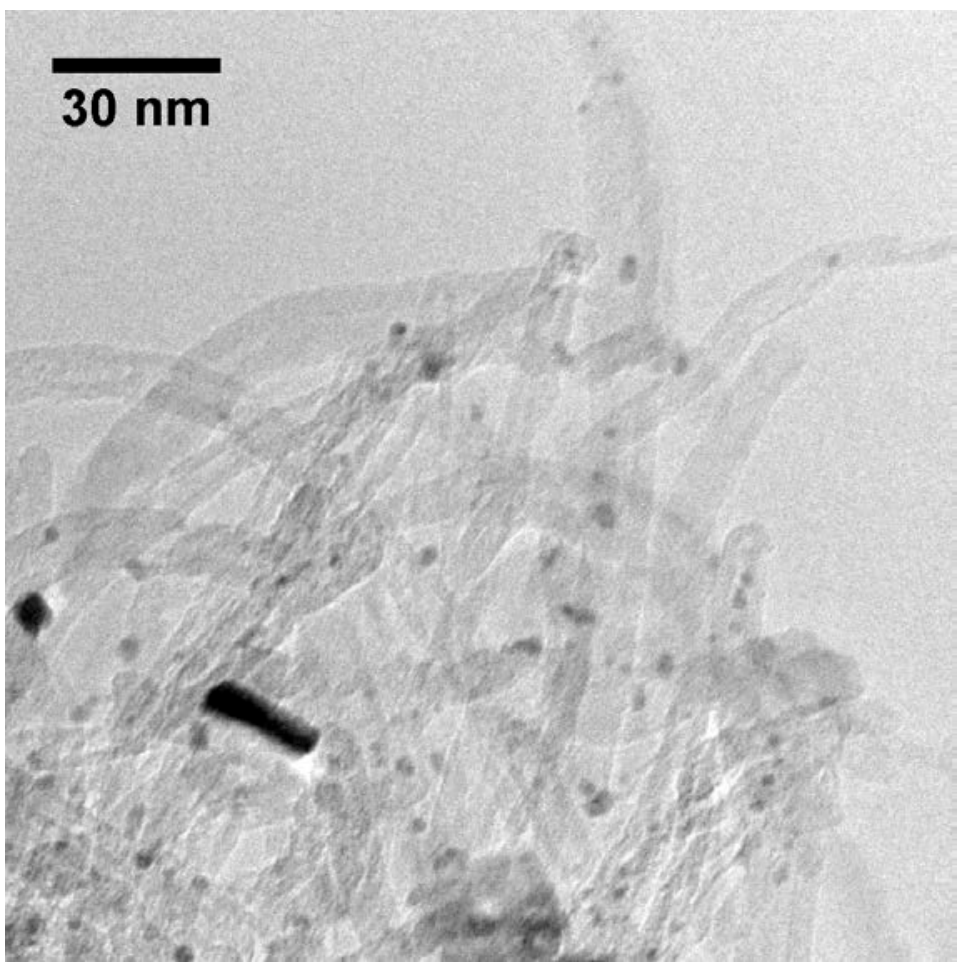

Figure 2.81. Higher Magnification TEM Micrograph of the Baseline Rh-Mn Sample Tested to $299^{\circ} \mathrm{C}$ (Run 82 in Table 2.4) Supported on Hyperion Fibril MWCNT

\subsubsection{Hyperion Fibril MWCNT-Supported Rh-Mn-Ir Baseline Catalysts}

The Rh-Mn-Ir catalyst supported on Hyperion Fibril MWCNT supports (CS-02C-063-XD Lot 38482) also was subjected to TEM measurements at various points in the catalyst lifecycle. The catalyst consisted of $11.78 \mathrm{wt} \% \mathrm{Rh}, 3.58 \mathrm{wt} \% \mathrm{Mn}$, and $2.19 \mathrm{wt} \%$ Ir (sample 60074-74-C in Table 2.4). The freshly prepared catalyst was first analyzed with TEM and a representative micrograph is shown in Figure 2.82. Similar to the freshly prepared MWCNT-supported Rh-Mn catalyst 60074-70-C shown in Figure 2.73, small black metal particles are observed to be relatively well dispersed on the carbon nanotubes. 


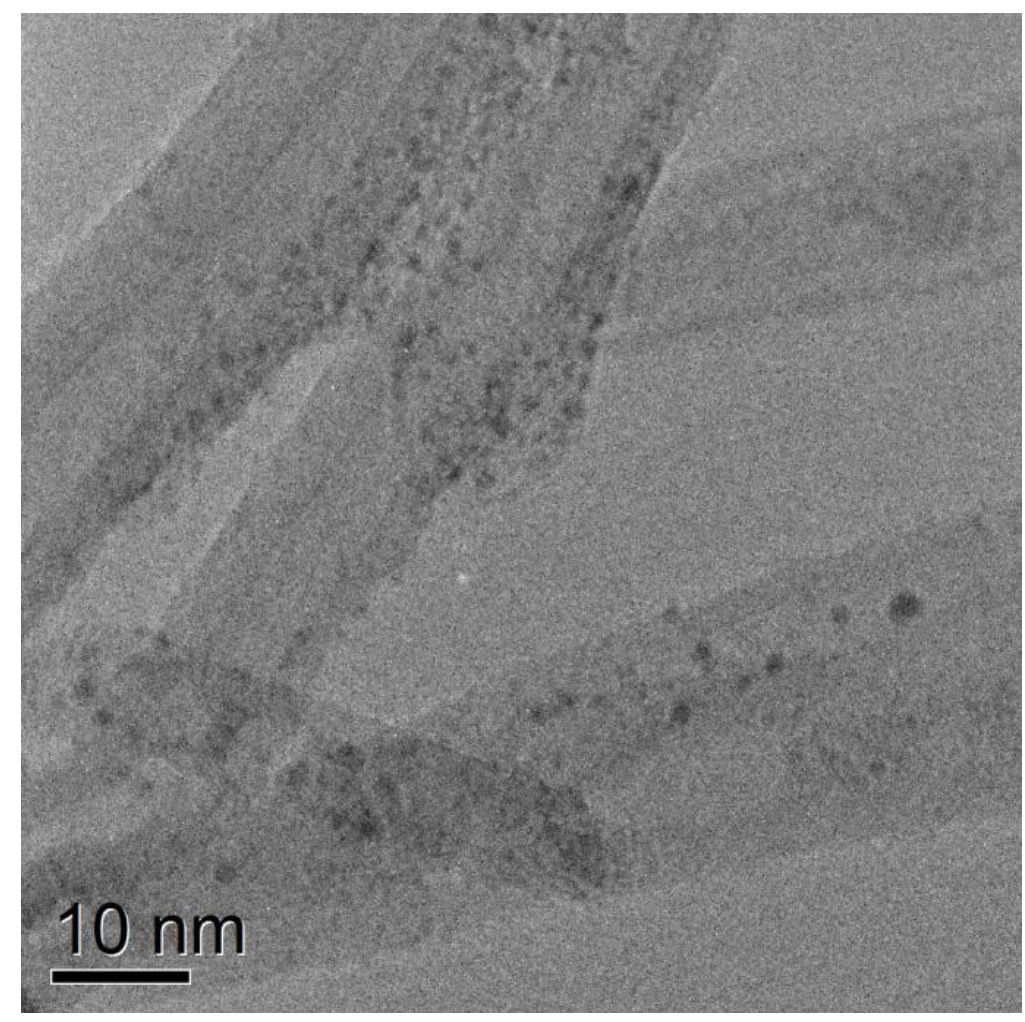

Figure 2.82. Higher Magnification TEM Micrograph of the Freshly Prepared Baseline Rh-Mn-Ir (sample 60074-74-C in Table 2.4) Supported on Hyperion Fibril MWCNT

While the freshly prepared Rh-Mn and Rh-Mn-Ir catalysts supported on MWCNTs were similar, there were starker differences when the catalysts were reduced. Whereas the Rh-Mn catalyst did not show significant grain growth or sintering after reduction (e.g., Figure 2.73 versus Figure 2.75), the reduced Rh-Mn-Ir catalyst (60074-59-C-R in Table 2.4) did not exhibit any distinct clusters of metal. Instead, the carbon nanotubes appeared to be coated with amorphous material. It is possible that the metal clusters were more raft-like (i.e., flat and close to the nanotube surface) than the larger $\sim 2 \mathrm{~nm}$ particles observed elsewhere. It also is possible that the metals were the amorphous material coating the nanotubes. This would require that the metal particles observed in the freshly prepared catalyst (Figure 2.82) dispersed over the tubes. Elemental analysis on the coated nanotubes was inconclusive. Initially, a small amount of Rh may have been observed, but after about a minute of being subjected to the electron beam a new peak formed that was near Mn, but did not exactly match Mn or any other reasonable element. It is likely that the amorphous coating is metallic as the tubes appear brighter in the TEM micrographs than would be expected for tubes composed solely of carbon.

The amorphous coating hypothesis may fit with the observation that some of the nanotubes were asymmetric with thicker walls on one side (see Figure 2.84). If metal is amorphously coating the outside of the MWCNTs during reduction, it is possible that in some instances the coating may be asymmetric, resulting in thicker walls on one side of a tube. Asymmetric MWCNTs were not observed in any other samples.

While it can be neither ruled out nor affirmed that the Rh-Mn-Ir metal mixture is dispersing under reduction conditions to coat the carbon nanotubes, more investigation is required to understand if sample 60074-74-C-R is anomalous or if the Rh-Mn-Ir mixture truly coats the reduced catalyst amorphously. 


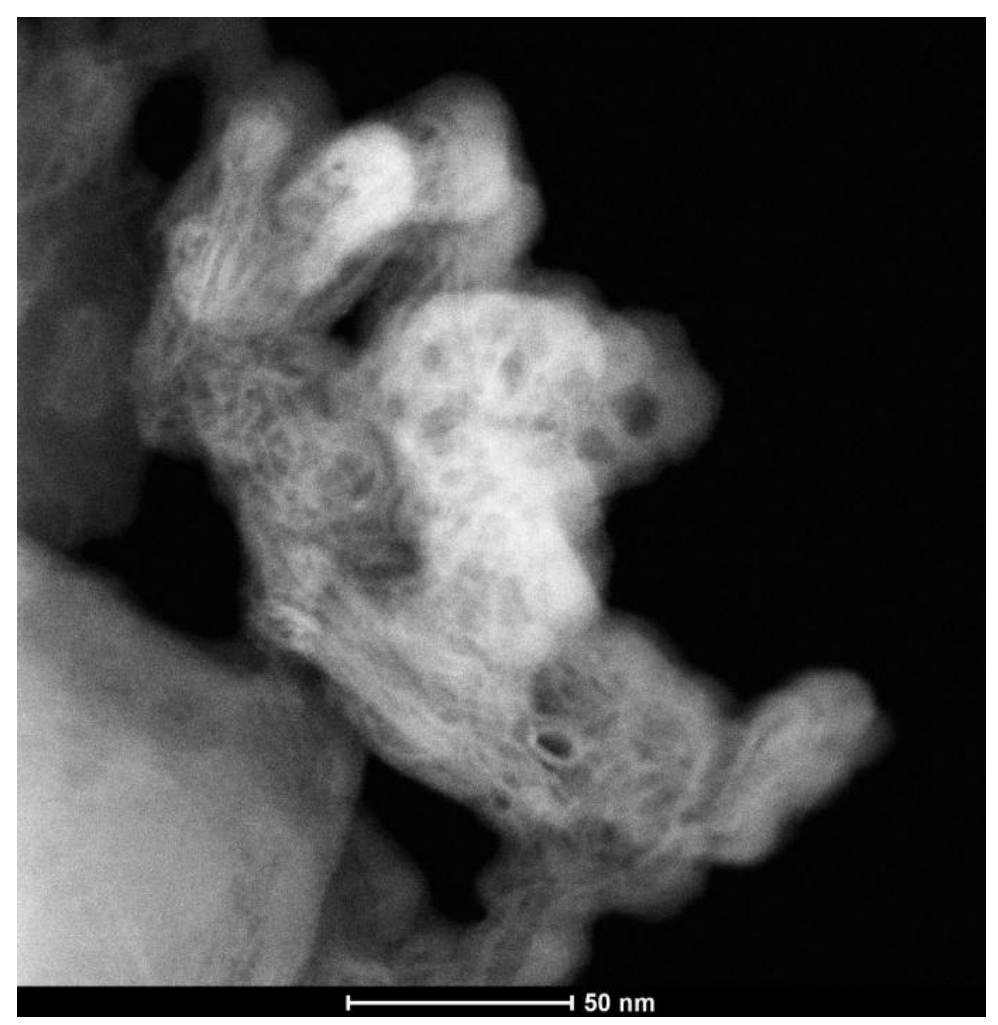

Figure 2.83. TEM Micrograph of the Reduced Baseline Rh-Mn-Ir (sample 60074-74-C-R in Table 2.4) Supported on Hyperion Fibril MWCNT 


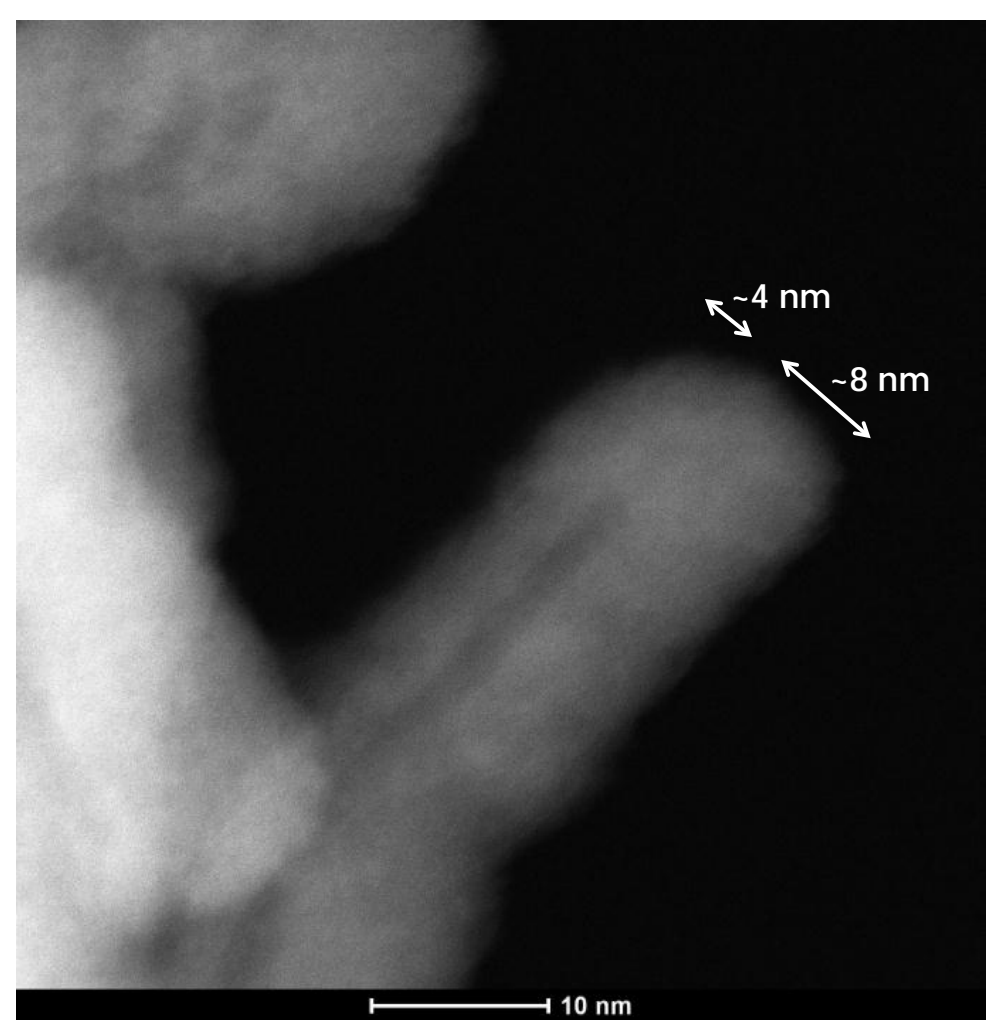

Figure 2.84. Higher Magnification TEM Micrograph of the Reduced Baseline Rh-Mn-Ir (sample 6007474-C-R in Table 2.4) Supported on Hyperion Fibril MWCNT. The scale dimensions in the micrograph illustrate the asymmetric nature of some of the carbon nanotubes.

Next, TEM measurements were performed on the MWCNT-supported Rh-Mn-Ir supported catalysts subjected to single-tube reactor testing up to $275^{\circ} \mathrm{C}$ (Run 89 in Table 2.4). As can be seen in Figure 2.85 and Figure 2.86, individual metal particles can be observed again unlike in the freshly reduced sample 60074-74-C-R. There is also the presence of very large metal particles on the size range of 10 to $100 \mathrm{~nm}$. The large particles seemed to consist of either large single crystals or from several grains stuck together, which would indicate sintering. A Z-contrast (image contrast based on atomic number) was performed, but there was no indication that different sized particles had different elemental compositions. For example, there was no evidence that the very large particles contained high concentrations of Rh, which would indicate preferential $\mathrm{Rh}$ rejection or sintering of $\mathrm{Rh}$. 


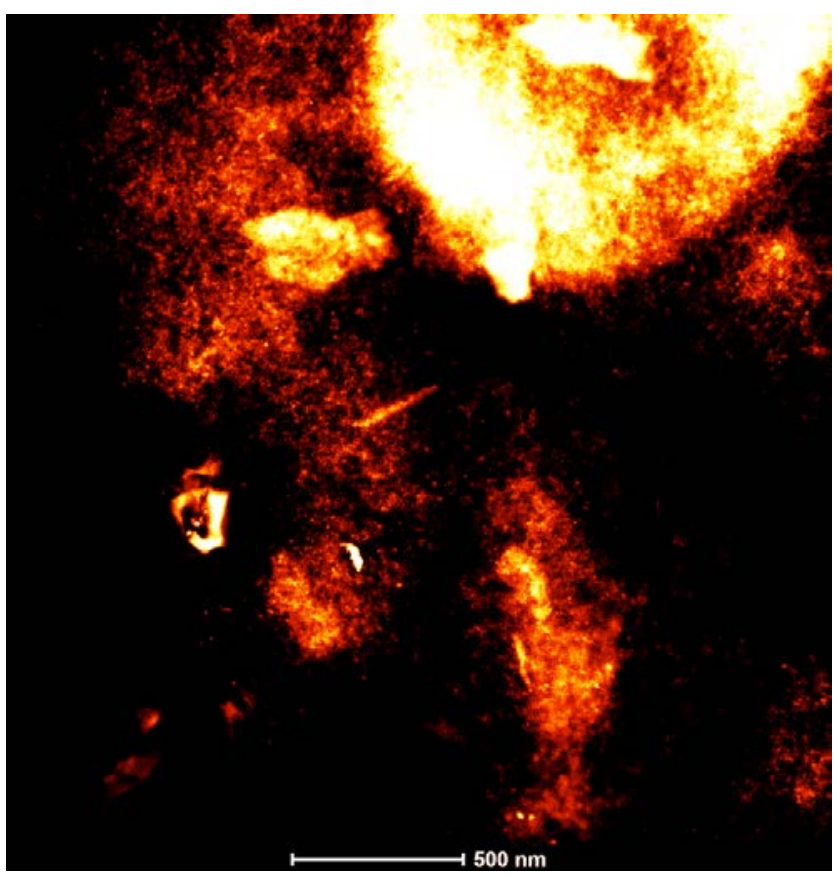

Figure 2.85. TEM Micrograph of the Baseline Rh-Mn-Ir that Experienced a Temperature of $275^{\circ} \mathrm{C}$ (Run 89 in Table 2.4) Supported on Hyperion Fibril MWCNT

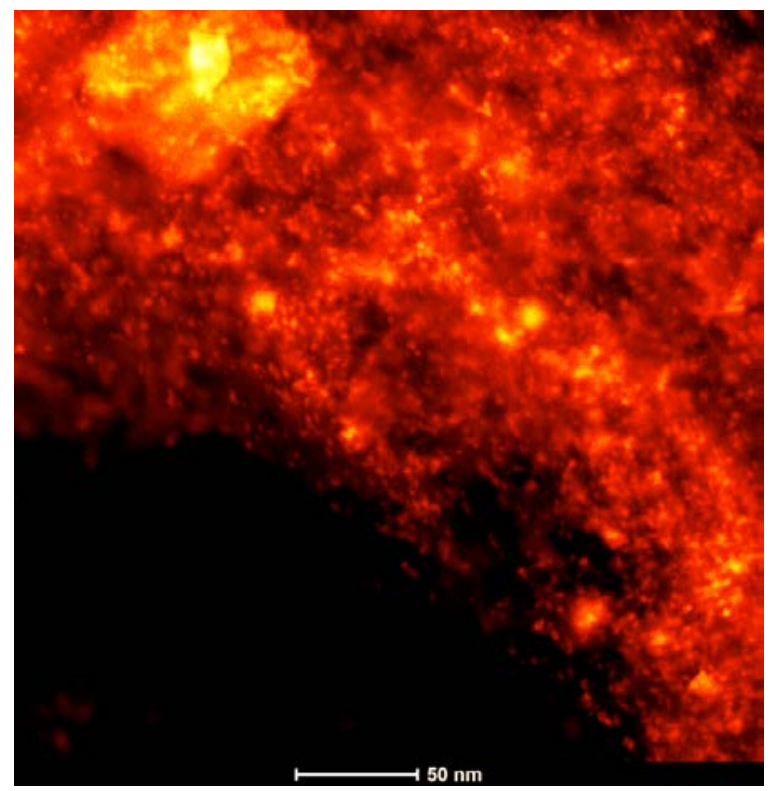

Figure 2.86. Higher Magnification TEM Micrograph of the Baseline Rh-Mn-Ir that Experienced a Temperature of $275^{\circ} \mathrm{C}$ (Run 89 in Table 2.4) Supported on Hyperion Fibril MWCNT

Figure 2.86 and Figure 2.87 show the MWCNT-supported Rh-Mn-Ir catalyst subjected to $\sim 314^{\circ} \mathrm{C}$ (Run 88 in Table 2.4). The size of the metal particles observed in Run 89 at $275^{\circ} \mathrm{C}$ and Run 88 at $314^{\circ} \mathrm{C}$ were similar, which suggests that increased sintering was not responsible for any differences between the catalytic behavior of the catalyst after testing at the two temperatures. Small relatively well-dispersed particles (Figure 2.87) as well as larger particles (Figure 2.88) were observed again. 


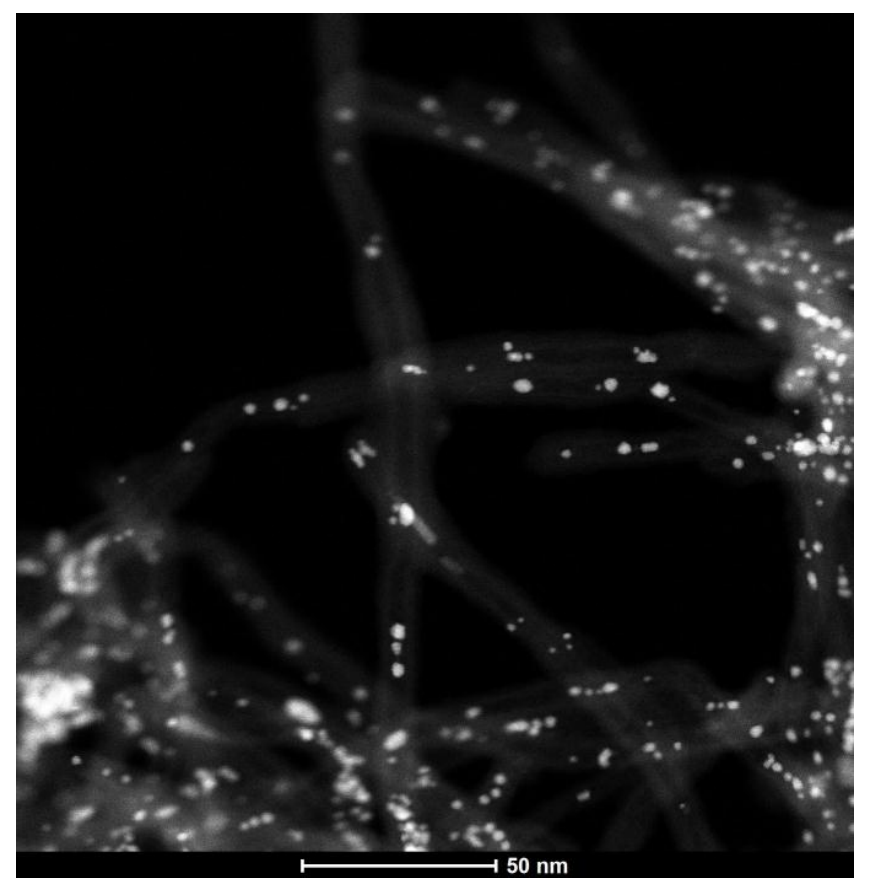

Figure 2.87. TEM Micrograph of the Baseline Rh-Mn-Ir that Experienced a Temperature of $314^{\circ} \mathrm{C}$ (Run 88 in Table 2.4) Supported on Hyperion Fibril MWCNT

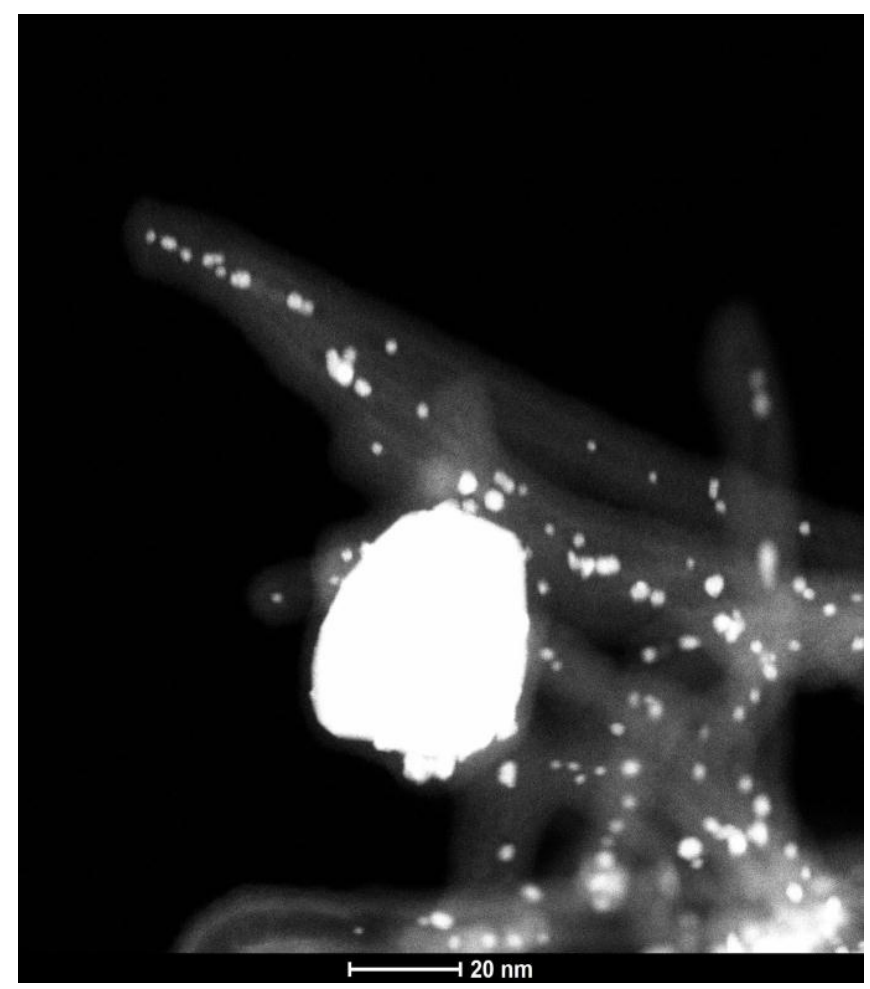

Figure 2.88. Higher Magnification TEM Micrograph of the Baseline Rh-Mn-Ir that Experienced a Temperature of $314^{\circ} \mathrm{C}$ (Run 88 in Table 2.4) Supported on Hyperion Fibril MWCNT. The large metallic clump in the center of the micrograph illustrates one instance of the metallic grain coarsening (sintering) observed after mixed alcohol synthesis. 
A final observation for all of the MWCNT samples was that it is highly possible that there are actually metal clusters on the inside as well as on the outside of the MWCNTs. Some metal clusters were clearly attached to the outside of the metal tubes (see the blue arrow in Figure 2.89). However, Figure 2.90 shows several metal clusters that appear to align with the MWCNT and also appear to be very near the width of the inner diameter of the nanotube as indicated by the lighter shading of the nanotube. While it is possible that all of the clusters are exactly on either the top or the bottom of the nanotube with the tube being relatively transparent, it does seem unlikely.

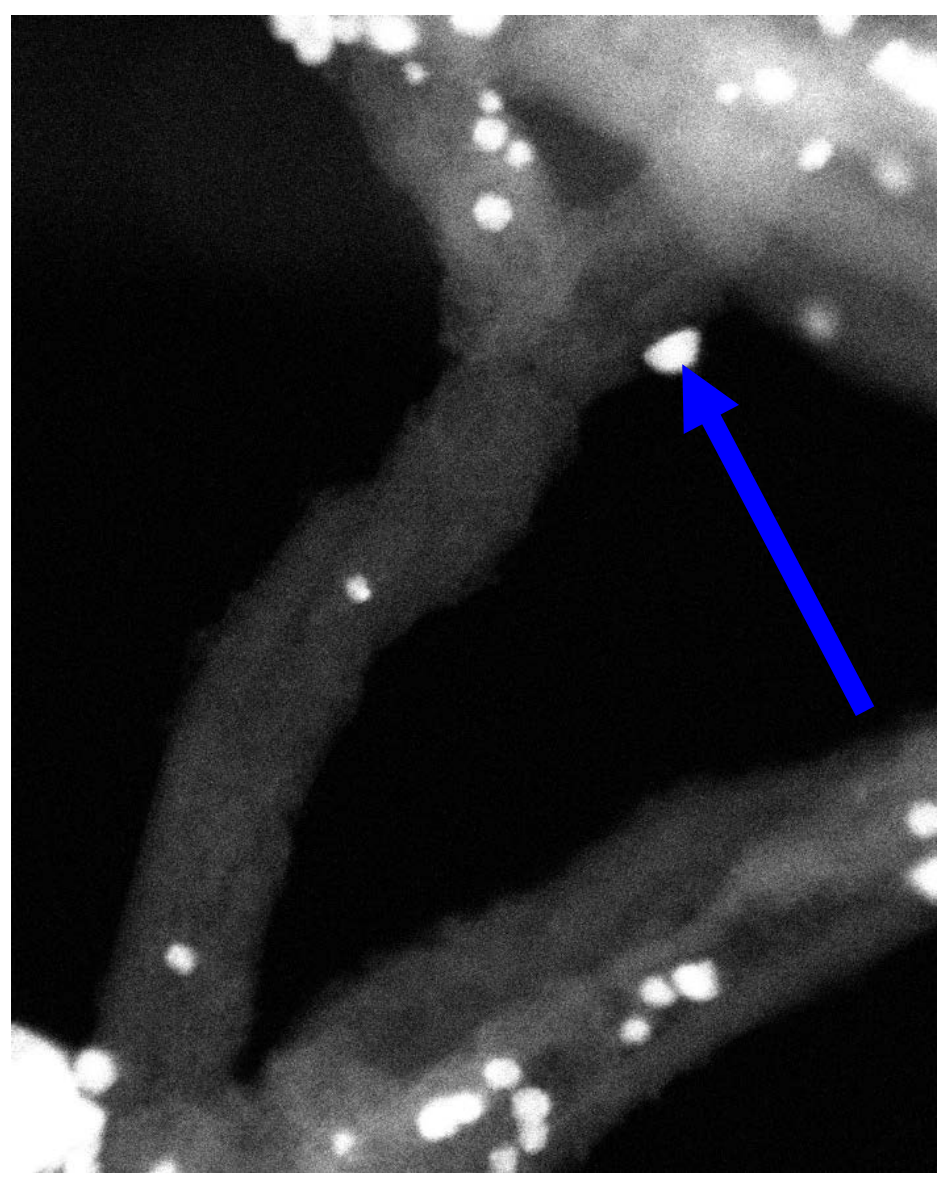

Figure 2.89. TEM Micrograph of the Baseline Rh-Mn-Ir that Experienced a Temperature of $314^{\circ} \mathrm{C}$ (Run 88 in Table 2.4) Supported on Hyperion Fibril MWCNT. The blue arrow indicates a metallic particle that is clearly on the outside of the carbon nanotubes. 


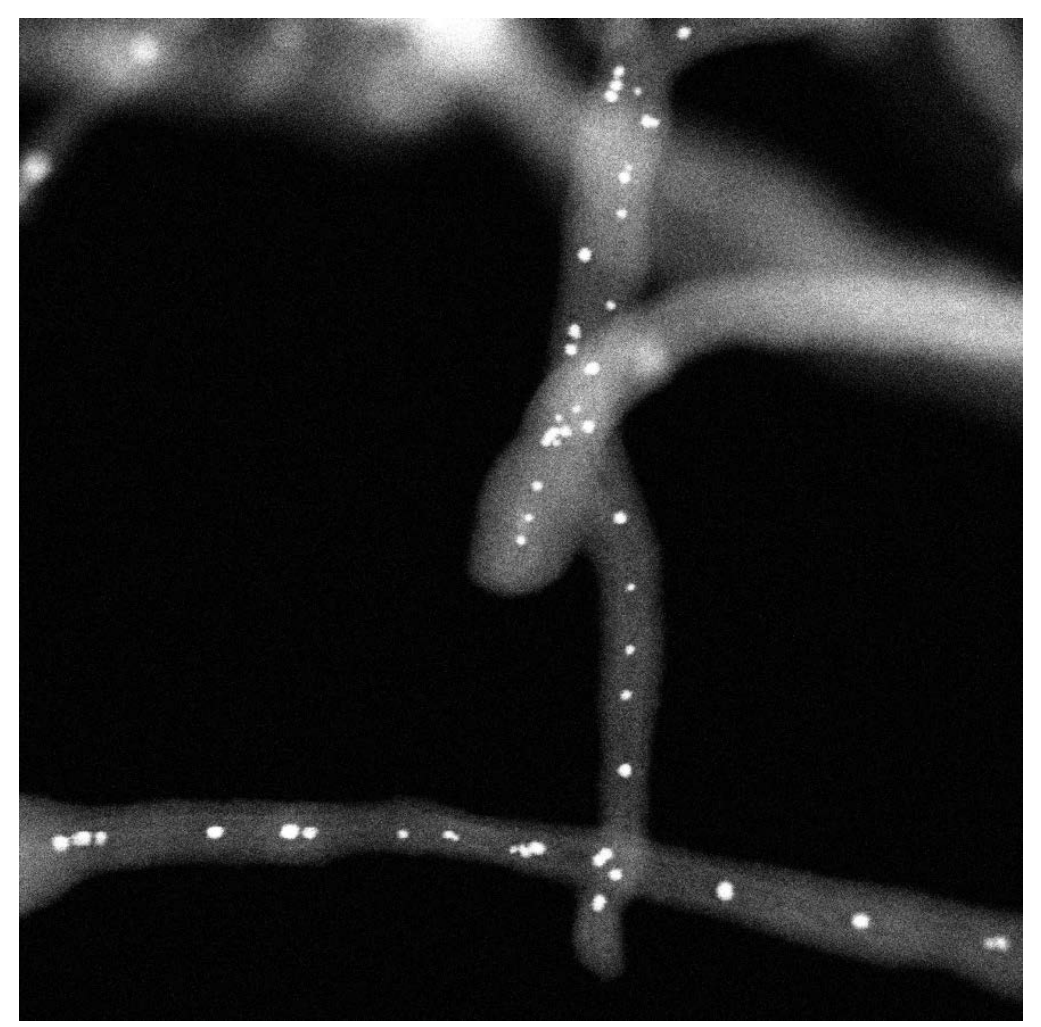

Figure 2.90. TEM Micrograph of the Baseline Rh-Mn-Ir that Experienced a Temperature of $314^{\circ} \mathrm{C}$ (Run 88 in Table 2.4) Supported on Hyperion Fibril MWCNT, Showing Metal Particles (bright spots) that are Likely Inside the MWCNTs

\subsubsection{Other Fresh and Spent Catalysts}

Catalyst samples listed in Table 2.6 were subjected to TEM. Selected micrographs of catalyst 60074-56-A, which consisted of $8.34 \mathrm{wt} \% \mathrm{Rh}$ and $2.54 \mathrm{wt} \% \mathrm{Mn}$ on sample KOA 6 (Hyperion 395-08), are shown in Figure 2.91 to Figure 2.93. Likewise, micrographs of catalyst 60074-59-C are shown in Figure 2.94 to Figure 2.96. Catalyst $60074-59-\mathrm{C}$ consisted of $11.778 \mathrm{wt} \% \mathrm{Rh}, 3.587 \mathrm{wt} \% \mathrm{Mn}$, and 2.189\% Ir on sample KOA 8 (Hyperion 384-82). It is worth mentioning that the metals in these catalysts are still present as nitrates ( $\mathrm{Rh}$ and $\mathrm{Mn}$ ) or chloride (Ir) as no calcination or reduction treatment had been performed on these catalysts.

The TEM micrographs of catalysts 60074-56-A and 60074-59-C are similar in that the metals are very well dispersed in all micrographs. The metal particles (presented as black dots due to the data collection mode of the TEM), which are on the size range of $\sim 2 \mathrm{~nm}$, appear as small black dots on the outside of the "spaghetti-like" carbon nanotubes in the micrographs with scale bars of $20 \mathrm{~nm}$ (Figure 2.93, Figure 2.95, and Figure 2.96). There is some evidence of higher concentrations of the impregnated metals where the density of the nanotubes is higher. In other words, it can be stated qualitatively that while the metals are very well dispersed on the carbon nanotubes, the concentration of the impregnated metals is higher where several carbon nanotubes are intertwined. The high dispersion and small particle size of the metal nitrates is consistent with Micro-XRD data, which revealed no crystalline component contributable to the metal precursors in the catalysts. 


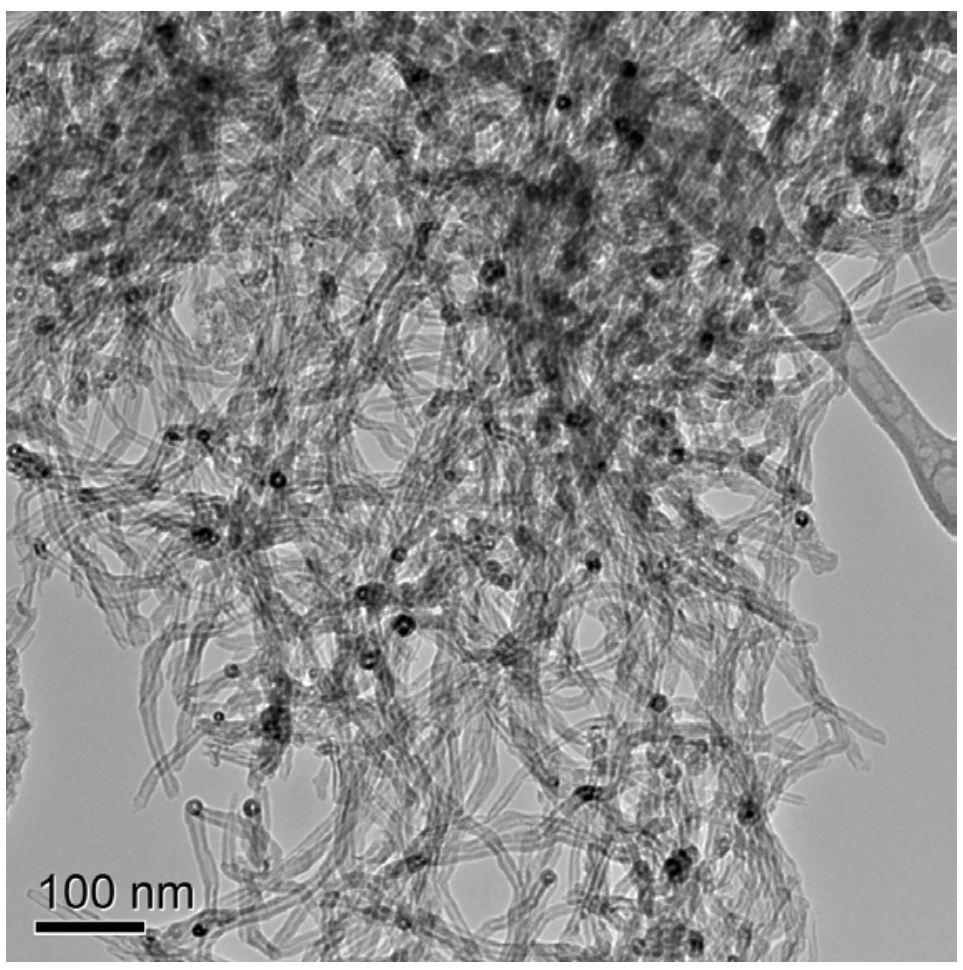

Figure 2.91. TEM Micrograph of Catalyst 60074-56-A, which Consists of $8.34 \mathrm{wt} \% \mathrm{Rh}$ and $2.54 \mathrm{wt} \%$ Mn on Hyperion 395-08

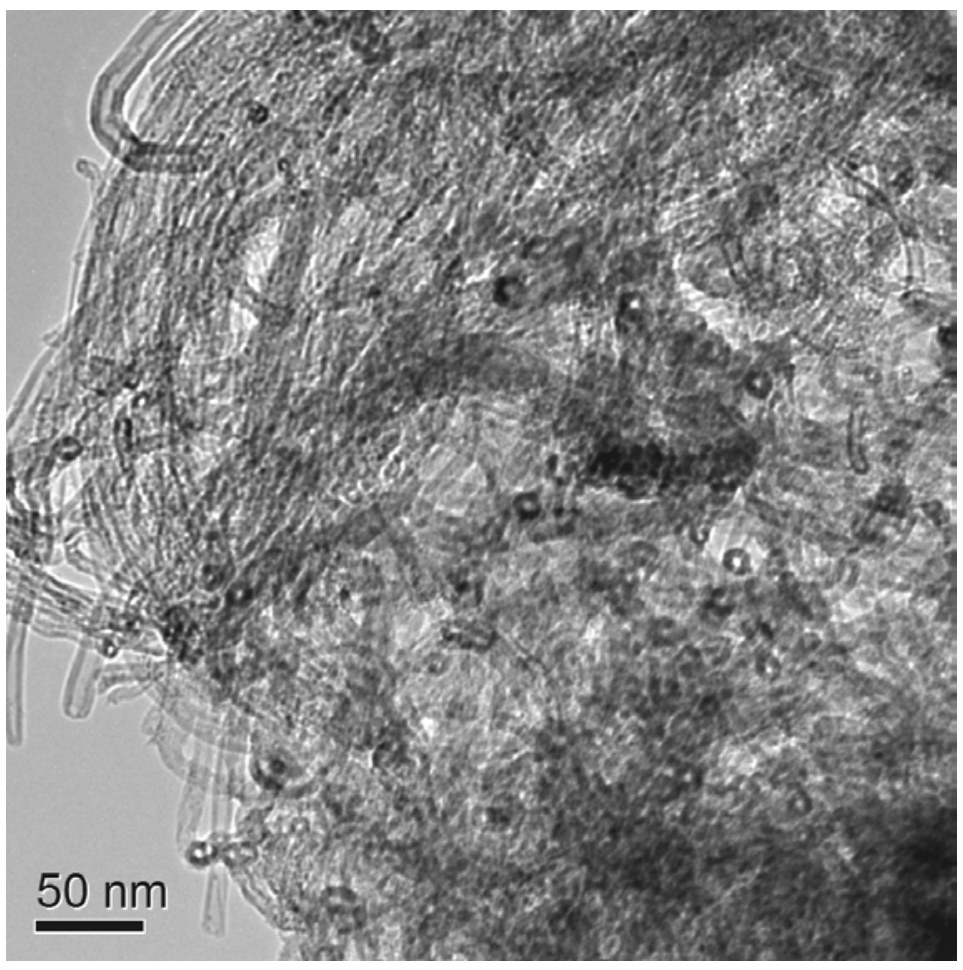

Figure 2.92. TEM Micrograph of Catalyst 60074-56-A, which Consists of $8.34 \mathrm{wt} \% \mathrm{Rh}$ and $2.54 \mathrm{wt} \%$ Mn on Hyperion 395-08 


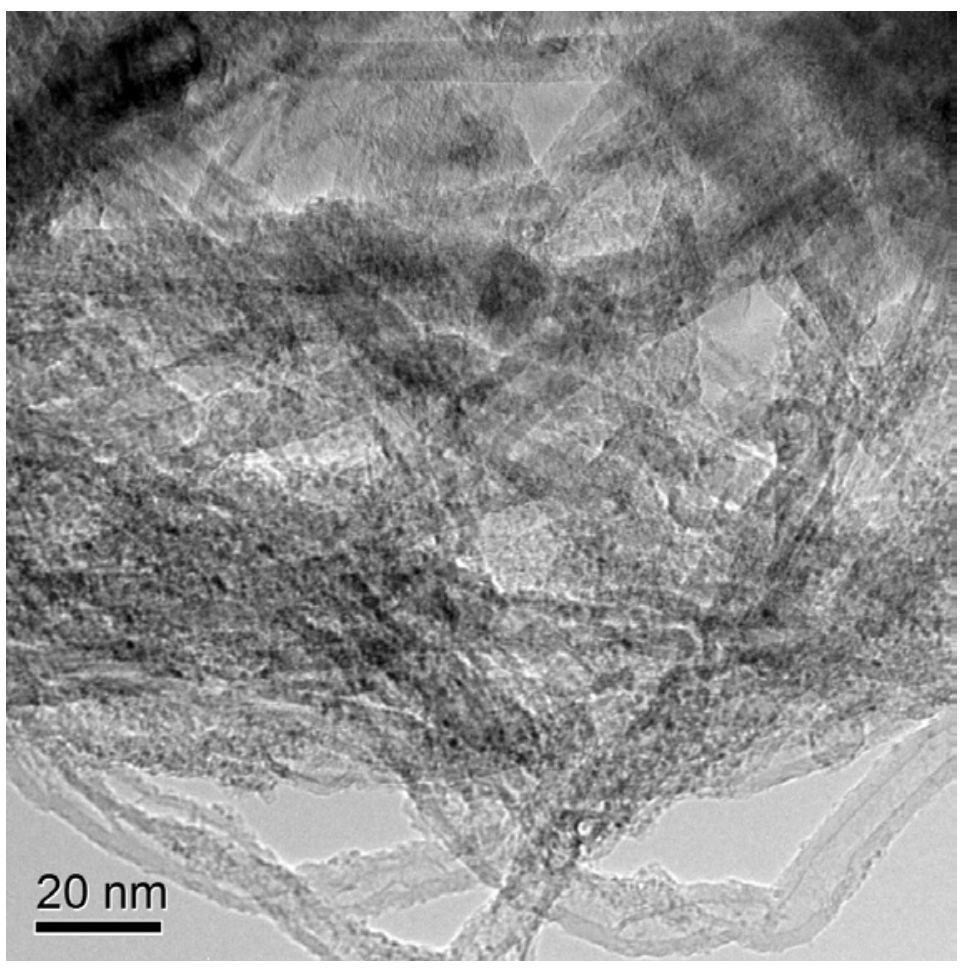

Figure 2.93. TEM Micrograph of Catalyst 60074-56-A, which Consists of $8.34 \mathrm{wt} \% \mathrm{Rh}$ and $2.54 \mathrm{wt} \%$ Mn on Hyperion 395-08

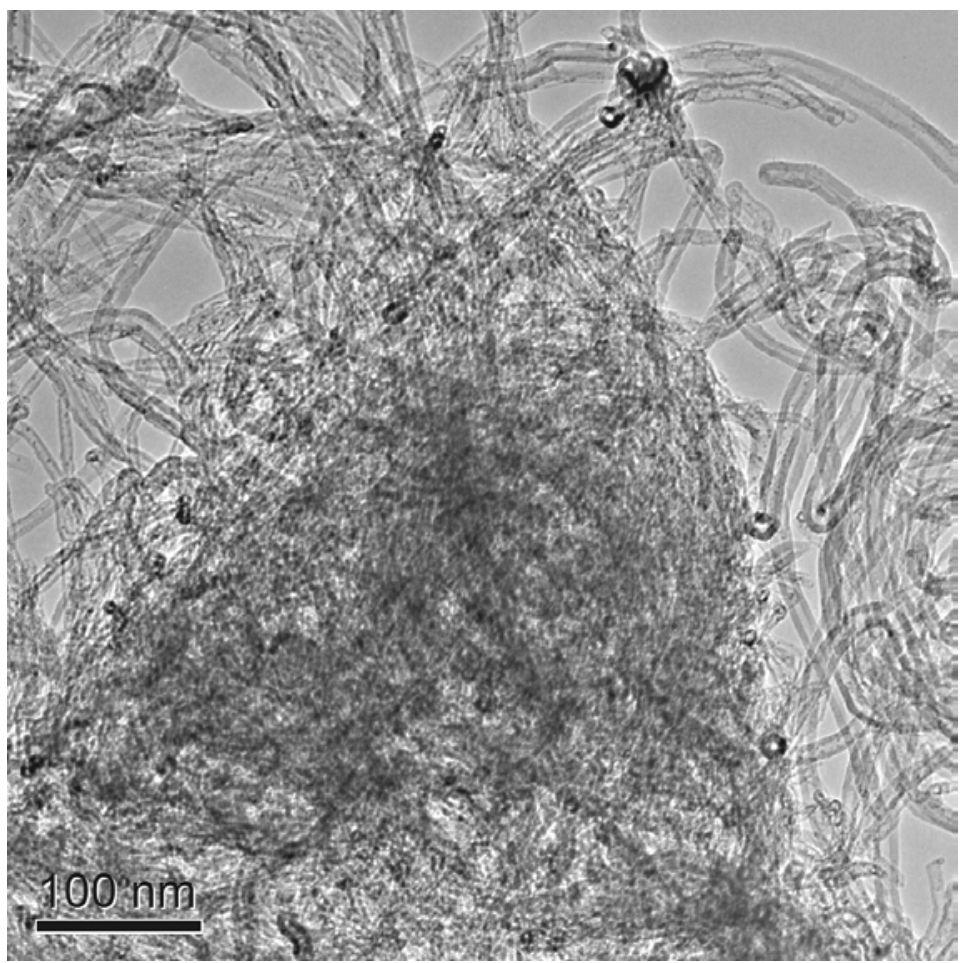

Figure 2.94. TEM Micrograph of Catalyst 60074-59-C, which Consists of 11.778 wt\% Rh, 3.587 wt\% Mn, and 2.189 wt\% Ir on Hyperion 384-82 


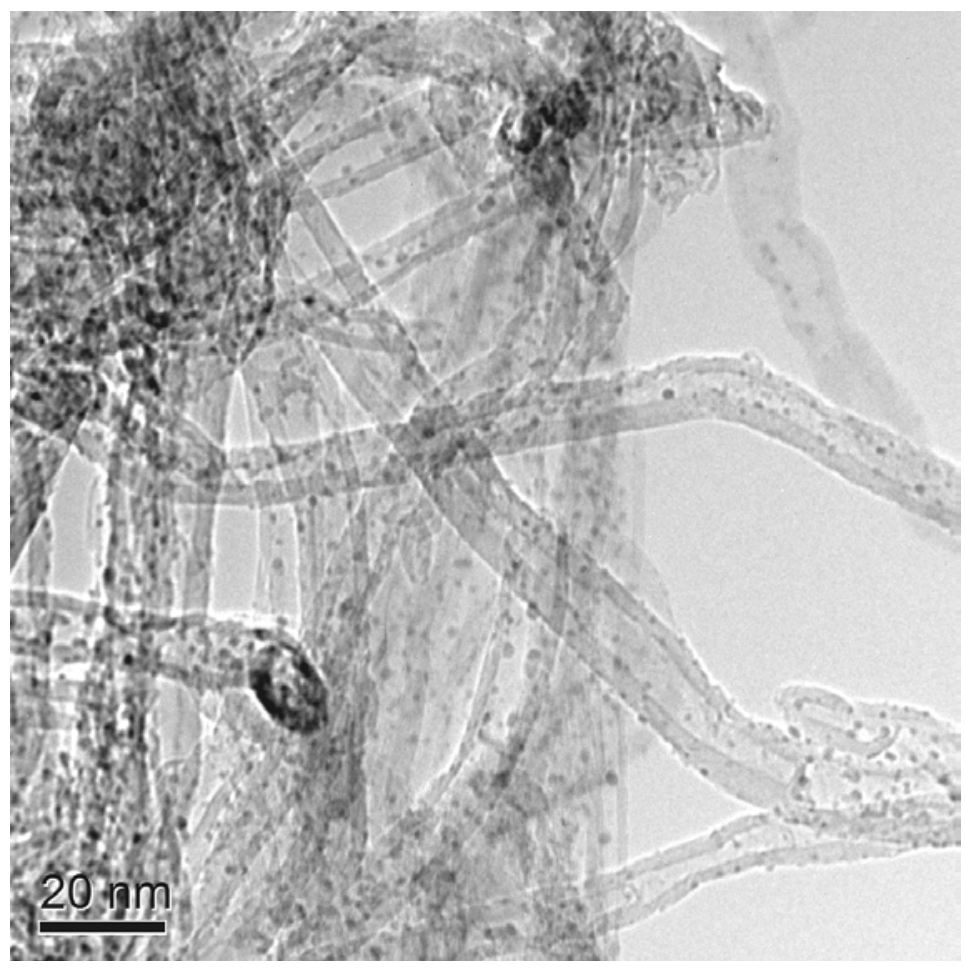

Figure 2.95. TEM Micrograph of Catalyst 60074-59-C, which Consists of $11.778 \mathrm{wt} \% \mathrm{Rh}$, 3.587 wt\% Mn, and 2.189 wt\% Ir on Hyperion 384-82

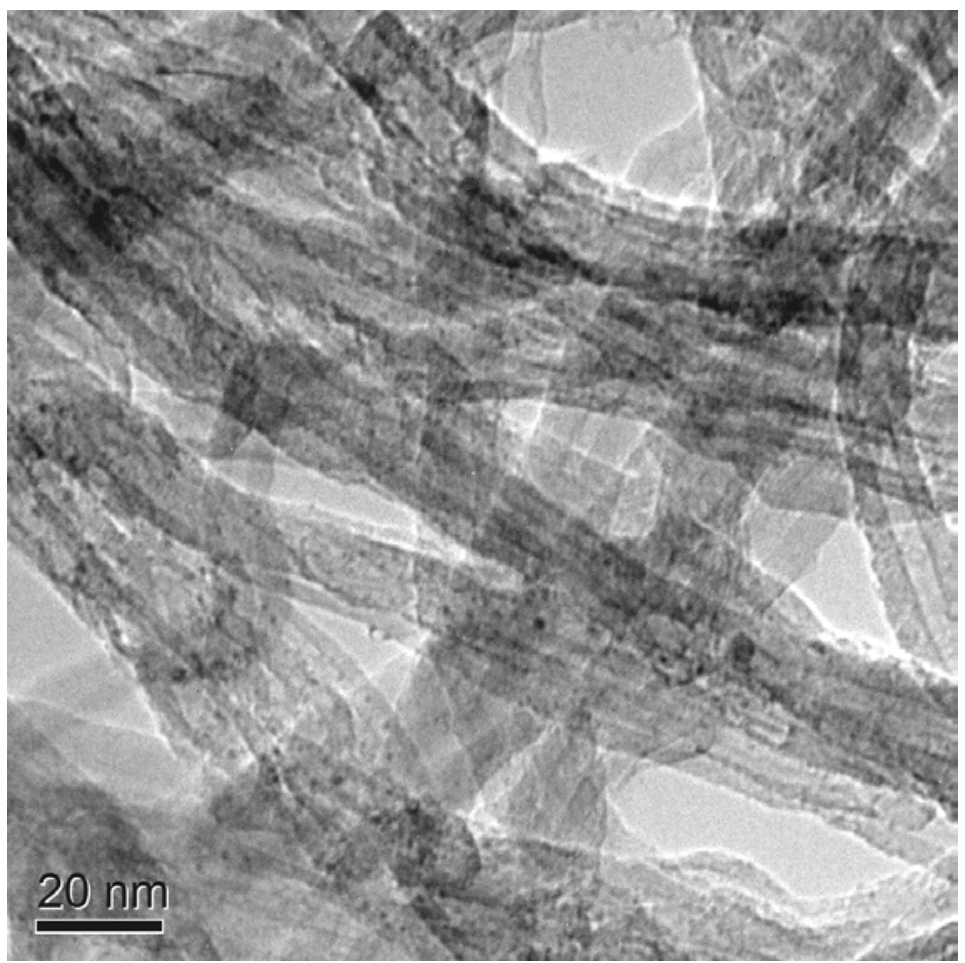

Figure 2.96. TEM Micrograph of Catalyst 60074-59-C, which Consists of $11.778 \mathrm{wt} \% \mathrm{Rh}$, 3.587 wt\% Mn, and 2.189 wt\% Ir on Hyperion 384-82 


\subsection{Baseline Catalyst Reaction Testing}

The baseline catalysts listed in Table 2.4 were tested in a single-tube, fixed-bed reactor. The test conditions each catalyst are outlined in Chapter 2.1.3.1. Figure 2.97 and Figure 2.98 illustrate the STY and converted carbon selectivity for the Rh-Mn-Ir catalysts supported on Davisil 645 silica. Two data points are presented for each run in Figure 2.97 and Figure 2.98. The first data point in each figure was taken at the third test condition in Table 2.5. The data points labeled "repeat" are points after the catalyst had been tested previously at $275^{\circ} \mathrm{C}$. For the low temperature-tested catalyst (Run 84), the repeat data is given after a longer time on stream at $275^{\circ} \mathrm{C}$ (Table 2.5, Run 84, Condition 4). For the high-temperature test, the repeat data point is given after the temperature in the reactor was increased to $298^{\circ} \mathrm{C}$ and subsequently returned to $275^{\circ} \mathrm{C}$ (Table 2.5 , Run 86 , Condition 5). Previous experience indicated that catalyst activity and selectivity can vary significantly after operation at higher temperature and/or after longer time on streams. Thus, comparing similar conditions at different times during a run can be informative.

Figure 2.97 is an excellent representation of the variability observed in the activity of a given catalyst. Although the parent catalyst was from the same parent batch (60074-53-A) and subjected to identical reduction and start-up conditions, the catalyst loaded for Run 84 exhibited greater activity than when loaded for Run 86. Interestingly, Run 84 exhibited increasing STY with respect to time as exhibited by the $\sim 23 \%$ greater STY of the repeat data. In contrast, the Davisil 645 silica-supported Rh-Mn-Ir catalyst loaded for Run 86 exhibited significantly lower activity that diminished slightly after the reactor had been heated to $298^{\circ} \mathrm{C}$.

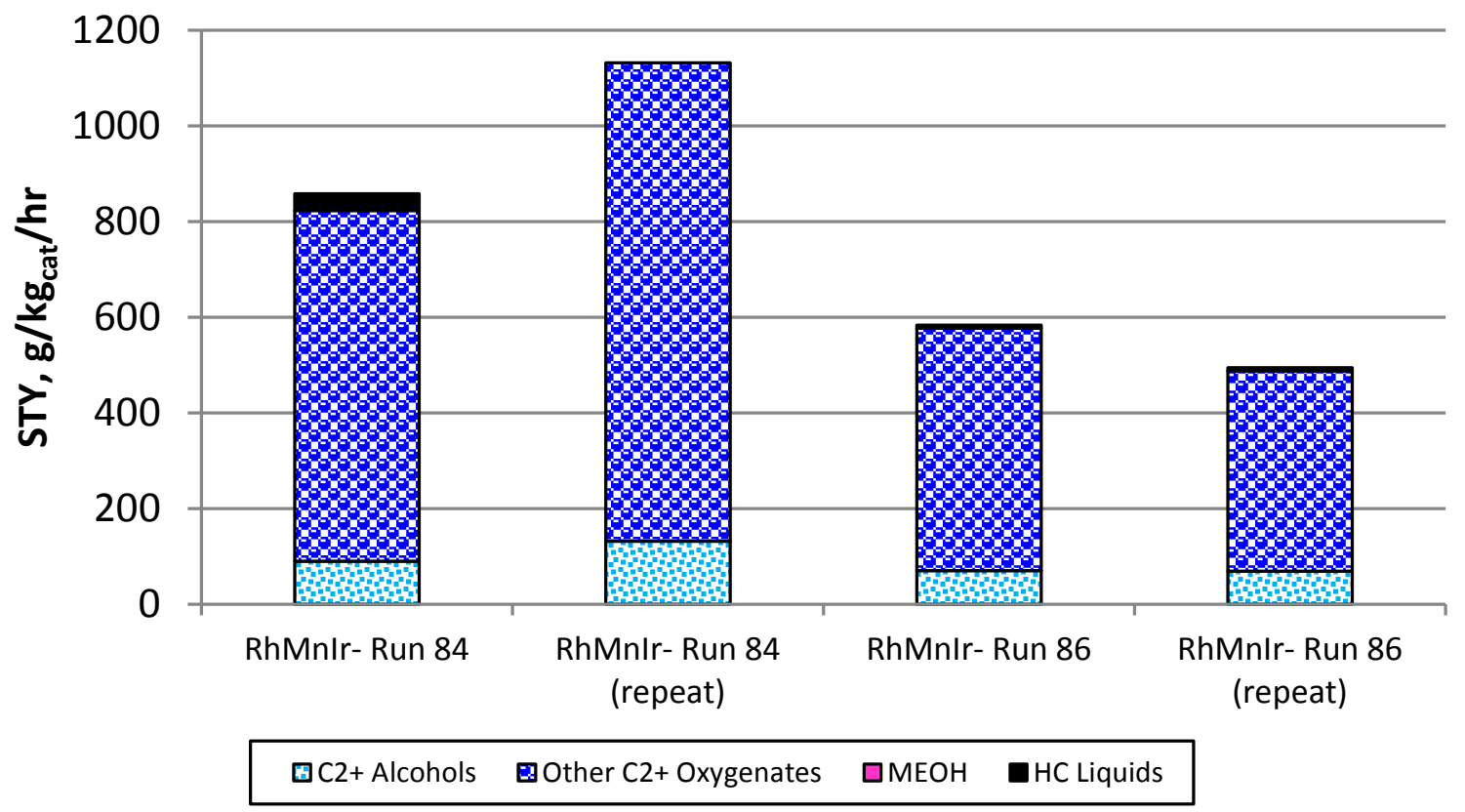

Figure 2.97. STYs at $275^{\circ} \mathrm{C}$ and $7900 \mathrm{~L} / \mathrm{Lcat} / \mathrm{hr}$ GHSV for Low- and High-Temperature Test Runs (Runs 84 and 86, respectively) using the Davisil 645 Silica-Supported Rh-Mn-Ir Catalysts 
In contrast to the variability of activity of the silica-supported catalysts, the converted carbon selectivity did not change significantly between runs or after higher temperature operation at $298^{\circ} \mathrm{C}$ in Run 86. Figure 2.98 illustrates that the selectivity to oxygenates was generally $\sim 45 \%$ and the selectivity to methane was $\sim 30 \%$. Selectivity to $\mathrm{C}_{2}+$ oxygenates did appear to improve with longer time on stream as evidenced in the Run 84 "repeat" data point. Interestingly, the increase in selectivity to oxygenates appeared to be due to a decreased selectivity to higher hydrocarbon gases, while the selectivity to methane remained relatively similar to the other data points at $30 \%$.

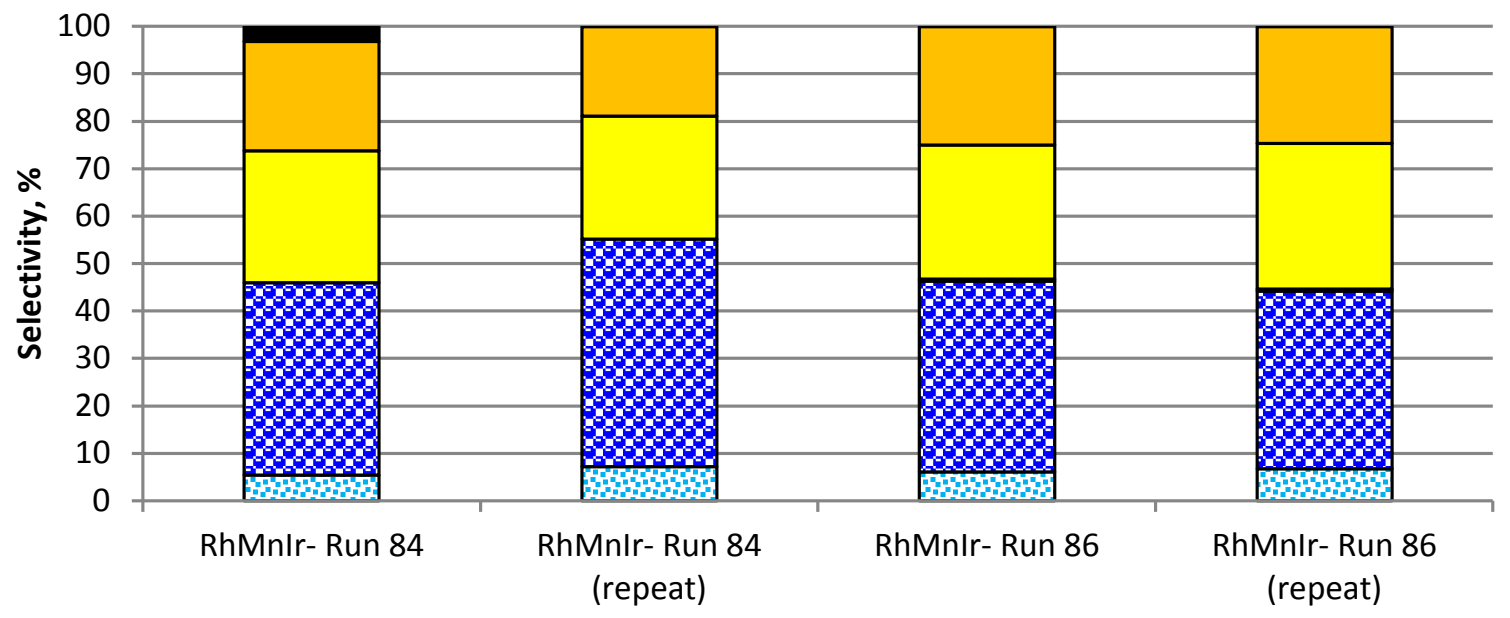

\begin{tabular}{|c|c|c|c|}
\hline 문 $2+$ Alcohols & 巴Other $\mathrm{C} 2+$ Oxygenates & $\square \mathrm{MEOH}$ & 口Other C1 Oxygenates \\
\hline 口Methane & $\square$ Higher HC Gases & - Higher HC Liquids (est) & \\
\hline
\end{tabular}

Figure 2.98. Converted Carbon Selectivities at $275^{\circ} \mathrm{C}$ and $7900 \mathrm{~L} / \mathrm{Lcat} / \mathrm{hr}$ GHSV for Low- and HighTemperature Runs (Runs 84 and 86, respectively) using the Davisil 645 Silica-Supported Baseline Rh-Mn-Ir Catalyst

Figure 2.99 and Figure 2.100 shows the $\mathrm{C}_{2}+$ oxygenate STYs and converted carbon selectivity for the four Hyperion carbon nanotube-supported catalysts. The repeat conditions at $275^{\circ} \mathrm{C}$ are at the fourth condition in Table 2.5 for the low-temperature Rh-Mn run (Run 80) and the fifth condition in the other three runs. Both runs using Rh-Mn catalysts used catalyst subsamples from the same master batch of catalyst, while the two runs using Rh-Mn-Ir catalysts used different master batches.

It should be noted that, in testing at the fourth condition for the high temperature run using the RhMn-Ir catalyst, the temperature had been set at $300^{\circ} \mathrm{C}$, but overnight the catalyst temperature slowly rose over an $8 \mathrm{hr}$ period and then spiked to $318^{\circ} \mathrm{C}$ for the remainder of the sample collection period ( $\left.16 \mathrm{hr}\right)$ for a time-weighted average temperature of $314^{\circ} \mathrm{C}$. Because the object of the test was to have one 24-hr sample period at $300^{\circ} \mathrm{C}$ before returning to $275^{\circ} \mathrm{C}$, the liquid product and gas samples at the fourth condition were used "as is" for comparison to the other test conditions, instead of taking a second sample at $300^{\circ} \mathrm{C}$. The phenomenon of increasing catalyst activity over $24 \mathrm{hr}$ has been shown to be significant at temperatures above $290^{\circ} \mathrm{C}$ for the Rh-Mn-Ir catalysts supported on carbon nanotubes. 


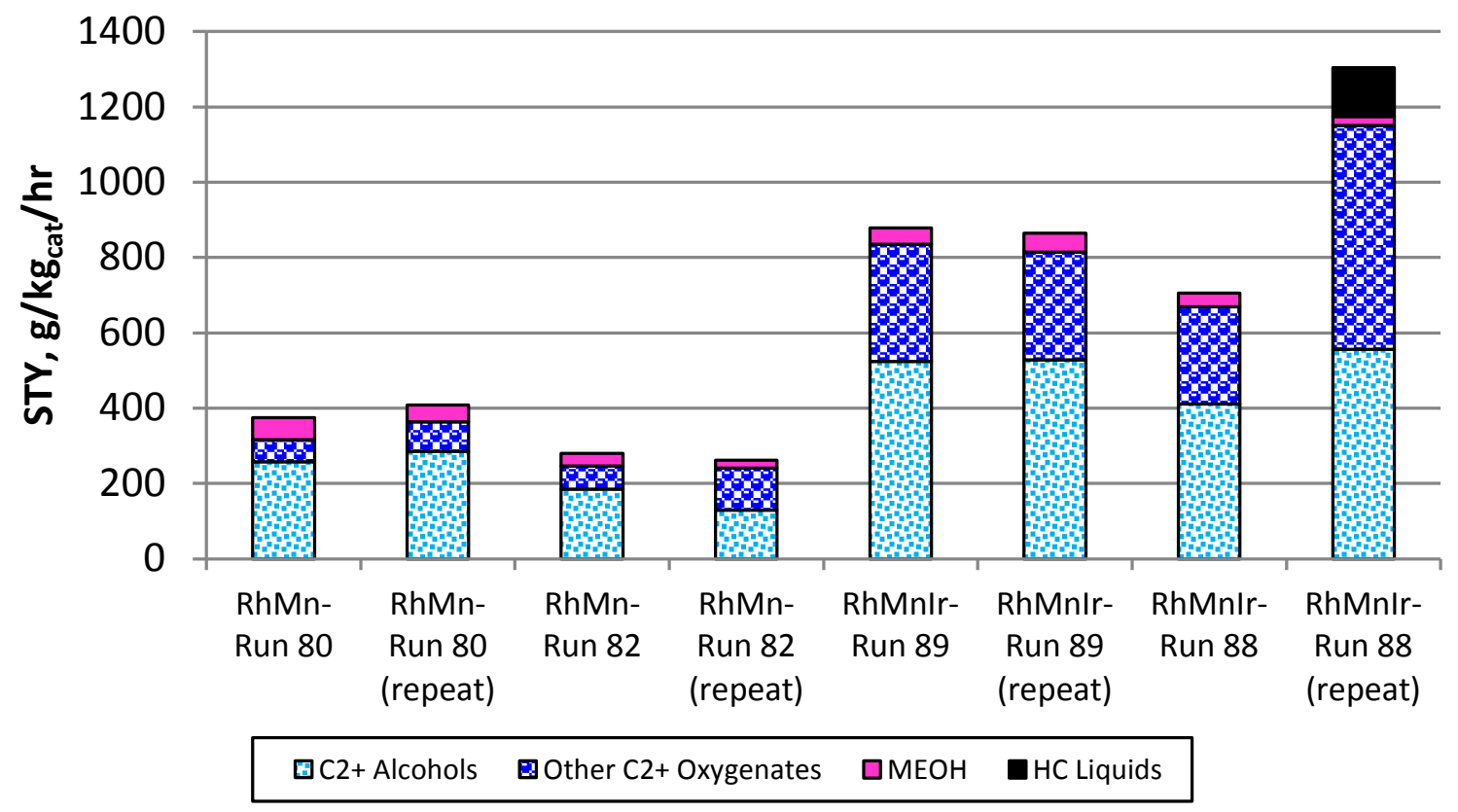

Figure 2.99. STYs at $275^{\circ} \mathrm{C}$ and $7900 \mathrm{~L} / \mathrm{Lcat} / \mathrm{hr}$ GHSV for Hyperion Carbon Nanotube-Supported Rh-Mn (Run 80 for low temperature; Run 82 for high temperature) and Rh-Mn-Ir Catalysts (Run 89 for low temperature; Run 88 for high temperature)

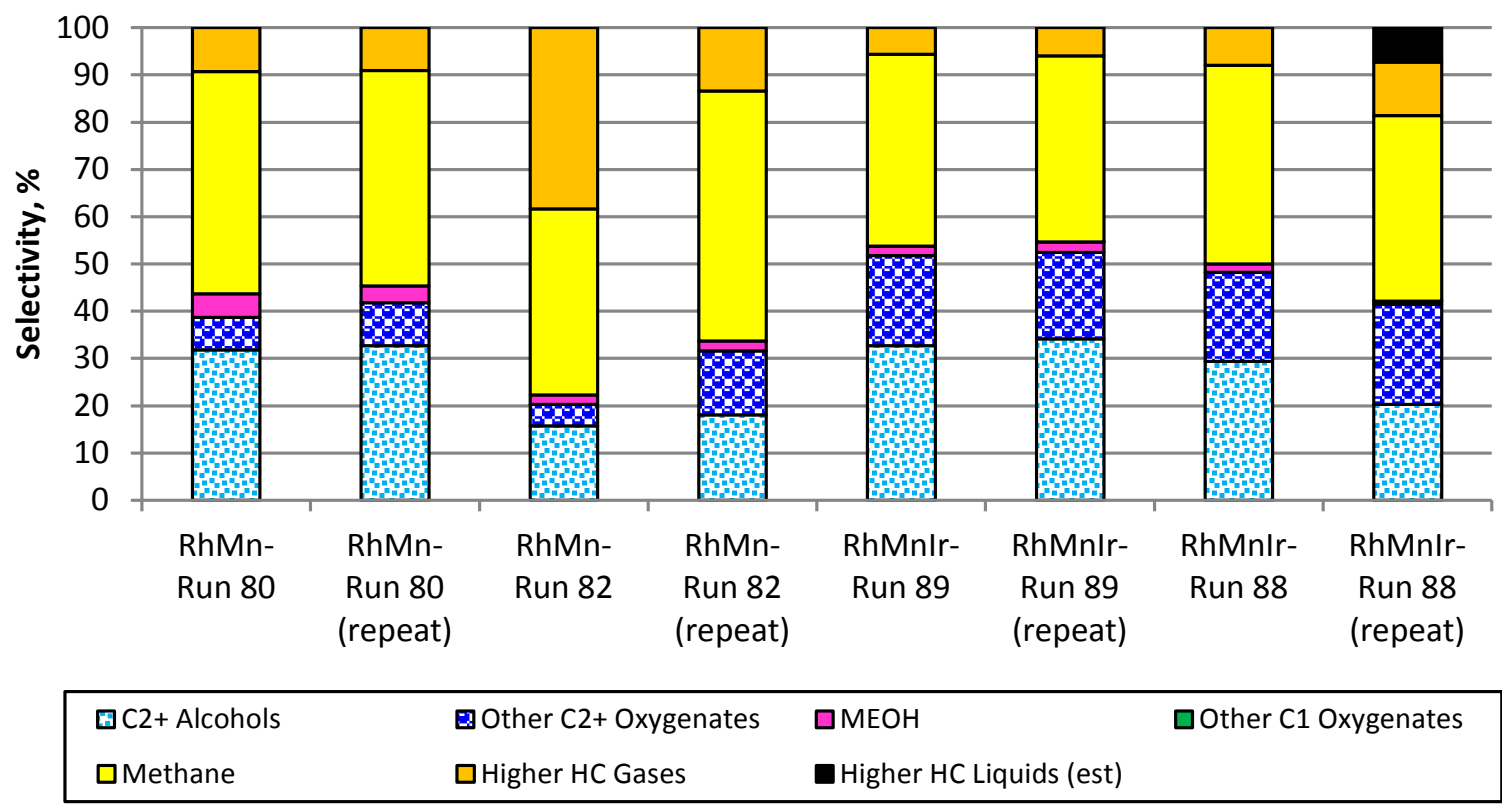

Figure 2.100. Converted Carbon Selectivities at $275^{\circ} \mathrm{C}$ and $7900 \mathrm{~L} / \mathrm{Lcat} / \mathrm{hr}$ GHSV for Hyperion Carbon Nanotube-Supported Rh-Mn (Run 80 for low temperature; Run 82 for high temperature) and Rh-Mn-Ir Catalysts (Run 89 for low temperature; Run 88 for high temperature) 
It should also be noted that. in Run 88 , conditions 4 and 5 in Table $2.5\left(314^{\circ} \mathrm{C}\right.$ and the repeat $275^{\circ} \mathrm{C}$ step), the carbon conversion was $65 \%$ and 51\%, respectively (75\% and 58\% CO conversion, respectively). Past experience has shown that the selectivity to $\mathrm{C}_{2}+$ oxygenates decreases significantly for these catalysts when the carbon conversion exceeds $\sim 35 \%$. If a higher GHSV had been used to reduce the carbon conversion at these test conditions for the Rh-Mn-Ir catalyst, it is likely that the $\mathrm{C}_{2}+$ oxygenate STYs and selectivity would have been higher.

Upon examination of the data for Runs 80 and 82, it is clear that their behaviors at the first test condition of $275^{\circ} \mathrm{C}$ are very different. Similar to the disparity in the activities of identical silicasupported catalyst tests above, the difference cannot be adequately explained.

Taking into account the caveats described above, examination of Figure 2.99 and Figure 2.100 clearly shows that adding Ir to the catalyst improves both the $\mathrm{C}_{2}+$ oxygenate STY and selectivity. It also appears that when Ir is added to the catalyst, increasing the temperature above $300^{\circ} \mathrm{C}$ for $24 \mathrm{hr}$ causes a significant increase in the $\mathrm{C}_{2}+$ oxygenate STY as demonstrated by the higher STY at the repeat $275^{\circ} \mathrm{C}$ test condition. No specific conclusions can be made on the effects of the high temperature on the catalyst selectivity because of the high carbon conversion at the repeat condition at $275^{\circ} \mathrm{C}$. However, other tests have shown that an increase in selectivity does occur when the test conditions are such that the carbon conversions, before and after a $24 \mathrm{hr}$ period at or above $300^{\circ} \mathrm{C}$, are are less than $\sim 35 \%$. 



\subsection{Computational}

Experiments based on first-principle calculations were initiated with two distinct goals in mind:

1. To garner fundamental insights and understanding into the complicated reaction network of CO and $\mathrm{H}_{2}$, especially for reaction steps (i.e., surface reactions) that are difficult to measure.

2. To develop a "feedback loop" whereby computational researchers could suggest catalyst modifications (e.g., promoters) that could be tested in the laboratory with feedback to the computational staff on the experimental results.

Development of the computational model was initiated with the development of a catalytic site consisting of a mixture of $\mathrm{Rh}$ and $\mathrm{Mn}$. Characterization efforts on early catalyst samples were initiated to support and direct development of theoretical catalyst surface model. Concurrent with the development of the surface model, a reaction network of numerous (i.e., 230) reaction steps of $\mathrm{CO}, \mathrm{H}_{2}$, and several adsorbed species was initiated to produce a "road map" for the multitude of reactions possible. The relative stability of transition state moieties identified by the reaction network on the catalyst surface was calculated. By calculating the stability of each transition state, activation barriers could be calculated. The activation barriers were employed in a KMC model to produce a kinetic model expected to be qualitatively consistent with experiential observations.

Next, the modeling effort shifted focus to the addition of Ir to the Rh-Mn system. Geometric considerations as to the behavior of the metal cluster and the location of $\mathrm{Rh}$ and $\mathrm{Mn}$ atoms in a metallic cluster when Ir was present were first considered. Once the Rh-Mn-Ir catalytic metal cluster was developed, activation barriers were again calculated. The activation barriers calculated were input to a micro-kinetic model.

Geometry optimizations and ab initio molecular dynamics simulations (AIMD) were performed on 50-atom nanoparticles ( 1-nm diameter) using a gradient-corrected functional. Norm-conserving pseudopotentials represented the core electrons, and Gaussian-type orbitals of double zeta quality that were tailor-made for this research represented the valence electrons. The wave functions (density) were expanded with an auxiliary plane-wave basis with a 200 to 300 Ry cutoff. The nanoparticles were placed in an $18 \AA$ cubic simulation box with periodic boundary conditions, allowing for $\sim 9 \AA$ between images. We used the climbing image nudged elastic band method to calculate reaction barriers using 7 to 10 points (replicas) along the path. Computations were done with cp2k (http://www.cp2k.org). The results, reported here, were based on reactivity studies on metal clusters with 50 total metal atoms.

\subsection{Catalytic Site Development}

\subsubsection{Rh-Mn/SiO ${ }_{2}$ Catalyst Characterization}

XPS, XRD, and TEM were used to investigate a Davisil 645 silica-supported Rh-Mn catalyst. The sample is referred to below as Sample 3. Preparation of the Sample 3 catalyst was similar to catalyst 60074-53-A in Table 2.4 (page 2.5) except that Ir was not added. Specific preparation conditions for Sample 3 as well as elaboration on the discussion below can be found in Mei et al. ${ }^{7}$ 
XPS measurements performed on catalyst samples similar to Sample 3 before and after use for mixed alcohol synthesis revealed the characteristic XPS Rh peak $3 \mathrm{~d}_{5 / 2}$ at $309.2 \mathrm{eV}$ for $\mathrm{Rh}_{2} \mathrm{O}_{3}$. After use, the $\mathrm{Rh}$ was at least partially reduced to $\mathrm{Rh}^{(0)}$ (peak at $307.4 \mathrm{eV}$ ). This is consistent with the interpretation that under operando conditions $\mathrm{Rh}$ exists as metal. Interestingly, the observation of the expected $\mathrm{Rh}^{(0)}$ binding energy was in contrast with spent samples of the Rh- $\mathrm{Mn}-\mathrm{Ir} / \mathrm{SiO}_{2}$ baseline catalyst (see Figure 2.48 and discussion on page 2.84).

Similar to XPS measurements on the baseline catalysts, conclusions regarding the oxidation state of Mn from XPS measurements are much less resolved, mainly due to the similarities between the 2p core hole energy between $\mathrm{MnO}$ and $\mathrm{Mn}^{(0)}$ metal. Although it is highly likely that $\mathrm{Mn}$ exists as an oxide (Mn $2 \mathrm{p}_{3 / 2}$ at $641 \mathrm{eV}$ ) for the fresh material, it is not clear if it has been partially reduced in the spent sample. Although the XPS spectra around the $2 \mathrm{p}_{3 / 2}$ peak between $645 \mathrm{eV}$ and $638 \mathrm{eV}$ could in principle have been deconvoluted into various Mn components of the signal to metallic Mn and oxide species, doing so is by no means a definitive way to prove the presence of metallic Mn. By the same token, the possibility that a small fraction of the Mn may exist in the metallic state under operando conditions cannot be discounted.

TEM was another characterization technique used to characterize the Rh-Mn silica-supported Sample 3 catalyst. Metal cluster sizes observed in catalyst Sample 3 before and after were qualitatively similar with observations of the baseline $\mathrm{Rh}-\mathrm{Mn}-\mathrm{Ir} / \mathrm{SiO}_{2}$-supported catalyst (e.g., see Figure 2.66 and Figure 2.69). Rh particles are $\sim 1$ to $2 \mathrm{~nm}$ in size and are well dispersed on the support in the fresh catalyst. On the used catalyst, only the larger Rh particles could be seen, although there was some evidence of the presence of mixed Rh/Mn particles (see Figure 3.1). However, the oxidation state of Mn in these species was indeterminate. No pure Mn-particles could be seen, although MnO could be detected in both the fresh and used catalysts.

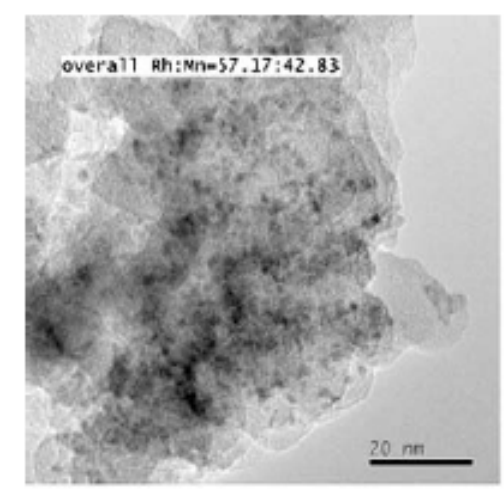

- $\quad$ S3:before

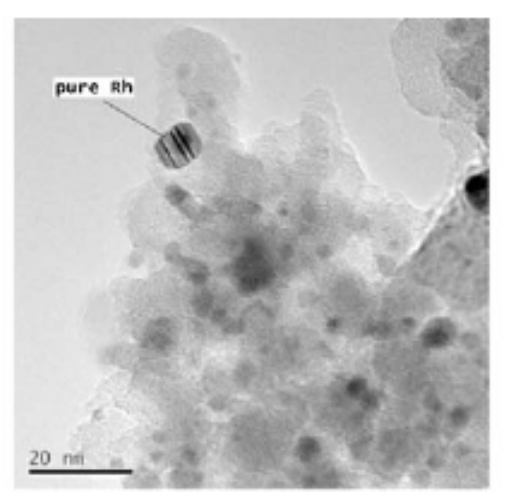

S3:after

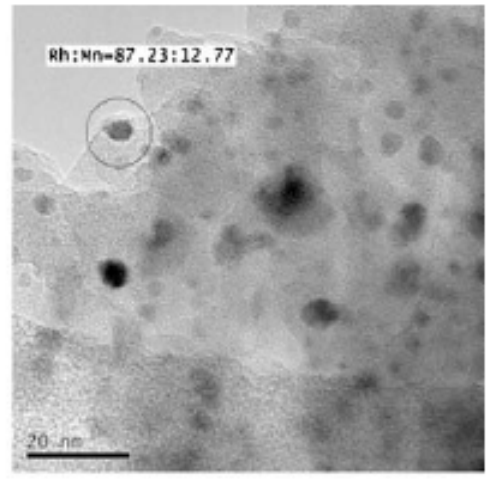

S3:after

Figure 3.1. TEM Images of Fresh and Used Rh/Mn/Silica Catalyst, Sample 3 (designated as S3)

XRD measurements of the $\mathrm{Rh}-\mathrm{Mn} / \mathrm{SiO}_{2}$ catalyst were also qualitatively similar to the baseline catalyst which contained Ir also (see Figure 2.28 and Figure 2.30). The XRD patterns mainly arise from the oxides, $\mathrm{Rh}_{2} \mathrm{O}_{3}, \mathrm{Mn}_{2} \mathrm{O}_{3}$, and $\mathrm{SiO}_{2}$ (support). The broad line patterns observed with the fresh $\mathrm{Rh}-\mathrm{Mn} / \mathrm{SiO}_{2}$ catalyst samples implied very small sized crystallites. The spent sample shows well-resolved peaks arising from the reduced state of the catalyst metals, containing powder Rh and possibly some small $\mathrm{Mn}_{3} \mathrm{Rh}$ inclusions, (111) peak. What is clear is that the components for the fresh sample are not present in the spent, and the single-component fit of the (111) peak suggests small particles of reduced Rh possibly with some Rh/Mn alloys in them as the catalysts age. It also is possible that the small crystallites 
sinter over time through aggregation on the support leading to metallic phase, which also is consistent with the $\mathrm{SiO}_{2}$-supported baseline $\mathrm{Rh}$-Mn-Ir catalyst observations.

While the experimental evidence for the state of Rh was almost certainly metallic, the situation was not as clear in the case of Mn. Although TEM evidence suggests that Mn can exist as an oxide in both fresh and used catalysts, reduction of bulk MnO would still be very difficult. However, small clusters or individual atoms of Mn oxide embedded in Rh could be reduced more easily. In our quest to understand the role of $\mathrm{Mn}$ as a promoter and clarify its oxidation state in these Rh catalysts, we employed statistical mechanics and quantum mechanical calculations.

\subsubsection{Determination of Mn Oxidation State}

The observation of Rh-Mn bimetallic particles in the TEM analysis on the order of 1 to 3nm in size was used as the starting point for the computational studies. However, prior to considering the role of reactive species on the catalytic sites, it became necessary to assign an oxidation state to Mn due to the indeterminacy of experimental measurements. Assuming bimetallic Rh-Mn alloy particles of $\sim 1 \mathrm{~nm}$ size, we employed density functional theory (DFT) to calculate the thermodynamic constants of certain simple chemical transformations typical of the CO hydrogenation process:

$$
\begin{gathered}
\mathrm{Rh}_{49} \mathrm{MnO}+\mathrm{H}_{2} \leftrightarrow \mathrm{Rh}_{49} \mathrm{Mn}+\mathrm{H}_{2} \mathrm{O} ; \Delta \mathrm{H}=-0.9 \mathrm{eV} \\
\mathrm{Rh}_{47} \mathrm{Mn}_{3} \mathrm{O}_{3}+3 \mathrm{H}_{2} \leftrightarrow \mathrm{Rh}_{47} \mathrm{Mn}_{3}+3 \mathrm{H}_{2} \mathrm{O} ; \Delta \mathrm{H}=-0.6 \mathrm{eV} \\
\mathrm{Rh}_{49} \mathrm{Mn}(\mathrm{C}+\mathrm{O}) \leftrightarrow \mathrm{Rh}_{49} \mathrm{Mn}(\mathrm{CO}) ; \Delta \mathrm{H}=-1.0 \mathrm{eV} \\
\mathrm{Rh}_{49} \mathrm{Mn}+1 / 2 \mathrm{O}_{2} \leftrightarrow \mathrm{Rh}_{49} \mathrm{MnO} ; \Delta \mathrm{H}=-1.5 \mathrm{eV} \\
\mathrm{Rh}_{50} \mathrm{O}+\mathrm{H}_{2} \leftrightarrow \mathrm{Rh}_{50}+\mathrm{H}_{2} \mathrm{O} ; \Delta \mathrm{H}=-0.8 \mathrm{eV} \\
\mathrm{Rh}_{50}+1 / 2 \mathrm{O}_{2} \leftrightarrow \mathrm{Rh}_{50} \mathrm{O} ; \Delta \mathrm{H}=-1.7 \mathrm{eV}
\end{gathered}
$$

Combining these reaction energies with estimates of the free energy components, we are able to estimate the likely state of the particle under catalytic conditions.

Using statistical mechanics, one can express the equilibrium constants of reactions (2) through (7) in terms of the canonical partition functions or reactants and products, their rotational and vibrational temperatures, and the ground-state energetics. For example, the equilibrium constant for reaction (2) can be written as:

$$
\mathrm{K}_{1}(\mathrm{~T})=\frac{q_{R h_{4} M n} q_{H_{2} O}}{q_{R h_{49} M n O} q_{H_{2}}}
$$

which can be further written as: 


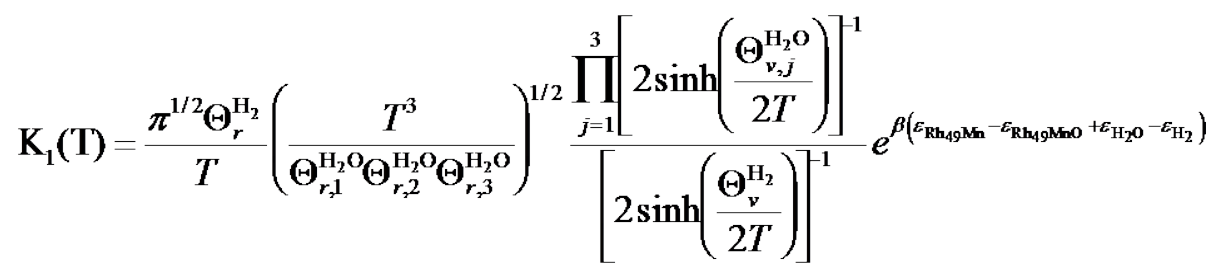

where $\Theta_{r}$ are the rotational temperatures, $\Theta_{v}$ are the vibrational temperatures, $\beta=1 / k_{B} T$, and $\varepsilon$ are the ground state energies determined from our DFT calculations using the cp2k code (www.cp2k.org). A full description of the equations solved can be found in Mei et al. ${ }^{7}$

Figure 3.2 shows the equilibrium constants calculated for all reactions (2) through (7) as a function of temperature in the range 0 to $1000 \mathrm{~K}$. In the range of the catalysts operation (400 to $700 \mathrm{~K}$ ), the equilibrium constants indicate that binary alloys of Rh-Mn are favored over the Rh-Mn oxides as the $\ln (\mathrm{K})$ of the equilibrium constant is greater than zero (R1 and R2 in Figure 3.2).

Therefore, we propose it is thermodynamically possible that $\mathrm{Rh}$ and Mn exist as a Rh-rich bimetallic alloy under reactor operation conditions. The existence of $\mathrm{Mn}$ in a metallic-alloy state with $\mathrm{Rh}$ under operating conditions is in contrast to a previous study that found $\mathrm{Rh}$ and $\mathrm{MnO}$ co-exist as separate phases when water is present. ${ }^{15}$ Interestingly, the same study did report that a $\mathrm{Rh}-\mathrm{Mn} / \mathrm{SiO}_{2}$ catalyst reduced in $\mathrm{H}_{2}$ at $500^{\circ} \mathrm{C}$ did not exhibit an electron paramagnetic resonance signal attributable to $\mathrm{Mn}^{2+}$ (or any other signal) suggesting no unpaired electrons. However, the signal attributed to $\mathrm{Mn}^{2+}$ re-occurred $\mathrm{CO}$ and $\mathrm{H}_{2} \mathrm{O}$ were introduced at room temperature. It is possible the $\mathrm{Mn}$ in the $\mathrm{Rh}-\mathrm{Mn} / \mathrm{SiO}_{2}$ catalyst studied in the electron paramagnetic resonance paper was reduced to metallic $\mathrm{Mn}$ and re-oxidized to $\mathrm{Mn}^{2+}$ when $\mathrm{H}_{2} \mathrm{O}$ and $\mathrm{CO}$ were introduced at room temperature. Our data do suggest that, under ambient oxygen partial pressure, the oxidized states are preferred (R4 and R6 in Figure 3.2) although we have not performed the calculation for the addition of water. Based on our thermodynamic calculations, we based the kinetic barrier calculations on silica-supported Rh-Mn binary alloy catalysts, which are likely to exist under operando conditions, where higher temperatures and reducing atmospheres are prevailing.

To further test the validity of thermodynamic analysis of the reduction level of Mn present in Rh-Mn catalytic particles, we performed benchmark analyses on the reducibility of $(\mathrm{MnO})_{50}$ and $\left(\mathrm{Rh}_{2} \mathrm{O}_{3}\right)_{25}$ particles. Similar to the study that produced the results in Figure 3.2, 1 nm wide (50 metal atoms) of both Rh and Mn oxide clusters were utilized. Detailed AIMD simulations were performed at high temperature conditions to simulate annealing of the clusters to generate energetically relaxed structures. The final structures are presented in Figure 3.3. 


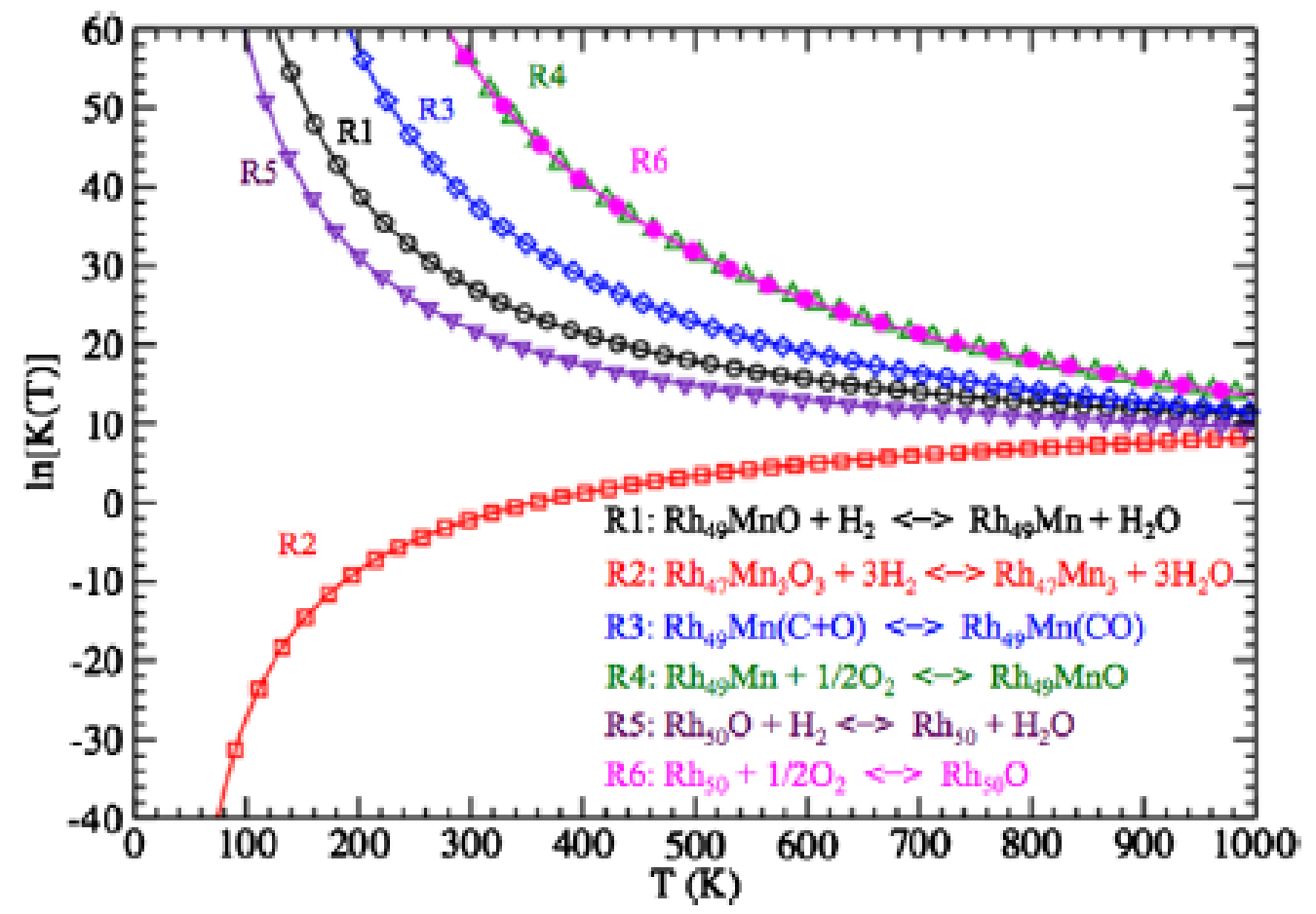

Figure 3.2. Calculated Equilibrium Constants for Elementary Reactions (2) through (7) as a Function of Temperature. Taken from Mei et al. ${ }^{7}$
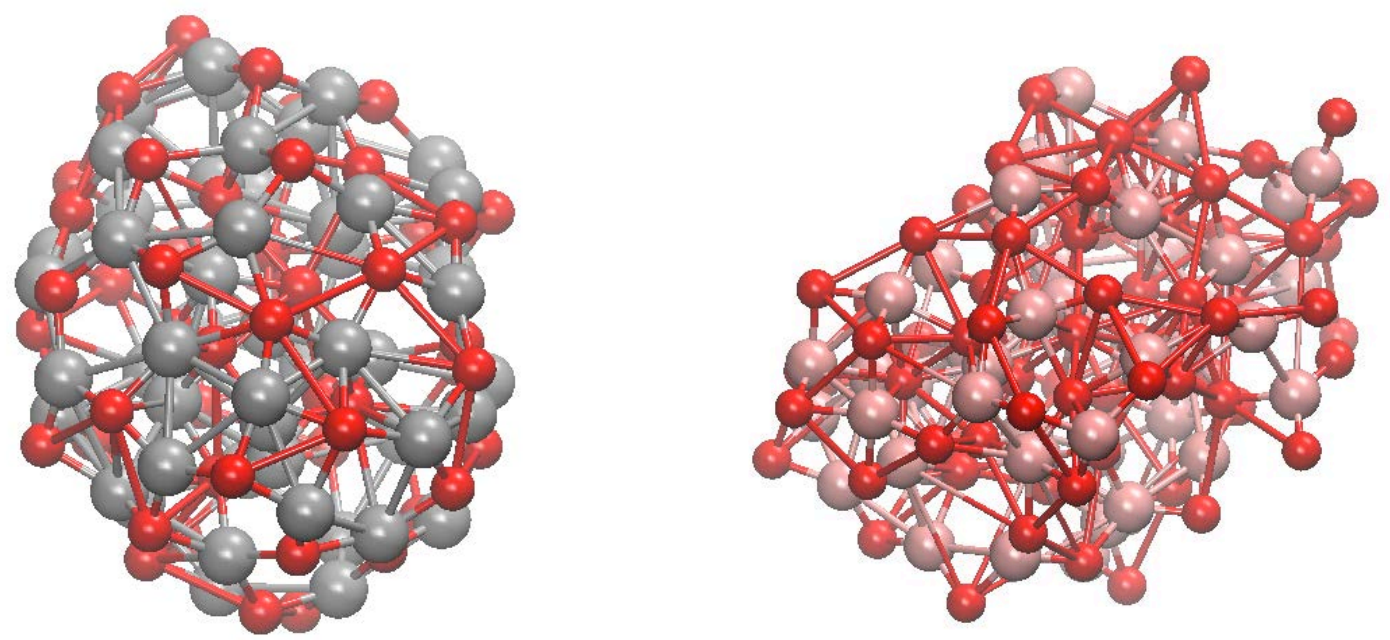

Figure 3.3. Structures of $(\mathrm{MnO})_{50}$ (left) and $\left(\mathrm{Rh}_{2} \mathrm{O}_{3}\right)_{25}$ (right) Particles Extracted from High-Temperature AIMD Simulated Annealing Studies. Gray spheres are Mn atoms (left); light red spheres are $\mathrm{Rh}$ atom (right); deep red spheres are oxygen atoms (left and right).

The energy required to reduce these particles was estimated from the chemical reactions:

$$
\begin{gathered}
(\mathrm{MnO})_{50}+\mathrm{H}_{2} \rightarrow \mathrm{Mn}(\mathrm{MnO})_{49}+\mathrm{H}_{2} \mathrm{O} \\
\left(\mathrm{Rh}_{2} \mathrm{O}_{3}\right)_{25}+\mathrm{H}_{2} \rightarrow \mathrm{Rh}_{50} \mathrm{O}_{74}+\mathrm{H}_{2} \mathrm{O}
\end{gathered}
$$


In this approximation, it was assumed that the initial reduction of the stoichiometric oxide particles would be the most energetically costly. After calculation, the initial reaction energies were converted into reaction-free energies and subsequently equilibrium constants by an analogous process as that performed on the $\mathrm{Mn}$ sites embedded in the $\mathrm{RH}_{49} \mathrm{Mn}$ and $\mathrm{Rh}_{47} \mathrm{Mn}_{3}$ particles to determine the extent of reduction under syngas at elevated temperatures.

The initial reduction of the (MnO) $)_{50}$ cluster (Reaction (8)) is found to be endothermic with an energy requirement of $160 \mathrm{~kJ} / \mathrm{mol}$. In comparison, $96 \mathrm{~kJ} / \mathrm{mol}$ of energy is produced via an exothermic reaction in the reduction of the for $\mathrm{Rh}_{49} \mathrm{Mn}$ cluster. Thus, the $\mathrm{Mn}$ in the $(\mathrm{MnO})_{50}$ cluster is much more resistant to reduction than $\mathrm{Mn}$ alloyed within the $\mathrm{Rh}_{49} \mathrm{MnO}$ particle. Likewise, the energy of Reaction (9) shows that reduction of $\mathrm{Rh}$ by syngas is appreciably more facile as the initial reduction is exothermic, with an energy of $130 \mathrm{~kJ} / \mathrm{mol}$. Applying the thermodynamic corrections to obtain a free energy as derived in Mei et al. ${ }^{7}$ would place a free energy correction of $\mathrm{T} \Delta \mathrm{S} \sim 40 \mathrm{k} \mathrm{J} / \mathrm{mol}$ at $\mathrm{T}=600 \mathrm{~K}$ (i.e., the conditions under which the catalytic experiments were performed). Approximating the free energy of these reactions as $\Delta \mathrm{G}=\Delta \mathrm{E}+\mathrm{T} \Delta \mathrm{S}$ (where $\Delta \mathrm{E}$ is the DFT-derived reaction energy), we determined that the reduction of the $\mathrm{Rh}_{2} \mathrm{O}_{3}$ clusters is favorable when $\Delta \mathrm{G}=-180 \mathrm{~kJ} / \mathrm{mol}\left(\mathrm{K}_{\mathrm{eq}}=\mathrm{e}^{(-\Delta \mathrm{G} / \mathrm{RT})} \sim 10^{16}\right)$, whereas reduction of MnO is not $\left(\Delta \mathrm{G}=220 \mathrm{~kJ} / \mathrm{mol}\left(\mathrm{K}_{\mathrm{eq}} \sim 10^{-20}\right)\right)$. This finding agrees with the TEM observation that MnO islands are not reduced to a metallic state in the spent catalyst samples but $\mathrm{Rh}_{2} \mathrm{O}_{3}$ ones are.

\section{2 $\mathrm{CO}+\mathrm{H}_{2}$ Reaction Network Development}

A reaction network consisting of elementary surface reactive steps for syngas conversion over Rhbased catalysts was constructed at the onset of computational investigations. DFT was employed. A schematic of the elementary reaction diagram is illustrated in Figure 3.4. The reaction network for CO transformation to ethanol was created taking into account experimental and theoretical studies from the literature and illustrates the level of complexity involved. Generally, the reaction begins with the associative adsorption of $\mathrm{CO}$ and the dissociative adsorption of $\mathrm{H}_{2}$. CO molecules adsorb at all surface sites (atop, bridge, fourfold hollow and threefold hollow) of both the (100) and the (111) facets. The adsorbed atomic $\mathrm{H}$ preferentially occupies either bridge sites or hollow sites on both facets. Adsorbed $\mathrm{CO}$ can further dissociate into atomic $\mathrm{C}$ and $\mathrm{O}$ that prefer to sit on the hollow sites of both facets. The $\mathrm{C}$ and $\mathrm{H}$ atoms recombine to form $\mathrm{CH}$ and further hydrogenation with the surface $\mathrm{H}$ atoms forming $\mathrm{CH}_{2}$ and $\mathrm{CH}_{3}$, and the major side product $\mathrm{CH}_{4}$. These $\mathrm{CH}_{\mathrm{x}}$ intermediates also can couple themselves, producing higher hydrocarbons. In this work, only $\mathrm{C}_{2}$ hydrocarbons are considered to simplify the model. The key elementary steps for $\mathrm{CO}$ hydrogenation to ethanol are the insertion of $\mathrm{CO}$ into the $\mathrm{CH}_{\mathrm{x}}$ (x = 1 to 3) species, forming $\mathrm{CH}_{\mathrm{x}} \mathrm{CO}$ species. The desired ethanol product will be generated by further hydrogenation of $\mathrm{CH}_{\mathrm{x}} \mathrm{CO}$. Meanwhile, the intermediate $\mathrm{CH}_{\mathrm{x}} \mathrm{CO}$ species also will be oxidized and hydrogenated to form other $\mathrm{C}_{2}$ oxygenates such as acetaldehyde, acetic acid, and ethyl acetate shown in Figure 3.4. 


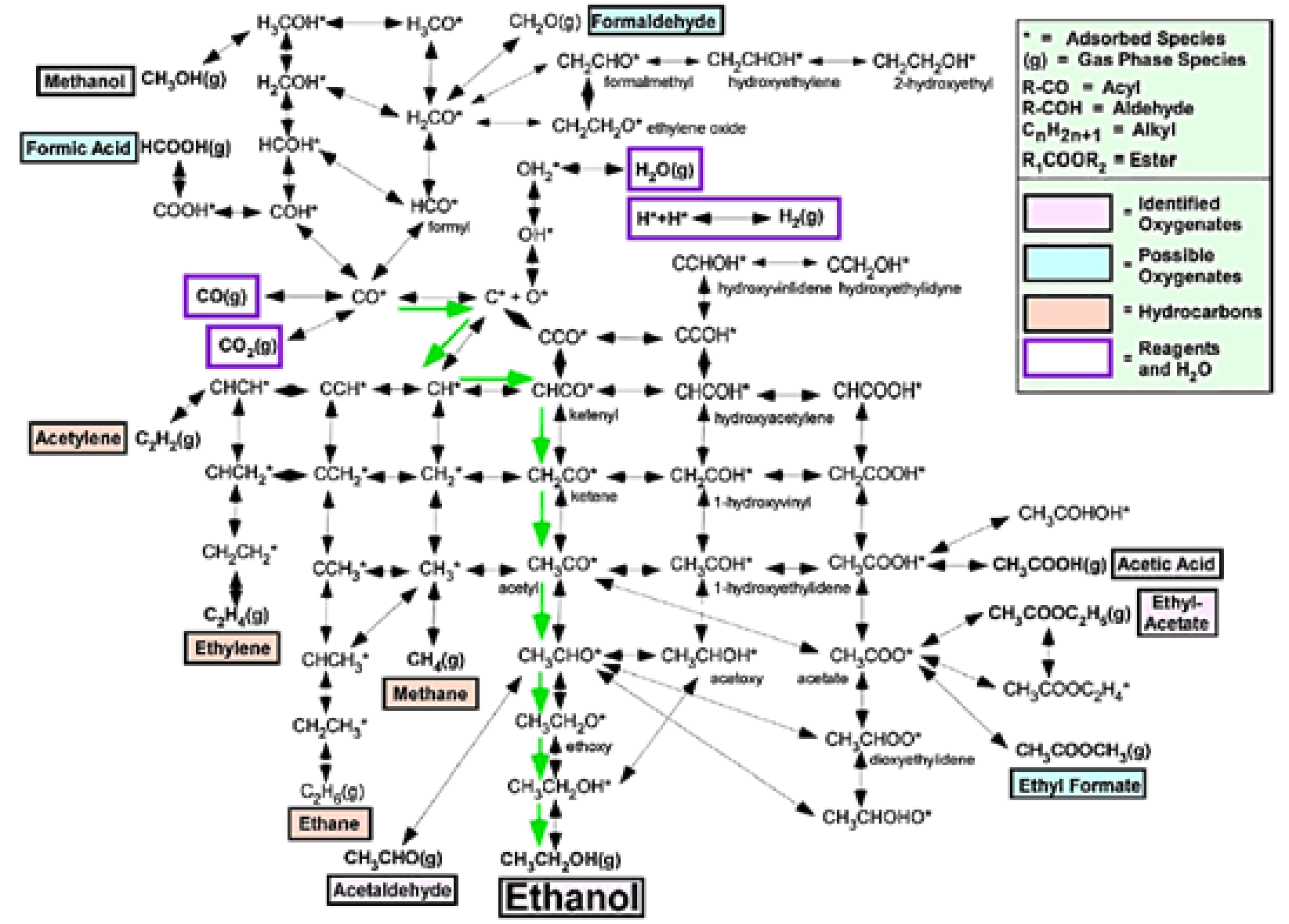

Figure 3.4. Elementary Reaction Diagram Showing Important Steps of Syngas Conversion as Complied from a Survey of the Literature and the Research Performed on this Project. Taken from Mei et al. ${ }^{7}$

Figure 3.5 shows the optimized adsorption configurations of important mechanistic steps on the Rh47Mn3 ( 30\% wt Mn) nanoparticle. The particles $\mathrm{Rh}_{50}$ and $\mathrm{Rh}_{49} \mathrm{Mn}(\sim 10 \%$ wt $\mathrm{Mn})$ also were used in our calculations to help understand the role of Mn. All atoms were allowed to relax during these geometry optimizations. The binding energies thus obtained are listed in Table 3.1. 

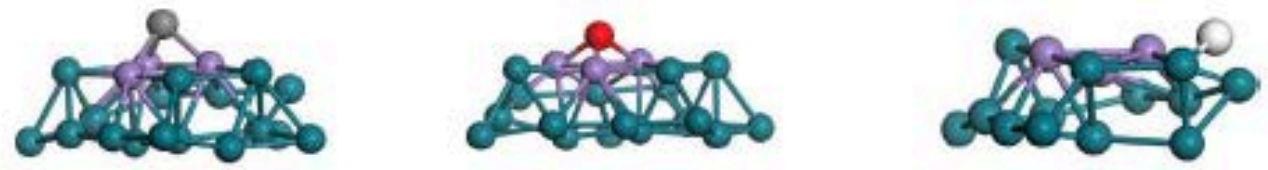

C

o

H

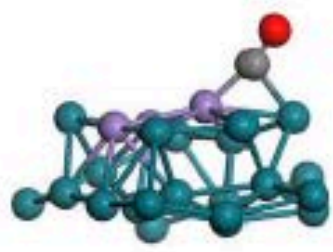

Co

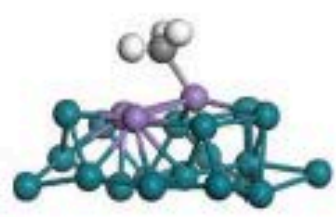

$\mathrm{CH}_{3}$

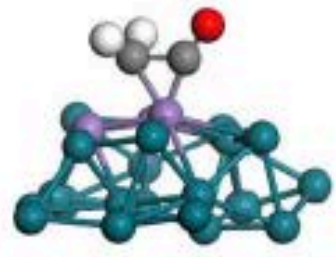

$\mathrm{CH}_{2} \mathrm{CO}$

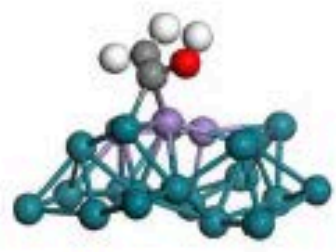

$\mathrm{CH}_{2} \mathrm{COH}$

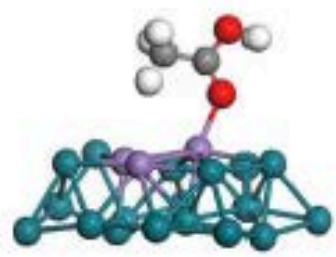

$\mathrm{CH}_{3} \mathrm{COOH}$

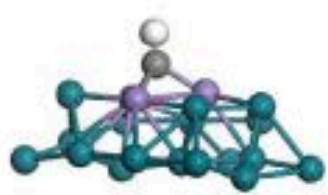

$\mathrm{CH}$

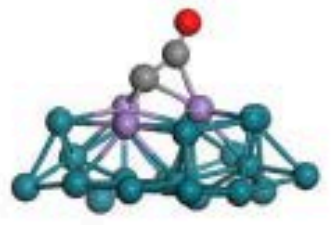

$\mathrm{CCO}$

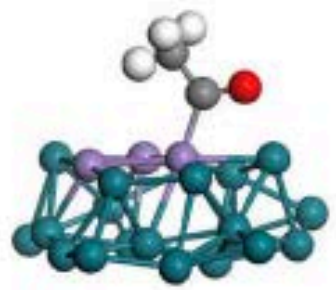

$\mathrm{CH}_{3} \mathrm{CO}$

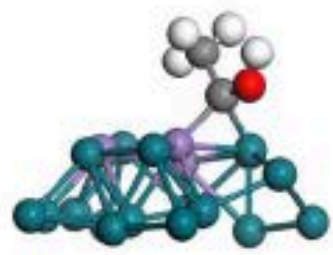

$\mathrm{CH}_{3} \mathrm{COH}$

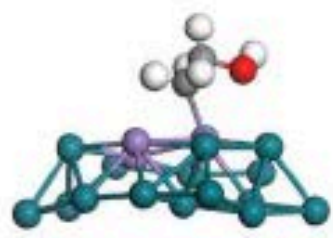

$\mathrm{CH}_{2} \mathrm{CH}_{2} \mathrm{OH}$

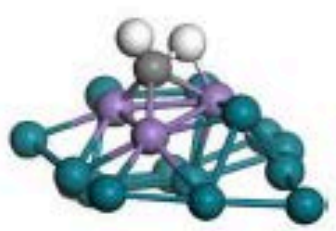

$\mathrm{CH}_{2}$

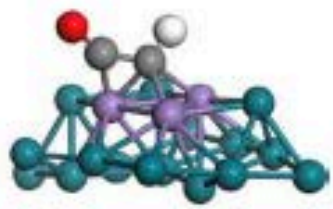

$\mathrm{CHCO}$

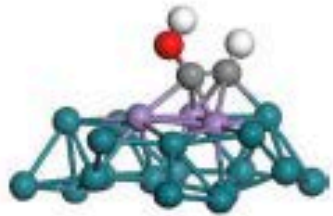

$\mathrm{CHCOH}$

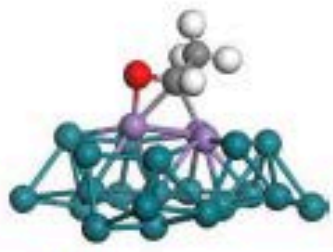

$\mathrm{CH}_{3} \mathrm{CHO}$

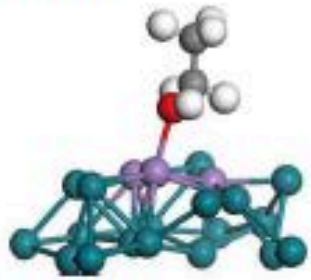

$\mathrm{CH}_{3} \mathrm{CH}_{2} \mathrm{OH}$

Figure 3.5. Optimized Adsorption Configurations on the Rh47Mn3 Nanoparticle (partially shown for clarity): Rh (green), Mn (purple), C (gray), O (red), H (white). Taken from Mei et al. ${ }^{7}$ 
Table 3.1. Binding Energies of Reactive Intermediates on Three Different Nanoparticles. "Na” indicates non-adsorption site. Taken from Mei et al. ${ }^{7}$

\begin{tabular}{|c|c|c|c|c|}
\hline \multirow{2}{*}{ Surface Intermediate } & \multirow{2}{*}{ Site } & \multicolumn{3}{|c|}{ Bind Energy $(\mathrm{eV})$} \\
\hline & & $\mathrm{Rh}_{50}$ & $\mathrm{Rh}_{49} \mathrm{Mn}$ & $\mathrm{Rh}_{47} \mathrm{Mn}_{3}$ \\
\hline $\mathrm{H}$ & Threefold fcc & -0.62 & -1.15 & -1.35 \\
\hline $\mathrm{C}$ & Threefold fcc & -6.58 & -6.34 & -6.21 \\
\hline $\mathrm{O}$ & Threefold fcc & -3.32 & -3.22 & -3.16 \\
\hline $\mathrm{CO}$ & Threefold fcc & -1.68 & -2.42 & -1.74 \\
\hline $\mathrm{CH}$ & Threefold fcc & -6.19 & -4.94 & -5.82 \\
\hline $\mathrm{CH}_{2}$ & Threefold fcc & -3.42 & -4.21 & -5.30 \\
\hline $\mathrm{CH}_{3}$ & Top & -1.11 & -2.96 & -3.91 \\
\hline $\mathrm{CH}_{4}$ & Top & -0.09 & na & na \\
\hline CCO & Threefold fcc & -5.11 & -4.29 & -4.11 \\
\hline $\mathrm{CHCO}$ & Threefold fcc & -3.37 & -3.21 & -3.14 \\
\hline $\mathrm{CH}_{2} \mathrm{CO}$ & Тор & -1.18 & -1.33 & -1.22 \\
\hline $\mathrm{CH}_{3} \mathrm{CO}$ & Тор & -1.89 & -1.73 & -1.54 \\
\hline $\mathrm{CHCOH}$ & Twofold bridge & -3.68 & -2.93 & -3.34 \\
\hline $\mathrm{CH}_{2} \mathrm{COH}$ & Twofold bridge & -2.82 & -3.06 & -3.35 \\
\hline $\mathrm{CH}_{3} \mathrm{COH}$ & Twofold bridge & -4.04 & -2.78 & -3.52 \\
\hline $\mathrm{CH}_{3} \mathrm{CHO}$ & Twofold bridge & -1.41 & -1.32 & -1.25 \\
\hline $\mathrm{CH}_{3} \mathrm{COOH}$ & Top & -0.72 & -0.36 & -1.00 \\
\hline $\mathrm{CH}_{2} \mathrm{CH}_{2} \mathrm{OH}$ & Top & -1.65 & -1.15 & -1.65 \\
\hline $\mathrm{CH}_{3} \mathrm{CH}_{2} \mathrm{OH}$ & Top & -0.93 & -0.93 & -1.01 \\
\hline
\end{tabular}

We first calculated the reaction path $\mathrm{CH}_{3}+\mathrm{H} \rightarrow \mathrm{CH}_{4}$, which is the final hydrogenation step for methane formation on the $\mathrm{Rh}_{50}, \mathrm{Rh}_{49} \mathrm{Mn}, \mathrm{Rh}_{47} \mathrm{Mn}_{3}$ nanoparticles. As shown in Figure 3.6, methane formation is exothermic on the $\mathrm{Rh}_{50}$ and $\mathrm{Rh}_{49} \mathrm{Mn}$ clusters while it is slightly endothermic on $\mathrm{Rh}_{47} \mathrm{Mn}_{3}$. This indicates that methane formation is thermodynamically more favorable than methane dissociation on $\mathrm{Rh}$ and $\mathrm{Rh} / \mathrm{Mn}$ nanoparticles. The calculated reaction energies of $\mathrm{CH}_{3}+\mathrm{H} \rightarrow \mathrm{CH}_{4}$ on $\mathrm{Rh}_{50}, \mathrm{Rh}_{49} \mathrm{Mn}$, and $\mathrm{Rh}_{47} \mathrm{Mn}_{3}$ are $-0.36,-0.22,+0.08 \mathrm{eV}$, respectively. The calculated activation barrier for $\mathrm{CH}_{3}+\mathrm{H} \rightarrow \mathrm{CH}_{4}$ is $0.60 \mathrm{eV}$ on the $\mathrm{Rh}_{50}$ cluster, which agrees with to the previously reported barriers of $0.65 \mathrm{eV} ; 0.63 \mathrm{eV}$ on $\mathrm{Rh}(111)$. By doping one $\mathrm{Mn}$ atom on the surface of the $\mathrm{Rh}_{50}$ cluster $\left(\mathrm{Rh}_{49} \mathrm{Mn}\right)$, the barrier for methane formation increases slightly to $0.70 \mathrm{eV}$. If three $\mathrm{Mn}$ atoms are doped onto the $\mathrm{Rh}_{50}$ cluster $\left(\mathrm{Rh}_{47} \mathrm{Mn}_{3}\right)$, this barrier decreases only slightly to $0.57 \mathrm{eV}$. This implies that doping $\mathrm{Mn}$ into Rh nanoparticles essentially does not change the activation barrier of methane formation although the isolated Mn (highly dispersed Mn on Rh-rich surface) may inhibit the methane formation to some extent by increasing the barrier of $0.1 \mathrm{eV}$. Considering the other hydrogenation steps of $\mathrm{CH}_{\mathrm{x}}$ (where $\mathrm{x}=0-2$ ) on $\mathrm{Rh}(111)$ have the similar low barriers between 0.47 to $0.69 \mathrm{eV}$, we can expect that the methane formation is still inevitable in $\mathrm{CO}$ hydrogenation by adding Mn onto Rh catalyst. 


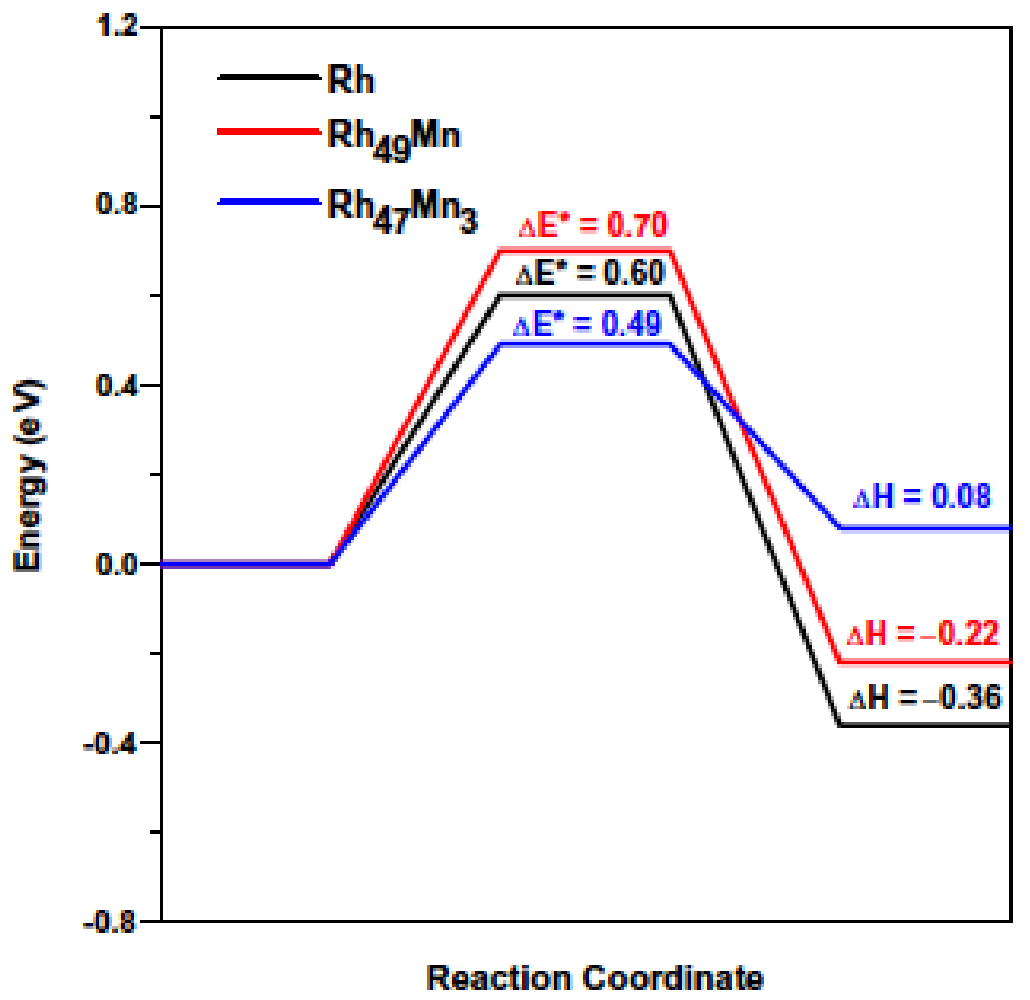

Figure 3.6. Relative Energies and Reaction Barriers for the Methanation Reaction on the Three Different Clusters

In other words, the addition of Mn onto Rh is predicted to have little effect on the catalytic performance with respect to the rate of methane formation because both the thermodynamic and kinetic factors for the hydrogenation of $\mathrm{CH}_{\mathrm{x}}$ are nearly the same for $\mathrm{Rh}$ and $\mathrm{Rh} / \mathrm{Mn}$ catalysts. This conclusion also is supported by our experimental results. As shown in Table 3.1, the methane selectivity on Rh-Mn on $\mathrm{SiO}_{2}$ supports under different experimental conditions are still high ( 31\% to $\left.60 \%\right)$. Previous experimental investigations of $\mathrm{CO}$ hydrogenation on the Mn-promoted Rh catalysts showed results similar to those of high methane selectivity. For example, Hu et al. ${ }^{16}$ observed that methane selectivity are in the range of 34 to $54 \%$. Ojeta et al. ${ }^{17}$ found that methane selectivity increased from 22 to $32 \%$ as the reaction temperature increased from 513 to $533 \mathrm{~K}$.

The role of other potential promoters on the methanation formation channel in the reaction network was also briefly studied. Specifically, we examined both alkali and alkali earth metals as potential third species. We found that lighter, harder, elements such as $\mathrm{Li}, \mathrm{Mg}$, and $\mathrm{Na}$ will exist in cationic form on the $\mathrm{Rh}$ nanoparticle and could serve as thermodynamic sinks for surface-bound $\mathrm{CH}_{3}$ groups, effectively retarding the methanation channel. However, this would come at a tradeoff of lower Co conversion (activity). Heavier, softer, species such as K, Cs, and Ca were less (or not at all) effective in achieving this result.

For $\mathrm{C}_{2}+$ oxygenated species to form, $\mathrm{CO}$ must insert into $\mathrm{CHx}(\mathrm{x}=0-3)$ prior to methane formation. Clearly, this will be feasible if the barriers to CO insertion are less or at least comparable to hydrogenation reactions. We examined the energetics and barriers to the following reactions on the three particles: $\mathrm{Rh}_{50}, \mathrm{Rh}_{49} \mathrm{Mn}$ and $\mathrm{Rh}_{47} \mathrm{Mn}_{3}$ : 


$$
\begin{gathered}
\mathrm{C}+\mathrm{CO} \rightarrow \mathrm{CCO} \\
\mathrm{HC}+\mathrm{CO} \rightarrow \mathrm{HC}-\mathrm{CO} \\
\mathrm{H}_{2} \mathrm{C}+\mathrm{CO} \rightarrow \mathrm{H}_{2} \mathrm{C}-\mathrm{CO} \\
\mathrm{H}_{3} \mathrm{C}+\mathrm{CO} \rightarrow \mathrm{H}_{3} \mathrm{C}-\mathrm{CO}
\end{gathered}
$$

For the reaction (10), our calculations show that the activation barriers are on the order of $2 \mathrm{eV}$ over all the catalyst particles studied here. The reason is that the bare $\mathrm{C}$ atom forms a very strong bond with surface $\mathrm{Rh}$ atoms. The binding energy of a $\mathrm{C}$ atom is $-6.54 \mathrm{eV}$ on the $\mathrm{Rh}_{50}$ and $-6.28 \mathrm{eV}$ on the $\mathrm{Rh}_{49} \mathrm{Mn}$ nanoparticles. As a result, we can safely disregard the $\mathrm{C}+\mathrm{CO}$ coupling reaction. Figure $3.7(\mathrm{a}-\mathrm{c})$ compares the reaction energies and activation barriers of $\mathrm{CH}_{\mathrm{x}}+\mathrm{CO}$ reaction.

Unlike methane formation, the $\mathrm{CH}_{\mathrm{x}}+\mathrm{CO}$ reaction is endothermic for the $\mathrm{Rh}_{50}$ and the $\mathrm{Rh}_{49} \mathrm{Mn}$ and almost neutral for the $\mathrm{Rh}_{47} \mathrm{Mn}_{3}$, indicating that insertion of $\mathrm{CO}$ into $\mathrm{CH}$ species is not thermodynamically favorable on pure $\mathrm{Rh}_{50}$ and $\mathrm{Rh}_{49} \mathrm{Mn}$ nanoparticles. For the $\mathrm{Rh}_{50}$ particle, the activation barrier of this reaction is $1.35 \mathrm{eV}$. However, we found that doping $\mathrm{Mn}$ into $\mathrm{Rh}$ dramatically lowers the insertion barriers to only $0.69 \mathrm{eV}$ for the $\mathrm{Rh}_{49} \mathrm{Mn}$ and $0.58 \mathrm{eV}$ for the $\mathrm{Rh}_{47} \mathrm{Mn}_{3}$. This suggests that $\mathrm{CO}$ insertion into $\mathrm{CH}$ reaction becomes a competing step with the methane formation on the $\mathrm{Rh}_{49} \mathrm{Mn}$ and $\mathrm{Rh}_{47} \mathrm{Mn}_{3}$ particles. We also noted that, because the reaction energy of $\mathrm{CH}+\mathrm{CO}$ is $0.52 \mathrm{eV}$, the $\mathrm{CHCO}$ formed on the $\mathrm{Rh}_{49} \mathrm{Mn}$ could easily dissociate back to $\mathrm{CH}$ and $\mathrm{CO}$ with a barrier of $0.17 \mathrm{eV}$. Nonetheless, the $\mathrm{CHCO}$ intermediate may survive as a short-lived kinetic intermediate that can be further hydrogenated to $\mathrm{C}_{2}+$ oxygenated species. As such, only the $\mathrm{Rh}_{47} \mathrm{Mn}_{3}$ particle provides a feasible promoting path for $\mathrm{CO}$ insertion into $\mathrm{CH}$ compared with the pure $\mathrm{Rh}_{50}$ nanoparticle. This latter observation indicates that although $\mathrm{Mn}$ sites alone do help in lowering the barrier to the formation of $\mathrm{CHCO}$, they are most effective when clustered, as in $\mathrm{Rh}_{47} \mathrm{Mn}_{3}$, indicating that reasonably high concentrations of $\mathrm{Mn}$ in the proposed $\mathrm{Rh} / \mathrm{Mn}$ alloy are required. It is noted however, that the propensity for cluster Mn to form oxides (which may phase segregate from the Rh particle onto or even into the silica) is higher when the Mn atoms are clustered as inferred from reaction (2) presented above.

The calculated reaction energies and activation barriers of reaction (12) on the $\mathrm{Rh}_{50}, \mathrm{Rh}_{49} \mathrm{Mn}$, $\mathrm{Rh}_{47} \mathrm{Mn}_{3}$ nanoparticles are shown in Figure 3.7(b). On pure $\mathrm{Rh}$ nanoparticles, the barrier is found to be approximately $1.5 \mathrm{eV}$ and is only marginally lower by $\sim 0.2$ to $0.3 \mathrm{eV}$ by inclusion of $\mathrm{Mn}$ into the nanoparticle. It is surprising that this insertion step is found to be highly endothermic with high barriers on all the particles, indicating the insertion of $\mathrm{CO}$ into the surface $\mathrm{CH}_{2}$ species is difficult. Likewise, insertion of $\mathrm{CO}$ into $\mathrm{CH}_{3}$ with reaction (13) on different Rh-based nanoparticles is shown in Figure 3.7 (c) where it is also found that the activation barriers of this step are still high ( 1.0 to $1.26 \mathrm{eV})$, although this step is nearly energy neutral for all catalyst nanoparticles. Compared with the barriers of $0.5 \sim 0.7 \mathrm{eV}$ for methane formation, it is expected that the insertion of $\mathrm{CO}$ into $\mathrm{CH}_{2}$ and $\mathrm{CH}_{3}$ is still kinetically inhibited even with the addition of Mn onto Rh. This also explains why methane is still the dominant side product in $\mathrm{CO}$ hydrogenation for the Rh/Mn catalysts. 

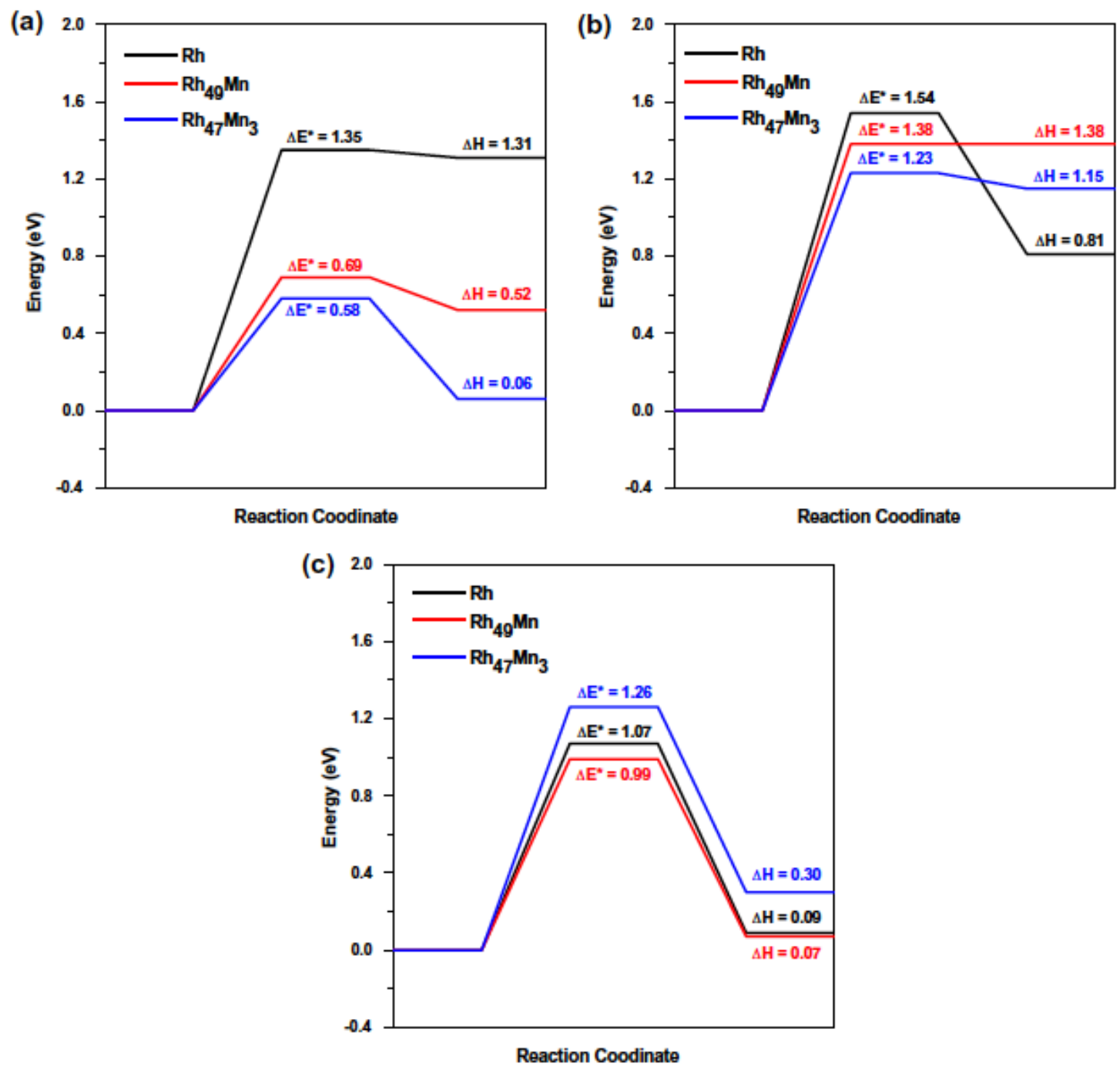

Figure 3.7. Energetics for $\mathrm{CH}_{\mathrm{x}}+\mathrm{CO}$ Reactions in the Three Different Nanoparticles, $\mathrm{x}=1$ for (a); $\mathrm{x}=2$ for (b); and $\mathrm{x}=3$ for (c). Taken from Mei et al. ${ }^{7}$

Clearly, for the all-Rh particle, the insertion of $\mathrm{CO}$ to $\mathrm{CH}_{3}$ is the most favorable for $\mathrm{CO}$ with any $\mathrm{CH}_{x}$ species. The endothermic nature of the $\mathrm{CO}+\mathrm{CH}$ reaction on the $\mathrm{Rh}_{50}$ cluster is highly endothermic, indicating any formed OCCH is likely to dissociate back to $\mathrm{CO}+\mathrm{CH}$. In the case of $\mathrm{CO}+\mathrm{CH}_{2}$ or $\mathrm{CO}+\mathrm{CH}_{3}$, the barrier of formation of the $\mathrm{OCCH}_{2}$ and $\mathrm{OCCH}_{3}$ species is still relatively high, but the energy difference between the reactants and products is not as endothermic, suggesting any formed $\mathrm{OCCH}_{2}$ and $\mathrm{OCCH}_{3}$ would be less likely to dissociate.

In the case of $\mathrm{CO}+\mathrm{CH}$, the presence of Mn makes the most significant difference, especially in the case of $\mathrm{Rh}_{47} \mathrm{Mn}_{3}$ particle: the barrier is only $0.6 \mathrm{eV}$ for the $\mathrm{CO}+\mathrm{CH}$ coupling and leads to an equally stable product. The least favorable reaction seems to be the $\mathrm{CO}+\mathrm{CH}_{2}$ coupling (Figure 3.7(b)), while $\mathrm{CO}+\mathrm{CH}_{3}$ coupling has an intermediate barrier and thermodynamically favorable product OCCH3. 


\subsection{Kinetic Monte Carlo Simulator}

To validate the reaction network model developed with DFT calculations via the cp2k code, we used a KMC model to perform a series of reaction kinetic calculations for the reaction of CO with $\mathrm{H}_{2}$ over silica-supported Rh-Mn particles operating under conditions similar to the mixed alcohol synthesis experimental reactors. The KMC simulator allows for comparisons involving promoters, reactive sites, reaction temperature and partial pressures of the reactive components. A snapshot showing the Rh/Mn particle supported on $\mathrm{SiO}_{2}$ in the presence of $\mathrm{C}, \mathrm{H}$, and $\mathrm{O}$ atoms is illustrated in Figure 3.8.

Each simulation was allowed to equilibrate to steady state in which the surface coverage for all of surface intermediates reached constant values with the exception of small fluctuations resulting from the stochastic nature of the simulation. After achieving steady state, the overall macroscopic kinetic values, such as the turnover frequencies (TOFs) of the major products and averaged surface coverages for the key surface intermediates were calculated. For example, the TOF for ethanol production was calculated by simply counting the number of ethanol molecules desorbed from the $\mathrm{Rh} / \mathrm{Mn}$ nanoparticle surface during a given time interval. The number of molecular desorption events as a function of time was fitted to a simple function that includes a first-order exponential term to describe the initial transient kinetics, followed by a straight line to model the long-time, steady-state behavior. The TOF values reported herein were determined from the long-time behavior in which the slope of the straight line was normalized based on the total number of surface $\mathrm{Rh} / \mathrm{Mn}$ atoms on the nanoparticle. The time scales of the simulations vary depending on the specific conditions, but steady state typically is realized somewhere between 0.01 and $10.0 \mathrm{~s}$. The results were obtained with a nanoparticle with $\sim 30 \mathrm{wt} \% \mathrm{Mn}$ as suggested by the experimental measurements. The selectivity to each product is defined as:

$$
S_{\bar{i}}=\frac{T O F_{\text {product }(i)}}{T O F_{\text {dll products }}}
$$

Further details relating to the KMC model can be found in Mei et al. ${ }^{7}$

In Figure 3.9, the temperature dependence of the TOFs of the major products (a), the level of $\mathrm{CO}$ conversion (b), the selectivity of $\mathrm{CH}_{4}$ versus $\mathrm{CH}_{3} \mathrm{CHO}$ (c), and the selectivity of $\mathrm{CH}_{3} \mathrm{OH}, \mathrm{CH}_{3} \mathrm{CH}_{2} \mathrm{OH}$, and $\mathrm{CH}_{3} \mathrm{COOH}$ oxygenates (d) is illustrated. The activities of all products increase with temperature (a), and the comparison of apparent activation barriers with values from the literature agree extremely well (see Table 3.2), giving us confidence of the validity of our kinetic model in reproducing the relavent trends in activity and selectivity of the species. 


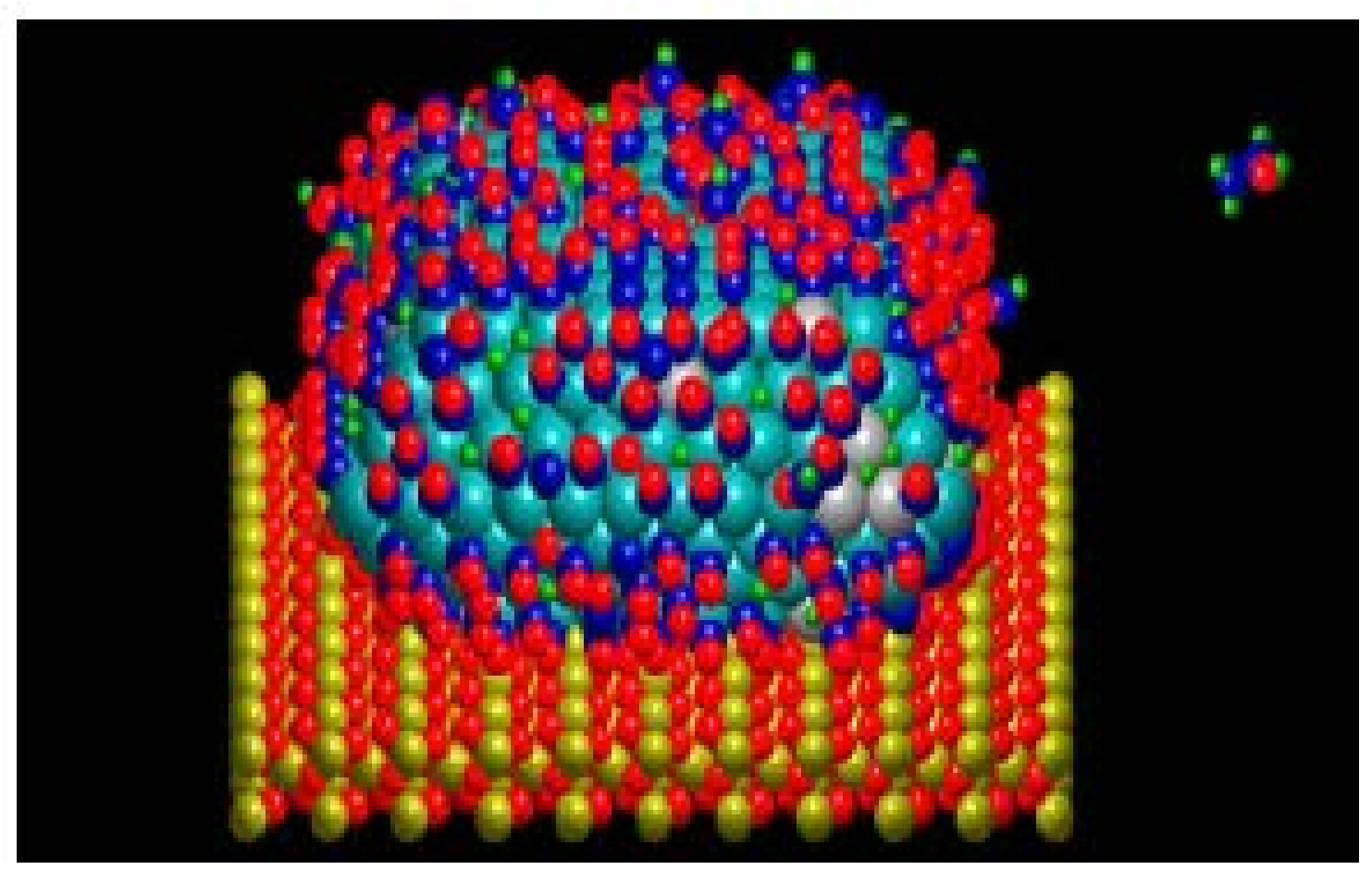

Figure 3.8. Snapshot of KMC Simulation of CO Hydrogenation to Ethanol: Si (yellow), O (red), Rh (cyan), Mn (grey), C (blue), and H (green). Taken from Mei et al. ${ }^{7}$ 

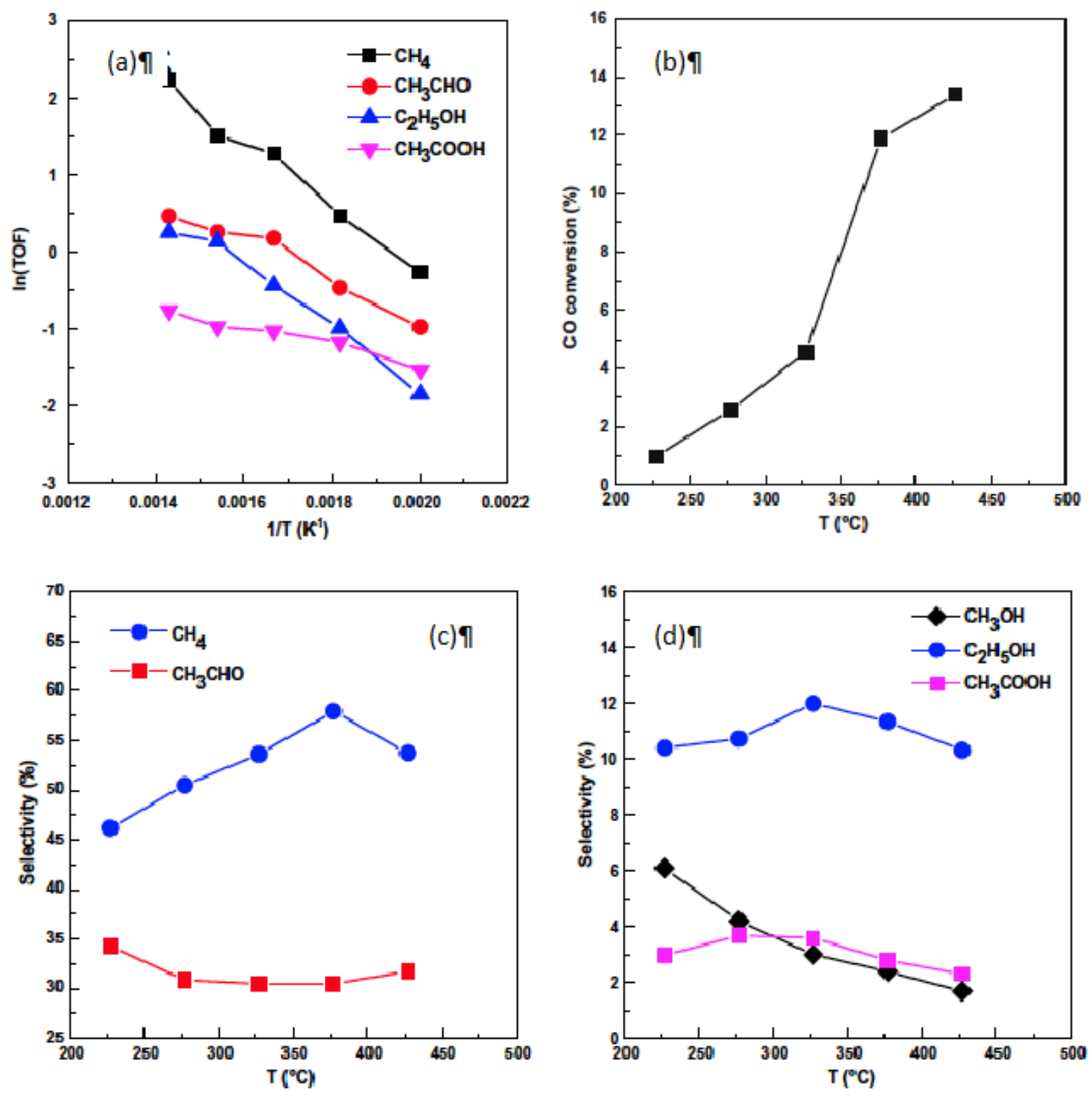

Figure 3.9. Temperature Dependence on TOF of Major Products (a); CO Conversion (b); Methane and Acetaldehyde Selectivity (c); and Methanol, Ethanol, and Acetic Acid Selectivity (d). Taken from Mei et al. ${ }^{7}$

Table 3.2. Activation Barriers For Reaction $\mathrm{CO}+\mathrm{H}_{2} \rightarrow$ Product. The "Theory" values are from "Progress in C1 Chemistry in Japan." "18 Energies are reported in $\mathrm{kJ} / \mathrm{mol}$. Taken from Mei et al. ${ }^{7}$

\begin{tabular}{llcc}
\hline Product & Theory & Exp. \\
\hline $\mathrm{CH}_{4}$ & 35 & 34 \\
$\mathrm{CH}_{3} \mathrm{CH}_{2} \mathrm{OH}$ & 37 & 45 \\
$\mathrm{CH}_{3} \mathrm{CO}$ & 24 & 19 \\
$\mathrm{CH}_{3} \mathrm{COOH}$ & 12 & 7.5 \\
\hline
\end{tabular}


Experimentally, Figure 3.9(b) illustrates that CO conversion shows a steep increase between 325 and $400^{\circ} \mathrm{C}$. Our model correctly predicts an increase in methane selectivity over that of oxygenates as temperature increases. Ethanol selectivity ranges between 10 and 12\%, showing an optimal temperature at $\sim 350^{\circ} \mathrm{C}$. The selectivity for the other oxygenates either varies very little $\left(\mathrm{CH}_{3} \mathrm{CHO}, \mathrm{CH}_{3} \mathrm{COOH}\right)$ or decreases somewhat with the temperature $\left(\mathrm{CH}_{3} \mathrm{OH}\right)$.

Other findings from our kinetic model show that the critical branching point in the reaction network is the $\mathrm{CH}+\mathrm{CO} \rightarrow \mathrm{CHCO}$ coupling step, as identified by DFT studies. There is a decrease of $0.2 \mathrm{eV}$ in apparent activation energy between the $\mathrm{Rh}$ and $\mathrm{Rh} / \mathrm{Mn}$ based catalysts. However, the overall $\mathrm{CO}$ conversion when $\mathrm{Mn}$ is present is higher because of the increased number of reaction channels available to CO under high coverage on the catalyst surface. Our model also predicts that methane to ethanol selectivity is more affected by pressure variations as illustrated in Figure 3.10. In fact, ethanol selectivity increases significantly in the 1 to $5 \mathrm{MPa}$ pressure range, while methane drops.
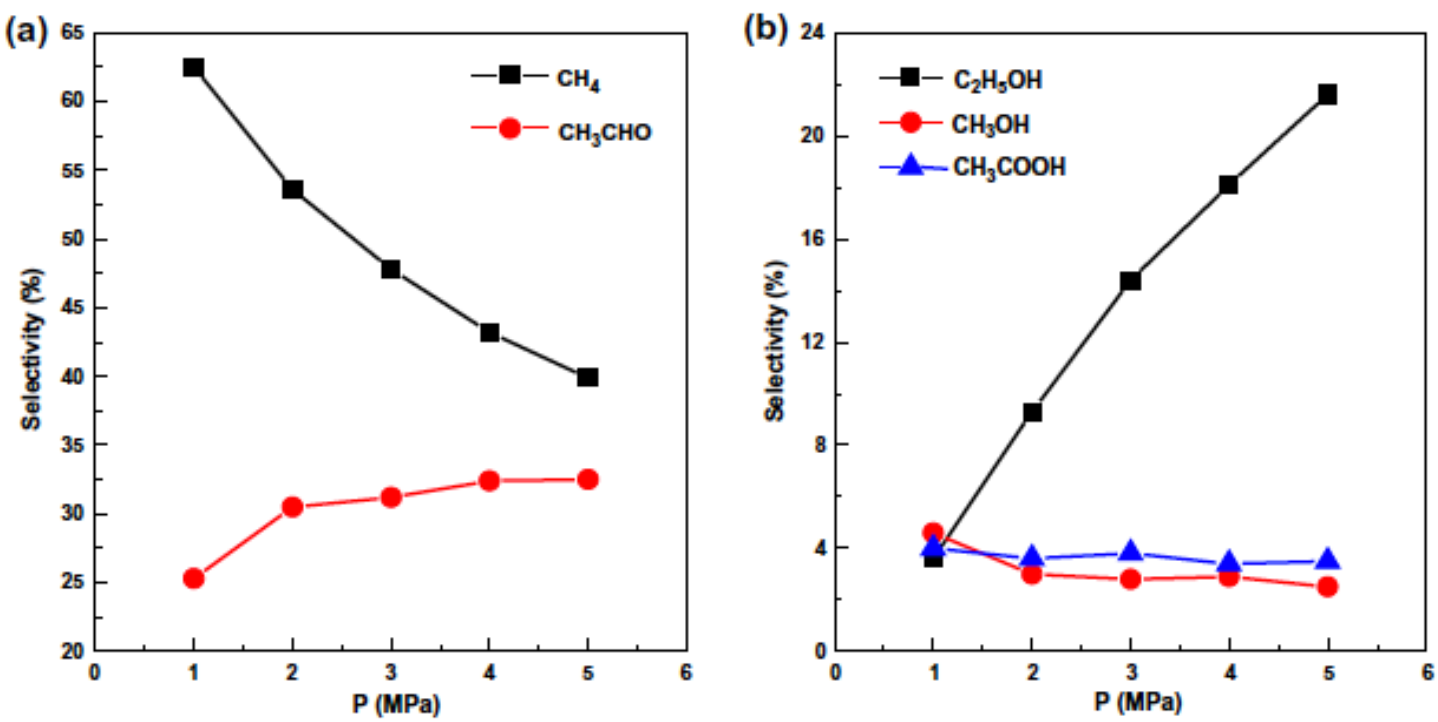

Figure 3.10. Pressure Dependence of Selectivity at $300^{\circ} \mathrm{C}$. Taken from Mei et al. ${ }^{7}$

Figure 3.11 shows the temperature dependence (KMC model data) for the TOFs of methane (in Figure 3.11 (a)) and ethanol (in Figure 3.11 (b)) production. Clearly, the presence of Mn significantly increases the ethanol production, although an increase in methane production also is observed when Mn is present due to higher overall CO conversion. 

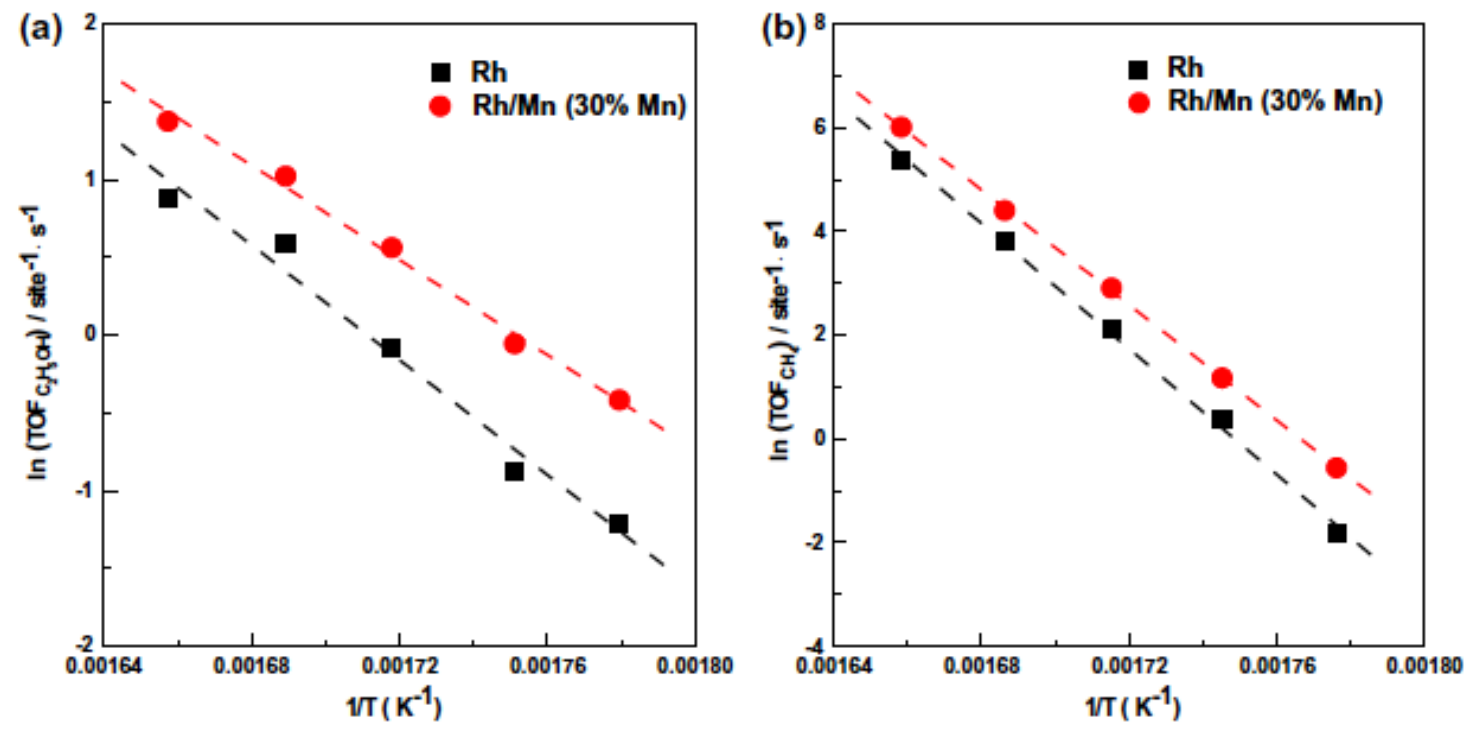

Figure 3.11. Arrhenius Plots (from the KMC model) Showing the $\mathrm{T}$ Dependence on the TOFs of (a) Methane and (b) Ethanol Production. Taken from Mei et al. ${ }^{7}$

\subsection{Investigation of Alternative Secondary Promoters}

The promotion effect resulting from adding the Rh-promoter (i.e., Mn) was investigated by calculating the relative reaction barriers for the $\mathrm{CO}+\mathrm{CH}$ insertion reaction over a nanoparticle containing other metals. The composition $\mathrm{Rh}_{49} \mathrm{M}$, where $\mathrm{M}=\mathrm{Ir}, \mathrm{Ga}, \mathrm{V}, \mathrm{Mn}, \mathrm{Ti}, \mathrm{Sc}$, Ca, and Li were investigated. Here, we assume for sake of argument that all elements will exist in a metallic (i.e., non-oxide) phase. We hypothesized that the difference in selectivity and productivity can be correlated with the difference in electronegativity between $\mathrm{Rh}$ and the metal promoter. This postulate is based on the observation that, for each Rh replaced with a promoter, the Rh matrix acquires a small partial positive charge, making it slightly Lewis acidic. Embedding the metal promoters in the Rh matrix in a sense alleviates some of the potential difficulty of having some of these metals in the reduced (metallic) state. Figure 3.12 shows the variation in the reaction barriers of the OCCH coupling reaction as a function of the difference in electronegativity between $\mathrm{Rh}$ and the metal promoters. The barrier is least for $\mathrm{Mn}$ and $\mathrm{Ti}$, as the electronegativity difference becomes $\sim 0.7$ (Pauling's electronegativity scale), illustrating an optimum value in the difference of electronegativity between $\mathrm{Rh}$ and the metal promoter. For elements with higher electronegativity differences, $\mathrm{CO}$ becomes more firmly attached to neighboring $\mathrm{Rh}$ atoms and there arises an energy penalty to bring $\mathrm{CO}$ to the promoter site to perform the insertion. This observation suggests that alloying $\mathrm{Ti}, \mathrm{V}$, or Mn into a Rh catalyst would be the best approach for increasing the selectivity for and productivity of ethanol via the current mechanism. 


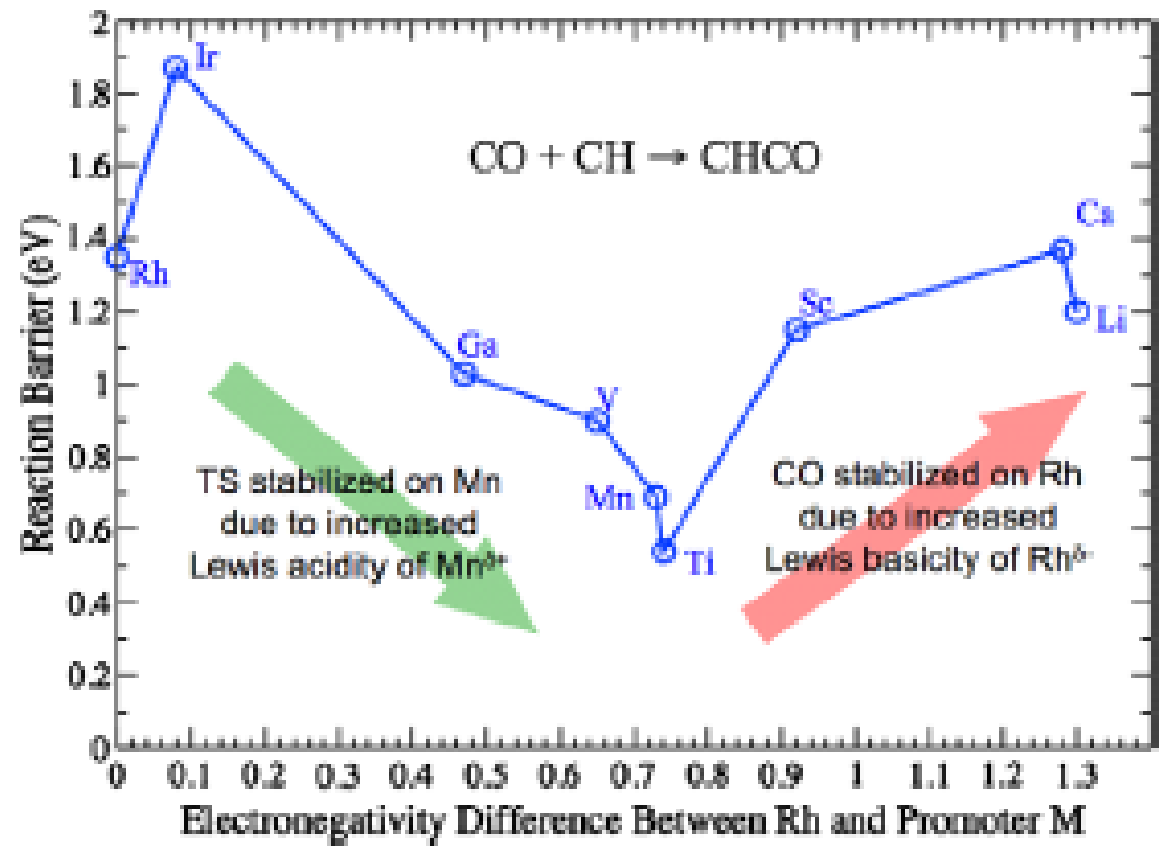

Figure 3.12. DFT Reaction Barriers as a Function of Electronegativity Differences between Rh and Metal Promoters. Taken from Mei et al. ${ }^{7}$

The observations of the computational $\mathrm{Rh} / \mathrm{Mn}$ catalytically active model, development of the reaction network with activation barriers, and results from the KMC model imply the following two options for catalyst improvement:

1. Increase the Mn contact with $\mathrm{Rh}$, This could be facilitated by using non-oxide supports such as various forms of carbon (where $\mathrm{MnO}$ may not preferentially phase segregate from $\mathrm{Rh}$ ).

2. Find an additional promoter that would open alternate reaction channels.

Both avenues were pursued experimentally, and our additional theoretical studies to support those experimental activities are described below.

\subsection{Role of Ir in Rh-Mn Catalysts}

In the course of experimental catalyst screening, Ir was discovered as a tertiary promoter to Rh-Mn catalysts that significantly enhanced the activity and selectivity of the catalysts toward $\mathrm{C}_{2}+$ oxygenates. The carbon-supported Rh-Mn and Rh-Mn-Ir baseline catalysts shown in Figure 2.99 illustrate the effect of Ir. Addition of Ir to the binary Rh-Mn catalysts resulted in an increase of the STY of $\mathrm{C}_{2}+$ oxygenates. For the Ir-containing catalysts, re-testing after heating at $575 \mathrm{~K}$ (Run 86, "Repeat" data point shown in Figure 2.99) caused the CO conversion to increase significantly (nearly double $\sim 57 \%$ ). The selectivity of the products at such relatively high CO conversion may have resulted in the appearance of the undesirable hydrocarbon liquid product. Thus, the selectivity to $\mathrm{C}_{2}+$ oxygenates may have been even greater under more controlled CO conversion. Furthermore, the increased activity of the Rh-Mn-Ir catalyst after higher-temperature testing suggests possible structural re-arrangement of $\mathrm{Rh}, \mathrm{Mn}$, and Ir at the higher temperature. 
Chemical calculations and theory were again employed to gain some insight for the increased activity and selectivity of the catalyst upon addition of Ir. It was first necessary to understand how well dispersed Mn and Ir were within the Rh-rich nanoparticles and how mobile these species were under operando conditions. Reactivity studies and AIMD simulations were performed on five different clusters of the general formula $\mathrm{Rh}_{\mathrm{x}} \mathrm{Mn}_{\mathrm{y}} \mathrm{Ir}_{\mathrm{z}}, \mathrm{x}+\mathrm{y}+\mathrm{z}=50$. AIMD simulation temperatures were in the range of 400 to $1800 \mathrm{~K}$. The positions and velocities of the atoms were used to extract metal distributions and overall structures as a function of temperature.

Figure 3.13 shows the metal distribution in two different composition clusters at high temperature $(1800 \mathrm{~K})$ and at $600 \mathrm{~K}$, which is nearer to the conditions of the mixed alcohols synthesis reactor operating temperature. The simulations showed that the clusters are "melted" (i.e., very plastic and almost fluid) at high temperatures. Generally, Ir shows a propensity for the interior of the cluster, but as Ir concentration increases, it can be found on the surface. Mn remains well mixed in all cases. Taking into account the simulations for all different $\mathrm{Mn} / \mathrm{Ir}$ compositions, we concluded that, for reasonably high $\mathrm{Mn}$ and moderate Ir concentrations, the nanoparticle will have all three metals on the surface.

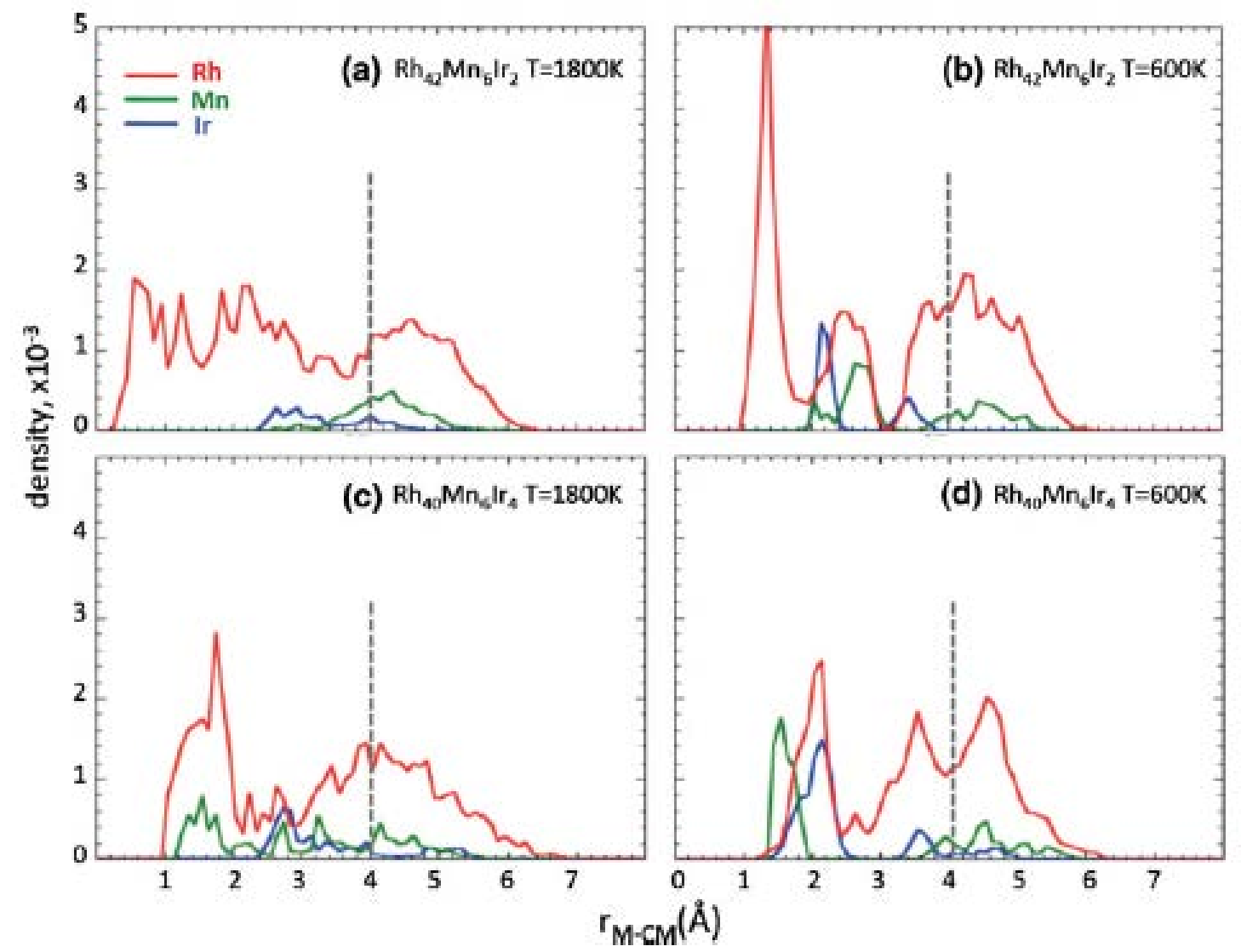

Figure 3.13. Density Distributions from the Center of Mass of the Cluster. The dotted line at $4 \AA$ roughly marks the interior of the cluster with a radius of $\sim 5 \AA$; distances to the right of the dotted line at $4 \AA$ are considered to be at or near the nanoparticle surface. Taken from Glezakou et al. ${ }^{8}$ 
An average coordination number that depicts the average distance between any two atom kinds in the particles also was calculated from radial distribution functions. The results are presented in Figure 3.14. The coordination number provides an estimate of the static and dynamic correlation between the atoms at different temperatures. Below approximatly $800 \mathrm{~K}$, the clusters become more structured and the coordination number decreases to about eight closest contacts for each atom, (red and blue lines). For the long-term neighbors, the root-mean-square fluctuation of their distance from a reference atom also was calculated. This parameter, also called the Lindemann criterion, provides an estimate of the amplitude of the thermal vibration, and ultimately the melting point of the cluster. In general, when interatomic distances fluctuate more than $\sim 10 \%$ (in the case of the clusters $\sim 0.3 \AA$ ), the particle starts to melt. This parameter is shown with the green and purple lines in Figure 3.14. Using the Lindemann criterion, the clusters melted at temperatures greater than $1000 \mathrm{~K}$. At $600 \mathrm{~K}$ they are essentially solids. However, with $\mathrm{d} \sim 0.2 \AA$ at $600 \mathrm{~K}$, the diffusion of metals through the cluster is still facile. This conclusion directly correlates with our experimetal results that show enhanced STY and selectivity after re-testing at $575 \mathrm{~K}$, implying that the increase in temperature causes diffusion and re-distribution on Ir and ultimately improves selectivity.

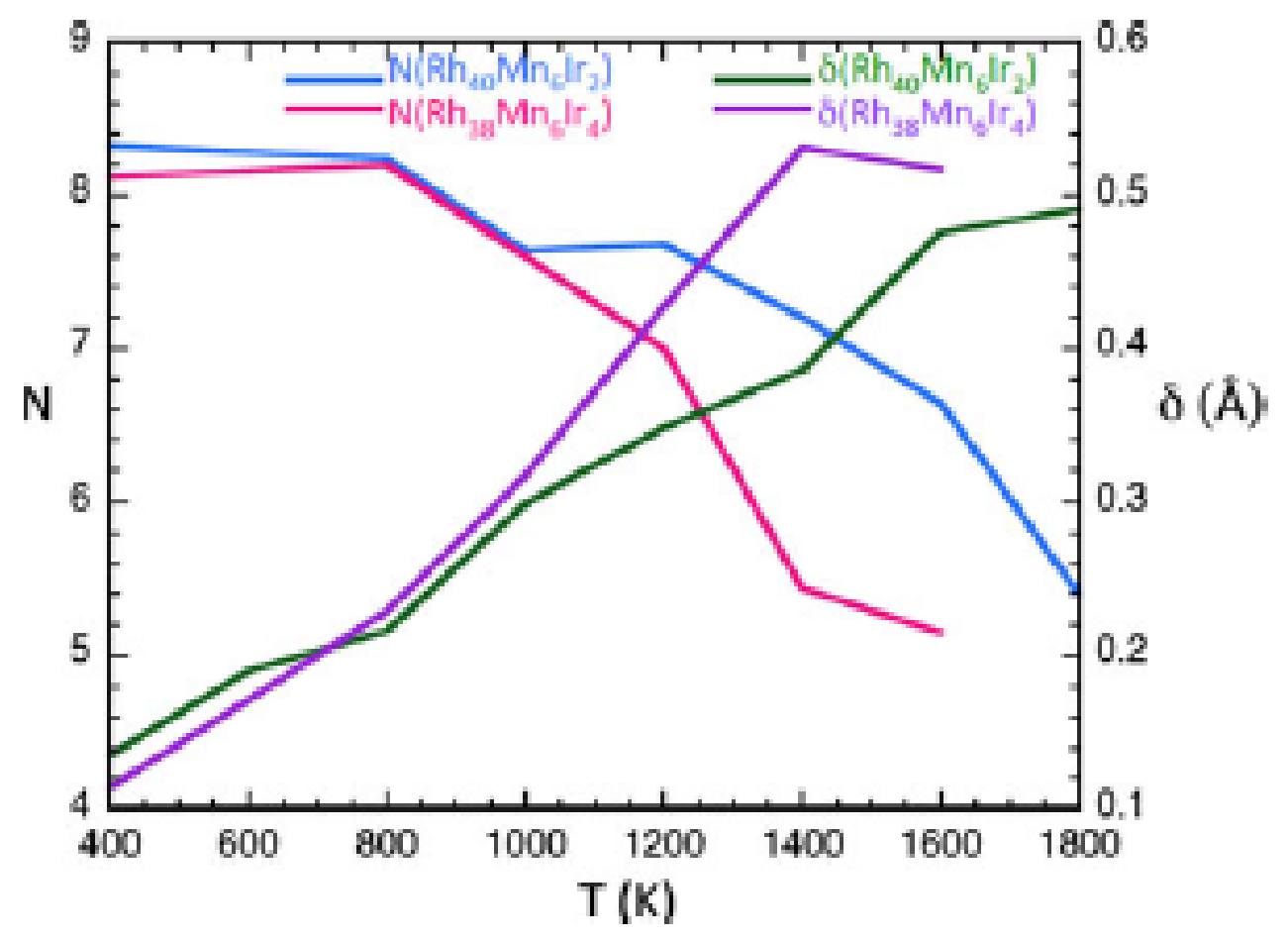

Figure 3.14. Figure C 16. Coordination Numbers $(\mathrm{N})$ and Bond Fluctuations $(\delta)$ Plotted Versus Temperature for Two Cluster Compositions

A set of reactivity studies was performed to help evaluate the role of Ir in the improved selectivity of the ternary clusters. Calculations were performed on the $\mathrm{Rh}_{40} \mathrm{Mn}_{6} \mathrm{Ir}_{4}$ nanoparticle after thermalization at $600 \mathrm{~K}$ and annealing. We note that evaluation of CO binding energy and redox properties of these clusters indicate that there is little depednece of the reactivity on the global concentration of these wellmixed clusters and that the leading indicator of reactivity is in fact the identity (either Rh, Ir or Mn) of the reactivtve site. AIMD simulations determined that a particle this size can adsorb up to two CO and three $\mathrm{H}_{2}$ molecules simulataneously, and these coverages were used to simulate the lowest energy path from $\mathrm{CO} / \mathrm{H}_{2}$ to $\mathrm{C}_{2}+$ oxygenates as shown in Figure 3.15 . 


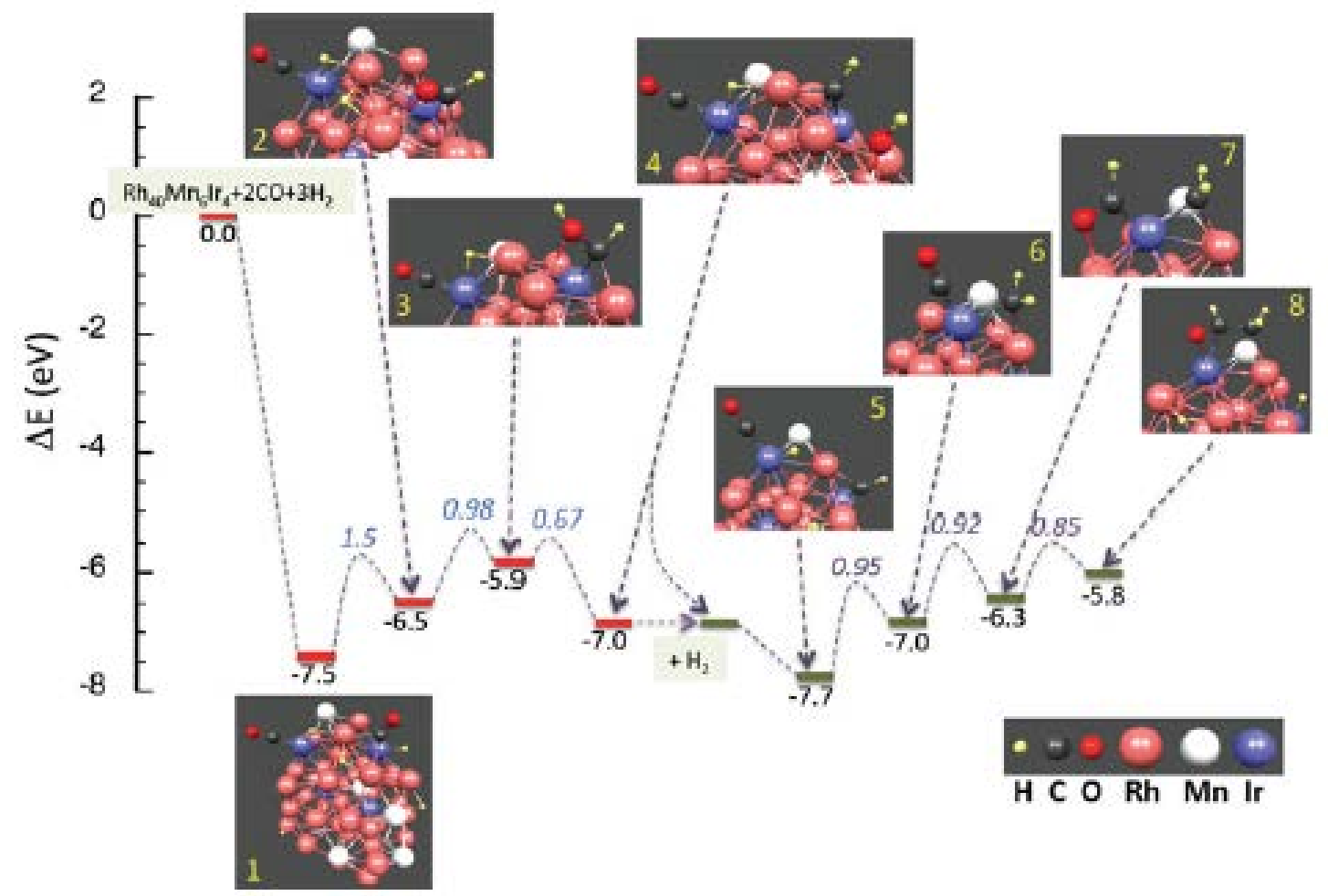

Figure 3.15. Initial Reactive Steps Leading to $\mathrm{C}_{2}$ Oxygenates on a Thermalized and Annealed $\mathrm{Rh}_{40} \mathrm{Mn}_{6} \mathrm{Ir}_{4}$ Nanoparticle. Taken from Glezakou et al. ${ }^{8}$

Reactive steps 1 through 4 in Figure 3.15 show the adsorption of the first two $\mathrm{CO}$ and three $\mathrm{H}_{2}$ (adsorbed as 6H). Our calculations also show how the particle morphology can affect the product formed: in general, CO preferably adsorbs on Ir sites, which also tend to simultaneously bind $\mathrm{H}$ atoms. The proximity of co-adsorbed $\mathrm{CO}$ and $\mathrm{H}$ at the same center facilitates the formation of HCO without the need to progress through CO dissociation, which has a very high activation barrier of over $2 \mathrm{eV}$. Ir on flat (terrace-like) parts of the particle tend to further react with more surface bound $\mathrm{H}$ to form $\mathrm{HCOH}$, which can eventually break into adsorbed $\mathrm{CH}$ and $\mathrm{OH}$ groups. On the other hand, Ir on edge-like sites may facilitate the formation of $\mathrm{HCO}$ moieties until they react with nearby $\mathrm{CH}$ groups. This new suggested pathway in the reaction network previously developed is illustrated by the red arrows in Figure 3.16 and may develop upon the addition of Ir. Furthermore, OCCH coupling is facilitated by the presence on Mn in the same way as in the binary $\mathrm{Rh} / \mathrm{Mn}$ catalyst. Therefore, we propose the Ir opens a new pathway to the generation of $\mathrm{C}_{2}+$ oxygenates in addition to the pathways present in the Rh-Mn catalyst. Our computational results correlate well with the experimental findings of increased CO conversion in the presence of Ir by making more reactive channels available. Also, C-C coupling is more likely to happen earlier in the conversion process, and by retaining the HCO groups in the initial stages without the prerequisite for $\mathrm{CO}$ dissociation, Ir helps direct the conversion toward $\mathrm{C}_{2}+$ oxygenates. 


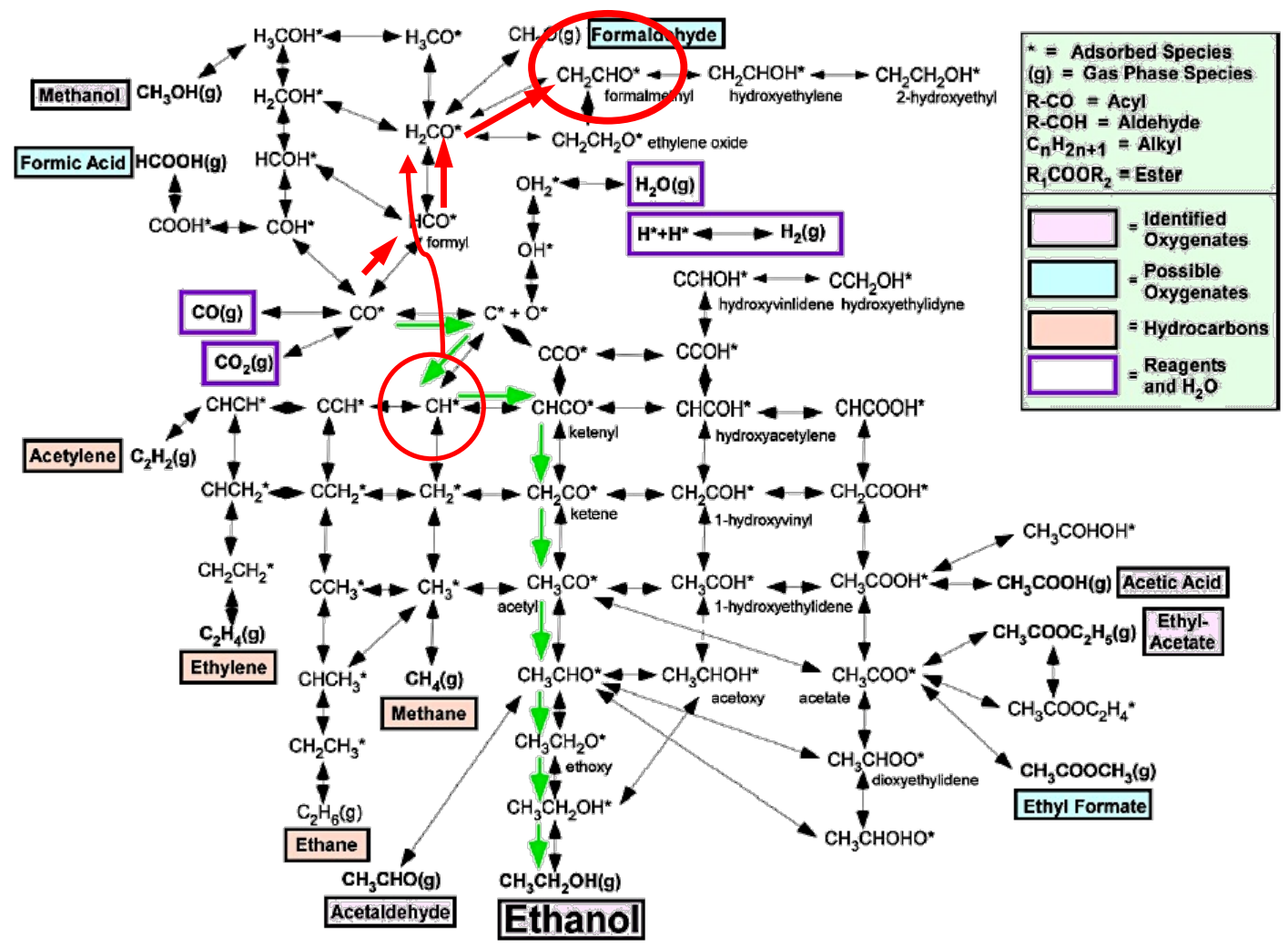

Figure 3.16. Elementary Reaction Diagram Showing Important Steps of Syngas Conversion. Red arrows represent the suggested pathway that opens upon the addition of Ir to the Rh-Mn catalyst.

\subsection{Effect of Catalyst Support on the Structure and Reactivity of Rh- Mn-Ir Catalyst Clusters}

Computational studies of the influence of the metal support materials on the structure of ternary (Rh, Mn, Ir) alloy particles were performed with AIMD simulations at temperature of $600 \mathrm{~K}$ on $1 \mathrm{~nm}$ $\mathrm{Rh}_{40} \mathrm{Ir}_{4} \mathrm{Mn}_{6}$ clusters. The metallic clusters were supported on either a (001) facet of $\alpha$-quartz or a graphene sheet as a model of a silica or carbon support, respectively. Two types of simulations were performed on both supports. The first set of simulations isolated the metallic clusters to investigate the influence of the support material alone on the alloy structure. The second set of simulations incorporated $10 \mathrm{H}_{2}$ and $10 \mathrm{CO}$ molecules to represent syngas adsorbed onto the metallic clusters to investigate the effect of the presence of reactants at high coverage with respect to their influence on catalyst structure.

Representative, well-equilibrated structures calculated from the first set of experiments undertaken to investigate the cluster shape as a function of each support are given in Figure 3.17. 

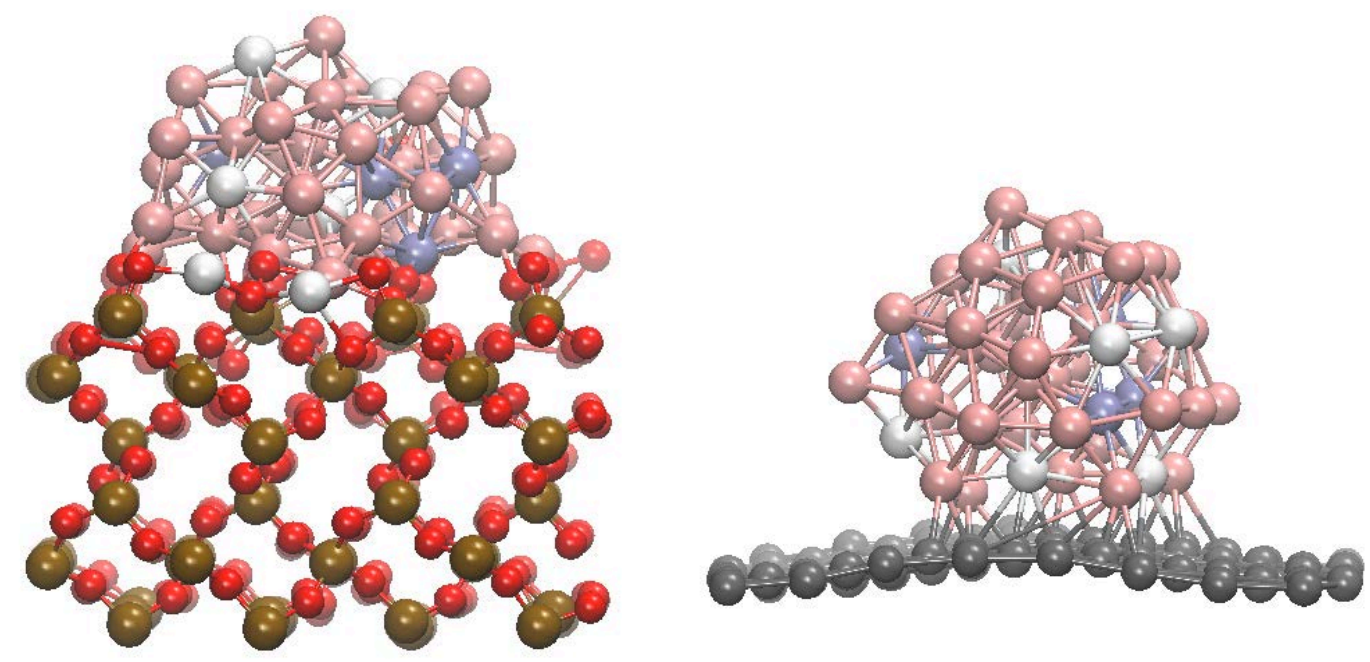

Figure 3.17. Structure of $\mathrm{Rh}_{40} \mathrm{Ir}_{4} \mathrm{Mn}_{6}$ on Silica (left) and Carbon (right) Quenched from a $20 \mathrm{ps}$ AIMD Simulation at T = $600 \mathrm{~K}$ : Rh (pink), Mn (white), Ir (blue), C (black), oxygen (red), Si (brown).

A few observations are evident from the structures shown in Figure 3.17. On carbon, the cluster remains approximately spherical ( $\mathrm{d}=1 \mathrm{~nm})$ with $\mathrm{Ir}, \mathrm{Rh}$, and $\mathrm{Mn}$ exposed on the surface. However, there was a tendency for two to three Ir atoms to remain sub-surface within the metallic particle. Likewise, one to two $\mathrm{Mn}$ atoms remain at the metallic cluster/graphene interface. Like the gas phase cluster developed in Mei et al. ${ }^{7}$, the diffusion of metal atoms is slow, but there is a slow exchange of sites between the bulk and the surface; approximately once every 5 ps. These results indicate that graphene has little effect on the cluster structure, and thus, it behaves similar to the isolated cluster model that has been used throughout this study to explain the reactivity.

On the other hand, the silica support had a much more profound impact on the cluster structure. Overall, the metal cluster wets the surface much more strongly, speading to be more of a hemispherical shape that is almost $1.4 \mathrm{~nm}$ in diameter at its base. In addition, a full two-thirds of the Mn atoms segregate to the metal/oxide interface and exist in a partially oxidized form. It is noticeable however that at least two of the Mn atoms remain within the Rh matrix as low oxidation state $\mathrm{Mn}$ in accord with our thermodynamic analysis based on isolated clusters. ${ }^{8}$ Ir behaves similarly to the gas phase, and graphene supported clusters in that one to two Ir atoms can be found at the surface at any given instant in time and remain in a low oxidation state within the $\mathrm{Rh}$ matrix. In general, the most striking influence of the support is on the amount of Mn available at the particle surface. Only a small fraction of Mn (approximately one-sixth) remains on the cluster surface in a low oxidation state, whereas on graphene, it is more than triple that value.

The second set of calculations simulated the effect of syngas on the silica- or carbon-supported clusters by adsorbing $10 \mathrm{CO}$ and $10 \mathrm{H}_{2}$ atoms onto the relaxed metal clusters. The supported clusters with $\mathrm{H}_{2}$ and $\mathrm{CO}$ adsorbed are presented in Figure 3.18. For these simulations, one CO molecule is broken into a $\mathrm{C}$ and $\mathrm{O}$ atom to simulate the products of a $\mathrm{CO}$ activation step (which would be kinetically slow on the AIMD time scale). It is notable that, in both simulations, all of the $\mathrm{CO}$ molecules adsorb onto the cluster while two to three $\mathrm{H}_{2}$ molecules remain in the gas phase at all times. This behavior is a result of the stronger binding of $\mathrm{CO}$ than $\mathrm{H}$ to the surface. 


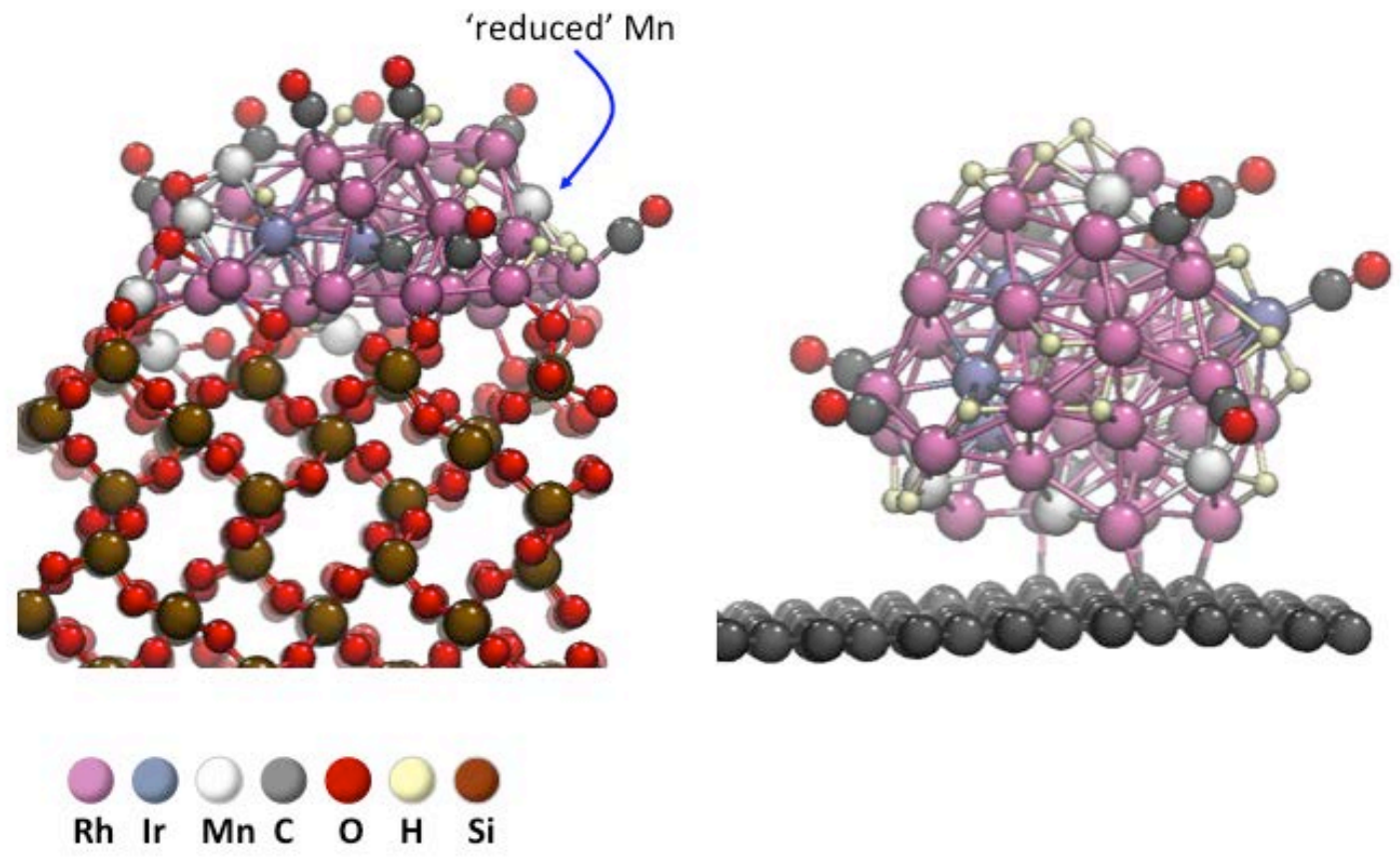

Figure 3.18. Structure of $\mathrm{Rh}_{40} \mathrm{Ir}_{4} \mathrm{Mn}_{6}$ on Silica (left) and Carbon (right) in the Presence of $\mathrm{H}_{2}$ and $\mathrm{CO}$ Adsorbates Quenched from a 20 ps AIMD Simulation at $\mathrm{T}=600 \mathrm{~K}$

On graphene, the metal atoms within the supported cluster remain distributed in a manner similar to the simulations without syngas. The Rh, Ir, and Mn remain available at the surface. Both $\mathrm{CO}$ and $\mathrm{H}$ prefer to reside on Ir and Rh sites, and freely diffuse on the cluster surface with $\mathrm{H}$ atoms hopping sites on the order of once every picosecond. The $\mathrm{C}$ of the dissociated $\mathrm{CO}$ remains at the surface in the vicinity of a $\mathrm{Rh}$ and $\mathrm{Mn}$ site but is not hydrogenated to $\mathrm{CH}$ on the AIMD time scale (although the hydrogenation is thermodynamically favorable in energy by $40 \mathrm{~kJ} / \mathrm{mol}$, it is not observed for kinetic reasons). The $\mathrm{O}$ adatom resides between two $\mathrm{Mn}$ sites, rendering them partially oxidized. Thus, even on graphene supports, Mn agglomeration may serve as a nucleation site for oxide growth.

The influence of syngas on the silica-supported particle is more profound. Adsorption of $\mathrm{CO}$ and $\mathrm{H}_{2}$ induces a larger surface reconstruction on the cluster and leads to further leaching of $\mathrm{Rh}$ and $\mathrm{Mn}$ onto the silica support. Ir is little influenced by the silica support and has multiple hydride adsorbates residing on it. Nonetheless, at least $1 \mathrm{Mn}$ sites remains well embedded in the $\mathrm{Rh}$ matrix in a reduced state though the five remaining $\mathrm{Mn}$ atoms are partially oxidized and slowly migrating toward the metal/oxide interface, which is indicative of phase segregation occurring over time periods longer than our simulations.

In summary, the results of the supported metal cluster AIMD studies suggest that, on supports such as graphene where weaker metal particle/support interactions occur, Rh, Ir, and $\mathrm{Mn}$ can remain at the cluster surface for reactivity. On the other hand, more strongly interacting supports such as silica alter the metal clusters substantially. Most notably, it becomes harder to maintain an appreciable concentration of Mn within the Rh nanoparticle on silica as there was appreciably more leaching of $\mathrm{Mn}$ toward the metal/oxide interface or onto the support. Segregation of Mn was enhanced by the presence of $\mathrm{CO}$ and $\mathrm{H}$ adsorbed onto the surface. 


\subsection{Summary}

The characterization and computational chemistry measurements and investigations undertaken in support of the development of a Rh-based mixed alcohol synthesis catalyst are documented in this report. Numerous measurements were performed to characterize several carbon catalyst supports to investigate features such as ash concentration and inorganic impurities, surface area and pore size distribution, and oxygenated surface functionality. Silica catalyst supports also were characterized to understand the concentration of non-silaceous impurities, surface area and pore size distribution, and surface acidity. Measurements performed on both the carbon and silica supports contributed to the selection, understanding, and comparison of the diverse supports in the course of catalyst development.

Several silica and carbon-supported catalysts also were measured and compared at distinct points in the life cycle of the catalysts. Freshly prepared catalysts, reduced catalysts, and catalysts subjected to low temperature $\left(275^{\circ} \mathrm{C}\right)$ and high temperature $\left(300^{\circ} \mathrm{C}\right)$ were prepared and investigated. XPS measurements of the metal concentration at the catalysts surface suggested that the presence of Ir as a promoter increased the concentration of $\mathrm{Mn}$ at the catalyst surface. TEM measurements suggested metal grain growth and sintering in samples subjected to the conditions of mixed alcohol synthesis as compared to the freshly reduced samples. Samples subjected to the conditions mixed alcohol synthesis at $300^{\circ} \mathrm{C}$ may have exhibited greater sintering. The higher degree of sintering after the catalysts had been used at $300^{\circ} \mathrm{C}$ is consistent with XRD measurements, which showed a greater degree of crystallinity in samples subjected to high temperature operando conditions. While several XRD phases were ambiguous or isomorphic with unlikely phases (i.e., the HfIr phase was identified but is unlikely to be present), the presence of metallic Rh and Ir and the discovery of crystalline $\mathrm{MnCO}_{3}$ all gave valuable insight into the nature of the spent catalyst samples.

The computational chemistry investigations were instrumental in helping development of our fundamental understanding of the nature of the catalyst from a first-principles perspective. The thermodynamically based deduction regarding the metallic state of Mn present in Rh-Mn clusters provided the basis for the observation that Mn opens a new pathway for C-C bond formation that does not have a large effect on carbon hydrogenation. Thus, the addition of Mn opened a new pathway for the formation of ethanol and other $\mathrm{C}_{2}+$ oxygenates without a significant effect on the rate of methane formation. Furthermore, calculations performed on the addition of Ir to the computationally investigated metallic cluster revealed the opening of yet an additional pathway to $\mathrm{C}_{2}+$ oxygenates. Calculations pertaining to the effect of a silica support versus a carbon support revealed that the silica support was likely acting as sink to the catalytically necessary Mn. Computational investigations of C supported RhMn-Ir particles revealed the lack of interaction with the support, even when a syngas simulant of CO and $\mathrm{H}$ atoms were added to the system.

Further development of the Rh-based mixed alcohol synthesis catalyst would benefit from a continuing effort to probe the nature of the metals on supported catalysts, especially pertaining to the nature of the active metal clusters. Directly observed evidence of the oxidation state of Mn has been difficult to ascertain, but state-of-the-art techniques such as x-ray adsorption near edge structure(XANES) may be useful in determining the oxidation state of $\mathrm{Mn}$ as well the local coordination environment of Rh, Mn, and Ir. The effect of electronic coupling (or decoupling) of the metal particles with the support would be a highly interesting combined computational and characterization investigation. For example, if the metal particles on the silica support are electronically decoupled as suggested by XPS measurements, 
it would be interesting to study the effect on the reactivity of the metal clusters and how decoupled clusters compare with electronically coupled clusters as was suggested of the metal clusters on the carbon support. Finally, the effect of adding a dopant or excess Mn to the silica supports before applying the RhMn impregnation liquid may inhibit the migration of catalytically active Mn into the silica support. The investigation of manganese silicates as catalyst supports could be investigated through reaction testing, characterization, and computational means. 


\subsection{References}

1. Gerber, M. A.; Gray, M. J.; Stevens, D. J.; White, J. F.; Rummel, B. L. Optimization of RhodiumBased Catalysts for Mixed Alcohol Synthesis -- 2009 Progress Report; PNNL-20115; Pacific Northwest National Laboratory: Richland, Washinton, 2010.

2. Gerber, M. A.; White, J. F.; Gray, M. J.; Stevens, D. J. Mixed Alcohol Synthesis Catalyst Screening 2007 Progress Report; PNNL-17074; Pacific Northwest National Laboratory: Richland, Washinton, 2007.

3. Gerber, M. A.; White, J. F.; Gray, M. J.; Stevens, D. J. Evaluation of Promoters for Rhodium-Based Catalysts for Mixed Alcohols Synthesis; PNNL-17857; Pacific Northwest National Laboratory: Richland, Washinton, 2008.

4. Gerber, M. A.; White, J. F.; Stevens, D. J. Mixed Alcohol Catalyst Screening; PNNL-16763; Pacific Northwest National Laboratory: Richland, Washinton, 2007.

5. Groen, J. C.; Peffer, L. A. A.; Pérez-Ramírez, J., Pore size determination in modified micro- and mesoporous materials. Pitfalls and limitations in gas adsorption data analysis. Microporous and Mesoporous Materials 2003, 60, (1-3), 1-17.

6. Aksoylu, A. E.; Freitas, M. M. A.; Figueiredo, J. L., Bimetallic Pt-Sn catalysts supported on activated carbon: I. The effects of support modification and impregnation strategy. Applied Catalysis A: General 2000, 192, (1), 29-42.

7. Mei, D.; Rousseau, R.; Kathmann, S. M.; Glezakou, V.-A.; Engelhard, M. H.; Jiang, W.; Wang, C.; Gerber, M. A.; White, J. F.; Stevens, D. J., Ethanol synthesis from syngas over Rh-based/SiO2 catalysts: A combined experimental and theoretical modeling study. Journal of Catalysis 2010, 271, (2), 325-342.

8. Glezakou, V.-A.; Jaffe, J.; Rousseau, R.; Mei, D.; Kathmann, S.; Albrecht, K.; Gray, M.; Gerber, M., The Role of Ir in Ternary Rh-Based Catalysts for Syngas Conversion to C\&lt;sub\&gt;2\&lt;/sub\&gt;\&lt;sup\&gt;+\&lt;/sup\&gt; Oxygenates. Topics in Catalysis 2012, 55, (7), 595-600.

9. Okpalugo, T. I. T.; Papakonstantinou, P.; Murphy, H.; McLaughlin, J.; Brown, N. M. D., High resolution XPS characterization of chemical functionalised MWCNTs and SWCNTs. Carbon 2005, 43, (1), 153-161.

10. Moulder, J.; Stickle, W.; Sobol, P.; Bomben, K., Handbook of X-Ray Photoelectron Spectroscopy Perkin-Elmer Corporation: 1992.

11. NIST X-ray Photoelectron Spectroscopy Database Version 3.5 (National Institute of Standards and Technology, In Gaithersburg, 2003) http://srdata.nist.gov/xps/.

12. Barr, T. L., Studies in differential charging. Journal of Vacuum Science \& Technology A: Vacuum, Surfaces, and Films 1989, 7, (3), 1677-1683. 
13. Polvinen, R.; Vippola, M.; Valden, M.; Lepistö, T.; Suopanki, A.; Härkönen, M., The effect of PtRh synergism on the thermal stability of rhodium oxide on pure alumina and Ce-ZrO2-modified alumina-supported catalysts. Journal of Catalysis 2004, 226, (2), 372-381.

14. Huang, J. H.; Rosén, E., Determination of Gibbs free energies of formation for the silicates MnSiO\&lt;sub\&gt;3\&lt;/sub\&gt;, Mn\&lt;sub\&gt;2\&lt;/sub\&gt;SiO\&lt;sub\&gt;4\&lt;/sub\&gt; and Mn\&lt;sub\&gt;7\&lt;/sub\&gt;SiO\&lt;sub\&gt;12\&lt;/sub\&gt; in the temperature range 1000-1350 K by solid state emf measurements. Physics and Chemistry of Minerals 1994, 21, (4), 228-233.

15. Wilson, T. P.; Kasai, P. H.; Ellgen, P. C., The state of manganese promoter in rhodium-silica gel catalysts. Journal of Catalysis 1981, 69, (1), 193-201.

16. Hu, J.; Wang, Y.; Cao, C.; Elliott, D. C.; Stevens, D. J.; White, J. F., Conversion of biomassderived syngas to alcohols and C2 oxygenates using supported Rh catalysts in a microchannel reactor. Catalysis Today 2007, 120, (1), 90-95.

17. Ojeda, M.; Granados, M. L.; Rojas, S.; Terreros, P.; García-García, F. J.; Fierro, J. L. G., Manganese-promoted Rh/Al2O3 for C2-oxygenates synthesis from syngas: Effect of manganese loading. Applied Catalysis A: General 2004, 261, (1), 47-55.

18. Progress in C1 Chemistry in Japan. Elsevier Science Ltd.: Amsterdam, The Netherlands, 1989. 


\section{Appendix A}

\section{Pore Volume Distributions of Carbon Supports KOA1 through 15 Obtained from $\mathrm{N}_{2}$ Adsorption/Desorption Testing}





\section{Appendix A}

\section{Pore Volume Distributions of Carbon Supports KOA1 through 15 Obtained from $\mathrm{N}_{2}$ Adsorption/Desorption Testing}

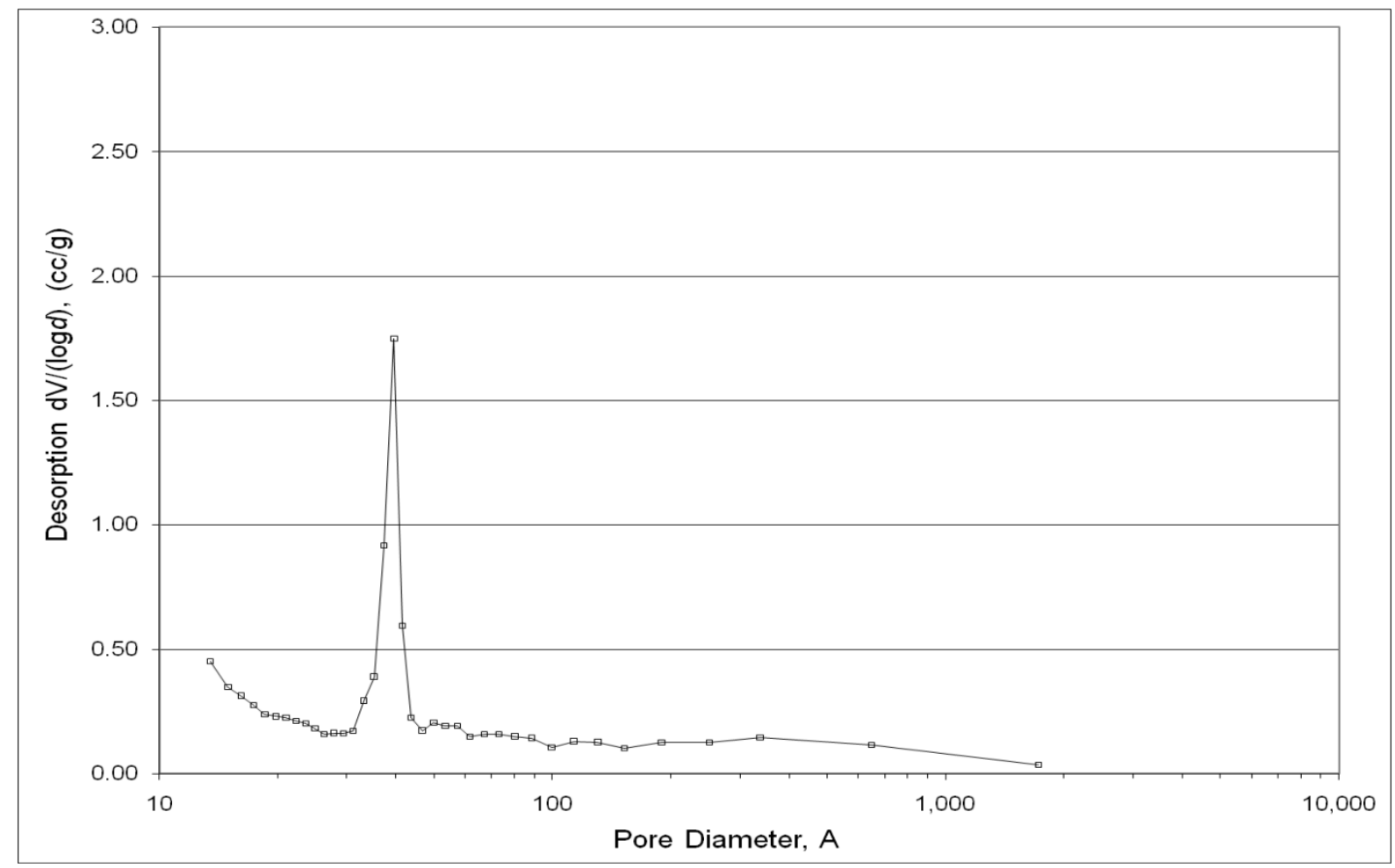

Figure A.1. Pore size distribution of KOA 1, which is Norit ROX 0.8. 


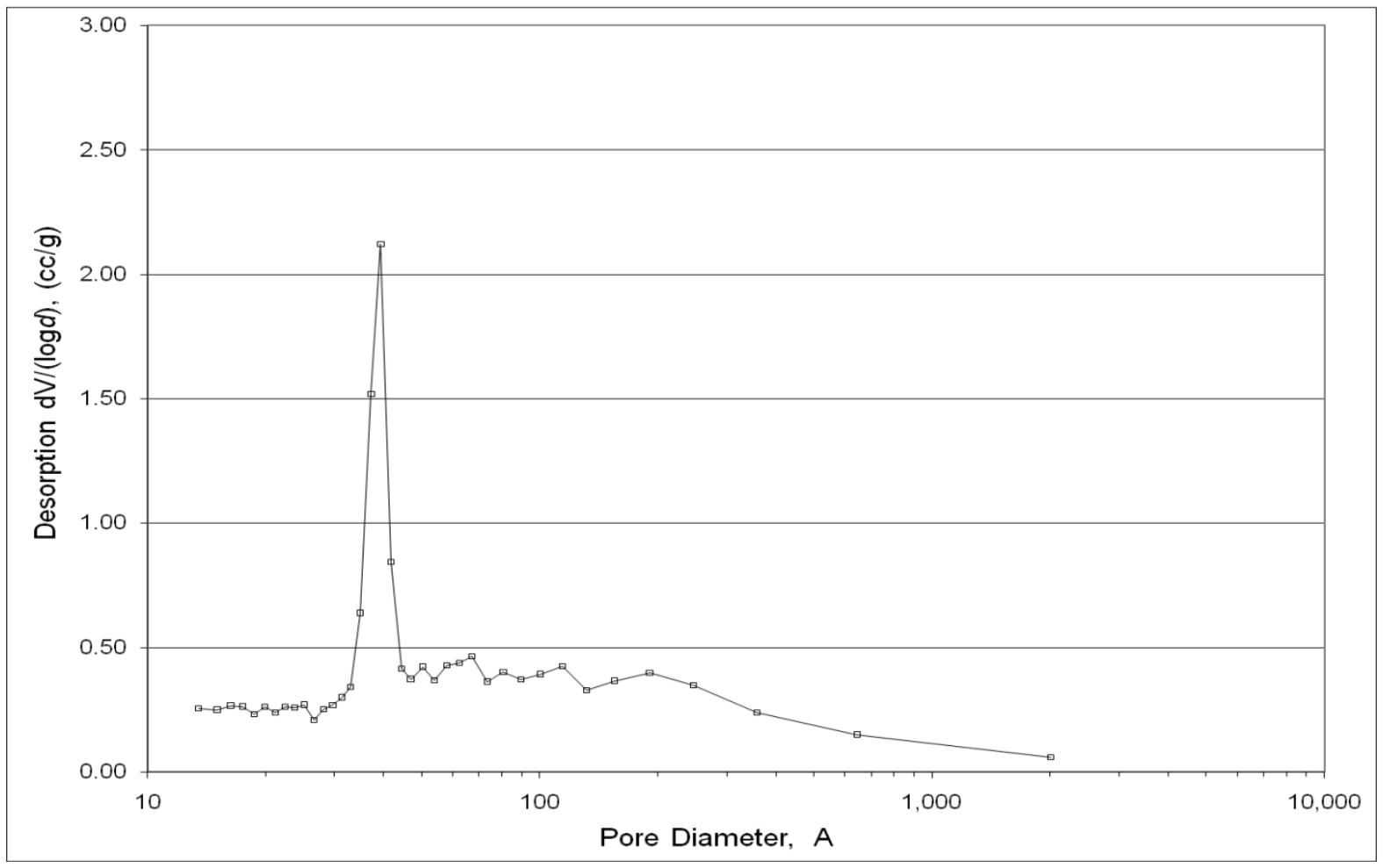

Figure A.2. Pore size distribution of KOA 2, which is Norit Darco-LS.

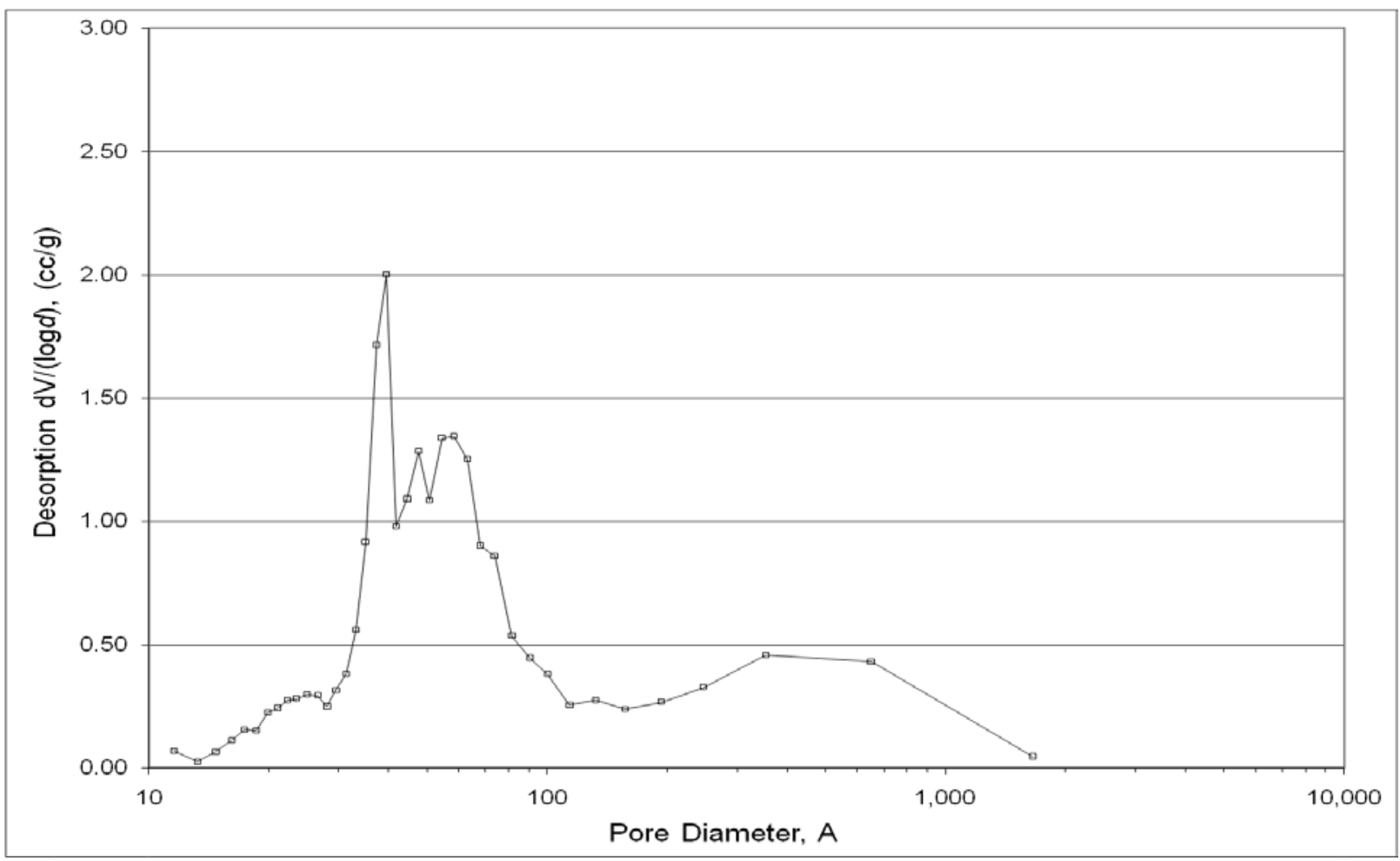

Figure A.3. Pore size distribution of KOA 3, which is Sibunit carbon support. 


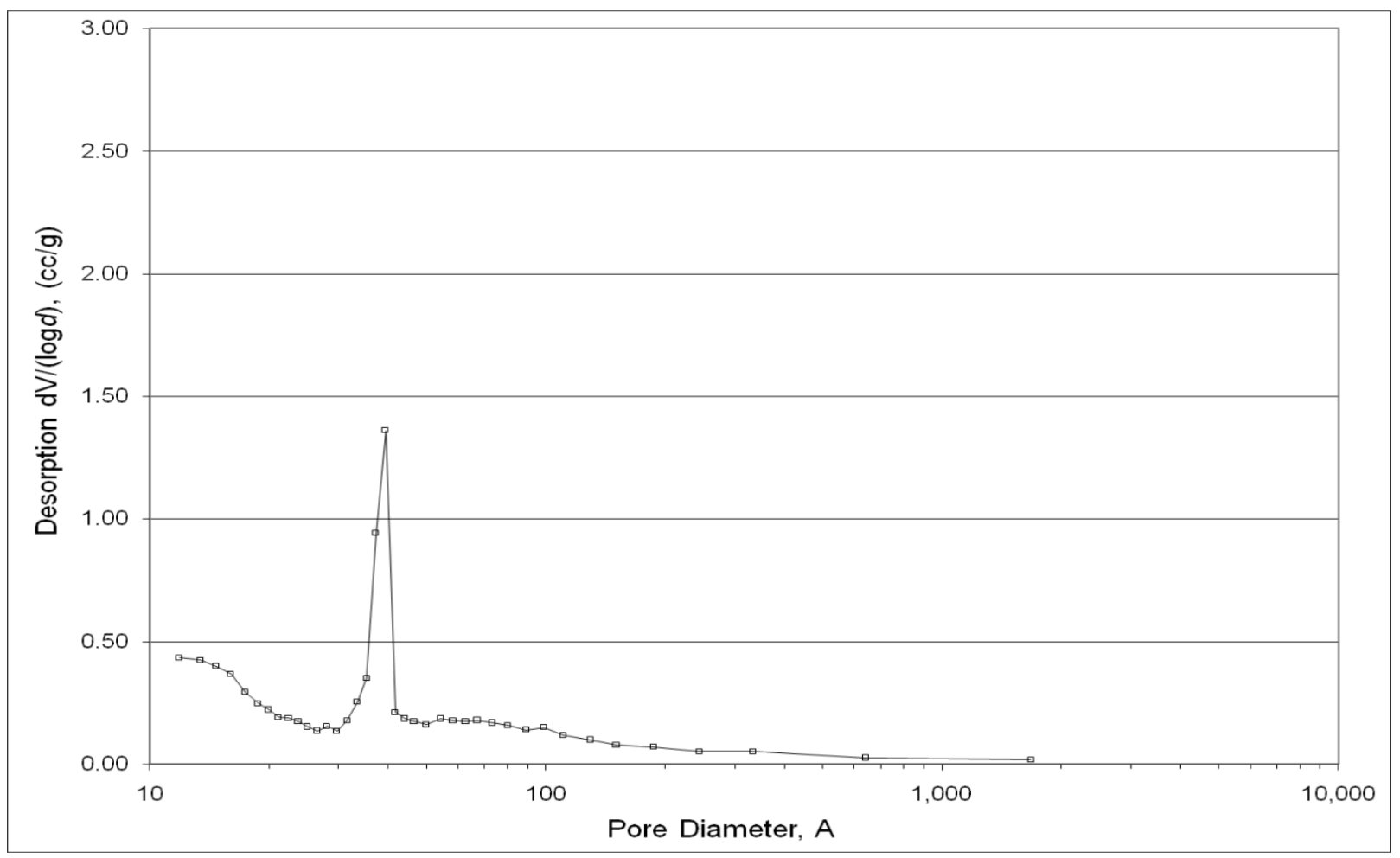

Figure A.4. Pore size distribution of KOA 4, which is Englehard High Surface Area Graphite (HSAG-1).

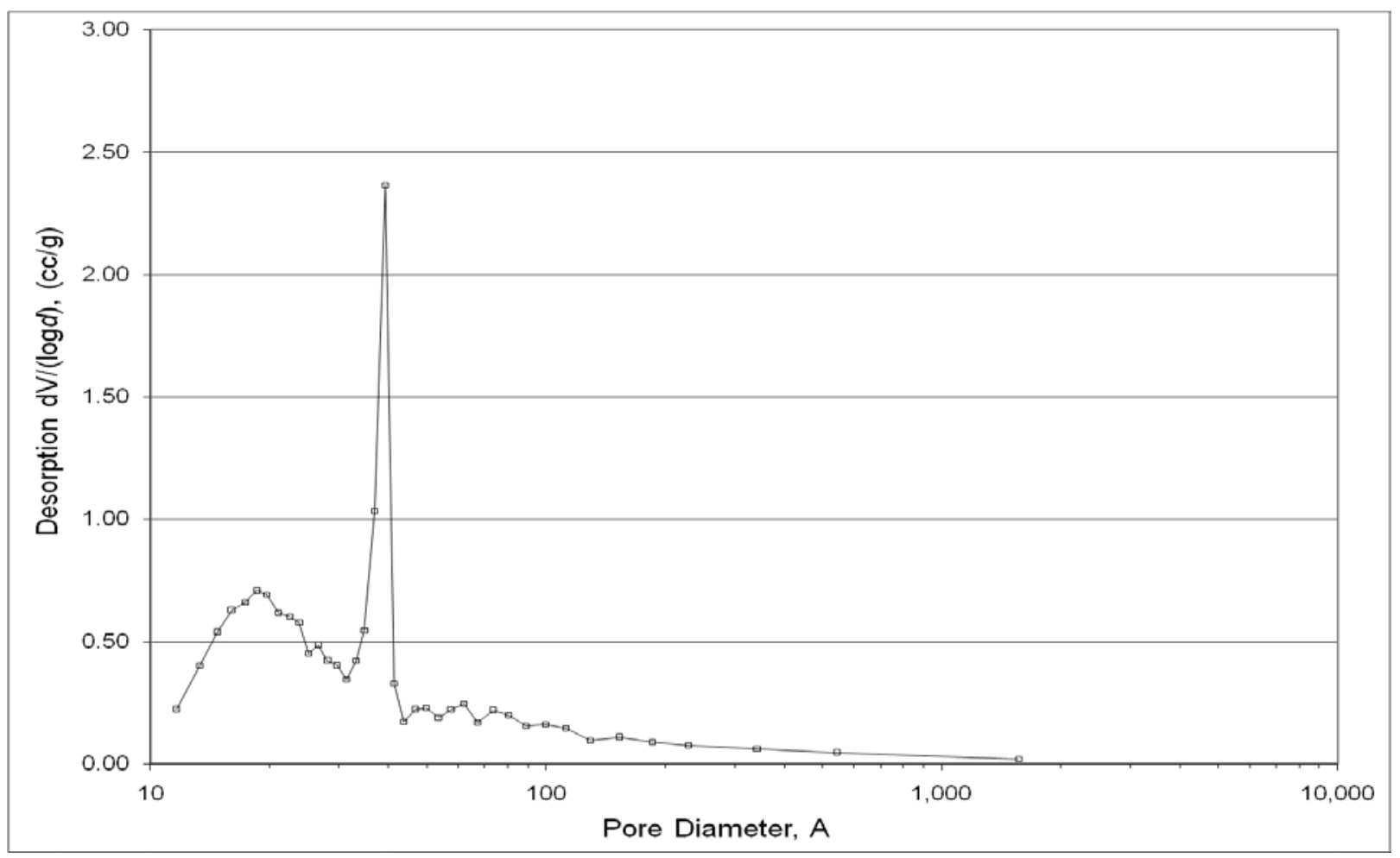

Figure A.5. Pore size distribution of KOA 5, which is Englehard High Surface Area Graphite (HSAG-2). 


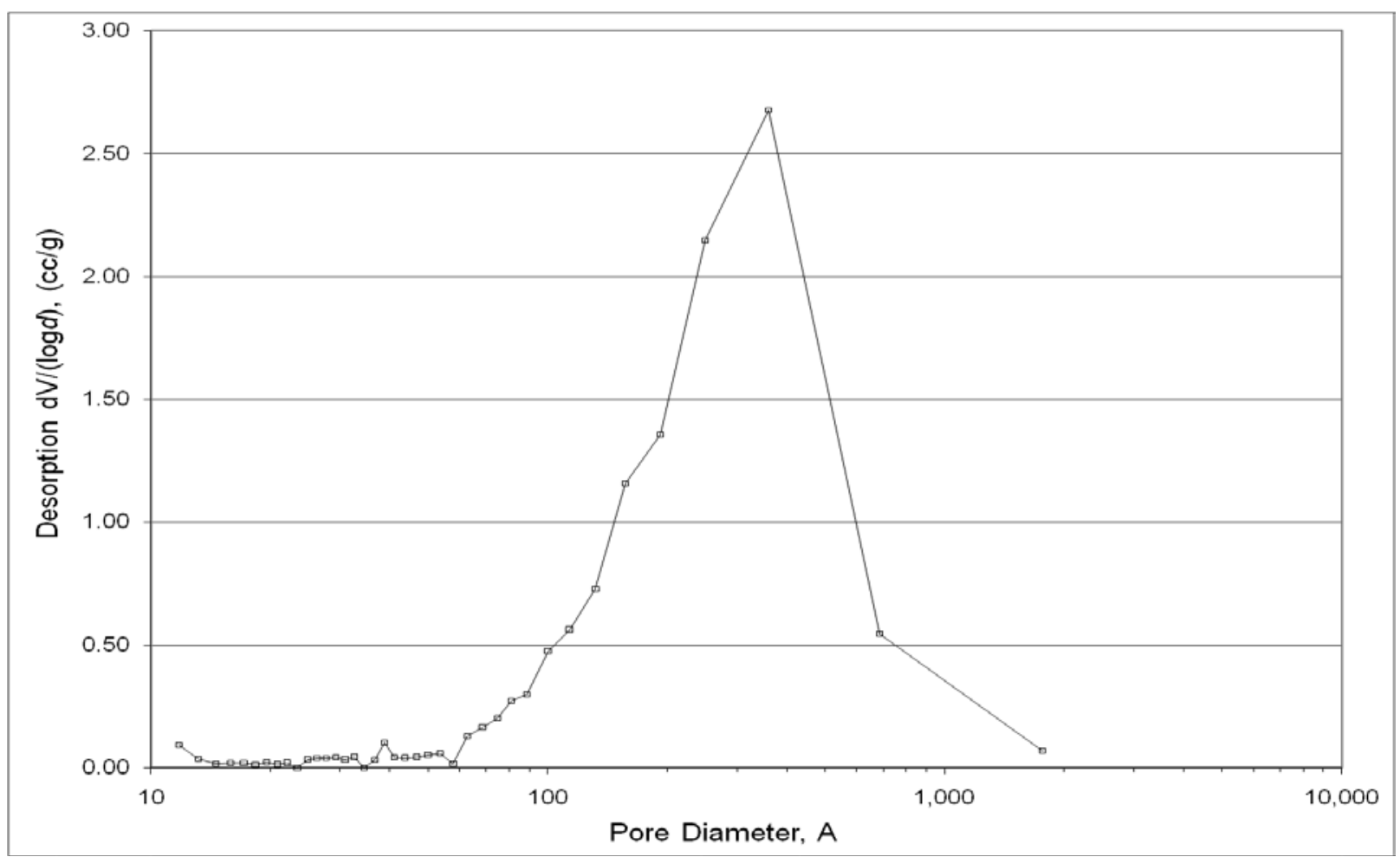

Figure A.6. Pore size distribution of KOA 6, which is the Hyperion Lot 395-08 carbon support.

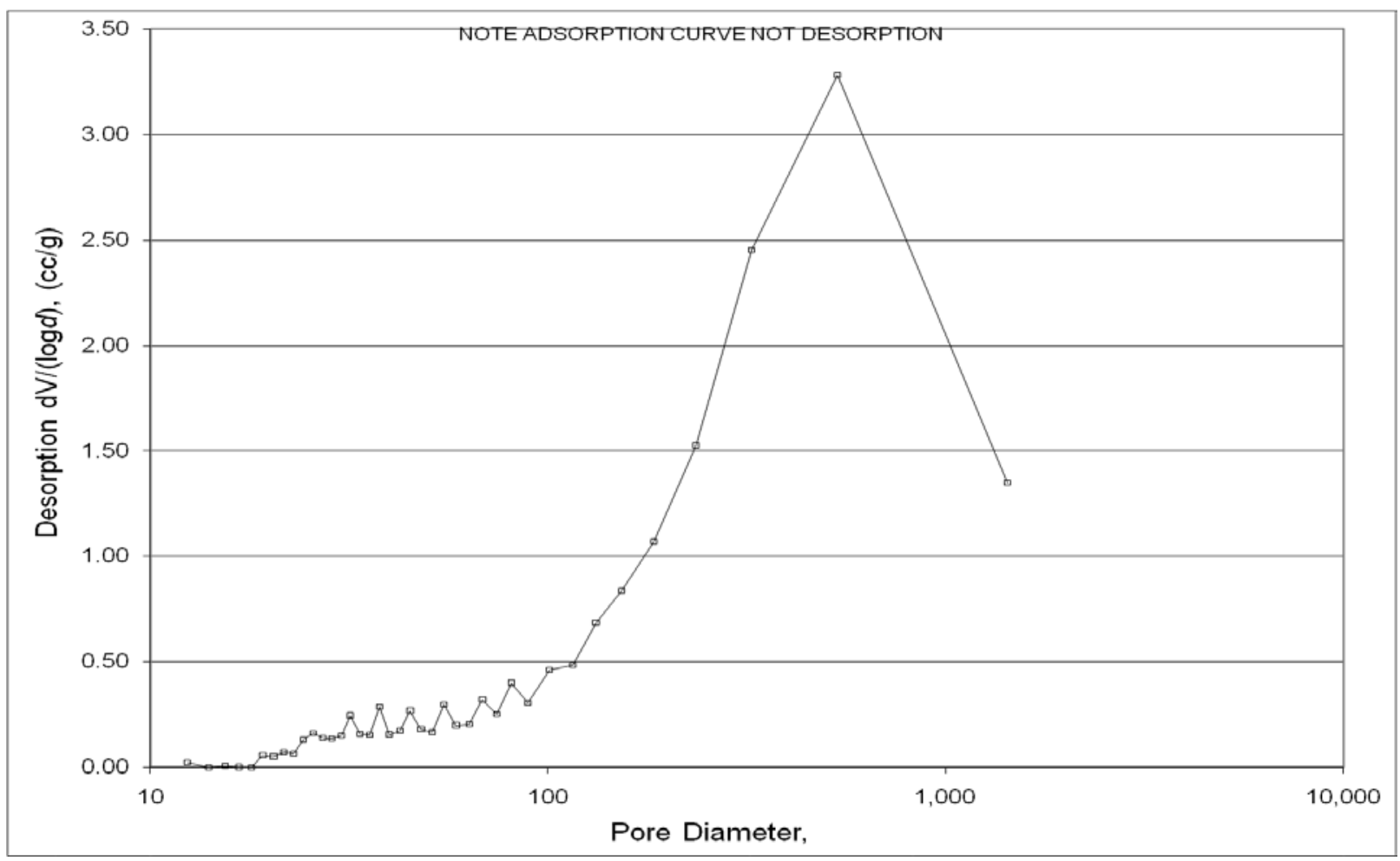

Figure A.7. Pore size distribution of KOA 7, which is the Hyperion Lot 395-10 carbon support. Note that this pore size distribution was determined from the adsorption of $\mathrm{N}_{2}$ as opposed to the desorption portion of the $\mathrm{N}_{2}$ test. 


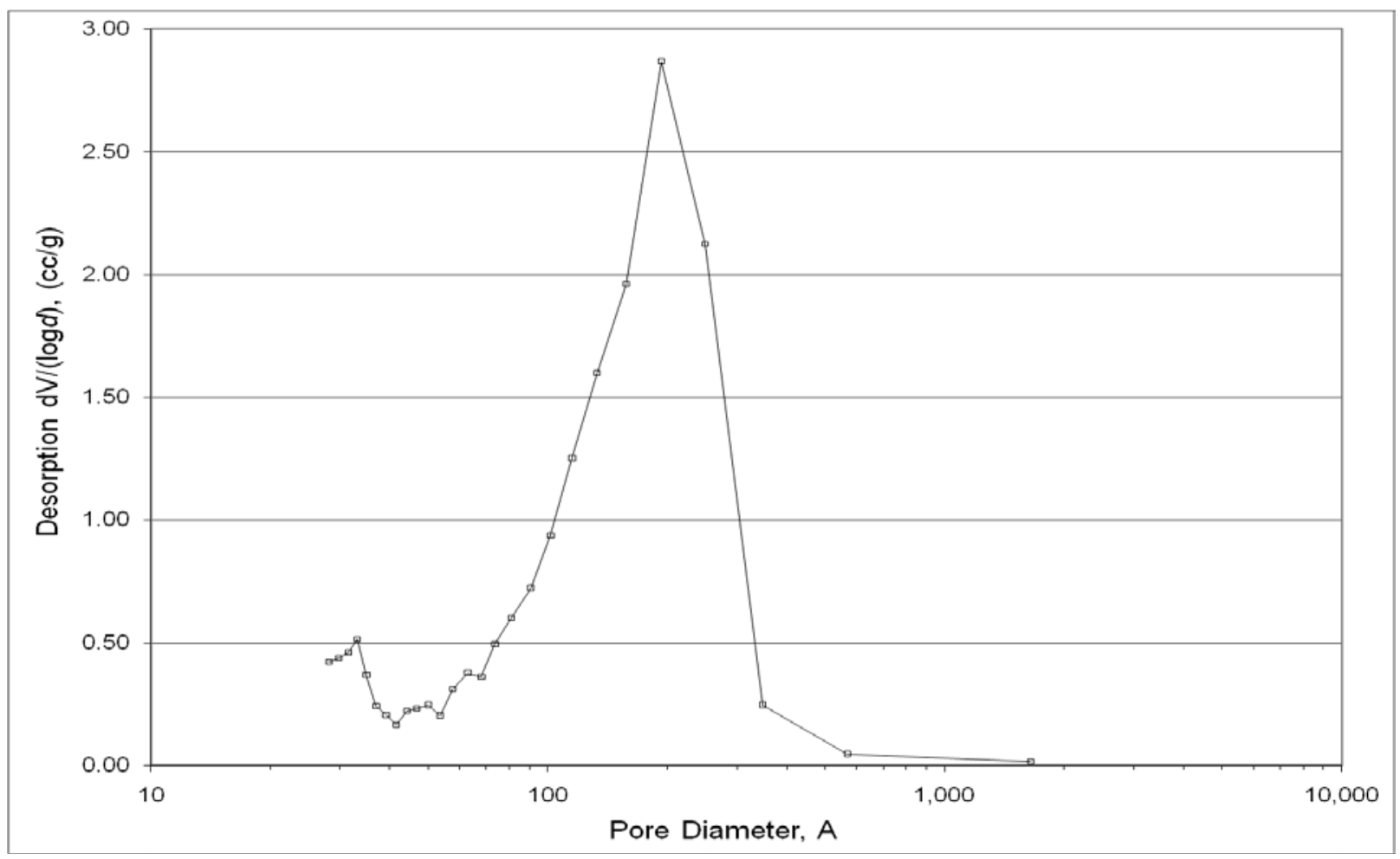

Figure A.8. Pore size distribution of KOA 8, which is the Hyperion Lot 384-82 carbon support.

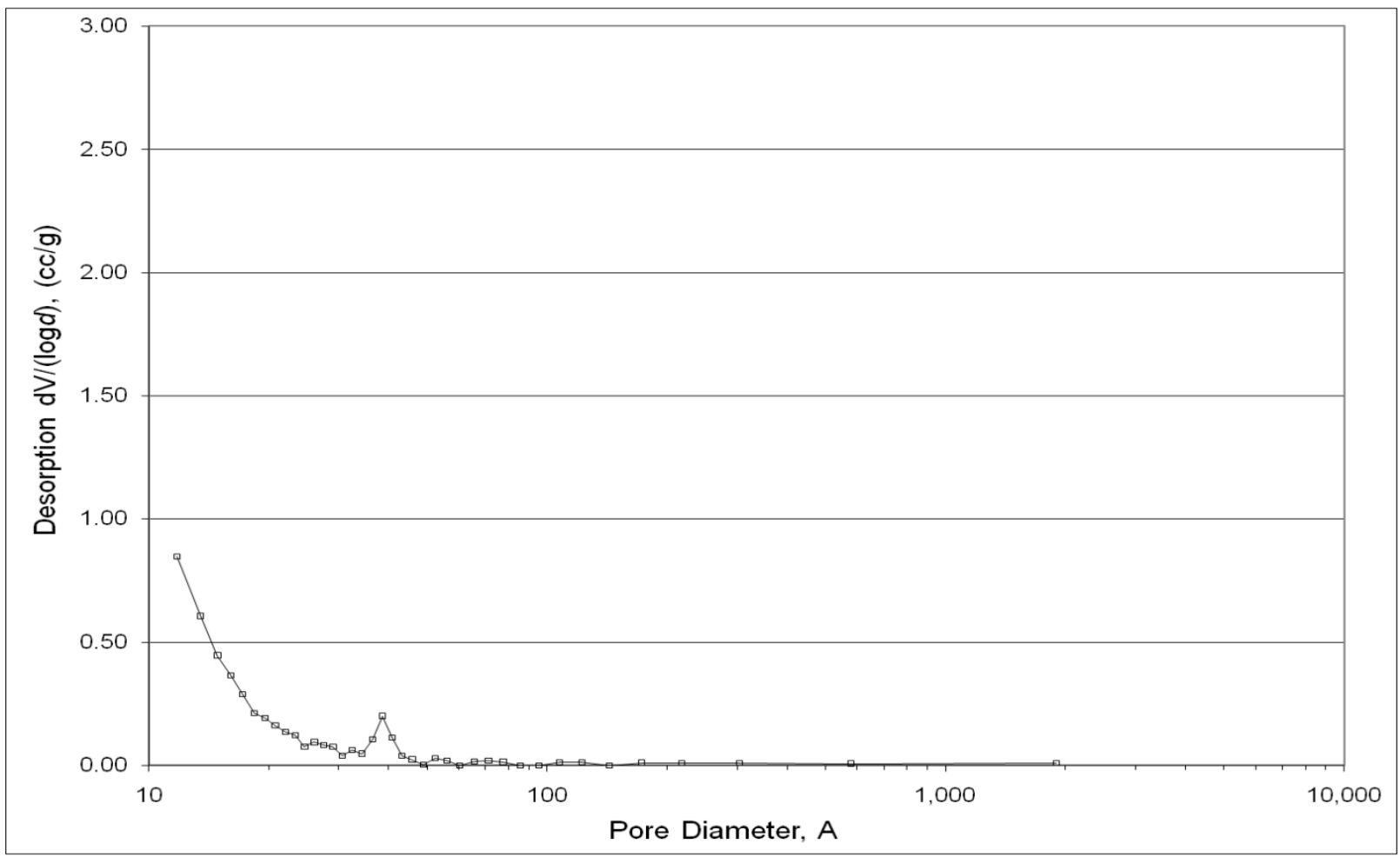

Figure A.9. Pore size distribution of KOA 9, which is Cummins-Moore 5559 S carbon support. 


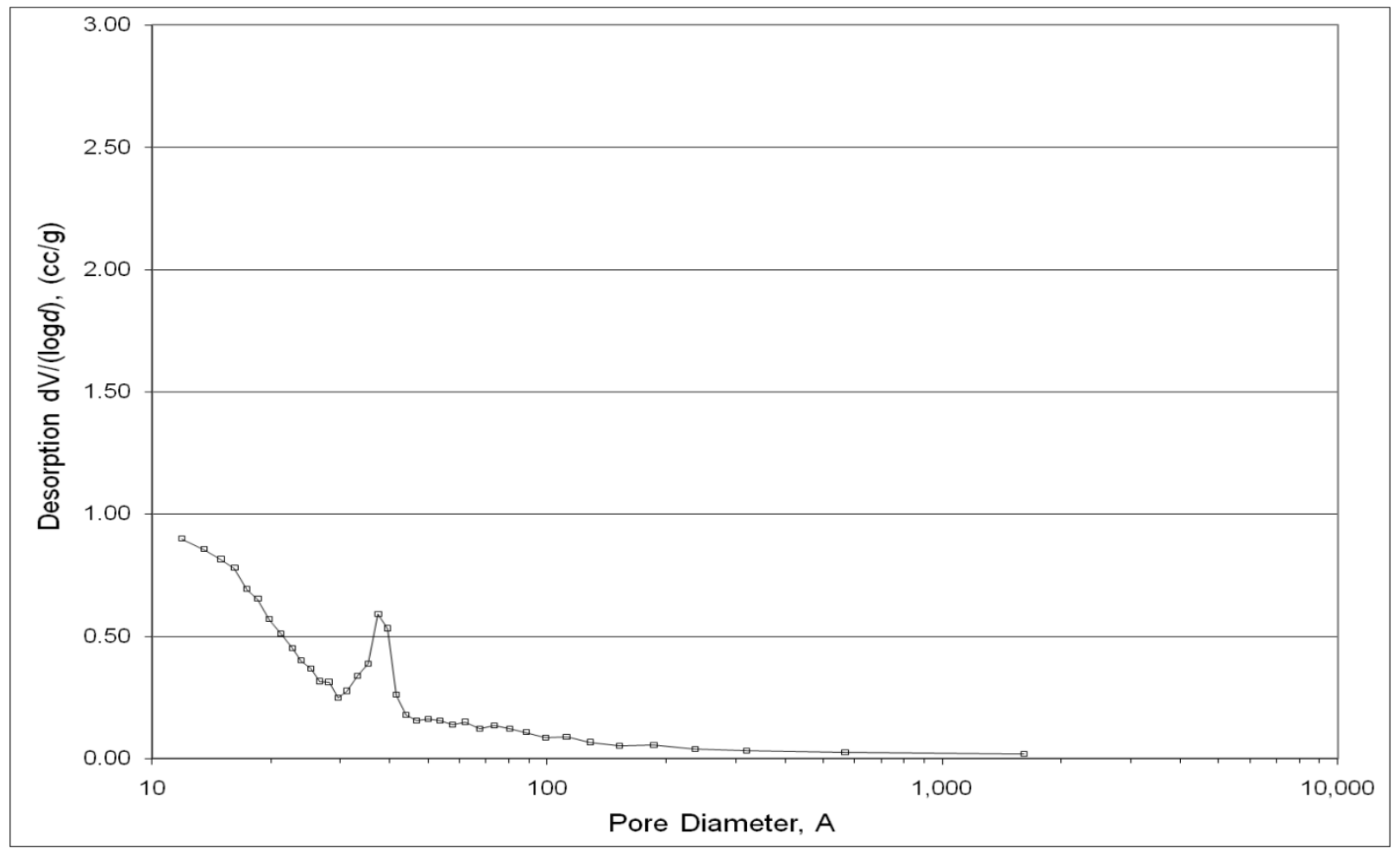

Figure A.10. Pore size distribution of KOA 10, which is Cummins-Moore 5586 AW carbon support.

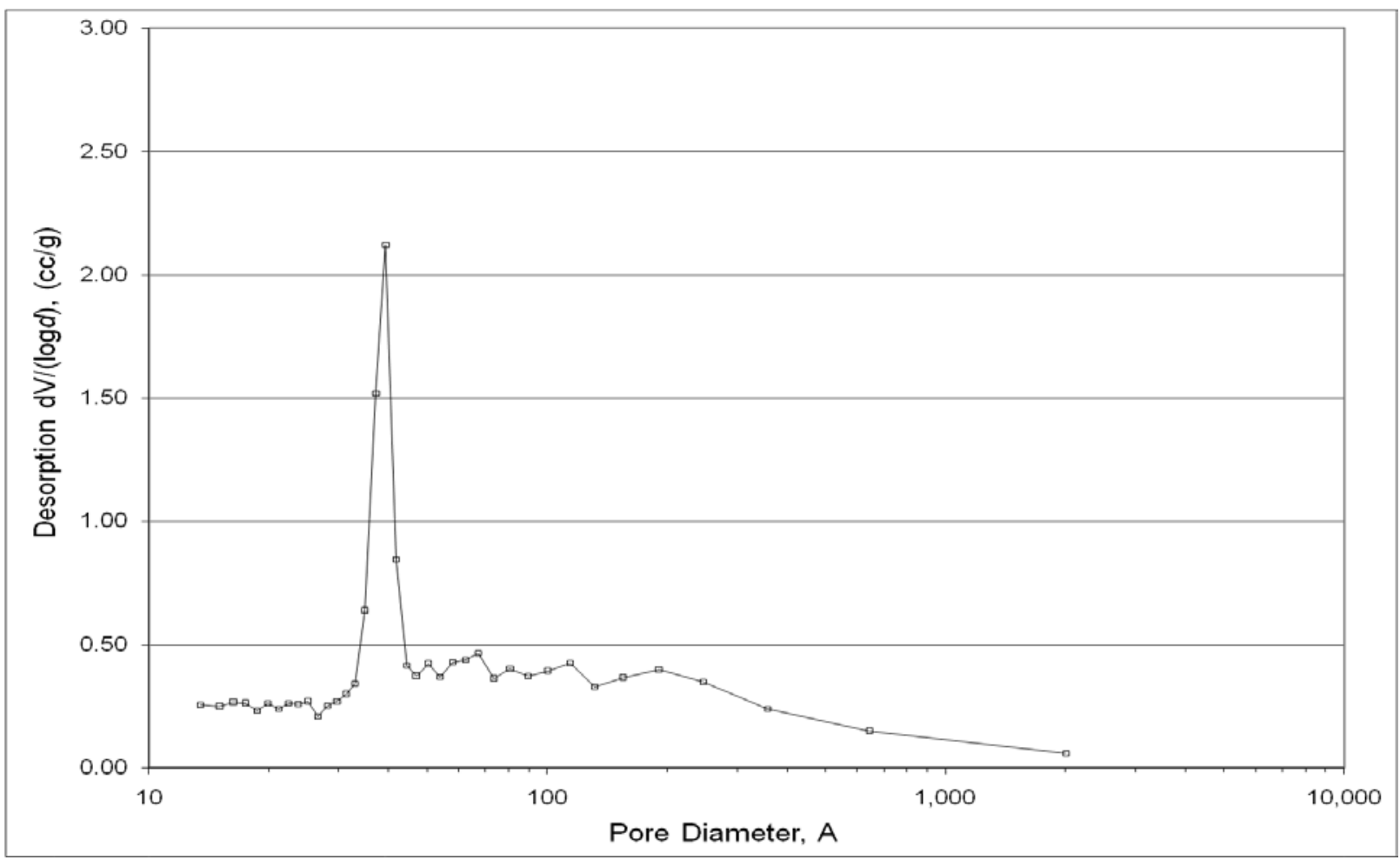

Figure A.11. Pore size distribution of KOA 11, which is Norit Darco-LS subjected to HF washing. 


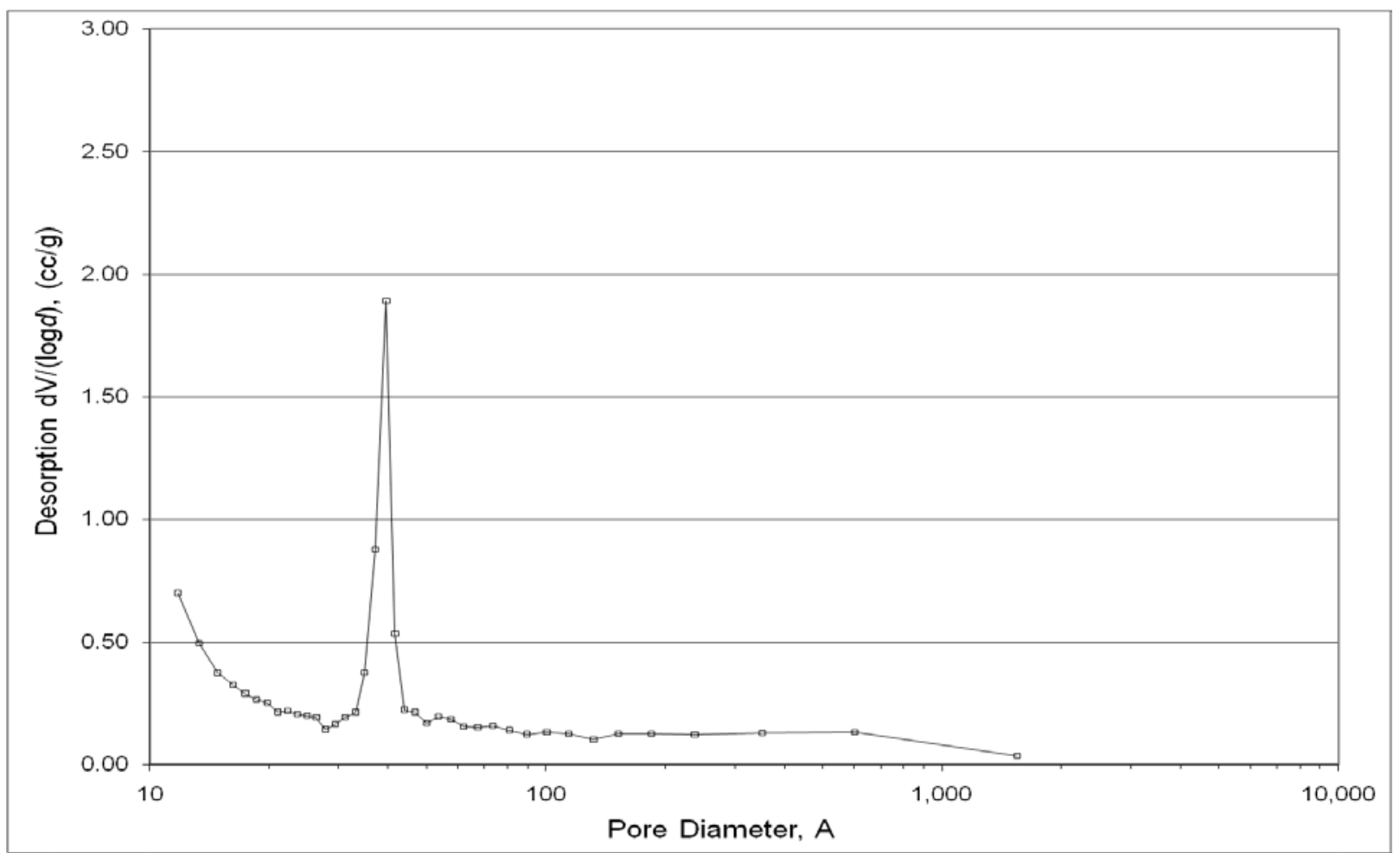

Figure A.12. Pore size distribution of KOA 12, which is Norit ROX 0.8 subjected to HF washing.

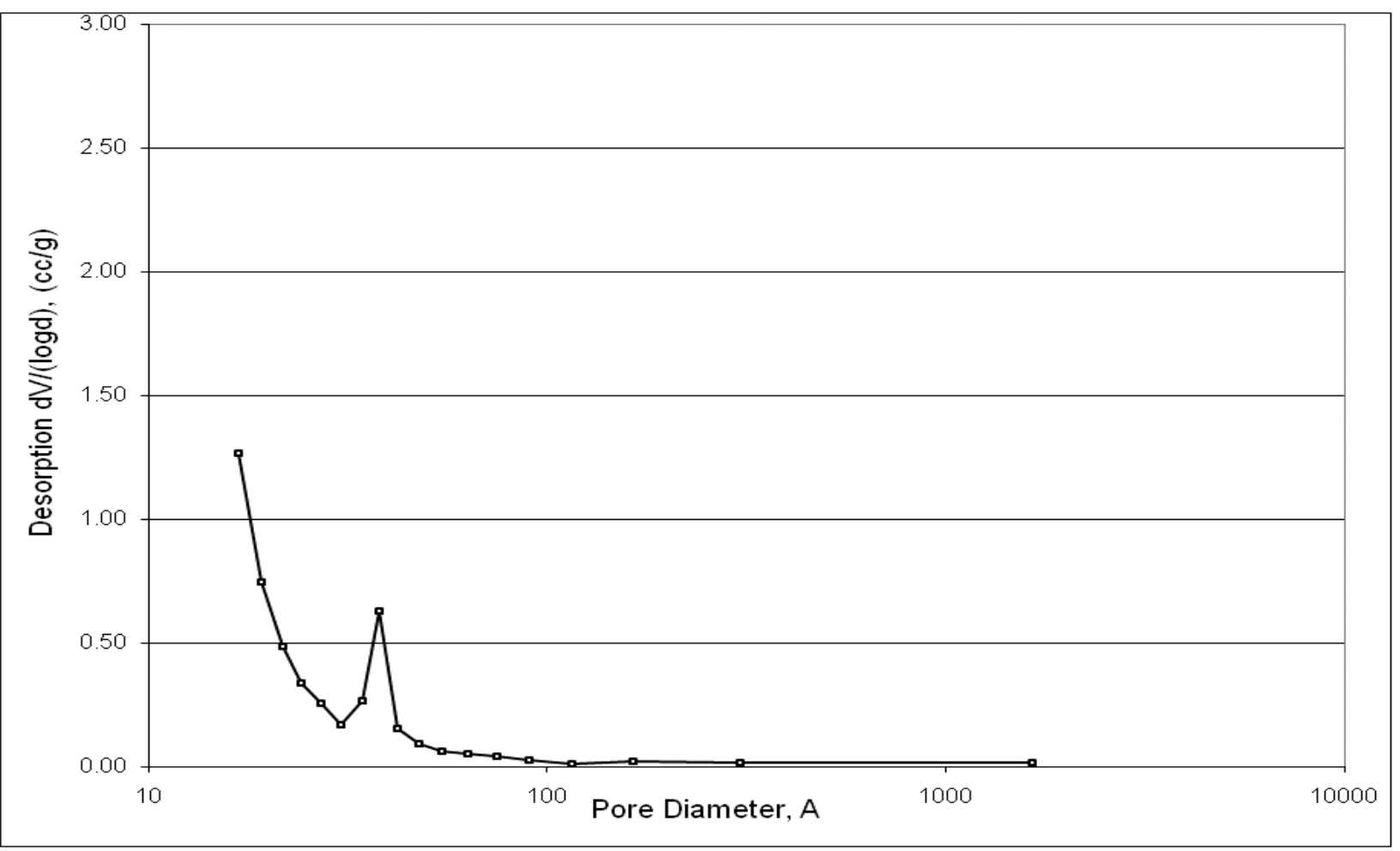

Figure A.13. Pore size distribution of KOA 13, which is the Pacco carbon support. 


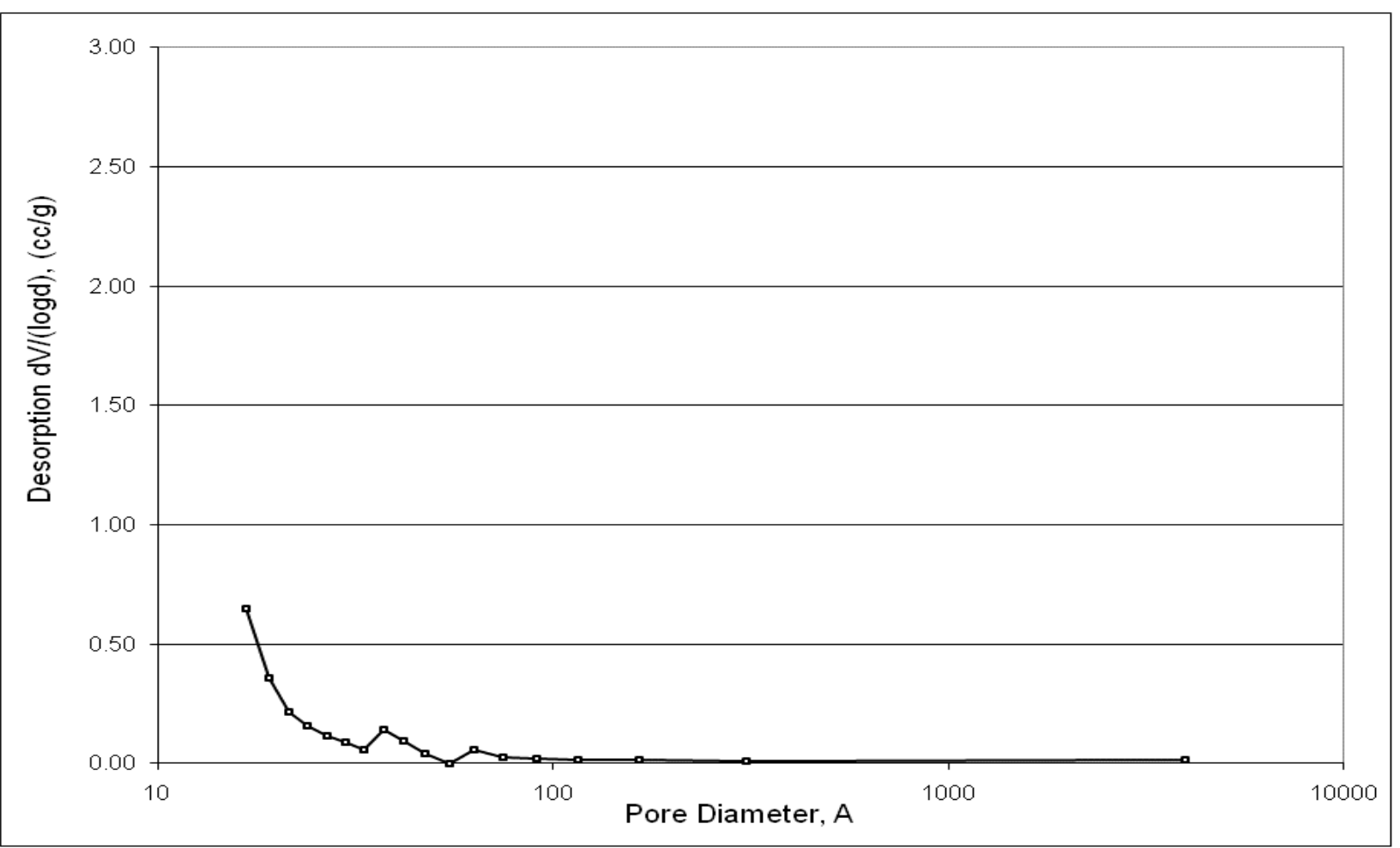

Figure A.14. Pore size distribution of KOA 14, which is the Kureha carbon support.

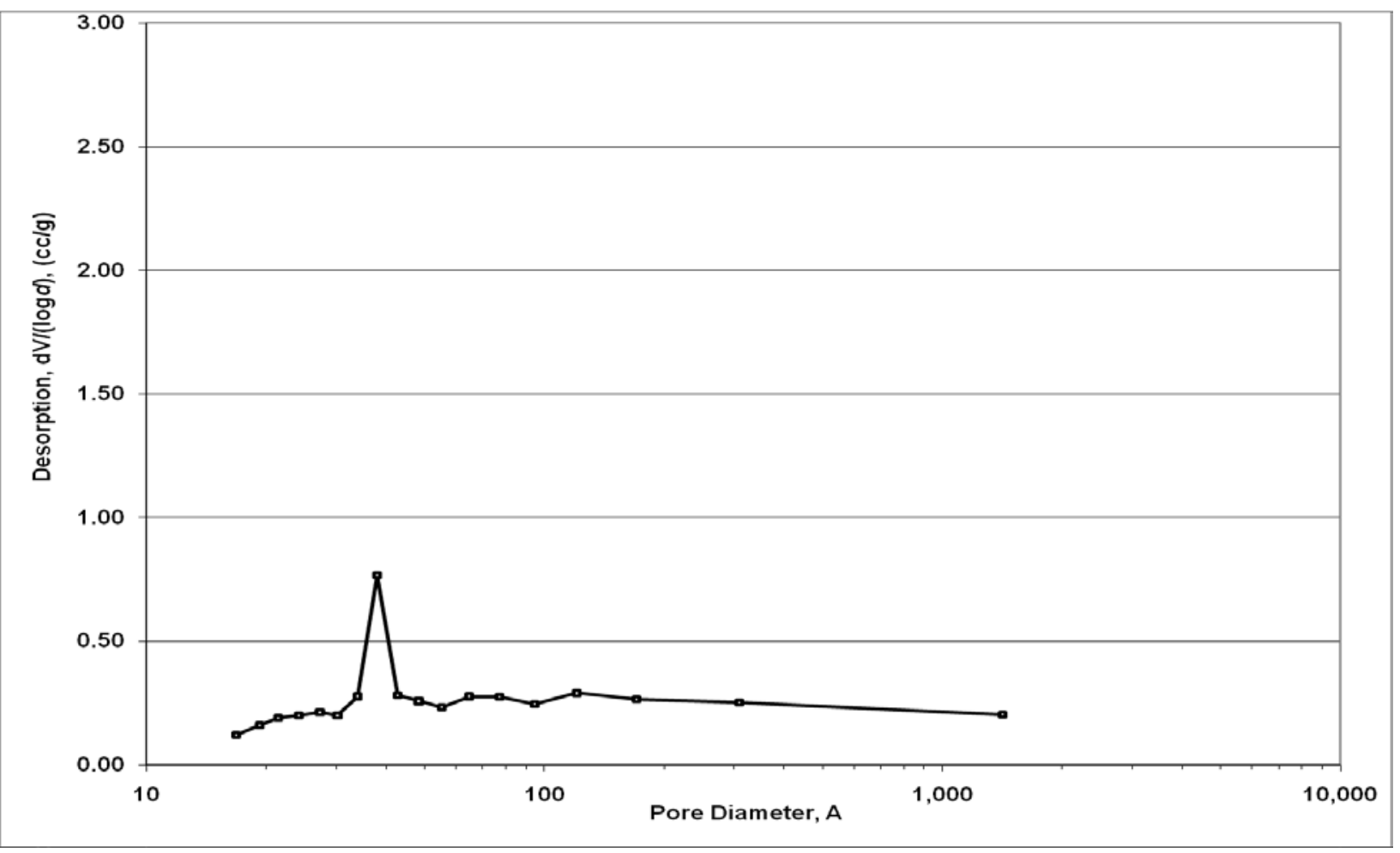

Figure A.15. Pore size distribution of KOA 15, which is the TimCal Timrex HSAG 300 carbon support. 


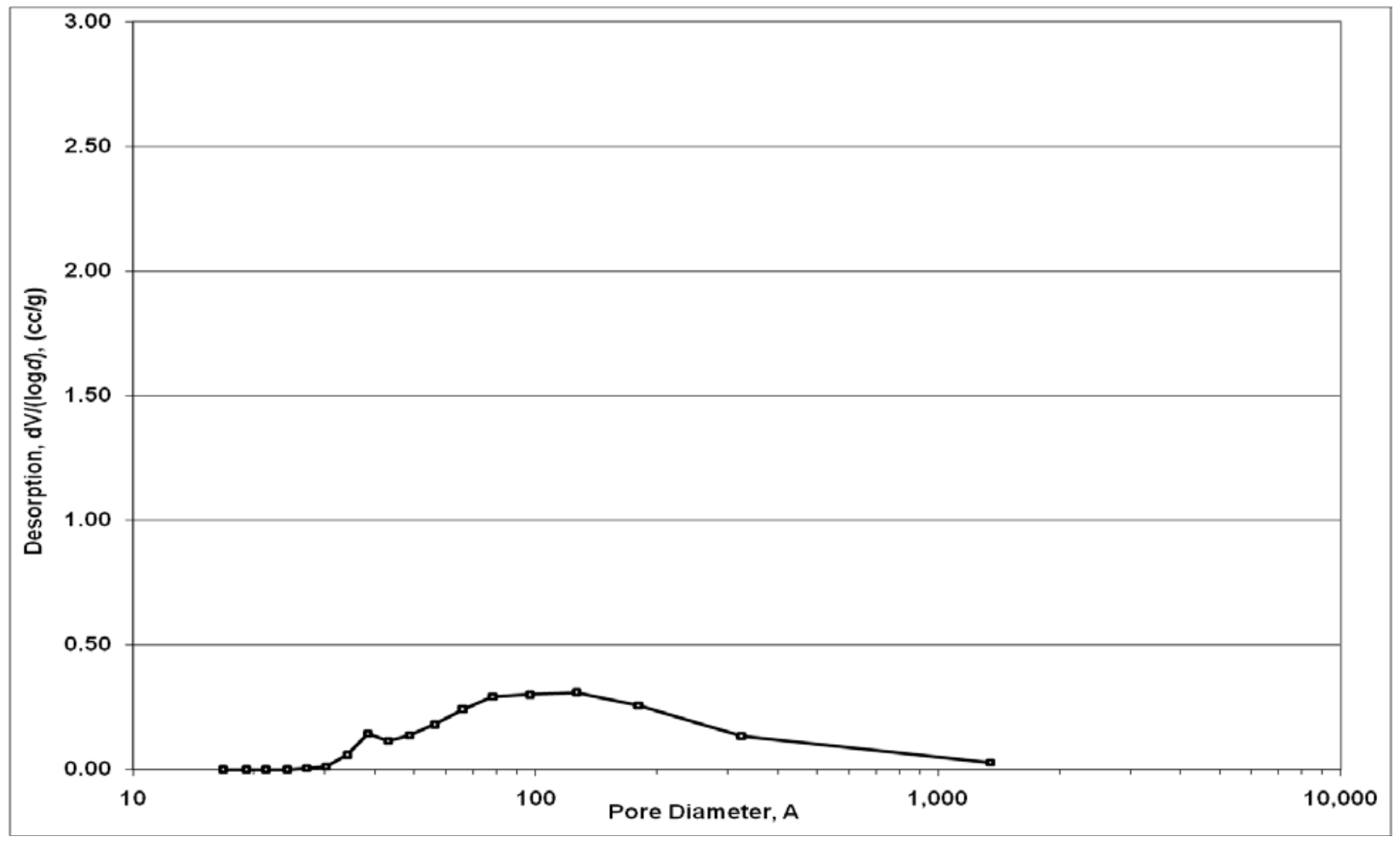

Figure A.16. Pore size distribution of the ICI low pressure methanol synthesis catalyst.

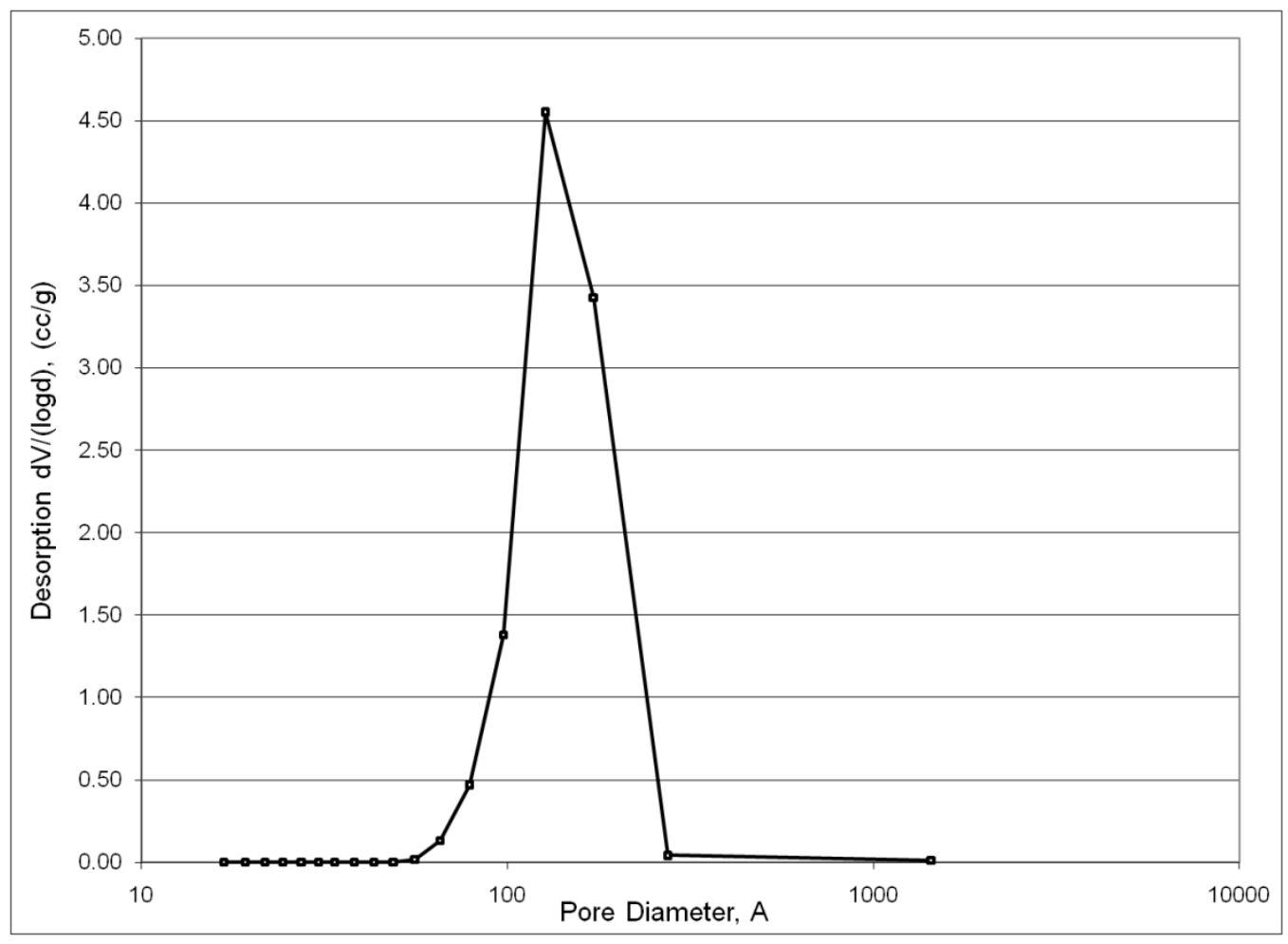

Figure A.17. Pore size distribution of Davisil 645. 


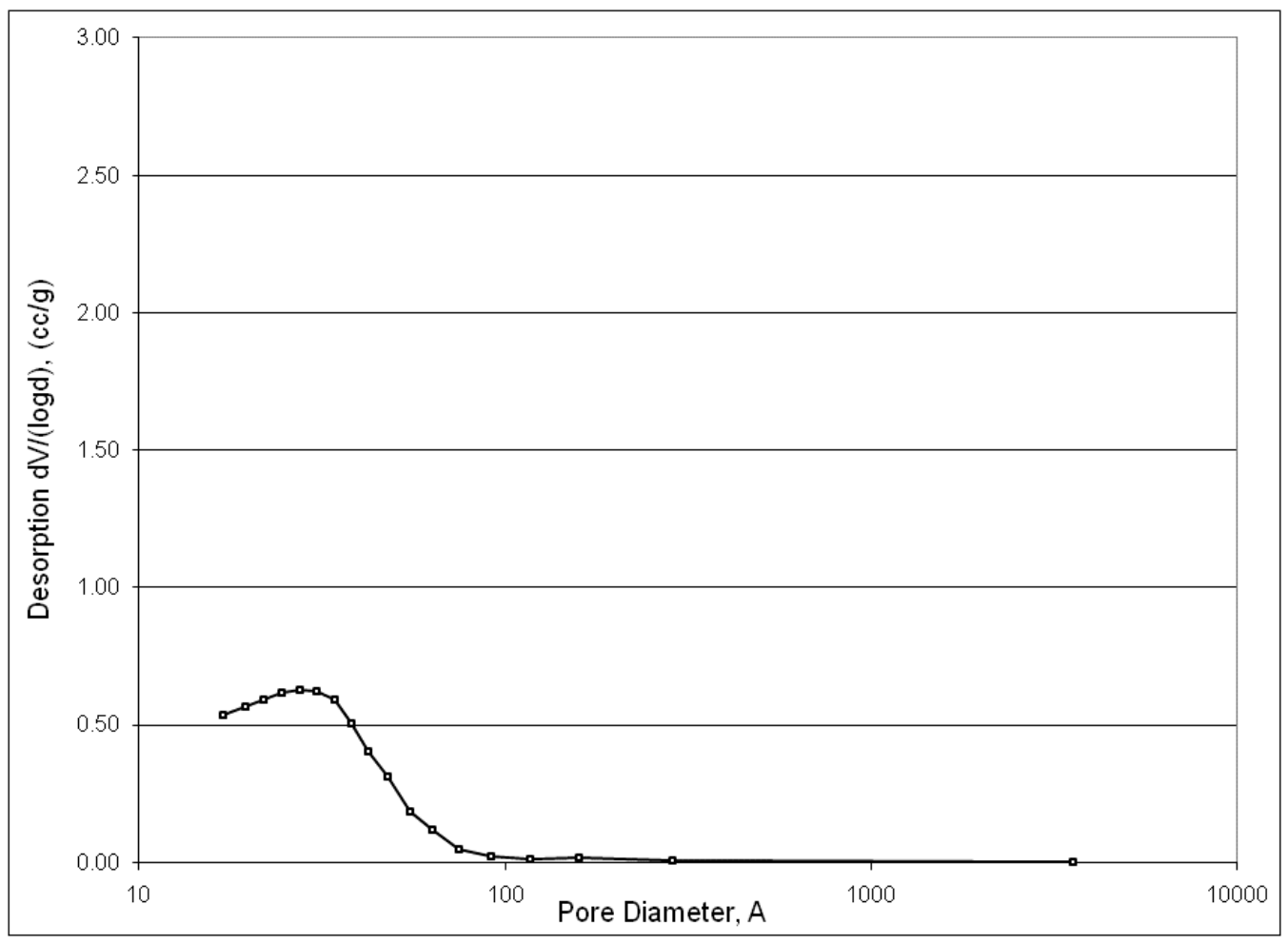

Figure A.18. Pore size distribution of Engelhard Mod D Silica

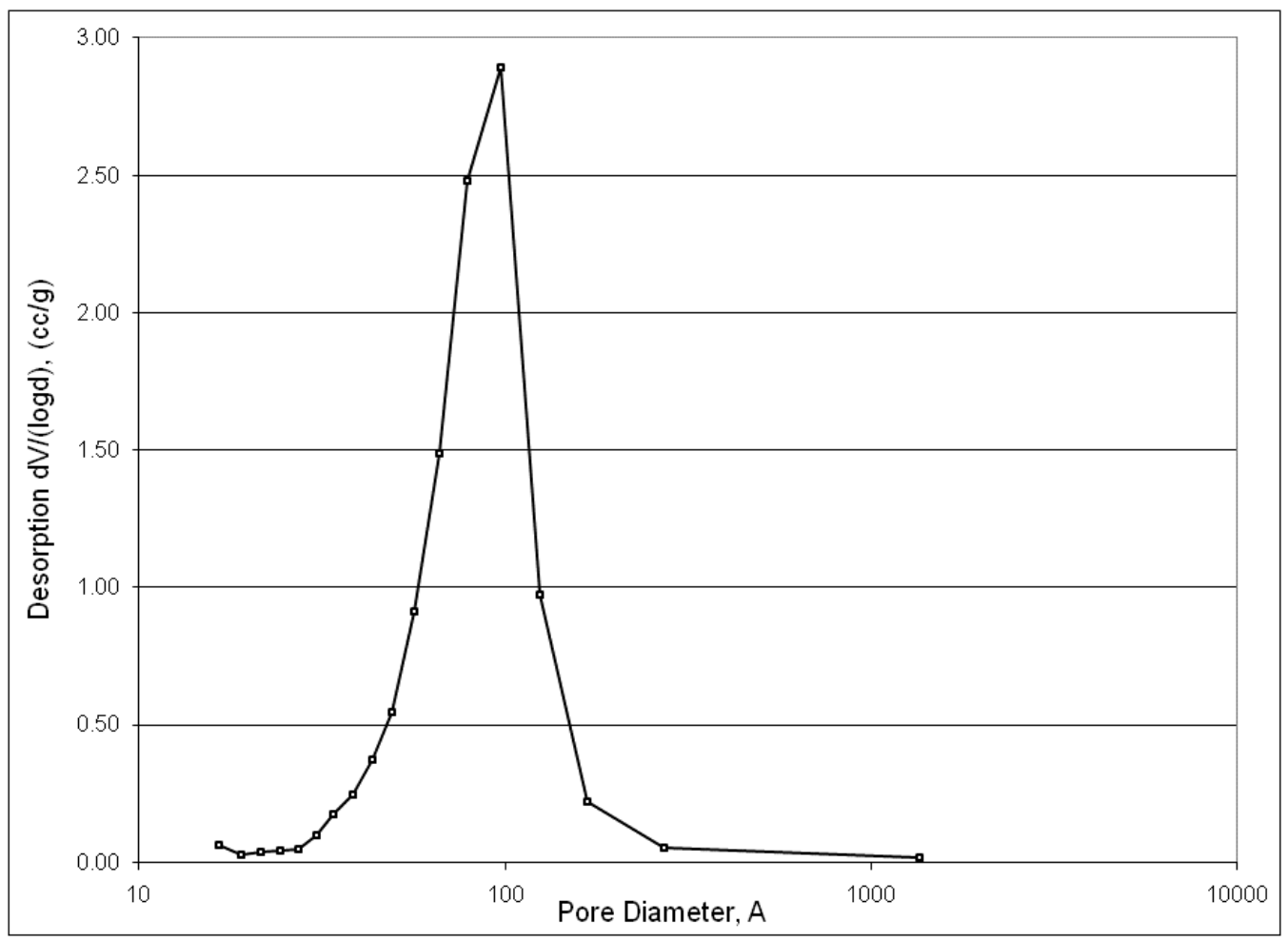

Figure A.19. Pore size distribution of Perlkat 97-0 


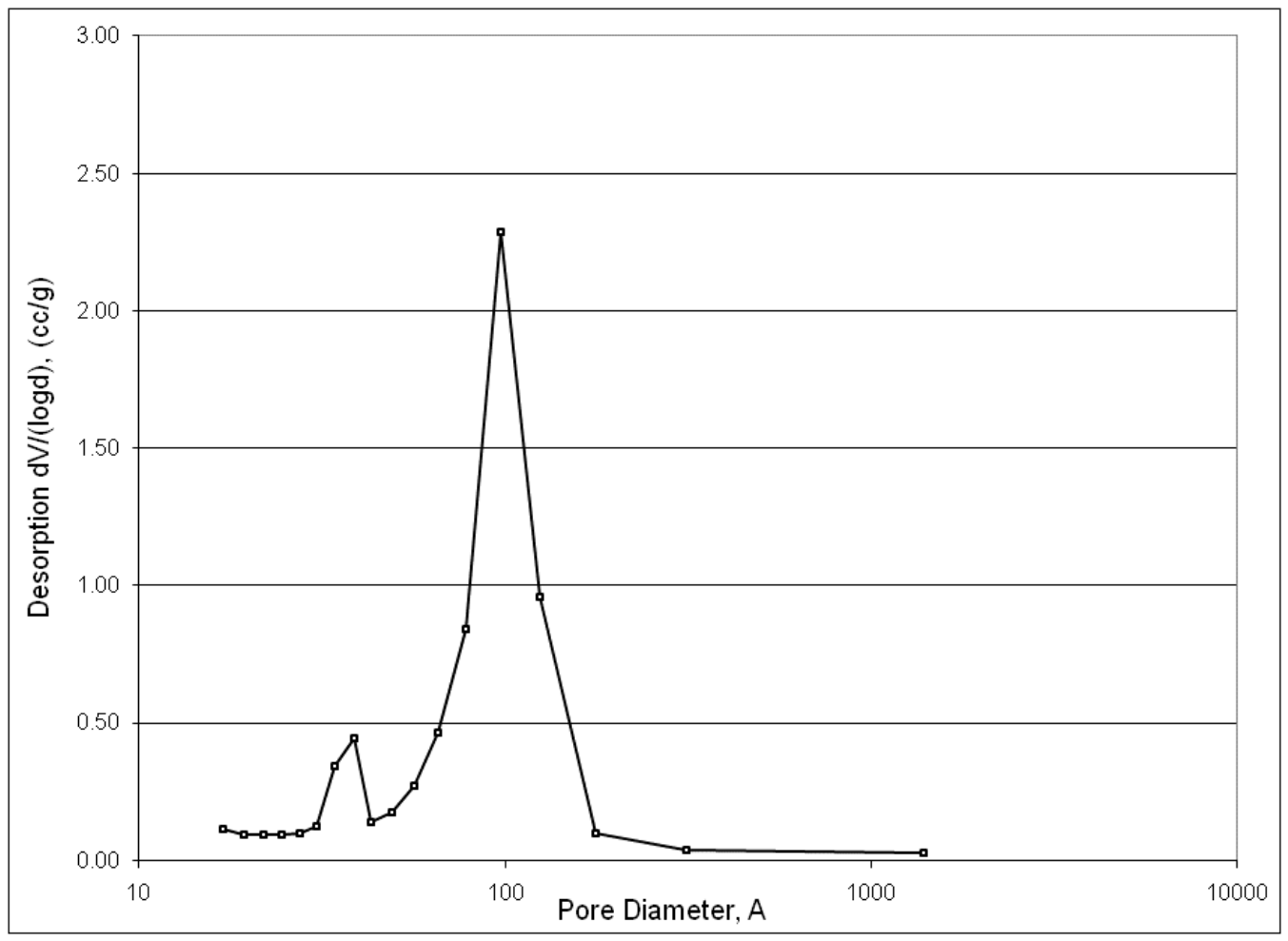

Figure A.20. Pore size distribution of Perlkat 79-3

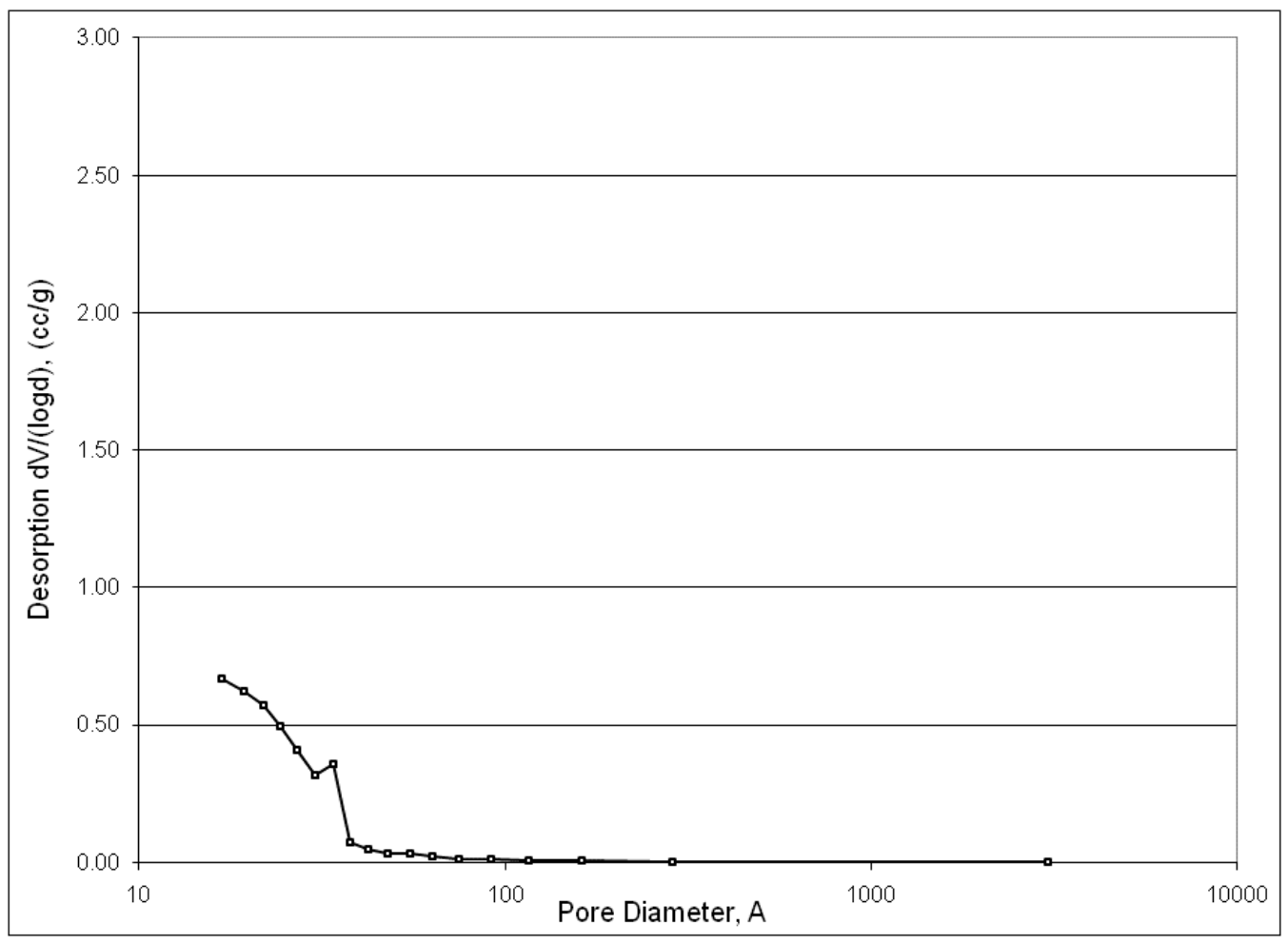

Figure A.21. Pore size distribution of Grace 408 


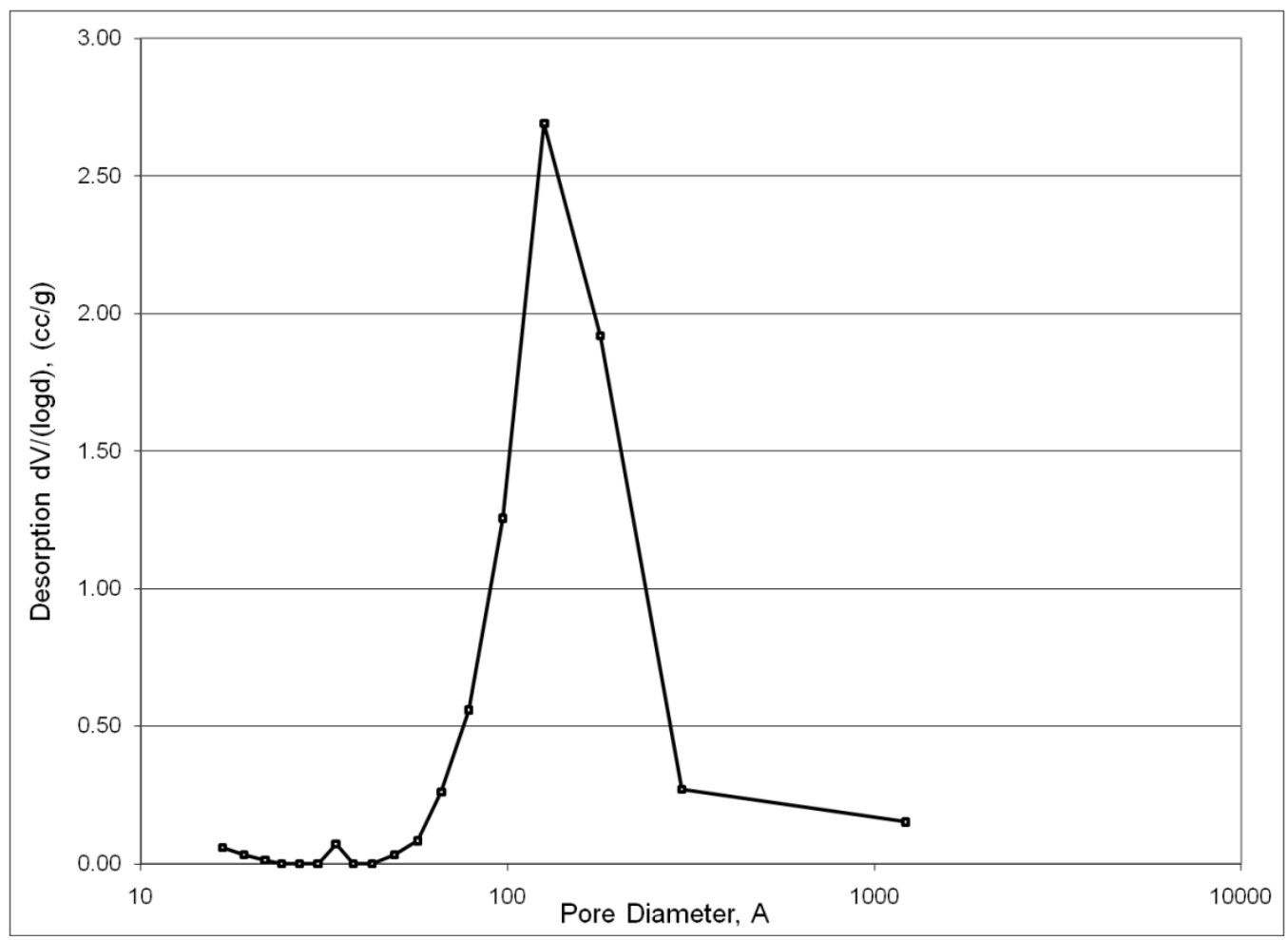

Figure A.22. Pore size distribution of Norpro SS61138.

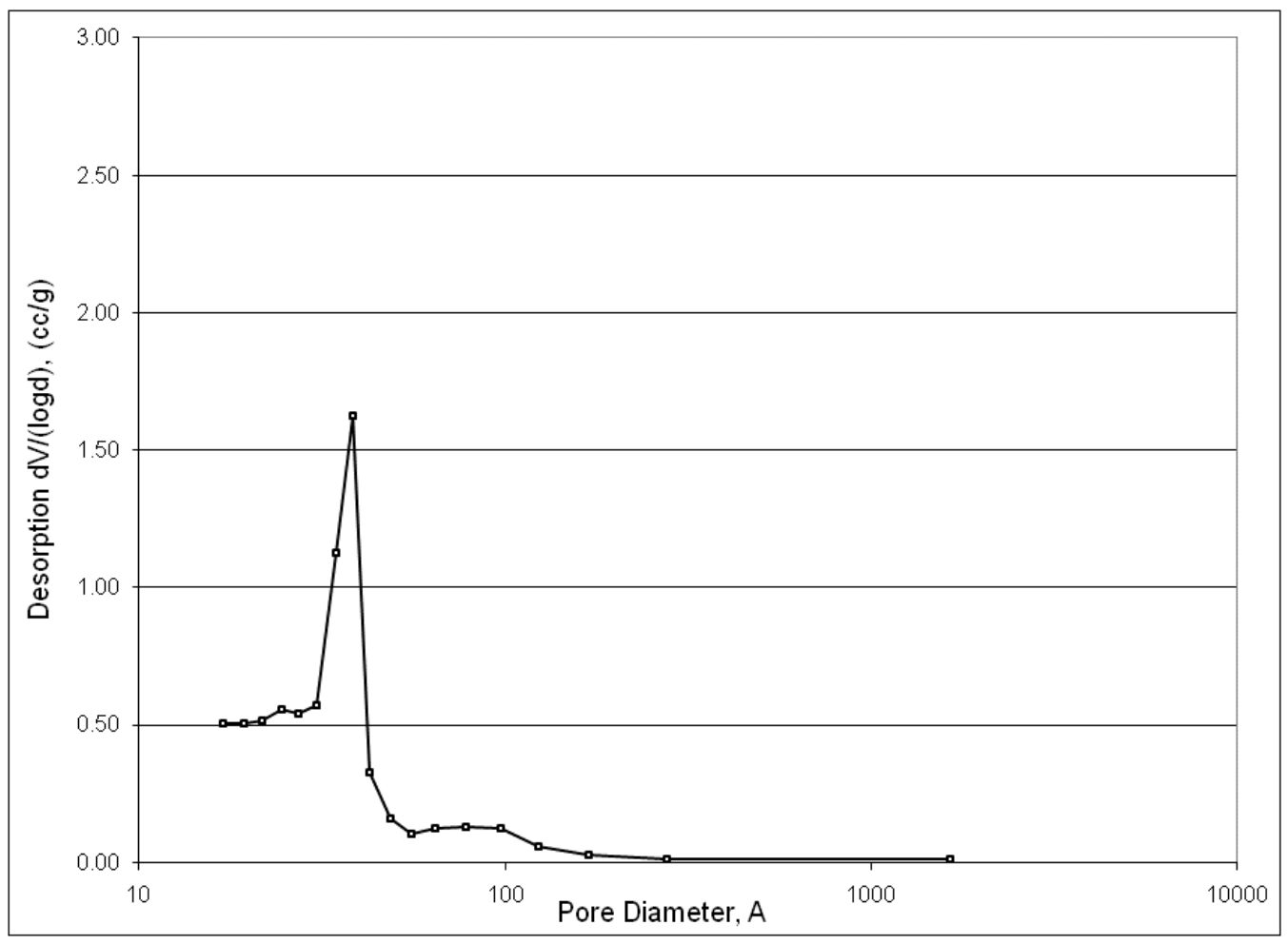

Figure A.23. Pore size distribution of Perlkat 29-3 


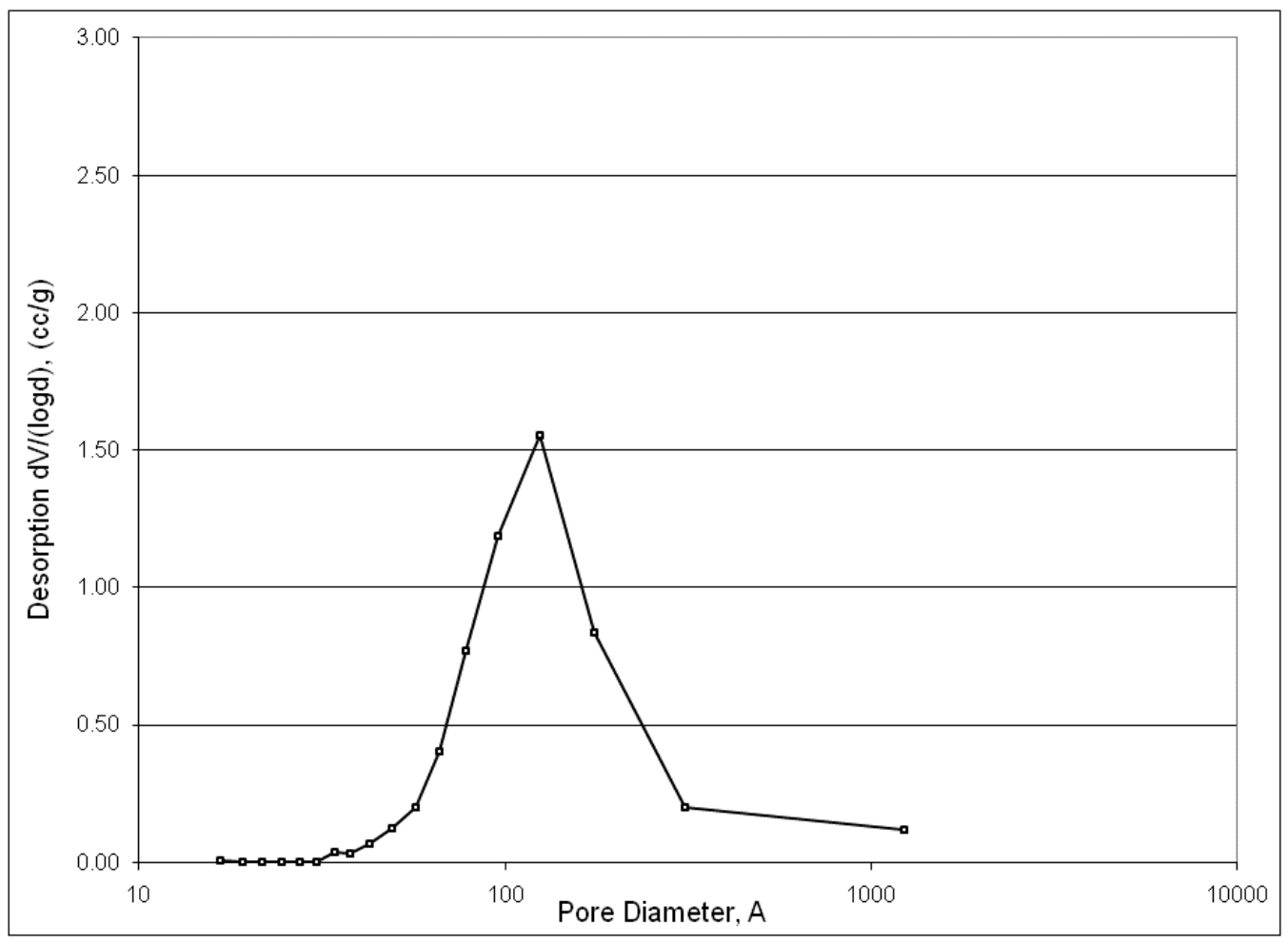

Figure A.24. Pore size distribution of Norpro SS61137

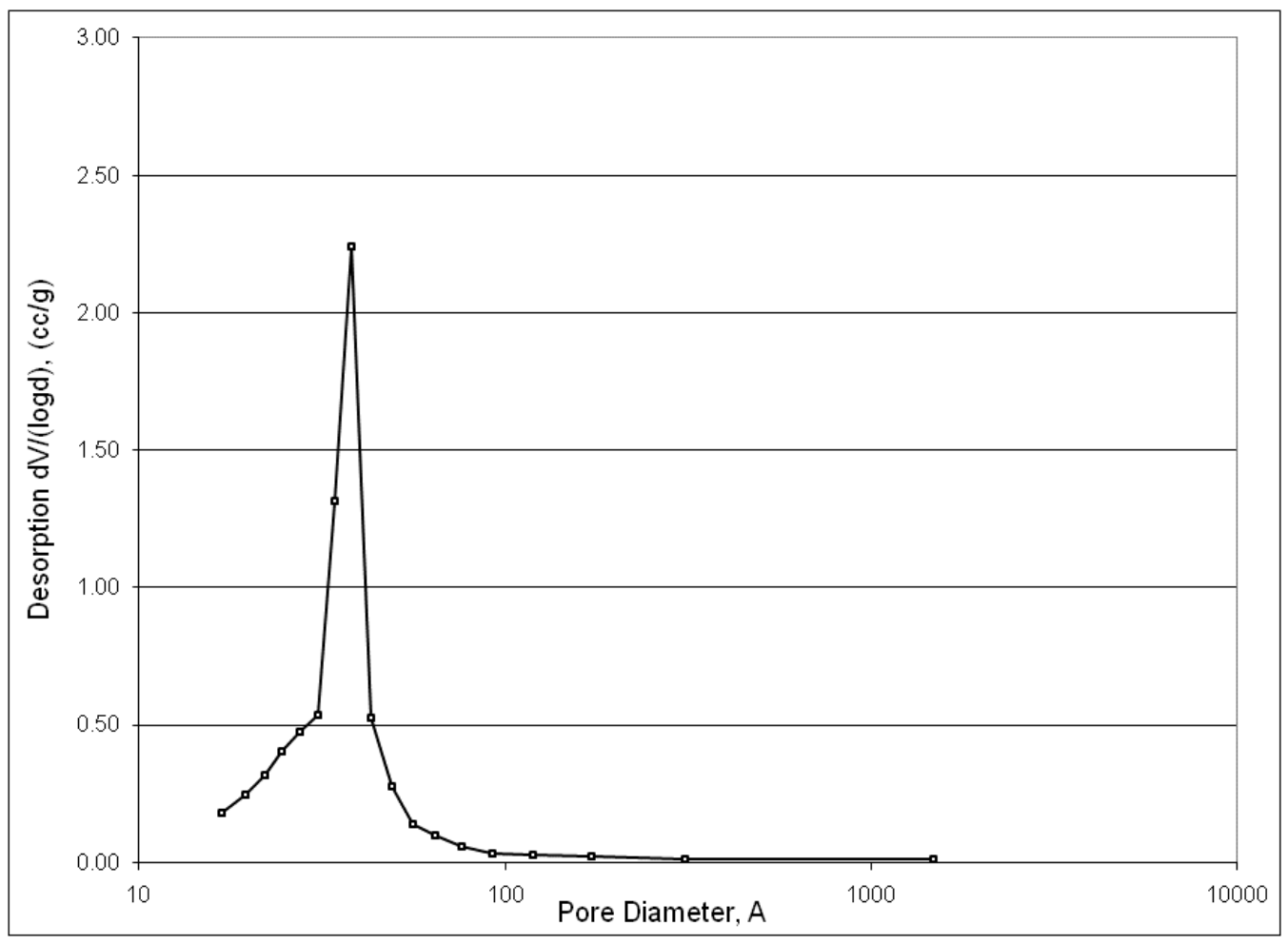

Figure A.25. Pore size distribution of Grace 980-25 Silica Alumina 


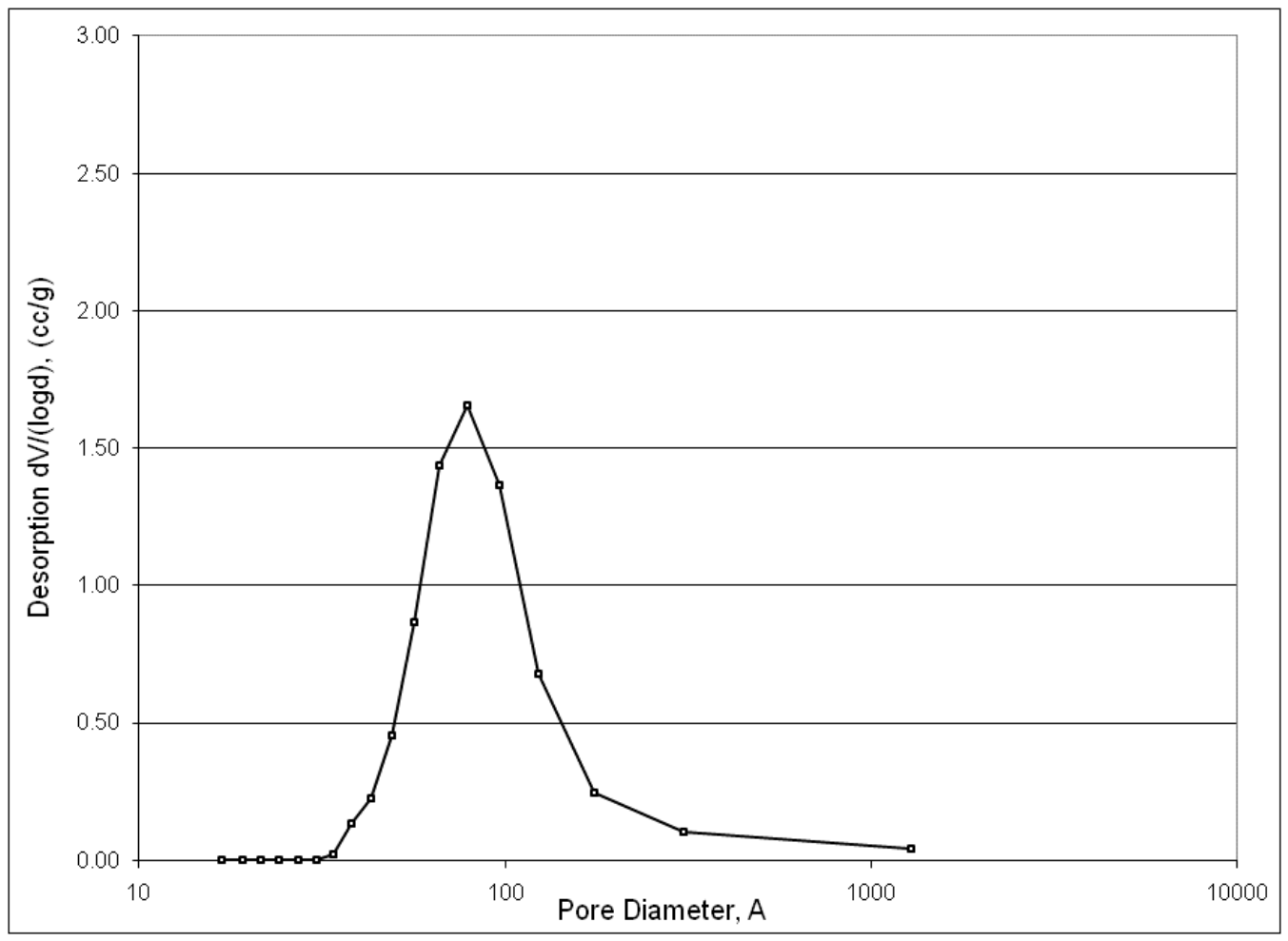

Figure A.26. Pore size distribution of BASF Al 5700 E 1-16 Ca on Alumina

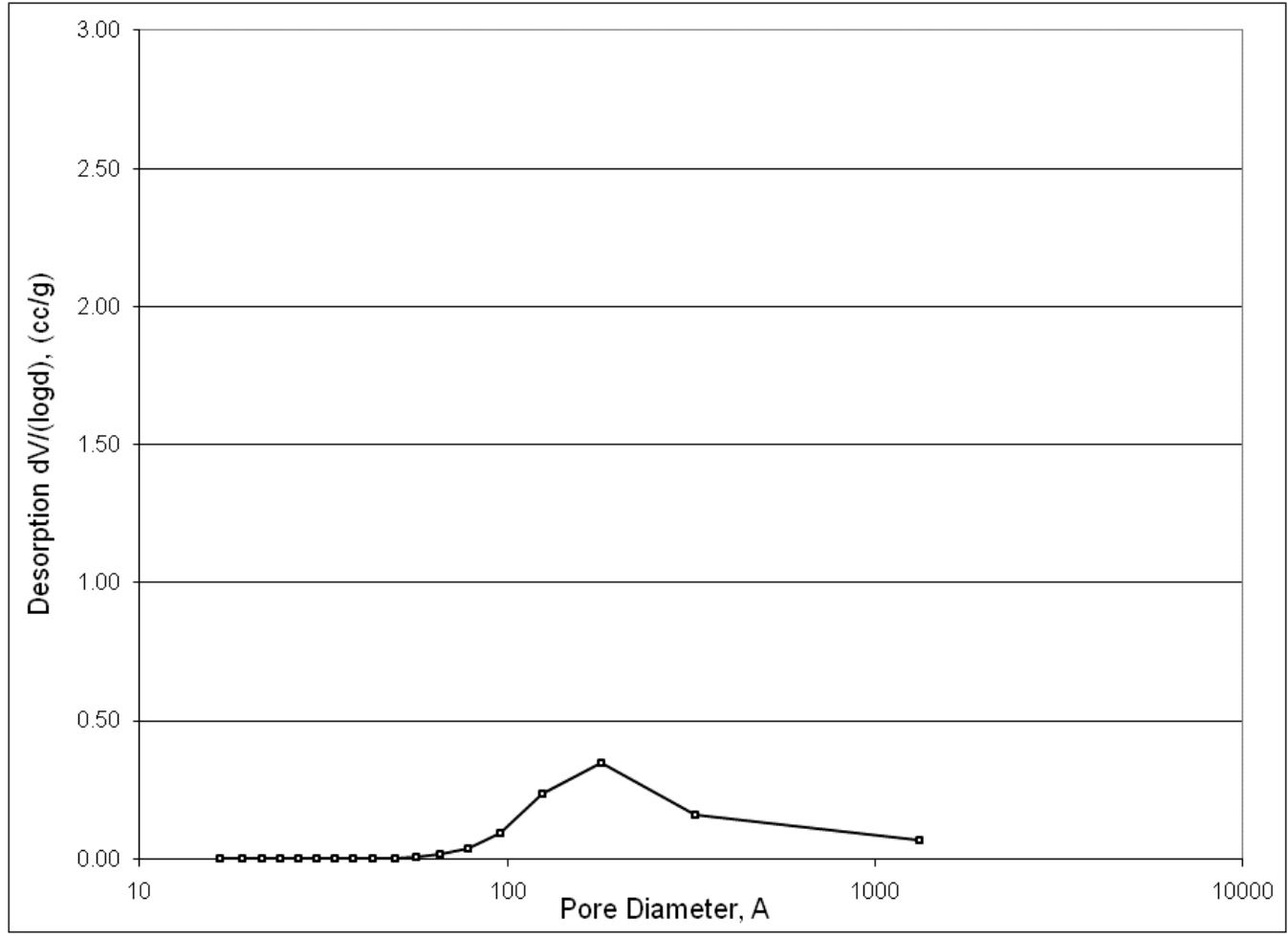

Figure A.27. Pore size distribution of Norpro ST 31119 Titania 


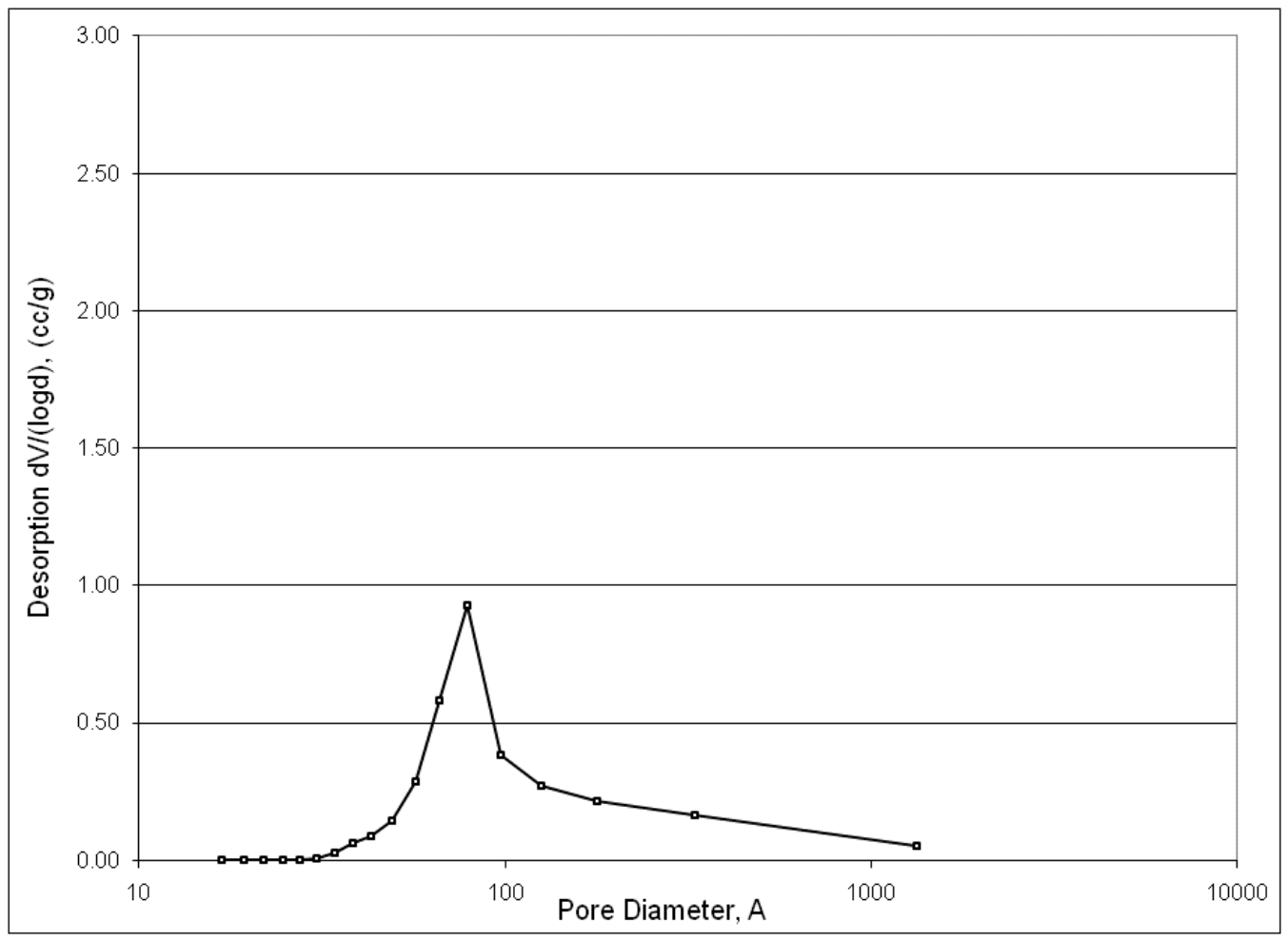

Figure A.28. Pore size distribution of Norpro SZ 31164 Zirconia

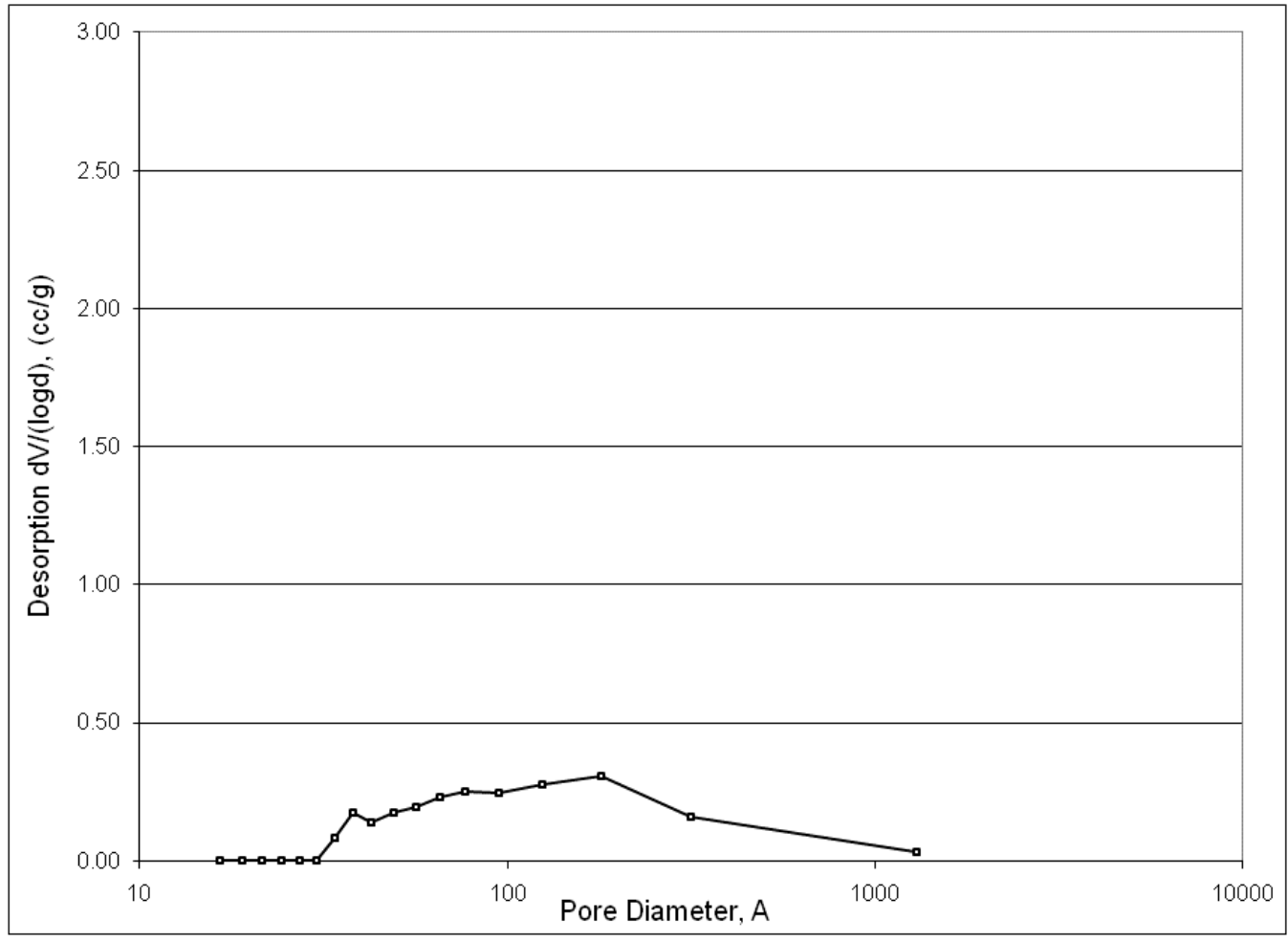

Figure A.29. Pore size distribution of BASF 264-19A MgO 


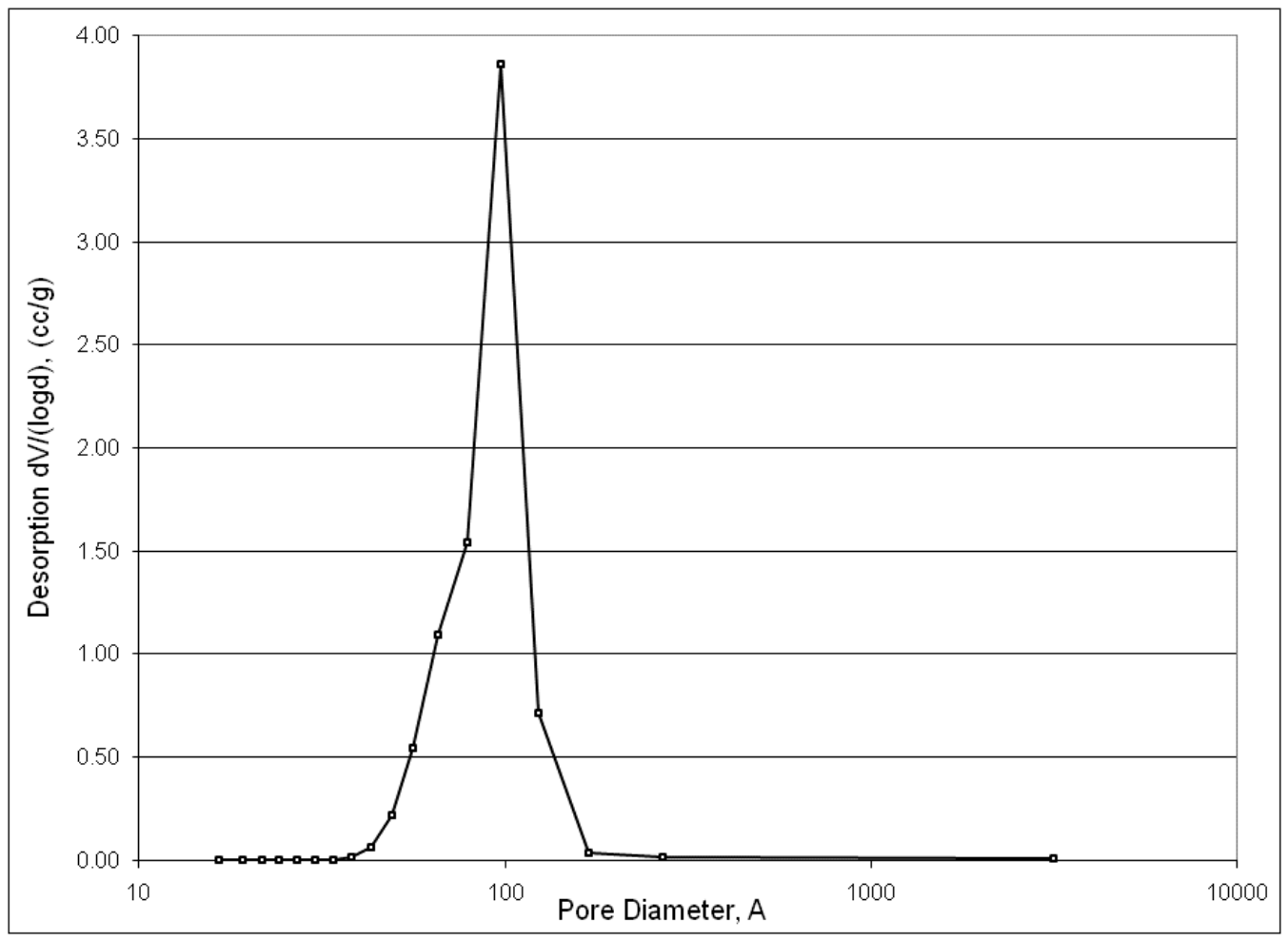

Figure A.30. Pore size distribution of Engelhard Al3945

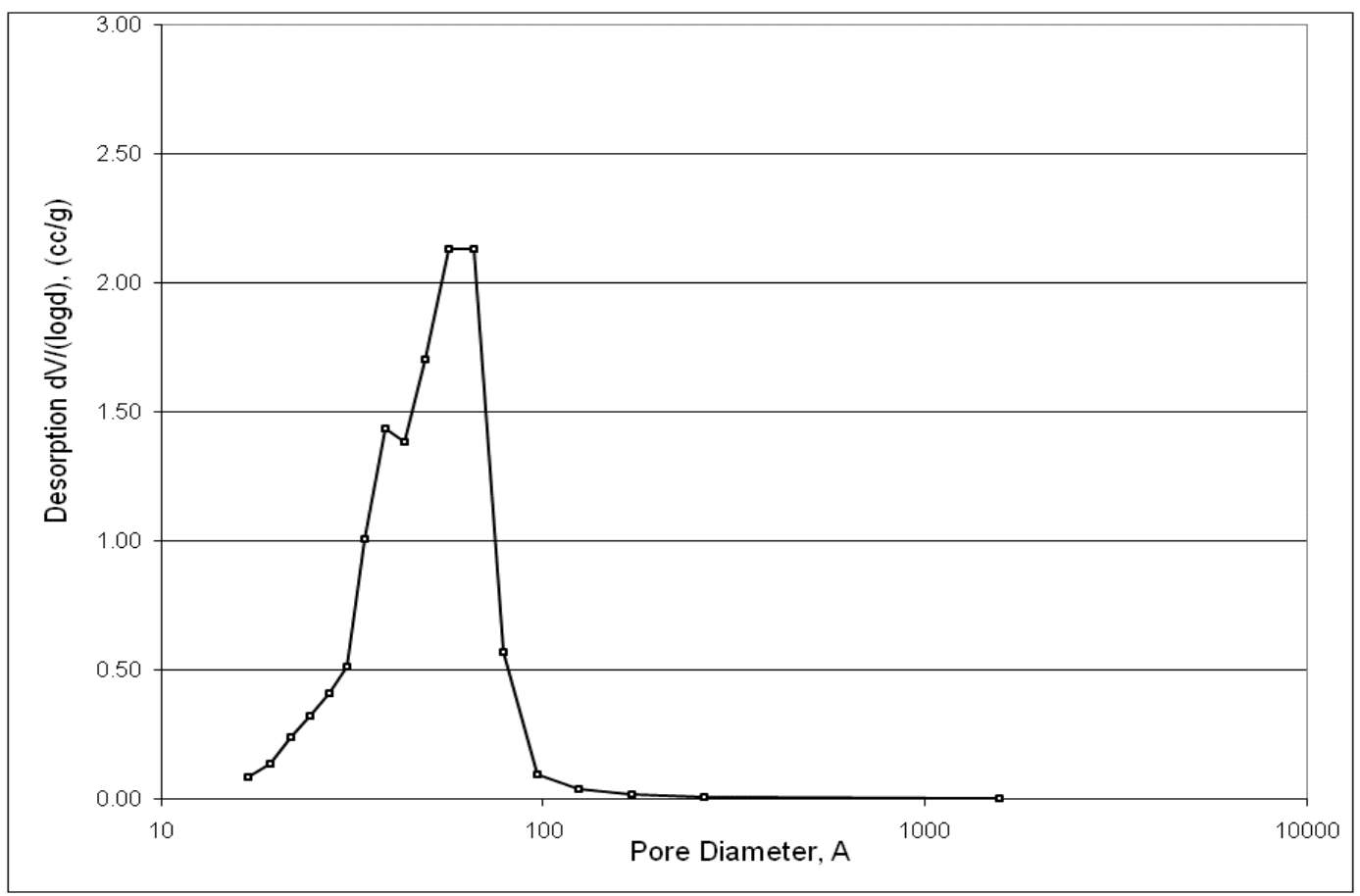

Figure A.31. Pore size distribution of Merck Aldrich Grade 60 silica. 


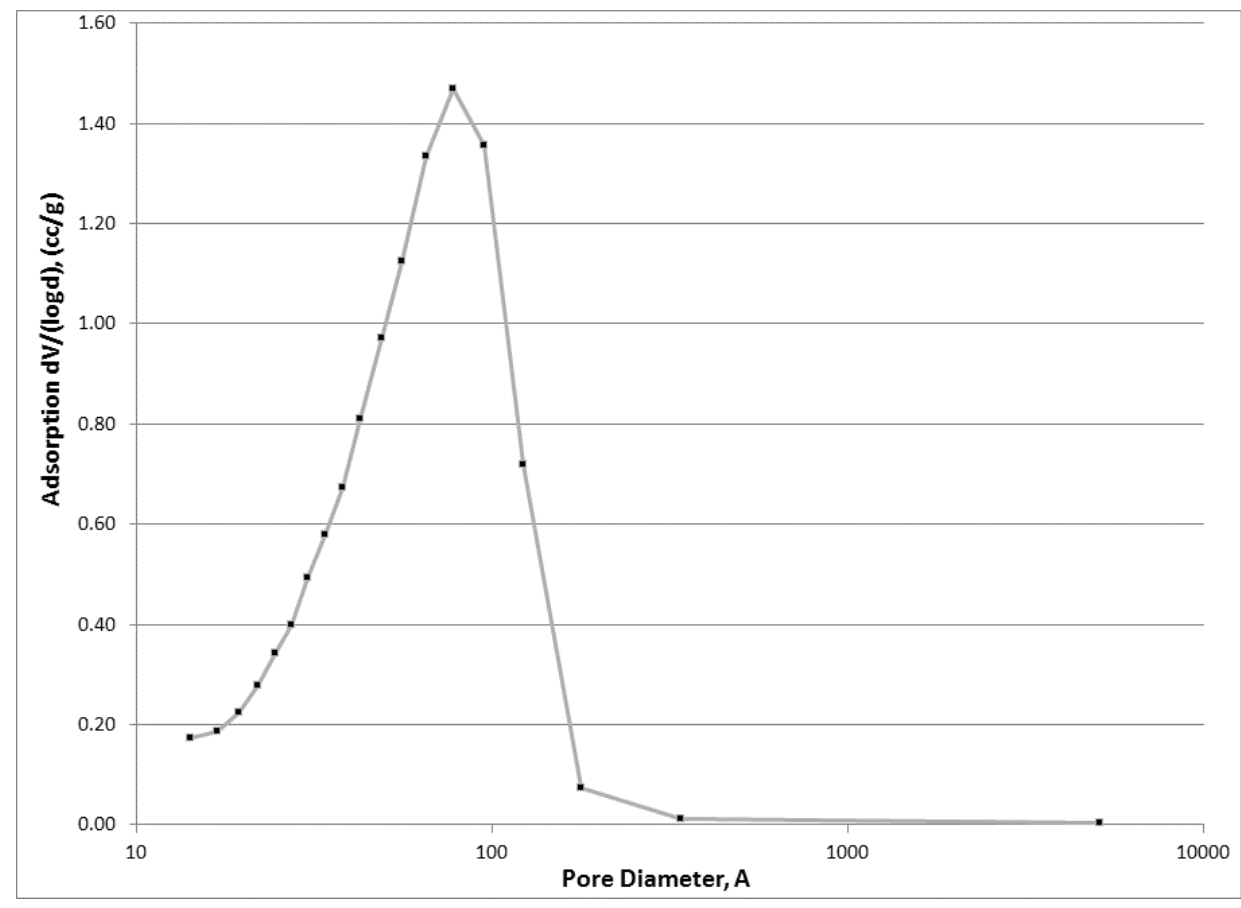

Figure A.32. Pore size distribution of Merck Aldrich Grade 7734 silica gel. Note that this is pore size distribution was calculated from the adsorption portion of the $\mathrm{N}_{2}$ adsorption/desorption test due to incomplete data on the desorption curve. Also note that the portion of the pore size distribution curve calculated from the available part of the desorption hysteresis curve is similar to that of the Merck Aldrich Grade 60 and Sigma-Aldrich Grade 7754 silica gels.

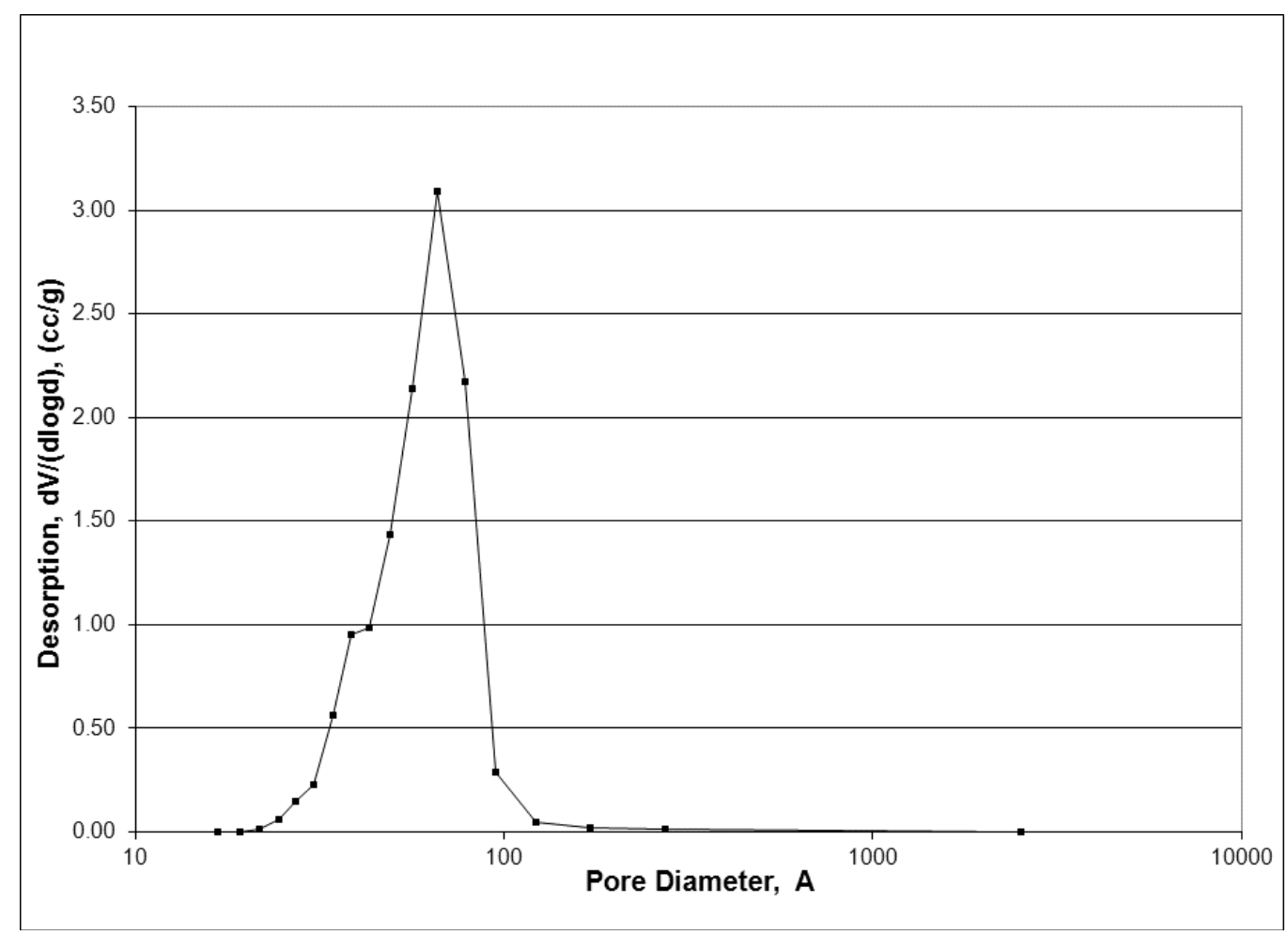

Figure A.33. Pore size distribution of Sigma-Aldrich Grade 7754 silica gel. 



\section{Appendix B}

$\mathrm{NH}_{3}$ Temperature Programmed Desorption Traces of Silica Mixed Alcohol Catalyst Supports 



\section{Appendix B}

\section{$\mathrm{NH}_{3}$ Temperature Programmed Desorption Traces of Silica Mixed Alcohol Catalyst Supports}

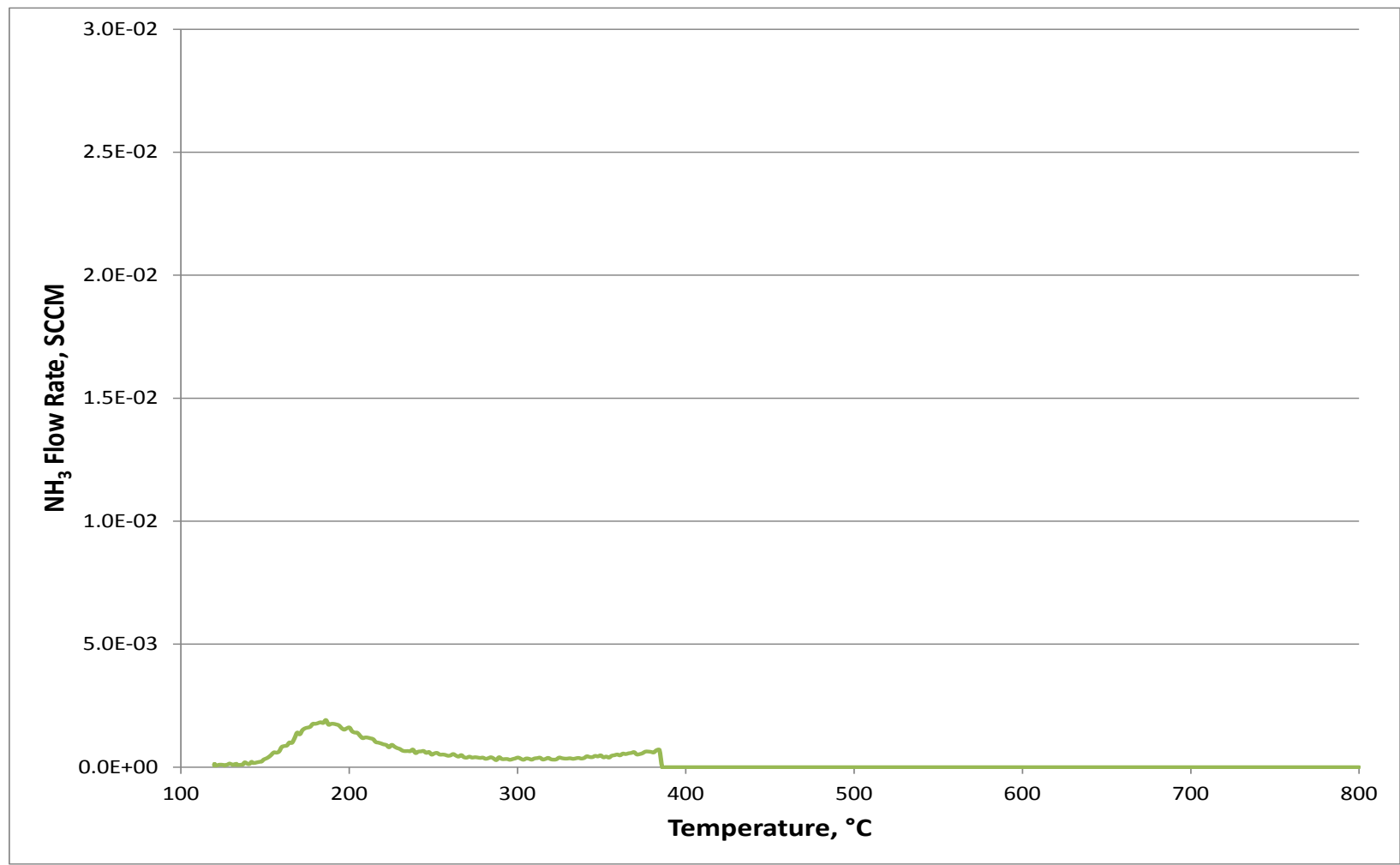

Figure B.1. $\mathrm{NH}_{3}$ TPD trace of the Sigma-Aldrich Grade 7754 silica gel pre-dried at $300^{\circ} \mathrm{C}$ for $2 \mathrm{hr}$. 


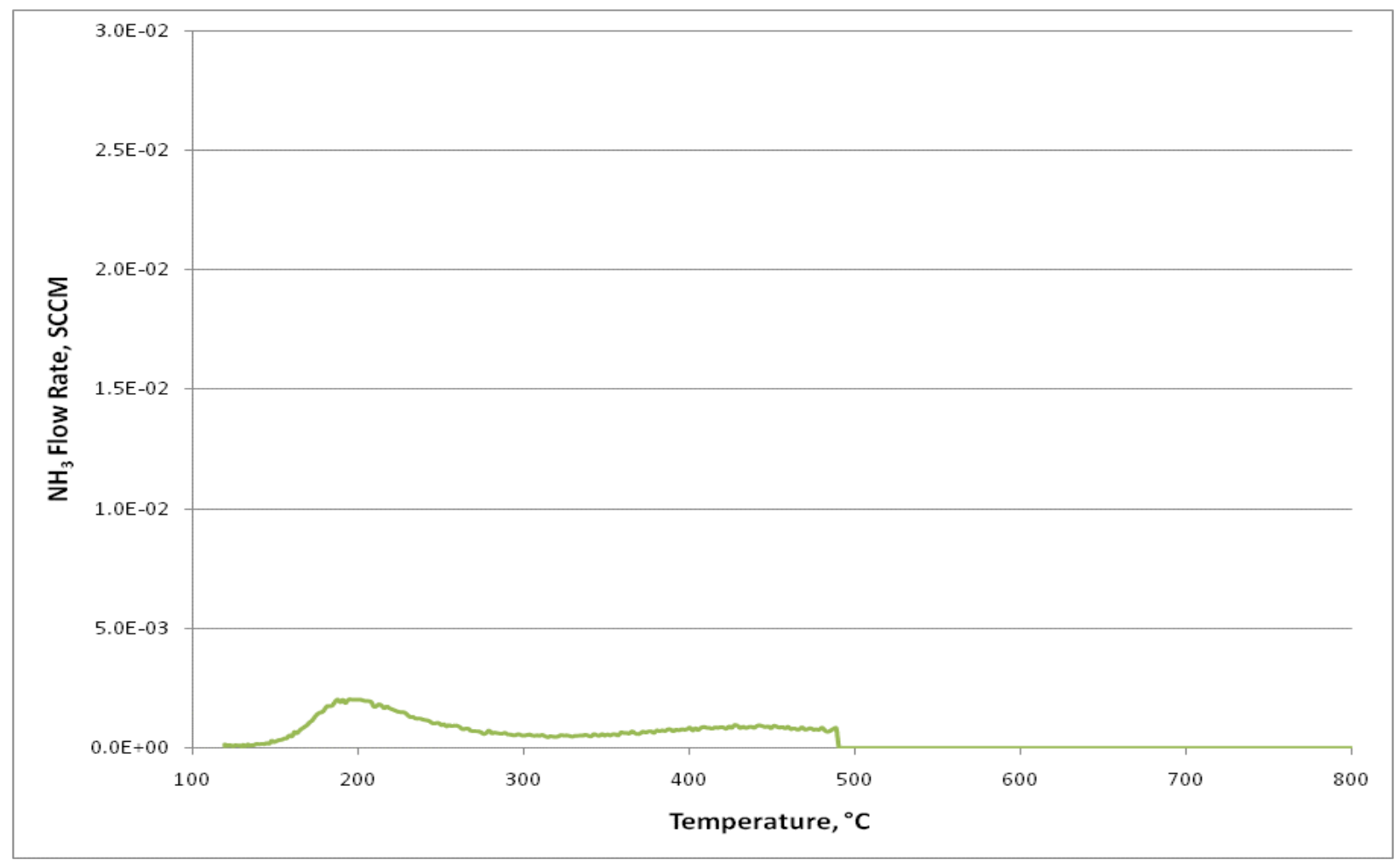

Figure B.2. $\mathrm{NH}_{3} \mathrm{TPD}$ trace of the Perlkat $97-0$ silica pre-dried at $300^{\circ} \mathrm{C}$ for $2 \mathrm{hr}$.

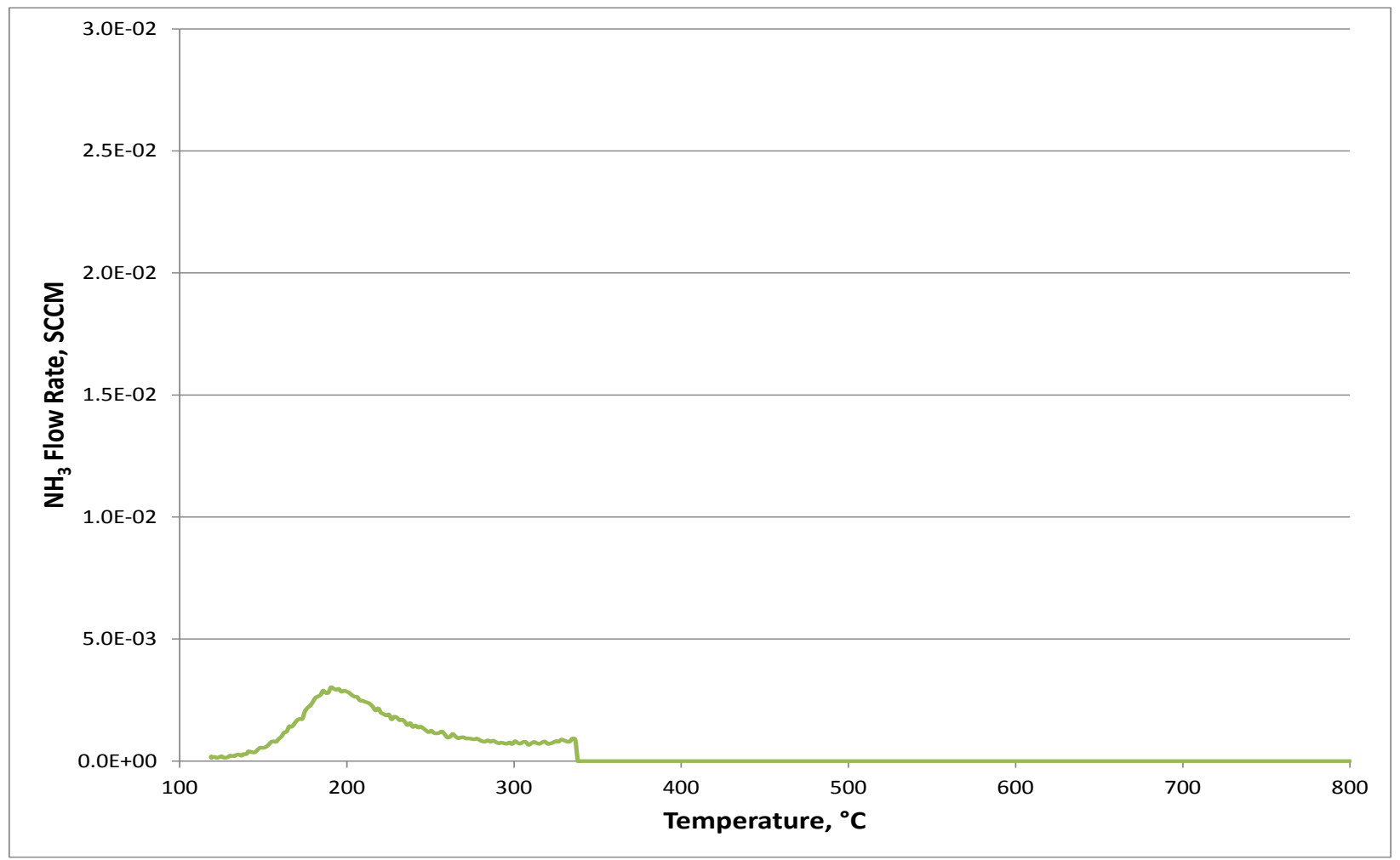

Figure B.3. $\mathrm{NH}_{3} \mathrm{TPD}$ trace of the Sigma-Aldrich Grade 7734 silica gel pre-dried at $300^{\circ} \mathrm{C}$ for $2 \mathrm{hr}$. 


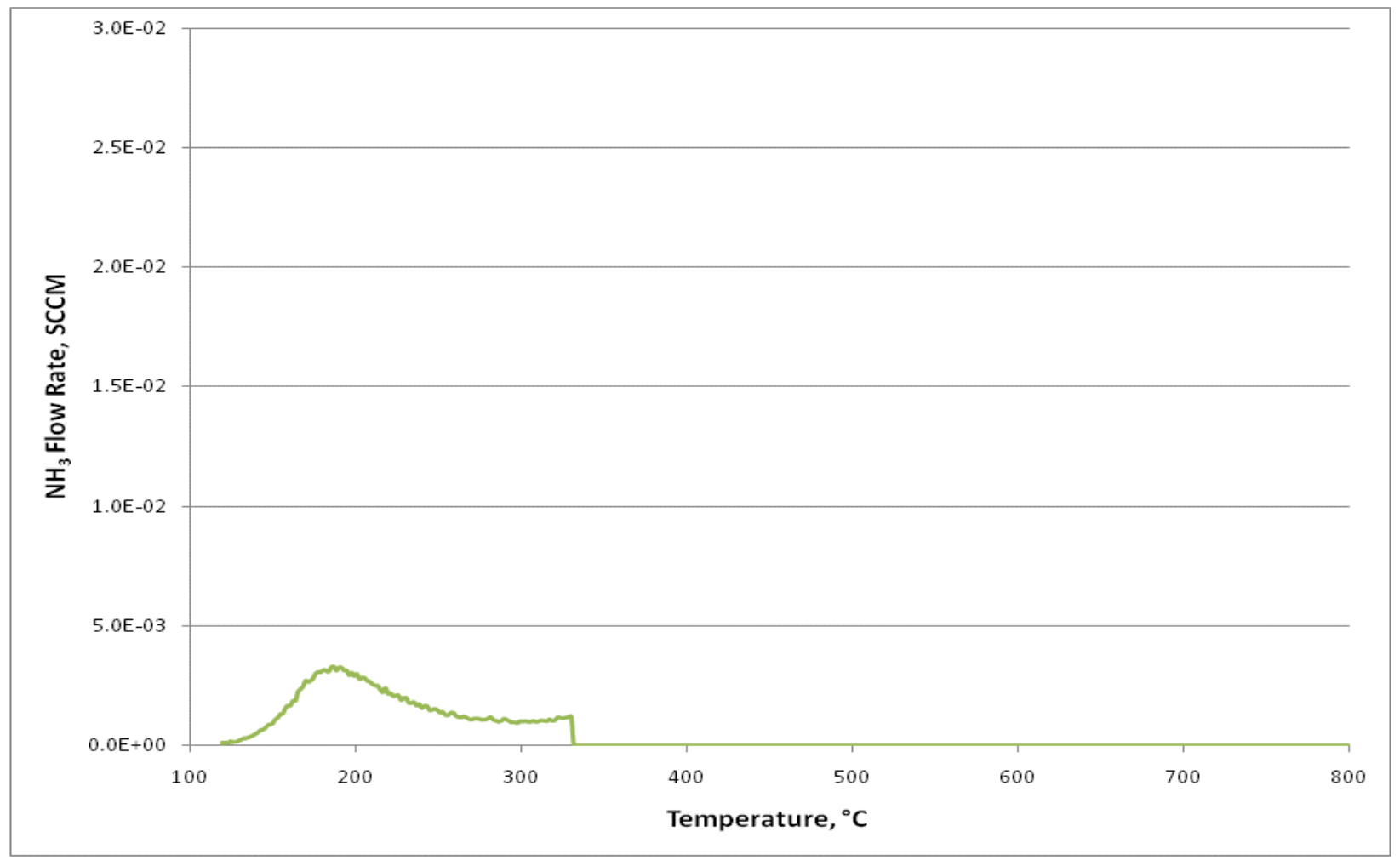

Figure B.4. $\mathrm{NH}_{3} \mathrm{TPD}$ trace of the Merck Aldrich Grade 60 silica pre-dried at $300^{\circ} \mathrm{C}$ for $2 \mathrm{hr}$.

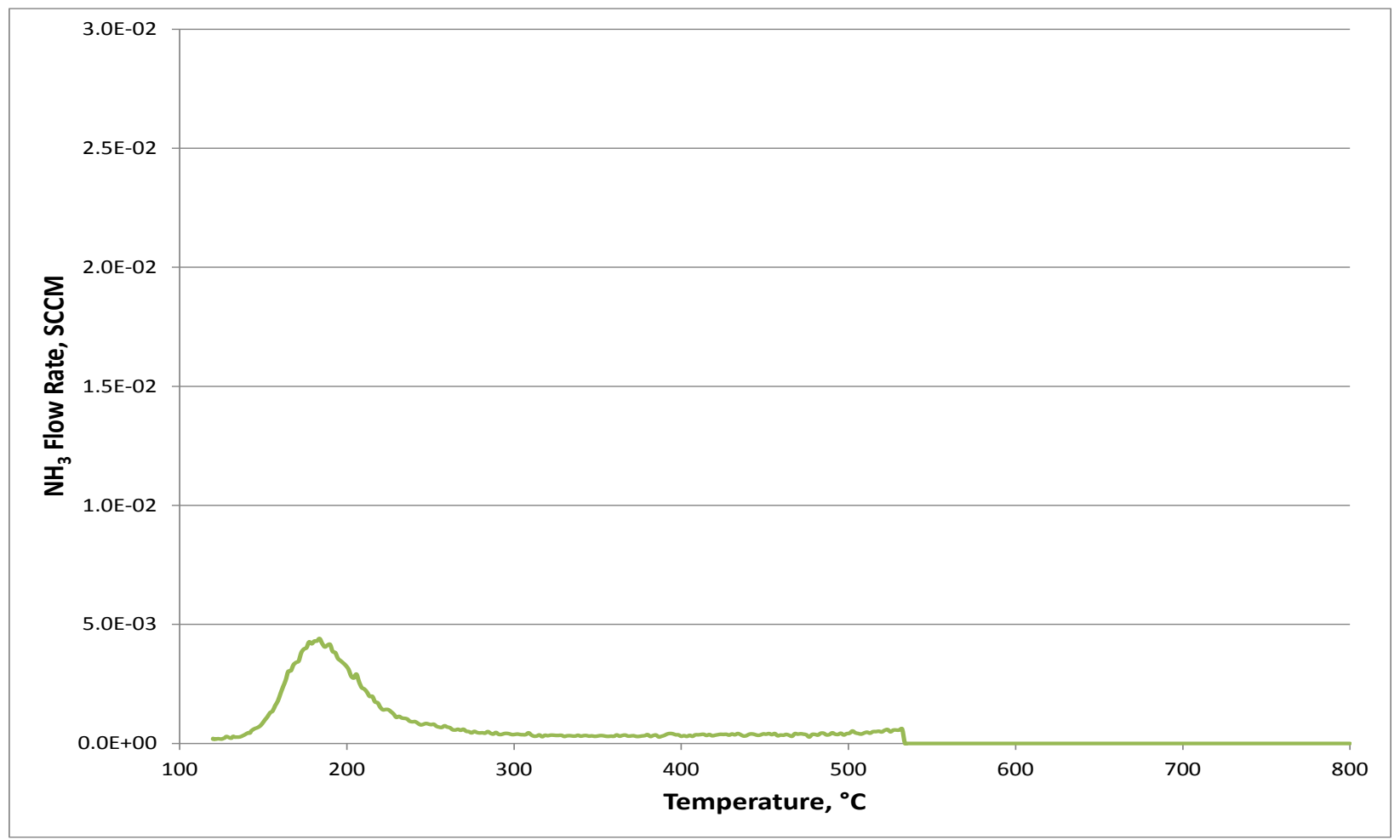

Figure B.5. $\mathrm{NH}_{3}$ TPD trace of the Davisil 645 silica washed in $10 \% \mathrm{HNO}_{3}$ and calcined at $500^{\circ} \mathrm{C}$ in air prior to testing. The silica was pretreated at $300^{\circ} \mathrm{C}$ for $2 \mathrm{hr}$ in flowing $\mathrm{He}$ prior to $\mathrm{NH}_{3} \mathrm{TPD}$ testing. 


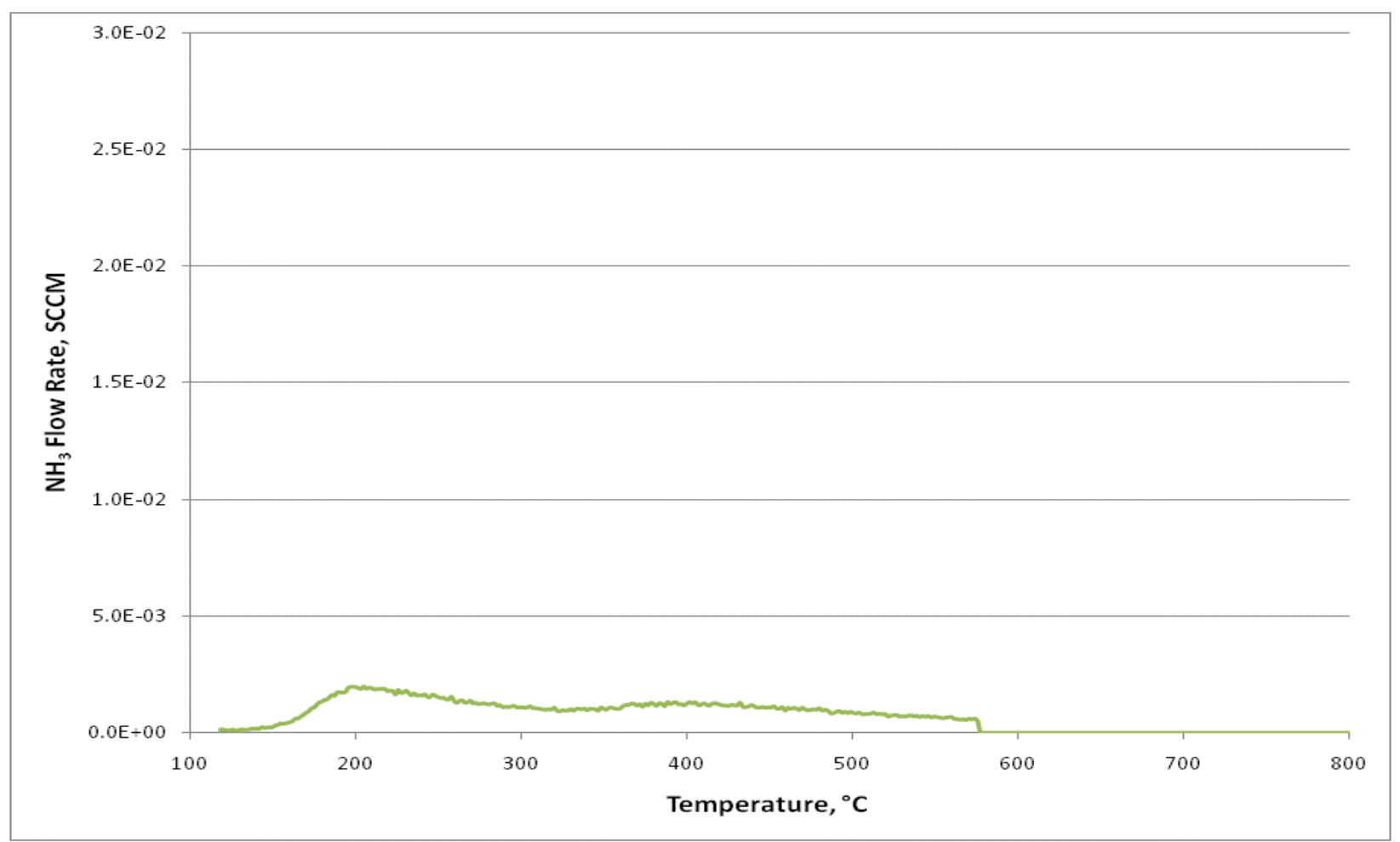

Figure B.6. $\mathrm{NH}_{3} \mathrm{TPD}$ trace of the Norpro 61137 silica pre-dried at $300^{\circ} \mathrm{C}$ for $2 \mathrm{hr}$.

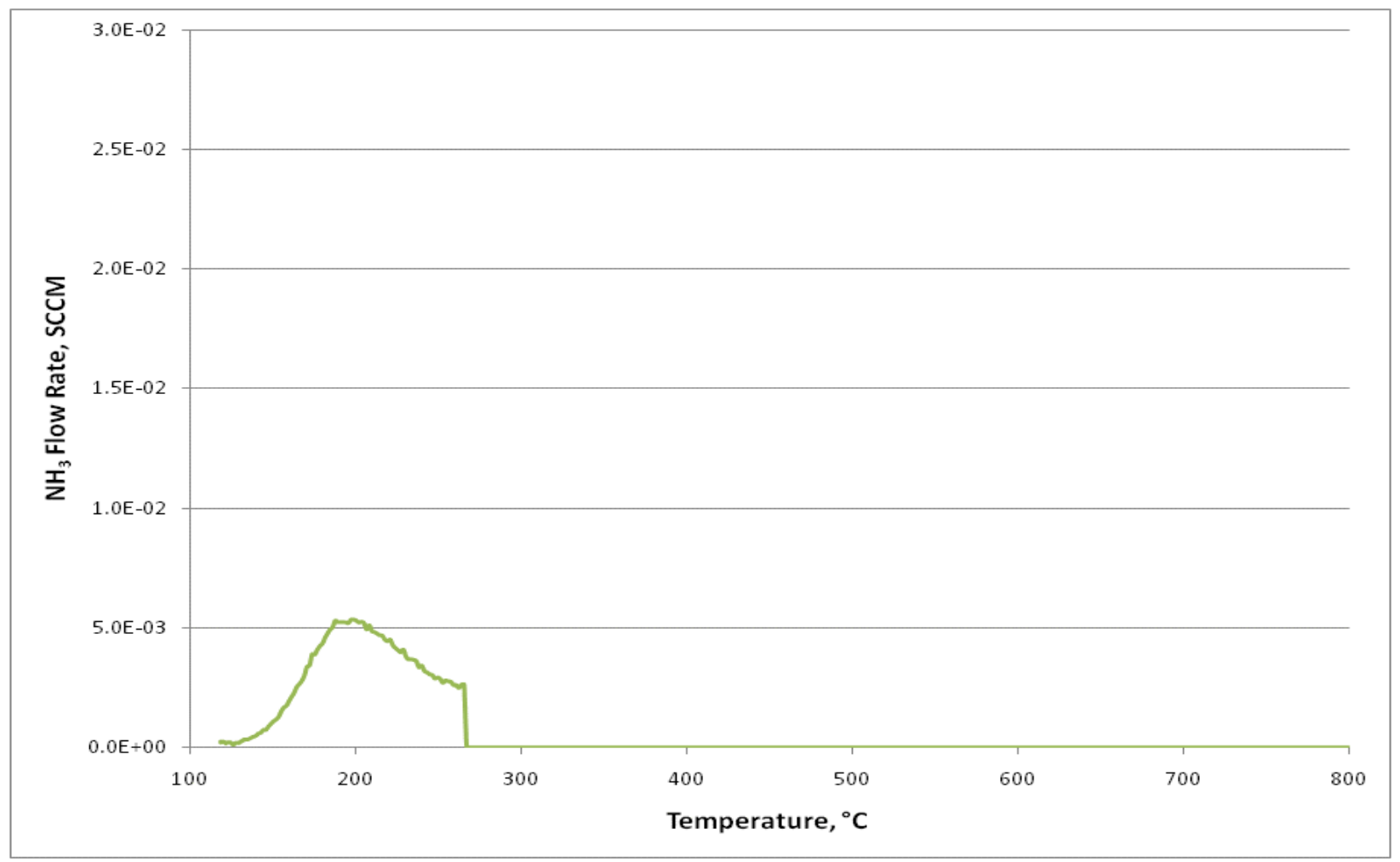

Figure B.7. $\mathrm{NH}_{3} \mathrm{TPD}$ trace of the Merck Aldrich Grade 60 silica pre-dried at $120^{\circ} \mathrm{C}$ for $2 \mathrm{hr}$. 


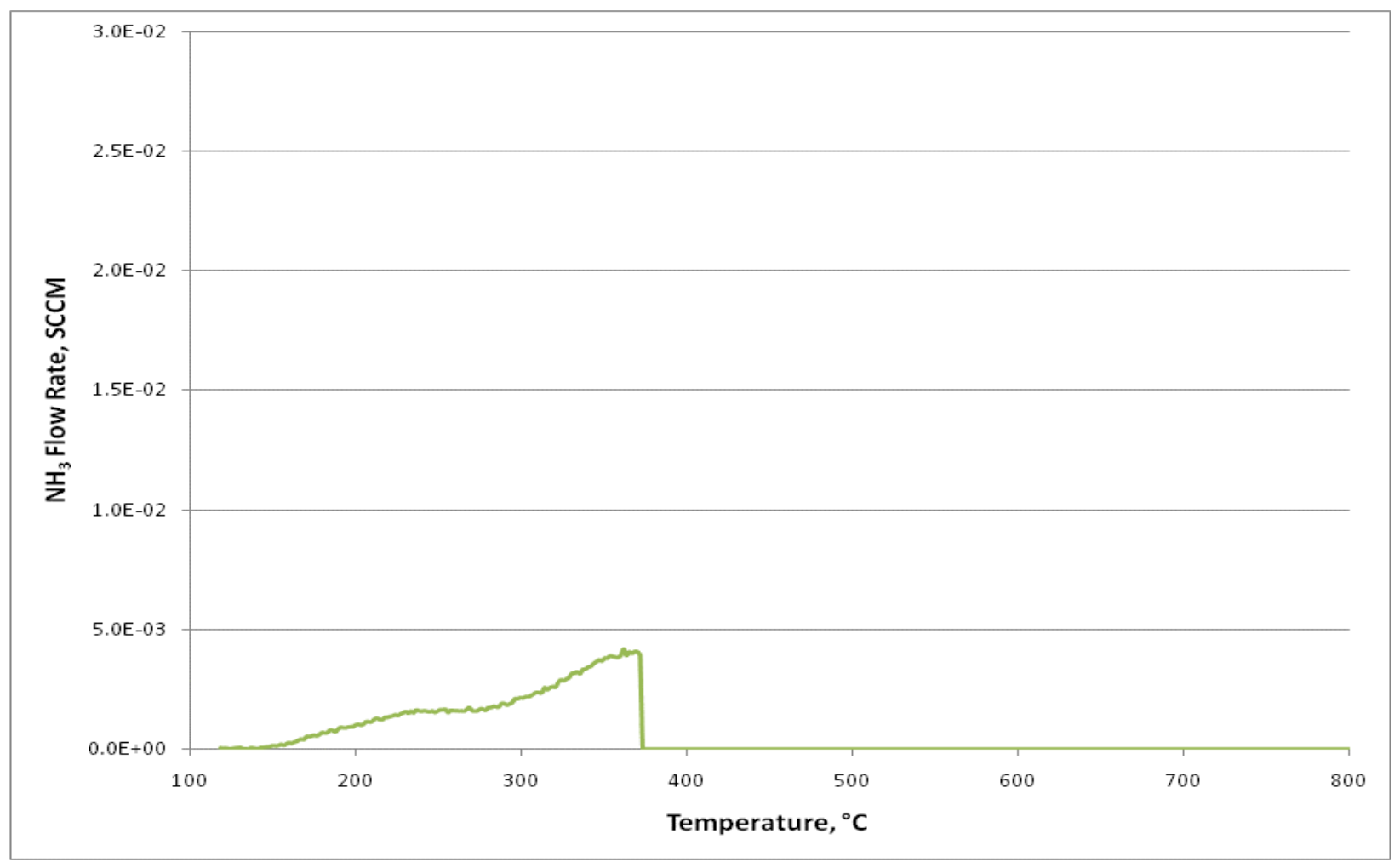

Figure B.8. $\mathrm{NH}_{3} \mathrm{TPD}$ trace of the Grace Grade 408 silica pre-dried at $300^{\circ} \mathrm{C}$ for $2 \mathrm{hr}$.

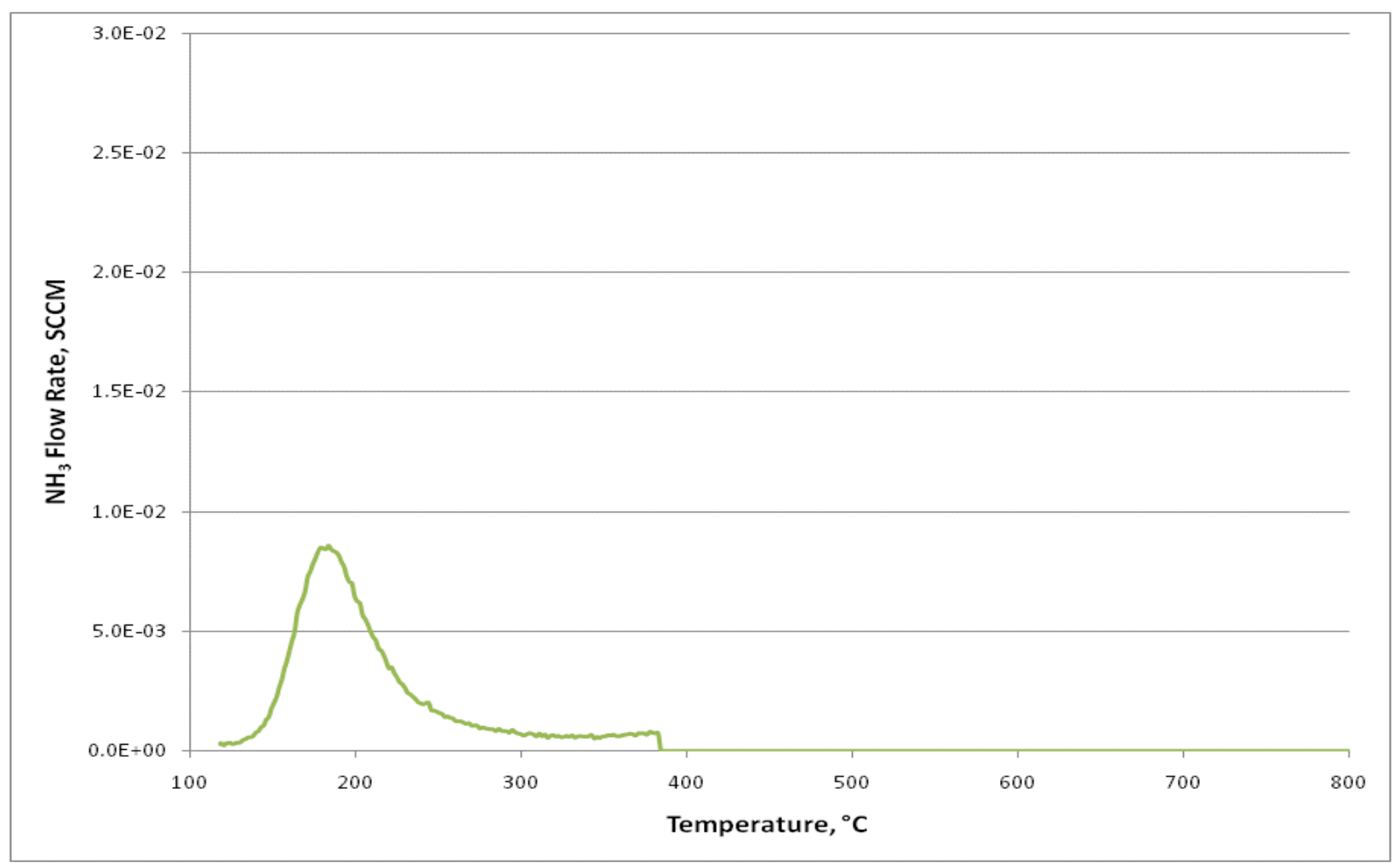

Figure B.9. $\mathrm{NH}_{3} \mathrm{TPD}$ trace of the Davisil 645 silica pre-dried at $300^{\circ} \mathrm{C}$ for $2 \mathrm{hr}$. 


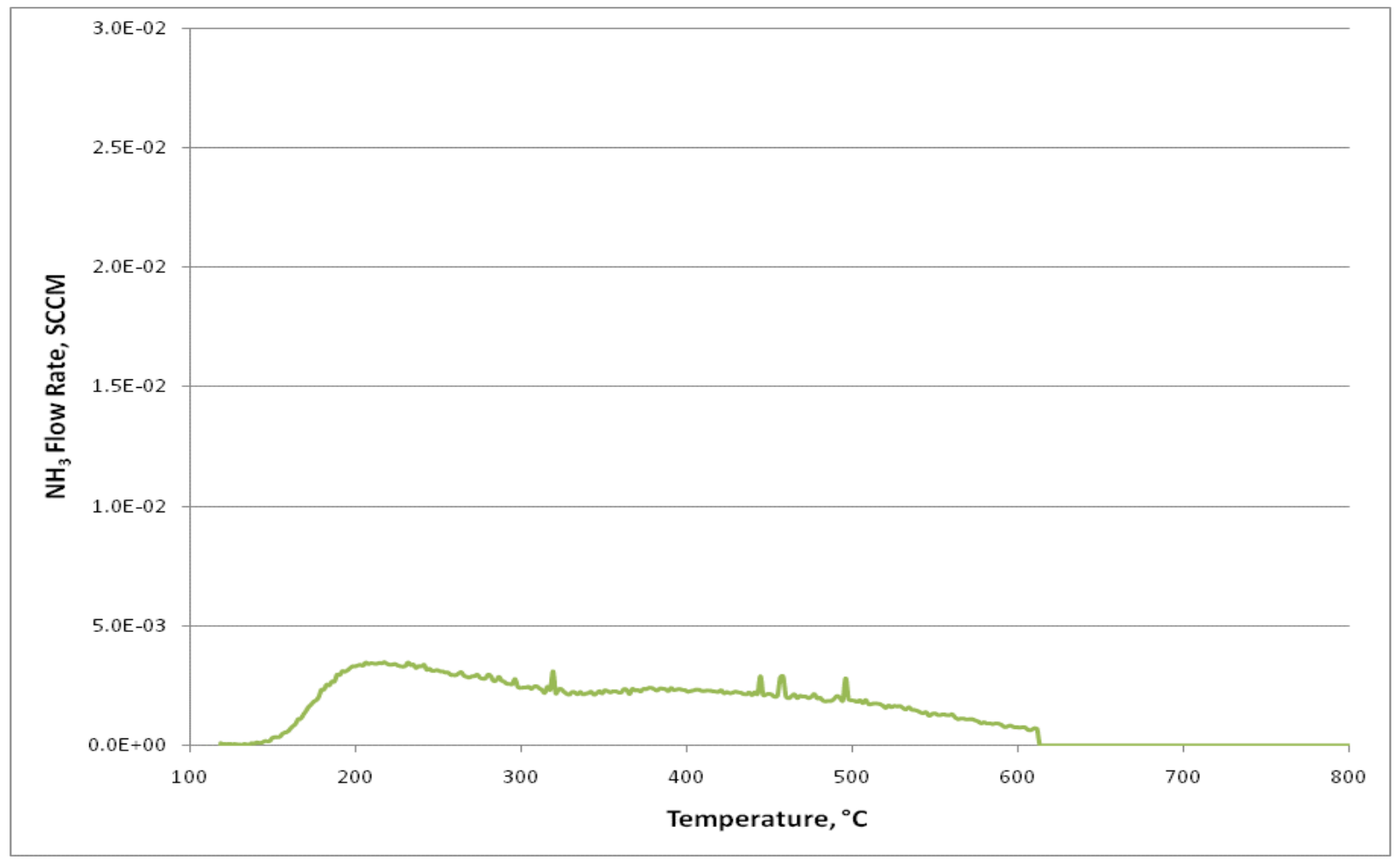

Figure B.10. $\mathrm{NH}_{3} \mathrm{TPD}$ trace of the Norpro 61138 silica pre-dried at $300^{\circ} \mathrm{C}$ for $2 \mathrm{hr}$.

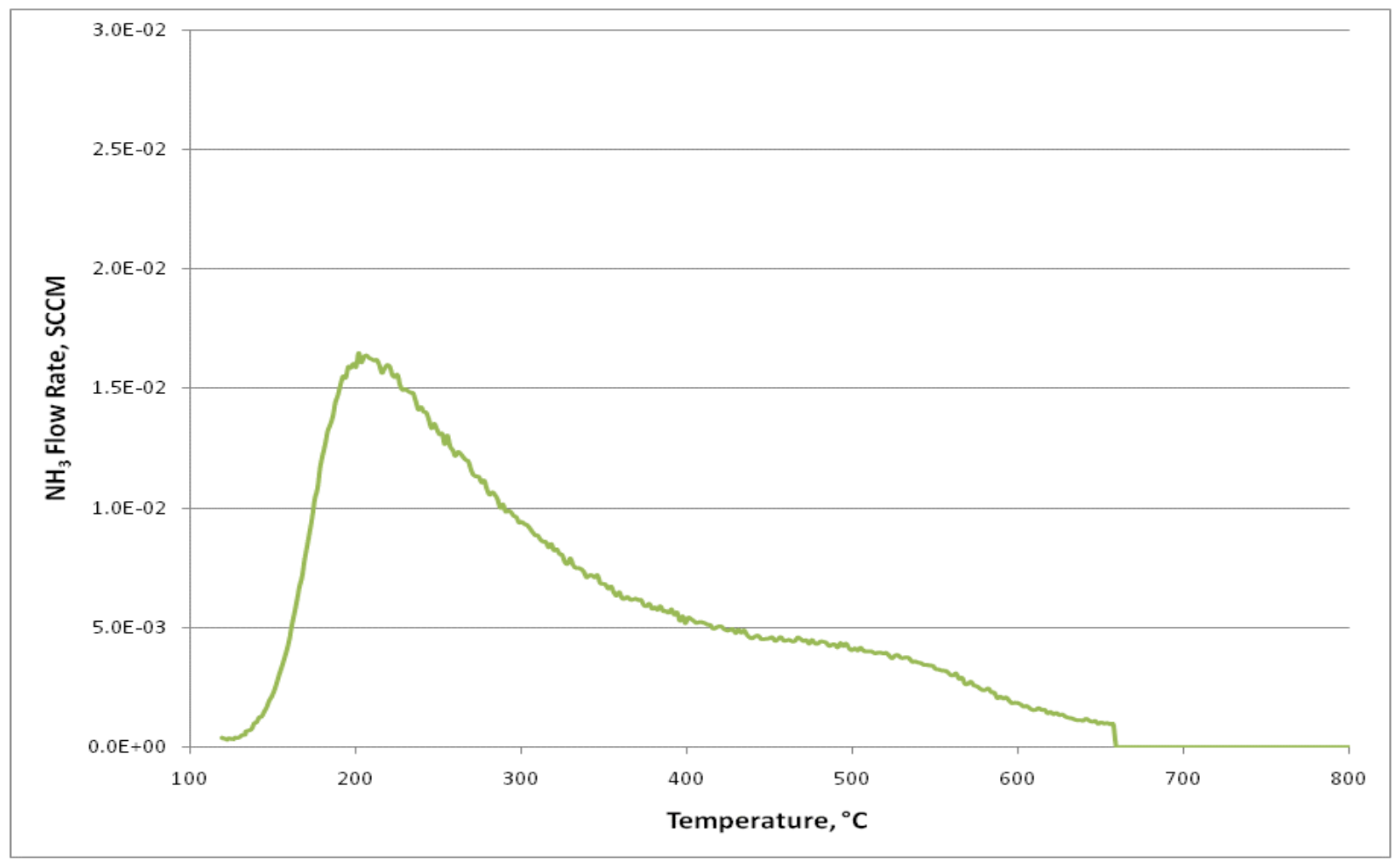

Figure B.11. $\mathrm{NH}_{3} \mathrm{TPD}$ trace of the Engelhard Mod D silica pre-dried at $300^{\circ} \mathrm{C}$ for $2 \mathrm{hr}$. 


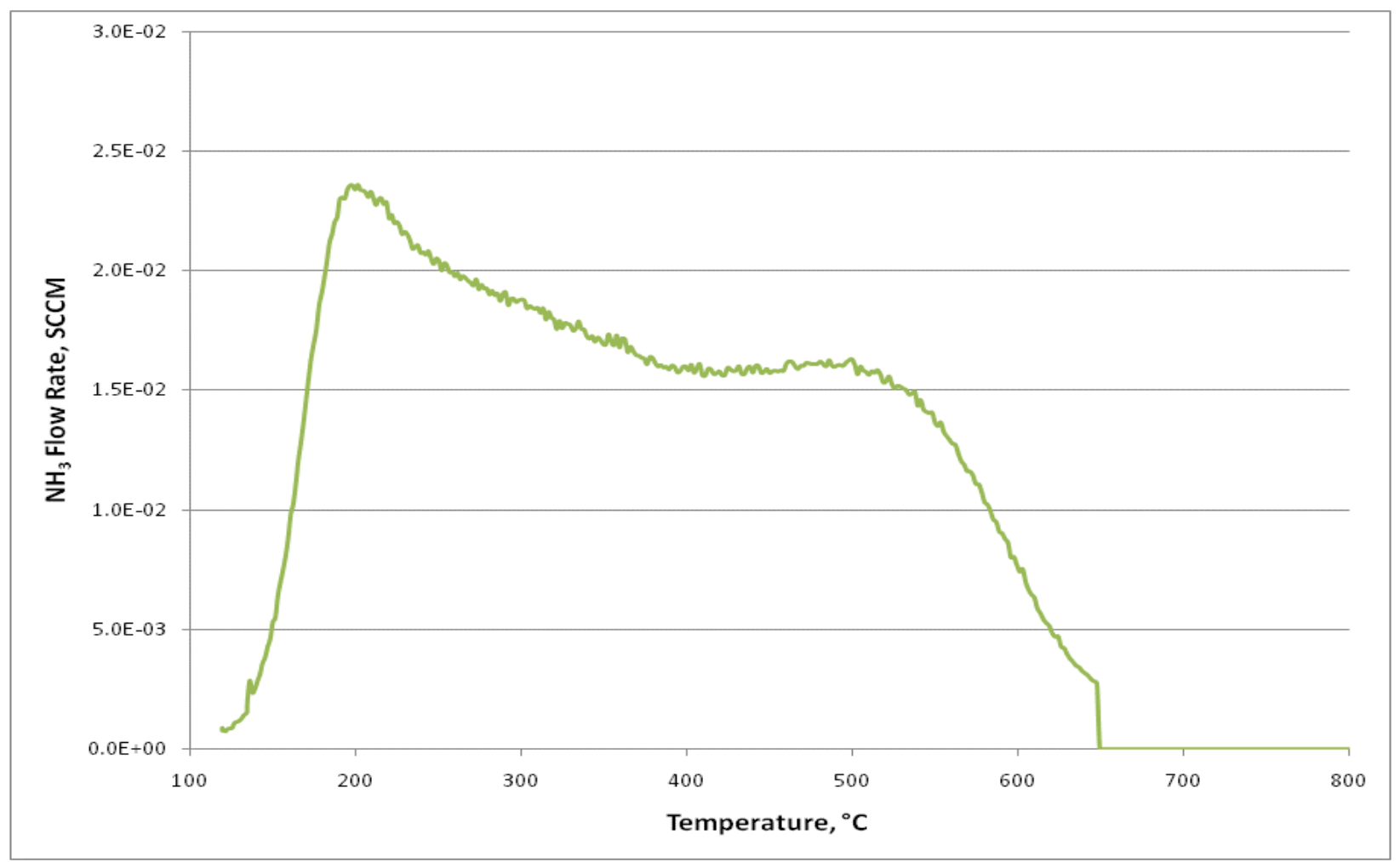

Figure B.12. $\mathrm{NH}_{3} \mathrm{TPD}$ trace of the Perlkat $79-3$ silica pre-dried at $300^{\circ} \mathrm{C}$ for $2 \mathrm{hr}$.

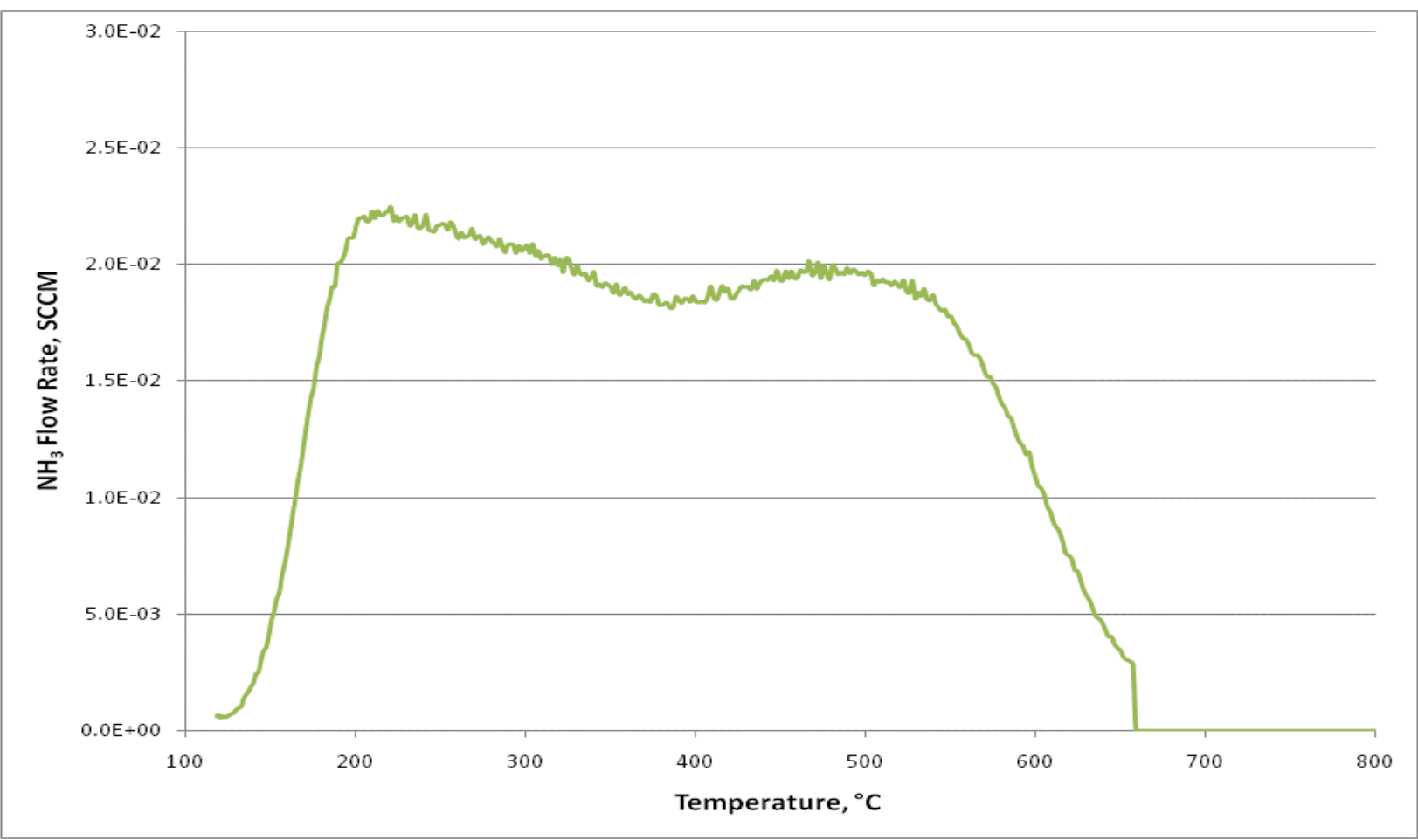

Figure B.13. $\mathrm{NH}_{3} \mathrm{TPD}$ trace of the Perlkat $29-3$ silica pre-dried at $300^{\circ} \mathrm{C}$ for $2 \mathrm{hr}$. 


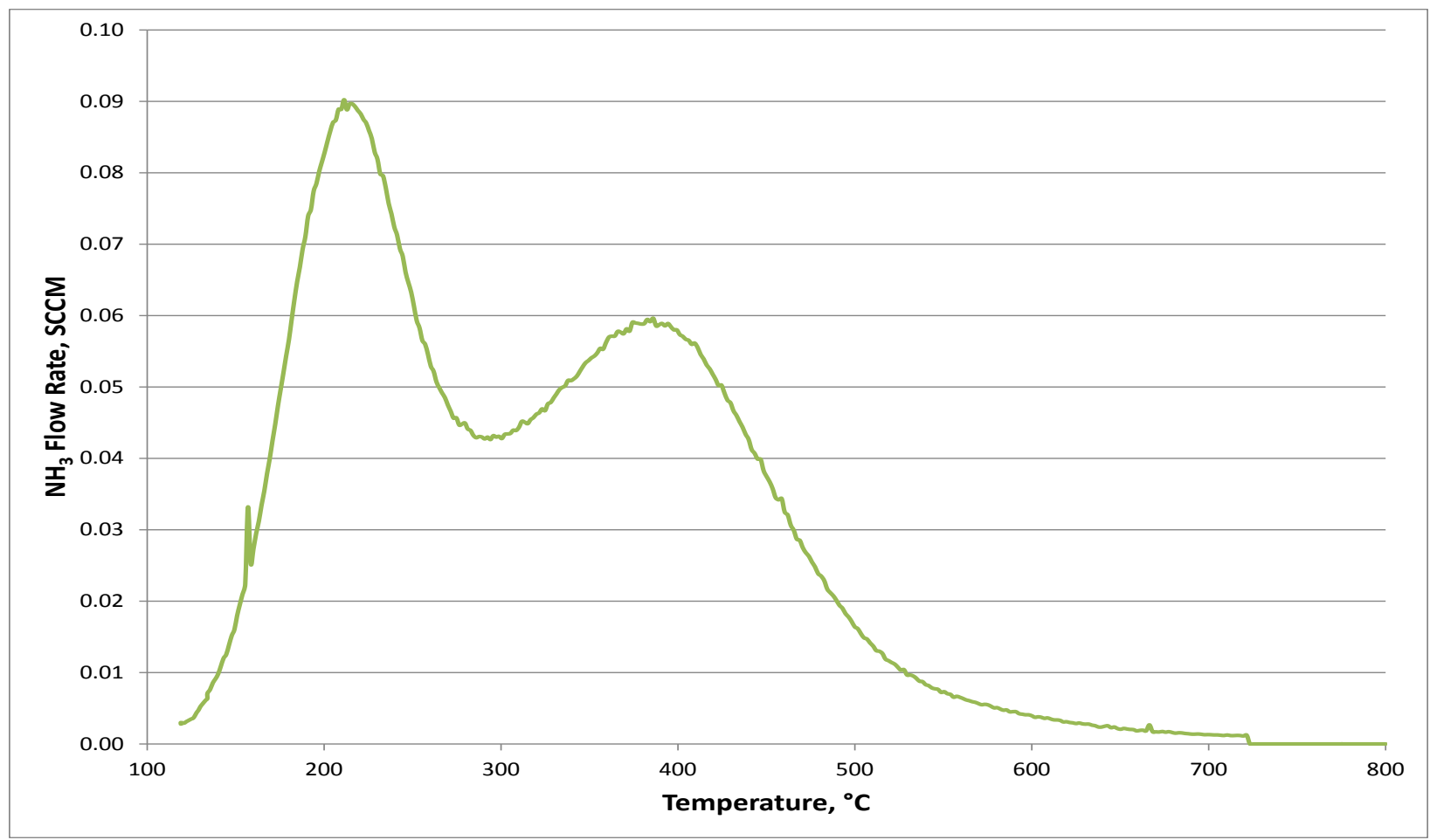

Figure B.14. $\mathrm{NH}_{3}$ TPD trace of a $\mathrm{H}-\mathrm{ZSM} 5(\mathrm{Si} / \mathrm{Al}=30)$ pre-dried at $500^{\circ} \mathrm{C}$ for $2 \mathrm{hr}$. The H-ZSM5 is considered a strong solid acid. This TPD trace is included as a basis for comparison with the mixed alcohols supports. 


\section{Appendix C}

Thermogravimetric and Mass Spectrometer Data from Temperature Programmed Desorption Testing on Samples KOA 1 through 12 



\section{Appendix C}

\section{Thermogravimetric and Mass Spectrometer Data from Temperature Programmed Desorption Testing on KOA Samples 1 through 12}

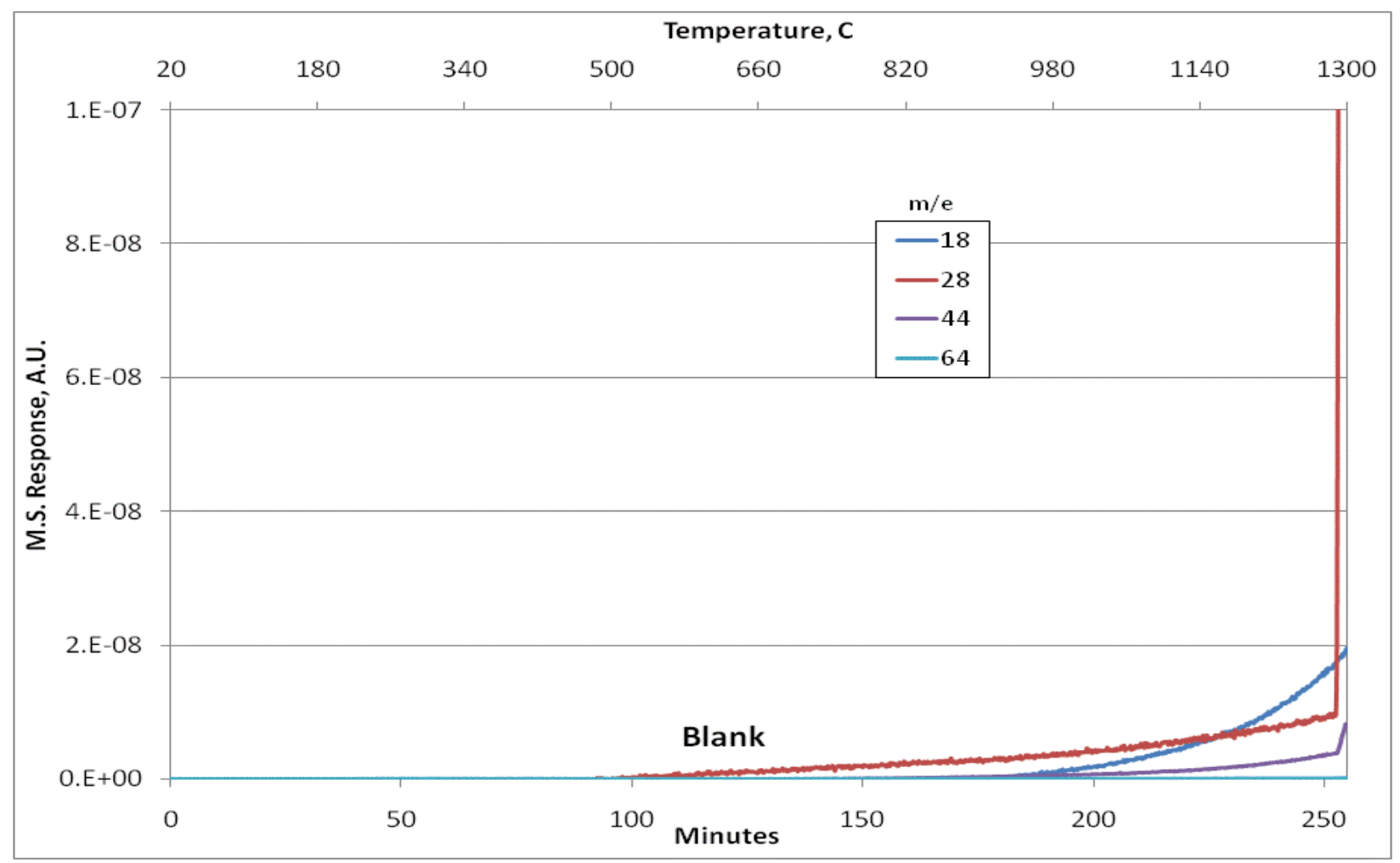

Figure C.1. A reference MS trace obtained from testing of an empty sample pan. 


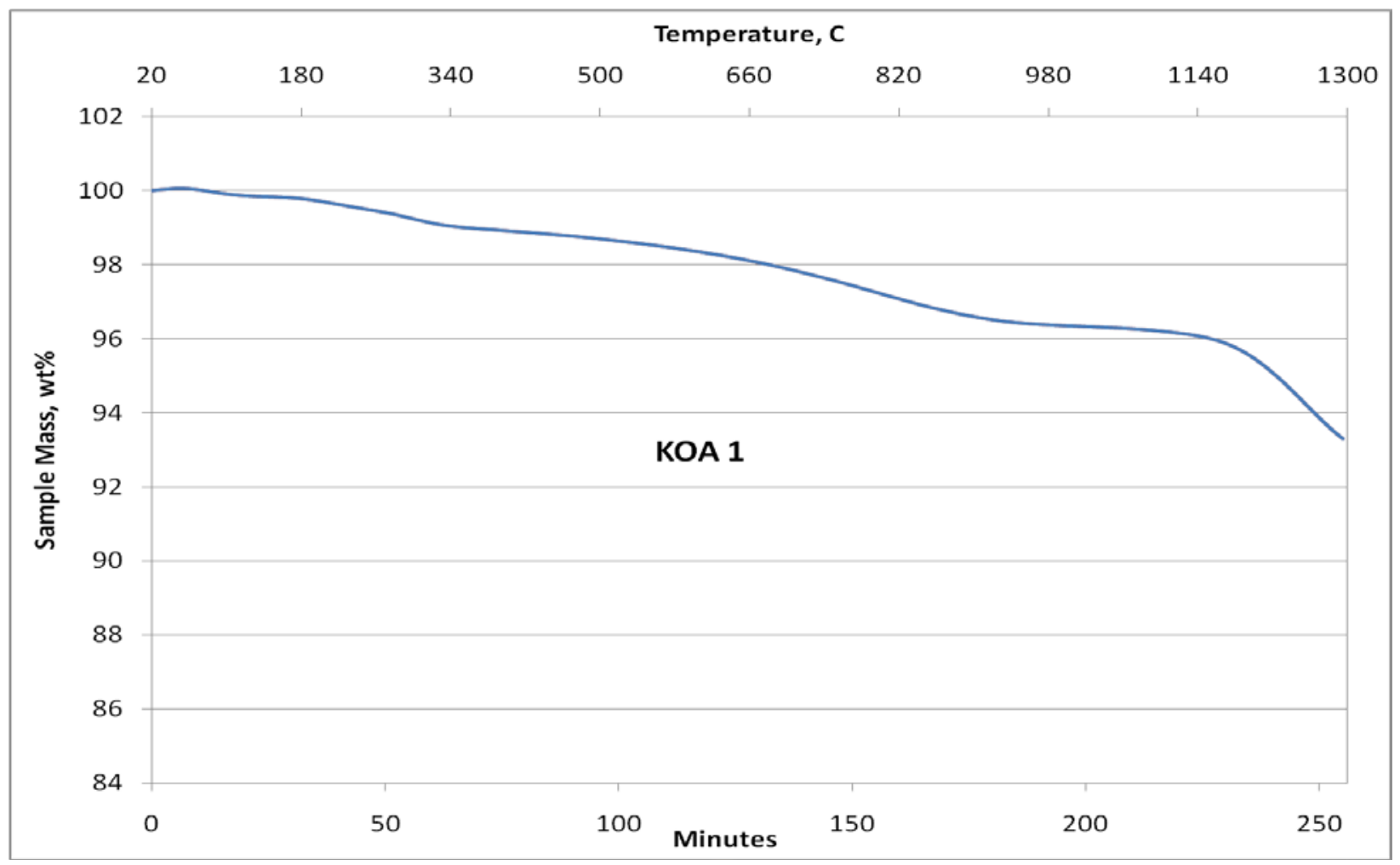

Figure C.2. The TG trace obtained from TPD testing of KOA 1, which is Norit ROX 0.8.

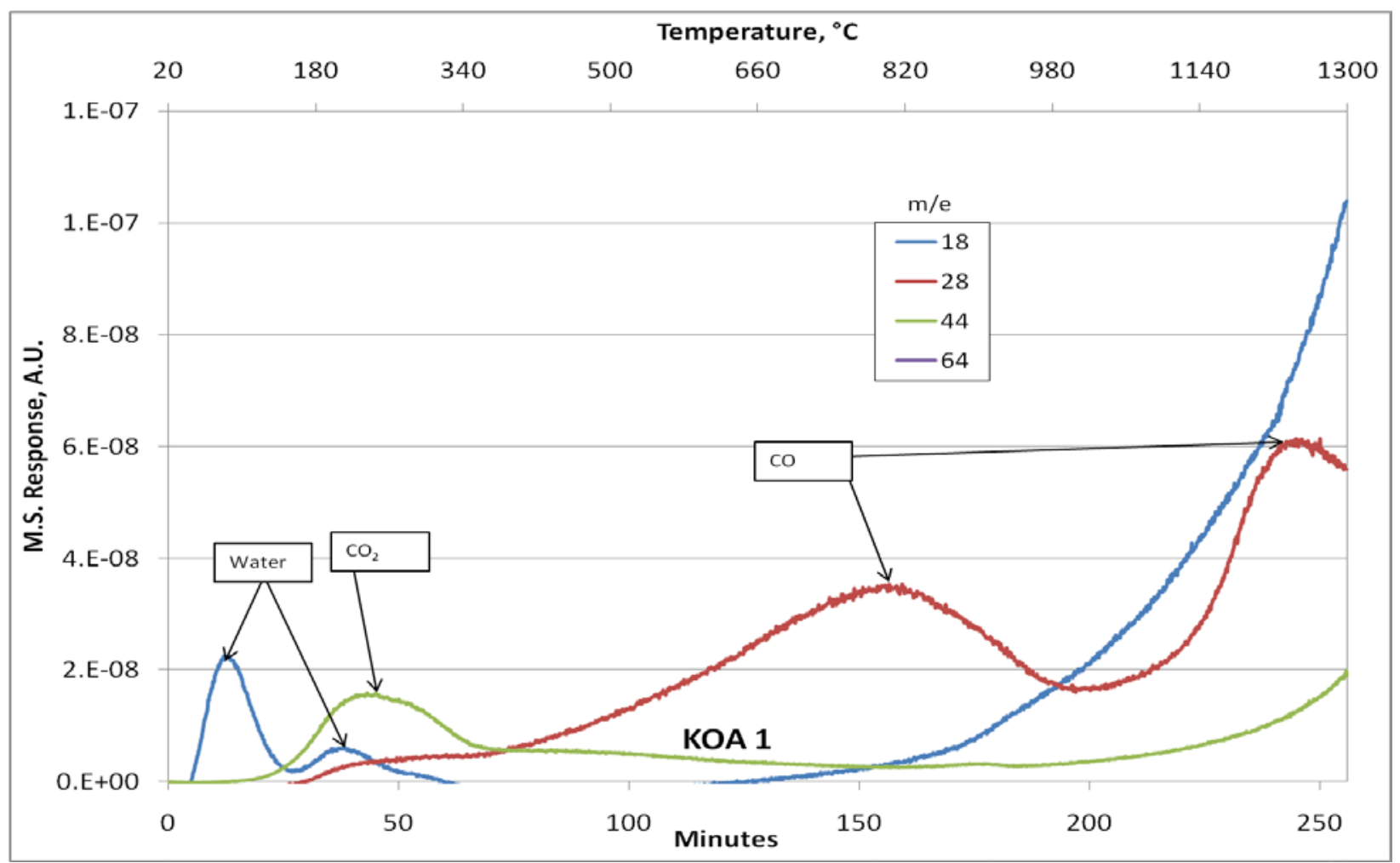

Figure C.3. The MS trace obtained TPD testing of KOA 1, which is Norit ROX 0.8. 


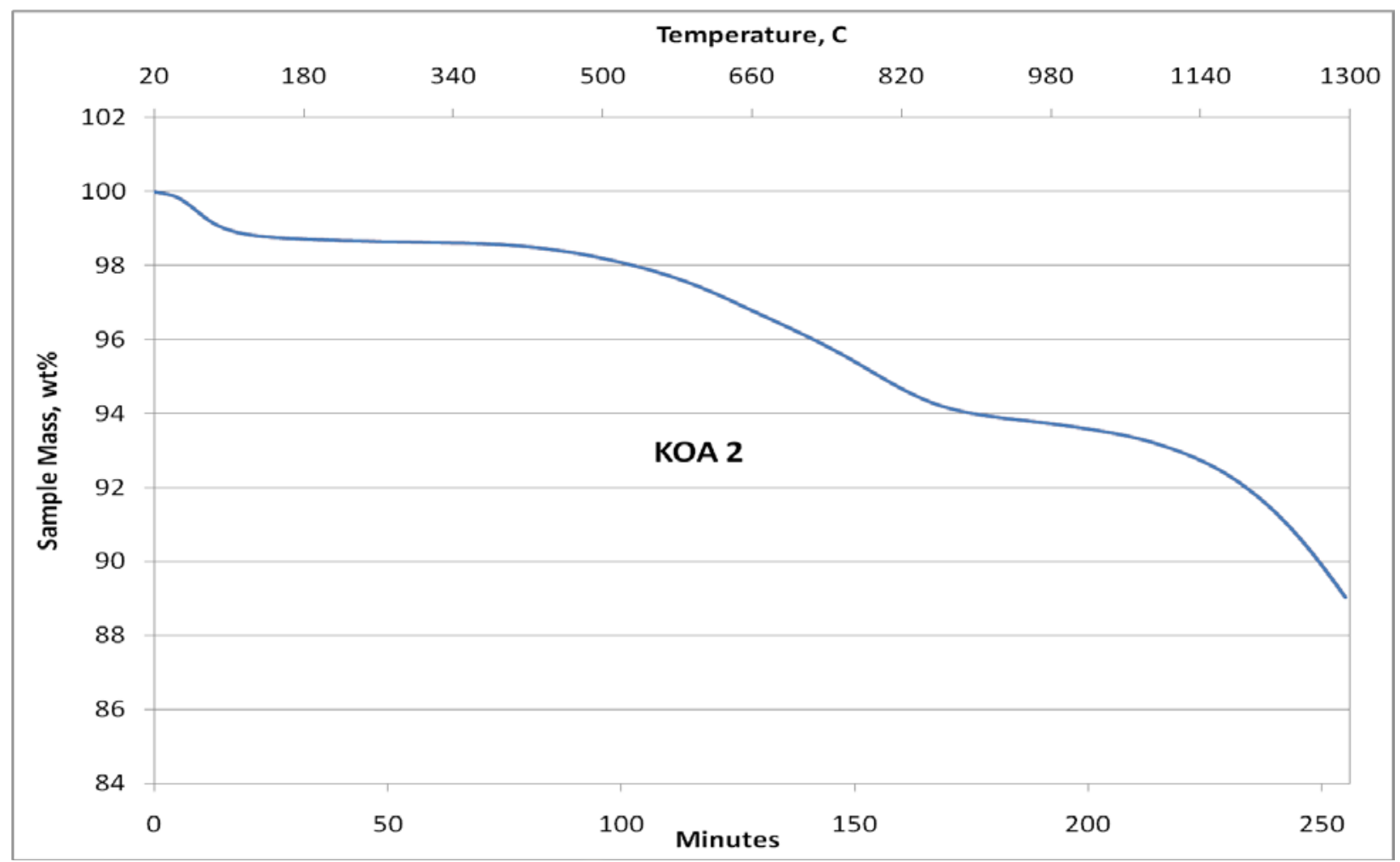

Figure C.4. The TG trace obtained from TPD testing of KOA 2, which is Norit Darco-LS.

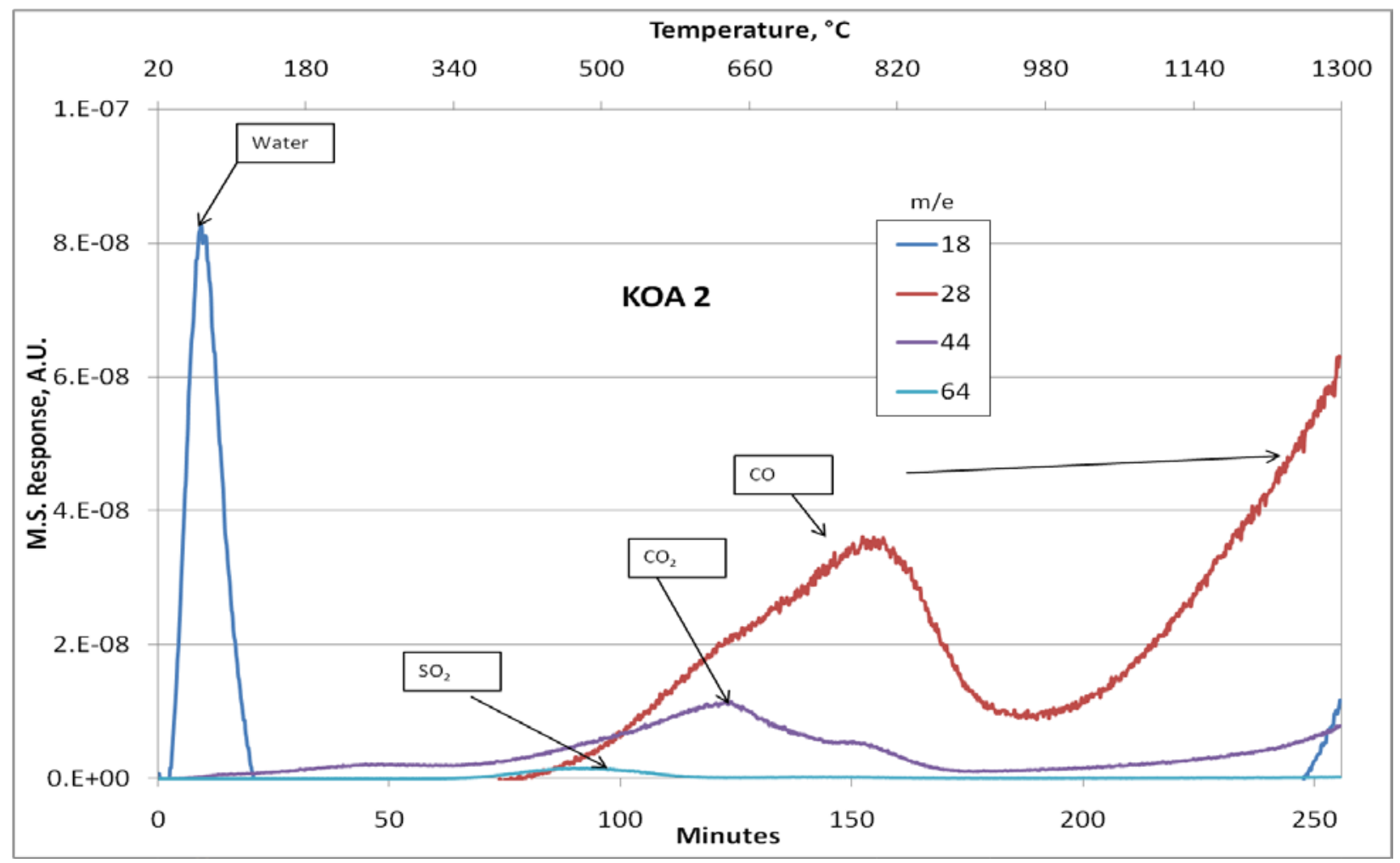

Figure C.5. The MS trace obtained TPD testing of KOA 2, which is Norit Darco-LS. 


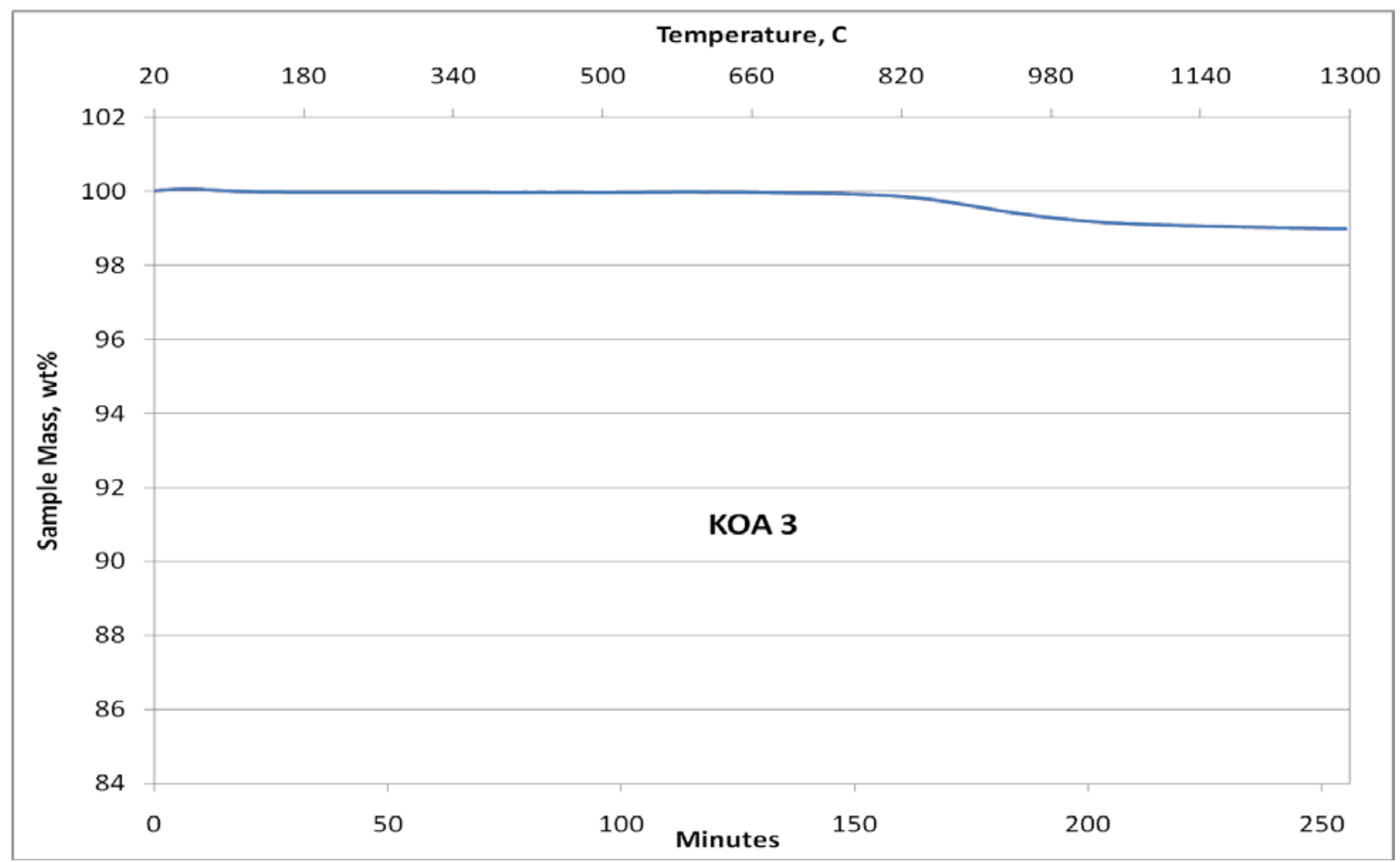

Figure C.6. The TG trace obtained from TPD testing of KOA 3, which is Sibunit carbon.

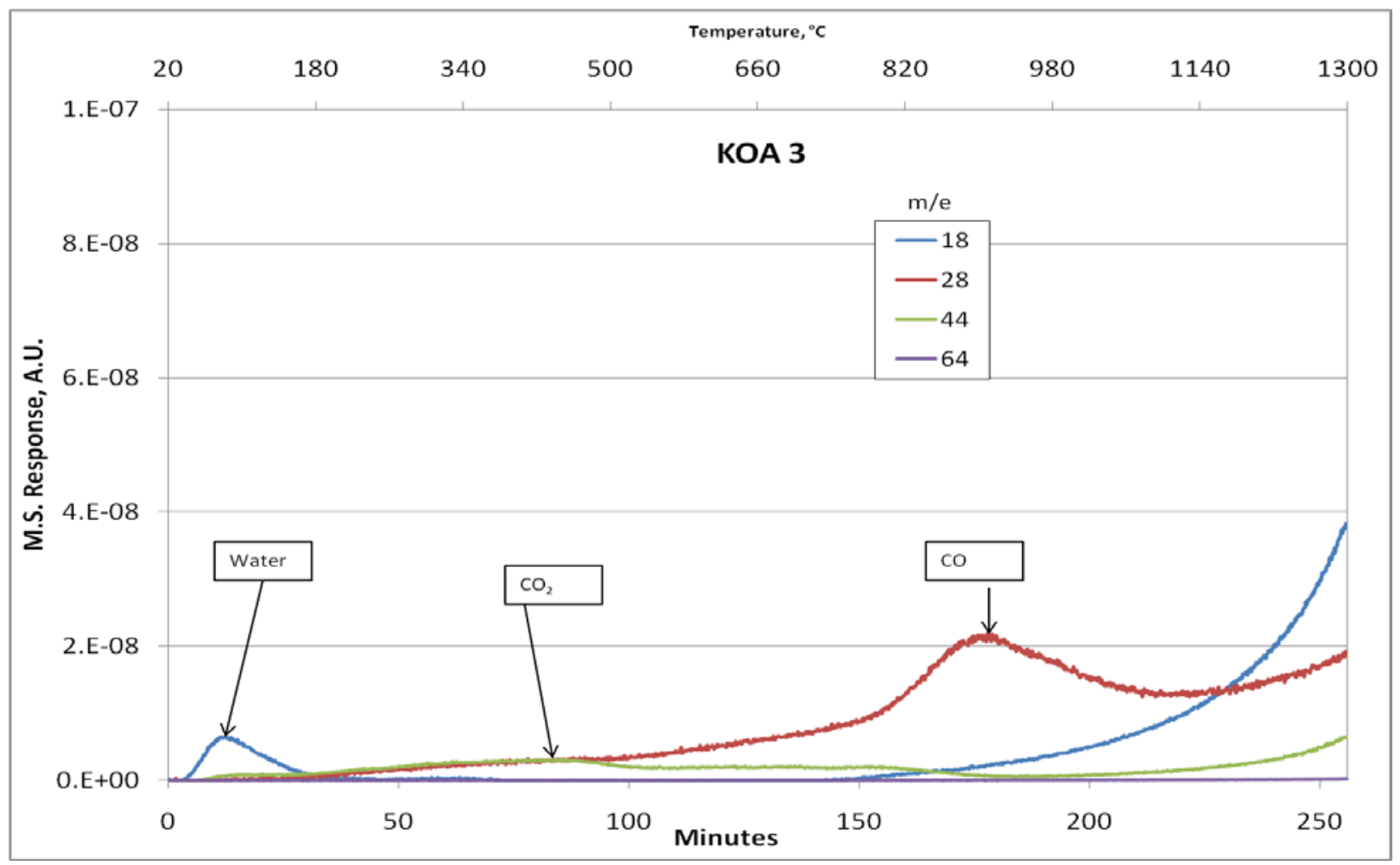

Figure C.7. The MS trace obtained TPD testing of KOA 3, which is Sibunit carbon. 


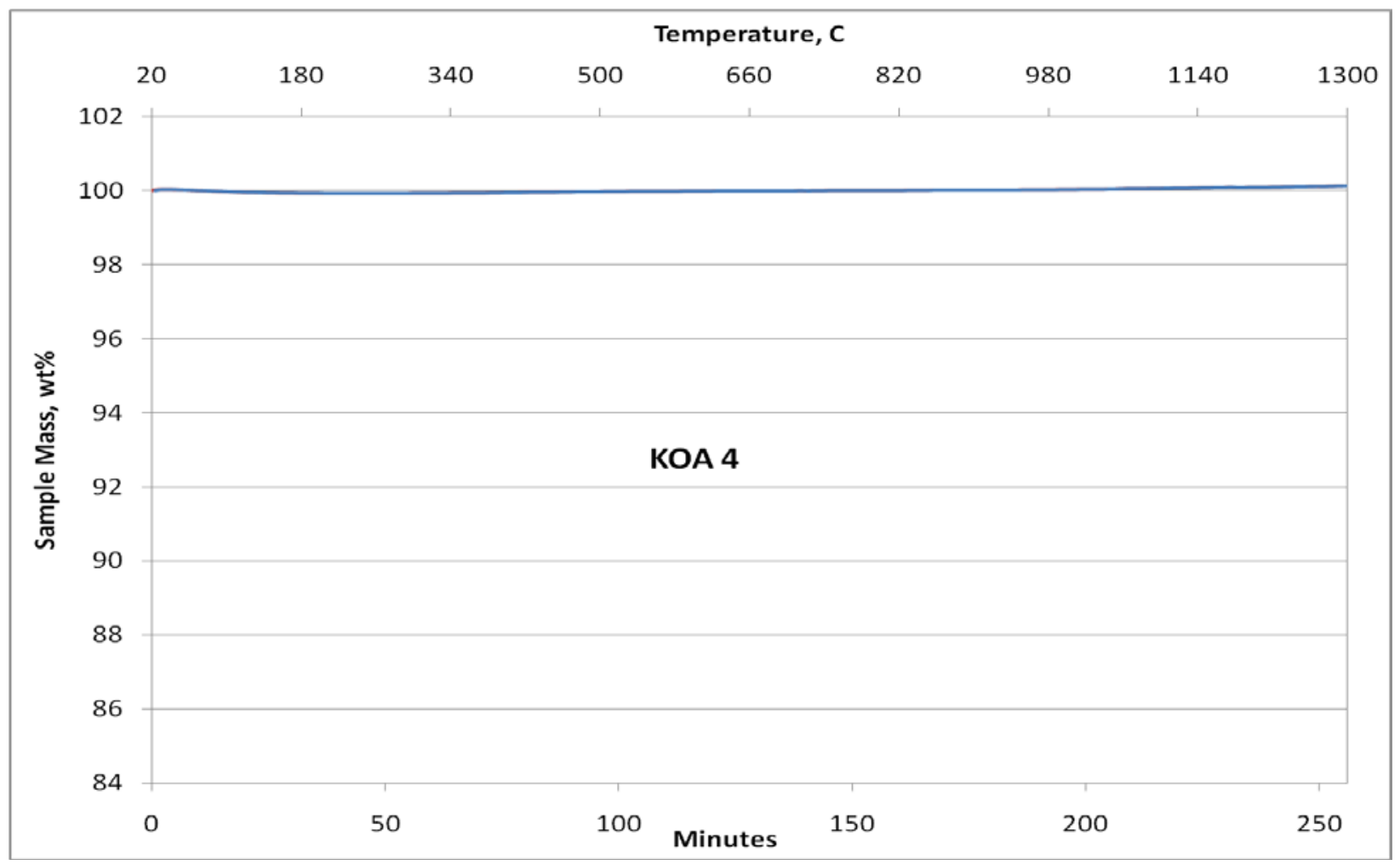

Figure C.8. The TG trace obtained from TPD testing of KOA 4, which is Englehard High Surface Area Graphite (HSAG-1).

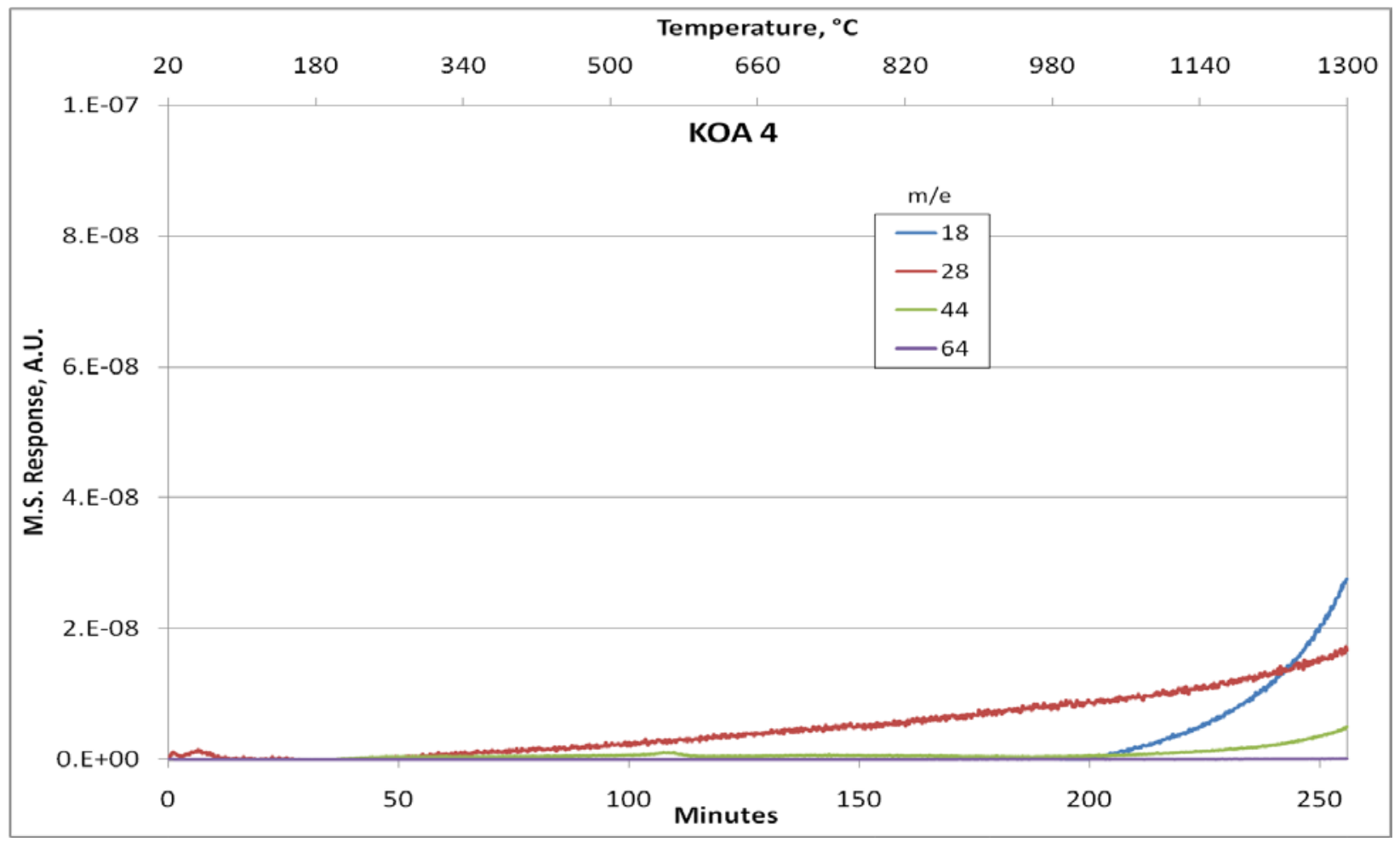

Figure C.9. The MS trace obtained TPD testing of KOA 4, which is Englehard High Surface Area Graphite (HSAG-1). 


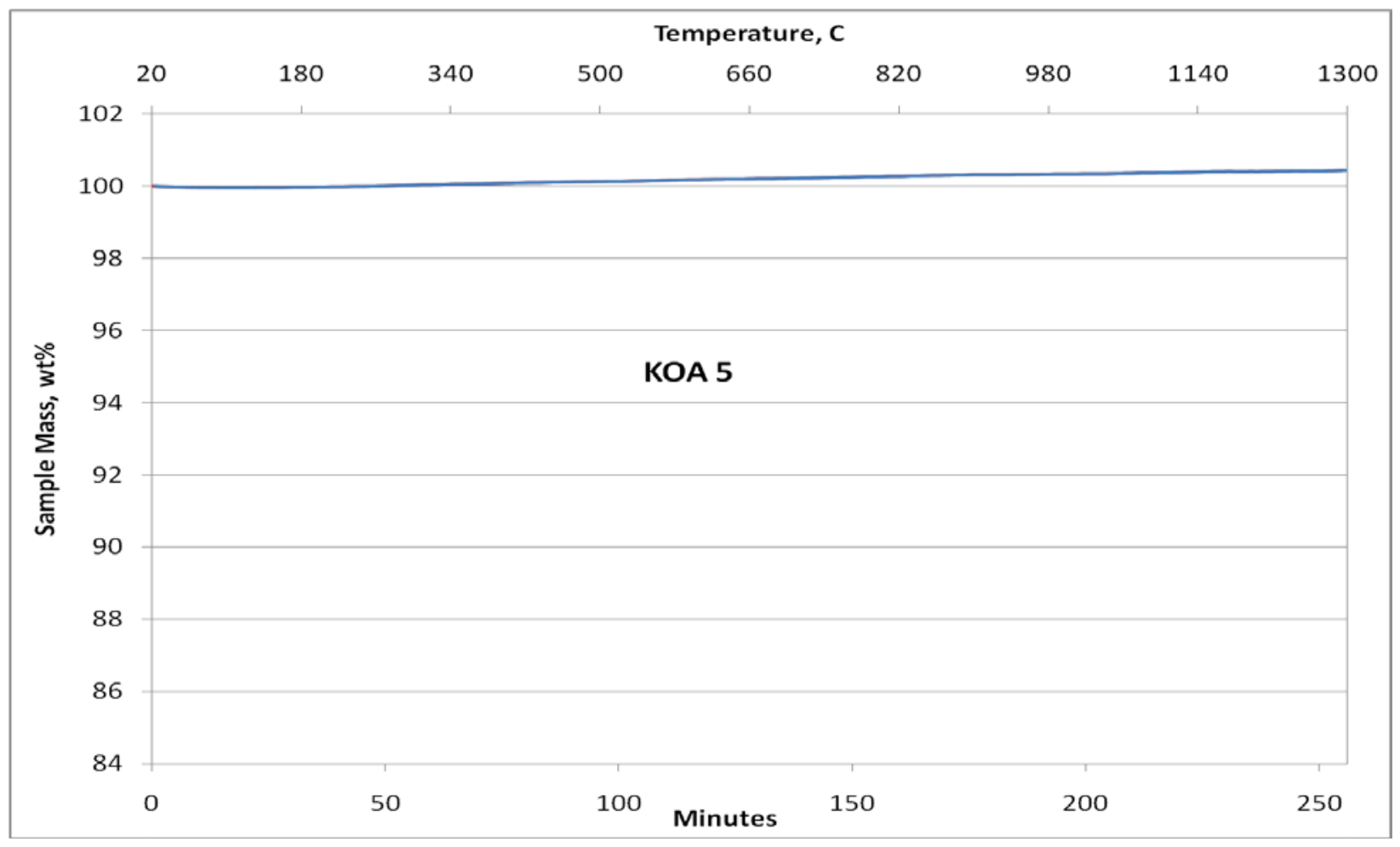

Figure C.10. The TG trace obtained from TPD testing of KOA 5, which is Englehard High Surface Area Graphite (HSAG-2).

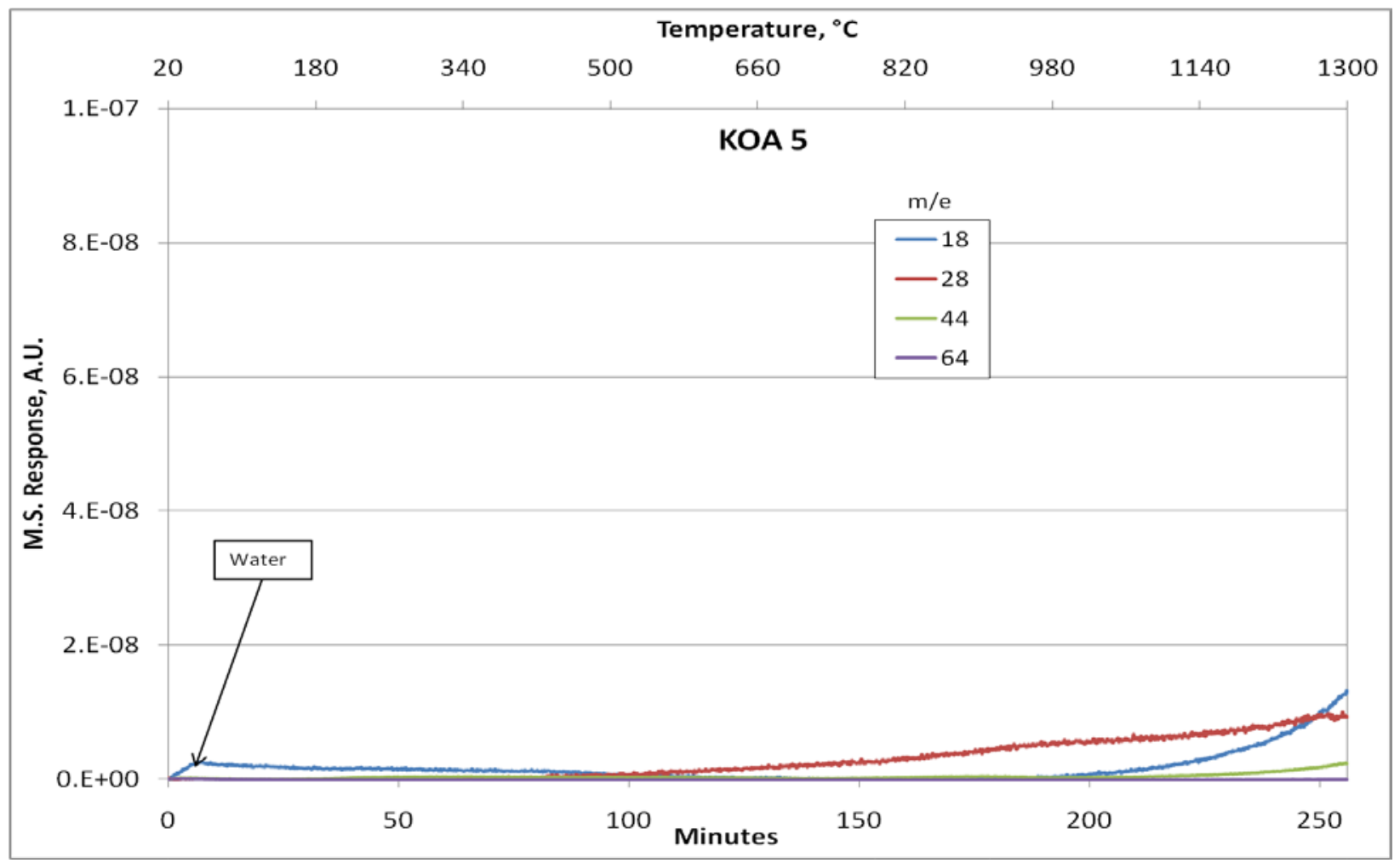

Figure C.11. The MS trace obtained TPD testing of KOA 5, which is Englehard High Surface Area Graphite (HSAG-2). 


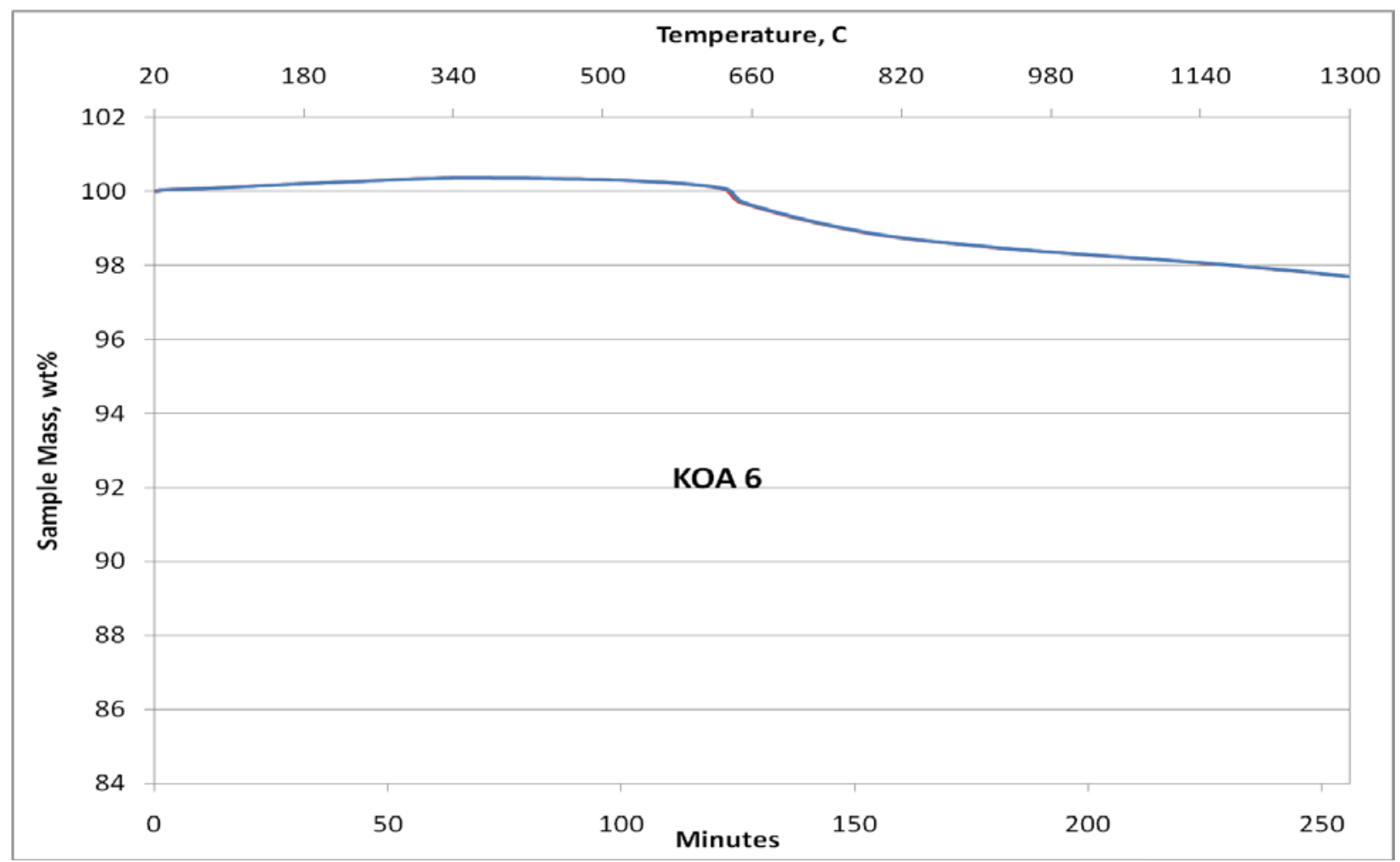

Figure C.12. The TG trace obtained from TPD testing of KOA 6, which is Hyperion Lot 395-08.

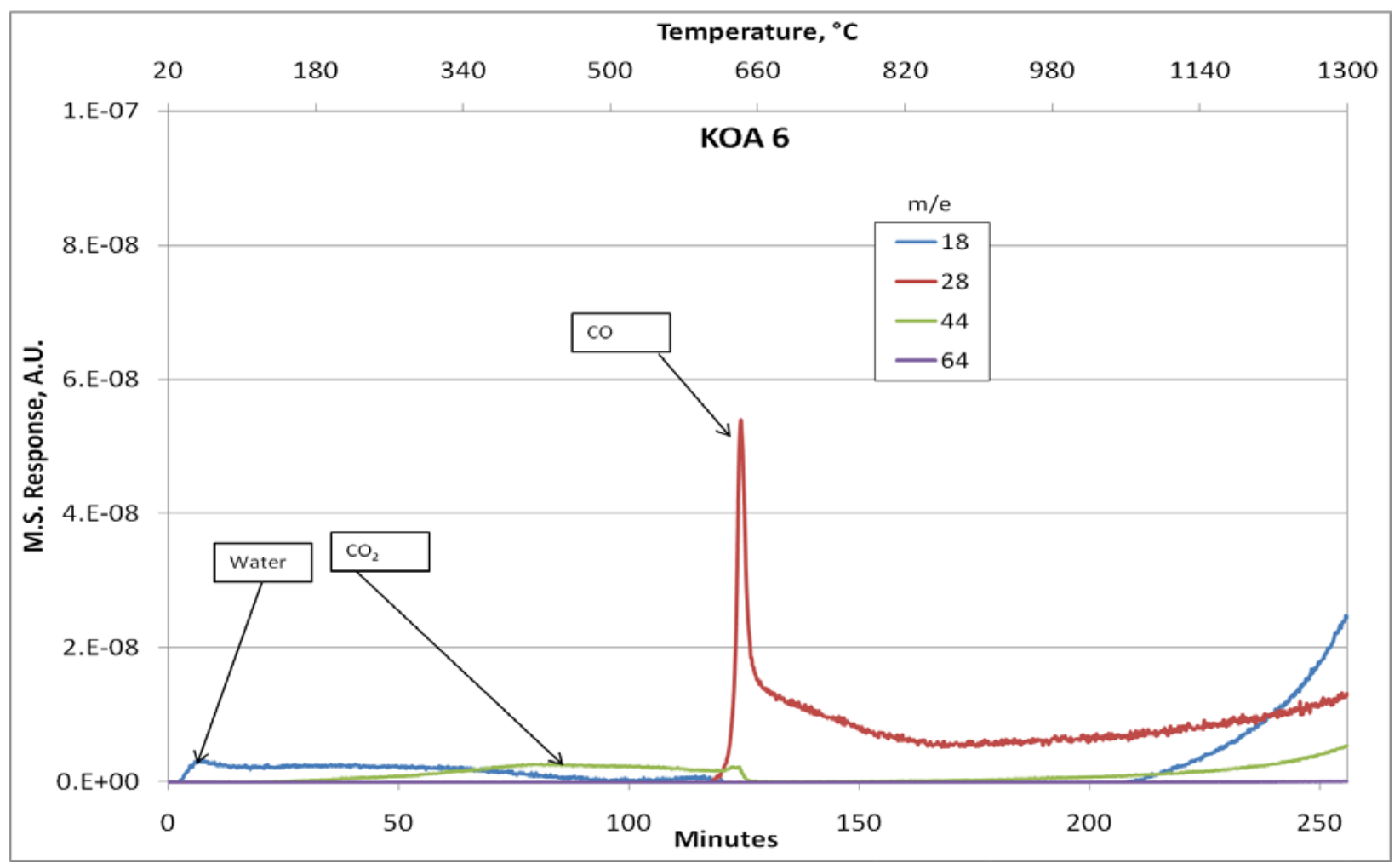

Figure C.13. The MS trace obtained TPD testing of KOA 6, which is Hyperion Lot 395-08. 


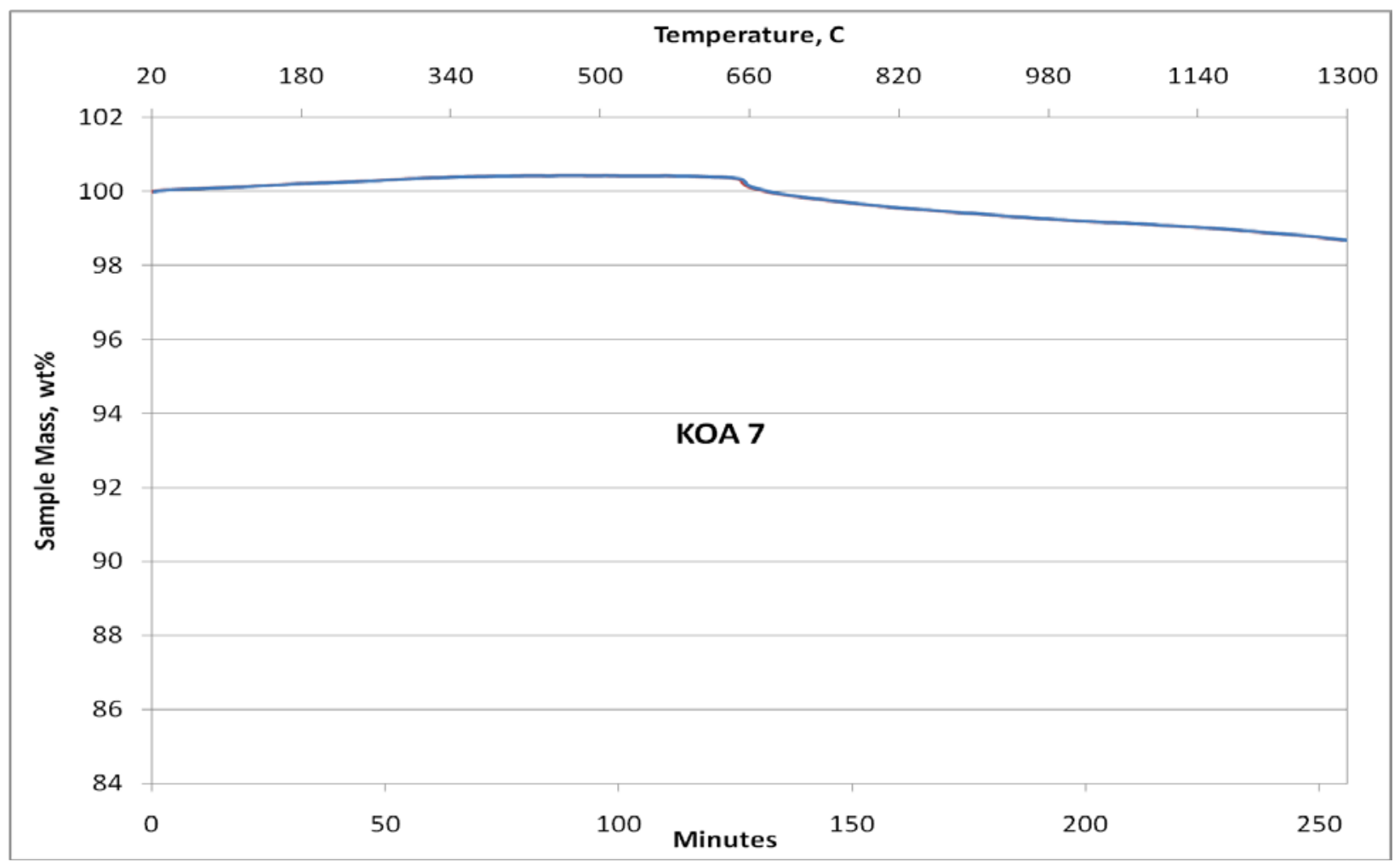

Figure C.14. The TG trace obtained from TPD testing of KOA 7, which is Hyperion Lot 395-10.

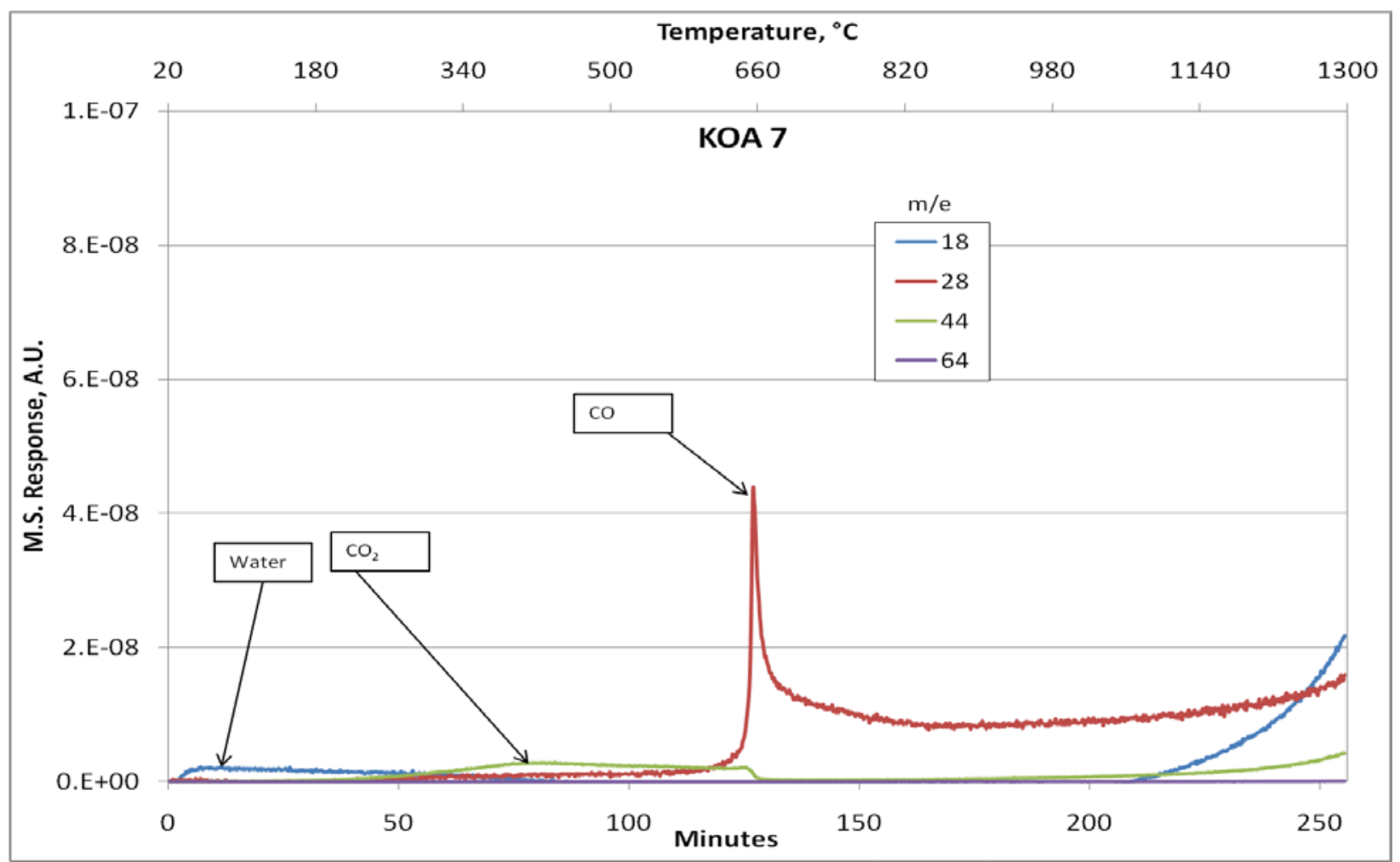

Figure C.15. The MS trace obtained TPD testing of KOA 7, which is Hyperion Lot 395-10. 


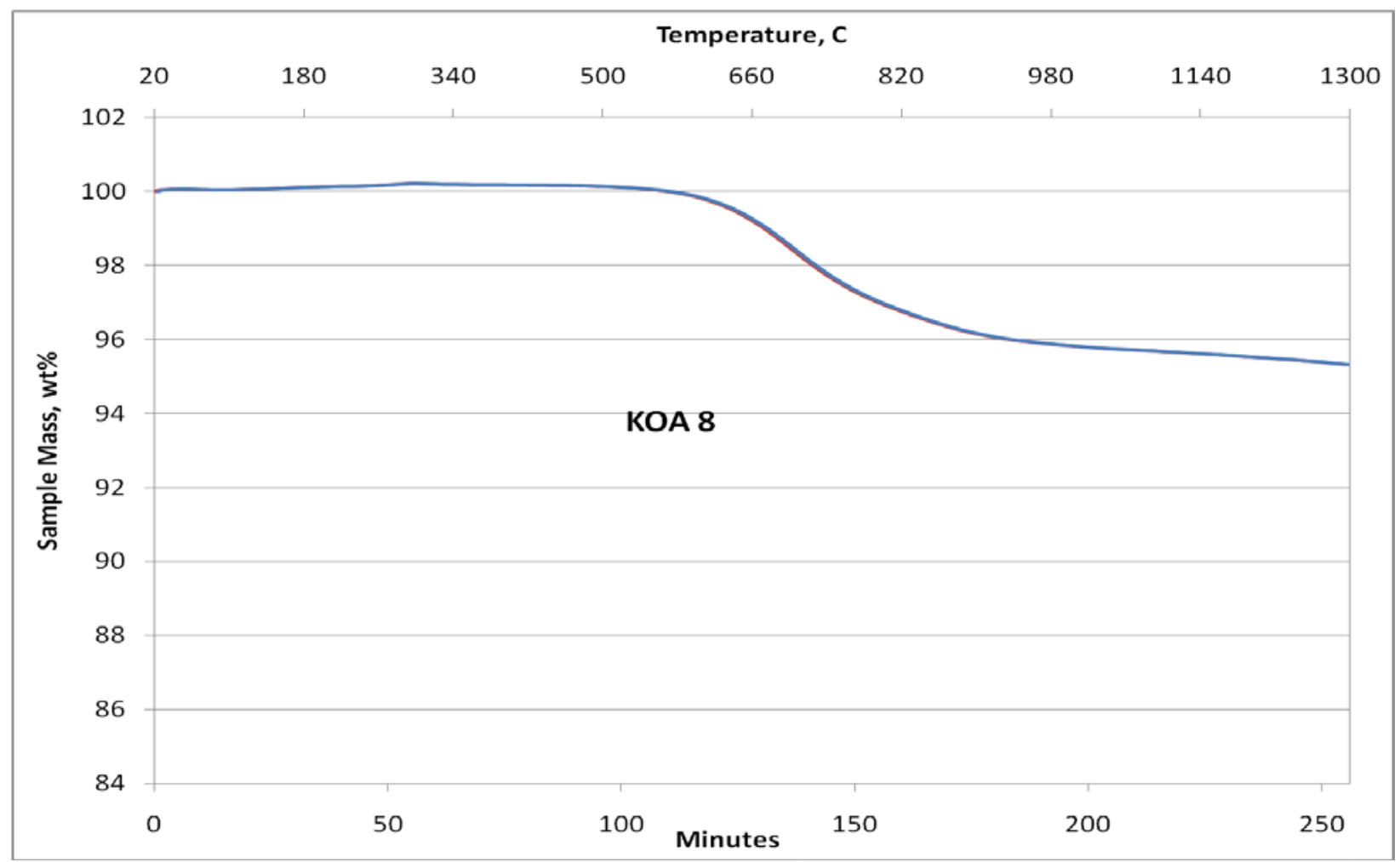

Figure C.16. The TG trace obtained from TPD testing of KOA 8, which is Hyperion Lot 384-82.

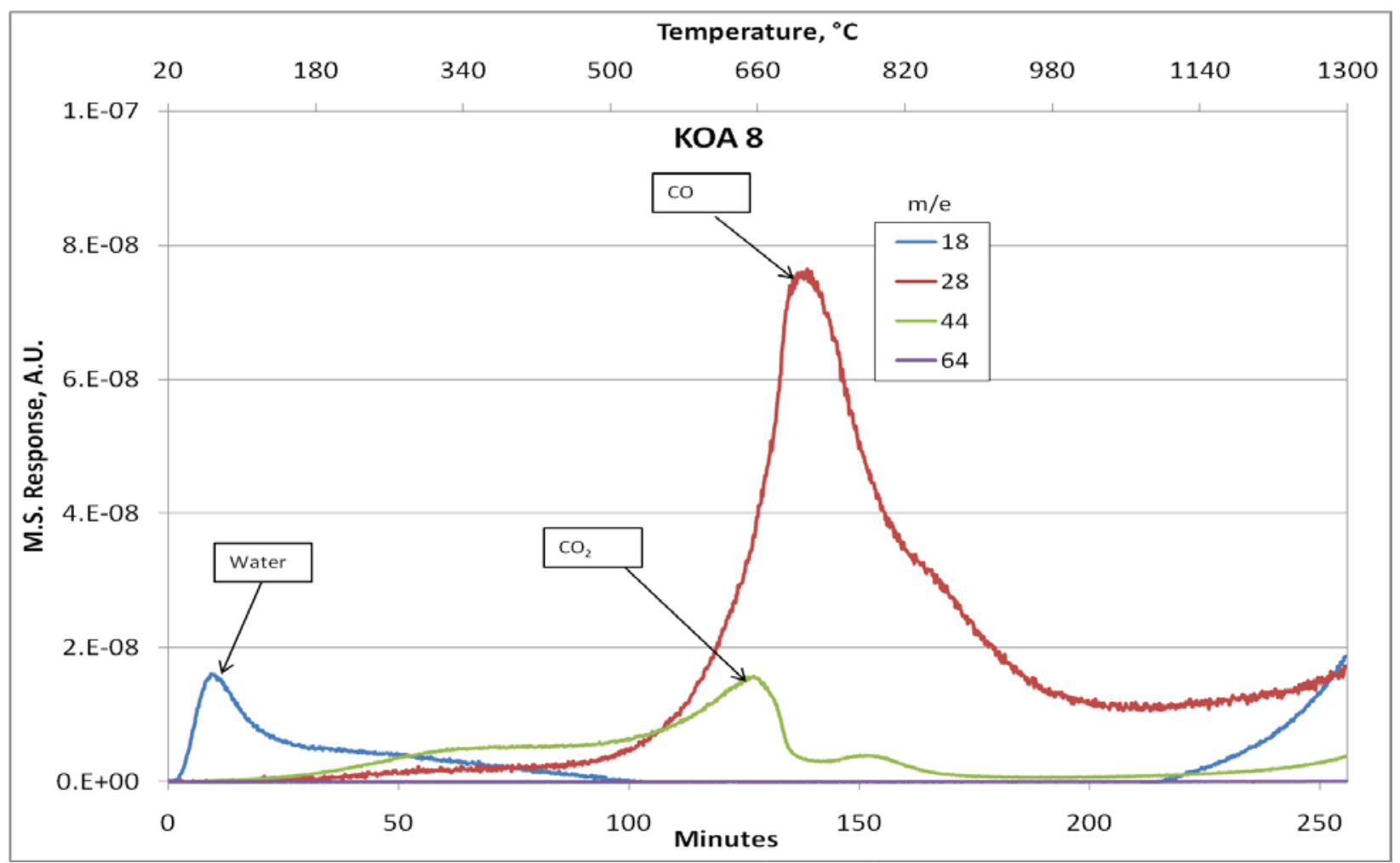

Figure C.17. The MS trace obtained TPD testing of KOA 8, which is Hyperion Lot 384-82. 


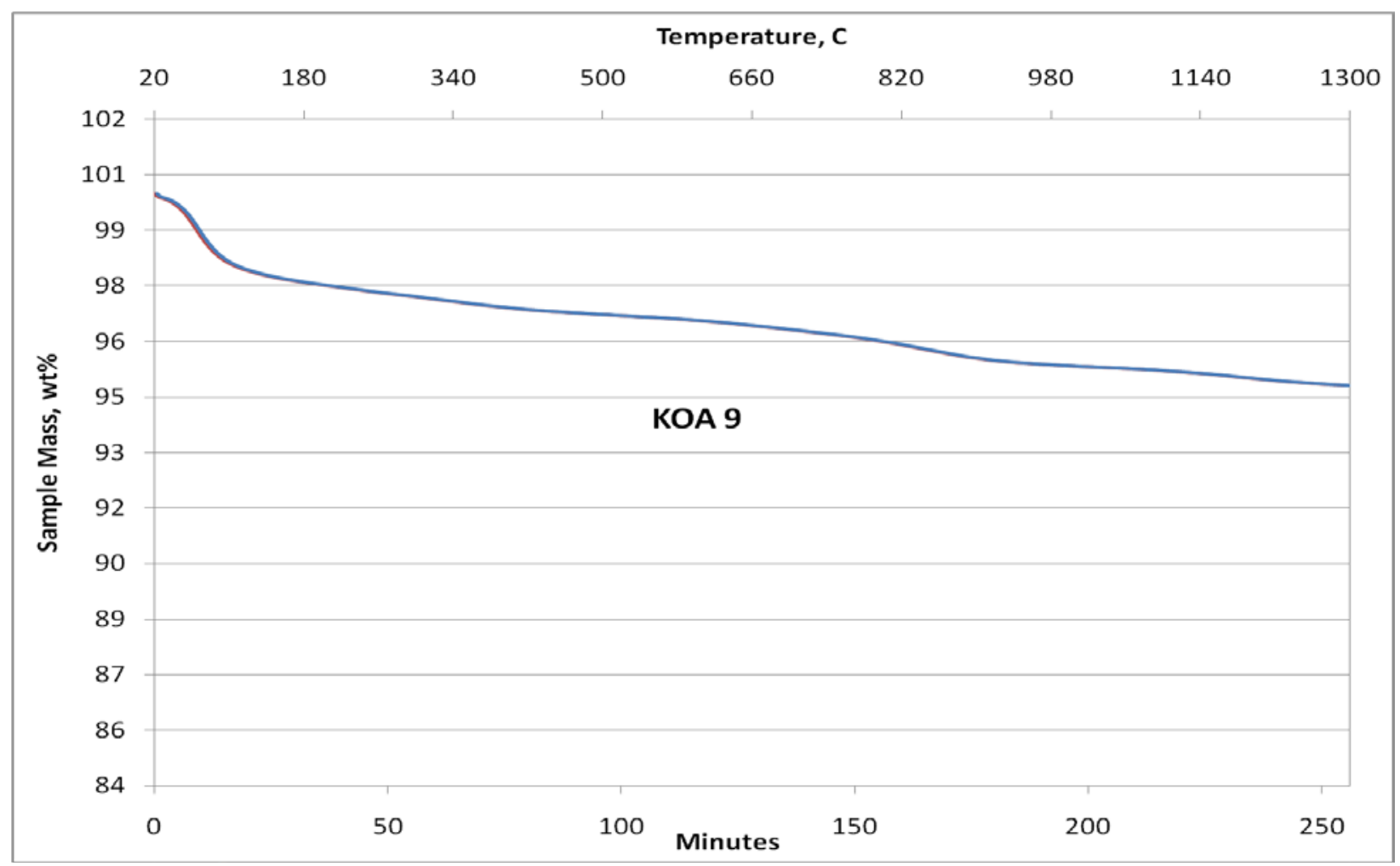

Figure C.18. The TG trace obtained from TPD testing of KOA 9, which is Cummins-Moore 5559 S.

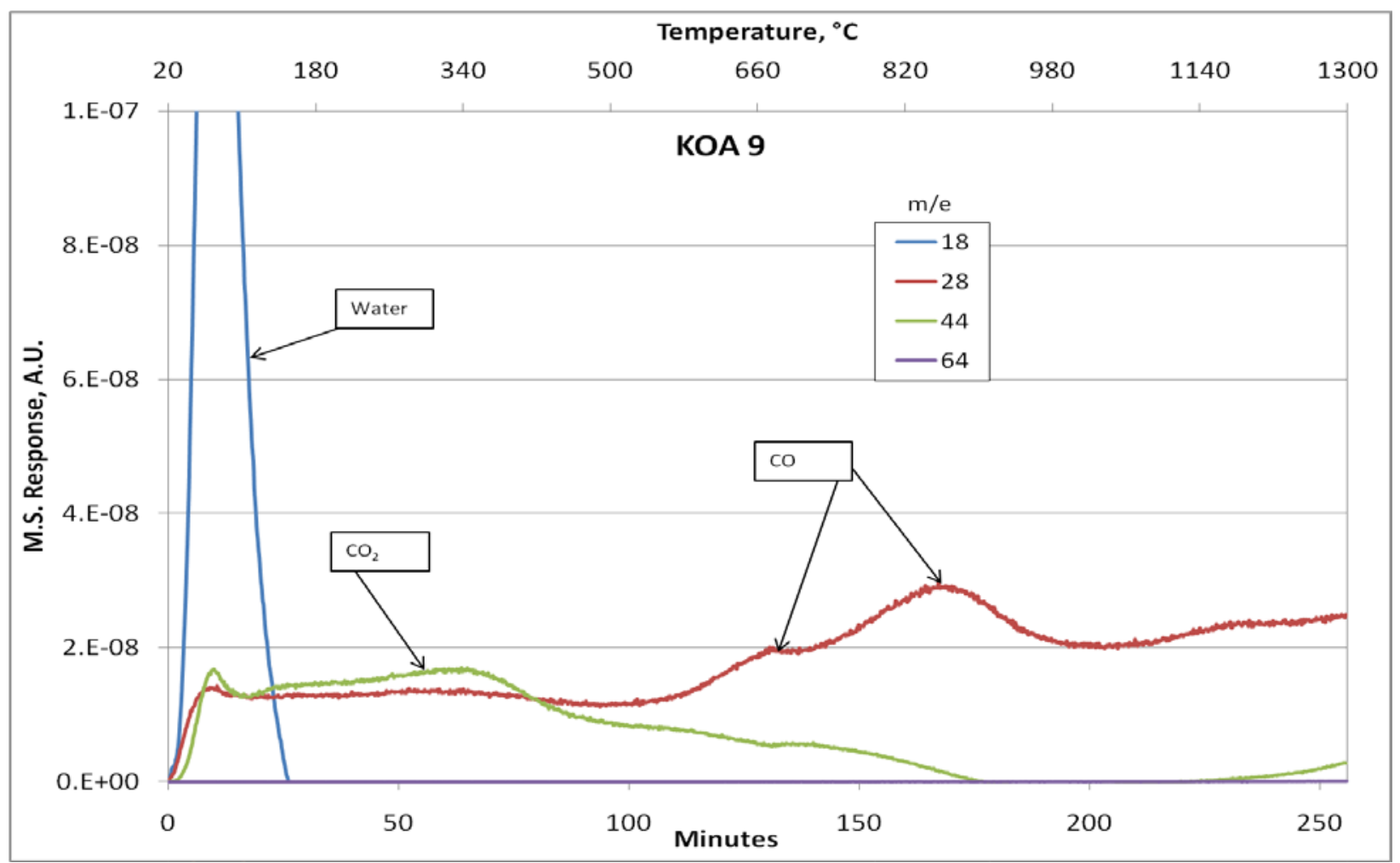

Figure C.19. The MS trace obtained TPD testing of KOA 9, which is Cummins-Moore 5559 S. 


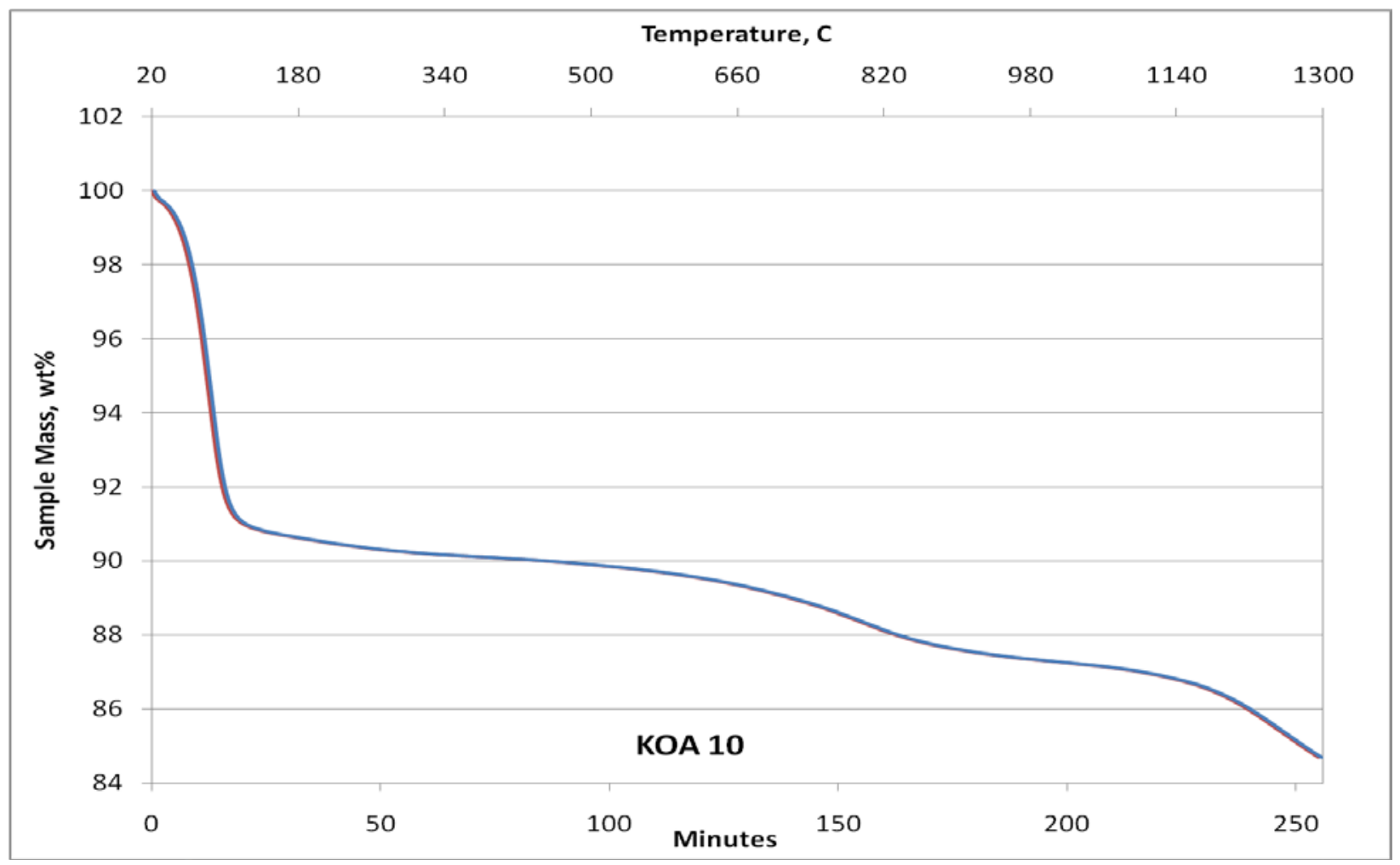

Figure C.20. The TG trace obtained from TPD testing of KOA 10, which is Cummins-Moore 5586 AW.

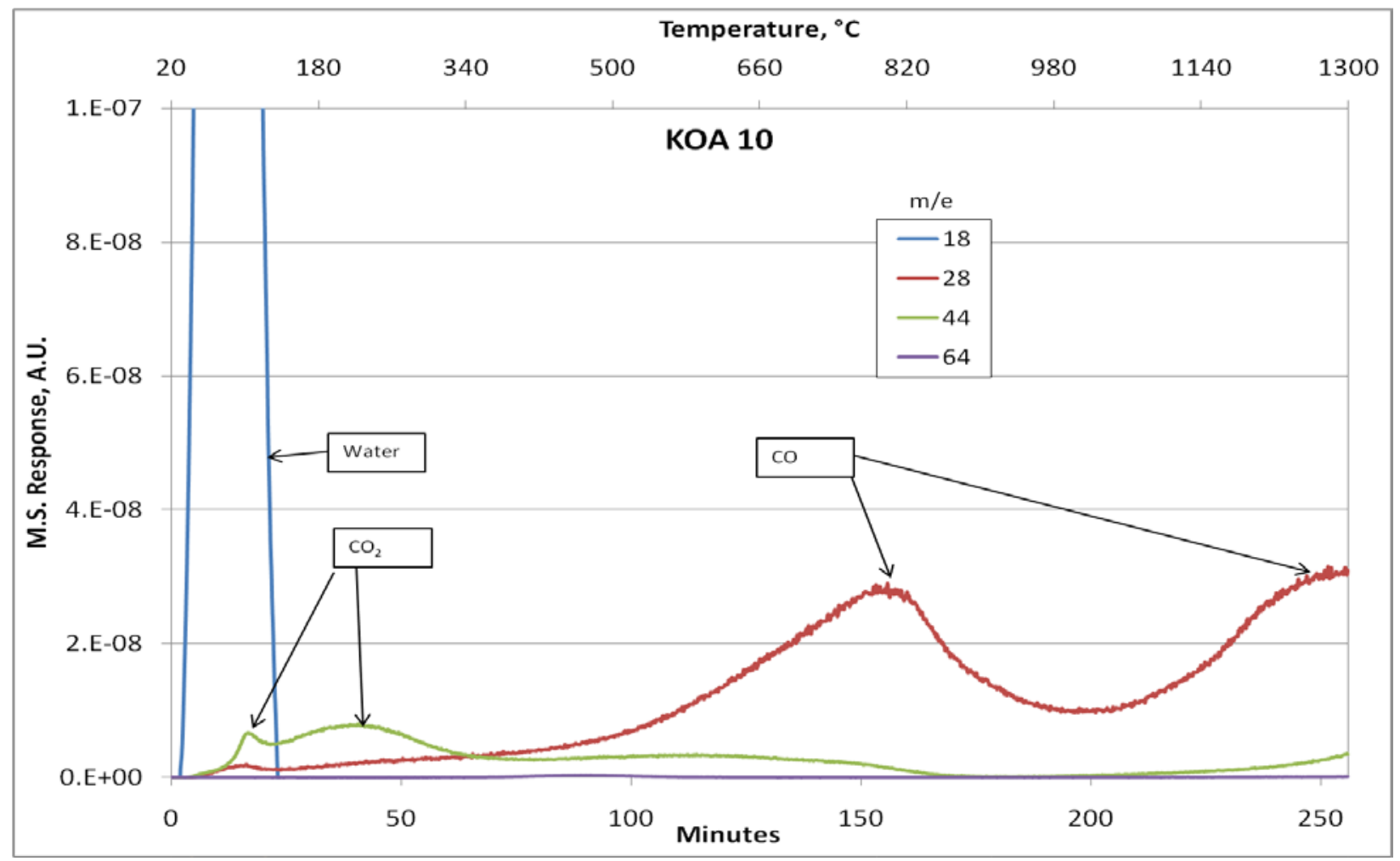

Figure C.21. The MS trace obtained TPD testing of KOA 10, which is Cummins-Moore 5586 AW. 


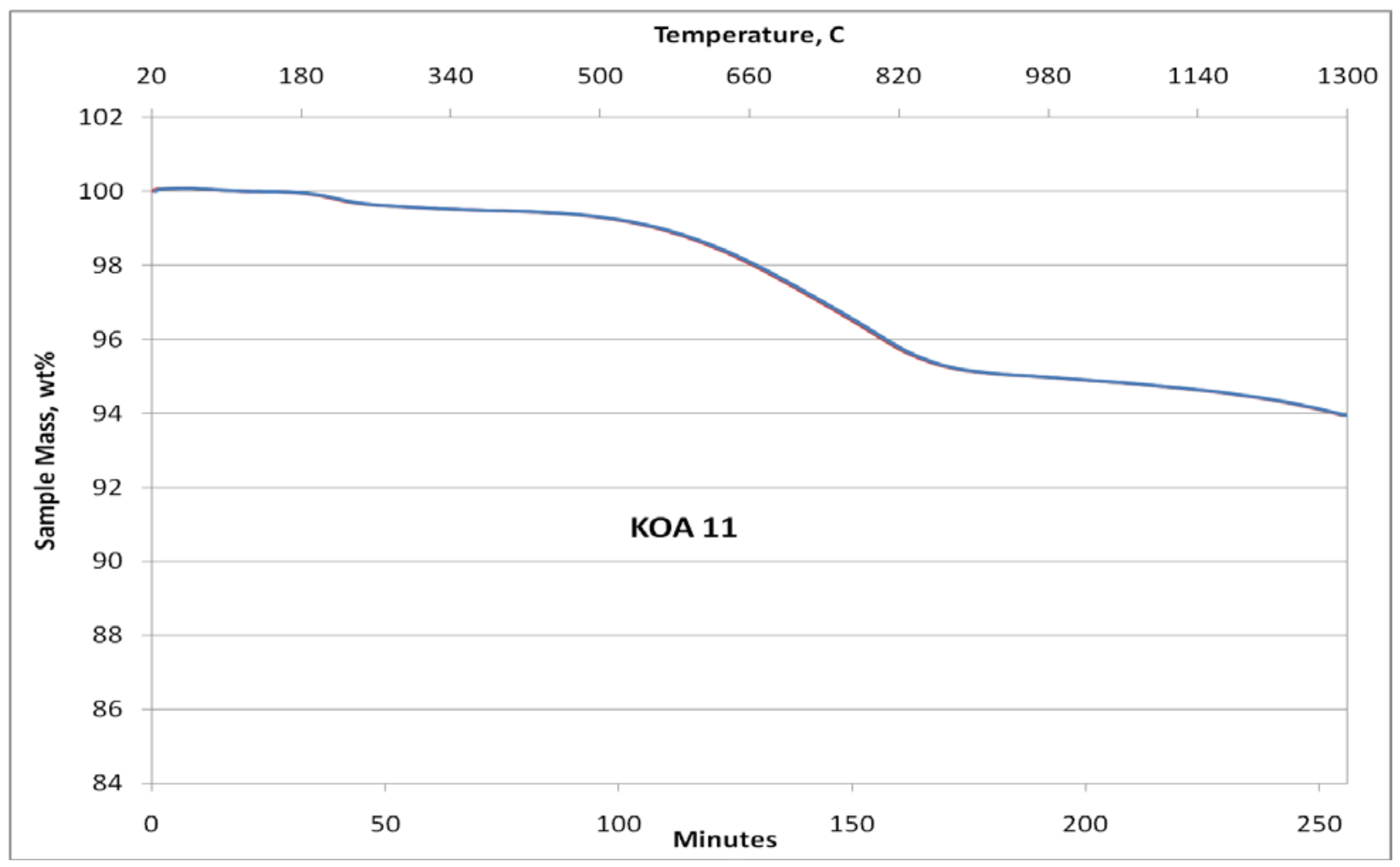

Figure C.22. The TG trace obtained from TPD testing of KOA 11, which is Norit Darco-LS HF-washed.

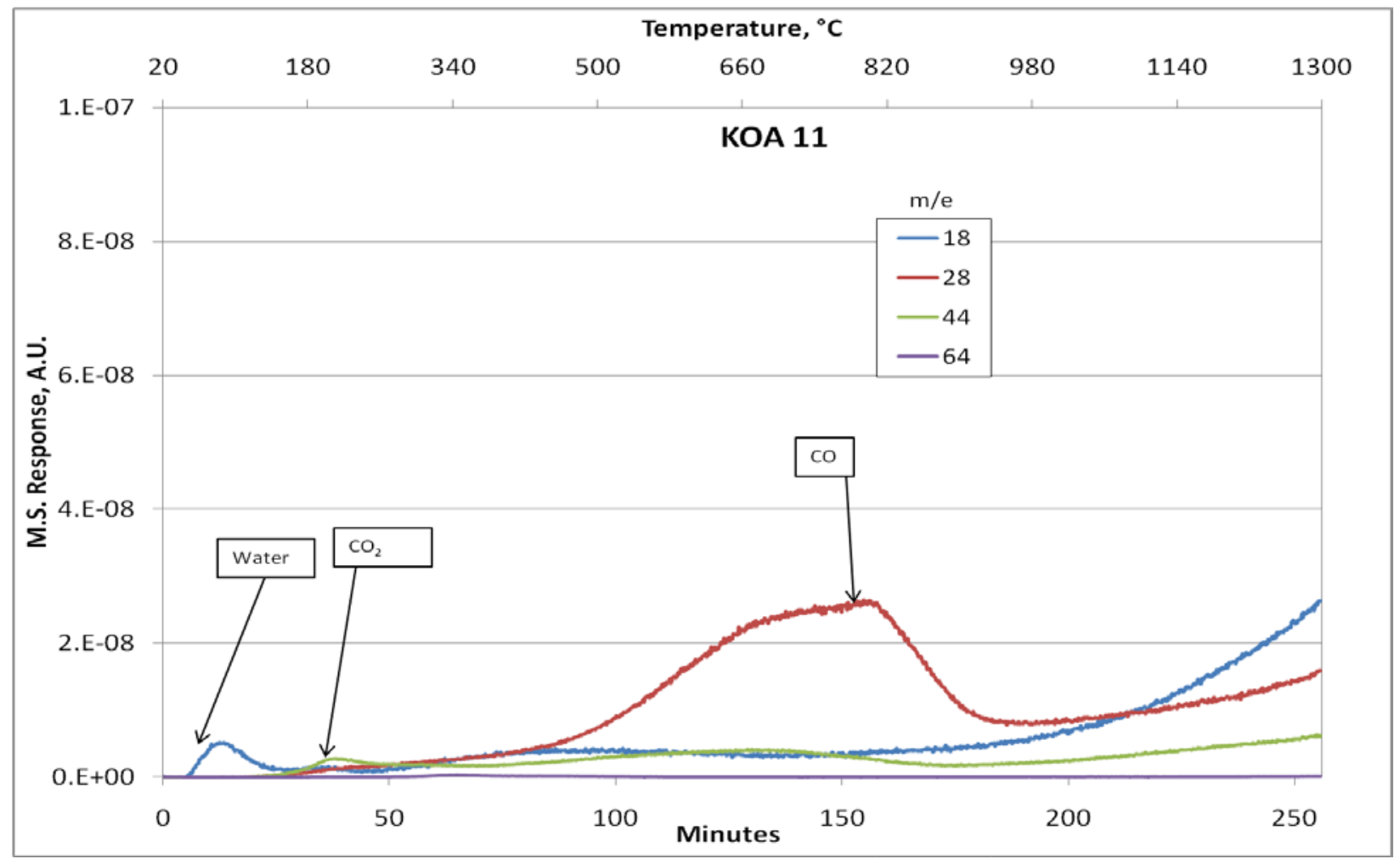

Figure C.23. The MS trace obtained TPD testing of KOA 11, which is Norit Darco-LS HF-washed. 


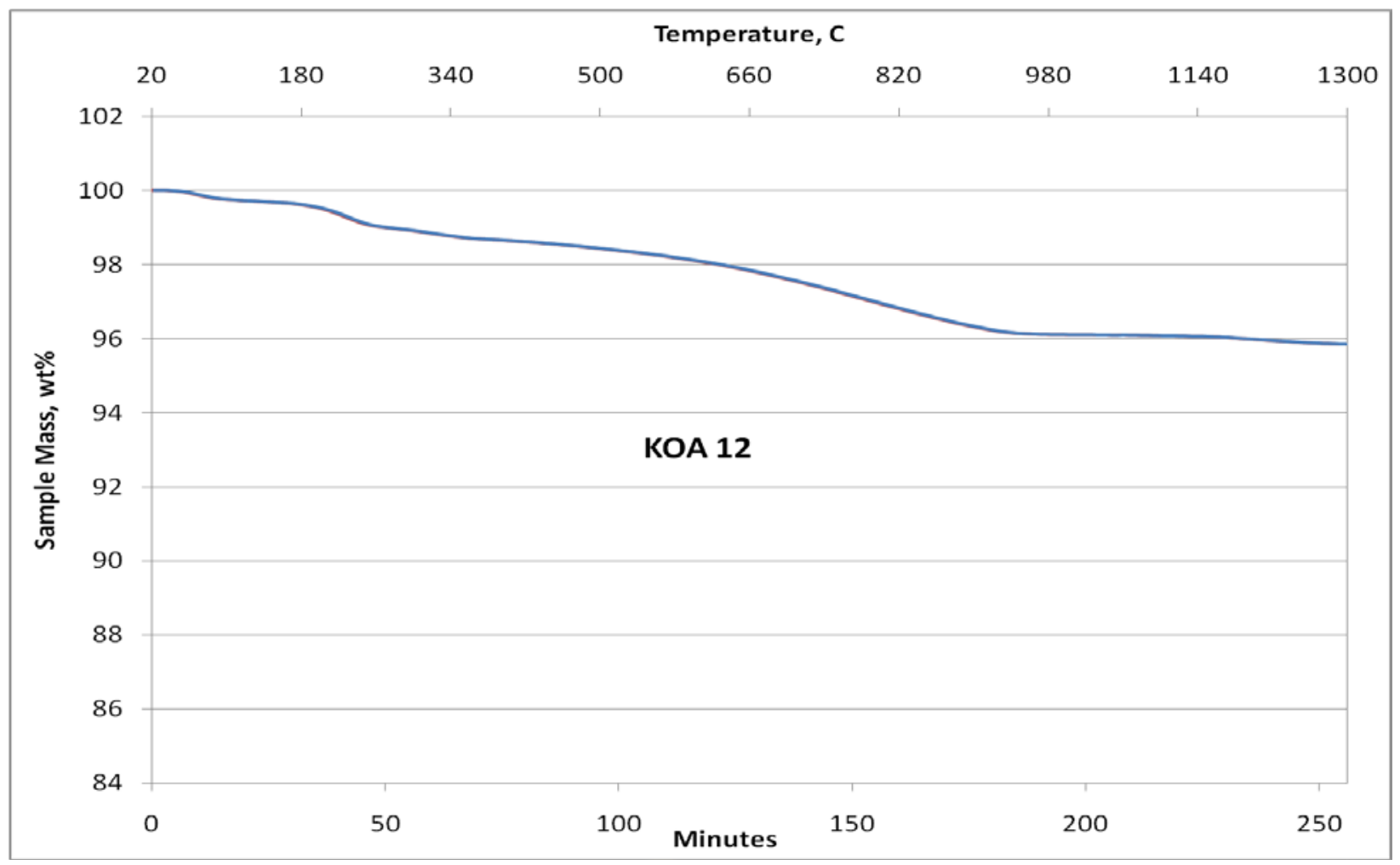

Figure C.24. The TG trace obtained from TPD testing of KOA 12, which is Norit ROX 0.8 HF-washed.

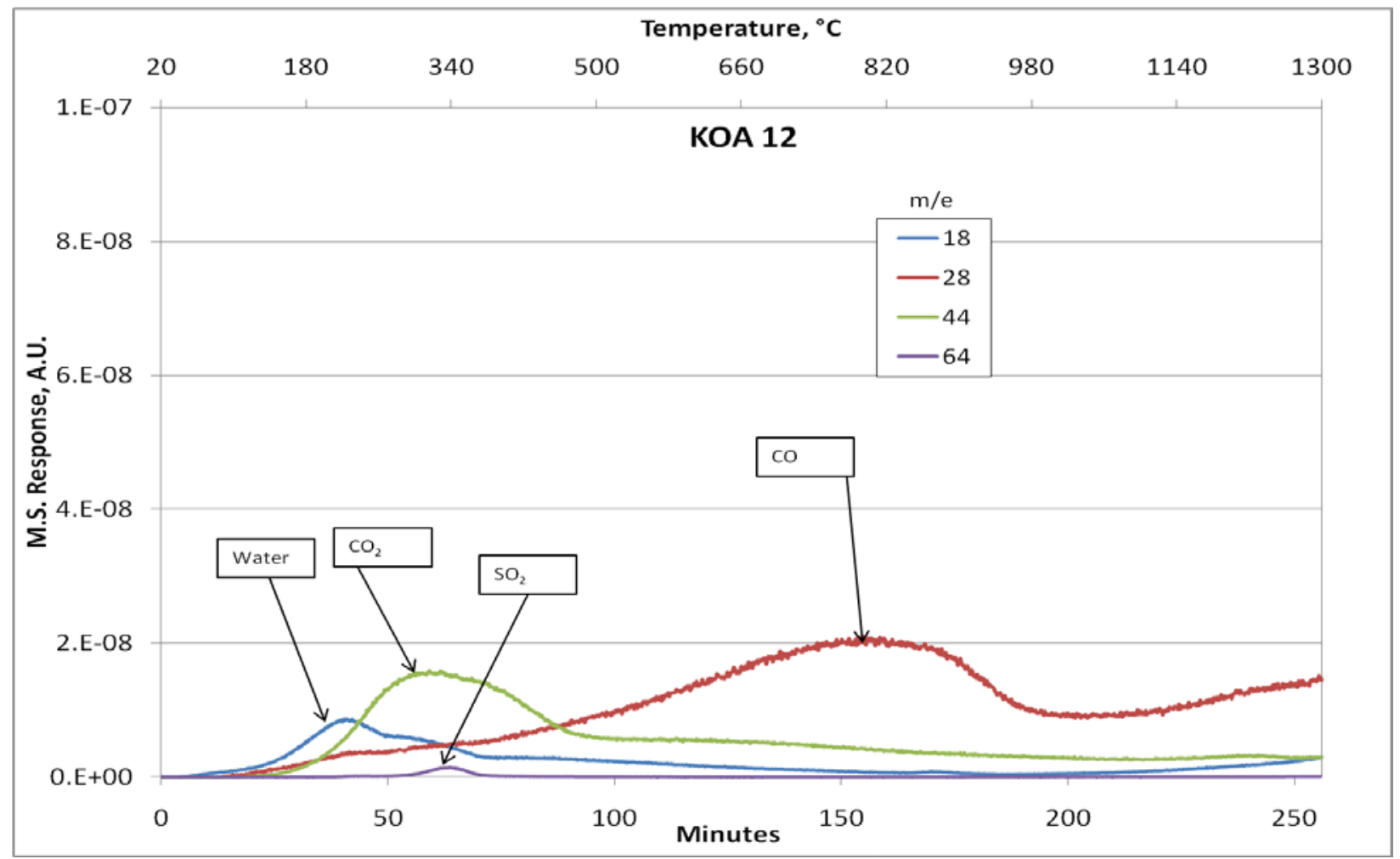

Figure C.25. The MS trace obtained TPD testing of KOA 12, which is Norit ROX 0.8 HF-washed. 

Appendix D

X-Ray Photoelectron Spectroscopy (XPS) Spectra of the Metal Impregnated Catalysts 



\section{Appendix D}

\section{X-Ray Photoelectron Spectroscopy (XPS) Spectra of the Metal Impregnated Catalysts}

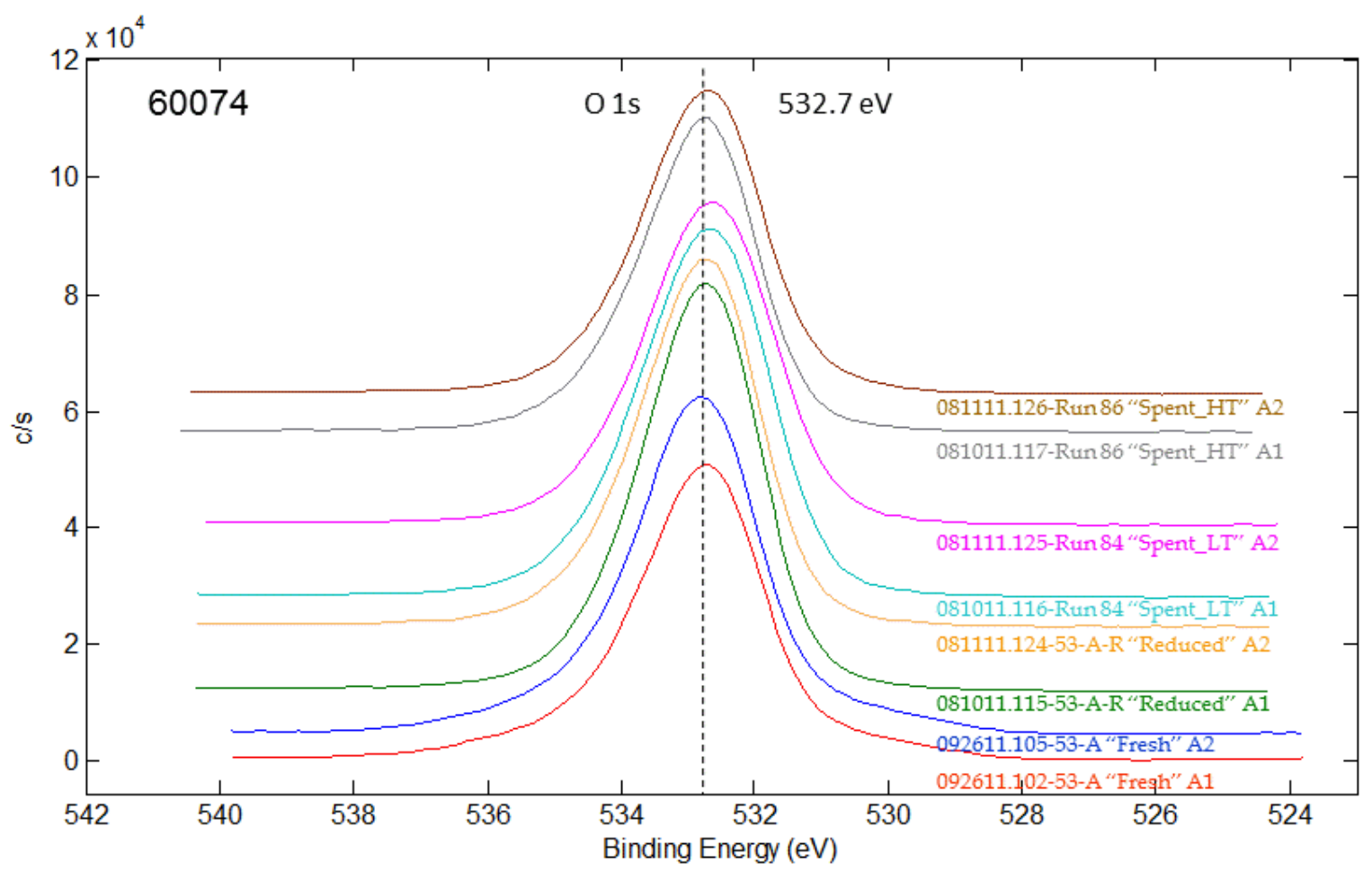

Figure D.1. High energy resolution XPS spectra of the O 1s region of the 1x Rh-Mn-Ir on Davisil 645 Silica baseline catalyst. The spectra are charge referenced using the Si $2 p$ line at $103.5 \mathrm{eV}$. 


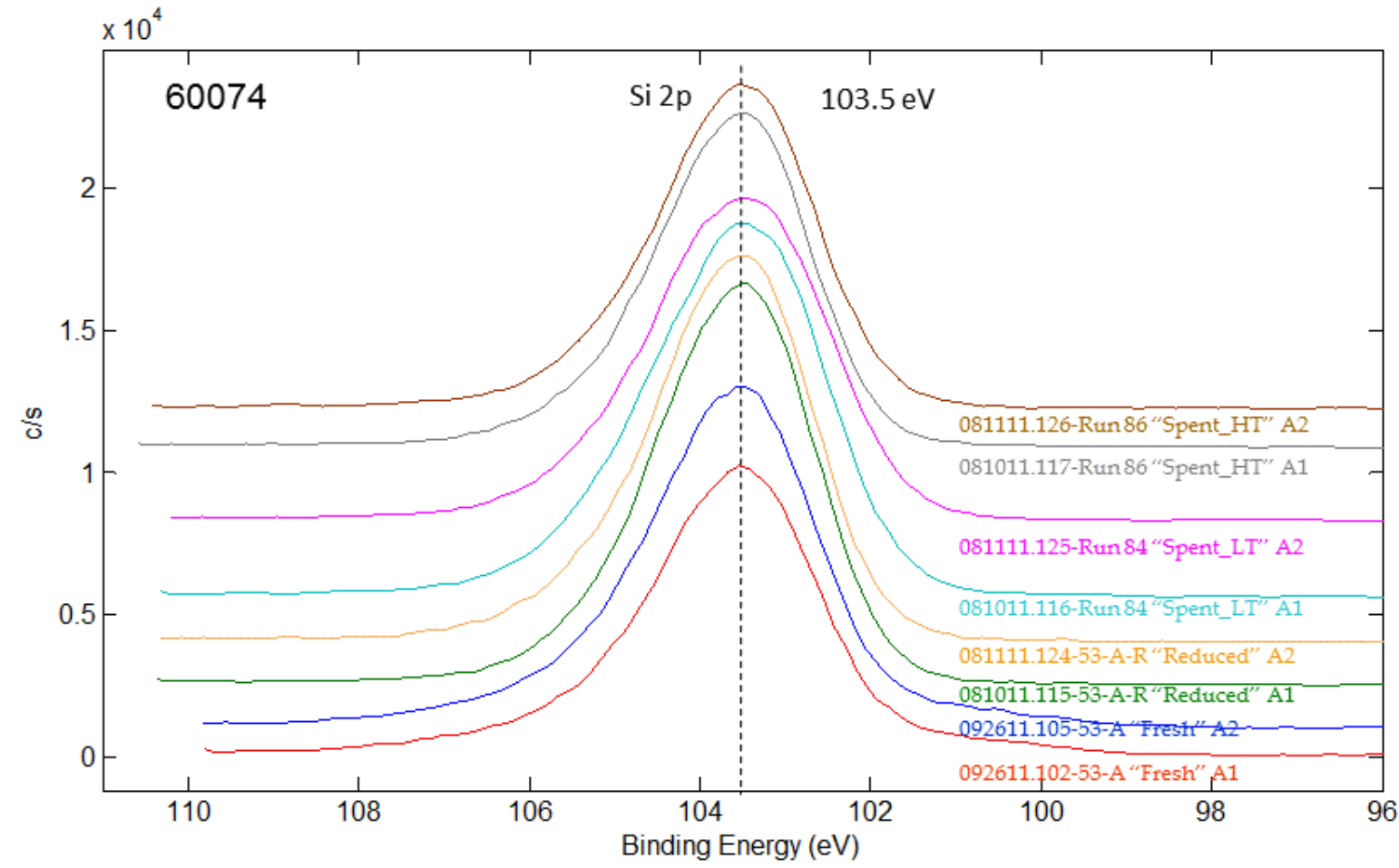

Figure D.2. High energy resolution XPS spectra of the Si 2p region of the 1x Rh-Mn-Ir on Davisil 645 Silica baseline catalyst. The spectra are charge referenced using the Si $2 \mathrm{p}$ line at $103.5 \mathrm{eV}$.

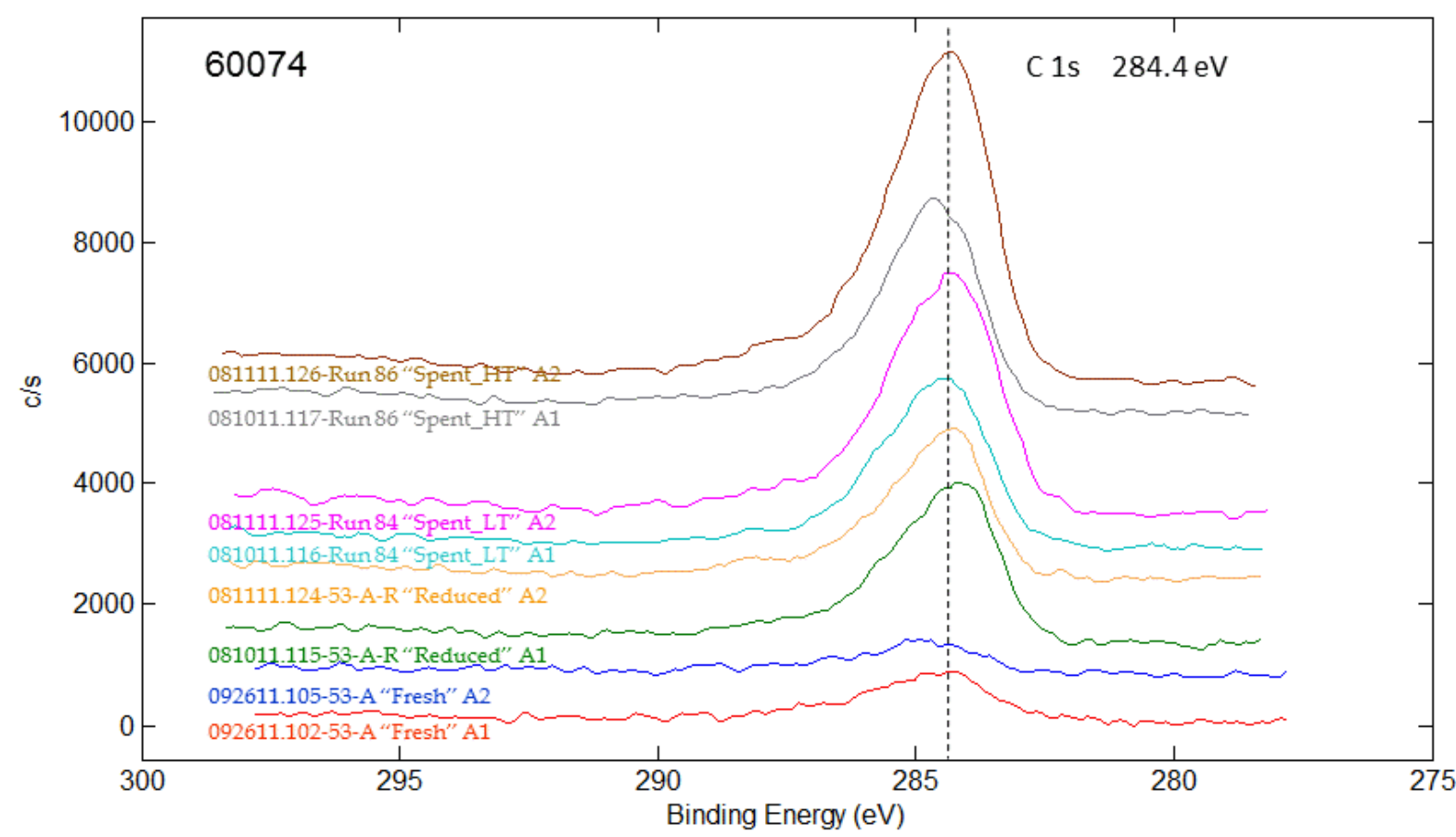

Figure D.3. High energy resolution XPS spectra of the C 1s region of the 1x Rh-Mn-Ir on Davisil 645 Silica baseline catalyst. The spectra are charge referenced using the Si 2p line at $103.5 \mathrm{eV}$. 


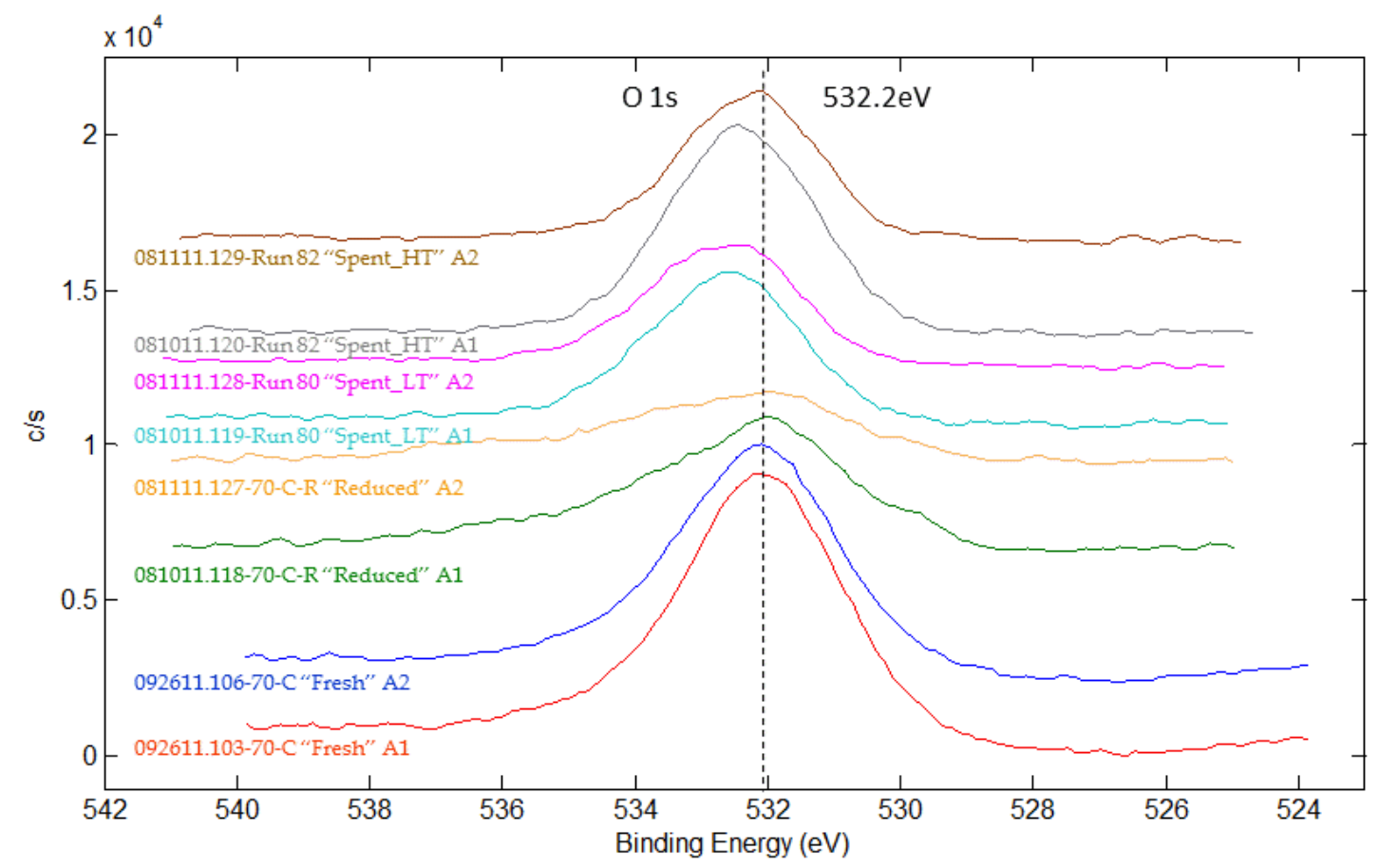

Figure D.4. High energy resolution (XPS) spectra of the $\mathrm{O} 1$ s region of the $2.11 \mathrm{x}$ Rh-Mn on Hyperion 384-82 MWCNT baseline catalyst. The spectra are charge referenced using the $\mathrm{C} 1 \mathrm{~s}$ line at $284.4 \mathrm{eV}$.

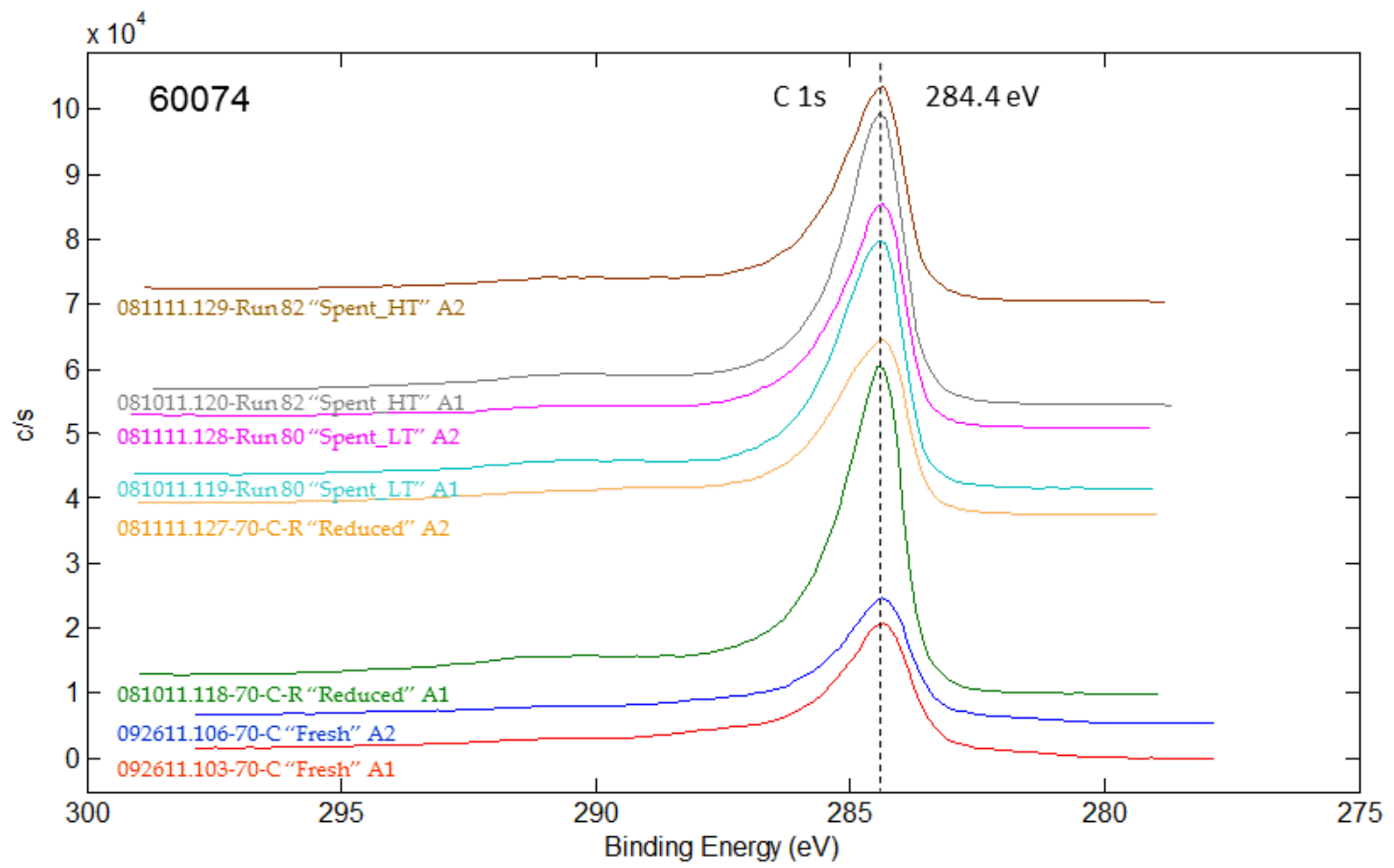

Figure D.5. High energy resolution (XPS) spectra of the C 1s region of the 2.11x Rh-Mn on Hyperion 384-82 MWCNT baseline catalyst. The spectra are charge referenced using the $\mathrm{C} 1 \mathrm{~s}$ line at $284.4 \mathrm{eV}$. 


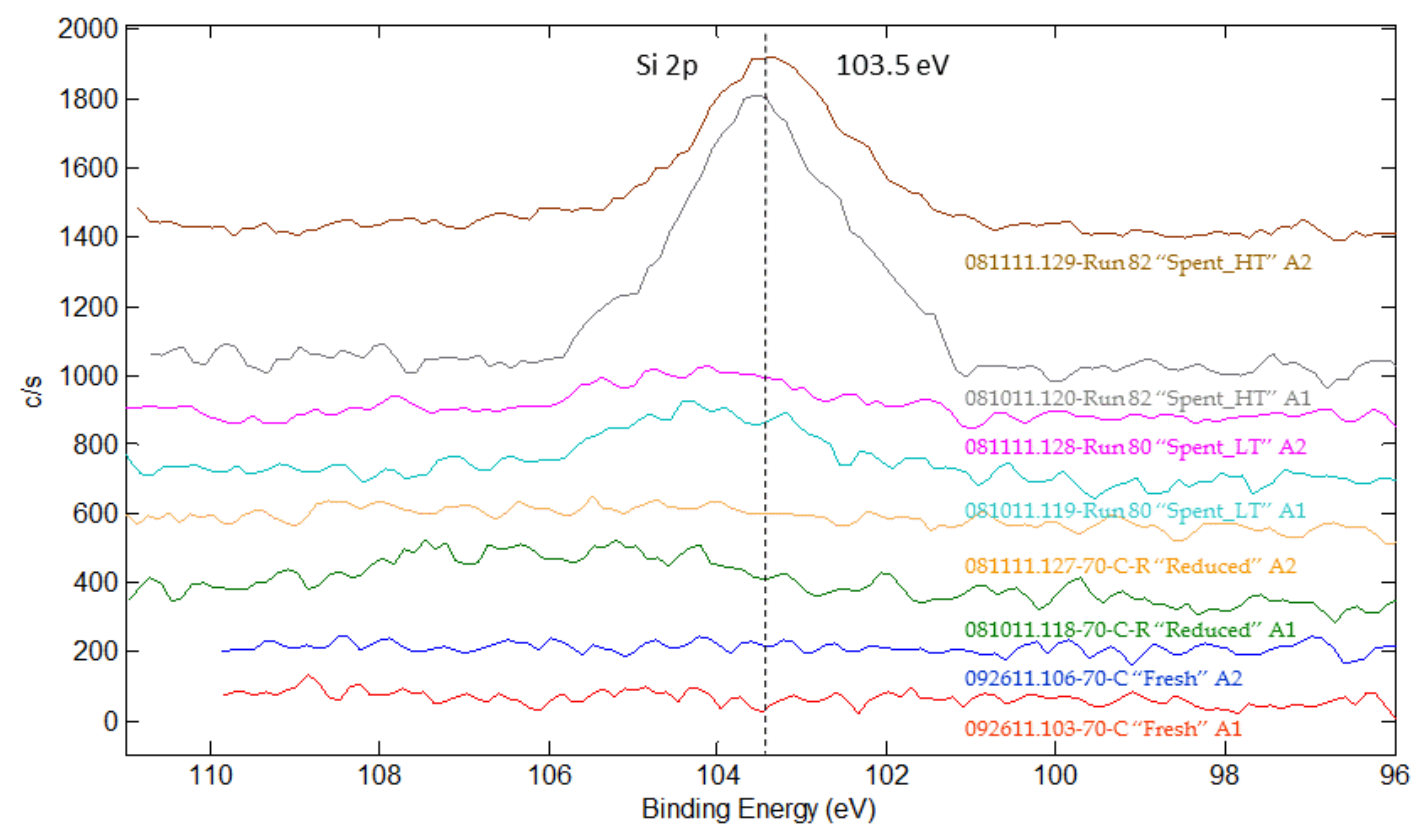

Figure D.6. High energy resolution (XPS) spectra of the Si 2p region of the 2.11x Rh-Mn on Hyperion 384-82 MWCNT baseline catalyst. The spectra are charge referenced using the $\mathrm{C}$ 1s line at $284.4 \mathrm{eV}$.

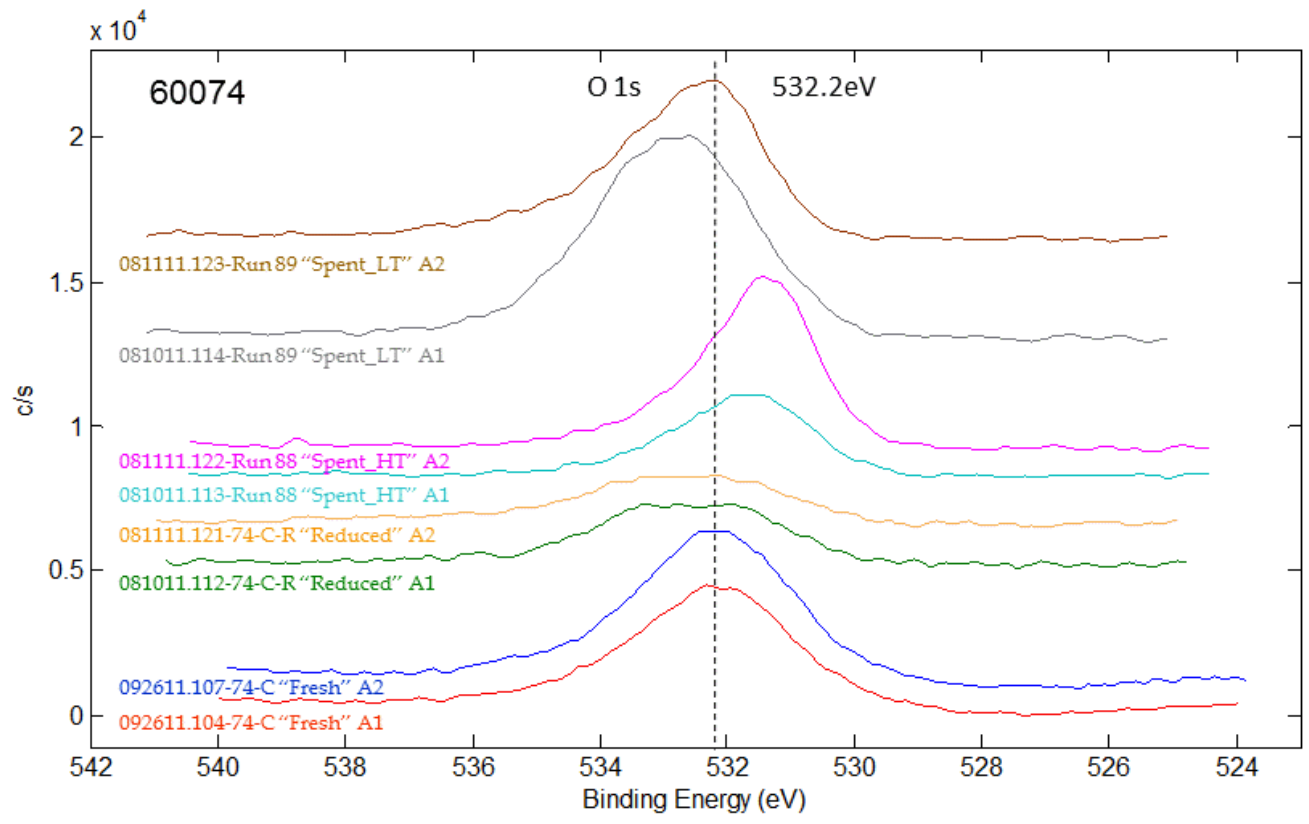

Figure D.7. High energy resolution (XPS) spectra of the O 1s region of the 2.11x Rh-Mn-Ir on Hyperion 384-82 MWCNT baseline catalyst. The spectra are charge referenced using the $\mathrm{C} 1 \mathrm{~s}$ line at $284.4 \mathrm{eV}$. 


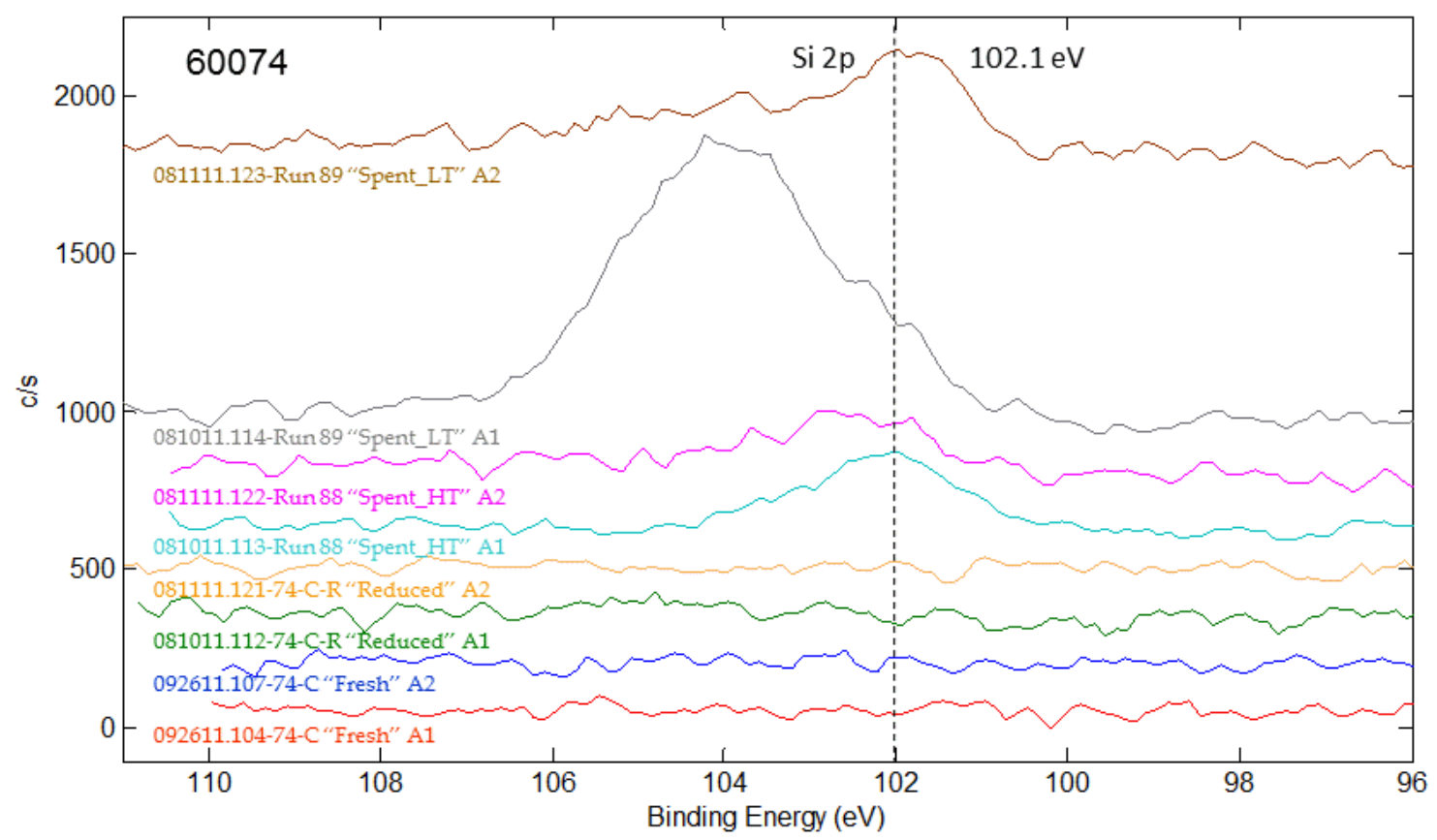

Figure D.8. High energy resolution (XPS) spectra of the Si 2p region of the 2.11x Rh-Mn-Ir on Hyperion 384-82 MWCNT baseline catalyst. The spectra are charge referenced using the $\mathrm{C} 1 \mathrm{~s}$ line at $284.4 \mathrm{eV}$.

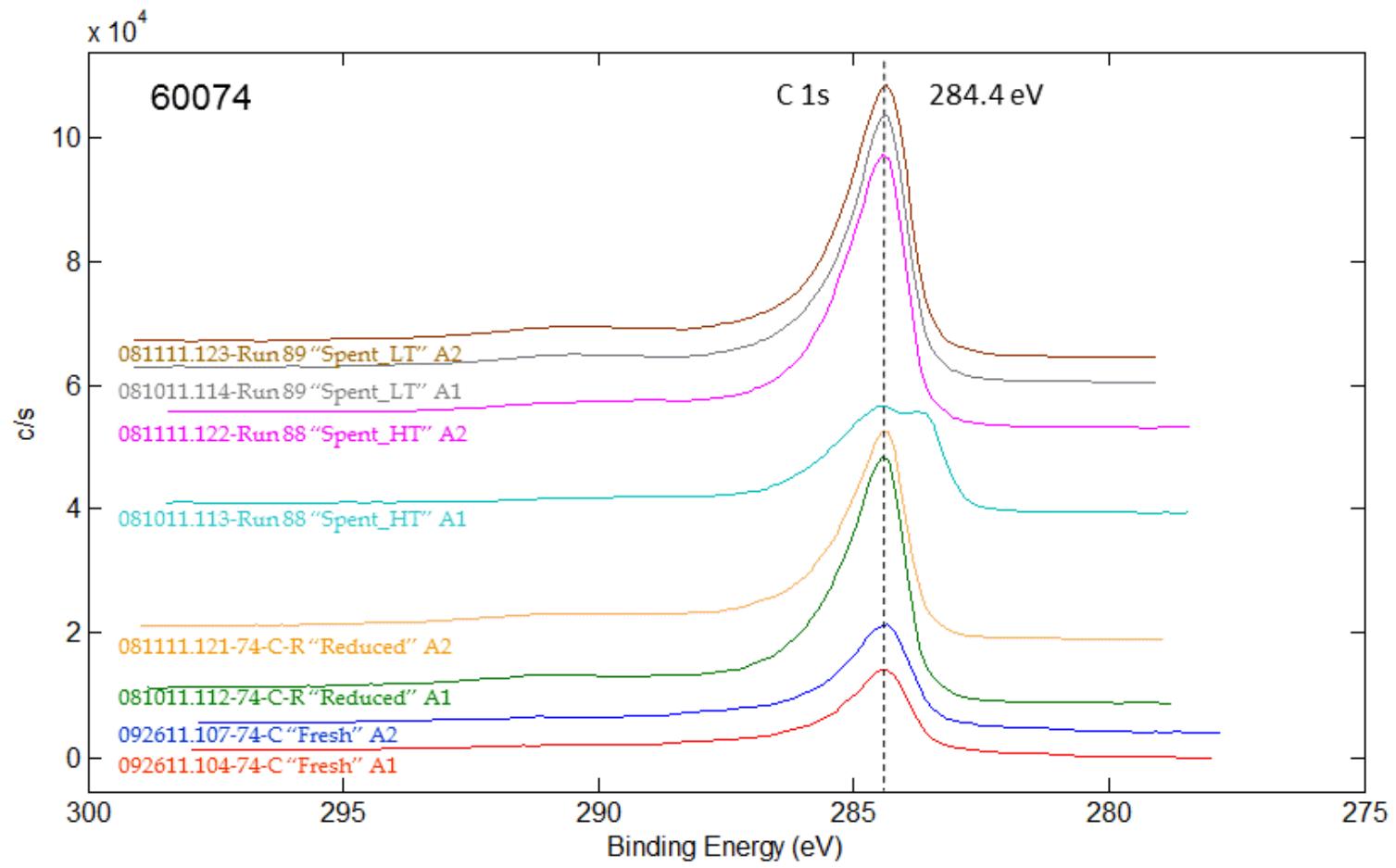

Figure D.9. High energy resolution (XPS) spectra of the C 1s region of the 2.11x Rh-Mn-Ir on Hyperion 384-82 MWCNT baseline catalyst. The spectra are charge referenced using the $\mathrm{C} 1 \mathrm{~s}$ line at $284.4 \mathrm{eV}$. 


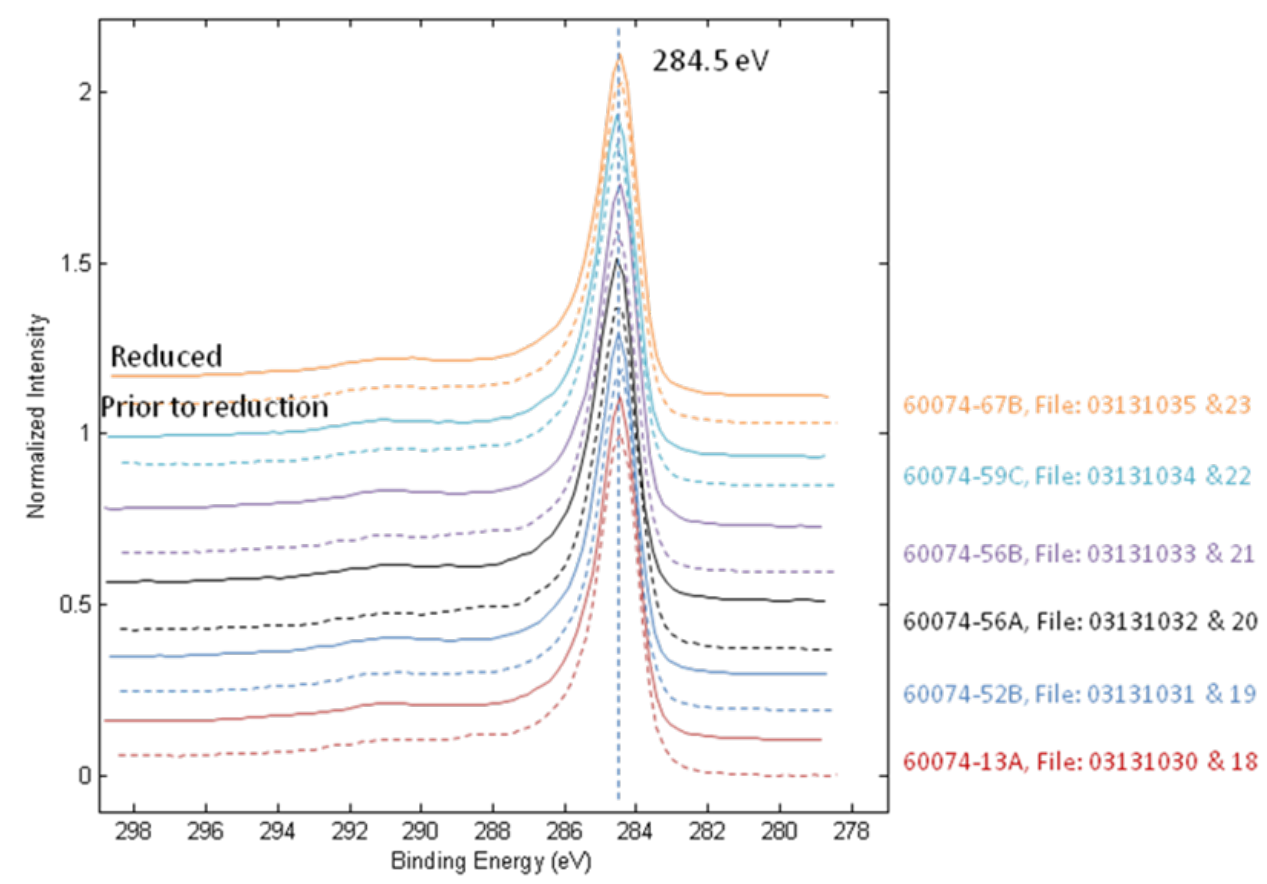

Charge referenced using the $\mathrm{C} 1$ s line at $284.5 \mathrm{eV}$ Reference: D. Kazachkin, et. al., "High resolution XPS characterization of chemical functionalized MWCNTs and SWCNTs "Carbon, V43, 2005 P 153.

Figure D.10. The C 1s electron region of the 6 fresh (unreduced - dashed lines) and reduced (solid lines) metal impregnated catalysts in Table 2.6.

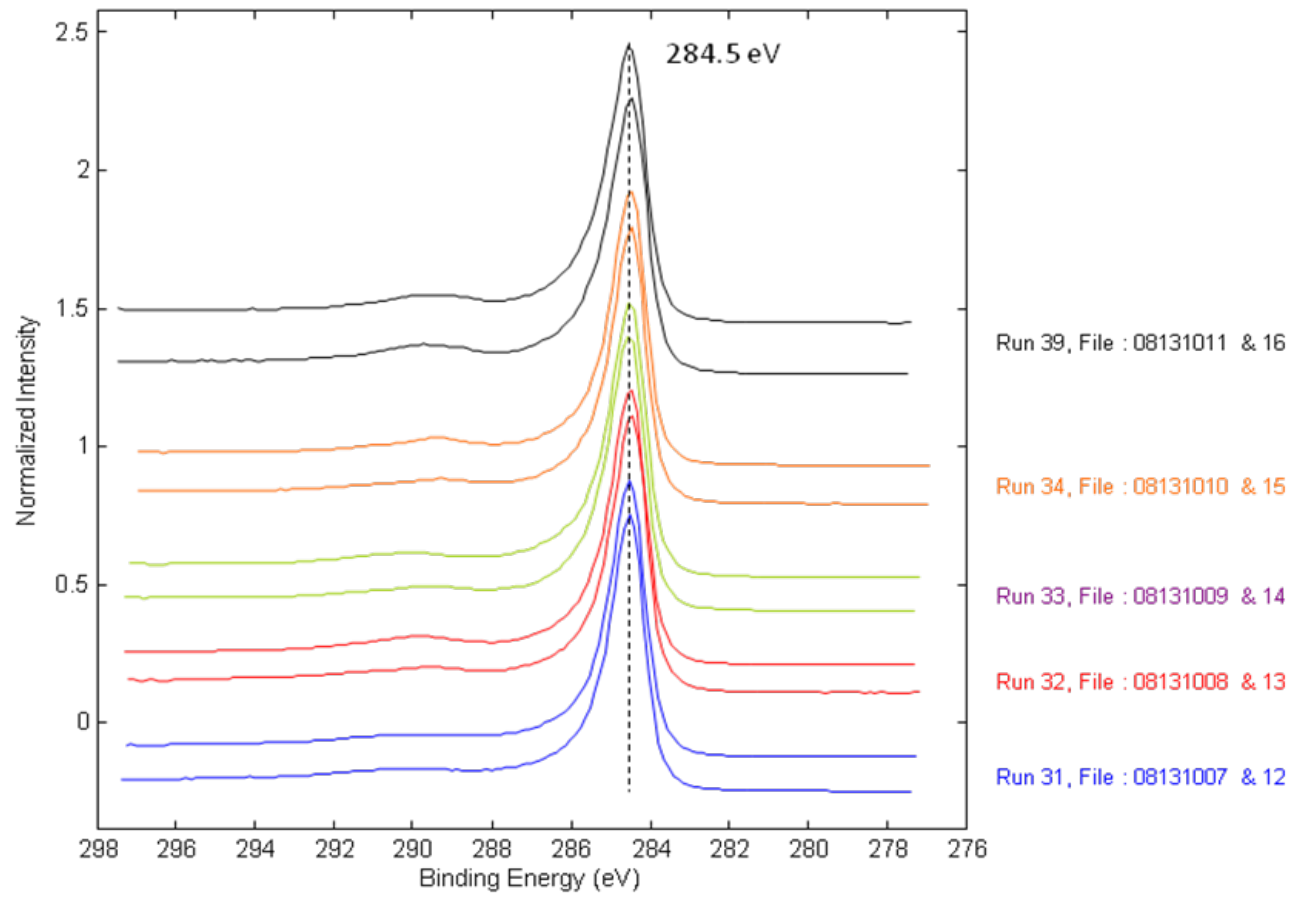

Charge referenced using the $\mathrm{C} 1 \mathrm{~s}$ line at $284.5 \mathrm{eV}$ Reference: D. Kazachkin , et. Al., "High resolution XPS characterization of chemical functionalised MWCNTs and SWCNTs" Carbon. V43. 2005 P 153

Figure D.11. The C 1s electron region of the 5 spent catalysts in Table 2.6. 


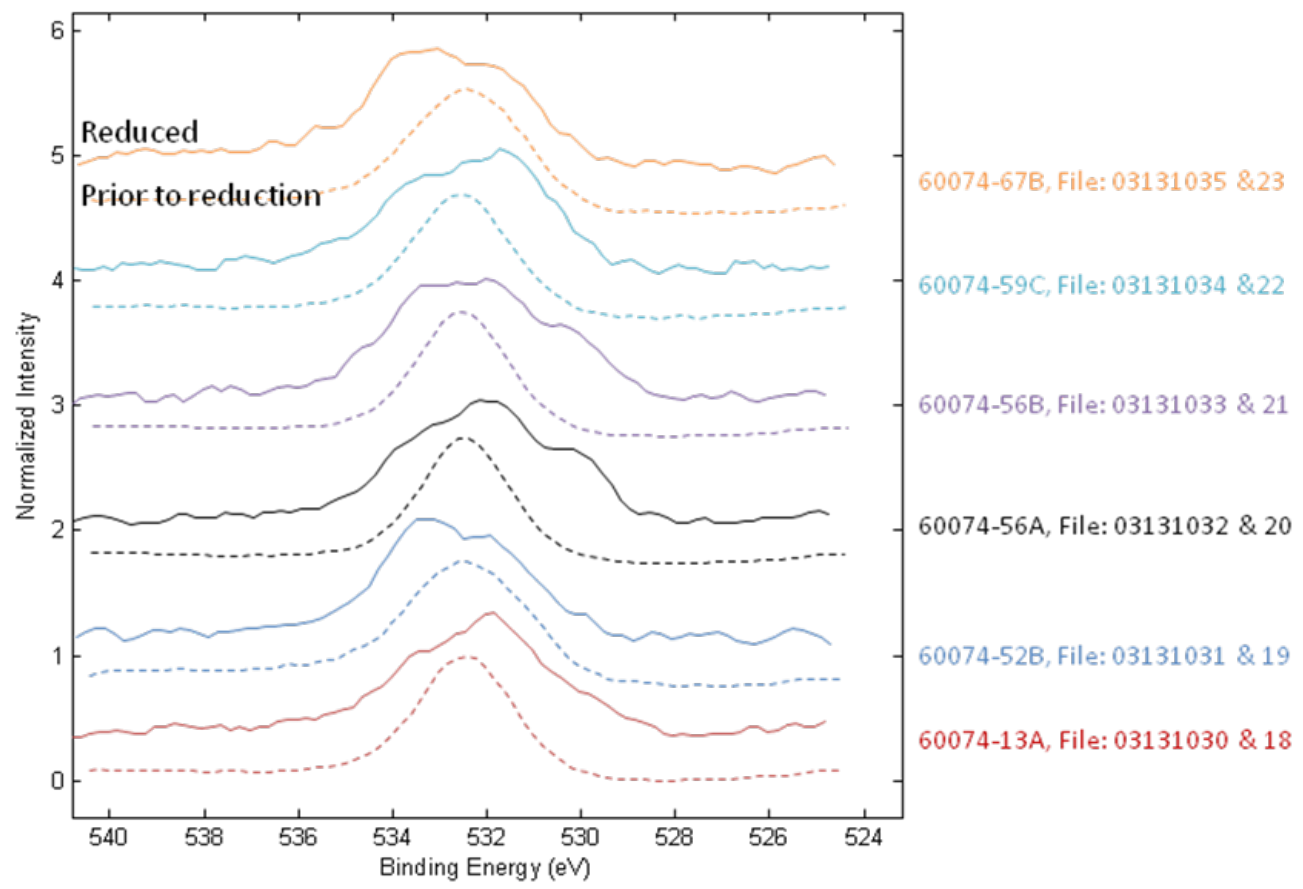

Charge referenced using the $\mathrm{C} 1 \mathrm{~s}$ line at $284.5 \mathrm{eV}$ Reference: D. Kazachkin, et. al., "High resolution XPS characterization of chemical functionalized MWCNTs and SWCNTs"Carbon, V43, 2005 P 153.

Figure D.12. The O 1s electron region of the 6 fresh (unreduced - dashed lines) and reduced (solid lines) metal impregnated catalysts in Table 2.6.

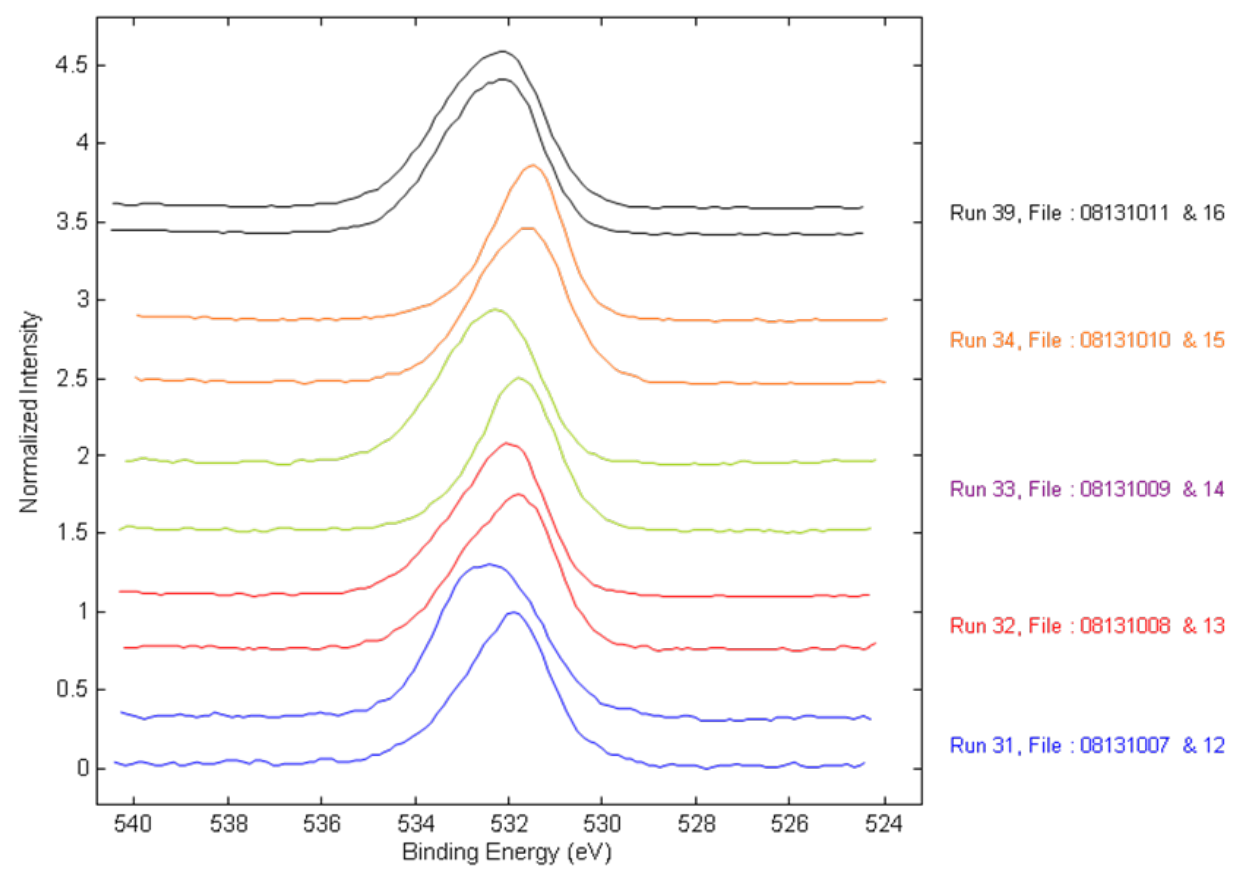

Charge referenced using the C 1 s line at $284.5 \mathrm{eV}$ Reference : D. Kazachkin ,et. Al., "High resolution XPS characterization of chemical functionalised MWCNTs and SWCNTS " Carbon. V43. 2005P 153

Figure D.13. The O 1s electron region of the 5 spent catalysts in Table 2.6. 



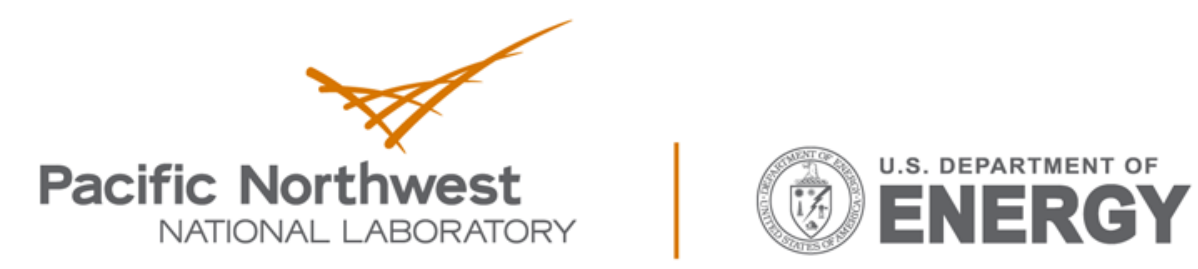

Proudly Operated by Battelle Since 1965

902 Battelle Boulevard

P.O. Box 999

Richland, WA 99352

1-888-375-PNNL (7665)

www.pnnl.gov 